Schimek, Bernhard [Hrsg.]; Kremsner, Gertraud [Hrsg.]; Proyer, Michelle [Hrsg.]; Grubich, Rainer [Hrsg.]; Paudel, Florentine [Hrsg.]; Grubich-Müller, Regina [Hrsg.]

\title{
Grenzen.Gänge.Zwischen.Welten. Kontroversen - Entwicklungen - Perspektiven der Inklusionsforschung
}

Bad Heilbrunn : Verlag Julius Klinkhardt 2022, 295 S.

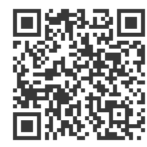

Quellenangabe/ Reference:

Schimek, Bernhard [Hrsg.]; Kremsner, Gertraud [Hrsg.]; Proyer, Michelle [Hrsg.]; Grubich, Rainer [Hrsg.]; Paudel, Florentine [Hrsg.]; Grubich-Müller, Regina [Hrsg.]: Grenzen.Gänge.Zwischen.Welten.

Kontroversen - Entwicklungen - Perspektiven der Inklusionsforschung. Bad Heilbrunn : Verlag Julius Klinkhardt 2022, 295 S. - URN: urn:nbn:de:0111-pedocs-238116 - DOI: 10.25656/01:23811

https://nbn-resolving.org/urn:nbn:de:0111-pedocs-238116

https://doi.org/10.25656/01:23811

in Kooperation mit / in cooperation with:

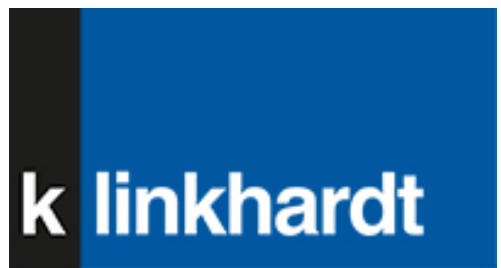

http://www.klinkhardt.de

\section{Nutzungsbedingungen}

Dieses Dokument steht unter folgender Creative Commons-Lizenz: http://creativecommons.org/licenses/by-nc-sa/4.0/deed.de - Sie dürfen das Werk bzw. den Inhalt unter folgenden Bedingungen vervielfältigen, verbreiten und öffentlich zugänglich machen sowie Abwandlungen und Bearbeitungen des Werkes bzw. Inhaltes anfertigen: Sie müssen den Namen des Autors/Rechteinhabers in der von ihm festgelegten Weise nennen. Dieses Werk bzw. der Inhalt darf nicht für kommerzielle Zwecke verwendet werden. Die neu entstandenen Werke bzw. Inhalte dürfen nur unter Verwendung von Lizenzbedingungen weitergegeben werden, die mit denen dieses Lizenzvertrages identisch oder vergleichbar sind.

Mit der Verwendung dieses Dokuments erkennen Sie die Nutzungsbedingungen an.

\section{Terms of use}

This document is published under following Creative Commons-License: http://creativecommons.org/licenses/by-nc-sa/4.0/deed.en - You may copy, distribute and transmit, adapt or exhibit the work in the public and alter, transform or change this work as long as you attribute the work in the manner specified by the author or licensor. You are not allowed to make commercial use of the work. If you alter, transform, or change this work in any way, you may distribute the resulting work only under this or a comparable license.

By using this particular document, you accept the above-stated conditions of use.

\section{Kontakt / Contact:}

\section{peDOCS}

DIPF | Leibniz-Institut für Bildungsforschung und Bildungsinformation Informationszentrum (IZ) Bildung

E-Mail: pedocs@dipf.de

Internet: www.pedocs.de

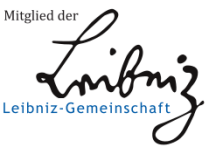




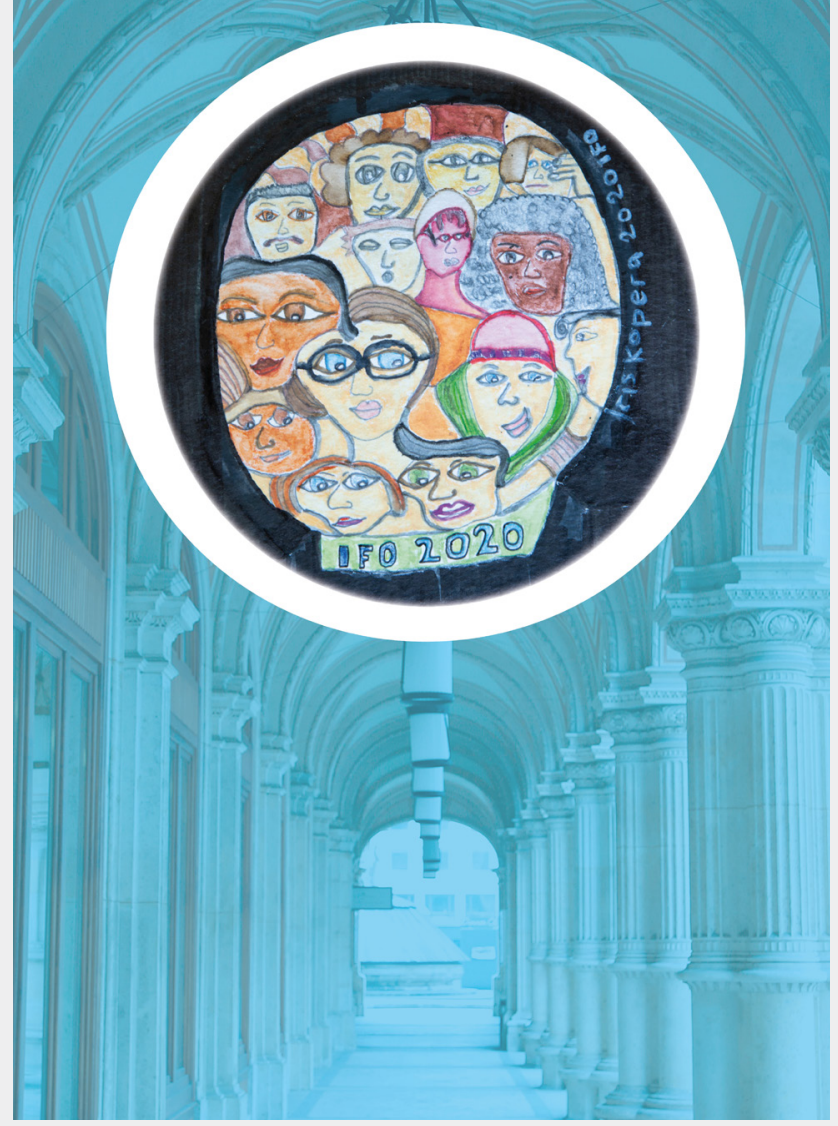

Bernhard Schimek / Gertraud Kremsner Michelle Proyer / Rainer Grubich Florentine Paudel / Regina Grubich-Müller (Hrsg.)

\section{Grenzen.Gänge. Zwischen.Welten.}

Kontroversen - Entwicklungen Perspektiven der Inklusionsforschung 
Schimek / Kremsner / Proyer / Grubich / Paudel / Grubich-Müller

Grenzen.Gänge. Zwischen. Welten. 



\section{Bernhard Schimek Gertraud Kremsner Michelle Proyer Rainer Grubich Florentine Paudel Regina Grubich-Müller (Hrsg.)}

\section{Grenzen.Gänge. Zwischen. Welten.}

\section{Kontroversen - Entwicklungen - Perspektiven der Inklusionsforschung}


Dieser Titel wurde in das Programm des Verlages mittels eines Peer-Review-Verfahrens aufgenommen. Für weitere Informationen siehe www.klinkhardt.de.

Bibliografische Information der Deutschen Nationalbibliothek Die Deutsche Nationalbibliothek verzeichnet diese Publikation in der Deutschen Nationalbibliografie; detaillierte bibliografische Daten sind im Internet abrufbar über http://dnb.d-nb.de.

2022.hg. () by Julius Klinkhardt.

Coverabbildung: Grafik: (C) Iris Kopera, Foto: kiyopayo/Adobe Stock.

Druck und Bindung: AZ Druck und Datentechnik, Kempten.

Printed in Germany 2022.

Gedruckt auf chlorfrei gebleichtem alterungsbeständigem Papier.

(c) (i)(3)(D) Die Publikation (mit Ausnahme aller Fotos, Grafiken und Abbildungen) ist veröffentlicht unter der Creative Commons-Lizenz: CC BY-NC-SA 4.0 International https://creativecommons.org/licenses/by-nc-sa/4.0/

ISBN 978-3-7815-5924-0 digital doi.org/10.35468/5924

ISBN 978-3-7815-2485-9 print 


\section{Inhalt}

Vorwort der Herausgeber*innen 9

Gertraud Kremsner, Bernhard Schimek und Michelle Proyer

Grenzen.Gänge.Zwischen.Welten.

Kontroversen - Entwicklungen - Perspektiven der Inklusionsforschung

\section{Kontroversen}

Georg Feuser

Die Bühne der Inklusion. Ein Prolog!

oder: Grenzgänge zwischen Welten

Mai-Anh Boger

Risse in der Landschaft der Inklusionsforschung -

Aktuelle Entwicklungen und offene Fragen

Sven Bärmig

Dialektik als Methode

Jan Jochmaring, Lena Bömelburg und Dirk Sponholz

Inklusive Berufsorientierung als Diskurs. Der ,scheinbare' Konsens:

gemeinsame Begriffe - unterschiedliche Ideen

Felix Kappeller

Prothetische Absenz, Articulating Gaps:

Repräsentationskritische Perspektiven auf visuelle Darstellungen

nicht an den Körper gefügter Prothesen

Jana York und Jan Jochmaring

Dilemmata einer inklusiven Arbeitswelt -

Menschen mit Behinderung zwischen Sondersystemen und

Gestaltungschancen einer Arbeitswelt 4.0

Juliane Gerland, Imke Niediek, Julia Hülsken und Marvin Sieger

Kontingenz von Differenzkonstruktionen in der inklusionsorientierten musikalischen Bildung am Beispiel des Umgangs

mit digitalen Musizier-Medien

Pierre-Carl Link

Zur Bedeutung einer befreiungspädagogischen Perspektive für die Inklusions- und Sonderpädagogik 
Katharina Hamisch und Robert Kruschel

Zwischen Individualisierungsversprechen und Vermessungsgefahr -

Die Rolle der Schlüsseltechnologie Künstliche Intelligenz in der inklusiven Schule

Felix Buchhaupt, Dieter Katzenbach, Deborah Lutz und Michael Urban

Zur Kontextualisierung der Inklusionsforschung

Lisa-Katharina Möhlen, Helena Deiß, Seyda Subasi Singh und Michelle Proyer

Lebenswelt(en der) Schule. Internationale Perspektiven zur Inklusion von

Schüler*innen mit Fluchterfahrungen. Eine Perspektive aus der Praxis

Thomas Hoffmann, Cathrin Reisenauer und Hendrik Richter

Helfen als individuelle Erfahrung und soziale Praxis zwischen

Deautonomisierung und Befähigung

\section{Entwicklungen}

Kathrin te Poel

Zur Bedeutsamkeit eigener schüler*innenbiografischer Erfahrungen von angehenden Lehrpersonen für den sich anbahnenden Lehrer*innenhabitus und seine Anerkennungsbezüge

Alina Quante und Oliver Danner

Grenzsetzungen bei Aufgabenbereichen von sonderpädagogischen und

allgemeinen Lehrkräften in inklusiven Settings

Edvina Bešić und Katharina Maitz

Das Boot: Eine Fluchtgeschichte -

Design-Based-Research in der Primarstufe

\section{Brigitte Kottmann}

Der Übergang von der Grundschule zur weiterführenden Schule als

(Soll-)Bruchstelle des Gemeinsamen Lernens

Anne Goldbach und Nico Leonhardt

Elemente von Macht im Kontext einer inklusionssensiblen

Hochschulentwicklung

Michaela Kaiser

Inklusionsbezogene Anforderungswahrnehmung -

Regulativ für (kunstpädagogische) Professionalisierung

Patrick Gollub, Silvia Greiten, Teresa Schkade und Marcel Veber

Schulpraktische Professionalisierung für den Umgang mit Heterogenität ein interdisziplinärer Blick aus hochschuldidaktischen Projekten 
Pierre-Carl Link, Cedric Steinert und Susanne Jurkowski

Implementierung von Inklusion als Querschnittsthema an der

Universität Erfurt durch das Kompetenz- und Entwicklungszentrum

für Inklusion. Inklusionsspezifische Professionalisierung der

Lehrer*innenbildung durch Team-Teaching, Fortbildung und

Online-Lernumgebung

\section{Perspektiven}

Timm Albers, Agnes Filipiak, Katja Franzen und Frank Hellmich

Kompetenzentwicklung im inklusiven Unterricht (KinU) -

eine internationale Perspektive

Katharina Maria Pongratz

Sehnsucht nach Bildung? Über den Einsatz systemischer Fragetechniken in der qualitativen Erwachsenenbildungs-/Weiterbildungsforschung bei Biografieträger*innen mit einer zugeschriebenen geistigen Behinderung

Laura Schwörer, Hannah van Ledden, Pia Algermissen und Mandy Hauser

Zusammenarbeit und Mediennutzung in einer

Partizipativen Forschungsgruppe

David Paulus, Patrick Gollub und Marcel Veber

Forschendes Lernen und Kasuistik. Grenzwelten und Zwischengänge

bezogen auf Reflexivität in der inklusionssensiblen Lehrer*innenbildung

Dietlind Gloystein und Ulrike Barth

Divers denken und handeln! - Theoretische Orientierungen und

Handlungsperspektiven für die Lehrkräftebildung

Ann-Christin Faix

Wie verändern sich die subjektiven Theorien von Lehramtsstudierenden über guten inklusiven Unterricht

Katja Baucke

Internationaler Vergleich als Reflexionsangebot. Eine explorative Studie zur Sicht von Hochschullehrenden auf schulische Inklusion in

Deutschland und Kanada

Bettina Amrhein, Benjamin Badstieber und René Schroeder

Zum Umgang mit als störend wahrgenommenen Handlungsweisen von

Schüler*innen in einem inklusionsorientierten Unterricht - Perspektiven

für die Lehrer*innenbildung (im Förderschwerpunkt emotionale und soziale Entwicklung) 
8 Inhaltsverzeichnis

Andrea Holzinger, Gerda Kernbichler, Silvia Kopp-Sixt, Mathias Krammer und Gonda Pickl

Profilierung für Inklusive Pädagogik (IP) im Lehramt der Primar- und

Sekundarstufe Allgemeinbildung

Timo Finkbeiner und Susanne Eibl

Kooperative Prozesse im technikbezogenen Unterricht 280

Autor*innenverzeichnis 287 


\section{Vorwort der Herausgeber*innen}

Die vorliegende Herausgeber*innenschaft versteht sich als thematischer Sammelband, dessen Konzeption auf der ,IFO2020 ${ }^{1}$ - der 34. Jahrestagung der Inklusionsforscher*innen - beruht und der nun den Versuch darstellt, die rund um diese Tagung begonnenen, weiterentwickelten oder vielleicht sogar in diesem Kontext überhaupt erst entstandenen Diskussionen zu bündeln und zumindest in Ansätzen zu systematisieren. Ins Zentrum der Auseinandersetzungen gerät dabei (notwendigerweise, wie im Verlauf des Bandes vielfach gezeigt werden wird) die Inklusionsforschung selbst. Obschon es mitunter leicht(er) gelingt, an der eigenen Disziplin Kritik zu üben, fällt es vielen von uns deutlich schwerer, diese nicht nur auf Inhalte von Kolleg*innen zu beziehen, sondern auf das eigene akademische Handeln zu übertragen und ernst zu nehmen - sei es in forschenden, lehrenden oder theoriebasierten Zusammenhängen. Wenig überraschend resultiert daraus, dass ein solches Unterfangen keinesfalls beliebig sein darf. Entsprechend sorgfältig haben wir darauf geachtet, dass wir für den vorliegenden Band ausschließlich solche Beiträge aufnehmen, die im Rahmen eines strengen Double-Blind-Peer-Review-Verfahrens als publikationswürdig erachtet wurden. Wir möchten uns entsprechend bei all jenen aufs Herzlichste bedanken, die als wissenschaftlicher Beirat durch ihre sorgfältige und konstruktive Kritik im Rahmen des Peer-Review-Verfahrens einen massiven Beitrag zum Gelingen dieses Buches geleistet haben: Sven Bärmig, Julia Biermann, Mai-Anh Boger, David Brehme, Jens Geldner, Ingeborg Hedderich, Andreas Hinz, Thomas Hoffmann, Claudia Kaluza, Gabriele Kulhanek-Wehlend, David Labhart, Reinhard Markowetz, Lisa Pfahl, Heribert Schopf, Marcel Veber, Benjamin Wagener, Monika Wagner-Willi, Anne Weidermann, Michael Wininger und Raphael Zahnd.

Die Erstellung des Buches selbst wäre ohne die tatkräftige Unterstützung von Felix Studencki, Wolfgang Osztovics und Tina Obermayr undenkbar gewesen. Sie haben uns als Herausgeber*innen durch ihre Gewissenhaftigkeit im Lektorat, ihr Organisationstalent und ihre administrativen und technischen Skills den Rücken für intensive inhaltliche Auseinandersetzungen freigehalten. Dafür kann gar nicht genug gedankt werden. Unser herzlicher Dank gilt auch Iris Kopera, die das (auch auf dem Buchumschlag zu findende) Logo der IFO2020 illustriert hat und das von Michael Oplatek grafisch aufgearbeitet und in Szene gesetzt wurde. Lukas Schnabel danken wir für die dynamische Grafik, die die vier Eckpunkte -

1 Die Tagungswebsite ist - auch mit näheren Infos zur Konzeption der Tagung - hier einsehbar: https://ifo2020.ssr-wien.at/ (letzter Zugriff: 05.08.2021) 


\section{Vorwort der Herausgeber*innen}

Grenzen, Gänge, Zwischen und Welten - visualisiert, die sich bereits auf dem Call for Papers fand und die auch - wenngleich unbewegt - in diesem Beitrag wiederzufinden ist.

Wie knapp wir mit der IFO selbst an einer Grenze vorbeigeschrammt sind, zeigt sich in der zeitlichen Nähe der Tagung zum Ausbruch der COVID-19-Pandemie - kaum vorstellbar, dass die IFO2020 nur knapp drei Wochen vor dem ersten Lockdown lag und die Tagung damit für viele von uns wohl eine der letzten Großveranstaltungen für lange Zeit war. Dieser historische Marker stellt vieles, was wir zunächst unhinterfragt als gegeben erachtet haben, infrage bzw. verstärkt viele markante Unterschiede hinsichtlich Ungleichheit massiv und gibt so vielfach Anlass zu Forschung und weiterführender Auseinandersetzung.

Wir wünschen eine anregende Lektüre!

Bernhard Schimek, Gertraud Kremsner, Rainer Grubich, Florentine Paudel, Michelle Proyer und Regina Grubich-Müller 
Gertraud Kremsner, Bernhard Schimek und Michelle Proyer

Grenzen.Gänge.Zwischen.Welten.

Kontroversen - Entwicklungen - Perspektiven der

Inklusionsforschung

\section{Grenzen.Gänge.Zwischen.Welten. Oder: Inklusionsforschung verorten?!}

Der Konzeption der IFO2020 lag die grafische Bestimmung eines Raums zugrunde, in dem Auseinandersetzungen mit Grenzen, Gängen, Formen des Zwischen und von Welten der Inklusionsforschung mithilfe unterschiedlicher Koordinaten verortet werden sollten:

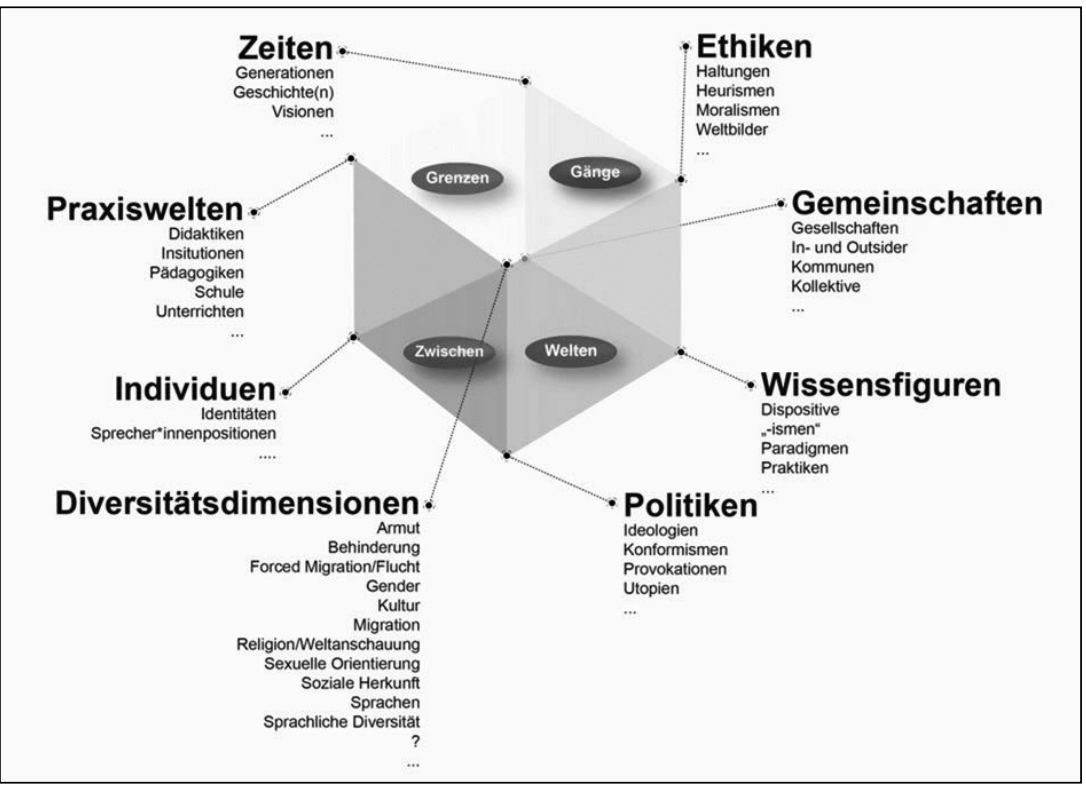

Abb. 1: Grenzen.Gänge.Zwischen. Welten. Dynamische Grafik zur Verortung divergierender Koordinaten der Inklusionsforschung

In Weiterführung der konzeptiven Überlegungen, die dem Titel und der Programmierung der Tagung zugrunde lagen, beschäftig(t)en uns auch bei der Konzeption und Rahmung dieses thematischen Sammelbandes folgende grundlegende Fra- 
gen, die nun auch auf gewisse Weise die Navigation durch die hier versammelten Beiträge anleiten: Wie bzw. inwieweit, durch wen und in welcher Form lässt sich Inklusionsforschung verorten? Lassen sich allgemeingültige Aussagen darüber formulieren, was die (eine und einzige?) Inklusionsforschung nun ist, oder liegt ihr Wesen gerade in ihrer Unbestimmtheit und Vielfältigkeit ihrer Zugänge? Worin liegt aber dann ihr Spezifisches, ihr Bezugspunkt, ihr Gegenstand? Und (nicht) zuletzt: Wie wird die strukturelle und disziplinäre Verankerung der Inklusionsforschung aufgefasst und welche (politischen) Implikationen können, sollen dadurch (nicht) abgeleitet werden? Wie ,geht' Inklusionsforschung eigentlich?

Diese und ähnlich gelagerte Grundsatzfragen sind den alljährlichen Tagungen der Inklusionsforschungen (kurz: der IFOs) inhärent und folglich vielfach diskutiert - und zwar sowohl sachlich, auf theoretische und empirische Erkenntnisse bezogen als auch in mitunter emotional besetzten Streitgesprächen und allen dazwischenliegenden Graubereichen. Die Intensität, mit der dieser Diskurs geführt wird, lässt auf die Dringlichkeit schließen, die Inklusionsforschung im deutschsprachigen Raum selbst einschließlich ihrer historischen Gewordenheit in den Blick zu nehmen und kritisch zu hinterfragen. Der vorliegende Sammelband ist demnach - freilich ohne Anspruch auf Vollständigkeit! - als Versuch einer Momentaufnahme zum präsentierten, diskutierten und angeregten Status quo der Inklusionsforschung zu verstehen. Den Dreh- und Angelpunkt hierfür bilden die Fragen danach, welche Grenzen innerhalb und außerhalb der Inklusionsforschung gezogen werden (können); welche Welten dadurch entstehen, sich eröffnen, geschaffen oder verunmöglicht werden und welche Gänge, sofern diese sich auftun, zur Überbrückung, Überwindung, Verbindung oder gar als Aus- und Umweg beschritten werden (sollen) oder sich als Irrwege erweisen. Ebenso (bzw. gerade auch?) zur Diskussion gestellt wird alles, das sich in einem Dazwischen verorten lässt, darin oszilliert oder als zu verhandelnd verbleibt.

Für derartige Auseinandersetzungen einen Impuls zu geben, haben wir von zwei einander in vielerlei Hinsicht unterschiedlichen Akteur*innen aus dem Kreis der Inklusionsforscher*innen erbeten: Beiträge von Georg Feuser und Mai-Anh Boger spannen einen breiten Bogen und schaffen Raum für ein Nachdenken und Infrage-Stellen, ermöglichen es uns, Grenzen (an) zu erkennen oder zu verhandeln. In ihren Beiträgen werden in unterschiedlicher Perspektivität, Intention und Intensität die oben grafisch gesetzten Bezugspunkte adressiert, womit das diskursive Feld pointiert umrissen wird. Sie eröffnen so nicht nur diesen Band, sondern - wie sich sogleich bemerkbar machen wird - auch die Diskussion, die wir weiter anregen möchten und die uns weiter zu führen notwendig erscheint. Die dritte im Rahmen der Tagung geladene Keynote von Wayne Veck war dem Anspruch geschuldet, den geografischen Raum der IFO auch über den deutschsprachigen Fokus hinauszuheben. Wenngleich aus zeitlichen Gründen der dritte Beitrag keinen Eingang in den Band finden konnte, weshalb im Folgenden der 
Fokus auf Feuser und Boger gelegt wird, bleibt die im Vortrag von Wayne Veck vorgenommene konzeptive Setzung gewahr, die Inklusive Bildung im Kontext von Nationalismus und Feindseligkeit gegenüber Menschen mit Flucht- und/oder Migrationserfahrung thematisierte und damit maßgeblich zur intendierten weiteren thematischen Öffnung des Diskurses beitrug.

Die Notwendigkeit, die Inklusionsforschung selbst in den Fokus grundständiger Kritik zu rücken, thematisiert Feuser (2021, S. 28 in diesem Band; Hervorhebung im Original), indem er den Vorwurf erhebt, dass die Inklusionsforschung keinen oder zumindest nur sehr wenig Beitrag zu einer sich verändernden Gesellschaft zu leisten vermag - und dies, obwohl er uns, den Inklusionsforscher*innen (zu denen letztlich auch er selbst zu zählen ist?), keineswegs Untätigkeit vorwirft: Er leitet seinen Beitrag mit dem Statement ein, dass sich auf unseren "Schreibtischen sicher sehr viel“ getan habe, wenn „Diversitätsdimensionen erwürfelt" oder „vielfältige Wissensfiguren erstellt“ worden seien, dazu „vielleicht ein vermarktbares Inklusionsschachspiel entwickelt und Praxiswelten aufgestellt und in Kombination beider vielleicht ein Inklusions-Monopoly" das Resultat unserer akademischen Tätigkeit bilden würde. Das, was sich ,in Sachen Inklusion“ für die dadurch adressierten Kinder und Jugendlichen getan habe - was davon also im Alltag behinderter Menschen ankomme -, bilanziert Feuser allerdings als „tief im Minus" (ebd., S. 29); nach wie vor sei die bestehende Ordnung und mit ihr das Bildungssystem hochgradig selektiv und exklusiv. Damit einhergehend fragt er sich nicht nur, was an diesem reichen akademischen Output tatsächlich inklusiv sei, sondern auch, was die derart forschenden Akteur*innen denn eigentlich antreibe (ins Spiel kommen Feuser folgend narzisstische Selbstwahrnehmungen ebenso wie neoliberale Verkaufsargumente), wenn sie nichts anderes zustande brächten, „als sich Inklusion auf Arme, Beine, Brust oder Rücken zu tätowieren und sie durch die Ohren, Nasenflügel, Lippen oder Brustwarzen zu stecken“ (ebd., S. 29). Mit Rückgriff auf Bourdieus (1998, 119 zit. nach Feuser 2021, S. 33) Beschreibung einer "doxischen Unterwerfung“ kommt er zu dem Schluss, dass auch „wir als Forschende und Lehrende in allen Systembereichen der Pädagogik dieser Indoktrination lebenslang nie entronnen, von ihr bis zur Sättigung durchtränkt [seien] und uns wie Inklusionsbürokrat*innen [verhalten würden] “ (Feuser 2021, S. 33). Dies führe letztlich dazu, dass es eine Art Tabubruch darstelle, das hierarchisch gegliederte und ständisch organisierte Schulsystem infrage zu stellen - mündend darin, dass das aktuelle Schulsystem „alle Werte [konkret: das Soziale, das Liberale, das Christliche und das Demokratische], die wir in unserer Kultur für staatstragend erachten, konterkariert, ja sie pervertiert" (ebd., S. 34). Nach einem umfassenden Exkurs auf die Auswirkungen dessen auf die "prekäre Klasse“ fordert Feuser (2021, S. 36-37) schließlich, dass wir uns „endlich einmal unseres Verstandes ohne die obrigkeitsstaatliche Anleitung und ordnungsstaatlichen Vorgaben bedienen" müssen (ebd. S. 38). 
Feusers Worte stellen viele der (akademischen) Bemühungen in zynisch zugespitzter Form an den Pranger und vermögen dadurch sicherlich auch zu verletzen. Manche mögen sie auch als anmaßend empfinden oder aber infrage stellen, ob derlei Aussagen Hand und Fuß hätten, wenn das eigene akademische Wirken doch all dem gewidmet ist, was Feuser ,den Inklusionsforscher*innen' in seinem Beitrag abspricht. Das Feld des auch emotional besetzten Streitgesprächs, auf welches eingangs auch im Kontext der IFOs bereits angespielt wurde, ist damit eröffnet - und Feuser selbst fordert am Ende seines Beitrags einen „streitbaren Dialog“ (ebd., S. 40) ein.

Außer Frage zu stellen ist der gemeinsame Bezugspunkt eines inklusiven Gesellschaftssystems - ja, wir beziehen uns dabei explizit auf das gesamte gesellschaftliche Leben und nicht, wie so oft, auf Schulsysteme alleine -, welches nach wie vor meilenweit entfernt scheint, weil Menschen in aller aufzubringenden Härte an die und über die gesellschaftlichen Ränder hinausgedrängt werden. Insofern MUSS Reflexion und auch Kritik am eigenen Handeln und Wirken erfolgen - auch wenn dies noch so weh tut. Entsprechend kann eine solche Auseinandersetzung ohne Emotionalität oder einem gewissen $\mathrm{Maß}$ an Involviertheit möglicherweise gar nicht erst auskommen. Dies gilt im Übrigen und selbstverständlich auch für das Werk Georg Feusers, das sich gleichermaßen einer solchen möglicherweise schmerzhaften Kritik zu stellen hat. Für unser aller akademisches Schaffen bleibt also zu hinterfragen, welchen Beitrag es für einen Wandel hin zu einer inklusiven (oder zumindest inklusiveren) Gesellschaft leistet und entsprechend tatsächlich nicht nur bei an den Rand gedrängten Personen ankommt, sondern (dies sei Feusers Überlegungen vielleicht hinzugefügt, um ggf. drohenden paternalistischen Helfer*innen-Szenarien entgegenzuwirken) von diesen auch gewollt wird - oder aber, ob der polemisch artikulierte Vorwurf Feusers Geltung hat und es sich bei unseren Bemühungen lediglich um kosmetische Verbesserungen handelt, die niemandem außer der eigenen akademischen Vita nutzen. Es stellt sich damit einhergehend eine nicht neue, aber für den Kontext der Inklusionsforschung wohl noch nicht beantwortete Frage: Wie viel und welche Verantwortung trägt Wissenschaft hinsichtlich gesellschaftlicher Umwälzungsprozesse? Dies steht in engem Zusammenhang mit Fragen der Partizipation, Agency und Aktion. In diesem Kontext bleibt die Frage virulent, ob - und wenn ja, wie - politischer Aktivismus und wissenschaftliche Reflexion inklusive der ihr innewohnenden Dilemmata (und Trilemmata, siehe Boger in diesem Band) in Einklang gebracht werden können oder sollen und worin ein Richtmaß einer ethischen Bewertung der einzelnen akademischen Bestrebungen überhaupt bestehen kann.

Abschließend scheint es uns relevant, kritisch und sehr direkt die Frage aufzuwerfen, was es bedeutet, wenn Feuser (2021, S. 31) die Behauptung aufstellt, dass die „Integrationsbewegung in den 1990er-Jahren ihre politische Bewusstheit verloren [hat] und in einer Art politischem Wachkoma [steckt]." Im Rahmen der Tagung 
führte dies zu angeregten Ad-hoc-Diskussionen und es bleibt zu hoffen, dass diese abseits von vereinheitlichenden generationalen und/oder äußerlichen Zuschreibungen, offen und in aller Direktheit im Sinne der Disziplin und letztlich zugunsten der Beförderung von wahrhaft inklusiver Gesellschaft weitergeführt werden.

An die Feuser'sche Kritik anschlussfähig, setzt sich Boger (2021, S. 43 in diesem Band) eingehend mit der Disziplin selbst auseinander, indem sie anhand der von ihr entwickelten trilemmatischen Kartentheorie durchaus scharf analysiert, „welche Pfade in Zukunft beschritten werden könnten bzw. welche Sackgassen derzeit am Horizont erscheinen." Ebenso wie Feuser geht es ihr letztlich darum, Betroffenengruppen sichtbar zu machen, allerdings schlägt sie dafür einen völlig anderen argumentativen Weg ein: U.a. mit Rückgriff auf die Geschichte von emanzipatorisch ausgerichteten Nachbardisziplinen unternimmt sie den Versuch, ihre notwendige Kritik an der Inklusionsforschung zu systematisieren und durch bewusst eingreifende Vorhersagen - sie bekennt sich offensiv dazu, aktives Mitglied der deutschsprachigen Inklusionsforschung und damit im Feld situiert zu sein - Weggabelungen aufzuzeigen, an denen Fallstricke einerseits oder aber das Potenzial für Veränderung andererseits verborgen liegen. Diese sind, Boger (2021, S. 46) folgend, allerdings immer in Dilemmata verstrickt und lassen sich entsprechend nie simpel entwirren oder gar lösen. Dafür warnt sie zunächst vor den Gefahren, die mit der durch Dekonstruktion hervorgebrachten Entpartikularisierung von Zuschreibungskategorien einhergehen (im Fall der Inklusionsforschung ist das zum Beispiel Behinderung). Dadurch gehe nämlich die „Tür für die systematische Produktion falscher Universalismen aufseiten jener, die eine Nicht-Benennung und Nicht-Beschäftigung mit den Anderen* aus diesem dekonstruktiven Impuls machen " (ebd., S. 45; Hervorhebung im Original) überhaupt erst auf. Die damit einhergehende Geste der Selbstannihilation könne in weiterer Folge mit Leichtigkeit dazu führen, dass spezifisch auf Behinderung bezogene (theoretische wie empirische) Auseinandersetzungen, Zugänge, aber auch Praktiken Sparmaßnahmen zum Opfer fielen könnten und dadurch erst recht unsichtbar gemacht werden würden. Als Folge einer konstruktivistischen Sackgasse würde überdies, wie in der zweiten Weggabelung dargestellt, eine vermeintlich bereits erfolgte „Umsetzung“ von Inklusion im Sinne der UN-BRK zusammenkonstruiert" (ebd., S. 47). Dies werde möglich, weil a) über das Verschwinden der Benennung von Behinderung Vielfaltsrhetoriken zum Tragen kommen würden, die sich als leer erweisen sowie b) durch die Betonung einer (ausschließlich) sozialen Konstruktion von Behinderung diese als „bitterlich-reale Tatsache“ (ebd., S. 47) völlig übersehen werde. Anders als bei Feuser stehen bei Boger nicht nur die Inklusionsforscher*innen selbst im Fokus, sondern vor allem die Akteur*innen emanzipatorischer Bewegungen - konkret: der Behinderten- wie auch der Elternbewegung. Mehr oder weniger parallel zum akademischen inclusive turn erfolgte in diesen Bewegungen ein academic turn, der die Aneignung nicht nur wissenschaftlicher Auseinander- 
setzungen, sondern auch akademischer Positionen durch Personen aus diesen politischen Bewegungen zur Folge hatte. Dabei gehe es, so Boger (2021, S. 49) weiter, aber nicht um eine simplifizierte Logik der Repräsentation (selbst wenn angemahnt werden muss, dass behinderte Menschen in der akademischen Wissensproduktion dennoch völlig unterrepräsentiert sind), sondern um eine fundamentale und vor allem epistemologisch ernst gemeinte Standpunktreflexion, ohne dabei in „Moralisierung zu verfallen oder die zuweilen selbstgefällig anmutende moralische Hierarchie der Partizipationsleiter der Partizipativen Forschung mit einem Qualitätskriterium für wissenschaftliches Arbeiten und sachliche Argumente zu verwechseln. " Dazu reiche es nicht aus, akademische Auseinandersetzungen bloß in leichter(e) Sprache zu übersetzen. Die damit einhergehenden Ambivalenzen und Komplexitäten erkennen und sie aushalten zu können sei grundständig notwendig. Eng damit zusammenhängend verweist Boger (2021, S. 52) schließlich darauf, dass die Kritik an sämtlichen Fähigkeitserwartungen sich zwar als Ableismuskritik tarne, tatsächlich jedoch ob der ihrer inhärenten entmündigenden Bewahrpolitik zutiefst ableistisch sei. Zu unterscheiden sei hier zwischen (zwingend zu kritisierenden!) naturalisierten Fähigkeitserwartungen und (tatsächlich sogar zu stärkenden) legitimen, bildungswirksamen Fähigkeitserwartungen, die Bildung als Entwicklung bzw. Entwicklung als Bildung implizieren. Mit Überlegungen rund um Konzepte posthumaner Relationalität und rekurrierend auf den ,Cyborg' als Beispiel von „Denkfiguren, die auch den Humanismus als Essentialismus dekonstruieren sollen“" (ebd., S. 54), schließt Boger ihre Analyse zu den gewählten Weggabelungen, denen sich die Inklusionsforschung aktuell beispielhaft zu stellen habe. Dass dabei aber nicht die Disziplin als Ganzes in kritische(re) (Selbst)Reflexion eintauchen muss, sondern jedes einzelne darin agierende Individuum - also wir als Inklusionsforscher*innen, Mai-Anh Boger explizit und durch sie selbst betont eingeschlossen - das eigene akademische Handeln und Wirken ernst gemeint und sorgfältig zu prüfen habe, sei im Sinne einer Revitalisierung der „Idee von Inklusion als emanzipatorischen Entwurf" (ebd., S. 56) unverzichtbar.

\section{Kontroversen. Entwicklungen. Perspektiven. Oder: Inklusionsforschung verorten!}

Die von Feuser wie auch von Boger angerissenen (und mit unseren Überlegungen verschränkten) Impulse bilden den Auftakt dafür, nun folgend und mit Betonung auf keineswegs gegebene Vollständigkeit den Versuch einer Bündelung jener Auseinandersetzungen zu unternehmen, die gemäß des Titels Grenzen.Gänge. Zwischen. Welten die Inklusionsforschung selbst (kritisch) in den Blick nehmen. Im Sinne einer zart und sicherlich oberflächlich angesetzten, keineswegs also 
,sauberen' Systematisierung haben wir alle angenommenen Beiträge großfl̈chig (und erneut ohne Anspruch auf Vollständigkeit oder method(olog)ische Sauberkeit!) verschlagwortet, um als Map einen groben Überblick darüber zu gewinnen, wie sich der Status quo der Inklusionsforschung im Kontext dieses thematischen Sammelbandes gestaltet. Als Ergebnis dieser Schlagworte erhalten wir nicht nur Hinweise darauf, was aktuell überhaupt thematisiert und diskutiert wird, sondern auch darauf,

- welche empirischen Ausrichtungen gegeben sind,

- welche theoretischen Grundlegungen bedient werden,

- welche (grundlegenden) Terminologien benutzt und ggf. begründet werden,

- welche paradigmatischen Ausrichtungen sich finden lassen,

- ob bzw. inwiefern interdisziplinäre Zusammenschlüsse vorhanden sind,

- welche Rolle Gesellschaftskritik im Kontext der Inklusionsforschung spielt,

- ob bzw. wenn, wie (Selbst-)Kritik an der Inklusionsforschung geübt wird,

- ob und wenn, wie Kritik an der pädagogischen Praxis geübt wird,

- welche intersektionalen Verwobenheiten zum Thema gemacht werden,

- welche Umsetzungsmöglichkeiten inklusiven Unterrichts thematisiert werden,

- welche Rolle inklusive Lehrer*innenbildung spielt,

- welche Adressat*innengruppen im Fokus stehen,

- welche (offensichtlichen) Leerstellen sich durch tatsächliche oder auch durch angemahnte Nicht-Nennungen ergeben,

- und zuletzt, wie all diese genannten Aspekte miteinander in Verbindung stehen.

Das Ergebnis daraus ist zwar (leider) keine trilemmatische Karte im Boger'schen Sinne, dennoch lässt sich eine Fülle an Schlagworten samt deren Vernetzungen zunächst grafisch als Map und in weiterer Folge ausformuliert darstellen. Im besten Falle bildet die derart gewonnene Map vielleicht sogar den Anlassfall für eine systematischere und methodisch sauberere Analyse: 


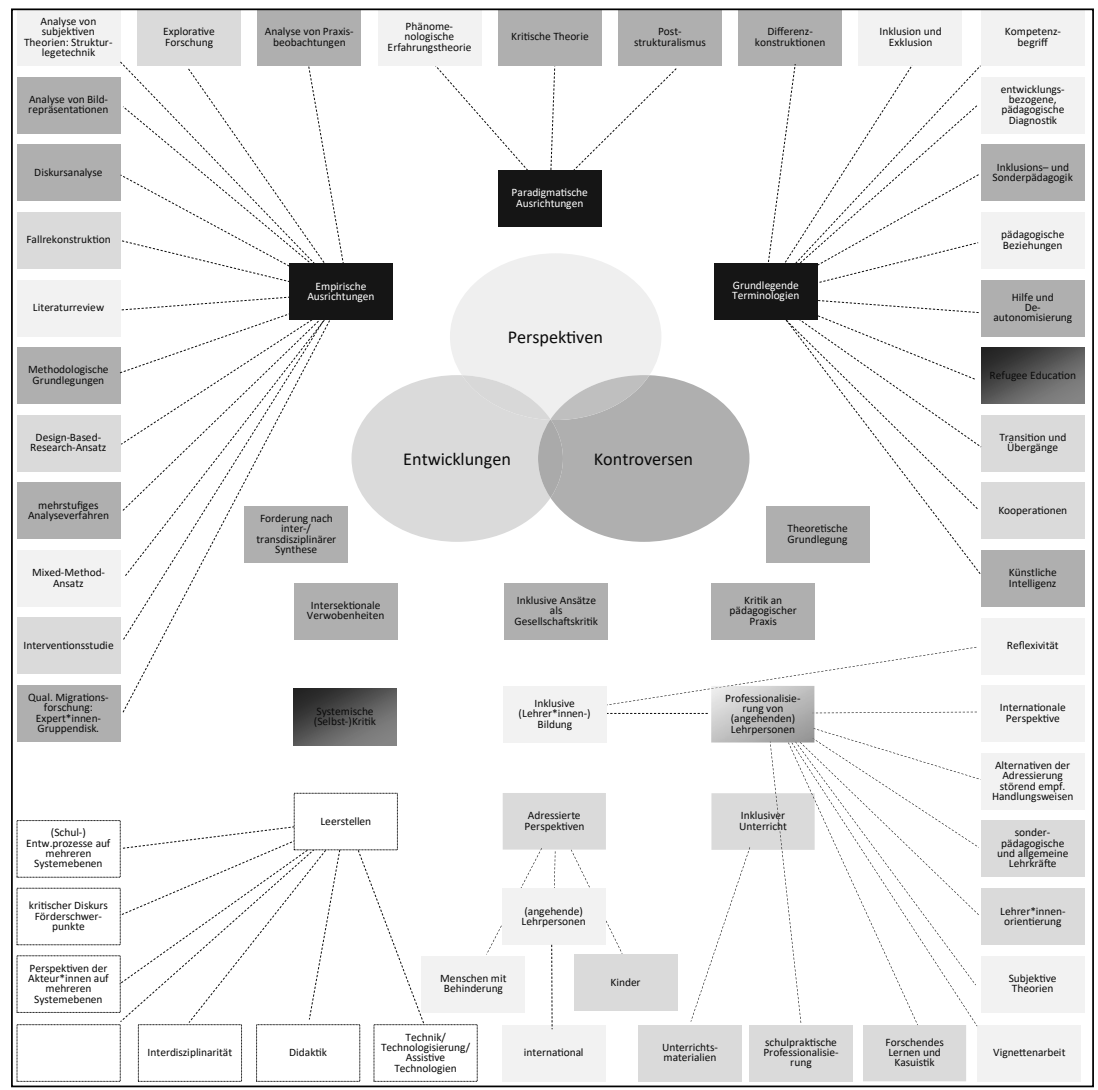

Abb. 2: Grenzen.Gänge.Zwischen. Welten. - Map zu aktuellen Auseinandersetzungen in der deutschsprachigen Inklusionsforschung

Den Ausgangspunkt unserer Map bildet zunächst die grobe Ordnung, der wir die Beiträge (übrigens auch als Subkapitel in diesem Band) zugeordnet haben:

Dabei werden in den Kontroversen Inklusionsforschung und Inklusive Pädagogik im inner- wie auch inter- und transdisziplinären Widerstreit verortet und diskursive Auseinandersetzungen eröffnet oder aufgegriffen. Kurzgefasst: Worum wird in unserem Feld gestritten bzw. worüber (leidenschaftlich) diskutiert? Bzw. auch: Welche Fragen bleiben (bewusst) ungeklärt?

Entwicklungen wiederum beschreiben und reflektieren Entwicklungslinien und Modelle inklusiver Praxen - nicht nur, aber insbesondere im Kontext schulischer Bildung wie auch der akademischen Lehrer*innenbildung. Kurzgefasst: Was wurde erprobt und was sind die Ergebnisse einer systematischen Reflexion dazu? 
Perspektiven bieten schließlich Ausblicke sowohl auf diskursive wie auch auf handlungsorientierte Weiterentwicklungen der Inklusionsforschung bzw. der Inklusiven Pädagogik. Kurzgefasst: Wohin entwickelt sich das Feld, wohin entwickeln wir uns als darin handelnde (akademische) Akteur*innen?

Welcher Beitrag sich in welchem Subkapitel in diesem Band wiederfindet, kann anhand des Inhaltsverzeichnisses nachvollzogen werden; die jeweiligen Zuordnungen wurden mit den Autor*innen abgesprochen. Keineswegs selbsterklärend ist jedoch die nachfolgende Verschlagwortung samt der Herstellung der sich jeweils ergebenden Verbindungen und Netzwerke. Sie wird nun kurz und jeweils exemplarisch an einzelnen Beiträgen skizziert begründet und erhebt keinen Anspruch auf Vollständigkeit. Vielmehr will die Darstellung Diskussionsanlass sein und Hinweise für implizite Gewichtungen und nicht adressierte Felder geben.

Empirische Ausrichtungen finden wir - mit einem Augenzwinkern gesprochen und ganz der Inklusionsforschung entsprechend - in einer bunten Vielfalt. Während Bärmig in seinem Beitrag „Dialektik als Methode“ (zu finden im Kapitel Kontroversen) nicht nur eine methodische, sondern auch eine methodologische Grundlegung diskutiert, bleibt festzustellen, dass sich die deutliche Mehrheit der eingereichten empirisch begründeten Beiträge der qualitativen Sozialforschung zuordnet. Beispielhaft angeführt werden können hier etwa diskursanalytische Ansätze (wie bei Jochmaring, Bömelburg und Sponholz in den Kontroversen), aber auch die Analyse von Bildrepräsentationen (wie bei Kappeller, ebenfalls in den Kontroversen) oder Fallrekonstruktionen (wie bei te Poel in den Entwicklungen). Abseits davon findet sich im Spektrum der empirischen Ausrichtungen allerdings auch eine Interventions-Studie (bei Quante und Danner in den Entwicklungen), eine entlang des Design-Based-Research-Ansatzes angelegte Studie (Bešić und Maitz in den Entwicklungen) sowie eine Mixed-Methods-Studie (Albers, Filipiak, Franzen und Hellmich in den Perspektiven). Pongratz setzt sich in den Perspektiven mit systemischen Fragetechniken auseinander, die in der qualitativen Erwachsenenbildungs- bzw. Weiterbildungsforschung bei Biografieträger*innen mit einer zugeschriebenen geistigen Behinderung eingesetzt werden. Schwörer, van Ledden, Algermissen und Hauser wiederum nehmen im selben Kapitel die eigene partizipative Forschungsgruppe zum Anlassfall, ihre Zusammenarbeit wie auch ihre Mediennutzung zu reflektieren.

Fundierte theoretische Grundlegungen finden wir z.B. bei York und Jochmaring (in den Kontroversen), die theoretisch äußerst fundiert Kritik an der für den Arbeitsmarkt rechtlich grundgelegten Etikettierungspraxis, dem damit verbundenen Wording und dem System der spezifisch ausdifferenzierten (Sonder-)Arbeitsmärkte vornehmen, das sie in aktuell unhinterfragten kapitalistischen Produktionsverhältnissen und Leistungsvorstellungen verankert und durch sie bedingt sehen. Aber auch Bärmig (ebenfalls in den Kontroversen) ist hier anzusiedeln: er 


\section{Gertraud Kremsner, Bernhard Schimek und Michelle Proyer}

setzt sich grundständig mit Adornos dialektischem Verfahren auseinander und macht dieses für die Inklusionsforschung nutzbar.

Die in den einzelnen Beiträgen Verwendung findenden und teilweise auch fundiert eingeführten grundlegenden Terminologien müssten, würden sie vollständig angeführt, in einer langen Liste aufgezählt werden. Daraus wird deutlich, dass sich die Inklusionsforscher*innen keineswegs eines einheitlichen Vokabulars bedienen, sondern über weite Strecken auf Terminologien zurückgreifen, die sie reflektiert und begründet verwenden. Gerland, Niediek, Hülsken und Sieger (in den Kontroversen) nutzen etwa den Begriff der ,Differenzkonstruktionen', den sie mit der Konzeption des ,Doing Difference' verschränken. Darüber hinaus finden sich weitere Auseinandersetzungen mit Begriffen; z.B. bei Paulus, Gollub und Veber (in den Perspektiven), die mit Rekurs auf ,klassische' theoretische Grundlegungen ein eigenes Kapitel der Frage danach widmen, welche Sprache für ,Inklusion` und ,Exklusion ‘ gefunden werden kann. Kottmann (in den Entwicklungen) wiederum beleuchtet den ,Übergang' aus rechtlicher und statistischer wie auch aus empirischer Hinsicht terminologisch. Bezugnehmend auf alle eingereichten Beiträge ist übrigens festzuhalten, dass weder die Inklusionsforschung noch die Inklusions-, die Integrations- oder die Sonderpädagogik begrifflich definiert oder auch operationalisiert werden. Wecken einzelne Begriffskonstruktionen unmittelbar den Wunsch nach Erläuterung und Diskurs, wie bei Link (in den Kontroversen) mit der Verwendung von ,Inklusions- und Sonderpädagogik', so bleibt insgesamt zu fragen, welche Formen der Verständigung in dieser Unbestimmtheit (dennoch) gelingen bzw. entzogen bleiben.

Bezüglich der paradigmatischen Ausrichtung der angenommenen Beiträge ist festzustellen, dass sich die Mehrheit derer im Poststrukturalismus verorten lassen beispielhaft angeführt seien die bereits in anderen Kontexten genannten Gerland, Niediek, Hülsken und Sieger, aber auch York und Jochmaring sowie Kappeller. Bärmig wiederum ließe sich mit seinen Bezugnahmen auf Adorno und damit die Kritische Theorie freilich der Kritischen Erziehungswissenschaft zuordnen. Sie alle sind im Kapitel zu den Kontroversen zu finden; für sie alle gilt - der paradigmatischen Ausrichtung entsprechend -, dass ihr Erkenntnisinteresse mit ihrem Wirksamkeitsinteresse korreliert und die derart verorteten wissenschaftlichen Erkenntnisse nicht ohne politische Bezugnahmen oder politische Forderungen zu denken sind. Anders ist dies bei der bei Gloystein und Barth (in den Perspektiven) nutzbar gemachten phänomenologischen Erfahrungstheorie: Hier geht es explizit darum, Theorie und Praxis von Bildung und Erziehung in Bezug zu setzen und anhand phänomenologischer Zugänge „(pädagogische) Erfahrungen im Vollzug in ihren zeitlichen, sinnlichen und weltlichen Dimensionen zu erfassen und zu beschreiben" (Gloystein \& Barth 2021, S. 239 in diesem Band). Auf hermeneutisch-phänomenologische Grundlagen beruft sich explizit übrigens auch Link (in den Kontroversen). 
Forderungen nach inter-bzw. transdisziplinärer Synthese werden u.a. bei Jochmaring, Bömelburg und Sponholz sowie bei Gerland, Niediek, Hülsken und Sieger (beide in den Kontroversen) laut. Besonders eindringlich wird dieser Zusammenschluss wohl aber bei Hamisch und Kruschel (ebenfalls in den Kontroversen) eingemahnt, die inklusive Schule und die ihr inhärenten Lehr-Lernsettings mit aus künstlicher Intelligenz resultierenden Möglichkeiten, aber auch Herausforderungen kontextualisieren.

Mit einer vergleichsweise großen Zahl an zuzuordnenden Beiträgen erkennen wir einen größeren Schwerpunkt in inklusiven Ansätzen als Gesellschaftskritik und Gegenbewegung zu systematischer Diskriminierung. Einige bereits genannte Beiträge (z.B. Bärmig; York \& Jochmaring; Jochmaring, Bömelburg \& Sponholz; Gerland, Niediek, Hülsken \& Sieger; Kappeller; Kottmann; Hamisch \& Kruschel) lassen sich hier einordnen. Besonders hervorgehoben werden sollen jedoch die Beiträge von Buchhaupt, Katzenbach, Lutz und Urban (in den Kontroversen) - sie kontextualisieren die Inklusionsforschung in Abhängigkeit zu einer von betriebswirtschaftlichen Aspekten und Ansprüchen auf Verwertbarkeit in der Praxis bestimmten Forschungsförderungslandschaft - sowie von Goldbach und Leonhardt (in den Entwicklungen), die Erfahrungen in der Zusammenarbeit mit Bildungs- und Inklusionsfachkräften zum Anlass nehmen, um über Elemente von Macht im Kontext einer inklusionssensiblen Hochschulentwicklung zu reflektieren. Beide wurden zusätzlich auch mit dem Schlagwort systemische (Selbst-)Kritik versehen.

Kritik an pädagogischer Praxis wird wiederum in Paulus, Gollub und Veber (in den Perspektiven) sowie bei Möhlen, Deiß, Subasi Singh und Proyer (in den Kontroversen) formuliert; darüber hinaus aber auch bei Hoffmann, Reisenauer und Richter (in den Kontroversen) systematisch bearbeitet. Sie beschäftigen sich gleich mit mehreren Spannungsfeldern, wenn sie Helfen als individuelle Erfahrung wie auch als soziale Praxis im Kontext von Deautonomisierung und Befähigung grundständig analysieren und reflektieren.

Intersektionale Verwobenheiten werden an und in mehreren Beiträgen sichtbar. Besonders deutlich Bezug genommen wird auf diesen Aspekt jedoch bei Kappeller (in den Kontroversen), der ,Anderskörperlichkeiten ' anhand visueller Darstellungen entlang einer Vielzahl an intersektionalen Zusammenhängen analysiert; den verbindenden Anlassfall bilden hier nicht an den Körper gefügte Prothesen. Möhlen, Deiß, Subasi Singh und Proyer (in den Kontroversen) wiederum richten den Blick auf die Lebenswelt(en) von Schüler*innen mit Fluchterfahrungen und erweitern so die ,klassischen' Bezugnahmen auf Behinderung auf eine ebenfalls durch Inklusive Pädagogik und Inklusionsforschung adressierte Personengruppe.

Ein deutlicher Schwerpunkt ist in Auseinandersetzungen mit der Umsetzung einer inklusiven Lehrer*innenbildung zu erkennen. Ansätze der Professionalisierung (angehender) Lehrkräfte werden in mehreren Beiträgen erarbeitet und diskutiert 
(z.B. in den Entwicklungen bei Quante und Danner sowie bei te Poel) und teilweise mit Fragen nach professioneller Reflexivität verschränkt (etwa bei Paulus, Gollub und Veber sowie Gloystein und Barth in den Perspektiven). Ausgehend von Ansätzen aus der rekonstruktiven Kasuistik verhandelt Kaiser (in den Entwicklungen) etwa inklusionsbezogene Anforderungswahrnehmungen im Kontext (kunstpädagogischer) Professionalisierungsprozesse. Im selben Kapitel angesiedelt, machen Gollub, Greiten, Schkade und Veber schulpraktische Professionalisierung zum zentralen Thema ihres Beitrags, wenn sie diese als schulpraktische Herausforderung mit einem interdisziplinären Blick aus hochschuldidaktischen Projekten verschränken. In thematischer Nähe und entsprechend ebenfalls in den Entwicklungen angesiedelt ist auch der Beitrag von Link, Steinert und Jurkowski, der sich mit Inklusion als Querschnittsthema in der Professionalisierung der Lehrer*innenbildung an der Universität Erfurt auseinandersetzt. In den Perspektiven angesiedelt, beschäftigt sich Faix wiederum damit, wie sich die subjektiven Theorien über guten inklusiven Unterricht von Lehramtsstudierenden verändern, während Albers, Filipiak, Franzen und Hellmich eine internationale Perspektive auf Auseinandersetzungen mit Kompetenzentwicklung im inklusiven Unterricht übertragen. Ebenfalls international ausgerichtet ist übrigens auch der Beitrag von Baucke im selben Kapitel, der die Sicht von Hochschullehrenden auf schulische Inklusion in Deutschland und Kanada miteinander vergleicht.

Ausgehend von der ,Pädagog*innenbildung Neu' arbeiten Holzinger, Kernbichler, Kopp-Sixt, Krammer und Pickl (in den Perspektiven) heraus, welche Gelingensfaktoren und Spannungsfelder sich aus der Profilierung für Inklusive Pädagogik im Lehramt der Primar- und Sekundarstufe Allgemeinbildung ableiten lassen. Mit spezifischem Fokus auf die Lehrer*innenbildung im Förderschwerpunkt emotionale und soziale Entwicklung setzen sich Amrhein, Badstieber und Schroeder (ebenfalls in den Perspektiven) damit auseinander, wie im inklusionsorientierten Unterricht mit als störend wahrgenommenen Handlungsweisen von Schüler*innen umgegangen wird.

Konkrete Umsetzungsmöglichkeiten im inklusiven Unterricht werden von Bešić und Maitz in den Entwicklungen erarbeitet, wenn sie anhand des Buches ,Das Boot: Eine Fluchtgeschichte' das Thema Flucht in der Primarstufe explorativ erforschen und damit einhergehend innovative Unterrichtsmaterialien entwickeln. Finkbeiner und Eibl stärken in den Perspektiven wiederum die Forderung nach kooperativen Prozessen im technikbezogenen Unterricht.

Ein Blick auf die eingenommenen Perspektiven eröffnet übrigens ebenfalls interessante Erkenntnisse: Die Sichtweise von Kindern steht bei Kottmann (in den Entwicklungen) im Zentrum, während diejenige von bzw. mit behinderten Menschen insbesondere bei Pongratz wie auch bei Schwörer, van Ledden, Algermissen und Hauser (allesamt in den Perspektiven) fokussiert wird. Der letztgenannte Beitrag ist übrigens auch der einzige in einem inklusiven Team verfasste. Angehende Lehr- 
personen und deren Perspektive werden in den Entwicklungen bei te Poel sowie bei Quante und Danner und in den Perspektiven bei Faix sowie bei Holzinger, Kernbichler, Kopp-Sixt, Krammer und Pickl herausgestellt.

Zuletzt sei - ohne jeglichen Anspruch auf Systematik oder Vollständigkeit - auf einige ausgewählte mögliche Leerstellen im Diskurs hingewiesen, welche uns auf den ersten Blick aufgefallen sind: Augenscheinlich wurde, dass trotz der expliziten Einladung im Call for Papers abseits von Behinderung nur wenige andere Diversitätsdimensionen im Sinne eines breiten Inklusionsbegriffs thematisiert wurden. Die Komplexität, welche sich aus dieser Erweiterung bzw. dem Blick auf Überschneidungen und Intersektionen notwendigerweise ergibt, rückt zwar immer wieder in den Blick der Disziplin, scheint im Kontext der Einreichungen und auch der Wiener IFO kein zentraler Fokus gewesen zu sein. Ähnlich verhält es sich mit den viel diskutierten Bereichen der Digitalisierung und didaktischen Fragen an der Schnittstelle zu Inklusion. Das bedeutet allerdings keineswegs, dass diese Perspektiven nicht relevant seien oder ausgeblendet bleiben, sondern - neben einer Vielzahl von anderen relevanten Betätigungsfeldern - inhärente Teile von Forschungsagenden oder -plänen sind. Auffällig ist bei der thematischen Auseinandersetzung mit Lehrer*innenausbildung im Kontext von Inklusion, dass die Perspektiven der Lehrpersonen selbst häufig keinen Fokus von Forschungsbemühungen darstellen. Gerade dann, wenn umfassende Bildungsreformen (wie derzeit in Österreich) Platz greifen, deren Bestreben der Erhöhung von Effektivität und Effizienz im Bildungssystem gewidmet sind, und wenn damit umfassende Qualitätsmanagementsysteme auf den Plan gerufen werden, entwickeln allfällige Leerstellen zu Inklusiver Schul(system)entwicklungsforschung eine gewisse Brisanz. Und ganz generell fiel uns bei der Betrachtung der eingereichten Beiträge auf, dass einige Stimmen nicht repräsentiert erscheinen bzw. andere mehr Platz einnehmen. Ein innerhalb der Disziplin laufend zu reflektierendes Fragefeld - wer erhält eine Stimme (möglicherweise auch stellvertretend, aber durch wen?) und wer wird gehört - soll also auch in diesem Eingangstext in Erinnerung gerufen und kritisch markiert werden. Vielleicht ist gerade mit dem (individuellen) bewussten Benennen des Wenig- oder Nicht-Beachteten, mit dem gezielten Wiederentdecken des Aus-dem-Blick-Geratenen und dem gewagten Gestalten neuer (interdisziplinärer) Verbindungen, jene geforderte Re-Fokussierung und -Politisierung der Inklusionsforschung auf den Weg gebracht.

\section{Fazit bzw. vielmehr Ausblick}

Das weit aufgespannte Feld, das die Tagung adressieren wollte, hat eine Vielzahl von kontroversen Positionen an einen gemeinsamen Tisch gebracht, aktuell sich Entwickelndes im Feld vor den Vorhang geholt und Möglichkeiten geboten, 


\section{4| Gertraud Kremsner, Bernhard Schimek und Michelle Proyer}

Neues und auch Bewährtes in Perspektive zu setzen oder Richtungen für weiterführende Diskurse aufzuzeigen.

Im Jahre 2021 musste die IFO erstmalig (?) ausfallen. Es bleibt abzusehen, wie sich diese Zäsur auf Forschungsagenden und politische Fragen rund um Inklusion ausgewirkt hat und weiter auswirken wird. Insbesondere wird es gelten, das Engagement auf jene gesellschaftlichen Sollbruchstellen zu richten, die im Brennglas der COVID-19-Pandemie und ihrer Effekte deutlich an Virulenz gewonnen haben und neu entstandene bzw. gewandelte Formen von Exklusion aufzuspüren und zu benennen. Wir gehen davon aus, dass die vier eröffneten Bezugspunkte - Grenzen, Gänge, Zwischen, Welten - wohl auch künftig als relevant erachtet werden können. Wir sind gespannt auf weitere Resonanz(en), die sich aus dieser unserer Auseinandersetzung und dem Nachklang der Tagung ergeben. 
Kontroversen 



\section{Georg Feuser}

\section{Die Bühne der Inklusion. Ein Prolog! oder: Grenzgänge zwischen Welten}

\section{Der erste Akt: Die Bühnen des Inklusionstheaters ${ }^{1}$}

Im Rahmen seiner Rede in der Wiener Hofburg zum Gedenktag gegen Gewalt und Rassismus vom 04. Mai 2018 sagte Michael Köhlmeier (2018, 9): „Ich habe lange darüber nachgedacht, was ich heute vor Ihnen sagen soll. Mir wäre lieber gewesen, man hätte mich nicht gefragt, ob ich hier sprechen will.“ Es erging mir genauso. Und er fährt fort: „Aber man hat mich gefragt, und ich empfinde es als meine staatsbürgerliche Pflicht, es zu tun“" (ebd.). Seine Begründung: Um den Opfern von Gewalt und Rassismus in die Augen sehen zu können - und auch sich selbst. Das möchte ich mir zu eigen machen, denn alle, die wir - und sei es aus bester Absicht und mit auf Hochglanz polierten Pseudoargumenten, Verwaltungsvorgängen und Verordnungen - noch immer in Verweigerung ihrer uneingeschränkten Anerkennung als aller Rechte eines Menschen berechtigt, von der aktiven Teilhabe an unserer Kultur, Gesellschaft und an den gesellschaftlichen Gütern und Institutionen ausschließen, bleiben und sind Opfer von struktureller und personaler Gewalt und eines Rassismus, der durch wohltönende Etikettierungen einer medizinisch-psychiatrischen, psychologischen und heil- und sonderpädagogischen Diagnostik, in IQ-Werten mathematisiert und als, sonderpädagogischer Förderbedarf deklariert, nicht zu übertünchen und ungeschehen zu machen ist; das sollten wir endlich begreifen.

Michael Köhlmeier beginnt seine Rede, deren Anfang ich für die meine gerne übernehme, wie folgt: „Sehr geehrte Damen und Herren, erwarten Sie nicht von mir, dass ich mich dumm stelle. Nicht an so einem Tag, nicht bei so einer Zusammenkunft. Ich möchte nur eines: den Ermordeten des NS-Regimes, von deren Leben die Schüler so eindringlich berichtet haben, in die Augen sehen zu können ..." (ebd., 7; Hervorhebung im Original). Und ich stelle für mich fest: Ich möchte jenen, die wir mit dem Begriff der Inklusion in Theoriebildung und Praxis noch immer exkludieren und in Sonderräume zwangsinkludieren, in Sonderklassen und -schulen, in Klein- und Reintegrationsklassen unterrichten, die wir in

1 Zur Eröffnung des Vortrags wurde der Prolog aus der Oper, Der Bajazzo“ von Ruggero Leoncavallo eingeblendet. Einige Textteile beziehen sich darauf. Die ungekürzte Vortragsfassung zur Eröffnung der 34. IFO in Wien vom 25.02.2020 finden Sie zum Download auf meiner Homepage: https://www.georg-feuser.com/die-buehne-der-inklusion-ein-prolog-oder-grenzgaenge-zwischenwelten/ (letzter Zugriff: 22.02.2021). 
Heimen und psychiatrischen Abteilungen unterbringen, in den Werkstätten des Sonderarbeitsmarktes unter dem Mindestlohnniveau beschäftigen, die mit ihren Sinnes- und motorischen Beeinträchtigungen ständig vor neuen Barrieren stehen oder durch die Schwere ihrer Beeinträchtigungen ihre räumliche Lage nicht selbst verändern können und zum Zweck basaler Stimulation unter irgendwelchen Mobiles platziert werden oder in Snoezelen-Räumen, exotisch beleuchtet und beschallt, eingehüllt von Düften, die aus Düsen strömen, der Aufrechterhaltung oder Wiedergewinnung der psychischen Stabilität derer dienend, die sie damit zu fördern meinen und dabei ein gutes Geschäft betreiben - also allen denen und auch jenen, die im Dunkel ihrer Lebensbühne unsichtbar geworden sind, ihnen allen möchte ich in die Augen sehen können.

Was hat sich also getan in Sachen Inklusion? Auf Ihren Schreibtischen sicher sehr viel: In den Instituten der Universitäten und Hochschulen wurden sicher Diversitätsdimensionen erwürfelt, weil es noch immer schwerfällt, zu verstehen, dass kein Mensch einem anderen je identisch ist, und doch ein jeder einem jeden wertschätzend und solidarisch verbunden sein kann, was die Kunst der Pädagogik dahingehend dimensioniert, einer jeden und einem jeden hinsichtlich der Möglichkeit seiner Persönlichkeitsentwicklung in Gewährung des dafür nötigen symbolischen pädagogischen Kapitals zu entsprechen - und das in Wahrung der Würde der*des Einzelnen und im Bewusstsein des Angewiesenseins eines jeden Menschen auf den Menschen, um diese zu keinem Lebensalter im Sinne einer ,entwicklungslogischen Didaktik' (vgl. Feuser 2011) aus ,kommunikationsbasierten Kooperationen an einem Gemeinsamen Gegenstand' (vgl. ebd.) herauslösbaren Entwicklungsprozesse durch ein gemeinschaftliches Lernen ermöglichen zu können. Was ist daran so schwer? Sicher wurden auch vielfältige Wissensfiguren erstellt; vielleicht ein vermarktbares Inklusionsschachspiel entwickelt und Praxiswelten aufgestellt und in Kombination beider vielleicht ein Inklusions-Monopoly. Von der Existenz von fächerspezifischen Inklusionskoffern für inklusiven Unterricht habe ich schon Kenntnis erhalten. Man könnte ja in Anbetracht unserer OECD-gelenkten und der Bertelsmannstiftung hörigen marktgerechten und PISA-getreuen BildungsPolitiken der Länder durchaus berechtigt auf die Idee kommen, ein börsennotiertes Bildungsimperium aufzubauen und damit eine inklusive Bildung, sofern sie es irgendwo gibt, in die Insolvenz treiben. Eine feindliche Übernahme der Inklusion durch das selektierende, ausgrenzende und segregierende Erziehungs-, Bildungs- und Unterrichtssystem (sEBU) ist ohnehin längst auf den Weg gebracht. Würde solches in der „Gesellschaft der Singularitäten“, wie sie Reckwitz (2017) beschreibt, der Steigerung der Attraktivität des eigenen Profils und der Schaffung eines Besonderen in der Vielfalt der ,Explosion des Besonderen' dienen? Oder könnte die Hoffnung auf ein Quäntchen politischer Zustimmung das Motiv dafür sein, dass die Inklusions-Community in masochistischer Weise über Jahre 
unentwegt den Prozess der Sisyphus-Arbeit der Integration der Inklusion in die Segregation betreibt? Ein solcher Anachronismus ist durchaus denkbar.

Meine Frage, was sich also getan hat in Sachen Inklusion, muss ich dahingehend präzisieren, dass es mir nicht darum geht, was sich in Sachen Inklusion für uns getan hat, für die sich damit beschäftigende akademische Elite, sondern für die Kinder, Jugendlichen und Erwachsenen, denen ich weiterhin in die Augen schauen können möchte? Bei allem, was bei mir dazu zusammenläuft, ist diese Bilanz in Relation zum verschriftlichten akademischen Inklusionsoutput nicht nur erschreckend gering, sondern tief im Minus. Ist man von sich selbst so eingenommen, berauscht und beeindruckt, um sich besser verkaufen zu können? In seiner Laudatio zur Verleihung des Friedenspreises des Deutschen Buchhandels von 2019 an Sebastião Salgado, und bezogen auf dessen fotografisches Werk, fragt Wim Wenders: „Wem geht es dabei noch um das Wohl von anderen, wer vertritt noch glaubhaft die eines Gemeinwohls? Oder wem geht es dabei nicht vor allem um sich selbst, um das eigene Image, die Unfehlbarkeit oder Glorie, eine Hybris, wie wir sie bei vielen der gerade Herrschenden zu scheußlichen Zerrbildern gesteigert sehen" (Wenders 2019, 39f.).

Heute ist alles inklusiv - die Unterrichtsfächer sind es, die Didaktik, die Methodik, die Lehrer*innenbildung. Was zum Teufel ist da inklusiv? Vermag die Erziehungswissenschaft nicht mehr, als sich Inklusion auf Arme, Beine, Brust oder Rücken zu tätowieren und sie durch die Ohren, Nasenflügel, Lippen oder Brustwarzen zu stecken? Inklusiv kann nur das Verhältnis der Menschen, Kulturen und Gesellschaften zueinander und untereinander sein - sonst nichts! Und wie wir das in den zahlreichen Feldern der Pädagogik bewältigen, ist unsere Aufgabe - Punktum! Glauben Sie, dass ein Kind inkludiert sei, wenn wir das Fach, in dem es gerade unterrichtet wird, als inklusiv etikettieren? Mit Sicherheit nicht. Wir machen uns mit solchen Begriffsgarnierungen im Feld der Wissenschaften unglaubwürdig und verursachen damit selbst, dass man im Verein mit anderen Wissenschaften eher auf der Bühne der Gaukler*innen platziert wird, wovon aber auch schnell eine Abgrenzung erfolgt, wenn es aufs System bezogen und strukturell ernst wird. Wann hören wir endlich auf, letztlich nur brauchbare "Zustimmungsfunktionäre“, „Techniker des praktischen Wissens“, „Angestellte der Herrschaft" zu sein und „Befriedungsverbrechen“ zu begehen (Basaglia \& Basaglia-Ongaro 1980) und die Funktion der Selektion, Exklusion und Zwangsinklusion von Kindern und Jugendlichen in die ihnen dann verordneten Bereiche des Erziehungs-, Unterrichts- und Bildungssystems (EBU) zu übernehmen und das wohl noch überwiegend im Glauben, damit die viel beschworene ,Chancengleichheit ${ }^{`} \mathrm{zu}$ befördern? Sie kann es aus Gründen aller zu bedenkenden Diversitätsdimensionen des Menschen und funktionsteiliger Gesellschaftsordnungen nicht geben. Deshalb ist der Begriff der Chancengleichheit total verblendend, gesellschaftspolitisch geht von ihm keine Gefahr der Strukturveränderung des EBU aus und die Auf- 
fassung, dass Inklusion im gegliederten System selektierend fraktioniert werden könnte, ist so verlockend, dass man des dahinter verdeckten Mottos: ,Jedem das Seine' nicht gewahr wird. Diese Aussage stand, ursprünglich rot gestrichen und in völliger Verkehrung des vom römischen Kaiser Justinian (um 482-565) - er kodifizierte das römische Recht in zwölf Büchern - aufgesetzten Rechtsgrundsatz ,suum cuique ${ }^{2}$, über dem Lagertor für die im KZ Buchenwald ermordeten 56.000 Juden und Jüdinnen, Kommunist*innen, Sozialdemokrat*innen, Schriftsteller*innen, Künstler*innen, Homosexuellen, Geistlichen u.a.; von den Nazis die ,Gemeinschaftsfremden' genannt. Nach der Befreiung des KZ Buchenwald auf dem Ettersberg bei Weimar, wo Goethe und Eckermann vermutlich spazieren gegangen waren, kamen die Vögel in den Buchenwald zurück, nachdem sich der Rauch der Tag und Nacht brennenden Krematoriumsöfen verzogen hatte; so berichteten Überlebende. Der erste Akt der Oper endet mit der Arie des Canio und der Aussage: „Ah - lach doch, Bajazzo, schneid tolle Grimassen, kennst kein Gefühl, bist nur ein Spielzeug zum Scherz!" (Hartmann o.J., 24).

\section{Der zweite Akt: Die Bühnen des Welttheaters}

Im Rahmen der vier mit Peter Haffner geführten Gespräche in seinem Haus in Leeds erklärte der im Alter von 92 Jahren 2017 verstorbene Soziologe Zygmunt Bauman (1925-2017), dass zu seiner Studienzeit der auf seinem Territorium souveräne Nationalstaat noch die oberste Institution gewesen war; kulturell, ökonomisch und militärisch. Dann ist die Macht aus der Politik in globalisierte Räume ausgewandert, wo transnationale Unternehmen und nicht demokratisch kontrollier- und steuerbare Konsummärkte herrschen; aber auch zu den Bürger*innen, die versuchen, gesellschaftliche Probleme auf privatem Weg zu lösen (vgl. Bauman 2017, 104), während die Beziehungen zwischen Kund*innen und Waren, wie er schon zu Beginn des Gesprächs betont, zum Muster der Beziehungen zwischen den Menschen wurden (vgl. ebd., 11). Die Menschen, für die wir Verantwortung zu übernehmen haben, nennen wir auch ,Klient*innen'. Ist, was wir ihnen zu bieten haben, dann nicht eine Ware?

Damit ist andeutungsweise beschrieben, was sich mit zunehmender Beschleunigung in den letzten 50 Jahren parallel zur Geschichte der Entwicklung der Integration bzw. Inklusion im deutschsprachigen Raum politisch, gesellschaftlich und kulturell in globaler Weise vollzogen hat - und das vor allem mit einer die Grundlagen der Demokratie und die zwischenmenschlichen Verhältnisse extrem zerstö-

2 Im Corpus iuris civilis heißt es: Die Gebote des Rechts sind diese: Ehrenhaft leben, den anderen nicht verletzen, jedem das Seine gewähren ... Siehe online unter: https://de.wikipedia.org/wiki/ Jedem_das_Seine (letzter Zugriff: 15.02.2020). 
renden Gewalt. Der staatsförmige Einfluss der Politik fokussierte sich zunehmend auf lokale Einflussfelder, unter denen in föderalistischen Strukturen sich die politische Macht, den allgemeinen Souveränitätsverlust kompensierend, verfestigte, vor allem bezogen auf das Bildungssystem. Ich erwähne nur nach Inkrafttreten der UN-Behindertenrechtskonvention in Deutschland $2009^{3}$ die Einigung der ,Ständigen Konferenz der Kultusminister der Länder in der Bundesrepublik Deutschland (KMK)' darauf, keine Strukturreform des Schulwesens zu diskutieren oder, wie jüngst in Bremen die rot-grün-rote Koalition beschlossen hat, die Grundstrukturen des Bildungssystems zu bewahren und das im Sinne eines Schulkonsenses bis 2028, was den Fortbestand des sEBU langfristig garantiert und damit die Sackgasse der Integration der Inklusion in die Segregation festschreibt. Auch die deutsche Bundesbildungsministerin, Frau Karliczek, argumentiert eindeutig dafür, Kinder in homogene Lerngruppen aufzuteilen (vgl. Lehmann \& Pauli 2019).

Einen Grund, in nostalgischer Euphorie auf den Beginn der Integrationsentwicklung in den 1970er-Jahren zurückzublicken, der durchaus mit unserer Selbstaufklärung über die Verbrechen des Nationalsozialismus, des Holocaust und der Euthanasie, den Aktivitäten der Außerparlamentarischen Opposition (APO), der 1968er-Bewegung und der großen Friedensbewegung, z.B. in Sachen der Pershing-Stationierung 1969-1983, um nur einige Momente zu benennen, zu tun hatte, gibt es nicht. Die Integrationsbewegung hat in den 1990er-Jahren ihre politische Bewusstheit verloren und steckt in einer Art politischem Wachkoma. Sie wurde im Sinne von Horst Eberhard Richter (1978) zu einem Machen, in dem nicht mehr angewendet wurde, was man erkannt hatte, weshalb man bis heute nicht zu erkennen vermag, was zu machen ist. Das charakterisiert aus meiner Sicht das Dilemma der Inklusion und erklärt zum Teil die Paradoxien, die sie hervorbringt - und das hat eine weitgehend desolate Wissenschaftskultur in Sachen Inklusion zu verantworten, die ihre Kraft und Potenziale in die Schaffung einer narzisstischen Hyperkultur investiert, in Folge die vor Ort tätigen Lehrer*innen und Fachkräfte im Stich lässt und die für die menschliche Persönlichkeitsentwicklung fundamentale Bedeutung inklusiver Lern- und Unterrichtsräume nicht an die Eltern zu vermitteln vermag und der Politik, wie angedeutet, längst zu Kreuze kriecht.

Die Inklusion - welch ein Theater - welch ein Narrativ! Eines, so nicht alle Kräfte auf eine grundlegende Transformation des sEBU in ein inklusives EBU fokussiert werden, dessen Ausgang ein dystopischer sein wird. Vielleicht, wie Reckwitz das in seiner Arbeit ,Das Ende der Illusionen“ (2019) thematisiert, kann die der

3 Siehe das Übereinkommen über die Rechte von Menschen mit Behinderungen der Vereinten Nationen, UN-BRK: online unter: http://www.bmas.de/SharedDocs/Downloads/DE/PDF-Publikationen/a729-un-konvention.pdf?_blob=publicationFile (letzter Zugriff: 22.02.2021). 
Realität entsprechende Desillusionierung auch eine Chance sein - auf Neubesinnung, Bündelung von Kräften und Potenzialen, Schaffung von Bündnissen und einer unverbrüchlichen Solidarität mit den Exkludierten und Zwangsinkludierten unseres Bildungssystems auf allen seinen relevanten Bühnen. Mir persönlich fehlt der Glaube, dass das gewollt, um- und durchgesetzt wird; aber ich irre mich gerne!

In der Oper verlagert das Vogellied der Nedda die Sehnsucht nach Ruhe und Frieden in eine Traumwelt. Diese hatte aber, wie angedeutet, als ,konkrete Utopie‘ im Sinne von Ernst Blochs (1885-1977), Prinzip Hoffnung' (1969), vor einem halben Jahrhundert über zwei, schließlich abnehmend über weitere drei Jahrzehnte hinweg, eine reale, gesellschaftsverändernde politische Gestalt und Macht, die es heute wiederzuerlangen gilt. Aber, der große Feind des Friedens in unserer Zeit ist, so Wim Wenders in seiner Laudatio zu Salgados Friedenspreis 2019, „, .. der brutale Niedergang des Mitgefühls, der Mitverantwortung, des Gemeinsinns, des grundsätzlichen Willens zur Gleichheit des Menschengeschlechts“ (2019, 42). Aber die Ursachen dieser massiven Verwerfungen in Gesellschaft und Kultur werden fast nur hinter vorgehaltener Hand diskutiert. Wozu man zu diesem Theater auf zahllosen Bühnen auch recherchiert, klafft eine gähnende Leere hinsichtlich einer Übereinkunft, im Sinne der zentralen Funktionen des Bildungssystems, in pädagogischer Einheit von Erziehung und Bildung, eine Persönlichkeitsbildung der Kinder und Jugendlichen anzustreben, die dem Aufbau tragfähiger Kommunikations- und Kooperationsfähigkeiten in Bezug auf Gemeinsinn und Solidarität einen zentralen Stellenwert einräumt. Das dürfte das wirksamste Instrument sein, diese Trends noch vor einem Point of no Return bewältigen zu können. Das erfordert, heute und jetzt, was der Begriff einer inklusiven Bildung zu umfassen vermag, pädagogisch umzusetzen.

Wolfgang Klafki (1927-2016) hat schon vor Jahrzehnten in seinen ,Studien zur Bildungstheorie und Didaktik' (1996) Bildung als demokratisches Grundrecht und Bedingung der Selbstbestimmung angesehen, nämlich als „Bildung für alle im Medium des Allgemeinen in allen Grunddimensionen menschlicher Interessen und Fähigkeiten "(ebd., 53f.; Hervorhebung im Original), die eine freie Entfaltung der Persönlichkeit mit den Zielen der ,Fähigkeit zur Selbstbestimmung' (vgl. ebd., 97f.), der ,Mitbestimmungsfähigkeit‘ (vgl. ebd.) und der ,Solidaritätsfähigkeit (vgl. ebd.) anhand der Konzentration des Unterrichts auf, epochaltypische Schlüsselprobleme' (vgl. ebd., 43ff.) zu gewährleisten vermag. Die Klafki’sche Pädagogik und Didaktik ist von mir von einer Objektwissenschaft zur Subjektwissenschaft der ,Allgemeinen Pädagogik und entwicklungslogischen Didaktik' (vgl. Feuser 2013 \& 2018) neu- und weiterentwickelt worden und vermag die Widersprüche und Paradoxien, wie sie der Inklusionismus in Feldern der Pädagogik produziert, zu überwinden und aufzuheben. Wie kann man in Anbetracht eines solchen Fundamentums der Pädagogik und Didaktik noch immer im Korsett der Fächer, in 
hierarchisch gegliederten Schulformen und Schulstufen nach ihnen zugeordneten bildungsreduktionistischen Curricula und in Jahrgangsklassen unterrichten, ohne in Verpflichtung auf den heute vorliegenden Erkenntnisstand in den Humanwissenschaften und die eigene Berufsethik gegen das selektierende, ausgrenzende, segregierende und an ständischen Vorstellungen orientierte Schulsystem gemeinsam aufzubegehren und Widerstand zu entfalten? Das ist mir eine ernste Frage. Ein zentrales - für mich das zentralste - und für einen zu erkämpfenden SystemChange über alle pädagogischen Funktionsebenen und Arbeitsbereiche hinweg überaus hemmendes Moment möchte ich mit Bezug auf Pierre Bourdieu (19302002) mit seinem Begriff des, Staatsgeistes' fassen, den er in einem Vortrag von Juni 1991, gehalten in Amsterdam, nicht als eine juristische Kategorie entfaltete, sondern bezogen auf die Mechanismen analysierte, die uns veranlassen, so zu denken, wie wir über Sachen und Menschen denken und wie deren Absicherung und Regulierung im Sinne des Staates als Allgemeinheit erfolgt. Dies auch bezogen auf Aspekte des ,Habitus', mit dem er sozialisierte Wahrnehmungs-, Denk- und Handlungsweisen begrifflich fasst, mit denen er unsere Unterwerfung unter die bestehende Ordnung als „Produkt der Übereinstimmung zwischen den kognitiven Strukturen, die dem Körper durch die Geschichte kollektiv (phylogenetisch) und individuell (ontogenetisch) in Fleisch und Blut übergegangen sind, und den objektiven Strukturen der Welt, auf die sie angewendet werden" (Bourdieu 1998, 118), beschreibt. Dies sind nicht Formen des Bewusstseins, sondern „Dispositionen des Körpers“ (ebd.) und auch keine „bewusste Zustimmung zu einer Ordnung bzw. Anordnung“ (ebd.). Er spricht von einer „doxischen Unterwerfung“ (ebd., 119), die „uns mit allen Fasern des Unbewußten an die bestehende Ordnung bindet“ (ebd.); auch von „inkorporierten kognitiven Strukturen“ (ebd., 120), die dazu führen, dass die "Sichtweise der Herrschenden (...) sich als die allgemeine Sichtweise darstellt" (ebd., 121); eben auch als die unsere. Die Hauptmacht des Staates sieht er darin, die Denkkategorien zu produzieren und durchzusetzen, die wir spontan auf jedes Ding der Welt und auch auf den Staat selbst anwenden (vgl. ebd., 93) - und das vor allem mit Hilfe des Bildungssystems. Im Grunde sind gerade wir als Forschende und Lehrende in allen Systembereichen der Pädagogik dieser Indoktrination lebenslang nie entronnen, von ihr wie ein Schwamm bis zur Sättigung durchtränkt, wir verhalten uns wie Inklusionsbürokrat*innen. Der in uns gedrungene Staatsgeist ist unser Lebenselixier und jeder Gedanke und Versuch, ihn kritisch zu hinterfragen, verbindet sich unbewusst mit dem Gefühl der Bedrohung, es zu verschütten oder gar zu verlieren. Allein der Gedanke einer kritischen Infragestellung des hierarchisch gegliederten und ständisch organisierten Schulsystems ist eine Art Tabubruch, der Ängste auslöst, weil der ,Herr' seine vermeintlich schützende Hand über uns zurückziehen oder uns bestrafen könnte. Diese Bedrohung scheint derart groß, dass wir jene, für die wir diese Profession gewählt haben, entgegen allen heute in den Humanwissenschaf- 
ten vorliegenden Erkenntnissen, in den sensibelsten Phasen ihrer Persönlichkeitsentwicklung einem Bildungs- und insbesondere einem Schulsystem ausliefern, das alle Werte, die wir in unserer Kultur für staatstragend erachten, konterkariert, ja sie pervertiert:

- das Soziale durch einen Gemeinsinn und Solidarität zerstörenden egomanen Leistungsbegriff,

- das Liberale durch ein Gespinst an Gesetzen, Verordnungen und Vorschriften, die jedes Detail der Organisation des Systems regeln, bis hin zu wer, was, wann, wo, wie schnell zu lernen und zu reproduzieren hat,

- das Christliche durch eine konkurrente Vernichtung der Empathie den Mitmenschen gegenüber und

- das Demokratische schließlich durch die Strukturvorgaben eines selektierenden, ausgrenzenden, mithin exkludierenden und segregierend-inkludierenden Schulsystems, das eine ständische Funktionsweise hoch favorisiert, die dem Anspruch einer Bildungsgerechtigkeit fundamental widerspricht.

Wir nehmen also hin, Generation für Generation psychisch zu verletzen und zu traumatisieren und sie mit den vorherrschenden Unterrichtsformen und Curricula bezogen auf die heute von der Menschheit zu bewältigenden ökologischen Aufgaben der Sicherung des Planeten Erde als Lebensraum für Menschen und der - auch damit verbundenen - ökonomischen Herausforderungen einer menschenwürdigen Verteilungsgerechtigkeit der Güter und Finanzen blind zu machen und dumm zu halten - und diese Auflistung ließe sich lange fortsetzen. Bude (2010) fasst das in seiner Arbeit über das Ende des Traums von einer gerechten Gesellschaft in Bezug auf Kinder und Jugendliche so zusammen: „Was sie können, braucht keiner, was sie denken, schätzt keiner, und was sie fühlen, kümmert keinen“" (ebd., 15) und das bringen wir dann unter den Begriff der Pädagogik.

Ein zweites Moment, seit wir im deutschsprachigen Raum mit der Integration begonnen haben, ist die in Fluss gekommene Umstrukturierung der westlichen Gesellschaften. Das dürfte kein Zufall sein und ist auch seitens der Inklusion bislang unerforscht geblieben. Was heute unübersehbar ist, war damals in seinen sich zunehmend deutlicher artikulierenden Anfängen - außerhalb der Diskurse im Rahmen der Entwicklung der ,kritischen und materialistischen Behindertenpädagogik' - nie hinreichend im Blickfeld der Pädagogik. Auch hat die sich dramatisch beschleunigende ,Flüchtige Moderne', wie sie Zygmunt Bauman (2003) beschreibt, mit der ,jede Art von Verbesserung nicht mehr kollektiv, sondern nur mehr individuell gedacht und bewertet wird" (ebd., 159), den Blick dafür verstellt, dass nicht Gleichheit und eine Art klassenlose Gesellschaft jeder und jedem alles ermöglicht, wenn sie*er nur ihren*seinen Fleiß und ihren*seinen Geist zu nutzen vermag, um ein befriedigendes Niveau zu erreichen und alles hinter sich zu lassen, was missfällt, wie er weiter schreibt (vgl. ebd.), sondern zu neuen 
Klassenbildungen geführt, die ihrerseits, wer u.a. eben Fleiß und Geist nicht wie gefordert zu nutzen vermag, brutal ausgrenzen. Andreas Reckwitz beschreibt das in seiner Arbeit ,Das Ende der Illusionen' (2019) auf der Basis seiner vorausgegangenen Analysen zur, Gesellschaft der Singularitäten und zum Strukturwandel der Moderne' (2017) in aller Deutlichkeit. Um es knapp zu skizzieren, entwirft er ein auf dem Hintergrund einer tief gespaltenen Gesellschaft der ,Hyperkultur ${ }^{`}$ und des ,Kulturessenzialismus` orientiertes Bild der gegenwärtig sich stark artikulierenden Klassen.

Die Hyperkultur bezeichnet - im Gegensatz zur Hochkultur des Bildungsbürgertums und einer eher homogenen Massenkultur der Nachkriegszeit - die Bezugnahme auf die Pluralität gesellschaftlicher Güter, die auf globalen Märkten zirkulieren und den Individuen Ressourcen für ihre Selbstverwirklichung zur Verfügung stellen, wobei letztlich alles in höchst variabler Weise als Gegenstand von Wert valorisiert werden kann - oder eben abgewertet wird (vgl. ebd., 36). Dies bei einer relativen Offenheit kultureller Praktiken und Güter, in Kontexten einer liberalen Kulturpolitik der Diversität und des Globalismus, meist zentriert in großen urbanen Zentren und basiert vor allem auf einem exzessiven Konsum unter Ausbeutung anderer Länder und Menschen und um den Preis der Zerstörung unserer Erde. Reckwitz (2019) spricht von einem Konsumentenkapitalismus (vgl. ebd., 85).

Der Kulturessenzialismus bildet nach Reckwitz eine Art Gegenfront fundamentalistischer Strömungen, deren Ausgangspunkt die kollektive Identität einer Gemeinschaft im Sinne eines Kommunitarismus ist (vgl. ebd., 42). Er schreibt: „Kultur erscheint als eine Essenz, und Zeit und Raum, also die Geschichte und der Herkunftsort, sind zwei wichtige Pfeiler, auf denen diese Kultur des Eigenen beruht“ (ebd., 44). In ihnen sammeln sich u.a. Personengruppen, die als „Modernisierungsverlierer" wahrgenommen werden und das sind zu erheblichen Anteilen auch Personen aus der alten Mittelklasse und nicht, wie fälschlicherweise gerne gedacht wird, jene aus dem Prekariat. „Die sich als unterlegen Fühlenden versuchen sich über eine kollektive Identität Überlegenheit zuzuschreiben und zu sichern" (ebd., 46). Ich denke, Ihre Gedanken dazu finden leicht Verankerungen in dem, was sich täglich in unserer Gesellschaft an Ereignissen spiegelt und sich u.a. in Wähler*innenverschiebungen nach rechts bis ins Rassistisch-Faschistoide niederschlägt und, last, but not least, unsere Verhaltensweisen mitbedingt.

Gerahmt von diesen Prozessen und jenseits der die Hälfte des Weltvermögens besitzenden Oberklasse, die, ohne zu arbeiten, sich alles zu leisten vermag, vollzog sich die Herausbildung einer Drei-Klassen-Kultur. Deren Transformation aus der alten Mittelklasse sieht er verursacht (a) durch die Ökonomie der Spätmoderne, die nur noch in geringem Umfang jene Arbeitsplätze zur Verfügung stellt, die den Wohlstand der Mittelklasse der industriellen Moderne (bis zu den 1970er-Jahren) sicherten, (b) durch die seit den 1970er-Jahren stattfindende Bildungsexpansion, 
bei dem es sich aber, nicht wie man annehmen könnte, um einen Prozess handelt, von dem jede und jeder profitiert und (c) durch den kulturellen Wertewandel. Reckwitz klassifiziert die neue Mittelklasse dominant als Akademikerklasse und als Trägerin der Bildungsexpansion sowie der Postindustrialisierung und Wissensökonomie. Wer diese nicht zu erreichen vermag, gerät in der postindustriellen Wirtschaft mit Niedriglohnsektoren und Unterbeschäftigung in die prekäre Klasse von Bildungsverlierern. Die gleichen Mechanismen - und das bitte ich Sie aufmerksam zur Kenntnis zu nehmen -, die gleichen Dynamiken der Postindustrialisierung und Bildungsexpansion, die die neue Mittelklasse aus der alten emporheben, treiben eine neue prekäre Klasse aus der alten Mittelklasse heraus (vgl. ebd., 87). Die alte Mittelklasse, belegt mit den Aussagen, dass sie alternativlos und Träger der gesellschaftlichen Hegemonie sei, gerät in Angst, Panik und Wut ob des Verlustes ihrer alten hegemonialen Bedeutung und Macht. Sie agiert entsprechend vor allem gegen die neue Unterklasse und eröffnet den Kampf gegen die Armen und nicht gegen die Armut. Das kulturelle Kapital (Bourdieu) der Hochqualifizierten, oft nur in formalen Bildungsabschlüssen repräsentiert, verwandelt auch die Erziehung und Bildung zu einer essenziellen Aufgabe, die mit Rankings, Kompetenzrastern und neuen Standardisierungen alles Menschliche, das mit Lernen und Entwicklungsprozessen zu tun hat, einer Quantifizierung unterzieht; auch das Soziale (vgl. Mau 2017). Das schafft, so Reckwitz (2019, 103), „paradoxe Negativeffekte". Damit driften die Entwicklungen des EBU mit gesteigerter Dynamik, von uns intensiv mitbewirtschaftet, in eine Singularitätspädagogik, die eben nicht ein individualisiertes Lernen im Kollektiv anstrebt, sondern ein individualistischegomanes in Konkurrenz einer*eines jeden zu einer*einem jeden. $\mathrm{Zu}$ welchen die Gesundheit der Kinder beeinträchtigenden Auswirkungen das führt, zeigen der Medikamentenkonsum, um die psycho-somatischen Folgen des Schulstresses unter Kontrolle zu bringen, der erschreckend hohe Anteil von im psychologischen Sinne behandlungsbedürftigen Schüler*innen, aber auch die Ausgaben, die Eltern, die ihrerseits dem Staatsgeist verhaftet sind, für Nachhilfe - in Deutschland jährlich rund 900 Millionen Euro ${ }^{4}$ - und außerschulische Bildungsangebote aufwenden, um nur drei Dimensionen zu benennen. ${ }^{5}$ Das ist nahezu eine Milliardenaussage über den desolaten Zustand schulischen Unterrichts. Hätten wir uns nicht dafür zu schämen, zumal die Ungleichheit dadurch noch weiter befördert wird? Es ist an der Zeit, alle Kräfte konzertiert auszurichten und viele Bereiche des

4 Siehe online unter: https:/www.bertelsmann-stiftung.de/de/themen/aktuelle-meldungen/2016/januar/ eltern-geben-jaehrlich-rund-900-millionen-euro-fuer-nachhilfe-aus/ (letzter Zugriff: 15.02.2020).

5 Siehe zu diesem Komplex die Sendung ,Kontext' des SRF2 vom 06. Juni 2016 mit der Thematik ,Nachhilfe'. 
akademischen, pseudoinklusiven und unterrichtspraktischen Unsinns, den wir machen, endlich aufzugeben. ${ }^{6}$

Die prekäre Klasse ist in der soziologischen Literatur, die wir vielleicht doch endlich in den Kernbestand pädagogischen Wahrnehmens, Denkens und Handelns aufnehmen sollten, schon lange als, ausgeschlossene', ,menschlicher Abfall' und ,überflüssige' (vgl. Bauman 2005; Bonet 2013; Trojanow 2015) kategorisiert. Dazu noch einmal Zygmunt Bauman (2003): „In prekären ökonomischen und sozialen Verhältnissen wird den Menschen beigebracht (oder sie lernen es möglicherweise auf die harte Tour) [und ich füge ein: auch in der Schule; Anm. G.F.], die Welt als einen Container voller Wegwerfobjekte, zum einmaligen Gebrauch bestimmt, zu verstehen - die ganze Welt, einschließlich anderer Menschen“ (ebd., 191; Hervorhebung im Original). Die längst ausrangierten und weggeworfenen Familien etikettieren wir dann in einer mir unerträglich abgehobenen und pseudowissenschaftlichen Weise als ,bildungsferne Familien' und machen sie für die Lernprobleme ihrer Kinder verantwortlich - ist doch in Wirklichkeit die Bildung diesen Menschen fern ... eine Schande!

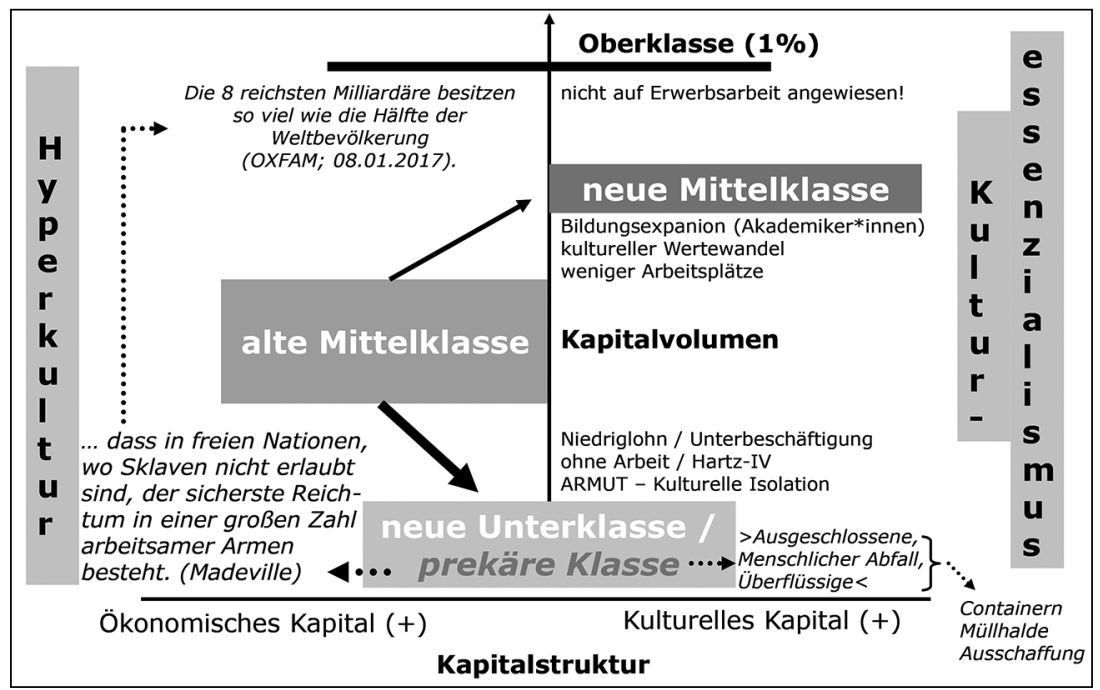

Abb. 1: Die Drei-Klassen-Struktur nach Reckwitz (2019, 86) [Ergänzungen G.F. in kursiv und gepunkteten Linien]

6 Ich meine damit z.B. vor allem empirische Studien, deren Fragestellung, würde man vor Ort gehen und die Methode der Verhaltensbeobachtung und -analyse beherrschen, nach einer Hospitationsstunde schlüssig beantwortet werden könnte; auch qualitative Studien, die sich mit der Darstellung ihrer Methode und Ergebnisse begnügen, wenn sie nur gerechnet und grafisch schön dargestellt sind, aber dort enden, wo es interessant werden könnte - nämlich hinsichtlich von Analysen und Schlussfolgerungen bezogen auf die Weiterentwicklung der Integration/Inklusion im Interesse der betroffenen Kinder, Schüler*innen, Lehrpersonen und Eltern. 
Auch wir haben uns die Frage zu stellen: Wer sind wir? ${ }^{47}$, mit der Ernst Bloch (1969) sein großes Werk ,Das Prinzip Hoffnung' einleitet. Wo befinden wir uns in den Strömen und Wirbeln der fließenden Gegenwart? An welchen Strohhalmen eines ausgedienten und selektierend-elitären, an der hegemonialen, alten Mittelklasse orientierten und von der Oberklasse gesteuerten Bildungssystems halten wir uns auf Kosten der uns in der Pädagogik allgemein und in besonderer Weise in den Bildungsinstitutionen anvertrauten Kinder und Jugendlichen fest? Von welcher Bühne aus betrachten und bewerten wir das Welttheater? Welche Ängste und gesinnungsmäßigen Borniertheiten hindern uns, die Turbulenzen gesellschaftlicher Veränderungen als Chance zu sehen und zu nutzen, um aktiv mit den Kindern ein bildungsgerechtes EBU zu schaffen? Wie lange wollen wir noch Kinder zu Nutzen einer kapitalistischen Raubwirtschaft eines nicht nur noch immer, sondern in neuer Weise rassistisch basierten imperialistischen Kolonialismus der weißen Herrenmenschen ausbilden, der noch immer Urstände feiert und sich materiell - z.B. in jedem Smartphone oder iPad, das wir in Händen halten - vergegenständlicht? Reckwitz (2019) resümiert: „Die spätmoderne Gesellschaft ist keine Gemeinschaft, kein homogenes Kollektiv und wird es auch niemals sein. Sie ist in Lebensstilen pluralisiert, in Klassen stratifiziert und multiethnisch. Die Herausforderung liegt vielmehr in der Konstitution eines gesellschaftlich Allgemeinen, das sich inmitten der sozialen Unterschiede und kulturellen Heterogenitäten zu behaupten vermag" (ebd., 290; Hervorhebung im Original).

Entsprechend geht es um die Schaffung eines pädagogisch Allgemeinen, das im Sinne einer Bildungsgerechtigkeit sich nicht in ständischer Manier von sozialen Unterschieden leiten lässt, wie sie mit der ,Allgemeinen Pädagogik‘ seit Jahrzehnten vorliegt (vgl. Feuser 2018) und die der so viel beschworenen Heterogenität in einer Schule für alle gerecht zu werden und aufgrund ihrer Potenzialität zur Persönlichkeitsbildung zu überzeugen und sich zu behaupten vermag. Es geht um nicht mehr und weniger als um die Gestaltung eines humanen und demokratischen Bildungssystems! Dafür werden wir Verantwortung zu übernehmen haben - jede und jeder von uns - und uns endlich dem Staatsgeist gegenüber als mündig erweisen müssen, indem wir uns auf dem Hintergrund einer nun viereinhalb Jahrzehnte langen Entwicklung der Integration resp. Inklusion im deutschsprachigen Raum endlich einmal unseres Verstandes ohne die obrigkeitsstaatliche Anleitung und ordnungsstaatlichen Vorgaben bedienen. Wenn nicht an den Universitäten und Hochschulen in Forschung und Lehre - wo dann?

7 Damit verweise ich auf den Film: ,Wer sind wir?" des Regisseurs Edgar Hagen. Er thematisiert u.a. auch die Problematik der Inklusion von Menschen, die bislang selbst in den Diskursen um Inklusion exkludiert blieben. Siehe online unter: https://www.cineman.ch/movie/2019/WerSindWir/ (letzter Zugriff: 01.03.2020). 


\section{Der Schluss: Das Spiel ist aus!?}

„Der postmoderne Neoliberalismus ist ein kalter und düsterer Ort, an dem persönliche Güte und die Fürsorge für andere einen zum Verlierer machen. Die Logik des Prekariats besteht nicht nur aus Ausbeutung und Entfremdung wie im klassischen Kapitalismus. Sie läuft auf eine groß angelegte Zerstörung des Sozialen hinaus", so Rendueles $(2018,236)$ in seinem Buch über den Kanaillen-Kapitalismus. „Doch das verwandelt ihn [den Menschen; Anm. G.F.] nicht in einen Proletarier, sondern in orientierungslose Vasallen“ (ebd., 76). „Aus dem Gesagten wird klar, dass in einer freien Nation, wo Sklaven nicht erlaubt sind, der sicherste Reichtum in einer großen Zahl arbeitsamer Armen besteht" (Madeville 1988, 274 zit. nach Rendueles 2018, 78). Am deutlichsten drückt sich das in Deutschland in der Hartz-IV ,Reform ' der rot-grünen Koalition von 2005 aus, durch die sich allein die Kinderarmut beinahe verdoppelt hat. „Die eigentlichen Profiteure der rot-grünen Arbeitsmarktreform“, so Butterwegge (2019, 5), „waren jedoch Unternehmen auf der Suche nach Arbeitskräften, die möglichst billig, willig und wehrlos sein sollten" - damit die Reichen noch reicher werden können. Unterricht, der in Fächern isoliert abrichtet und dadurch komplexe Zusammenhänge ausblendet, anstatt in Projekten zu arbeiten, die auf Erkenntnisgewinn und Analysefähigkeiten gerichtet sind, aus denen sich das erforderliche Wissen generiert, das dann auch verstanden werden kann, stellt die billigen und wehrlosen Arbeitskräfte zur Verfügung. So werden die Reichen noch reicher und die Armen noch zahlreicher. Kommt es dann noch zur unleidigen Verknüpfung der Sozial- mit der Migrationspolitik, wird der ,Feldzug' gegen die Armen weiter perfektioniert. Das nennt sich Demokratie!

Ich lese in der Zeitung: „Absaufen! Absaufen!', skandiert der Mob, als bei einer fremdenfeindlichen Demonstration in Dresden Fernsehbilder von in Seenot geratenen Migranten gezeigt wurden. Ausländer, ,Asylanten', Flüchtlinge seien unerwünscht, schreit die rechtspopulistische ,Alternative für Deutschland', und in der aufgewiegelten Bevölkerung dröhnt ein vieltausendstimmiges Echo" (Grill 2020, 1).

,Mob', ,Rechtspopulismus' - sind das nicht die Wirklichkeit der gesellschaftlichen Verwerfungen verharmlosende Begriffe, Begriffe der Distanz und Ausgrenzung, anstatt der längst überfälligen Kommunikation? Und sind nicht die, die wir ,Mob' und ,Rechtspopulist*innen' nennen, durch unsere Schulen, Hochschulen und Universitäten gegangen? Und wo sind wir mit einem vieltausendstimmigen inklusiven gesellschaftlichen Gegenentwurf? Hegen wir, wer auch immer im gängigen sEBU unsere Erwartungen und pädagogisch geheiligten und geadelten Vorschriften verletzt, nicht in Modelle kolonial geprägter Weltbilder in rassistischer Weise in unsere gelobten bildungshierarchischen Institutionen - und Sonderinstitutionen - ein? Wann kapieren wir endlich, dass jede Form von Ausgren- 
zung auf Unrecht beruht? Wann erfassen wir endlich, wie Enrique Dussel (2013) das in seinen ,20 Thesen zur Politik' akribisch analysiert, dass Institutionen, wie er begründet, zwar notwendig sind, dies jedoch nicht bedeutet, dass sie deshalb ewig, immerwährend und unveränderlich wären. „Das, worauf es ankommt, ist $\mathrm{zu}$ wissen, wann eine Institution weiterarbeiten soll, wann eine partielle, oberflächliche, tiefgreifende Transformation oder auch eine völlige Veränderung der partikularen Institution oder selbst des ganzen institutionellen Systems notwendig ist" (ebd., 134). Haben wir uns damit schon hinreichend befasst? Oder haben wir die Bühnen des Welttheaters schon verlassen - oder sie möglicherweise in unseren Gedankenwelten noch nicht einmal betreten? Es kann sogar Letzteres angenommen werden.

Nehmen wir die Masken ab und zeigen wir uns als die, die wir wirklich sind und stellen uns einem streitbaren Dialog! Denn (und das dürfte das höchste Gut sein): „Es kann keinen Frieden ohne soziale Gerechtigkeit und ohne Arbeit geben, es kann keinen Frieden ohne Anerkennung der Menschenwürde geben, und ohne die Beendigung der unnötigen Zustände von Armut und Hunger und es kann keinen Frieden geben, ohne dass wir die Schönheit und Heiligkeit unserer Erde achten“, so Wenders $(2019,35)$ an Salgado. Köhlmeier (2018, 8; Hervorhebung im Original) stellt fest: „Zum großen Bösen kamen die Menschen nie mit einem großen Schritt, sondern mit vielen kleinen, von denen jeder zu klein schien für eine große Empörung. Erst wird gesagt, dann wird getan.“

Auch wenn heute, wie auch Zygmunt Bauman (2017) feststellt, kaum ein*e Politiker*in, Unternehmer*in oder sonstige Machtträger*innen auf Intellektuelle hören, gilt es, unsere Stimme sach- und fachbezogener, deutlicher und kritischer denn je zu erheben; Ihre Stimme, die Stimme einer jeden und eines jeden. Im Interview mit Peter Haffner sagte Zygmunt Baumann (ebd., 117): „Wenn ich in Kürze sterben werde, weil ich ein sehr alter Mann bin, werde ich unerfüllt und unglücklich sterben. Weil es eine Frage gibt, mit der ich gerungen habe, um eine überzeugende Antwort zu finden, und mir das nicht gelungen ist. Ich weiß, dass ich die Antwort nicht mehr finden werde, ich habe keine Zeit mehr. Die Frage ist sehr simpel: Wie macht man die Welt frisch?" ${ }^{\text {"8 }}$ Vielleicht bedarf es dazu, wie Paul Mason (2019) fordert, einer „radikalen Verteidigung des Humanismus“.?

8 Mit diesem Begriff bezieht sich Zygmunt Bauman auf das Neue Testament und auf die Offenbarung des Johannes: „Seht, ich mache alles neu“. Damit stellt sich die Frage, wie man das Wort in Taten verwandelt, wozu er keine einzige Antwort gefunden habe, obwohl er diese Frage heute für dringlicher hält denn je. Das erinnert auch an die Aussage von Karl Marx in seinen, Thesen über Feuerbach'von 1845, dass die Philosoph*innen die Welt nur verschieden interpretiert haben, es aber darauf ankommt, sie zu verändern. Siehe online unter: https:/www.projekt-gutenberg.org/marx/feuerbac/feuerbac.html (letzter Zugriff: 21.01.2021).

9 Ein Nachtrag: Im Nachgang zu meinen Ausführungen kam es zu einem von der Tagungsplanung nicht vorgesehenen Workshop. Dessen Ausgangspunkt war der Bericht zum Schulversuch 


\section{Ob das Welttheater der Inklusion so ausgeht wie die Oper, wird auch an Ihnen liegen, an allen von uns! ${ }^{10}$}

\section{Literatur}

Basaglia, F. \& Basaglia-Ongaro, F. (1980): Befriedungsverbrechen. In: Basaglia, F. \& Basaglia-Ongaro, F. (Hrsg.): Befriedungsverbrechen. Über die Dienstbarkeit der Intellektuellen. Frankfurt am Main, $11-61$.

Bauman, Z. (2003): Flüchtige Moderne. Frankfurt am Main.

Bauman, Z. (2005): Verworfenes Leben. Die Ausgegrenzten der Moderne. Hamburg.

Bauman, Z. (2017): Das Vertraute unvertraut machen. Ein Gespräch mit Peter Haffner. Hamburg.

Bloch, E. (1969): Das Prinzip Hoffnung. Drei Bände. Frankfurt am Main.

Bonet, A. (2013): Die Würde des Mülls. Berlin.

Bourdieu, P. (1998): Praktische Vernunft. Zur Theorie des Handelns. Frankfurt am Main.

Bude, H. (2010): Die Ausgeschlossenen. Das Ende vom Traum einer gerechten Gesellschaft. München.

Butterwegge, C. (2019): Deutschland nach Hartz IV: Zwei Perspektiven. In: Beilage zur Wochenzeitung „Das Parlament“, 69, H.44-45 vom 28.10.2019, 4-7.

Dussel, E. (2013): 20 Thesen zur Politik. Berlin.

Feuser, G. (2011): Entwicklungslogische Didaktik. In: Kaiser, A., Schmetz, D., Wachtel, P. \& Werner, B. (Hrsg.): Didaktik und Unterricht. Band 4 des Enzyklopädischen Handbuchs der Behindertenpädagogik: Behinderung, Bildung, Partizipation. Stuttgart, 86-100.

Feuser, G. (2013): Die „Kooperation am Gemeinsamen Gegenstand“ - ein Entwicklung induzierendes Lernen. In: Feuser, G. \& Kutscher, J. (Hrsg.): Entwicklung und Lernen. Stuttgart, 282-293.

Feuser, G. (2018): Wider die Integration der Inklusion in die Segregation. Zur Grundlegung einer Allgemeinen Pädagogik und entwicklungslogischen Didaktik. Berlin.

Grill, B. (2020): Bröckelnde Amnesie. In: Das Parlament, 70, H.2-3 vom 06.01.2020, 1.

Hartmann, L. (o.J.): Der Bajazzo. London.

Klafki, W. (19965): Neue Studien zur Bildungstheorie und Didaktik. Weinheim/Basel.

Köhlmeier, M. (2018): Erwarten Sie nicht, dass ich mich dumm stelle. Reden gegen das Vergessen. München.

Primus-Schule (= Primarstufe und Sekundarstufe I; Schuljahre 1-10 in Mehrstufenklassen) in NRW, der Gefahr läuft, politisch beendet zu werden, ehe er sich voll entfalten konnte. Seitens der Wissenschaft fühlt man sich im Stich gelassen. Dies auch, weil die Ergebnisse wissenschaftlicher Studien oft nicht hinsichtlich ihrer Bedeutung für die Entwicklung einer entsprechenden Unterrichtspraxis weitergedacht werden. Meinerseits warf ich die Frage auf, ob sich Forschung allein daraus legitimieren kann, dass sie der Methodologie nach heutigen Standards Rechnung trägt. Ich betonte mein Wissenschaftsverständnis dahingehend, dass Wissenschaft Wissen zu schaffen habe, das den Menschen Aufklärung und die Verbesserung ihrer Lebensweisen ermöglicht; nicht zuletzt, weil sie seitens öffentlicher Mittel und Steueraufkommen finanziert wird, was nicht negiert, dass sie als solche unabhängig zu bleiben hat. Die aufgeworfenen Fragen blieben in diesem Kreis unbeantwortet. Es zeigte sich aber, dass ein erheblicher Bedarf an Diskursen zu Grundsatzfragen in Kontexten der Inklusionsforschung besteht, die praktisch nicht geführt wurden und werden.

10 Die Oper ,Der Bajazzo` endet damit, dass Canio, der Bajazzo, seine Frau Nedda und Silvio tötet. Taddeo, der zu Beginn der Oper den Prolog vorgetragen hat, beendet die Oper mit den Worten: „Geht ruhig heim - das Spiel ist aus ...!"Wäre das für eine wirkliche Inklusion mit einem Ausrufeoder einem Fragezeichen zu versehen? 


\section{Georg Feuser}

Lehmann, A. \& Pauli, R. (2019): Das passt überhaupt nicht zu unserem Ziel. Interview mit der Bundesbildungsministerin Anja Karliczek. In: TAZ am Wochenende vom 07./08. Dezember 2019, 04 Politik.

Mason, P. (2019): Klare, lichte Zukunft. Eine radikale Verteidigung des Humanismus. Berlin.

Mau, S. (2017): Das metrische Wir. Über die Quantifizierung des Sozialen. Berlin.

Reckwitz, A. (2017): Die Gesellschaft der Singularitäten. Berlin.

Reckwitz, A. (2019): Das Ende der Illusionen. Politik, Ökonomie und Kultur in der Spätmoderne. Berlin.

Rendueles, C. (2018): Kanaillien-Kapitalismus. Frankfurt am Main.

Richter, H.-E. (1978): Engagierte Analysen. Reinbek bei Hamburg.

Salgado, S. (2019): Ansprachen aus Anlass der Verleihung des Friedenspreises des Deutschen Buchhandels. Frankfurt am Main.

Trojanow, I. (2015): Der überflüssige Mensch. München.

Wenders, W. (2019): Kann Photographieren ein Akt des Friedens sein? In: Salgado, S. (Hrsg.): Ansprachen aus Anlass der Verleihung des Friedenspreises des Deutschen Buchhandels 2019. Frankfurt am Main, 29-43. 


\section{Mai-Anh Boger}

\section{Risse in der Landschaft der Inklusionsforschung - Aktuelle Entwicklungen und offene Fragen}

Vorliegender Beitrag fokussiert verblasste Punkte in der Theorie- und Forschungslandschaft der Inklusionspädagogik. In seinem Vorgehen geht er zurück auf die Theorie der trilemmatischen Inklusion (vgl. Boger 2019a), die sich als eine Kartenarbeit im Sinne einer rhizomatischen Topographie (vgl. Deleuze \& Guattari 1977 ) versteht. Verzeichnet werden darin unterschiedliche Verständnisse von ,Inklusion' als einem emanzipatorischen Projekt, das gegen Unterdrückung und Diskriminierung arbeitet. Auf diese Weise wird ein transdisziplinäres Geflecht assembliert, das Konzepte aus verschiedensten emanzipatorischen Bewegungen und disziplinären Diskursen versammelt. Es wird daher auch dazu eingeladen, durch Analogien eine interdisziplinäre Vermittlung zu stiften, in der aufscheinen kann, was die Behinderten- und Inklusionspädagogik zum Beispiel von den Frauenstudien/Gender Studies und den Critical Race/Postcolonial Studies lernen kann. Viele der dort beschriebenen Sackgassen und Wendepunkte betreffen nämlich jedes akademische Feld, das sich einer unterdrückten/diskriminierten Gruppe widmet. Aus der Geschichte der Nachbardisziplinen lässt sich daher etwas darüber lernen, welche Problemstellungen vielleicht auch im eigenen Feld im Kommen sind. Im Fokus dieses Beitrags steht nicht eine erneute Einführung in die trilemmatische Kartentheorie, sondern die Benutzung dieser Karte, um Vorhersagen zu treffen, die sich aus der gesellschaftstheoretischen Analyse und dem interdisziplinären Vergleich ergeben. ${ }^{1}$ Aus der Karte lassen sich Hinweise auslesen, welche Pfade in Zukunft beschritten werden könnten bzw. welche Sackgassen derzeit am Horizont erscheinen.

Wissenschaftstheoretisch gedacht funktioniert eine solche Vorhersage freilich nicht wie eine statistische Vorhersage eines Erwartungswerts im positivistischen Sinne. Vielmehr handelt es sich um eine Form des ,eingreifenden Denkens' (sensu

1 Dies geschieht auch deshalb, da in dem Keynote-Vortrag, Grenzgänge zwischen Generationen und durch das Rad der Geschichte - Zur trilemmatischen Kartographierung einer neuen/alten Generation Inklusionsforschung' vom 27.02.2020, auf den dieser Beitrag zurückgeht, der andere Weg gewählt und auf spontanen Antrag hin nochmals eingeführt wurde. Im Schriftlichen ist dies nicht nötig, da man die Grundlagen vielerorts bereits nachlesen kann. Dieser Beitrag holt daher jenes nach, was im Februar 2020 nicht gesagt werden konnte. Absatz 4 zu gängigen Aporien der Forderung nach zugänglicherer/leichterer Sprache kann durchaus im Widerhall darauf gelesen werden. 
Brecht), die selbst versucht, auf das Zukünftige Einfluss zu nehmen und es mitzugestalten. Statt von einem objektiven oder neutralen Beobachter*innenstandpunkt auszugehen, ist jede Kritik im rhizomatischen Kartenmodus eine immanente, die selbst in dem Feld situiert ist, das sie beobachtet. Für eine Prognose im positivistischen Sinne ist sie daher viel zu involviert (und motiviert). Viel eher liefen diese ,Prognosen' im Falle des Eintretens auf selbsterfüllende Prophezeiungen hinaus: $\mathrm{Ob}$ der gebotenen Kürze werden exemplarisch sechs solcher Sackgassen bzw. Trendwenden kurz angerissen; und sollte sich jemand über eine dieser sehr kurzen Überpointierungen 40.000 Zeichen lang auslassen wollen, gibt es wieder einen Diskussionsbeitrag mehr zu den hier, prognostizierten' Themen der Zukunft. Diese Form der Prognostik hat demnach spielerischen oder schelmischen Charakter: Sie gibt offen zu, zu wollen, was sie, vorhersagt'. Sechs markante Weggabelungen sollen nun in diesem Modus ausgeführt werden - in der Hoffnung, dass man sich über diese Verkürzungen sehr lange und in größter Ausführlichkeit ärgern wird, eben weil es wichtige Fragen der Zukunft sind, die man viel genauer und differenzierter betrachten müsste.

\section{Umkämpfte Grundbegriffe und leere Signifikanten}

Es gibt eine latente Fragestellung, die der im Folgenden dargelegten Analogienkette zugrunde liegt und die sich durch diesen Aufsatz zieht wie ein heimlicher roter Faden: „Sollte ,Behinderung“ ein Grundbegriff unserer Disziplin sein?“ Ich schreibe dabei ganz diffus , unsere Disziplin`, da die Bezeichnung ,Behindertenpädagogik die Frage schon beantworten würde - jedenfalls dann, wenn es einem als wenig sinnig erscheint, eine Disziplin nach etwas zu benennen, das kein Grundbegriff derselben sein soll. Die analoge Frage der Frauenstudien lautete entsprechend: „Sollte ,Frau' (und/oder, Weiblichkeit') ein Grundbegriff unserer Disziplin sein?“. Wie kam es von dieser Frage ausgehend zu jenem Feld, das den meisten Menschen heutzutage unter dem Namen ,Gender Studies' (und eben nicht mehr unter dem Namen ,Frauenstudien') bekannt ist?

Zwei Punkte will ich hier herauspicken: Erstens geht es um die Tatsache, dass jene, die durch dekonstruktive Manöver zu Unbenannten und Unversammelten werden, außerhalb der Disziplin, die sich mit dieser Dekonstruktion befasst, im öffentlichen Diskurs zumeist schlichtweg vergessen und unsichtbar gemacht werden. Zweitens geht es um die Gefahr, aus der Forderung nach ,Abschaffung' einer verbesonderten sowie verbesondernden und spezialisierten Expertise eine Sparmaßnahme zu machen.

Hark spricht in ihrem Werk, Dissidente Partizipation ‘ bezüglich der ersten Aporie von einer „integrativen Desintegration“ (Hark 2005, 118) feministischer Impulse 
in akademischen Kreisen. Wie auch in der rhizomatischen Karte ist hier von Ungleichzeitigkeiten die Rede: „Doch ungeachtet disziplinär bedingter Ungleichzeitigkeiten ist zu konstatieren, dass die geschlechtlich kodierte Dichotomie von Universalem/Partikularem im akademischen Feld eher mühelos reproduziert statt transformiert wird: In einer aufs Ganze gesehen schier reibungslos funktionierenden geschlechtlichen Arbeitsteilung bestellen männliche Wissenschaftler das Allgemeine der jeweiligen Disziplin, während Frauen- und GeschlechterforscherInnen den ,Sonderfall' Geschlecht bearbeiten“ (ebd., 129). Ebendies gilt analog auch für Behinderung: Während das (selbsternannt) Allgemeine die Dichotomie von Universalem/Partikularem weiterträgt, zeichnen sich Behindertenpädagog*innen mit und ohne Behinderung dadurch aus, dass sie versuchen, diese zu irritieren oder zu unterlaufen. Der Versuch der eigenen Entpartikularisierung durch Dekonstruktion öffnet dabei jedoch die Tür für die systematische Produktion falscher Universalismen aufseiten jener, die eine Nicht-Benennung und Nicht-Beschäftigung mit den Anderen* aus diesem dekonstruktiven Impuls machen. Sodann wird gewissermaßen aus den falschen Gründen Abstand von der Kategorie ,behinderte Menschen' genommen, nämlich um sie weiterhin zu ignorieren. Daher resümiert Hark, dass es ist, als würde man „am eigenen Erfolg scheitern“ (ebd.). Man kann über den Diskurs, den man dekonstruktiv zu irritieren versucht, nicht verfügen: Was aus diesen Impulsen gemacht wird, entgleitet uns.

Die Entnennung der Frau als Frau kann daher wider Willen in eine Verwässerung des feministischen Impulses münden: Nicht über Frauen als Frauen zu sprechen, mag innerhalb der Kreise der Gender Studies als dekonstruktive Praxis verstanden werden. Im Feuilleton ist es jedoch der leichteste Weg, sich nicht mit der Unterdrückung und Diskriminierung von Frauen zu befassen. Ebendies gilt auch für Behinderung und m.E. stehen wir dort vor derselben Aporie: Nicht über Behinderung als Behinderung zu sprechen, mag innerhalb unserer Reihen emanzipatorisch gemeint (gewesen) sein. Im öffentlichen Diskurs ist es hingegen schlichtweg eine weitere Möglichkeit, nicht über Behinderung zu sprechen.

Derzeit lassen sich zwei analoge Benennungspraxen im Feld Behinderung finden: Erstens schreiben manche statt Disability Studies lieber Dis/Ability Studies, um zu betonen, dass hier nicht die (konstruierte) Gruppe der Behinderten* im Zentrum steht, sondern die Hervorbringung der Konstruktion selbst. Dies wäre also das Analog zur Bezeichnung, Gender Studies'. Zweitens wird Behinderung zunehmend zu einer Heterogenitätsdimension unter vielen. Dies geht häufig mit einer disziplinären Selbstbezeichnung als inklusionspädagogisch oder aber als schlichtweg allgemeinpädagogisch einher. Das Partikulare wird dabei in der Benennung fallengelassen; der Begriff Behinderung taucht nicht mehr auf oder wird lediglich als eine Dimension von vielen miterwähnt (man denke an die Listen, die bei dem Versuch entstehen, den sog. , breiten Begriff von Inklusion darzulegen). 
Was sich darin artikuliert, ist gewissermaßen eine Geste der (avisierten) Selbstabschaffung oder der Selbstannihilation: ,Wäre die allgemeine Pädagogik tatsächlich allgemein, bräuchte es uns nicht'. Ebendies wurde auch in den Gender Studies diskutiert: So analysiert Hark in diesem Werk auch, inwiefern die beiden Ziele, erstens die Frauenstudien/Gender Studies als eigene Disziplin zu etablieren und zweitens geschlechtersensibles Denken und Handeln in alle Disziplinen hineinzutragen, gegenläufig sind oder Hand in Hand gehen (können). Auch aus diesen Darlegungen lassen sich zahlreiche fruchtbare Impulse ziehen (vgl. Boger 2018), für die ob der gebotenen Kürze hier kein Platz ist. Vorweggenommen sei daher nur die Pointe: Das Risiko, das im Kampf um die Dissemination der Theorien und (Forschungs-) Praktiken zu Geschlechtergerechtigkeit nicht vergessen werden darf, besteht in der Tatsache, dass die Geste der Selbstannihilation im Medium des Allgemeinen (z.B. der Allgemeinen Pädagogik) jederzeit zu einer Abschaffung als Sparmaßnahme pervertiert werden kann. Das besondere* Fach wird sodann gestrichen - aber nicht aus einem emanzipatorischen Impuls heraus, sondern im Gegenteil, weil man es unsichtbar machen will.

Aus den genannten Gründen lohnen sich die derzeit wieder häufiger werdenden Rückgriffe auf u.a. differenzfeministische Schriften, in denen Frau-Sein/Weiblichkeit weder als reine Konstruktion noch als ahistorische Kategorie gedacht wird. Dies geschieht z.B. über Konzepte wie jene des "Symptoms“ (Casale 2020, 20) oder über eine ereignistheoretische Analyse des essentiell Gewordenen. ${ }^{2}$ Dies ist z.B. in meinen Arbeiten der Fall - weswegen ich das Wörtchen wesentlich bzw. essentiell entgegen einer jahrhundertelangen Tradition der Philosophiegeschichte auch im Komparativ verwende: wesentlicher/essentieller. Dies ermöglicht es, zum Beispiel zu sagen, dass eine Differenz in einem historischen Prozess zu einem (un) wesentlicheren Unterschied wurde (qua Sedimentierung und Habitualisierung). Analog läge es nahe, auch im Bereich Behinderung von der Schlichtheit des sozialen Modells wegzukommen und sich vertieft der Theoriebildung zu widmen (vgl. Waldschmidt 2020).

\section{Die konstruktivistische Sackgasse}

In der Theorie der trilemmatischen Inklusion geht es stets um das Denken in Dilemmata, in gegenläufigen Vektoren, die sich in dissonanter Gleichzeitigkeit kreuzen. Auf diese Weise erscheint die jeweilige Schattenseite eines jeden Konzepts, das sich zu einer gegebenen Zeit, in einer gegebenen Generation zu sicher

2 „Im Sinne Freuds geht es auch bei den Denkerinnen der sexuellen Differenz nicht um eine Biologisierung bzw. Essentialisierung einer physischen Differenz. Die Differenz im Geschlecht ist weder Akzidenz noch Essenz, sie ist somatisch, sie ist ein Symptom“ (Casale 2020, 20). 
ist, emanzipatorische Wirkung zu entfalten. Die bereits angedeutete Sackgasse konstruktivistischer und dekonstruktiver Epistemologien zeigt, dass es nicht nur Dekonstruktionen im progressiven, emanzipatorischen Sinne gibt, sondern auch Dekategorisierungen von Behinderung, die dazu dienen, eine Betroffenengruppe unsichtbar und mundtot zu machen, indem man ihre Kollektivierung unter einem gemeinsamen Zeichen unterbindet. Sodann wirken ,Dekonstruktionen“ anti-emanzipatorisch, insofern sie die Wirkmacht dieser Kategorien und Kategorisierungen verschleiern (vgl. Köbsell 2015).

Dies zeigt sich auch in der Frage, wann die Entnennung von Behinderung (ohne Sternchen, ohne Anführungsstriche und ohne dekonstruktive gaps, also als bitterlich-reale Tatsache) zugunsten des Hohelieds auf die ,bunte Vielfalt aller' einer Verwässerung emanzipatorischer Impulse zuarbeitet. Ein Beispiel für eine empirische Studie hierzu liegt mit der Dokumentenanalyse von Lambrecht (2020) vor, in der gezeigt werden konnte, dass im Zuge der (Pseudo-),Umsetzung' der UN-BRK von der transnationalen Ebene über die nationale bis hin zur kleinsten Ebene der Einzelschule der Trilemma-Code Empowerment behinderter Menschen (die dazu auch als solche benannt werden müssen) immer seltener wird, während dekonstruktive Aussagen in Richtung einer leeren Vielfaltsrhetorik (ND-Linie, deren Gegenspieler der verlorene Punkt E gewesen wäre) zunehmen. Gerade durch die Betonung darauf, dass Behinderung sozial konstruiert sei, wird sich sodann eine bereits erfolgte ,Umsetzung' von Inklusion im Sinne der UN-BRK zusammenkonstruiert. ${ }^{3}$

In einem politik-philosophischen Wortspiel ließe sich zusammenfassend fragen: Hätte ein realistisches Bild von Behinderung derzeit vielleicht die größeren Chancen, emanzipatorische Wirkung zu erzielen und zum Empowerment behinderter Menschen beizutragen? In jedem Fall scheint es lohnenswert, sich wieder stärker mit nicht-konstruktivistischen (z.B. materialistischen oder phänomenologischen) Behinderungsbegriffen und Forschungsperspektiven zu befassen. So zeigt gerade der Vergleich mit Debatten um Geschlecht: Die Nicht-Bearbeitung dieser konstruktivistischen Sackgasse hinterlässt im öffentlichen Diskurs eine offene Flanke, die sodann allzu gerne mit reaktionären oder schlichtweg naiven Essentialismen gefüllt wird. In diesem Sinne gilt es, einen (Neuen) Realismus zu denken, der zugleich nicht von den altbekannten Essentialismen getragen ist.

Eine weitere zu bearbeitende Sackgasse des Konstruktivismus widmet sich der Sorge, dass insbesondere zu radikal ausgelegte Konstruktivismen das deliberative oder diskursive Moment des Demokratischen zersetzen können: Der akademische Weg zu dieser Diskussion führt in der Regel über die Konstruktivismuskritik in der Diskursethik nach Habermas (2001) oder über materialistische Einwürfe (vgl. Badiou 2012a \& 2012b) oder aber durch jenes Feld, das man derzeit unter

3 Für eine intergenerationale Betrachtung zu diesem Thema s.a. Boger und Jantzen (2020). 
dem Namen Neuer Realismus findet. Der Alltagsverstand stolpert intuitiv in das Erkennen dieser Sackgasse, wann immer man vor einem gestandenen Verschwörungstheoretiker mit Alu-Hut auf dem Kopf steht und sich wünscht, man könne (wieder) sagen: Das ist nicht wahr (statt nur zu sagen, dass er die Welt wohl anders konstruiert als man selbst).

\section{Standpunkte beim Gang durch die Institutionen}

Die dritte ,Prognose' hat zwei Aspekte. Der erste ist durch die zuvor genannten Argumente von Hark (2005) bereits vorgezeichnet: Bei der Etablierung eines neuen wissenschaftlichen Feldes aus einer emanzipatorischen Bewegung heraus, beim Gang durch die Institutionen wird dieser emanzipatorische Impetus verwässert, eingehegt und disziplin(aris)iert. In Anlehnung an die Unterscheidung Harks lässt sich dabei von einem academic turn der Behindertenbewegungen und Elternbewegungen für gemeinsamen Unterricht und einem inclusive turn der Akademie sprechen (ausführlicher in Boger 2018). Ersteres beschreibt die Akademisierungsbewegung der zuvor marginalisierten Subjekte und ihrer Diskurse: die durch sie entstehenden Theoriebildungen, die zunächst praxisnahen, dann abstrakter werdenden und disziplinär eingebundenen Forschungsprojekte etc. Letzteres beschreibt die Dezentrierung der bereits etablierten Disziplinen, die sich diesen zuvor veranderten Themen sodann zuwenden (wie z.B. die etablierte Allgemeine Didaktik, die sich den Themen der Integrationsbewegung zuwendet und sich durch diese verändern lässt). In beiden Bewegungen finden komplexe Prozesse der (Fehl-)Aneignung statt: Im Zuge des academic turns setzen sich manche Teile oder ,Flügel' der politischen Bewegung stärker durch als andere; es kann zur Etablierung einer ,Betroffenenhierarchie‘ kommen, bei der manche Betroffenengruppen stärker wahrgenommen und andere übergangen werden. Dies betrifft insbesondere jene, denen ein Zugang zu Bildung, Forschung und damit Akademisierung noch stärker erschwert ist als anderen. Im Zuge des inclusive turns der Etablierten wiederum kann es passieren, dass nur einzelne - bevorzugt pflegeleicht integrierbare - Elemente aus den theoretischen und praktischen Impulsen der emanzipatorischen Bewegung herausgepickt werden, während andere als ,nicht anschlussfähig' oder ,nicht umsetzbar/zu radikal' abgetan werden. In beiden Prozessen ist daher viel Platz für Verschiebungen, kommunikative Missverständnisse und Unverhältnisse - und eben auch für ein Wiedereinziehen hierarchisierender und exkludierender Figuren.

Mit diesen Prozessen einhergehend werden neue Plätze geschaffen - sowohl im symbolischen Sinne von neuen Positionen in einem rearrangierten Diskurs als auch ganz konkret im Sinne neuer (Mittelbau-)Stellen und Professuren. Während Hark für die Frauenstudien nun rekonstruiert, wann, wie viele und welche 
Frauen sich die ersten Professuren innerhalb und außerhalb der Frauenstudien erkämpften (vgl. Hark 2005, 119ff.), gilt für die Behindertenpädagogik nach wie vor, dass sie größtenteils eine Stellvertreterwissenschaft ist. Sowohl innerhalb als auch außerhalb dieser sind behinderte Menschen unterrepräsentiert. Der zweite Aspekt, den es zukünftig zu reflektieren gilt, betrifft die Kritik an dieser Tatsache der Stellvertreterwissenschaft und der allfälligen Normalisierung dieser Blickordnung.

Dabei geht es nicht um eine schlichte Selbstrepräsentation um der Selbstrepräsentation willen. Gerade dies würde zu einer harschen Re-Essentialisierung von Behinderung und einer Reproduktion der Ordnung zugesprochener und abgesprochener Expertise führen, wie z.B. auch Homann und Bruhn $(2020,85)$ betonen. Daher gilt es einerseits, in Zukunft auf eine Kritik des bezüglich Behinderung normalisierten Stellvertretertums zu bestehen: „Es mag trivial erscheinen, aber was für Frauen- und Geschlechterstudien oder Antirassismusforschung gilt, gilt auch für Disability Studies: Jene, an denen sich der Tatbestand Behinderung vollzieht, die also selbst-betroffen sind, können prinzipiell am besten Auskunft darüber geben, welchen Diskriminierungserfahrungen sie ausgesetzt sind. Daher ist (nicht nur) für Disability Studies die Betroffenenperspektive zentral“ (ebd.; Herv. i.O.). Andererseits aber zeigen auch Selbstbetroffene Zeichen internalisierter Diskriminierung (vgl. ebd.) - und zudem kann die subjektive Erfahrung die fachliche Expertise weder ersetzen, noch darf sie mit ihr in eins gesetzt werden. So erinnern Homann und Bruhn daran, dass entgegen der trivialisierten Formel von ,Expert*innen in eigener Sache' in seriösen Konzepten der Selbstvertretung wie z.B. der UN-BRK stets auf fachlich einschlägige Expertise beharrt wurde: „An keiner Stelle wird [im Kontext der UN-BRK-Gremien; Anm. M.B.] Selbstbetroffenheit als Expert*innentum im Sinne einer fachlichen Qualifikation per se konstruiert" (ebd.).

Statt einer simplifizierten Logik der Repräsentation, die zudem nicht bruchlos vom Register des Politischen in das Register der Wissenschaft übersetzt werden kann, gilt es daher, sich verstärkt mit Standpunktreflexionen zu befassen (vgl. Haraway 1995; Harding 1986/1991 \& 1994). Bei diesen Reflexionen geht es weder um (Sprech-)Verbote noch um die falsche Überzeugung, dass Deutungshoheiten unmittelbar oder zwangsläufig aus einer Sprechposition folgen würden (vgl. Boger 2019b). Viel eher gilt es, im Kontext von Wissenschaft und Forschung die epistemologische Frage der Standpunkttheorie als epistemologische ernst zu nehmen, ohne in eine Moralisierung zu verfallen oder die zuweilen selbstgefällig anmutende moralische Hierarchie der Partizipationsleiter der Partizipativen Forschung mit einem Qualitätskriterium für wissenschaftliches Arbeiten und sachliche Argumente zu verwechseln. So betonen zum Beispiel auch Beresford und Rose (2009, 13) im Kontext der Mad Studies, dass betroffenenkontrollierte Forschung auf demselben Niveau und mit derselben methodischen Strenge zu erfolgen habe: 
Standpunkttheoretische epistemologische Reflexionen sollen die Forschung besser machen, indem sie wissenschaftstheoretische Debatten und selbstkritische Praktiken anregen. Forschung wird also nicht per se ,besser' oder ,moralisch höherwertig', wenn Betroffene an ihr partizipieren; erst die im Dialog vollzogene Reflexion der Positionierungen und Perspektivierungen aller Beteiligten vermag es, zu dieser Qualitätssteigerung beizutragen.

\section{Leichtere Sprache, Praxisnähe, praktische Vernunft und wann sie reaktionäre Wirkungen entfaltet}

Vor dem Hintergrund des vorherigen Absatzes benutze ich nun den Begriff ,Leichte(re) Sprache' aus behindertenpädagogischer Perspektive auf betont schiefe Weise, nämlich so, dass in aller Deutlichkeit erscheint, wie wenig er (kausal) mit Behinderung zusammenhängt. Stattdessen soll es um diskursethische Fragen gehen, die mit vielfachen intersektionalen Überkreuzungen einhergehen können: Alle unsere Nachbar*innen aus Kontexten emanzipatorischer Wissensproduktion können bezeugen, dass Anti-Intellektualismus ein beliebtes Mittel der Be_Hinderung der Etablierung gesellschaftskritischer Theorien ist. Auch hier gilt es daher, in Dilemmata zu denken (statt einer moralisierenden Vereindeutigung anheimzufallen). Die dissonante Gleichzeitigkeit unterdrückerischer Vektoren hat hier folgende Form: Einerseits muss es Teil inklusiver Bewegungen sein, akademischen Elitarismus zu kritisieren und die typischerweise in solipsistische Nabelschau mündende Entkopplung von emanzipatorischen Bewegungen zu verhindern. Andererseits blockieren gewisse Formen des aggressiven Einforderns von ,Praxisnähe und zugänglicher, leichter Sprache' die Entfaltung kritischer Wissensproduktion. Dies rührt daher, dass - wie es sich in phänomenologischem Vokabular und/oder mit Bourdieu gesprochen zeigen lässt - das Altbekannte, das Sedimentierte und Habitualisierte, das längst Fleisch geworden ist, leichtgängiger erzählbar und vermittelbar ist als jenes, das uns in kritischer Distanz zu jenen Selbstverständlichkeiten in seinem Anspruch zu befremden vermag. Dies ist gerade mit Bezug auf eine (Schul-)Praxis, die tatsächlich versucht, Inklusion zu leben, leicht einsehbar: Womit hat man versucht, die Etablierung des Gemeinsamen Unterrichts zu verhindern? Indem man sagte, diese sei eine ,praxisferne, nicht umsetzbare Ideologie'.

In der Theorie der trilemmatischen Inklusion geht es stets darum, auf den ersten Blick gegenläufige Formen des Leidens zu konzertieren: So finden sich in der Geschichte der IFO-Tagung in der alten Garde sowohl Menschen, die diese Erfahrung - die Erfahrung, für den eigenen inklusiven/emanzipatorischen Anspruch als weltfremd-abstrakt-schwurbelnde Elfenbeinturm-Wissenschaftler*innen angegangen zu werden - teilen, als auch Menschen, denen von den Gegner*innen 
des Gemeinsamen Unterrichts das Gegenteil vorgeworfen wurde, nämlich dass sie ,theoretisch unterbelichtet' seien und mit ihrer praxisnahen Aktionsforschung (bzw. handfesten Schulbegleitforschung) den akademischen Standards empirischer Forschung nicht genügen würden. Kopflose Gutmenschen hat man sie genannt. Oder eben: gefährlich radikal in ihren ,abstrakten' Theoriebewegungen.

Daher muss man stets sehr genau auf den Kontext achten, in dem eine Kritik an zu leichter oder aber zu schwerer (akademisch-abgeriegelter) Sprache erklingt, da sich weder von dem einen noch von dem anderen Vorwurf apriorisch sagen lässt, ob er inklusiv/emanzipatorisch wirkt oder das Gegenteil hervorbringt.

Eine Form des Selbstzweifels wird auf diese Weise in jedes forschende Subjekt eingeführt, egal ob es den einen oder den anderen Vorwurf öfter gehört hat (oder beide, denn das schließt sich ja nicht aus: Leicht ist es vorstellbar, dass sogar in den meisten akademischen Biographien beide Vorwürfe vorkommen). Vorbildlich in diesem Gestus arbeitet Bourdieu, wenn er mit Blick auf den von ihm selbst entfalteten Begriff praktischer Vernunft reflektiert, dass dieser nicht per se einem progressiven oder emanzipatorischen Register zufalle: „So fragt man sich schließlich, ob konservative und der rationalistischen Tradition feindlich gegenüberstehende Denker (...) nicht bloß deswegen einige Eigenschaften praktischer Erkenntnis mit dem Ziel benennen konnten, die Überlieferung gegen den ausschließlichen Glauben an die Vernunft aufzuwerten, weil soziale Instinkte sie dazu motivierten“ (2001, 105). Praxisnähe und die Aufwertung einer praktischen Vernunft gegenüber dem Akademisch-Abstrakten kann ebenso gut im Dienste eines anti-emanzipatorischen Konservatismus und Traditionalismus stehen; und ebendies gilt auch für eine falsch verstandene zugängliche, leichte‘ Sprache.

Dies betrifft auch die Frage, wie ,inklusiv' bzw. diskriminierend die akademische Welt mit Blick auf Forschende aus deklassierten Gruppen ist: Es ist kein Zufall, dass derselbe Pierre Bourdieu, der einst Sartre vorwarf, dass dieser seine Klassenprivilegien nicht reflektiere (für eine biographische Analyse/Deutung s. Eribon 2017, 79), so viel komplizierter schreibt als Sartre, dem höchste literarische Würden für sein zugängliches philosophisches Schreiben zuteil wurden. Bezeichnenderweise lässt sich diese These Bourdieus nicht in einem griffigen, einfachen Blockzitat wiedergeben, aber wenn man zwei Stunden Zeit auf die Schachtelsätze der beiden hier zitierten Seiten verwendet, wird man in diesen einen Bourdieu finden, der als ,sozialer Aufsteiger' immer noch dagegen ankämpft, dagegen ankämpfen muss, dass die etablierte Bourgeoisie ihn für unfähig hält, sprachlich restringiert und eben: habituell deplatziert. Ebendies gilt freilich auch für andere minoritäre Gruppen: So wird migrantischen Menschen und Personen of Color, die sich für eine zugänglichere Sprache entscheiden, gerne unterstellt, sie seien wohl zu komplexerem Deutsch nicht in der Lage. Leichte Sprache wird dabei zur Vorlage für ihre Abwertung und Diskreditierung als fähige Wissenschaftler_innen; und dasselbe gilt für behinderte Forscher_innen, deren Exklusion aus dem Wissenschafts- 
betrieb häufig genau dadurch erfolgt, dass man ihnen nicht mehr an Fähigkeiten zuspricht als zugängliche, nette Geschichten aus dem eigenen Leben zu erzählen. Es ist also nicht so, dass eine leichtere Sprache Wissenschaft für alle zugänglicher und weniger diskriminierend machen würde. Mitunter ist sogar das Gegenteil der Fall. In dieser Komplexität und Ambivalenz muss man es halten.

\section{Zur Dialektisierung der Ableismuskritik: Bildungsanspruch als Fähigkeitserwartung}

Eine ähnlich bedenkliche Vereinseitigung zeigt sich in Analysen, die sich als ableismuskritisch verstehen und dabei in einer Verkürzung dieses theoretischen Konzepts jedwede Fähigkeitserwartung als oppressiv deuten. Dies steht einerseits in Zusammenhang mit der bereits erwähnten de_konstruktivistischen Sackgasse und andererseits mit dem vorherigen Punkt: Bildungsdeprivierte und sprachlich restringierte Menschen als, anders befähigt` (differently-abled) zu bezeichnen, kann nicht nur emanzipatorisch gelesen werden, sondern auch als Eingangspforte in eine entmündigende Bewahrpädagogik, welche die Verwerfung der Bildsamkeit behinderter Menschen wiederholt.

Aus einer diskriminierungskritischen Perspektive wären daher lediglich naturalisierte Fähigkeitserwartungen ins Visier zu nehmen, um ebenjene Naturalisierung und Biologisierung und das davon ausgehende Othering zu kritisieren; es geht um eine „Dekonstruktion vermeintlich ,natürlicher' Fähigkeitskonstruktionen“" (Kaiser \& Pfahl 2020, 101) - und nicht etwa undifferenziert aller. Eine Kritik sämtlicher Fähigkeitserwartungen führt nämlich tragischerweise in ebenjene Wiederholung der Verwerfung der Bildsamkeit behinderter Menschen, die sich sodann zwar als Ableismuskritik tarnt, aber in Wahrheit zutiefst ableistisch ist. Bildung lässt sich nicht ohne Fähigkeitserwartungen denken. Dies ist ganz offensichtlich der Fall: Wir hoffen und vertrauen auf die Fähigkeit zur Entwicklung bzw. zum Lernen unserer Kinder/Schüler*innen/Klient*innen. Ihre Fähigkeiten zu entwickeln und zu fördern ist oberste Aufgabe der Bildungswissenschaften. Wer sich länger und differenziert mit Ableismuskritik befasst, weiß daher um diese Ambivalenzen und diskutiert Möglichkeiten der Dialektisierung oder Verschiebung dieses Dilemmas (vgl. Buchner \& Pfahl 2017; Buchner, Pfahl \& Traue 2015). Auch hier gilt das Spivaksche Diktum, dass es stets verdächtig ist, wenn eine*r zu sicher glaubt, zu wissen, was Empowerment/Ermächtigung ist: „Eigentlich (und sehr praktisch) ist eine Zwickmühle in weit weniger gefährlicher Weise ermächtigend als die Unilateralität gelöster Dilemmata" (Spivak 2014, 179). Man muss die Frage wieder so stellen, dass sie weh tut: Wie lässt sich mit der Tatsache umgehen, dass die Unterstellung von Bildsamkeit eine Fähigkeitserwartung ist und dass 
diese Fähigkeitserwartung just in dem Moment, in dem man die Bildsamkeit aller verteidigt, zu einer naturalisierten Fähigkeitserwartung (im Sinne einer anthropologischen Universalie) wird? Oder als These formuliert: Ableismuskritik im Sinne einer Kritik und Dekonstruktion naturalisierter Fähigkeitserwartungen steht in einem dilemmatischen Verhältnis zur Affirmation der Bildsamkeit aller, da die Bildsamkeitsunterstellung selbst eine naturalisierte - ja sogar anthropologisierte Fähigkeitserwartung darstellt.

Zwei wichtige Schattierungen müssen in diesem Sinne bei einer Analyse (tatsächlich) ableistischer Fähigkeitserwartungen bekräftigt werden: Erstens werden wir in Zukunft darauf achten müssen, dass die Unterscheidung zwischen ableistischen Fähigkeitserwartungen und legitimen, bildungswirksamen Fähigkeitserwartungen im Sinne der Unterstellung von Bildsamkeit und der pädagogischen Hoffnung auf Entwicklung nicht vergessen, sondern sogar stark gemacht wird. Erstere zu bekämpfen und Letztere hochzuhalten ist Aufgabe der Pädagogik. Zweitens geht es um die feine, aber pädagogisch hoch relevante Schattierung, eine Fähigkeitserwartung als pädagogische Als-ob-Unterstellung denken und verstehen zu können. Es gibt zahlreiche allgemein-pädagogische Klassiker, mit denen sich die Bedeutung dieser Als-ob-Unterstellung herleiten lässt. Exemplarisch sagen wir es mit Heydorn: „Mit dem Begriff Bildung wird die Antithese zum Erziehungsprozeß entworfen; sie bleibt zunächst unvermittelt“, denn sie „begreift sich als entbundene Selbstätigkeit, als schon vollzogene Emanzipation" (Heydorn 2004, 9). Bildung setzt Mündigkeit voraus, und Mündigkeit Bildung. Aus diesem Zirkel kommen wir nur heraus, indem wir mit einer Als-ob-Unterstellung in ihn hereinbrechen.

Aber auch in der bereits erwähnten Habermas'schen Diskursethik (2001) geht es darum: Indem wir unseren Zöglingen begegnen als ob sie bereits im Diskurs orientiert und zur Artikulation einer vernünftigen Meinung fähig wären, ermöglichen wir es ihnen, sich darin zu erproben und es dadurch zu werden.

Eine Verwerfung von Bildsamkeit und (detranszendentalisierter, also immanenter) Vernunft als handlungsleitendem Prinzip führt zu nichts - oder ins Dunkel. Auch hier sieht man es deutlich, wenn man an die derzeit virulenten Probleme der Gesellschaft denkt: Verschwörungstheorien, Anti-Intellektualismus, Wissenschaftsfeindlichkeit einerseits und naive Wissenschaftsgläubigkeit andererseits (die sich Wissenschaft als monolithischen Block ohne diskursiven Charakter vorstellt) sind aktuelle Gefahren der Demokratie. Es ist daher nicht anti-inklusiv, sondern im Gegenteil Möglichkeitsbedingung inklusiven Handelns daran zu erinnern, dass es Bildung braucht, um sinnhaft und vernünftig mitdiskutieren zu können. Dies zu sagen, ist keine ableistische Fähigkeitserwartung, sondern unsere Aufgabe als Pädagog*innen und als Wächter*innen der Demokratie. 
Ableistisch ist lediglich die pauschale Unterstellung, dass es nur sog. ,behinderte Menschen beträfe, dass diese durch Fähigkeitserwartungen von demokratischer Partizipation ausgeschlossen seien - während in Wahrheit tagtäglich sog. ,nichtbehinderte‘ Menschen die Medien mit ihrer Unfähigkeit, an vernünftigen, demokratischen Diskursen zu partizipieren, auf Trab halten.

\section{Von der Fleischwerdung des Cyborgs und posthumaner Relationalität}

Vor ein paar Monaten stand an dieser Stelle noch: Offensichtlich wird Digitalisierung der neue Trend in Forschung und Praxis. Die entsprechenden Fördermittel und Ausschreibungen dazu sind bereits da oder in Planung. Dann kam die COVID-19-Pandemie. Die Aussage ist damit noch trivialer geworden bzw. die im Februar 2020 aufgestellte Prognose, dass es zu einer vermehrten Beschäftigung mit Online-Lehre und Digitaler Kultur kommen wird, hat sich auf eine für viele von uns nicht immer einfache Weise bewahrheitet.

Nun sind sie also da - die Zeiten, in denen wir fragen: Was bedeutet interkorporale Resonanz? Was ist eine körperliche Präsenz überhaupt? Warum/Wie ist sie zwischenleiblich vernehmbar? Und was unterscheidet diese Zwischenleiblichkeit in einem nicht-virtuell geteilten Erfahrungsraum von der virtualisierten Zwischen-Avatar-igkeit? Es gilt, präzise zu schauen, woher die Irritation der Virtualisierung sozialer Beziehungen kommt - und insbesondere die Phänomenologie bietet dazu sehr geeignete Mittel. Ein anderer hat lange vor mir darauf gewettet: „Die Transformation der Phänomenologie in allgemeine Medientheorie lässt bedeutende Konsequenzen für das Selbstverständnis des postmodernen Menschen erwarten. Sie wird dazu beitragen, Subjektivität von den traditionellen Dualismen von Präsentation und Repräsentation, von Eigentlichkeit und Entfremdung, von Unmittelbarkeit und System, von Körper und Maschine und wie die Oppositionen alle heißen, zu befreien" (Fellmann 2006, 176f.).

Gerade in behinderungsbezogenen Disziplinen und Studien wird es sich sicherlich lohnen, wieder an einem Begriff von Körper zu arbeiten, der diesen nicht auf seinen Charakter als Zielscheibe und Formungsobjekt von Diskursen reduziert. In besagtem Februar endete der Vortrag mit der Idee, im Zuge der Digitalisierungsdebatten ein Zeitfenster für (anti-)anthropologische Reflexionen freizuhalten, denn auch in diesem Feld zeigt sich das Verblassen eines Gegenspielers bzw. eine ungünstige Vereinseitigung: Konzepte posthumaner Relationalität, die Figuration des behindert_enthinderten Körpers als ,Cyborg' und ähnliche Denkfiguren, die auch den Humanismus als Essentialismus dekonstruieren sollen, wurden entworfen (vgl. Goodley, Lawthom, Liddiard \& Runswick-Cole 2019). Damit 
steht die Frage im Raum, ob diese emanzipatorisch sind oder eher nicht. Maskos (2020) zum Beispiel spricht, um dieser Vereinseitigung zu entgegnen, in Anlehnung an Spivaks (1990) Konzept des „strategischen Essentialismus" von einem „strategischen Humanismus“, an dem man zukünftig festhalten müsse: In Anbetracht der brutalen Tatsache, dass eine Dehumanisierung behinderter Menschen immer noch häufig zu finden ist, erscheint auch diese Dekonstruktion verfrüht (ähnlich der in den Absätzen 1-3 thematisierten Schattenseiten übereilter/verkürzter Dekonstruktionen). Auch außerhalb behinderungsbezogener Debatten wurde diese Denkfigur bereits eingeführt. So spricht zum Beispiel Alain Badiou davon, dass es gälte, einen „praktischen Humanismus“ mit einem „theoretischen Anti-Humanismus" zu dialektisieren (einführend siehe Pluth 2012, 17). Wer in emanzipatorischen Kämpfen auf die Menschenwürde und Menschenrechte rekurrieren will, muss dazu an der Figuration ,Mensch' festhalten. Gleichzeitig eröffnen besagte Konzepte des Cyborgs und der posthumanen Relationalität fruchtbare Gedanken zu einer Verabschiedung des Glaubens an ein autonomes Subjekt.

In anderen Diskursräumen sind diese posthumanistischen Gedanken freilich noch nicht relevant geworden. Hier zeigt sich eher die gegenläufige Vereinseitigung: Ein (teilweise nostalgisch wirkendes) Festhalten an humanistischen Ideen, das die Kritik an diesen kaum wahrhaben kann. Allein der Begriff, ,Anti-Humanismus erscheint sodann als eine Provokation, insofern er aus einer Perspektive, die den Schatten des humanistischen Erbes nicht betrachten will oder kann, als Angriff auf das Gute vernommen wird. Eine Vermittlung wird sich daher für beide Formen des vereinseitigten Diskurses lohnen: Das Konzipieren eines Humanismus als strategische Operation erlaubt es, einem praktischen Humanismus die Treue zu halten, ohne die theoretischen Irrläufer falscher Universalismen fortzuschreiben.

\section{Fazit: Kartentheorien als Re_Orientierungsmittel}

Die Theorie der trilemmatischen Inklusion geht als Kartentheorie nach Deleuze und Guattari (1977 \& 1992) davon aus, dass wir es mit ungleichzeitigen Verschiebungen im Rad der Geschichte zu tun haben. Die auf dieser Landkarte verzeichneten Inhalte oder Punkte sind in historischen Momenten manchmal stärker und manchmal schwächer vertreten. Durch die trilemmatische Struktur wird deutlich, dass das gänzliche Vergessen oder Verblassen einzelner Punkte uns in historisch kontingente Sackgassen treiben lässt. Der Versuch, diese Sackgassen zu verlassen, verführt uns sodann dazu, die verblassten Punkte aus der Geschichte wieder aufzugreifen, sie in eine verschobene Wiederholung zu bringen, sie zu revitalisieren. Alle oben genannten Thesen verweisen auf solche Wendepunkte, die im Kommen sind: 
1) Vielleicht widmen wir uns aktuellen Gründen, das Wort Behinderung ohne Anführungsstriche und Dekonstruktionssternchen angstfrei zu verwenden, es als emanzipatorisches Zeichen zu affirmieren - oder es zu lassen?

2) Vielleicht ist es Zeit für einen $n /$ Neuen Realismus, der sicherlich an vielen Stellen sehr alt sein wird?

3) Vielleicht sind wir bereits an dem Punkt, an dem wir - wie Hark es formulierte - an unserem eigenen Erfolg gescheitert sind? Ist es Zeit für eine neue Strategie in Anbetracht der Tatsache, dass das Wörtchen Inklusion ,erfolgreich' an tausenden Orten eingeführt wurde, die dem ursprünglichen kritischen Anspruch dieser Bewegung nicht ansatzweise gerecht werden? Was sagt dies über das Werden einer Bewegung, die einst einer radikaldemokratischen Versammlung entsprang? Wie ließe sich daran wieder anknüpfen?

4) Vielleicht wäre es ratsam, zu einer Theorie der Praxis zu kommen, in der sich die Sprache der Praxis als eine unfasslich schwere und die akademische Sprache als verdächtig leichte zeigt? Könnte sich ein anderes Denken darüber, was eine Sprache schwer oder leicht macht, lohnen?

5) Vielleicht nehmen wir uns mehr Zeit, Ableismuskritik nicht nur im Zuge empirischer Forschungen zu durchdenken, sondern sie auch an allgemeinpädagogische Theorien (von Bildsamkeit als Fähigkeitserwartung) zurückzubinden? Vielleicht wieder mehr Habermas (Fähigkeitserwartung als (pädagogische) Als-ob-Unterstellung) und weniger Foucault (Fähigkeitserwartung als diskursive Konstruktion)? Vielleicht - und hoffentlich! - geht dies einher mit neuen_alten demokratietheoretischen Entwürfen?

6) Vielleicht ist es Zeit für phänomenologische Entwürfe, die sich zwischen den Dichotomien von Leib und Seele, Körper und Leib, online/virtuell und offline bewegen? Vielleicht - und hoffentlich! - gelingt eine Dialektisierung von praktischem Humanismus und theoretischer Humanismus-Kritik.

Die Vereinseitigungen bzw. Vereindeutigungen - man könnte auch sagen: EntDialektisierungen oder mangelnden Dialektisierungen -, auf die hier hingewiesen wurden, vernachlässigen freilich das Singuläre eines jeden von uns als Diskursteilnehmer*in. Neben dieser diskursiven Ebene, auf der sich gewisse Trends ausmachen lassen, gibt es auch eine persönliche Ebene, auf der sich fragen lässt: Was habe ich (als singuläres Subjekt) verblassen lassen? Welche Punkte sind mir auf meiner Reise verloren gegangen? In diesem Sinne bleibt mir zum Abschluss nur zu sagen, dass ich einem jeden Subjekt, das der Idee von Inklusion als einem emanzipatorischen Entwurf die Treue halten will, solche Momente der Revitalisierung wünsche. Sie werden auch uns Andere revitalisieren. Und vielleicht - hoffentlich! - sind darunter noch ganz andere Punkte, die ich in diesen singulären Ausführungen habe verblassen lassen. 


\section{Literatur}

Badiou, A. (2012a): Das demokratische Wahrzeichen. In: Suhrkamp Verlag (Hrsg.): Demokratie? Eine Debatte. Berlin, 13-22.

Badiou, A. (2012b): Logiken der Welten. Zürich.

Beresford, P. \& Rose, D. (2009): Introduction and Background - Chapter 2: Background. In: Sweeney, A., Beresford, P., Faulkner, A., Nettle, M. \& Rose, D. (Hrsg.): This is Survivor Research. Monmouth, 11-21.

Boger, M. (2018): Die Politik und das Politische der Inklusionsforschung. In: Walm, M., Häcker, T., Radisch, F. \& Krüger, A. (Hrsg.): Empirisch-pädagogische Forschung in inklusiven Zeiten. Bad Heilbrunn, 64-75.

Boger, M. (2019a): Die Methode der sozialwissenschaftlichen Kartographierung und die Theorie der trilemmatischen Inklusion zum Mitfühlen - Mitdiskutieren - Mitdenken (Buchreihe in 4 Bänden). Münster.

Boger, M. (2019b): ,Du musst ihnen sagen, dass das falsch ist. ' - Eine Geschichte vom existentiellen Wert der Theoriebildung. In: Dederich, M., Ellinger, S. \& Laubenstein, D. (Hrsg.): Sonderpädagogik als Erfahrungs- und Praxiswissenschaft. Leverkusen, 127-153.

Boger, M. \& Jantzen, W. (2020): Wahrhaft allgemeine Bildung: Erst war es unmöglich... dann ging es verdächtig schnell. Vortrag auf der 54. Sektionstagung Sonderpädagogik der DGfE. 25.09.2019, Wuppertal. Online unter: http://www.basaglia.de/Artikel/boger_jantzen_2020_wahrhaft_allg_ bild_onlineversion.pdf (letzter Zugriff: 20.06.2020).

Bourdieu, P. (2001): Meditationen - Zur Kritik der scholastischen Vernunft. Frankfurt am Main.

Buchner, T. \& Pfahl, L. (2017): Ableism und Kindheit: Fähigkeitsorientierte Praktiken in Medizin und Pädagogik. In: Amirpur, D. \& Platte, A. (Hrsg.): Handbuch Inklusive Kindheiten. Opladen, 210-224.

Buchner, T., Pfahl, L. \& Traue, B. (2015): Zur Kritik der Fähigkeiten. Ableism als neue Forschungsperspektive für die Disability Studies und ihre Partner_innen. In: Zeitschrift für Inklusion-Online. net, 2, o.S. Online unter: https://www.inklusion-online.net/index.php/inklusion-online/article/ view/273 (letzter Zugriff: 20.03.2021).

Casale, R. (2020): Verkörperte Bildung: Leidenschaft, Krankheit, Geschlechtlichkeit. In: Casale, R., Rieger-Ladich, M. \& Thompson, C. (Hrsg.): Verkörperte Bildung - Körper und Leib in geschichtlichen und gesellschaftlichen Transformationen. Weinheim, 10-21.

Deleuze, G. \& Guattari, F. (1977): Rhizom. Berlin.

Deleuze, G. \& Guattari, F. (1992): Anti-Ödipus. Frankfurt am Main.

Eribon, D. (2017): Gesellschaft als Urteil. Klassen, Identitäten, Wege (Edition suhrkamp. Sonderdruck). Frankfurt am Main.

Fellmann, F. (2006): Phänomenologie - Zur Einführung. Hamburg.

Goodley, D., Lawthom, R., Liddiard, K. \& Runswick-Cole, K. (2019): Provocations for Critical Disability Studies. In: Disability \& Society, 34, H.6, o.S. Online unter: https://www.tandfonline.com/ doi/full/10.1080/09687599.2019.1566889 (letzter Zugriff: 20.03.2021).

Habermas, J. (2001): Kommunikatives Handeln und detranszendentalisierte Vernunft. Ditzingen.

Haraway, D. (1995): Ein Manifest für Cyborgs. In: Haraway, D. \& Hammer, C. (Hrsg.): Die Neuerfindung der Natur. Primaten, Cyborgs und Frauen. Frankfurt am Main, 33-72.

Harding, S. G. (1986/1991): Feministische Wissenschaftstheorie. Zum Verhältnis von Wissenschaft und sozialem Geschlecht. Hamburg.

Harding, S. G. (1994): Das Geschlecht des Wissens. Frauen denken die Wissenschaft neu. Frankfurt am Main.

Hark, S. (2005): Dissidente Partizipation. Eine Diskursgeschichte des Feminismus. Frankfurt am Main.

Heydorn, H.-J. (2004): Über den Widerspruch von Bildung und Herrschaft. Wetzlar. 
Homann, J. \& Bruhn, L. (2020): Wer spricht denn da? - Kritische Anmerkungen zum Konzept der Selbstbetroffenheit. In: Brehme, D., Fuchs, P., Köbsell, S. \& Wesselmann, C. (Hrsg.): Disability Studies im deutschsprachigen Raum - Zwischen Emanzipation und Vereinnahmung. Weinheim, 82-88.

Kaiser, M. \& Pfahl, L. (2020): Ableism und Verletzlichkeit - Überlegungen zur ,Erfindung neuer Formen von Subjektivität'. In: Brehme, D., Fuchs, P., Köbsell, S. \& Wesselmann, C. (Hrsg.): Disability Studies im deutschsprachigen Raum - Zwischen Emanzipation und Vereinnahmung. Weinheim, 96-102.

Köbsell, S. (2015): Disability Studies und Inklusion oder: Warum Inklusion die Disability Studies braucht. In: Schnell, I. (Hrsg.): Herausforderung Inklusion. Theoriebildung und Praxis. Bad Heilbrunn, 116-128.

Lambrecht, J. (2020): Warum machen wir nicht einfach Inklusion? Entwicklung einer Theorie schulischer Inklusion. (Perspektive Schule, 1). Bielefeld. Online unter: https://doi.org/10.3278/6004774w (letzter Zugriff: 20.03.2021).

Maskos, R. (2020): Posthuman Risks? Some Thoughts on Posthuman Disability Studies and 'Strategic Humanism'. In: Atkinson, R. \& Goodley, D. (Hrsg.): Humanity under duress. Sheffield, 46-49. Online unter: http://eprints.whiterose.ac.uk/155940/3/Humanity_Under_Duress\%20WRRO.pdf (letzter Zugriff: 20.03.2021).

Pluth, E. (2012): Badiou - Eine Philosophie des Neuen. Hamburg.

Spivak, G. C. (1990): The Post-Colonial Critic: Interviews, Strategies, Dialogues. New York.

Spivak, G. C. (2014): Kritik der postkolonialen Vernunft - Hin zu einer Geschichte der verrinnenden Gegenwart. Stuttgart.

Waldschmidt, A. (2020): Jenseits der Modelle - Theoretische Ansätze in den Disability Studies. In: Brehme, D., Fuchs, P., Köbsell, S. \& Wesselmann, C. (Hrsg.): Disability Studies im deutschsprachigen Raum - Zwischen Emanzipation und Vereinnahmung. Weinheim, 56-73. 


\section{Sven Bärmig}

\section{Dialektik als Methode}

\section{Methode}

Die folgenden Ausführungen sollen zeigen, dass mit der Dialektik eine ,Methode existiert, die sehr anregend und herausfordernd das Tagungsthema des ,Zwischen oder der ,Grenzen ' bearbeitet. Mit ,Methode` ist nach Jürgen Ritsert (2011) dabei eine einer bestimmten Ordnung folgende Weise des Denkens bei Theodor W. Adorno gemeint. Dessen dialektisches Verfahren analysiert Gesellschaft als Beziehung von Allgemeinem und Besonderem mit dem Primat der Gegenstände und Themen. Sie ist als Reflexion der Erkenntnisse angelegt und bezieht sich gleichfalls selbstkritisch auf die eigene Position. Insbesondere in komplexen und schwer durchschaubaren sozialen Verhältnissen wird dies bedeutsam (vgl. ebd.). Dann zeigt sich, dass bspw. eine Kategorie wie Inklusion nur scheinbar eindeutig ist und deshalb immer wieder dahingehend neu analysiert werden muss, ob sie das trifft, was damit ausgesagt werden soll. Allein aus dieser kurzen Hinführung ergeben sich einige wesentliche Merkmale dialektischen Denkens.

\section{Vom Begriff aus}

$\mathrm{Zu}$ bedenken ist, „dass die Begriffe immer schon etwas an uns heranbringen, was wir keineswegs erst stiften und dem wir uns in der Sprache selber gleichsam zu unterwerfen haben“ (Adorno 2015, 281). Dabei gilt zunächst: „Denken heißt Identifizieren“ (Adorno 2003, 17), was aussagt, mit einem Begriff auch eine bestimmte Sache zutreffend zu beschreiben/zu benennen.

Die Begriffe selbst lassen sich trotz ihrer Bestimmtheit sui generis nicht auf einen Inhalt ,ohne Weiteres' vereidigen, „das Bedürfnis der Definition“ (Adorno 2015, 281) verengt bereits. Dialektisches Denken ist deshalb für Adorno grundlegend verknüpft mit der Einsicht, dass die Gegenstände im Begriff nicht aufgehen und so in Widerspruch zur anvisierten Identifizierung geraten.

a. Verhältnis Begriff und Sache - Im begrifflichen Bemühen um den (historischen) Ursprung einer Sache, dem Ursprungsdenken, kommt es zur Verkennung, weil es eigentlich keine Phänomene gibt, die so statisch sind, dass sie als ewige Wahrheiten gelten könnten. Das hat immer einen Aspekt von der „Geschichtlichkeit der Welt (...), die grundsätzliche Erfahrung davon, dass es eigentlich nichts gibt, zwischen Himmel und Erde, was einfach so sei, sondern dass alles, was ist, 
eigentlich als ein Bewegtes und als ein Werdendes zu begreifen ist“ (ebd., 20). Gleiches gilt für das Telos: Auch ein endgültiges Ziel als ,Letztbegründung ist damit nicht zwangsläufig anzugeben bzw. ebenso problematisch. Die Kunst, vor die der Gebrauch der Begriffe die dialektische Methode stellt, ist also, das in jedem Begriff Enthaltene zu bewahren, es nicht abzuschneiden, nicht durch willkürliche Setzungen oder Festlegungen zu verdecken, aber es gleichzeitig so zum Bewusstsein zu erheben, dass es doch eben aus der Sphäre der Zweideutigkeit heraustritt. Diese nach Adorno innere Vermittlung zielt „auf den Sachverhalt, dass es Phänomene geben kann, die in einem strengen Gegensatzverhältnis zueinander stehen und bei denen dennoch der jeweils eine Pol den anderen (oder zumindest Merkmale des jeweils anderen) in sich enthält" (Ritsert 2011, 224). Eine Sache verweist zugleich auf das ihr Entgegengesetzte: Inklusion auf Exklusion, Bildung auf Dummheit ${ }^{1}$, Normalität auf A/Normalität, Zuschreibung von Behinderung auf Zuschreibung von Fähigkeiten; und zugleich ist Exklusion nicht gleich Inklusion usw. Dialektik ist damit „eine Veranstaltung des Denkens“ (Adorno 2015, 279), um „eben diesem Eigentümlichen des Begriffs gerecht zu werden, ihn weder als ein Unbestimmtes und Vages also zu nehmen, noch durch die Willkür der Definition ihn einfach abzuschneiden" (ebd.). In der ständigen Konfrontation des Begriffs mit der Sache, verstanden als immanente Kritik, gilt es, die begriffliche Definition als unzulänglich zu kennzeichnen. Bereits im Bemühen, genauer zu beschreiben, ergibt sich durchaus eine Änderung der Sache selbst (vgl. ebd., 18f.).

b. Verhältnis Allgemeines und Besonderes - Hinzu tritt das Verhältnis vom Ganzen als System und seinen Teilen, welches Adorno als komplex beschreibt und in dem das Einzelne nicht selbstständig (autonom) ist, weil: ohne das Ganze nicht zu denken, jedoch auch nicht durch die Totalität determiniert. Es ist nicht möglich, alles lediglich (begrifflich) in ein Ganzes (Theorie) einzufügen, „die Beschränktheit des Gedankens übersteigen und den Gedanken an seine richtige Stelle bringen“ (ebd., 53), sondern, „dass der Gedanke sich wirklich auf Gnade und Ungnade, unbarmherzig in die Dialektik selber hineinbegeben muss" (ebd.). Durch Denken des Teils ist das Ganze ebenso wenig bereits positiv gegeben wie umgekehrt, „sodass sie sich also unablässig fragen müssen, wie sie diese beiden Dinge eigentlich zusammenbringen“ (ebd., 129). Denn sie sind "nicht gleichsam zeitlos, dinghaft, gleichzeitig miteinander da“ (ebd.), sondern durch eine dynamische Beziehung charakterisiert. Für kritische Theorie heißt das bspw., dass Erkenntnis nie allein (Besonderes) stattfindet, sondern immer in einem größeren Zusammenhang (Allgemeines) gesehen werden

1 Horkheimer und Adorno (1989) sprechen von der ,Dummheit als Wundmal', etwas, was einer Person von anderen ,geschlagen ' wird. In diesem Sinne ist Dummheit als Ergebnis der Zueignung das Gegenteil von Bildung. 
muss. Auch Wissenschaft, und mit ihr Pädagogik und Inklusionsforschung, hat in einer spezifischen Gesellschaft eine spezifische Funktion. „Die Kontrolle wissenschaftlicher Urteile muss vielfach erfolgen, indem man Schritt für Schritt sich klar macht, wie man jeweils zum Urteil gelangte“" (Adorno 2003, 195).

c. Widerspruch - Widersprüche zeigen sich, da die Sache bzw. der Gegenstand nicht im Begriff, in der Definition aufgehen, sondern Beziehungen darstellen. Was der Begriff nicht fassen kann, das Nichtidentische, muss mitgedacht werden. „Kein Begriff, kein Urteil, kein System von gegenstandsbezogenen Sätzen kann die unendliche Mannigfaltigkeit der Merkmale jemals ausschöpfen, welche dem interessierenden Gegenstand zukommen und/oder zukommen könnten. Was ist, ist mehr, als es für uns ist und jemals sein kann" (Ritsert 2011, 228). Denn dort, wo die Dinge sich selbst gleichbleiben und miteinander identisch sind, besteht keine Notwendigkeit, die Gesetze der Entwicklung ${ }^{2}$ zu durchschauen, sondern nur dort, wo sie sich selbst widersprechen. Für die Dialektik gilt deshalb, die Unvermeidlichkeit der Widersprüche in der Sache selbst und das Weitertreibende der Widersprüche, die „Konsequenz des Gedankens" (Adorno 2015, 48), ernst zu nehmen. Widerlegung hat keine äußeren Gründe, die an den Begriff herangetragen werden, sondern das zu Erklärende (die Welt) ist in sich widersprüchlich, als Begriff für die widersprüchliche Praxis. Ein Gedanke, ein Prinzip, eine Definition, sich absolut setzend, ist bereits falsch. Definitionen, von Inklusion bspw., sind nicht durch Spielereien mit verschiedenen Ansichten - einen „sophistischen Meinungsstreit“ (ebd.) zwischen/unter Befürworter*innen oder Kritiker*innen - zu entkräften, sondern mit der Kritik der dahinterstehenden Erkenntnismethode. Wie wird Inklusion beschrieben, welche analytischen Kategorien werden benutzt? Welche Beziehungen zu anderen Begriffen werden hergestellt?33

2 Deshalb spricht materialistische Behindertenpädagogik (vgl. Jantzen 2007) davon, in Prozessen zu denken und nicht dinghaft. Das ist der Grund, weshalb die jeweilige Entwicklungssituation mit ihren Prozessen von Partizipation oder Isolation sowie die Zonen der aktuellen und nächsten Entwicklung im Mittelpunkt stehen. An der Entwicklungstatsache (vgl. Bernfeld 1973) gibt es kein Vorbei.

3 Als ,Paradebeispiel' sehe ich noch immer den Text ,Was ist Inklusion?‘ von Grosche (2015), in dem nicht einmal erwähnt wird, dass der Begriff aus der soziologischen Systemtheorie stammt und Exklusion als Komplementärbegriff zu verstehen ist. Stattdessen erfolgt die Darstellung einer historischen Abfolge von Begriffen (?), an deren Ende 28 Definitionen (!) vorgeschlagen werden, die verwendet werden können. 


\section{Konstellationen}

Im Aufeinander-verwiesen-Sein von Wahrnehmung (Forschung?) und Denken Letzteres ist erst die Reflexion des Denkens, die unbedingt notwendig ist - verortet Adorno die Idee von Subjektivität und Objektivität, die oftmals im falschen Sinne begriffen wird. Objektivität ist weniger wertneutrale denn möglichst umfassende Beschreibung und Analyse des Vorgefundenen ( $\mathrm{a}$ und b), wobei es die forschenden Subjekte sind, die ein Objekt beschreiben, analysieren, kritisieren. Zunächst heißt das, Erkenntnisoperationen und Sprachspiele machen Abstraktionen - das Abstrakte ist das für sich allein Stehende, ohne Beziehung - unvermeidbar. Daraus folgt, dass eine Beschreibung zum Referenten von Aussagen mit einer Fülle von Merkmalen und Beziehungen und daraus wiederum folgend von Schlüssen und Urteilen wird. Nur durch ein Subjekt (Forscher*in) ist ein Objekt erkennbar. „Wir beziehen uns ständig mit Sprache auf Themen (linguistisches Material), mit dem Geist auf geistige Phänomene. Doch daraus, dass uns alles Gegebene und Vorgegebene immer nur durch Empfindung, Wahrnehmung, Denken und Sprache gegeben sein kann, folgt logisch überhaupt nicht, dass alle Gegenstände Produkte unserer Empfindungen, unserer Gedanken, unserer Sprache wären“ (Ritsert 2011, 226). Hier laufen nach Ritsert verschiedene Themen und Thesen in Adornos Philosophie zusammen. Entscheidend ist, dass man sich nur dann einen klaren Begriff von Gegebenheiten machen kann, wenn man die Sensibilität für möglichst viel von dem entwickelt, woran der einzelne Begriff nicht heranreicht (Konsequenz aus Punkt a)). So wird das Nichtidentische zum Inbegriff dessen, was im ,jeweiligen System der Feststellungen nicht erfasst wurde und darin grundsätzlich nicht aufgehen kann" (ebd., 228).

Die einzelnen Wesenheiten sind als objektiver Zusammenhang (Totalität) zu erfassen, aber der objektiven Bestimmtheit nach als sich verändernd, als dynamische Prozesse, zu begreifen ${ }^{4}$. Vermittlung, das Verhältnis von Sein und Werden, gilt es ernst zu nehmen und damit ist die ,absolute Wahrheit " - bspw. ,Inklusion war schon immer dies oder jenes oder soll dies und jenes sein ' - ausgeschlossen. Wahrheit als objektives Kriterium entspricht keinem Einzelmoment der Erkenntnis und keine Einzelerkenntnis kann ihre ganze Wahrheit einlösen, sondern Erkenntnis und Wahrheit sind aufeinander verwiesen (vgl. Adorno 2015, 281). Wahrheit gibt es entsprechend „nur im Prozess, in der Konstellation der Momente, nicht in der Reduktion auf ein Urprinzip“ (ebd., 157) oder ein letztgültiges Telos. In dieser Konstellation richtet sich Dialektik als Kritik an der Erkenntnismethode

4 Diesen komplexen Zusammenhang hat Wolfgang Jantzen (2005) im Anschluss an die kulturhistorische Schule der sowjetischen Psychologie immer wieder beschrieben. Als kurze Einführung hier am besten: „Die Funktion des Ganzen ist aufgebaut als integrierende Tätigkeit, der hochdifferenzierte, hierarchisch verbundene, dynamische interzentrale Beziehungen zu Grunde liegen“ (ebd., o.S.). 
gegen eine geradlinige kontinuierlich verlaufende Entwicklung, sieht die Sachen selbst als widersprüchlich.

Inklusion (und Exklusion) als gesellschaftstheoretische Kategorie(n) können als angemessen betrachtet, ihr analytischer Gehalt muss immer wieder neu herausgestellt werden. Damit wird bedeutsam, ob ich im Zusammenhang mit Schule Begriffe wie ,Mündigkeit', ,Bildung', ,Lehren', ,Lernen` benutze oder ,Kompetenz', ,Sonderpädagogischer Förderbedarf ', ,Classroom-Management' oder aber, ob mit ,Teilhabe' dasselbe gemeint ist wie mit ,Inklusion'?

So wird Dialektik als Methode zu einer Sache der Erkenntnistheorie. Dieser Zusammenhang ist historisch und systematisch verschieden. Es kann Fortschritte und Rückschritte der menschlichen Erkenntnisfähigkeit in der Theorie, in den Begriffen geben. Die Zerlegung der Erkenntnis ist nicht schon die Erkenntnis selbst. Wir müssen Adorno zufolge die Beschränkung durchmachen, dass die kritische (Denk-)Bewegung bereits für die Wahrheit gehalten wird. Aber nur in der Bewegung, Vermittlung ist so etwas wie Wahrheit, Entstehendes, Entspringendes möglich. Es geht daher nicht (ausschließlich) um die Falschheit eines Satzes (einer Definition), sondern um dessen (deren) erkenntnistheoretischer Unzulänglichkeit und damit auch darum, die Erkenntnismethode selbstkritisch zu reflektieren: „Erkenntnisgewinn als Naivitätsverlust“ (Steinert 2007, 177). Das ist mit ,Bewegung' gemeint, denn ,Falschheit' ist wiederum nichts Zufälliges, wir geraten „in die Falschheit notwendig“" (Adorno 2015, 47) hinein.

\section{Materialismus oder Verhältnis Theorie - Praxis}

Die Konstellation der Begriffe und die Vermittlung von Allgemeinem und Besonderem verweisen in einem weiteren Schritt auf ein materialistisches Theorie-Praxis-Verhältnis. Denn hier gilt: Wenn alles gleich bleibt, still gestellt ist, gibt es keine Entwicklung, keine Bewegung. Dieser auf Marx bezogene Materialismus meint eine „handelnde Veränderung der Welt“ (ebd., 127), da konkrete Substanzen, soziale Formen' (vgl. Hirsch 1995), die Welt ausmachen. Zu dieser Materie (Institutionen, Organisationen) tritt der Mensch in ein Verhältnis, sinnlich tätig, in dem er einerseits dadurch geformt wird, aber auch Urheber*in dessen ist, und beruht ebenfalls auf geschichtlichen Entwicklungsschritten. „Das Bewusstsein kann nie etwas anderes sein als das bewusste Sein und das Sein des Menschen ist ihr wirklicher Lebensprozess ... Nicht das Bewusstsein bestimmt das Leben, sondern das Leben bestimmt das Sein“" (Marx \& Engels 1846, 26 zit. nach Aufenanger 1993, 104). Deshalb ist es wichtig, wie die Menschen arbeiten (Arbeitsteilung) oder lernen, sich miteinander in Beziehung zu setzen (funktional über bestimmte Rollenzuweisungen wie Konsument*in, Konkurrent*in, Kolleg*in, Bürger*in, 
Schüler*in, Behinderte*r, Ausländer*in ...), sich zu bilden, kurz: Es geht darum, wie sie ihre Gesellschaft gestalten. Kritische Theorie schärft den Blick dafür, sich nicht zu behaglich einzurichten.

Für kritische Theorie ist dabei charakteristisch, die Bedingungen der Möglichkeiten zu analysieren und mit der Realität ins Verhältnis zu setzen. Im Widerspruch geht es um die unabgegoltenen Ansprüche bzw. kann man das auch als verpasste Möglichkeiten der Entwicklung benennen, bspw. warum Menschen noch ausgeschlossen, diskriminiert werden, weshalb Inklusion gescheitert ist und welche Gründe dafürstehen, dies zu behaupten? Heinz Steinert (2007) verweist darauf, dass Adornos Theorie „kritisiert (...), indem sie auf die Versprechungen wie die Versagungen der bürgerlichen Gesellschaft hinweist und die Rückfälle auch der Intellektuellen aufzeigt. Sie macht uns keine Vorschriften, sie warnt uns" (ebd., 141). Kritik ist dabei „ein Instrument der Analysis. Als solches zielt sie in erster Linie auf sorgfältige Unterscheidungen von Merkmalen, klare Abgrenzungen von Begriffen sowie auf Schlüssigkeit der Urteile“ (Ritsert 2011, 230). Kritisches Denken im Sinne Adornos muss sich „nicht nur von jenen verdinglichten sprachlichen und kognitiven Rastern befreien, hinter denen der Wille zur Macht, zur Kontrolle und der stumme Zwang der Verhältnisse stehen, sondern sich so weit wie nur irgend möglich für die ,Vielheit der Verschiedenen' (Adorno), also diejenigen Bestimmungen der interessierenden Sachverhalte offen halten, welche durch das jeweils benutzte begriffliche Ordnungsmuster nicht erfasst wird“ (Ritsert 2014, 12). Dies legt zwei Dinge nahe. Erstens widerspricht das einem Monismus, der alles erfassen kann, und zweitens dem Relativismus, der alles als gleichgültig betrachtet. Dialektik hat jedoch eine normative Dimension, immer dann, wenn durch das Nichtidentische die Zwangsmechanismen, Macht- und Herrschaftsverhältnisse offengelegt werden, der negative Zustand der Welt „bei seinem eigenen Namen" (Adorno 2015, 106) benannt wird. Denn die Frage der Humanität, also die Diskussion um die Bedingungen des Zusammenlebens, ihrer institutionellen Ausgestaltung und deren Auswirkungen auf das einzelne Individuum, bleibt immerwährende Aufgabe der Menschen. Dies gilt auch für die Inklusionsforschung und damit ebenso für die Fragen nach Mündigkeit und Bildung als Menschenrecht (vgl. Gruschka 2015).

Festzuhalten ist: Der negative Zustand der Welt lässt sich am individuellen Leid des einzelnen Individuums (Besonderes) festmachen und den bestehenden gesellschaftlichen Möglichkeiten (Allgemeines) gegenüberstellen. Kritische Theorie, und mit ihr dialektisches Denken als Methode, ist eine Instanz, die dazu verhilft, dies auszusprechen (vgl. Bärmig 2017). Dialektik, wie sie als ,Methode‘ hier vorgestellt wird, heißt also, die Begriffe zwar ernst zu nehmen, sie jedoch nicht als abzuarbeitende oder zu erreichende Definitionen stillzustellen. Es geht bspw. darum, ob ,Inklusion' tatsächlich das beschreibt, was damit gemeint ist, weil es im Besonderen für eine Person als Begriff selbst nicht hinreicht, oder darum, welche 
Teile der Gesellschaft Inklusion durch den Einschluss in eine Institution, also als Ausschluss, erfahren ${ }^{5}$.

Dabei ist - negativ formuliert - festzuhalten, was unterbleiben soll oder ,zu unterlassen ist', z.B. ,Einschluss ist nicht Inklusion'. Das Positive wiederum kann zwar anvisiert, doch nie festgehalten werden. Es würde zu einem statischen Phänomen, selbst wenn Veränderung stets eine „wie immer auch erklärungsbedürftige Vorstellung vom Richtigen" (Ritsert 2011, 232) braucht. Reflexivität ist ein zentrales Element dialektischen Denkens. Sie kommt nicht von außerhalb, sie erkennt den Herrschaftszusammenhang und ist vor allem der Anwendung nach in einem naiven Theorie-Praxis-Verhältnis entgegengesetzt, da Widerspruch und Nicht-Identität nicht im bloßen Denken aufhebbar sind (vgl. ebd., 228). Adornos Methodenverständnis, so Ritsert weiter, sein Denken und Schreiben, lässt sich mit „keiner metalogischen Position vereinbaren, die Form und Inhalt trennt, wenn nicht dichotomisiert. Er hält diese strikte Trennung - (...) - für schlechthin undurchführbar" (ebd., 223). Ritsert betont, dass es keine Schnittmenge mit einer ,zufällig' immer gleichen Aufteilung gibt, es nicht um einen Moderantismus als einheitlichen Standpunkt einer, harmonischen' Mitte, mengenlogisch betrachtet, geht. Was wäre auch die Mitte von Inklusion, Normalität oder Bildung?

\section{Literatur}

Adorno, Th. W. (2003): Negative Dialektik/Jargon der Eigentlichkeit. Frankfurt am Main.

Adorno, Th. W. (2015): Einführung in die Dialektik. Frankfurt am Main.

Aufenanger, J. (1993): Bertelsmann Lexikon Philosophie. Gütersloh.

Bärmig, S. (2017): Behindertenpädagogik und kritische Theorie. In: Lütje-Klose, B., Boger, M., Hopmann, B. \& Neumann, P. (Hrsg.): Leistung inklusive? Inklusion in der Leistungsgesellschaft. Band 1. Bad Heilbrunn, 127-138.

Bernfeld, S. (1973): Sisiphos oder die Grenzen der Erziehung. Frankfurt am Main.

Grosche, M. (2015): Was ist Inklusion? - Ein Diskussions- und Positionsartikel zur Definition von Inklusion aus Sicht der empirischen Bildungsforschung. In: Kuhl, P., Stanat, P., Lütje-Klose, B., Gresch, C., Pant, H. A. \& Prenzel, M. (Hrsg.): Inklusion von Schülerinnen und Schülern mit sonderpädagogischem Förderbedarf in Schulleistungserhebungen. Bielefeld, 17-39.

Gruschka, A. (2015): Verstehen lehren. In: Schnell, I. (Hrsg.): Herausforderung Inklusion - Theoriebildung und Praxis. Bad Heilbrunn, 223-232.

Hirsch, J. (1995): Der nationale Wettbewerbsstaat - Staat, Demokratie und Politik im globalen Kapitalismus. Berlin.

Horkheimer, M. \& Adorno, Th. W. (1989): Dialektik der Aufklärung - Philosophische Fragmente. Leipzig.

Jantzen, W. (2005): Was ist der Mensch? Konturen einer marxistischen Anthropologie. Online unter: http://www.basaglia.de/Artikel/Mensch-MBl-2005.pdf (letzter Zugriff: 23.02.2021).

5 Es ist erstaunlich, dass alle großen Träger der Behindertenhilfe in ihren schriftlich fixierten Leitbildern von Inklusion sprechen, Heime jedoch oftmals eher mit Exklusion in Verbindung zu bringen sind. Auch diese thematischen Widersprüche in verschiedenen Bereichen der Gesellschaft, die weniger frei sind als andere, sind charakteristisch für gesellschaftliche Entwicklung. 


\section{6 | Sven Bärmig}

Jantzen, W. (2007): Kritisch-materialistische Behindertenpädagogik. In: Greving, H. (Hrsg.): Kompendium Heilpädagogik. Band 2. Troisdorf, 86-95.

Ritsert, J. (2011): Methode. In: Klein, R., Kreuzer, J. \& Müller-Doohm, S. (Hrsg.): Adorno Handbuch. Leben - Werk - Wirkung. Darmstadt, 223-232.

Ritsert, J. (2014): Themen und Thesen kritischer Gesellschaftstheorie. Weinheim/Basel.

Steinert, J. (2007): Dialektik der Aufklärung als Forschungsprogramm. Münster. 


\section{Jan Jochmaring, Lena Bömelburg und Dirk Sponholz}

\section{Inklusive Berufsorientierung als Diskurs. Der ,scheinbare' Konsens: gemeinsame Begriffe - unterschiedliche Ideen}

Der Begriff inklusive Berufsorientierung gewinnt zunehmend Bedeutung in der wissenschaftlichen Diskussion. Der vorliegende Beitrag analysiert den Diskurs zur inklusiven Berufsorientierung und fokussiert mit der Legitimations-, Stellvertretungs- und Gestaltungsformation drei zentrale Aspekte der Diskursorganisation, die es spezifischer zu betrachten gilt. ,Grenz.Gänge` ergeben sich dabei neben dem inhaltlichen Bezug zur Transitionsforschung vor allem durch die Interdisziplinarität des Diskurses. Deutlich wird der nur scheinbare Konsens im Diskurs, dessen konstitutives Merkmal vielmehr eine Begriffsvielfalt ist.

\section{Inklusive Berufsorientierung - Entstehungskontext}

Die inklusive Berufsorientierung ist ein junges Diskursfeld, das in den letzten Jahren eine wachsende Bedeutung erfährt. Der Diskurs um inklusive Berufsorientierung ergibt sich aus der Verbindung der zentralen Begriffe Inklusion und Berufsorientierung. Zunächst ist festzuhalten, dass es keine klare disziplinäre Verortung für den Diskurs gibt. Beteiligte Fachdisziplinen sind unter anderem Sonderpädagogik und Erziehungswissenschaft, Soziale Arbeit, die Fachdidaktik(en), Berufspädagogik sowie Soziologie und Psychologie. Trotz thematischer und fachdisziplinärer Unterschiede und Themengewichtungen lässt sich unabhängig davon ein disziplinübergreifender Konsens feststellen: Die inklusive Berufsorientierung wird als Herausforderung wahrgenommen (vgl. Nentwig, Sponholz \& Jochmaring 2019).

Um die Entwicklung der zentralen Diskursereignisse zu umreißen, sind zunächst die wichtigsten bundesweiten Ereignisse zu beleuchten. Grundlegend ist die sogenannte ,Hauptschuldebatte ' der 1960er-Jahre in Deutschland. Aus ihr ist die erstmalige Verankerung der vorberuflichen Bildung als schulischer Schwerpunkt hervorgegangen. Es folgt im Jahr 1993 die Empfehlung der Kultusministerkonferenz, die Berufsorientierung als Pflichtaufgabe aller Schulformen der Sekundarstufe I und II vorsieht. Darüber hinaus gibt es bundeslandspezifische Initiativen, die auf Verankerung einer verbindlichen und strukturierten, schulform- und schulab- 
schlussübergreifenden Berufsorientierung abzielen (u.a. vgl. MAGS NRW 2018). Die UN-Behindertenrechtskonvention 2009 bildet den gesetzlichen Fixpunkt zur Inklusion. Mit den Artikeln 24 und 27 sind die Forderungen eines inklusiven Schulsystems und Arbeitsmarktes verbunden.

Beide Diskursfelder der Berufsorientierung und der Inklusion sind in den letzten Jahren durch eine zunehmende Dynamik gekennzeichnet. Dies geht einher mit einer steigenden Bedeutsamkeitszuschreibung. In ihrer (begrifflichen) Verbindung wird die inklusive Berufsorientierung zum neuen Diskursfeld. Den Ausgangspunkt der Diskursanalyse bildet eine Recherche in der Datenbank FIS-Bildung. Sie umfasst alle Teilbereiche des Bildungswesens im deutschsprachigen Raum. Die Ergebnisse verdeutlichen quantitative Unterschiede zwischen den einzelnen Schlagworten. Der Begriff der Berufsorientierung verzeichnet 2.727 Treffer, in der Kombination Inklusion und Berufsorientierung werden lediglich 39 Treffer angezeigt. Mit dem Begriffspaar Inklusion und Beruf werden mit 653 Treffern deutlich mehr gekennzeichnet. Bei der Verwendung des zentralen Diskursgegenstandes inklusive Berufsorientierung werden in der Datenbank FIS-Bildung gar keine Treffer angegeben. Betrachtet man die gelisteten Veröffentlichungen zu Inklusion und Berufsorientierung genauer, fällt die zeitliche Verteilung auf. Von den 39 Treffern für den Zeitraum 2000-2020 fällt jeweils ein Treffer in das Jahr 2007 und 2008, alle anderen finden sich in der Phase ab 2011. Es ist davon auszugehen, dass mit der Verabschiedung der UN-BRK 2009 ein Bedeutsamkeitsanstieg verbunden ist, welcher sich durch die Vielzahl der Publikationen in den 2010er-Jahren zeigt.

\section{Aktueller Diskurs - Themenfelder und Schwerpunkte}

Die Diskursanalyse verweist auf drei zentrale Themenfelder: Grundlegend zählt dazu die Auseinandersetzung mit der Frage, was inklusive Berufsorientierung im engeren Sinn eigentlich ist (vgl. Günther 2012; Koch 2014; 2015; 2017). Ein zweites Themenfeld fokussiert die Übergangsprozesse mit enger Anbindung an die Benachteiligtenförderung (vgl. Bojanowski \& Eckert 2012; Fiegert, Graalmann \& Kunze 2016). Das dritte umfasst die berufliche Bildung im Allgemeinen (vgl. Bylinski \& Rützel 2016; Erdsiek-Rave \& John-Ohnesorg 2015; Euler \& Severing 2015; Gillen \& Wende 2017).

Es sind zudem zwei weitere Schwerpunkte des Diskurses auszumachen: Erstens wird Berufsorientierung als (explizit) schulische Aufgabe verstanden - hier liegt der Schwerpunkt auf dem Praxisbezug, in dem sowohl Fragen der schulischen Umsetzung als auch theoretische Grundannahmen bearbeitet werden. Zweitens wird die Untersuchung der Transition Benachteiligter in die Arbeitswelt thema- 
tisiert. Dazu liegen nur wenige empirische Studien mit kleinen Stichproben und explorativen Designs vor.

Als konstitutives Merkmal des Diskurses - sowohl in Bezug auf die Themenfokusse als auch die weiteren Untergliederungen in Schwerpunkte - ist grundlegend eine Begriffsvielfalt/-divergenz der Begriffe Inklusion und Berufsorientierung zu konstatieren. Als konstitutives Merkmal des Diskurses bildet dies den Ausgangspunkt der theoretischen Aufarbeitung der professionsübergreifenden Perspektiven im Rahmen des vorliegenden Beitrags.

\section{Forschungskonzeption der Diskursanalyse}

Bei der Vielzahl der verschiedenen Variationen der Diskursanalyse (vgl. Fegter, Kessl, Langer, Ott, Rothe \& Wrana 2015) ist die gemeinsame Basis der interdisziplinäre Blick und das Ziel, Wissens- bzw. Sinnproduktion zu analysieren. In der Orientierung am grundlegenden Verständnis sozialwissenschaftlicher Diskursanalysen (vgl. Traue, Pfahl \& Schürmann 2019, 567ff.) geht es im Weiteren um die Analyse der Wissensformen in einem spezifischen Verwendungszusammenhang. Die sozialwissenschaftliche Diskursanalyse zeichnet sich durch eine handlungstheoretische Orientierung aus, die aus diskursiven Praktiken besteht. Der Diskurs ist geprägt durch ambivalente und heterogene Diskurseinheiten (vgl. ebd., 566f.). Unterschieden werden kann der Diskurs als diskursive Praxis der geregelten Produktion von Aussagen und das durch Regeln charakterisierte Ordnungssystem selbst. In Bezugnahme auf Foucault (1981) hat eine Diskursanalyse das Ziel, diese Bezogenheit und Strukturierung zu analysieren. Innerhalb des diskursiven Feldes existieren spezifische Regelhaftigkeiten, indem diskursive Effekte wirken. Hier konstituieren sich verschiedene Diskursgefilde - die Gruppierungen von Aussagen wiederum werden als diskursive Formationen verstanden, die sich ähneln und sich in der Thematik aufeinander beziehen. Von Interesse ist hierbei vornehmlich, wie sich verschiedene Diskursgefilde konstituieren. Das können zum Beispiel die spezifischen Diskurse zu Inklusion oder Berufsorientierung sein (vgl. Nentwig et al. 2019) oder wie bei der wissenssoziologischen Subjektivierungsanalyse von Pfahl, Schürmann und Traue (2015) die sonderpädagogischen Diskurse um Lernbehinderung. Als diskursive Formation(en) werden die Abbildungen spezifischer Bedeutungen und Regelhaftigkeiten in einzelnen Diskursgefilden bezeichnet (vgl. Foucault 1992). Hier zielt der wissenschaftliche Blick auf theoretische Konzepte, zusammenfassende Begriffe, auf Systematisierungen und Erklärungsangebote.

Die vorliegende Diskursanalyse richtet den Fokus auf die produktive Seite des Diskurses: Verwendet wird im Kontext der inklusiven Berufsorientierung ein enger Themenbegriff als Basis, und zwar mit dem Ziel, den wissenschaftlichen 
Metadiskurs zum Vorschein zu bringen. Der Textkorpus der Diskursanalyse stützt sich infolge des Ausschlusses fehlerhafter Verschlagwortungen mit anderen Kontextbezügen auf 23 der oben angeführten 39 Treffer aus der Schlagwortsuche Inklusion und Berufsorientierung.

\section{Ergebnisse}

Im Folgenden werden drei ausgewählte diskursive Formationen zur inklusiven Berufsorientierung vorgestellt: die Legitimations-, Stellvertretungs- und Gestaltungsformation.

Im Fokus steht die Legitimierung des Diskurses, die sowohl (bildungs-)politisch als auch forschungsmethodisch argumentiert wird. Es ist festzustellen, dass stets die UN-Konvention als Referenzpunkt in den Artikeln benannt wird. Diese dient dazu, die Relevanz des Aufgabenfeldes zu benennen: Inhärent ist der Verweis auf die Bedeutsamkeit der Thematisierung. Beispielhaft wird von Koch (2017) die Notwendigkeit der Berufsorientierung betont. Die Relevanz des Aufgabenfeldes legitimiert sich implizit aus dem genannten Inhalt: ,Jugendliche, die voraussichtlich Schwierigkeiten haben werden, nach der Schule in eine berufliche Ausbildung einzumünden, sind besonders von den Folgen unzureichender oder fehlender schulischer Berufsorientierung betroffen" (ebd., 116).

Außerdem wird ein weiterer Forschungsbedarf abgeleitet. Begründet wird dieser wiederum mit der Notwendigkeit desselbigen. Verwiesen wird auf das bestehende Forschungsdesiderat hinsichtlich der Wirksamkeit der Berufsorientierung: „Man geht davon aus, dass Berufsorientierung zur Persönlichkeitsentwicklung beiträgt und den Weg in den Beruf ebnet, allerdings ist hierzu kein überzeugendes empirisches Material vorhanden“" (Heister 2016, 173).

Als weitere Legitimationsformation ist in einigen Artikeln die Benennung spezifischer ,Bedarfe' der Schüler*innen auszumachen, welche als Notwendigkeit für weitere Forschung herangezogen werden: „Jugendliche mit den sonderpädagogischen Förderbedarfen Lernen, soziale und emotionale Entwicklung sowie Sprache haben zur erfolgreichen Bewältigung des Übergangs von der Schule in den Beruf besondere Unterstützungsbedarfe" (Koch 2015, 284). Eine nähere Spezifikation steht jedoch aus.

Bei der Stellvertretungsformation wird die inklusive Berufsorientierung in Relation zu ,etwas anderem' gesetzt. Anstelle einer konkreten Definition erfolgt eine Begriffserweiterung. So wird sie als eine neue Aufgabe bzw. als Schulentwicklungsaufgabe interpretiert. 
„Setzt sich eine Schule zum Ziel, ihre Berufsorientierung inklusiven Maßstäben anzupassen, steht sie vor einer Schulentwicklungsaufgabe. Inhalte, Methoden, Strukturen und Strategien des Lernens in der Übergangsphase sind an die Bedürfnisse der Jugendlichen anzupassen, wobei in einer inklusiven Schule davon auszugehen ist, dass alle Lernenden einer Altersgruppe dazugehören“" (Koch 2017, 124).

Eng damit verbunden ist die Formation der inklusiven Berufsorientierung als Herausforderung, wie es in Aussagen von Koch (2015) und Gillen und Wende (2017) fast wortgleich zum Ausdruck kommt: „In einer inklusiven Schule steht die Berufsorientierung allerdings aufgrund der zunehmend heterogen zusammengesetzten Lerngruppen vor den besonderen Herausforderungen" (Gillen \& Wende 2017, o.S.).

Weiterhin findet sich der gerichtete Fokus auf die Barrieren und Probleme. Hier werden zum Beispiel sowohl die Unübersichtlichkeit der Berufsorientierung als auch Schwierigkeiten im Transitionsprozess benannt (vgl. Heister 2016; Koch 2014). Zudem wird ein Spannungsfeld zwischen Individuum und Arbeitswelt eröffnet und (thematisch) um Probleme am Übergang von der Schule in den Beruf erweitert, wie es Wagner $(2014,348)$ zum Ausdruck bringt:

„Individuelle Bildungsentscheidungen an Übergängen und daran anknüpfend ganze Bildungsverläufe von Jugendlichen erscheinen so wesentlich zum einen von den Zwecken und dem Aufbau des gesamten Bildungssystems und zum anderen vom Handeln seiner Organisationsmitglieder bestimmt."

Als weitere Barrieren der Berufsorientierung werden ein „schier undurchschaubarer Dschungel an Programmen, Veröffentlichungen und Materialien" (Schmidt \& Zauner 2014, 74 zit. nach Heister 2016, 170) identifiziert.

Ergänzende Themen sind soziale Ausgrenzungsmechanismen sowie der Zuweisungsprozess. Hierzu passt das Zitat von Rosenberger $(2017,101)$, die zu dem oben angeführten Transitionsprozess und den Zuweisungsprozessen kritisch anmerkt, „dass bei diesen Jugendlichen kaum von individueller Entscheidungsfreiheit und tatsächlich vorhandenen Auswahloptionen gesprochen werden kann.“

Bei der Gestaltungsformation geht es um die konkreten Handlungsaufträge in der Berufsorientierung - einfacher formuliert: Was ist zu tun?

Auszumachen ist die Thematisierung der Heterogenität. „Alle Schüler/-Innen sollen einen Zugang zu qualitativ hochwertigen Bildungsangeboten zur Berufsorientierung erhalten und entsprechend unterstützt werden" (Heister 2016, 170). Im Fokus steht folglich die gesamte Schüler*innenschaft.

Weiterhin wird die Kooperation thematisiert. In Bezug auf die Forderung der Kooperation merkt Koch $(2015,285)$ an, „dass eine inklusive Berufsorientierung nicht an einzelne Personen zu delegieren ist, sondern eine Aufgabe darstellt, die 
von einem multiprofessionellen Team getragen wird." Die zentrale Forderung bezieht sich demnach auf die Netzwerkarbeit und den multiprofessionellen Austausch.

Projekte und konkrete Ausgestaltungen bilden einen weiteren Strang. Vorschläge sind u.a. außerschulische Lernorte, z.B. Betriebe, Berufsinformationszentren und Werkstätten (vgl. ebd.) oder auch Schüler*innenfirmen (vgl. Balli 2015; Sponholz \& Lindmeier 2017). Grundsätzlich wird durch diese Formation die Forderung nach veränderten Konzepten und handlungspraktischen Angeboten deutlich.

\section{Organisation des Diskurses zu inklusiver Berufsorientierung - Diskussion und Fazit}

Die Gesamtheit der Ergebnisse zeigt eine zunehmende Ausdifferenzierung des Diskurses rund um inklusive Berufsorientierung. Unter der spezifischen Zielsetzung, die Wissensformierung dieses Gegenstands zu untersuchen, bilden die drei herausgearbeiteten diskursiven Formationen die zentralen Organisationseinheiten des Diskurses ab.

Die fehlende Eindeutigkeit des zentralen Begriffs ist zum einen konstitutives Merkmal des Diskurses, bedingt zum anderen aber auch seine spezifische Ausprägung durch die aufgedeckten Formationen. Prägnant zeigt sich eine inhaltliche Ausdifferenzierung des zentralen Diskursgegenstandes, verdeutlicht durch die Stellvertretungsformationen. Das Sprechen über inklusive Berufsorientierung schafft in der Folge eine Begriffserweiterung, die keiner Begriffsklärung im Rahmen der Einzelartikel zuträglich ist und zudem die Frage nach dem ,Inklusiven der Berufsorientierung unbeantwortet lässt. Weder die Einzelbegriffe der Inklusion und Berufsorientierung noch deren gemeinsame Verwendung werden im engeren Sinne spezifiziert. Dies zieht sich durch die Gesamtheit des Diskurses. Eine begriffliche Annäherung erfolgt vorrangig implizit. Angenommen wird ein konsensuales Grundverständnis, das jedoch nicht verschriftlicht wird. Zugleich verschwimmen die Grenzen zur allgemeinen Berufsorientierung. Es fehlt eine einheitliche, argumentativ starke Legitimierung für das Besondere der inklusiven Berufsorientierung. Die Legitimation findet sowohl über Kontextthematiken innerhalb der allgemeinen Inklusionsdebatte als auch über die allgemeine Berufsorientierung und Übergangssystematik statt. Es scheint, als sei an vielen Stellen das Spezifische des Begriffs kein Thema. Vielmehr steht eine grundlegende Auseinandersetzung unter einer neuen Überschrift im Fokus.

Konsensual ist eine positive Perspektive bzw. Notation des Begriffs festzustellen. Sie speist sich aus der wiederkehrenden Betonung der Schwierigkeiten und Hürden sowie den daraus resultierenden Bedarfen. Dies erklärt den Diskursschwerpunkt 
der Gestaltungsformation, die mit sprachlichen Mustern der Handlungsaufforderung einhergeht. Gekennzeichnet sind die Ausführungen durch Falldarstellungen und normative Forderungen, die aus den Einzelfällen abgeleitet werden. Ein argumentativer Diskurs, einhergehend mit der Übernahme bzw. Ablehnung von Positionen, findet sich an keiner Stelle. Einziger Bezugspunkt sind z.T. ähnliche Argumentationsansätze, die jedoch nicht explizit durch Verweise gekennzeichnet sind. So ist die steigende Veröffentlichungszahl nicht mit einer Zunahme neuer Erkenntnisse verbunden. Es finden sich Doppelungen bzw. ,Kopien' der Forderungen, die aus der allgemeinen Berufsorientierung auf die Inklusion übertragen werden.

Die in diesem Beitrag ausgearbeiteten Diskursformationen als organisatorisches Merkmal des Begriffs zeigen zusammenfassend die Multiplikation des Begriffs. Infolge der Annäherung über ausgewählte Formationen erfolgt eine stetige ,Umkreisung' des inneren Kerns der Thematik, die keine Auseinandersetzung tatsächlich aufgreift und auflöst; weder innerhalb der Fachdisziplinen noch im interdisziplinären Kontext. Zugleich zeigt sich in der gesamten Analyse die Verwobenheit der ausgewählten diskursiven Formationen.

Der vorliegende Beitrag ist als Aufschlag der diskursanalytischen Annäherung an die inklusive Berufsorientierung einzuordnen. Mit den herausgearbeiteten Formationen werden erste Ansatzpunkte des Diskurses aufgezeigt. Insgesamt ist in den kommenden Jahren ein Zuwachs an Veröffentlichungen im Themenfeld zu erwarten. Die Diskursanalyse ist diesbezüglich fortlaufend zu erweitern und in ihrer Entwicklung zu beobachten. Einen Ansatzpunkt bietet zudem die Synthese der Einzeldiskurse über Inklusion und Berufsorientierung - unter Berücksichtigung ihrer methodischen, theoretischen und gegenstandsbezogenen Differenzen (vgl. u.a. Pfahl 2011; Truschkat 2015).

\section{Literatur}

Balli, S. (2015): Inklusion im WAT-Unterricht. In: Forum Arbeitslehre, 14, 35-46.

Bojanowski, A. \& Eckert, M. (Hrsg.) (2012): Black Box Übergangssystem. Münster.

Bylinski, U. \& Rützel, J. (Hrsg.) (2016): Inklusion als Chance und Gewinn für eine differenzierte Berufsausbildung. Bielefeld.

Erdsiek-Rave, U. \& John-Ohnesorg, M. (Hrsg.) (2015): Inklusion in der beruflichen Ausbildung. Berlin.

Euler, D. \& Severing, E. (2015): Inklusion in der beruflichen Bildung. Umsetzungsstrategien für inklusive Ausbildung. Gütersloh.

Fegter, S., Kessl, F., Langer, A., Ott, M., Rothe, D. \& Wrana, D. (Hrsg.) (2015): Erziehungswissenschaftliche Diskursforschung. Empirische Analyse zu Bildungs- und Erziehungsverhältnissen. Wiesbaden.

Fiegert, M., Graalmann, K. \& Kunze, I. (Hrsg.) (2016): Schulische Übergänge gestalten - Brücken bauen. Osnabrück.

Foucault, M. (1981): Archäologie des Wissens. Frankfurt am Main.

Foucault, M. (1992): Die Ordnung des Diskurses. Frankfurt am Main. 


\section{Jan Jochmaring, Lena Bömelburg und Dirk Sponholz}

Gillen, J. \& Wende, J. (2017): Inklusion in der beruflichen Bildung. Status Quo, Konsequenzen und Potenziale für Forschung und Lehre. In: Zeitschrift für Inklusion, 3, o.S.

Günther, J. (2012): Gemeinsam(e) Perspektiven schaffen. Aspekte der Berufsorientierung von Jugendlichen mit Lernschwierigkeiten im Kontext der UN-Konvention für die Rechte von Menschen mit Behinderungen (UN-BRK). In: Forum Arbeitslehre, 9, 49-55.

Heister, M. (2016): Allen Schülerinnen und Schülern einen Zugang zu qualitativ hochwertiger Berufsorientierung ermöglichen! In: Bylinski, U. \& Rützel, J. (Hrsg.): Inklusion als Chance und Gewinn für eine differenzierte Berufsausbildung. Bielefeld, 169-182.

Koch, B. (2014): Berufsorientierung in einer inklusiven Schule. In: Berufs- und Wirtschaftspädagogik Online, 27, 1-18.

Koch, B. (2015): Inklusive Schule - und dann? Was Schulen zu einer inklusiven Berufsorientierung beitragen können. In: Wirtschaft \& Erziehung, 8, 283-288.

Koch, B. (2017): Teilhabechancen von Jugendlichen durch inklusive Berufsorientierung. In: Burow, O. A. \& Gallenkamp, C. (Hrsg.): Bildung 2030 - Sieben Trends, die die Bildung revolutionieren. Weinheim, 116-127.

MAGS NRW (Ministerium für Arbeit, Gesundheit und Soziales Nordrhein-Westfalen) (2018): Kein Abschluss ohne Anschluss. Übergang Schule - Beruf in NRW. Zusammenstellung der Instrumente und Angebote. Düsseldorf.

Nentwig, L., Sponholz, D. \& Jochmaring, J. (2019): Diskursanalyse inklusive Berufsorientierung. In: Lindmeier, C., Fasching, H., Lindmeier, B. \& Sponholz, D. (Hrsg.): Inklusive Berufsorientierung und berufliche Bildung - aktuelle Entwicklungen im deutschsprachigen Raum. 2. Beiheft Sonderpädagogische Förderung heute. Weinheim, 54-72.

Pfahl, L. (2011): Techniken der Behinderung. Bielefeld.

Pfahl, L., Schürmann, L. \& Traue, B. (2015): Das Fleisch der Diskurse. Zur Verbindung von Biographie- und Diskursforschung in der wissenssoziologischen Subjektivierungsanalyse am Beispiel der Behindertenpädagogik. In: Fegter, S., Kessl, F., Langer, A., Ott, M., Rothe, D. \& Wrana, D. (Hrsg.): Erziehungswissenschaftliche Diskursforschung. Wiesbaden, 89-106.

Rosenberger, H. (2017): Inklusion im Übergang von der Schule in Ausbildung und Arbeit? In: Gercke, M., Opalinski, S. \& Thonagel, T. (Hrsg.): Inklusive Bildung und gesellschaftliche Exklusion. Wiesbaden, 99-120.

Sponholz, D. \& Lindmeier, C. (2017): Inklusive schulische Berufsorientierung aus habitustheoretischer Perspektive. In: Vierteljahresschrift für Heilpädagogik und ihre Nachbargebiete, 86, 285-297.

Traue, B., Pfahl, L. \& Schürmann, L. (2019): Diskursanalyse. In: Baur, N. \& Blasius, J. (Hrsg.): Handbuch Methoden der empirischen Sozialforschung. Wiesbaden, 493-508.

Truschkat, I. (2015): Kompetenz im Übergang? Methodologische Reflexion eines dispositivanalytischen Zugangs in der Übergangsforschung. In: Fegter, S., Kessl, F., Langer, A., Ott, M., Rothe, D. \& Wrana, D. (Hrsg.): Erziehungswissenschaftliche Diskursforschung. Wiesbaden, 249-262.

Wagner, S. (2014): Wirtschaft statt Werkstatt: Berufsorientierung und Übergänge in Ausbildung für Jugendliche im sonderpädagogischen Förderschwerpunkt Lernen im Zeichen der Inklusion. In: Sonderpädagogische Förderung heute, 59, 343-356. 
Felix Kappeller

\section{Prothetische Absenz, Articulating Gaps: Repräsentationskritische Perspektiven auf visuelle Darstellungen nicht an den Körper gefügter Prothesen}

In diesem Beitrag beschäftige ich mich mit der visuellen Repräsentation des mit Prothesen versehenen Körpers, den Bedingungen, unter welchen ihm Sichtbarkeit zuteilwird, wie auch den Bedeutungen, die seine Darstellung zu produzieren vermag. Nachgegangen wird dem anhand zweier exemplarischer Fotografien, in denen Prothesen nicht an den Körper gefügt, aber im Bild befindlich sind - scheint diese Darstellung der Absenz von Prothesen doch gegenläufig zu dominanten Repräsentationsweisen. Ich zeige, wie sich solche Darstellungen und ihre Potenziale in einem Spannungsfeld referenzieller Verfangenheit und fragiler Artikulationsmöglichkeit bewegen.

\section{Körper und Prothetik im Zeichen neoliberaler Bildpolitiken}

Sichtbarmachungen von Körper und Prothetik wie auch ihre Bedeutungsproduktionen bewegen sich seit jeher auf spannungsreichen, sich historisch wandelnden Terrains. Gegenwärtig begegnen sie uns in kulturellen Aufführungen wie den Paralympics und ihren Medienkampagnen, auf Laufstegen renommierter Designer*innen oder in TV-Formaten wie Dancing with the Stars. Beerben diese Darstellungen ihnen vorgängige Modi des Zeigens und (An-)Sehens wie jene der Schaumedizin und sogenannter Freakdiskurse zwar in Teilen, indem das als anderskörperlich Diskursivierte visuell und inszenatorisch exponiert wird, ${ }^{1}$ sind sie doch durch neuartige repräsentationale Qualitäten gekennzeichnet. So zeigt z.B. Karin Harrasser (2013, 16ff.) bzgl. der Darstellungen und Selbstthematisierungen der doppelbeinamputierten Athletin Aimee Mullins und des dop-

1 Adressiert sind zwischen Verwissenschaftlichung und Spektakularisierung oszillierende Kontexte des (Be-)Deutens, des Sehens und der Wissensproduktion, die im 19. Jahrhundert Konjunktur hatten. Während etwa Fehlbildungen und Krankheitsverläufe anhand von Präparaten in Schausammlungen und pathologischen Museen präsentiert wurden, etablierte sich das spektakularisierende, schaustellerisch-gewerbliche Inszenieren von Behinderung, ethnischer und geschlechtlicher Differenz unter dem Label ,Freak' (vgl. im Weiteren z.B. Dederich 2007, 85ff.). 


\section{Felix Kappeller}

pelbeinamputierten Bionikers Hugh Herr, dass sich solche Repräsentationen in der Regel durch eine Affirmation und Verkörperung von Wettbewerbslogiken, Leistungsbereitschaft und Selbstdisziplin auszeichnen. Sie stünden verschlungen mit einer heteronormativ kodierten „Idee von Schönheit, die den gegebenen Körper als empfangsfähiges und modellierbares Material behandelt" (ebd., 21), und scheinen dem spätmodernen Narrativ einer das ganze Selbst betreffenden Entrepreneurship verpflichtet.

Als behindert diskursivierte Körper und Subjekte werden in diesen Darstellungen zuvorderst anhand eines Überwindens von Widrigkeiten, als inspirierend, faszinierend und Spektakel des (An-)Sehens inszeniert. Prothesen sind dabei in der Regel an den Körper gefügt und avancieren inszenatorisch zu Garanten und Vehikeln eines Gelingens.

Solche Darstellungen lassen sich damit als beispielhaft für flexibel-normalistische Repräsentationsregimes und ihre neoliberalen Bildpolitiken perspektivieren: Anderskörperlichkeit und zuvor ausgeschlossene Positionen stünden nicht mehr im Zeichen des Verworfenen und nicht für Sichtbarkeit Qualifizierten. Vielmehr komme es zu einer ,hochgradigen Kommerzialisierung und Individualisierung von Körpern“ (Raab 2010, 88), die das bisher Minorisierte einschlössen. Differenz werde dabei „als erfreuliches Spektakel oder als kulturelles Kapital“ (Engel 2009, 13), als virtuos gestaltbar und vermarktbar präsentiert.

\section{Prothetische Absenz repräsentationstheoretisch und -kritisch}

Gemäß einem diskurstheoretischen Verständnis von Repräsentation als bedeutungsherstellender Praxis (vgl. Hall 2013) rücken diese Darstellungen bzgl. ihrer wirklichkeitsproduzierenden Effekte in den Blick. Als Bestandteile, Ausdrücke und Effekte diskursiver Praktiken und epistemischer Ordnungen lassen sie sich als an der Produktion von Wissen, Wahrnehmungs- und Deutungsordnungen mitsamt in ihnen prozessierter Subjektpositionen und Identifikationsangebote beteiligt verstehen (vgl. ebd., 26ff.; Schaffer 2008, 81). Die Produktion und Dekodierung von Bedeutungen, visuellen und andersmedialen Repräsentationen erscheinen damit weniger als Resultate intentional orchestrierter Akte, sondern als durch kulturell herrschende Kodes, Darstellungsvokabulare und Repräsentationsgrammatiken reguliert und formatiert (vgl. Hall 2013, 39). Kaja Silverman (1996) beschreibt dies durch das Konzept des kulturellen Bildrepertoires als Set historisch spezifischer, kulturell zur Verfügung stehender Bilder, Vorstellungen, Repräsentationsparameter und -weisen sowie Modi des Wahrnehmens und Wahrgenommenwerdens. Zwar fasse dieses Repertoire gleichermaßen dominante und normative, subkulturelle und oppositionelle Bilder (vgl. ebd., 179), bestimmte 
würden sich in Produktion und Deutung jedoch förmlich aufdrängen, da sie qua Wiederholung hochgradig konventionalisiert, naturalisiert und damit in den Worten Silvermans vor-gesehen sind (vgl. ebd., 221).

Werden die angeführten Darstellungen in Richtung dominanter Bedeutungsweisen und eines Vor-Gesehenen perspektiviert, lässt sich im Sinne einer diskurstheoretisch informierten Repräsentationskritik fragen, welchen Verkörperungsmodi von Prothetik Sichtbarkeit zuteilwird, -werden kann und ferner: wo, wie und unter welchen Bedingungen sowie durch welche Unsichtbarmachungen? Heike Raab $(2010,88)$ und Monika Windisch (2014) verweisen darauf, dass neuartige Formen der einschließenden Sichtbarkeit und der Anerkennung einer (Re-) Affirmation persistenter normativer Kriterien, „die als relativ beständige Indikatoren für ,Normalität‘ und Erfolg gelten“ (ebd., 87), verpflichtet blieben, somit also spezifische Normen zitieren und sich letztlich über Ausschlüsse verwirklichen (müssen). Hierfür beispielhaft resümiert Marquard Smith $(2007,66)$ bzgl. der Repräsentation der female amputee, dass spezifische Sichtbarmachungen von Vulnerabilität und Instabilität (noch) nicht für den Diskurs der Prothese „with its overwhelming imperatives of rehabilitation, empowering, and resolute unshakablity" qualifiziert seien.

Hiervon ausgehend werden im Folgenden beispielhaft zwei Fotografien analysiert, die Amputiertenkörper ohne an den Körper gefügte, aber im Bild befindliche Prothesen zeigen. Diese Darstellungen sind in mehrerlei Hinsicht geeignet, um die aufgezeigte Ambivalenz von Ein- und Ausschluss, Sichtbarkeit und Unsichtbarkeit für die visuelle Repräsentation von Körper und Prothetik und ihre Bedingungen zu betrachten. Solche Sichtbarmachungen scheinen zumindest potenziell dominanten Darstellungen, die Körper und Prothese zumeist in einem gelingenden Gemeinsam inszenieren, zu widersprechen und damit Raum für Differenziertheit, Brüchigkeit und Mehrdeutigkeit zu schaffen. Dies lässt sich einerseits in der durch die Absenz, aber Kopräsenz des Prothetischen erwirkten Gleichzeitigkeit der Dargestellten als Amputierte und als Subjekte der Prothese verorten; andererseits vor allem in den sich auftuenden Zwischenräumen zwischen Körper und technischem Artefakt, den gaps.

In verflochtener Form soll gefragt werden, unter welchen Bedingungen prothetischer Absenz Sichtbarkeit verliehen wird und welche Bedeutungen solche Darstellungen über Körper und Prothetik produzieren (können). Aufgrund ihrer Verwicklung in Mobilität, bzw. im Falle ihrer Absenz potenzieller Immobilität, wird hierbei Beinprothetik fokussiert. 


\section{Erste Lektüre prothetischer Absenz}

Die erste Fotografie ist Bestandteil des Hunger Games-Franchise und fand sich im Zuge der Veröffentlichung des dritten Filmteils, Mocking Jay 1, auf der indes offline geschalteten Homepage thecapitol.pn. Bei der Seite handelte es sich um ein fiktives Selbstrepräsentationsformat des filmisch dargestellten Regimes und Regierungssitzes der in Distrikte eingeteilten Nation Panem, dem Capitol. Über diese Plattform wurde sich an fiktive Bürger*innen gewendet. Die Fotografie ist eines von sieben Plakaten, die je eine*n Held*in einer der Distrikte zeigen. Das Plakat gehört zu Distrikt 7, dem Lumber District, wo vornehmlich der Holzfällerei nachgegangen wird. Die abgebildete Person, Alex Minsky, im realen Leben US-amerikanischer Kriegsveteran, wird als Elias Haan präsentiert:

Die hochformatige Fotografie zeigt eine zügig als Mann identifizierbare Person mit kurzen Haaren, Bart und freiem, tätowiertem und muskulösem Oberkörper. Er ist Betrachter*innen frontal zugewandt, sein Blick strebt leicht gen oben, zum rechten Bildrand. Er sitzt auf einer Art Holzbank. Zu seiner Rechten ist ein rot-schwarz-kariertes Flanellhemd abgelegt, zur Linken befindet sich ein Stapel Holz, an die Bank ist eine Doppelaxt gelehnt. Er trägt verschlissen wirkende, leicht fleckige Jeans, deren rechtes Bein über dem Knie abgetrennt ist, wodurch das rechte Bein als unter dem Knie amputiert preisgegeben wird. Linksseitig trägt er einen abgenutzt wirkenden Schnürstiefel. Der Bildhintergrund wirkt wie ein blassgrauer, kahler Raum. Von oben links scheint scheinwerferartig Licht einzufallen, wodurch Partien des Oberkörpers glänzen und einzelne Schweißperlen zu sehen sind. Auf den leicht gespreizten Oberschenkeln liegt in der Waagerechten ein als Unterschenkel bzw. Unterschenkelprothese gefertigtes Holzobjekt mit plastischem Fußelement mitsamt Knöchel, Zehen und Zehennägeln. Oben rechts ist in Rot ein Logo positioniert, das in Weiß die Zahl 7 und einen Baum mit zwei überkreuzten Äxten abbildet. Darunter, ebenso in weißer Schrift, steht geschrieben: ,The Capitol salutes citizens in the Lumber District' und darunter, kleiner: ,Your diligent work makes us proud. Love your labor. Take pride in your task. Our future is in your hands.'

Bildbeschreibung zu: Elias Haan, District 7 (Alex Minsky für Bildkampagne von The Hunger Games: Mocking Jay (Teil 1)), 2014, Urheber*innenschaft: Lionsgate. Bildquelle: https:/www.thecapitol.pn/ downloads/posters/district07.jpg (letzter Zugriff: 20.11.2019)

Die Fotografie scheint eine Verflechtung von Männlichkeit, Leistung/Arbeit, Prothetik und Patriotismus zu zitieren, wie sie im Besonderen im Kontext von Nachkriegsprothetiken kultiviert wurde. Beispielhaft zeigt David Serlin (2002) für die USA der späten 1940er- und der 1950er-Jahre, dass gängige Darstellungen Amputiertenkörper einerseits als Symbole eines „unequivocal heroism“ (ebd., 51) figuriert hätten. Andererseits hätten vor allem spätere Repräsentationen Versehrte mit Prothesen bei der Verrichtung zeitgenössisch populärer, männlich kodierter Tätigkeiten gezeigt und auf eine visuelle Evidenz der Erwerbspraktikabilität spe- 
ziell gefertigter Prothesen gesetzt (vgl. ebd., 53ff.; 63ff.). Mit Prothesen als Vehikel sollten körperliche wie seelische Traumata qua Maskulinisierung und (Hetero-) Sexualisierung, zumindest im Gewand medial-diskursiver Verheißung, überwunden werden.

In der Fotografie finden sich diese Inszenierungsweisen wieder, jedoch neuartig verhandelt. Nicht nur durch den textlichen Verweis, sondern vor allem durch die offensichtliche Zitation des vor-gesehenen und ikonisch gewordenen Bildes eines Lumberjack, eines Holzfällers, wird offeriert, die Person auf einen ersten Blick als Arbeiter zu dekodieren: Blue Jeans, rot-schwarzes Karohemd und Bart sind Arbeitsbekleidung, Axt und Holz Werkzeug und Material. Die so erwirkte tradierte Konnotation viriler Männlichkeit findet sich auch in der durch Lichteinfall betonten, einer Sexualisierung zuarbeitenden muskulösen Verfasstheit von Oberkörper und Armen bestätigt.

Auf einen zweiten Blick jedoch verwirklicht sich diese Referenz auf Arbeit visuell insofern in differenzierter Form, als kein konkretes Arbeit-Tun dargestellt wird: Die Person sitzt, die Hände ruhen, die Axt lehnt. Stattdessen scheinen die glänzende, schweißbelegte Haut, das abgestreifte Hemd, das gehackte und gestapelte Holz Zeugnisse einer stattgefundenen, verrichteten Arbeit zu sein. So handelt es sich scheinbar um eine Darstellung eines erwerbskontextuellen Pausierens oder Unterbrechens. Hier scheint sich das als Unterschenkel gefertigte Holzobjekt einzufügen. Die räumliche Nähe von hölzerner Versatzstelle und Amputationsort impliziert eine Lektüre als Prothese; als Prothese, die ganz ähnlich dem Hemd während des referenziell im Bild angezeigten Arbeitens angelegt war und im Moment des Pausierens abgelegt wurde.

Diese Darstellung prothetischer Absenz ist gewissermaßen und mindestens in zweierlei Form ambivalent. Zunächst wird durch sie einerseits, und das macht sie produktiv, das vor-gesehene und intertextuell operierende, mit dem Prothetischen verwobene Bild eines Tuns, Bewegens und Arbeitens differenziert, indem ein Tun, Bewegen und Arbeiten eben nicht abgebildet wird. Andererseits bleibt die prothetische Absenz diesem Bild aber verhaftet, insofern impliziert ist, sie verzeitlicht in ein praktisches Vollzugskontinuum einzuordnen, das auch ein Wiederanlegen der Prothese zu Verrichtungs- bzw. Arbeitszwecken fasst. Prothetische Absenz erlangt hier Sinnfälligkeit, indem sie normativ ausgerichtet einen dominanten Bedeutungshorizont unangefochten lässt und qua Referenz bestätigt. Ähnlich verhält es sich mit einer Lesart, die das als Unterschenkel geformte Holzobjekt als durch die als Fachmann für Holz inszenierte Person produziert deutet. Überspitzt formuliert würde so nicht nur ein tatkräftiger, seinen Körper fit haltender Arbeiter, sondern auch ein Subjekt gezeigt, das nahezu selbstumsorgerisch seine eigene prothetische Versorgung orchestriert. Dass das Objekt ob der Gelenklosigkeit, der feinen und makellosen Textur eher wie ein kunstvoll gefertigtes, behutsam vor Verschleiß zu schützendes Artefakt denn wie eine einsetzbare Prothese wirkt, bildet 
hierbei unbestreitbar ein Irritationsmoment, das in meinem Dafürhalten jedoch der ikonisch operierenden, sich aufdrängenden Arbeiterreferenz eher anheimzufallen droht.

Schließlich muss das in der Fotografie angelegte, wenn auch ambivalente Potenzial zur Differenzierung insoweit als herausgefordert gelten, als die Darstellung durch den Repräsentationskontext sowie die gestalterischen Mittel droht, ins Irreale und Fiktive abzudriften. Die Website, auf der das Foto in Plakatform zu sehen gegeben wurde, evoziert als rahmender Ort des $\mathrm{Zu}$-sehen-Gebens eine Lektüre des Bildes als Bestandteil der im Film für das Regime als typisch inszenierten Propagandapraktiken. Dem geschmeidig zuarbeitend wirkt die Darstellung vor allem durch Licht und abgebildetes, an einen White Cube erinnerndes Setting szenografisch stark komponiert, gar artifiziell. Weichzeichnung und Kontrastierung verunklaren, ob es sich nicht eher um ein plastisches Gemälde denn um eine Fotografie handelt. Damit droht prothetische Absenz, sich eher als gestalterisches Mittel eines künstlerischen Gestus preiszugeben, statt etwas über sich selbst zu verlautbaren.

Durch diese erste Lektüre ist angezeigt, inwiefern neben Gestaltungsmitteln und Präsentationskontext vor allem eine referenzielle, sinnfällig machende Einbindung prothetischer Absenz sich mitunter als gratwanderungsförmig erweisen kann. Folgend wird kommentierend eine zweite Fotografie betrachtet, welche die Disability-, Queer- und Fat-Aktivistin Nomy Lamm zeigt.

\section{Zweite Lektüre prothetischer Absenz und abschließende Überlegungen}

Die querformatige Fotografie bildet in Zentralperspektive einen hochwandigen Raum mit pastellgelb gestrichenen Wänden und Dielenfußboden ab. Er ist durch einen Erker oder eine Auslucht mit Fenster begrenzt. Ein Blick aus dem Fenster ist durch einströmendes Licht verunmöglicht. Auf den Simsen des sich an der linken Wand befindlichen Kamins befindet sich eine Vielzahl kleiner Gegenstände und Figuren wie auch Puppen und Plüschtiere. An den Wänden finden sich verschiedenformatige gerahmte Bilder und ornamentierte Bilderrahmen sowie Regale. Auf dem Boden, an den Wänden sind neben einer Lampe und einem Regal unterschiedliche Instrumente (z.B. E-Piano, Trommeln, Akkordeon, Verstärker) positioniert. Im Bildzentrum und durch das von hinten einströmende Licht exponiert steht eine Person in aufrechter Position auf dem rechten Bein, ein linkes ist nicht zu sehen. Der Kopf ist leicht schräg gelegt, sie wirkt freundlich und lächelt. Ihr Blick nimmt den der Betrachtenden auf. Ihre Arme sind der Länge nach schräg vom Körper abgewinkelt. Die linke Hand ist flach angewinkelt, in der rechten hält sie einen kleinen Kunstblumenstrauß. Die Bekleidung mutet weiblich konnotiert, verspielt, gar rockabillyesque an (applizierte Rüschen an Ärmelsäumen, mehrfach gebahnter, knielanger, gerüschter Rock, pinker Kniestrumpf, rosafarbener Riemchenschuh, zu Zöpfen gefloch- 
tene Haare mit roten Schleifen). Rechts auf dem Boden, in einer Diagonalen und mit dem Schaft in den Bildhintergrund gerichtet, liegt eine Oberschenkelprothese in Inkarnatton. Wie auch das rechte Bein der Person ist der Unterschenkel der Prothese in einen pinken Strumpf und das Fußelement in einen rosafarbenen Riemchenschuh gehüllt.

Bildbeschreibung zu: Nomy Lamm, Farbfotografie, o.J., Urheberinnenschaft: Caldwell Linker. Bildquelle: https:/queerfatperformerfilm.wordpress.com/about/nomy-lamm/ (letzter Zugriff: 20.11.2019)

Mit Blick auf die erste Fotografie lässt sich damit liebäugeln, wie eine Verhandlung von Körper, Prothese und prothetischer Absenz hier erfolgt: Nicht nur verwehrt sich die Darstellung einem Abdriften ins Irreale und Fiktive, indem der Betrachter*innenblick in einem Raum installiert wird, der sich atmosphärisch als behaglicher Wohnraum mit Liebe zum Detail gibt und indem die Fotografie durch ihre Bildqualitäten amateur*innenhaft wirkt. Mehr noch sind es die Art und Weise des $\mathrm{Zu}$-sehen-Gebens von Nomy Lamm und ihre Körperpräsentation, die das Bild produktiv machen. Das rechtsseitige einbeinige Stehen und das überspitzte Abwinkeln der Arme stehen durchaus in Referenz zu dem vor-gesehenen Bild einer als Ballerina gestalteten Figurine. Nomy Lamm geht jedoch nicht in Gänze in diesem posenhaften Zitat eines Objekts auf, das dazu bestimmt ist, zu zieren, dem Blick dargeboten zu sein und angesehen zu werden: Sie blickt direkt und scheinbar völlig gewahr um das Angesehen- und Fotografiert-Werden in die Kamera. Sie wird damit als zweites Subjekt des Sehens und auch als Person erfahrbar. Es verunklart sich, ob sie betrachtet wird oder vielmehr Betrachtende beim Betrachten betrachtet. Es ist vor allem dieser Blick, der erwirkt, dass Nomy Lamm dabei gesehen wird, wie sie nichts anderes tut, als fotografiert zu werden und sich fotografieren zu lassen. Es handelt sich also um eine Darstellung des Sich-Darstellens in seiner Momenthaftigkeit. Im Begrenzen auf diese Momenthaftigkeit scheint kaum auf etwas davor Erfolgtes oder danach Erfolgendes verwiesen. Dies wiederum informiert die auf dem Boden liegende Oberschenkelprothese. Durch die gleiche Einkleidung in Strumpf und Schuh wie Nomy Lamms Standbein und die Eingelassenheit in den Raum kann sie zum einen als Nicht-an-den-KörperGefügtes, zum anderen als eines von vielen im Raum versammelten Dingen gelesen werden. Zentral ist, dass sich das Verhältnis beider Lesarten, also Körperding und/oder Objekt, durch eine Gleichzeitigkeit auszeichnet, die durch die Darstellung des Sich-Darstellens in seiner Momenthaftigkeit erwirkt wird und erhalten bleibt. Prothetische Absenz verbleibt im Status eines ergebnisoffenen, nicht aktualisierten Potenzials: Was mit der Prothese im Anschluss passiert, an den Körper gefügt werden, liegen bleiben oder weder noch, bleibt offen.

Anders als in der ersten Fotografie wird prothetischer Absenz hier eine Artikulationsform zugestanden, die nicht vorschnell durch vor-gesehene Referenzrahmen vereinnahmt zu werden droht. Mit der ersten Fotografie ist beispielhaft angezeigt, dass die Affirmation eines solchen Rahmens potenziell Bedingung der Sichtbar- 
keit von prothetischer Absenz ist. Die produktive Differenzierung der dort aufgerufenen Bilder von Prothetik, Männlichkeit und Arbeit entspricht gewissermaßen einer Flexibilisierung der Normen und Intelligibilitätsmuster, die das Feld der Sichtbarkeit durchziehen. Dieses Flexibilisieren, jedoch schlussendliche Unangefochten-Lassen und Bestätigen dominanter (Be-)Deutungsweisen ist im Sinne einer „Anerkennung im Konditional“ (Schaffer 2008, 60) ambivalent, insofern implizit Positionen und Sichtbarkeiten disqualifiziert werden, die sich nicht in diese dominanten Formen einfügen (können oder wollen).

Auch Nomy Lamms Sich-Präsentieren mag einer dominanten Repräsentationsweise verpflichtet sein, indem es sich als Performance trotz oder mit Amputation darbietet. Jedoch ist es das Zurückblicken, durch das sie einem quasi-voyeuristischen Blick, der eine Performance von ihr verlangen mag, aktiv begegnet. Im Darbieten einer solchen Performance scheint sie diesen Blick aufzugreifen und das Verlangte überspitzt zu verkörpern. Im Sinne eines staring back (vgl. Garland-Thomson 2000) mögen Betrachtende so über ihr wohlmöglich invasives und verlangendes Blicken, das bzgl. Behinderung als kulturell eingehegt gelten kann, informiert werden. In diesem Mobilisieren dominanter Repräsentationsund Sehweisen werden diese zugleich angefochten. Indem Nomy Lamm hierbei als Person erfahrbar und in einer Sphäre des Authentischen inszeniert wird, mag dies stärker wirken als es für die erste, durch Komponiertheit und Fiktionalität gekennzeichnete Fotografie möglich wäre.

Gleichwohl ist zu fragen, inwieweit sich eine solche Repräsentation und ihre Potenziale für öffentlichere Bildsphären qualifizieren können, wenn ein vorgängiges „Gitter der Lesbarkeit“" (Butler 2012, 73) Möglichkeiten der Sichtbarkeit begrenzen und potenziell dominante Darstellungs- und Deutungsmodi und spektakularisierte Bilder erzwingen mag. Insofern diese Fotografie von Caldwell Linker, deren Euvre queere Subkultur zentralisiert, auf der Homepage einer Independent-Dokumentation über die Arbeit fat queerer Performancekünstler*innen zu sehen ist, ist nicht nur auf begrenzte Reichweite, sondern auch subkulturell informierte Rezeptionsöffentlichkeiten und Modi des Sehens verwiesen. Damit sind Potenziale von Darstellungen einer sich über Zwischenräume (gaps) artikulierenden prothetischen Absenz nicht nur bzgl. ihrer Produktion herausgefordert. Auch sind Lektüren adressiert, die sich im Sinne eines produktiven Blickens (vgl. Silverman 1996, 180ff.) für eine Wahrnehmung von Differenziertheit und Andersheit öffnen müssten. Wenn aber Blicken, als selbst bedeutungsgenerierende Praxis, größtenteils unbewusst operiert und „oftmals an hegemonialen Idealen“" (Schaffer 2008, 156) orientiert ist, steht zur Disposition, für welche Darstellungen, wo und wie sich solche selbstreflexiv bemühten Akte des Blickens verwirklichen können. 


\section{Literatur}

Butler, J. (2012): Die Macht der Geschlechternormen und die Grenzen des Menschlichen. 2. Aufl. Frankfurt am Main.

Dederich, M. (2007): Körper, Kultur und Behinderung. Eine Einführung in die Disability Studies. Bielefeld.

Engel, A. (2009): Bilder von Sexualität und Ökonomie. Queere kulturelle Politiken im Neoliberalismus. Bielefeld.

Garland-Thomson, R. (2000): Staring Back. Self-Representations of Disabled Performance Artists. In: American Quarterly, 52, H.2, 334-338.

Hall, S. (2013): The Work of Representation. In: Hall, S., Evans, J. \& Nixon, S. (Hrsg.): Representation. 2. Aufl. Los Angeles u.a., 1-47.

Harrasser, K. (2013): Körper 2.0. Über die technische Erweiterbarkeit des Menschen. Bielefeld.

Raab, H. (2010): Shifting the Paradigm: „Behinderung, Heteronormativität und Queerness“. In: Jacob, J., Köbsell, S. \& Wollrad, E. (Hrsg.): Gendering Disability. Intersektionale Aspekte von Behinderung und Geschlecht. Bielefeld, 73-94.

Schaffer, J. (2008): Ambivalenzen der Sichtbarkeit. Über die visuellen Strukturen der Anerkennung. Bielefeld.

Serlin, D. (2002): Engineering Masculinity. Veterans and Prosthetics after World War Two. In: Ott, K., Serlin, D. \& Mihm, S. (Hrsg.): Artificial Parts, Practical Lives. Modern Histories of Prosthetics. New York/London, 45-74.

Silverman, K. (1996): The Threshold of the Visible World. New York/London.

Smith, M. (2007): The Vulnerable Articulate. James Gilingham, Aimee Mullins and Matthew Barney. In: Smith, M. \& Morra, J. (Hrsg.): The Prosthetic Impulse. From a Posthuman Present to a Biocultural Future. 2. Aufl. Cambridge, 43-72.

Windisch, M. (2014): Vom Leben im Trotzdem. Gedanken zu den Paralympischen Spielen 2012. In: FKW // Zeitschrift für Geschlechterforschung und visuelle Kultur, o.A., H.56, 83-93. 
Jana York und Jan Jochmaring

\section{Dilemmata einer inklusiven Arbeitswelt - Menschen mit Behinderung zwischen Sondersystemen und Gestaltungschancen einer Arbeitswelt 4.0?!}

Der normative und politische Anspruch einer inklusiven Arbeitswelt steht dem aktuellen Status quo der Beschäftigung von Menschen mit Behinderung im ersten Arbeitsmarkt und in Sondersystemen, wie der Werkstatt für behinderte Menschen, entgegen. Unter dem Label ,Arbeit 4.0` werden insbesondere Substituierbarkeitspotenziale und Automatisierungsprozesse im Zuge der Digitalisierung, die Pluralisierung von Beschäftigungskonstellationen sowie die fortschreitende Bildungsexpansion und Qualifikationsentwertung hinsichtlich neuer Exklusionsund Inklusionsmechanismen der Arbeitswelt in den Blick genommen.

\section{Politische Zielsetzungen und Ansprüche einer inklusiven Arbeitswelt}

Mit der UN-Behindertenrechtskonvention (UN-BRK) ist durch Artikel 27 (Arbeit und Beschäftigung) die Forderung formuliert, einen inklusiven Arbeitsmarkt zu schaffen. Dies umfasst das Recht von Menschen mit Behinderung auf Arbeit, die frei gewählt oder angenommen wird, in einem offenen Arbeitsmarkt fernab von Sondersystemen (vgl. Trenk-Hinterberger 2015). Durch das Bundesteilhabegesetz (BTG) werden die Prinzipien der UN-BRK in die bundesdeutsche Gesetzgebung überführt. Es soll zu einer Verbesserung der Teilhabe von Menschen mit Behinderung am Arbeitsleben führen, z.B. durch die Einführung des Budgets für Arbeit. Flankiert wird es durch bestehende Gesetzwerke - wie das Gesetz zur Gleichstellung von Menschen mit Behinderung (BGG) und das Allgemeine Gleichbehandlungsgesetz (AGG) -, welche wiederum das Ziel einer Stärkung gesellschaftlicher Teilhabechancen anvisieren. Der politische und gesetzliche Anspruch bildet bislang jedoch nicht die aktuelle Beschäftigungsrealität am Arbeitsmarkt ab. 


\section{Status quo zur Arbeitssituation von Menschen mit Behinderung}

\subsection{Beschäftigung von Menschen mit Behinderung im ersten Arbeitsmarkt}

Für eine Analyse der Beschäftigungssituation von Menschen mit Behinderung ist zunächst zu klären, wer als ,behindert' etikettiert und auch statistisch erfasst wird. Das Label ,Mensch mit Behinderung' ist aktuell eher ein Sammelbegriff als eine empirische Kategorie. Genaue Begriffsbestimmungen und Abgrenzungen sind notwendig, um die Arbeitssituation von Menschen mit Behinderung differenziert darstellen zu können. Unterschieden werden muss zwischen anerkannter (Schwer-)Behinderung nach SGB IX, einer chronischen Krankheit (vgl. BMG 2019) sowie der weiten Begriffsfassung der Beeinträchtigung (welche die beiden vorher genannten Konstrukte einschließt) und u.a. im zweiten Bundesteilhabebericht der Bundesregierung Anwendung findet. Weiter ist in diesem Kontext das Konstrukt ,Erwerbsfähigkeit' nach $\$ 8$ des SGB II relevant: „Erwerbsfähig ist, wer nicht wegen Krankheit oder Behinderung auf absehbare Zeit außerstande ist, unter den üblichen Bedingungen des allgemeinen Arbeitsmarktes mindestens drei Stunden täglich erwerbstätig zu sein."

Die Erwerbstätigenquote aller Personengruppen, mit oder ohne Beeinträchtigung sowie differenziert in chronisch Kranke und Schwerbehinderte, ist in den letzten Jahren deutlich angestiegen (vgl. BMAS 2016). Die Gründe dafür sind - neben der demografischen Entwicklung - ein Rückgang der Frühverrentungen, ein erhöhter Aktivierungsdruck zur Aufnahme einer Beschäftigung sowie die Entstehung eines breiten Niedriglohnsektors als Folge der Agenda 2010. Im Jahr 2017 waren von 3.254.905 schwerbehinderten Menschen im erwerbsfähigen Alter (15 bis 65 Jahre) 1.051.492 erwerbstätig, 162.373 arbeitslos und 2.041.040 erwerbslos (vgl. BA 2019). Für das Jahr 2017 ergibt sich eine Beschäftigungsquote von 32,3\% bei den Schwerbehinderten sowie von 75,2\% bei der Allgemeinbevölkerung. Von den beeinträchtigten Menschen verdienen $40 \%$ ihren Lebensunterhalt überwiegend aus Erwerbseinkommen im Gegensatz zu 75\% der nicht beeinträchtigten. Es zeigt sich ein substanzieller Unterschied im Ausmaß der atypischen Beschäftigung: 75\% der nicht beeinträchtigten und 66\% der beeinträchtigten Personen sind vollzeitbeschäftigt. Entsprechend ist der Anteil der Teilzeitbeschäftigten mit $22 \%$ und der Anteil geringfügig Beschäftigter mit 12\% in der Gruppe der Personen mit Beeinträchtigung größer (vgl. BMAS 2016). Aus diesen Zahlen ist jedoch keine Aussage über gewünschte oder auch beeinträchtigungsbedingt erzwungene Teilzeitbeschäftigung ableitbar. 


\subsection{Beschäftigung in Werkstätten für behinderte Menschen}

Bei den Werkstätten für behinderte Menschen (WfbM) handelt es sich um ein Sondersystem mit eingeschränkten Wettbewerbsbedingungen. Leistungsberechtigt für den Arbeitsbereich der WfbM nach $\$ 219$ Abs. 1 SGB IX sind Menschen, die „ein Mindestmaß wirtschaftlich verwertbarer Arbeitsleistung erbringen werden." Trotz des gesetzlichen Auftrags, dass der Übergang in den allgemeinen Arbeitsmarkt gefördert und vorbereitet werden soll, ist die WfbM de facto eine berufliche Sackgasse. Die Übergangsquote in den ersten Arbeitsmarkt liegt bei 0,1-0,2\%, dies ist mit den Forderungen der UN-BRK (Artikel 27: gleiches Recht auf Arbeit) nicht vereinbar (vgl. Jochmaring 2019). Die Zahl der Werkstattbeschäftigten hat sich in den letzten 15 Jahren verdoppelt: Aktuell liegt die Zahl bei über 312.000 (vgl. BAG WfbM 2019). Unterhalb des Leistungsspektrums der WfbM existieren darüber hinaus noch heilpädagogische Tages-/Förderstätten, die knapp 40.000 Menschen betreuen (Zahlen ohne Bundesland Nordrhein-Westfalen), die aufgrund der Schwere ihrer Beeinträchtigung nicht in der Werkstatt beschäftigt werden können (vgl. BAGüS 2019). Von einem weiteren Anstieg der Beschäftigung in Sondersystemen ist in den nächsten Jahren auszugehen, sind die Werkstätten doch faktisch das ,Auffangbecken' für Personen, deren Arbeitskraft am Arbeitsmarkt nicht benötigt wird bzw. nicht eingesetzt werden kann.

\section{Arbeit 4.0 und sozialstrukturelle Veränderungsprozesse}

Unter dem Label Arbeit 4.0 werden aktuelle Wandlungsprozesse der Arbeitswelt sowie denkbare Zukunftsszenarien von vernetztem, digitalisiertem und flexibilisiertem Arbeiten diskutiert (vgl. BMAS 2017). Der Mehrwert des Diskussionsansatzes Arbeit 4.0 liegt in der Verzahnung unterschiedlicher Aspekte des Wandels in einer handlungspraktischen Perspektive, der jedoch empirisch-analytische Grenzen gesetzt sind. Vermag es der Ansatz zwar durchaus abstrakte Leitbilder, übergeordnete Normen und Wertvorstellungen einer zukünftigen Arbeits- und Lebenswelt lose miteinander zu verbinden und einer kritischen Diskussion zuzuführen, ist eine konkrete Operationalisierung ausgewählter Aspekte des Ansatzes voraussetzungsvoll. Dieser Beitrag legt den Fokus auf die technischen Potenziale, die Pluralisierung von Beschäftigungskonstellationen und die Bildungsexpansion und Qualifikationsentwertung.

\subsection{Technische Potenziale}

Die Prozesse der Digitalisierung bzw. Computerisierung und Automatisierung sind für das Phänomen Arbeit 4.0 konstitutiv und verändern die Arbeitswirklichkeiten grundlegend. Im Fokus der Betrachtung stehen die technischen Auto- 
matisierbarkeitspotenziale, die in der Pionierarbeit von Frey und Osborne (2013) für die USA ermittelt wurden. Mit der Übertragung dieser Studie auf Deutschland (vgl. Bonin, Grogory \& Zierahn 2015) liegen auch Berechnungen und Szenarien zu den technischen Automatisierbarkeitspotenzialen für den deutschen Arbeitsmarkt vor (vgl. ebd.; Möller \& Walwei 2017). Sicher ist, dass das Risiko für geringqualifizierte Beschäftigte und Geringverdiener*innen deutlich größer ist als für höherqualifizierte Beschäftigte. Für Menschen ohne Sekundarschulabschluss liegt die Automatisierungswahrscheinlichkeit bei ca. $80 \%$, mit beruflichen Abschlüssen bei ca. 50\%, bei Akademiker*innen immerhin noch bei über 25\% (vgl. Bonin et al. 2015). Berechnungen des Instituts für Arbeitsmarkt- und Berufsforschung (IAB) (vgl. Dengler \& Matthes 2015) zeigen ein hohes Substituierbarkeitspotenzial nach Berufssegment u.a. in Fertigungs-(technischen) Berufen von bis zu 75\%. Ein niedriges Substituierbarkeitspotenzial weisen Gesundheitsund Dienstleistungsberufe von 10-20\% auf. Fachkräfteberufe haben mit $44 \%$ ein fast gleichhohes Substituierbarkeitspotenzial wie Helfer*innenberufe (vgl. ebd.).

\subsection{Pluralisierung von Beschäftigungskonstellationen}

Die Erosion des Normalarbeitsverhältnisses kennzeichnet die Arbeitswelt der vergangenen Jahrzehnte. Das Konstrukt ,Normalarbeitsverhältnis' fungiert keineswegs nur als empirische Kategorie, sondern ist auch ein normiertes Leitbild tradierter Standards. Rein quantitativ haben atypische Beschäftigungsverhältnisse zugenommen und gleichzeitig hat das Normalarbeitsverhältnis nicht nur relativ, sondern auch absolut an Bedeutung verloren. Mit den Veränderungen der Arbeitsrealitäten geht ein Zuwachs an sozialrechtlich schlechter abgesicherten atypischen und prekären Erwerbsarbeitsstrukturen bei gleichzeitigem Rückgang von Beschäftigung in sogenannten Normalarbeitsverhältnissen einher (vgl. Seils \& Baumann 2019; York 2019). Frauen sind sehr viel häufiger atypisch beschäftigt als Männer: Aktuell gehen 30,5\% aller kernerwerbstätigen Frauen einer atypischen Beschäftigung nach, jedoch nur 12,2\% aller Männer. Von allen Kernerwebstätigen ohne anerkannten beruflichen Abschluss sind 36,6\% atypisch beschäftigt (vgl. Seils \& Baumann 2019).

\subsection{Bildungsexpansion und Qualifikationsentwertung}

Mit der allgemeinen Höherqualifizierung der Bevölkerung verändert sich das Qualifikationsprofil der Arbeitnehmer*innen in Deutschland insgesamt (vgl. Autorengruppe Bildungsberichterstattung 2018). Die aktuelle Phase der Bildungsexpansion ist durch einen lebenslangen Qualifikations- und Kompetenzwettlauf gekennzeichnet (vgl. Quenzel \& Hurrelmann 2019). Die individuelle Wettbewerbsfähigkeit hängt noch stärker als bisher von individueller Variabilität, räumlicher Mobilität und beruflicher Weiterbildung ab. Der entstandene Druck 
der ständigen ,Höher- und Weiterqualifizierung' aufgrund des technologischen Wandels führt jedoch auch zu einem widersprüchlichen Effekt: Das exponentielle Wissenswachstum führt paradoxerweise zu einer permanenten Entwertung von Qualifikationen, was einen ständigen Wettbewerb um gefragte Bildungszertifikate nach sich zieht (vgl. Hirsch-Kreinsen 2014).

\section{Menschen mit Behinderung zwischen Sondersystemen und Gestaltungschancen einer Arbeitswelt 4.0}

Die technischen Potenziale von Arbeit 4.0 bieten neben Risiken, die sich aus der Substituierbarkeit menschlicher Arbeitskraft ergeben, auch Vorteile. Die Prozesse der Automatisierung und Digitalisierung eröffnen neue Teilhabechancen in der Arbeitswelt, z.B. durch die Reduktion körperlich belastender Tätigkeiten und den Einsatz neuer Kommunikationstechnologien (vgl. Engels 2016; Mikfeld 2016). Als problematisch sind die Beschäftigungschancen für Geringqualifizierte zu sehen, die aller Wahrscheinlichkeit nach weiter deutlich abnehmen werden. Das gilt vor allem für das weitere Schrumpfen des Arbeitskräftebedarfs im produzierenden Gewerbe, wobei im Dienstleistungssektor ein konstanter Bedarf an niedrig entlohnten qualifizierten und einfachen Arbeitskräften besteht (vgl. Quenzel \& Hurrelmann 2019). Die Prognosen zur Arbeitsmarktentwicklung sind jedoch aufgrund der Algorithmen zur Berechnung der Substituierbarkeitspotenziale kritisch einzuschätzen (vgl. Wajcman 2019).

Die Pluralisierung von Beschäftigungskonstellationen eröffnet Chancen einer Entwicklung neuer geschlechtergerechter, ökologischer und sozialpolitischer Konstitutionselemente. Die Veränderungen tradierter Beschäftigungskonstellationen gehen jedoch gleichzeitig mit neuartigen Prekaritäts- und Gesundheitsrisiken, wie einer Einkommensreduktion oder einer unvollständigen oder gänzlich fehlenden Einbindung in die Sozialversicherungssysteme und/oder in das System des Arbeits- und Gesundheitsschutzes, einher (vgl. York 2019).

Die beschriebenen Veränderungsprozesse der Bildungsexpansion und die damit verbundene relative Qualifikationsentwertung bieten Chancen einer beruflichen Um- oder Neuorientierung durch die Verbreiterung der vielfältigen Bildungs- und Qualifizierungsoptionen. Trotzdem ist das benannte Risiko hoch, dass Menschen mit Behinderung häufig Verlierer*innen des skizzierten Wettlaufs um Bildung sein werden. Die gesteigerten Qualifikationsanforderungen, die Nachfrage nach höherqualifizierter Arbeitskraft und der Druck des, lebenslangen Lernens' verschärfen die Beschäftigungssituation nicht nur für Menschen mit Behinderungen, sondern auch für weniger qualifizierte Menschen im Erwerbsalter (vgl. Quenzel \& Hurrelmann 2019). 
Die Digitalisierungs- und Automatisierungsprozesse werden für Menschen mit Behinderung mehr Beschäftigungsoptionen einschränken, als dass sie neue kreieren werden (vgl. Engels 2016). Zu erwarten ist, dass es immer mehr überflüssige Arbeitskräfte geben wird, die am Arbeitsmarkt nicht benötigt werden. Das Rehabilitationssystem bietet zum einen für Menschen mit Behinderung und geringem Leistungsvermögen überhaupt erst die Möglichkeit, in der Arbeitswelt teilzuhaben, u.a. durch Inklusionsbetriebe, Unterstützte Beschäftigung oder die WfbM. Zum anderen erfüllt es die Funktion, speziell die WfbM, nicht brauchbare Arbeitskräfte auf Ersatzarbeitsmärkten unterzubringen und mit Arbeit ,zu versorgen'. Auf diesen Zusammenhang wurde bereits in der politökonomischen Interpretation von Jantzen (1974) hingewiesen. Das Rehabilitationssystem absorbiert somit nicht brauchbare ,überzählige‘ Arbeitskräfte vom Arbeitsmarkt, die bei entsprechender Nachfrage als ,Reservearmee' eingesetzt werden können (vgl. ebd.). Auch das Angebot an Berufen für Menschen mit Behinderung ( $\$ 66 \mathrm{BBiG} / \$ 42 \mathrm{HwO}$ ) sinkt seit Jahren kontinuierlich. Die Entwicklung auf dem Ausbildungs- und Arbeitsmarkt steht der Einlösung der Verpflichtung aus der UN-BRK und der normativen Forderung nach einer inklusiven Arbeitswelt zentral entgegen (vgl. Autorengruppe Bildungsberichterstattung 2018; Jochmaring 2019).

Die Janusköpfigkeit besteht genau darin, dass einerseits exkludierende Sondersysteme wie die WfbM expandieren und andererseits der Abbau eben dieser gefordert wird. Ein gesetzlicher und politischer Anspruch, einen inklusiven Arbeitsmarkt zu verwirklichen, schafft keine neuen Berufs- oder Beschäftigungsoptionen. Ganz im Gegenteil: Diese werden sich, wie oben dargestellt, für den Personenkreis ,Menschen mit Behinderung' weiter reduzieren. In der Konsequenz bedeutet dies, dass auf Sondersysteme zur Teilhabe am Arbeitsleben auch prospektiv gar nicht verzichtet werden kann. Dieser zentrale Widerspruch zwischen politischer, gesetzlicher und normativer Forderung einer inklusiven Arbeitswelt und der aktuellen Technologie-, Beschäftigungs- und Wissensentwicklung spitzt sich in der Entwicklung zur Arbeitswelt $4.0 \mathrm{zu}$. Es sind die bestehenden Produktionsverhältnisse kapitalistischer Arbeitsgesellschaften, welche die Vorstellungen von Leistung bzw. Leistungsfähigkeit hervorbringen und konstituieren (vgl. Buchner, Pfahl \& Traue 2015). In einer kapitalistischen Produktionsweise wird der Begriff ,Fähigkeit' daher diskursiv determiniert und in die Verwertungsethik eingehegt, da ,produktive Arbeit' diejenige Form der Arbeit ist, die einen messbaren (Mehr-)Wert erzeugt. Schlussendlich wird es um die ,große Frage' gehen, wie viel ,dis-ability in einem kapitalistischen Wirtschaftssystem zugestanden wird. Eine ,inklusive Arbeitswelt' muss zwangsläufig auf der Ebene einer Differenzfeststellung zwischen ,Sollen und Sein' verhaftet bleiben, sofern kapitalistische Produktionsverhältnisse und Leistungsvorstellungen nicht angetastet werden. Schließlich generieren bloße Inklusionsansprüche keineswegs praktische Teilhabechancen, sondern verbleiben auf dem Niveau rechtlicher und politischer Forderungen (vgl. Wansing 2012), welche deutlich an die Grenzen aktueller Realität der Arbeitswelt stoßen. 


\section{Jana York und Jan Jochmaring}

\section{Literatur}

Autorengruppe Bildungsberichterstattung (2018): Bildung in Deutschland 2018: Ein indikatorengestützter Bericht mit einer Analyse zu Wirkungen und Erträgen von Bildung. Bielefeld.

BA (Bundesagentur für Arbeit) (2019): Statistik der Bundesagentur für Arbeit. Berichte: Blickpunkt Arbeitsmarkt- Situation schwerbehinderter Menschen. Nürnberg.

BAG WfbM (Bundesarbeitsgemeinschaft Werkstätten für behinderte Menschen e.V.) (2019): Menschen in Werkstätten. Online unter: www.bagwfbm.de/page/25 (letzter Zugriff: 11.02.2021).

BAGüS (Bundesarbeitsgemeinschaft der überörtlichen Träger der Sozialhilfe) (2019): Kennzahlenvergleich Eingliederungshilfe der überörtlichen Träger der Sozialhilfe. Online unter: www.kennzahlenvergleich-eingliederungshilfe.de/images/berichte/2019-04-08\%20BAGS\%20Bericht\%20 2017_barrierefrei_final.pdf (letzter Zugriff: 11.02.2021).

BMAS (Bundesministerium für Arbeit und Soziales) (2016): Zweiter Teilhabebericht der Bundesregierung über die Lebenslagen von Menschen mit Beeinträchtigungen: Teilhabe - Beeinträchtigung - Behinderung. Berlin.

BMAS (Bundesministerium für Arbeit und Soziales) (2017): Weissbuch Arbeiten 4.0. Arbeit weiterdenken: Diskussionsentwurf. Online unter: www.bmas.de/SharedDocs/Downloads/DE/PDFPublikationen/a883-weissbuch.pdf?_blob=publicationFile\&v=4 (letzter Zugriff: 11.02.2021).

BMG (Bundesministerium für Gesundheit) (2019): Chronisch kranke Menschen. Online unter: www.bundesgesundheitsministerium.de/service/begriffe-von-a-z/c/chronisch-kranke-menschen. html (letzter Zugriff: 11.02.2021).

Bonin, H., Grogory, T. \& Zierahn, U. (2015): Übertragung der Studie von Frey/Osborne (2013) auf Deutschland: Kurzexpertise Nr. 57. Online unter: ftp.zew.de/pub/zew-docs/gutachten/Kurzexpertise_BMAS_ZEW2015.pdf (letzter Zugriff: 11.02.2021).

Buchner, T., Pfahl, L. \& Traue, B. (2015): Zur Kritik der Fähigkeiten: Ableism als neue Forschungsperspektive der Disability Studies und ihrer Partner_innen. In: Zeitschrift für Inklusion, 2, o.S.

Dengler, K. \& Matthes, B. (2015): Folgen der Digitalisierung für die Arbeitswelt: In kaum einem Beruf ist der Mensch vollständig ersetzbar. In: IAB-Forschungsbericht, 24, 1-7. Online unter: http://doku.iab.de/kurzber/2015/kb2415.pdf (letzter Zugriff: 11.02.2021).

Engels, D. (2016): Chancen und Risiken der Digitalisierung der Arbeitswelt für die Beschäftigung von Menschen mit Behinderung. Köln.

Frey, C. B. \& Osborne, M. (2013): The future of employment: How susceptible are jobs to computerisation? Online unter: www.oxfordmartin.ox.ac.uk/downloads/academic/The_Future_of_Employment.pdf (letzter Zugriff: 11.02.2021).

Hirsch-Kreinsen, H. (2014): Digitalisierung von Arbeit. Folgen, Grenzen und Perspektiven. Soziologisches Arbeitspapier Nr. 43. Dortmund.

Jantzen, W. (1974): Sozialisation und Behinderung: Studien zu sozialwissenschaftlichen Grundfragen der Behindertenpädagogik. Gießen.

Jochmaring, J. (2019): Übergänge von Schüler/innen mit sonderpädagogischem Förderbedarf in die Berufsausbildung: Eine Auswertung von Sekundärstatistiken. In: Zeitschrift für Pädagogik, 3, 335354.

Mikfeld, B. (2016): Zur Einführung: Trends, Diskurse, Klärungsbedarfe. Arbeiten 4.0 in der digitalen Transformation. In: Bundesministerium für Arbeit und Soziales (Hrsg.): Werkheft 01. Digitalisierung der Arbeitswelt. Berlin, 16-20. Online unter: www.issuu.com/support.bmaspublicispixelpark. $\mathrm{de} /$ docs/werkheft-01?e=26749784/43070415 (letzter Zugriff: 11.02.2021).

Möller, J. \& Walwei, U. (Hrsg.) (2017): IAB-Bibliothek: Vol. 363. Arbeitsmarkt kompakt: Analysen, Daten, Fakten. Bielefeld.

Quenzel, G. \& Hurrelmann, K. (2019): Ursachen und Folgen von Bildungsarmut. In: Quenzel, G. \& Hurrelmann, K. (Hrsg.): Handbuch Bildungsarmut. Wiesbaden, 3-25. 
Seils, E. \& Baumann, H. (2019): Trends und Verbreitung atypischer Beschäftigung: Eine Auswertung regionaler Daten. Online unter: www.boeckler.de/pdf/p_wsi_pb_34_2019.pdf (letzter Zugriff: 11.02.2021).

Trenk-Hinterberger, P. (2015): Arbeit, Beschäftigung und Ausbildung. In: Degener, T. \& Diehl, E. (Hrsg.): Handbuch der Behindertenrechtskonvention. Teilhabe als Menschenrecht - Inklusion als gesellschaftliche Aufgabe. Bonn, 105-117.

Wajcman, J. (2019): Automatisierung: Ist es diesmal wirklich anders? Eine Sammelbesprechung. In: Butollo, F. \& Nuss, S. (Hrsg.): Marx und die Roboter. Vernetzte Produktion, Künstliche Intelligenz und lebendige Arbeit. Berlin, 22-35.

Wansing, G. (2012): Inklusion in einer exklusiven Gesellschaft. Oder. Wie der Arbeitsmarkt Teilhabe behindert. In: Behindertenpädagogik, 4, 381-396.

York, J. (2019): Arbeitsweltbezogene Gesundheitspolitik bei atypischer Erwerbsarbeit. Eine Rekonstruktion von Gestaltungschancen zur Reduzierung von Ungleichheit im Arbeitsleben. Wiesbaden. 


\section{Kontingenz von Differenzkonstruktionen in der inklusionsorientierten musikalischen Bildung am Beispiel des Umgangs mit digitalen Musizier-Medien}

Der vorliegende Beitrag fokussiert Differenzkonstruktionen im Bereich der musikalischen Bildung. Er diskutiert Ergebnisse der ersten Phase des Verbundprojektes ,be_smart-Bedeutung spezifischer Musik-Apps für die Teilhabe von Jugendlichen und jungen Erwachsenen mit komplexen Behinderungen an kultureller Bildung ${ }^{11}$ im Hinblick auf die Frage, welche Konstruktionsweisen von Behinderung, Musik und Talent im Kontext der Nutzung digitaler Musizier-Medien in inklusionsorientierten musikpädagogischen Settings wirksam werden. Wir bedienen uns dazu in der Ergebnisdarstellung differenztheoretischer und ableismuskritischer Theoriefragmente.

Im Zentrum stehen zwei Ergebnisfelder: Zum einen geht es um die Frage, welche Verständnisse von Behinderung in der Analyse der Interviews herausgearbeitet wurden. Zum anderen um die Frage, welches Verständnis von digitalen Musikinstrumenten die Interviewpartner*innen zugrunde gelegt haben. Im Vordergrund stehen dabei Verschränkungen und Wechselbeziehungen zwischen beiden Feldern und ihre möglichen Auswirkungen auf inklusive musikpädagogische Settings.

\section{Untersuchungsdesign}

In dem transdisziplinär angelegten Projekt (Musikpädagogik, Inklusive Pädagogik, Soziologie, Rehabilitationswissenschaften) werden Potenziale und Herausforderungen der Verwendung von Musizier-Medien für die Bildungsteilhabe von Jugendlichen und jungen Erwachsenen mit komplexen Behinderungen in unterschiedlichen musikpädagogischen Settings untersucht. Im Zentrum steht die Frage, in welcher Weise und unter welchen Bedingungen Musizier-Apps und digitale Musikinstrumente Zugänge zu kultureller Bildung und damit Voraussetzungen für Teilhabe schaffen können, wie sie Dietrich (2017) als

1 Gefördert vom Bundesministerium für Bildung und Forschung (Förderlinie: Digitalisierung in der kulturellen Bildung) 
prozedurale Gerechtigkeit beschreibt. Daher verstehen wir mit Nohl (2011) musikpädagogische Settings als soziodingliche Kollektive, in denen Menschen und Dinge aufeinander (ab)gestimmt werden und so konjunktive Transaktionsräume entstehen.

Das Untersuchungsdesign sieht ein mehrstufiges Vorgehen mit qualitativen Verfahren vor: Von einer Sondierungsphase über eine Phase der Rekonstruktion subjektiver Deutungsmuster und konjunktiver Erfahrungsräume der Beteiligten mittels Interviews und Gruppendiskussionen hin zur Analyse der konkreten Handlungspraxen (vgl. Wulf et al. 2007) durch die Analyse videografierter Beobachtungen von Unterrichtspraxis. Schließlich steht die Rückbindung der Ergebnisse an kulturwissenschaftliche und erziehungswissenschaftliche Überlegungen im Kontext von Digitalisierungs- und Inklusionsdiskursen im Zentrum der Studie. Damit zeigt sich das Vorhaben anschlussfähig an Hirschauer (2014), der dazu aufruft, die verschiedenen Sinnschichten zur Herstellung, Überlagerung und Außerkraftsetzung kultureller Differenzierungen zu analysieren.

Von der These ausgehend, dass relevantes Wissen in der Praxis zum Forschungsgegenstand aktuell relativ unverbunden und in beruflichen bzw. disziplinären Feldern voneinander abgegrenzt vorhanden ist, wurden in der ersten Projektphase von Januar bis Mai 2018 insgesamt zwölf Akteur*innen telefonisch befragt, von denen vertiefte Praxiskenntnis zu Aspekten des Forschungsgegenstands zu erwarten war. Je drei Expert*innen sind dem Feld einer ,inklusiven musikpädagogischen Praxis' (schulisch und außerschulisch) zuzuordnen. Drei Expert*innen arbeiten als Musikpädagog*innen vor allem mit Kindern und Jugendlichen mit komplexer Behinderung. Drei Expert*innen wurden wegen ihrer Expertise für die Anpassung digitaler Medien für Bildungsprozesse von Kindern und Jugendlichen mit schwerer Behinderung und drei Expert*innen mit besonderer Expertise im Bereich App-Musik befragt. Die Interviews wurden wortgetreu transkribiert und sequenzanalytisch analysiert.

\section{Theoretische Sensibilisierung}

Einen relevanten theoretischen Bezugspunkt stellt der Begriff der Differenz dar, wie ihn Tervooren (2001) versteht, nämlich als Relation zwischen zwei Begriffen, die voneinander abhängig sind und sich aus diesem Grunde wechselseitig bestimmen. Mit Hirschauer (2014) schließen wir hier das Konzept des ,Doing Difference' an. Er beschreibt mit Doing Difference die sinnhafte Selektion aus einem Set konkurrierender Kategorisierungen, die einen Unterschied schafft (vgl. ebd.). Das zentrale Moment des Doing Difference ist dabei nicht die Unterscheidung an sich, sondern die Frage, ob eine Unterscheidung soziale Relevanz entfaltet, indem im Verlauf sozialer Prozesse die Unterscheidung wieder aufgenommen wird (vgl. 
ebd.). Hirschauer $(2001,216)$ beschreibt mit dem Begriff ,Undoing Difference‘ dementsprechend die Prozesse des Nicht-Vollziehens einer Differenzierung im Sinne von ,inaktiven Kategorien“ bzw. von „Differenzverstärkung oder -minimierung“ (ebd., 185), wenn die Vollzüge der Akteur*innen die soziale Relevanz der Differenz verändern. Diese von Hirschauer beschriebene Kontingenz von Differenzkonstruktionen zeigt sich auch im erhobenen Datenmaterial.

Außerdem zeigt sich das Datenmaterial anschlussfähig an ableismuskritische Diskurse. Ableismus soll verstanden werden als „all jene sozialen, soziotechnischen und technischen Prozesse, die Individuen, Gruppen oder Dingen Fähigkeiten und Begabungen zuschreiben“" (Buchner, Pfahl \& Traue 2015, o.S.). Meißner (2015) weist darauf hin, dass in ableistischen Strukturen Fähigkeiten in ganz spezifischer Weise als individuelle Qualitäten eines Subjekts konstituiert werden - und zwar mit positiver und negativer Konnotation. Gerade Behinderung wird häufig mit mangelnden oder besonderen Fähigkeiten assoziiert und behinderte Menschen werden anhand dieser abgesprochenen oder zugeschriebenen Fähigkeiten bewertet (vgl. Williams 2011).

Vor diesem Hintergrund der vorgestellten theoretischen Rahmung(en) sollen im Folgenden die Ergebnisse zu den Verständnissen von Behinderung und von digitalen Musizier-Medien dargestellt werden.

\section{Verständnisse von Behinderung}

In allen Interviews nimmt ein dichotomes Behinderungsverständnis eine zentrale Rolle ein. Behinderung/Nicht-Behinderung gilt als Differenz, die sprachlich immer wieder von den Interviewpartner*innen in unterschiedlicher Weise hervorgebracht wird. Die interviewten Personen positionieren sich dabei vor allem auf der Seite der ,Nicht-Behinderung' und teilen die Musikschüler*innen in Gruppen von behindert/nicht-behindert ein. Wie Tervooren (2001) beschrieben hat, finden auch wir diese Differenz als Relation vor, d.h. es gelingt den Interviewpartner*innen nur durch Bestimmung eines - wie auch immer gearteten - Behinderungsverständnisses einen Teil der Schüler*innen und sich selbst als nicht-behindert zu kategorisieren. Unterschiede ergeben sich aber durchaus in der Konstruktion dessen, was als Behinderung wahrgenommen wird und wie dann Differenzlinien beschrieben werden. Hier lassen sich drei zentrale Bestimmungen ausmachen:

1.) Die deutlichste Bestimmung konstruiert Behinderung als vermindertes musikalisches Potenzial. Es handelt sich also um ein individualistisches und defizitorientiertes Behinderungsverständnis, welches allerdings pädagogisch gerahmt ist. Ausgangspunkt sind die der Person zugeschriebenen Möglichkeitsräume, sich mit den individuellen Bedingungen (körperlichen, sensorischen, sozial-emotionalen, kognitiven Dispositionen) in dem vorhandenen musikpädagogischen Set- 
ting musikalisch auszudrücken und musikalisch weiterzuentwickeln. Im Zentrum steht die Frage, inwiefern der Person zugetraut wird, ein Instrument zu erlernen und so eine musikalische Expertise zu entwickeln. Besondere (musik-)pädagogische Unterstützung kann dabei be-hindertes oder verschüttetes Potenzial in einem gewissen Maß kompensieren oder besonders fördern. Solange dies möglich ist, spielen Beeinträchtigungen der Schüler*innen für einen gemeinsamen (inklusiven) Musikunterricht auch keine nennenswerte Rolle, da (um mit schulischen Kategorien zu sprechen), zielgleich“ unterrichtet werden kann. Erst ein vermindertes musikalisches Potenzial, das auch durch besondere (musik-)pädagogische Unterstützung einen ,Leistungsunterschied' zu anderen Schüler*innen nicht ausgleichen kann, wird als ,Behinderung' für den Musikunterricht relevant, stellt dann aber auch die Möglichkeit gemeinsamen Lernens und Musizierens infrage.

2.) Die zweite Bestimmung von Behinderung sieht genau in der Frage dieses Passungsverhältnisses den Bestimmungsort für ,Behinderung'. Anders als in der ersten Bestimmung wird dabei aber betont, dass Behinderung nicht auf der Seite der individuellen Dispositionen der Schüler*innen entsteht, sondern durch das musikpädagogische Setting erst hervorgebracht wird. Ausgangspunkt ist der Gedanke, dass grundsätzlich allen Schüler*innen ein künstlerisch/musikalisches Ausdruckspotenzial zugesprochen wird. Im Fokus des Unterrichts steht dann weniger die individuelle musikalische Leistung (und das individuelle Lernen) der Schüler*innen, sondern die Möglichkeit des gemeinsamen Musizierens.

3.) Die dritte Bestimmung von Behinderung war in den Interviews zwar weniger stark vertreten, aber dennoch nachweisbar. Behinderung wird hier nicht in Defiziten oder in Relation zu üblichen musikalischen Leistungsbegriffen definiert, sondern als besondere Daseinsqualität, die einen eigenen (behinderungsbedingten) musikalischen Ausdruck hervorbringt und als spezifischer ästhetischer Gewinn definiert wird. Diese Ausdrucksqualität kann sogar als Inspiration oder auch ,Spiegel' der eigenen musikalischen Praxis aufgefasst werden. Auch diese Bestimmung von Behinderung beschreibt damit einen Othering-Prozess im Sinne einer Abweichung von einer nicht näher beschriebenen Normalität. Für musikpädagogische Settings stellt sich dabei die Frage, wie mit dieser ,Andersartigkeit' umzugehen ist, da hier sowohl soziale als auch pädagogische und in besonderer Weise musikalisch-ästhetische Dimensionen angesprochen werden.

\section{Verständnisse von digitalen Musizier-Medien}

Die beschriebenen Differenzierungslinien bringen in der Folge unterschiedliche Begründungszusammenhänge für die Verwendung digitaler Musikinstrumente und Apps hervor, die Auswirkungen darauf haben, welche Bedeutungen die Interviewpartner*innen digitalen Musizier-Medien im Musikunterricht zuweisen. 


\section{Juliane Gerland, Imke Niediek, Julia Hüllsken und Marvin Sieger}

In diesem zweiten Zugang, nämlich über Verständnisse von digitalen MusizierMedien, zeigen sich die Ergebnisse deutlich anschlussfähig an aktuelle musikpädagogische Diskurse bezüglich der Frage, was ein Musikinstrument eigentlich ist bzw. wodurch ein Gegenstand zu einem solchen (gemacht) wird (vgl. Godau 2018; Ismaiel-Wendt 2016; Randles, Ruiz, Strouse \& Griffis 2014). Im Wesentlichen lassen sich zwei Bedeutungen digitaler Musizier-Medien rekonstruieren:

1.) Überwiegend werden digitale Musikinstrumente als (schlechterer) Ersatz oder Substitut von konventionellen Instrumenten verstanden. Die Orientierung in diesem Verständnis erfolgt entlang konventioneller musikbezogener und musikpädagogischer Wissensbestände und der praktischen Erfahrungen in der Nutzung von konventionellen Musikinstrumenten (klanglich, visuell und haptisch): So wird eine Tablet-App nur schwerlich die Gesamterfahrung widerspiegeln können, die für die Person beim Spielen einer Geige oder eines Schlagzeugs wahrnehmbar ist.

2.) Seltener ließ sich in den Interviews ein zweites Konzept wiederfinden. Digitale Musikinstrumente werden hier als eigene Klasse von Musikinstrumenten eingeführt, die auch eine eigene musikalische Gattung, digitaler Musik' sowie spezifische Spielweisen und ästhetische Erfahrungen hervorbringt und weiterentwickelt. Hier wird zum Ausdruck gebracht, dass sowohl die Produktionsweisen wie auch die musikalischen Ergebnisse nur begrenzt mit einem konventionellen Musikverständnis vergleichbar sind und daher auch die notwendigen Aneignungs- und Bildungsprozesse im Umgang mit den Medien eigene Anforderungen an die Lehrenden und Lernenden stellen.

Die herausgearbeiteten Bedeutungen von Behinderungen und digitalen Musikinstrumenten gehen dabei in den Interviews spezifische Verbindungen ein. So hängen Behinderung als vermindertes musikalisches Potenzial und ein Verständnis von digitalen Musizier-Medien als kompensatorischer Ersatz für konventionelle Instrumente in den von uns untersuchten Interviewsequenzen eng zusammen. Einigen Schüler*innen wird das Potenzial abgesprochen, ein ,normales' Musikinstrument zu erlernen oder an regulären musikalischen Interaktionen teilzunehmen. Musik-Apps und digitale Musizier-Medien können dann eine Alternative darstellen. Damit geht allerdings auch eine hierarchische Ordnung einher: Vorrangiges Ziel ist die Beherrschung eines konventionellen Instruments, ein digitales Instrument ist nur ein minderwertigeres Substitut. Umgekehrt bleibt damit aber auch den Schüler*innen der Zugang zu digitalen Musizier-Medien versagt, denen das Potenzial zugeschrieben wird, ein ,echtes ' Musikinstrument zu erlernen - da ihnen ja die konventionellen Musikinstrumente vorbehalten sind.

Die kompensatorische Funktion digitaler Musizier-Medien steht auch dort im Vordergrund, wo die Beeinträchtigung weniger in den individuellen Dispositionen der Schüler*innen als vielmehr im musikpädagogischen Setting gesucht wird. Pate steht hier der Gedanke, mithilfe digitaler Instrumente ein Setting zu ent- 
wickeln, das voraussetzungslose Teilhabe und gehaltvolles Erleben musikalischer Praxis für alle ermöglicht. Dabei gerät allerdings die Frage der Ermöglichung von Bildungsprozessen gegenüber der Frage der sozialen Teilhabe in den Hintergrund. Die Rolle von digitalen Musizier-Medien als eigenständige Musikinstrumentenklasse zeigt sich schließlich im Material am ehesten in Verbindung mit der Zuschreibung von Behinderung als Daseinsaspekt mit eigener Ausdrucksqualität. Auch hier verschränken sich ,Besonderungen', die zwar positiv gerahmt werden, aber zugleich Differenzlinien beschreiben, sehr deutlich miteinander.

\section{Rückbindung an Spannungsfelder inklusiver Bildung}

Letztlich erscheinen die hier skizzierten Ergebnisse überaus anschlussfähig an allgemein beschreibbare Spannungsfelder inklusiver Bildung:

Im ersten Spannungsfeld wird die Frage verhandelt, inwiefern es unter inklusiven Vorzeichen vertretbar ist, Behinderung im Sinne eines Otherings, das durchaus auch positiv, also ästhetisch wertvoll intendiert sein kann, explizit sichtbar bzw. hörbar zu machen. Darf Behinderung grundsätzlich thematisiert werden oder widerspricht dies den Ansprüchen an eine inklusive Musikpädagogik, da letztlich die von den Akteur*innen angenommene Differenzlinie Dis-/Ability erneut bestätigt wird?

Im zweiten Spannungsfeld stehen sich einerseits die Konstruktion ,musikalisches Talent' und die Forderung nach ,voraussetzungsloser Teilhabe' gegenüber. ,Musikalisches Talent' erscheint im Datenmaterial immer wieder als eine Art persönliches Kapital, das unabhängig von möglichen Behinderungen existent sein kann und das es zu fördern gilt. Voraussetzungslose Teilhabe verschiebt dagegen den Fokus auf die sozial-affektive Bedeutung musikalischer Bildung. Dadurch können formale und kompetenzorientierte Ziele musikalischer Bildung in den Hintergrund rücken.

Individuelle Bildungsprozesse und gemeinsames Musizieren bilden die Pole des dritten Spannungsfeldes. Verhandelt wird hier einerseits die Bedeutung individueller musikbezogener Lern- und Auseinandersetzungsprozesse. Andererseits sind unter dem Aspekt der Inklusionsorientierung insbesondere Praktiken des gemeinsamen Musizierens relevant, da wir Inklusion letztlich als ein sozial-interaktionales Phänomen verstehen. Für dieses dritte Spannungsfeld bedeutet dies, dass abgewogen werden muss, wann die Anregung individueller Bildungsprozesse und wann die Ermöglichung des gemeinsamen Musizierens - und damit komplexe Abstimmungsleistungen mit anderen - im Zentrum steht.

Das vierte und letzte Spannungsfeld bildet den Raum zwischen Inputorientierung und Output- bzw. Produktorientierung ab. Hier gilt es, abzuwägen, inwieweit der Blick auf individuelle Entwicklungspotenziale gelenkt wird (Inputorientierung) 
oder ob sich die musikbezogenen Ziele aus übergeordneten äußeren Sachverhalten ergeben, beispielsweise die konkrete Vorbereitung auf ein Vorspiel im Rahmen eines Schulkonzerts (Output-/Produktorientierung).

\section{Ausblick}

In der hier diskutierten ersten Phase des Projektes konnten lediglich die reflexiv zugänglichen Momente von Differenzkonstruktionen rekonstruiert werden, die bei Wagner-Willi (2018) als kommunikative Dimension beschrieben werden. Wie Lindmeier (2018) herausarbeitet, erfolgt die Herstellung und Bearbeitung von Differenz aber innerhalb sozialer Interaktionsprozesse. Deshalb sind für die zweite Projekthälfte Videografien inklusionsorientierter, musikpädagogischer Unterrichtssettings vorgesehen, um Praktiken des gemeinsamen Musizierens mit digitalen Musizier-Medien im Sinne, konjunktiver Transaktionsräume' (vgl. Nohl 2011) zu analysieren und dadurch auch Prozesse wechselseitiger Annäherung herausarbeiten zu können. So gilt es, danach zu fragen, wann und wie es den Beteiligten in der Situation gelingt, Differenzunterscheidungen - temporär - nicht zu vollziehen und welchen Anteil daran digitale Musizier-Medien haben können.

\section{Literatur}

Buchner, T., Pfahl, L. \& Traue, B. (2015): Zur Kritik der Fähigkeiten: Ableism als neue Forschungsperspektive der Disability Studies und ihrer Partner_innen. In: Zeitschrift für Inklusion, 2, o.S. Online unter: https://www.inklusion-online.net/index.php/inklusion-online/article/view/273 (letzter Zugriff: 01.02.2020).

Dietrich, C. (2017): Teil haben - Teil sein - Anteil nehmen. Anthropologische Argumente der Zugehörigkeit. In: Miethe, I., Tervooren, A. \& Ricken, N. (Hrsg.): Bildung und Teilhabe. Zwischen Inklusionsforderung und Exklusionsdrohung. Wiesbaden, 29-46.

Godau, M. (2018): Inklusion und Appmusik - wie die Integration von Apps in den inklusiven Musikunterricht gelingen kann. In: Potsdamer Schriftenreihe zur Musikpädagogik, 6, H.2, 97-120.

Hirschauer, S. (2001): Das Vergessen des Geschlechts. Zur Praxeologie einer Kategorie sozialer Ordnung. In: Heintz, B. (Hrsg.): Geschlechtersoziologie. Wiesbaden, 208-235.

Hirschauer, S. (2014): Un/doing Differences. Die Kontingenz sozialer Zugehörigkeiten. In: Zeitschrift für Soziologie, 43, H.3, 170-191.

Ismaiel-Wendt, J. (2016): post_PRESETS. Kultur, Wissen und populäre MusikmachDinge. Hildesheim.

Lindmeier, C. (2018): Differenz, Inklusion, Nicht/Behinderung. Stuttgart.

Meißner, H. (2015): Studies in Ableism. Für ein Vorstellungsvermögen jenseits des individuellen autonomen Subjekts. In: Zeitschrift für Inklusion, 2, o.S. Online unter: http://www.inklusion-online. net/index.php/inklusiononline/article/view/276/259) (letzter Zugriff: 01.02.2020).

Nohl, A.-M. (2011): Pädagogik der Dinge. Bad Heilbrunn.

Randles, C., Ruiz, J. V., Strouse, J. \& Griffis, S. A. (2014): Using the iPad in musical performance: New pathways. Kansas Music Review. Online unter: http://kmr.ksmea.org/?issue=201314s\&section=articles\& page=ipad (letzter Zugriff: 01.02.2020). 
Tervooren, A. (2001): Pädagogik der Differenz oder differenzierte Pädagogik? Die Kategorie Behinderung als integraler Bestandteil von Bildung. In: Fritzsche, B., Hartmann, J., Schmidt, A. \& Tervooren, A. (Hrsg.): Dekonstruktive Pädagogik. Erziehungswissenschaftliche Debatten unter poststrukturalistischen Perspektiven. Wiesbaden, 201-218.

Wagner-Willi, M. (2018): Schülerinnen und Schüler: Inklusion und Differenz in mehrdimensionaler Perspektive. In: Sturm, T. \& Wagner-Willi, M. (Hrsg.): Handbuch schulische Inklusion. Opladen, 315-330.

Williams, V. (2011): Disability and Discourse. Analysing Inclusive Conversation with People with Intellectual Disabilities. Chichester.

Wulf, Chr., Althans, B., Blaschke, G., Ferrin, N., Göhlich, M., Jörissen, B., Mattig, R., NentwigGesemann, I., Schinkel, S., Tervooren, A., Wagner-Willi, M. \& Zirfas, J. (2007): Lernkulturen im Umbruch. Rituelle Praktiken in Schule, Medien, Familie und Jugend. Wiesbaden. 


\section{Zur Bedeutung einer befreiungspädagogischen Perspektive für die Inklusions- und Sonderpädagogik}

Vorliegender Beitrag widmet sich der Bedeutung einer befreiungspädagogischen Perspektive für die Inklusions- und Sonderpädagogik. Skizziert wird dabei eine phänomenologische Pädagogik, die vom Subjekt aus denkt und damit einen anderen Ansatzpunkt wählt als bspw. eine dekategorisierende Pädagogik der Systemlogiken.

\section{Zur Überhörbarkeit des Subjekts}

Innerhalb des Inklusionsdiskurses sind Subjekte mit psychosozialen Beeinträchtigungen und Subjekte mit mehrfach-komplexen Behinderungen eine Klientel, die zunehmend entweder aus dem Blickfeld gerät oder in besonderer Weise als sogenannte ,Systemsprenger*innen' sichtbar werden (vgl. Baumann 2012/2019). Das inklusive Bildungsideal zerbricht nicht selten an schwer und schwerst belasteten Subjekten, die aufgrund ihrer bisherigen Erfahrungen und Subjektlogiken mitunter selbst- und fremdschädigendes Verhalten zeigen. Innerhalb einer Regelschule sind viele von ihnen nur temporär beschulbar, aufgrund ihrer inneren psychischen Notwendigkeiten und Dynamiken in besonderer Weise erziehungsbedürftig. Von Bedürftigkeit ist deshalb die Rede, weil jedes Kind in seiner Entwicklung auf erwachsene Andere angewiesen bleibt (vgl. Ahrbeck 2004). Subjekte, die aufgrund brüchiger oder sogar schädigender Beziehungserfahrungen psychisch leiden, sind in einem besonderen Maße auf Erwachsene angewiesen, mit denen sie andere hinreichend gute Erfahrungen machen können, die ihnen anerkennend, ermunternd, unterstützend zur Seite stehen, aber auch lenkend und begrenzend (vgl. Ahrbeck 2020, 8).

Die Bedürftigkeit und Angewiesenheit der durch die sonderpädagogischen Disziplinen adressierten Subjekte werden auch durch die gegenwärtigen beschleunigten Lebensverhältnisse des 21. Jahrhunderts verschärft, die sich, psychisch und sozial auswirken' und zunehmend zu Risiken und Überforderungen führen (vgl. ebd., 16f.). Unter Berücksichtigung der Studien von Dornes $(2010,1008)$ postuliert Ahrbeck, dass beim Subjekt „[a]uf der einen Seite (...) ein Zugewinn an innerer Freiheit [steht], (...) starre innere Strukturen aufgelöst und rigide Verpflichtun- 
gen aufgeweicht wurden“ (Ahrbeck 2020, 19). Damit geht jedoch eine erhöhte Verwundbarkeit des Subjekts einher, „[d]ie inneren Strukturen sind durchlässiger und störanfälliger geworden, sodass die Gefahr eines Scheiterns [auch an Inklusion; Anm. d. Autors] wächst“ (ebd.).

Hoffmann (2018), der einer der Ersten ist, der Inklusionspädagogik als Befreiungspädagogik zu lesen beginnt, entwirft hierfür fünf Thesen. Die erste These von Hoffmann ist an dieser Stelle angesprochen: „(...) [D]er Universalitätsanspruch einer inklusiven Pädagogik als, Allgemeine Pädagogik‘ oder ,Pädagogik für alle [läuft] angesichts der Heterogenität sozialer Exklusionspraktiken Gefahr, die gesellschaftliche Realität von Behinderung und sozialer Benachteiligung zu verfehlen“" (ebd., 19f.). Dabei betont er die besondere Angewiesenheit von Menschen mit geistiger Behinderung, komplexer Beeinträchtigung, psychischen Störungen und Verhaltensauffälligkeiten, die den "harten Kern von Exklusion“ (ebd., 41) bilden, auf einen Dialog seitens der Disziplinen, weil ein Ausbleiben eines solchen Dialogs, verstanden als Resonanzraum, auch eine Ursache für (weitere) Verhaltensauffälligkeiten bilden könne. Eine weitere These Hoffmanns, und damit Anliegen seines Forschungsvorhabens, ist, dass Inklusive Pädagogik weder Allgemeine Pädagogik noch Sonderpädagogik sein könne, vielmehr müsse sie zu einer Pädagogik der Befreiung werden (vgl. ebd., 32).

Auch Dederich (2013a) äußert die Sorge über das, Verschwinden der Menschen im Inklusionsdiskurs. Die Stimme des vulnerablen, leidenden Subjekts kann auch überhört werden. Damit die*der Andere nicht überhört wird, ist die Einnahme einer philosophischen Perspektive unerlässlich, der es gelingt, „die Logik solcher Prozesse [der disziplinären Hervorbringung und Formung des Gegenstands und seiner Ordnungen; Anm. d. Autors] zu hinterfragen und hinsichtlich ihrer Legitimation kritisch zu prüfen (...) [,und zwar mit dem Ziel; Anm. d. Autors] Verschränkungen sichtbar und bewusst zu machen und auf ihre Voraussetzungen, Implikationen und Folgen hin zu bedenken" (Dederich 2013b, 26). Neben der Inklusionspädagogik bedarf aber auch die Sonderpädagogik dieser selbstkritischen Analyse, denn sie ist „als Disziplin und Profession (...) Sachwalterin und Kritikerin der historisch-gesellschaftlichen Konstruktion und Verbesonderung von Menschen mit Behinderung" (ebd., 28).

Gerade eine dezidiert phänomenologisch-hermeneutische wissenschaftstheoretische Grundlegung, die bis auf wenige Ausnahmen (vgl. Stenger 2002) in der Sonderpädagogik noch nicht vertreten ist, ,kann stärker in den Blick rücken, dass der Prozess der Forschung und Erkenntnisgewinnung wesentlich als ein Umlernen zu deuten ist und dass Forschung angesichts von menschlichen und sächlichen Ansprüchen keineswegs ein schlichtes ,Erfinden“ oder ,Konstruieren', sondern responsiv ist (...), also eine [mögliche; Anm. d. Autors] Antwort auf vorgängige Ansprüche darstellt“ (Dederich 2013b, 27f.). 
Bleibt eine solche interdisziplinäre selbstkritische Betrachtung aus, läuft die Philosophie selbst, aber auch die Sonder- und Inklusionspädagogik Gefahr, der Disziplinierung des Subjekts Vorschub zu leisten und ihre eigene „Tendenz zu Totalisierungen“ (ebd., 28) zu verkennen und damit „die Produktion von Normalisierungseffekten und gesellschaftlichen Ordnungen philosophisch (...) zu legitimieren, die Menschen benachteiligen, marginalisieren und ausgrenzen" (ebd.) können.

\section{Zur Hörbarkeit des vulnerablen Subjekts und seiner Befreiung}

Damit sich Inklusions- und Sonderpädagogik ihrer eigenen Ambivalenzen und Machtdiskurse in Hinblick auf Disziplinierung und Subjektivation bewusster werden, bedarf es nicht nur der Philosophie und der Disability Studies, sondern auch der Befreiungspädagogik. Diese kann in ihrer historischen Einmaligkeit eine Perspektive der Pädagogik der Unterdrückten zur Verfügung stellen, die sich innerhalb eines diktatorischen Systems entwickelt und gegen herrschende Machtverhältnisse aufbegehrt hat. Diese Lesart, Sonder- und Inklusionspädagogik unter den Aspekten von Unterdrückung und Befreiung zu interpretieren, kann dabei helfen, die Hörbarkeit des Subjekts in disziplinären Diskursen zu verstärken.

Wenn Sonder- oder Inklusionspädagogik zumindest temporär auch als Herrschaftsinstrument ihrer adressierten Subjekte gelten kann, wird die Perspektive der Befreiungspädagogik interessant. Denn die These der Theorie der Befreiung ist, dass jedes Herrschaftsinstrument immer auch ein Instrument der Befreiung sein kann. In diesem Fall kommt auch der Inklusions- und Sonderpädagogik eine für die Subjekte befreiende Wirkung zu. Die Diskurse um Unterdrückung sind, wenn auch politisch und soziokulturell heute anders gelagert, brandaktuell (vgl. Ofner 2009, 18). Denkt man an den neoliberalen Selbstoptimierungswahn der Subjekte in Hinblick auf bspw. Leistung und Körper, wird deutlich, dass das Subjekt, ganz im Sinne Foucaults, sich selbst regiert und unterwirft. Die Macht ist in das Subjekt inkarniert, leiblich eingeschrieben, und somit muss die Freiheit, die dem Subjekt möglich ist, mit vielen Grenzen betrachtet werden. Derzeit herrschen unterschiedliche Vollzüge der psychologischen Disziplinierung. Die Psyche herrsche laut diesem historischen Epistem über den Körper und ist somit - im Gegensatz zum Leib - der Kern des Subjekts. Diese ganz bestimmte historisch bedingte Subjektivation findet sich bei Foucault in Hermeneutik des Subjekts (2009) als Disziplinierung. Trescher $(2019$, 48) verweist darauf, dass ein poststrukturalistischer Verstehenszugang, wie er durch Foucault möglich wurde, Diskurs und Subjekt an jeder Stelle immer wieder neu entstehen lasse. Das Subjekt kann damit auf seine Teilhabe- und Handlungsmöglichkeiten hin befreit werden, 
aber auch selbst als befreiungsfähig und „teilhabefähig hervorgebracht werden“ (ebd.). Freires (1973) Pädagogik der Unterdrückten speist sich aus der Lebenspraxis und aus Erfahrungen der Beherrschung und Befreiung des Subjekts, sie ist darum bemüht, das Subjekt zu befähigen und zu seiner möglichen Freiheit und Befreiung hin zu ermächtigen. Folgt man Treschers $(2019,48)$ Argumentation unter poststrukturalistischer Perspektive weiter, dann kann „[d]ie theoriegeleitete, reflexive Auseinandersetzung mit dieser erfahrungswissenschaftlich zugänglich gemachten Lebenspraxis (...) dazu bei[tragen], Praxen infrage zu stellen, Strukturen anders zu denken und somit sowohl Lebenspraxis als auch Theorie selbst zu verändern."

„Die Anwendung [des befreiungspädagogischen Ansatzes; Anm. d. Autors] setzt eine genaue Analyse der Benachteiligung, Ausgrenzung und partiellen Einbeziehung jener Personen und Schichten voraus, die gestärkt werden sollen, um ihre Teilnahme an den gesellschaftlichen Prozessen zu ermöglichen oder zu erweitern" (Ofner 2009, 18).

In Kritik am und Abgrenzung vom „realen Sozialismus“ (ebd.) und seiner negativen Auswirkungen auf das Subjekt gibt Ofner unter Bezug auf Freire einen Hinweis auf mögliche Fehlentwicklungen zunehmend inklusiver Bildungspraxen (vgl. ebd.). Er weist darauf hin, „dass die Unterdrückten in ihrem Kampf um Befreiung nicht selbst zu Unterdrückern werden dürfen, da in diesem Fall wieder eine inhumane Gesellschaft entstehen würde“ (ebd.; vgl. hierzu auch Baquero, Knauth \& Schröder 1998, 16).

Mit Befreiung kann unter befreiungspädagogischen Gesichtspunkten nicht nur die Inklusion in eine bestehende gesellschaftliche Ordnung gemeint sein, sondern sie sei mit einer Gesellschaftsveränderung verbunden, mit der Etablierung einer „neuen Ordnung“ (Ofner 2009, 18f.), in der Unterdrückung und Benachteiligung ausgeschlossen seien (vgl. hierzu auch Hoffmann 2018). Die prekären Lebenslagen von Menschen mit Behinderung, ihr Leben in unterdrückenden und ermöglichenden Strukturen befreien, insofern sie zu Widerspruch und Widerstand animieren (vgl. Baquero et al. 1998, 16). Dabei ist eine Dialektik von ,sich befreien' und ,befreit werden' inhärenter Bestandteil des Befreiungsprozesses (vgl. ebd.). Insofern ist die fünfte These von Hoffmann $(2018,30)$ angesprochen: „(...) [E]ine inklusive Pädagogik [sollte] keineswegs die Forderung nach einer Schule für alle aufgeben, [muss] sich aber der zum Teil widersprüchlichen Anforderungen bewusst sein [...], die an sie gestellt werden, um diese in ihren Konzepten zu berücksichtigen." 


\section{Zuhören und Lauschen als Voraussetzung für einen interdisziplinären Dialog oder ,die Offenhaltung des Blicks für den anderen Menschen“ (Dederich 2013b, 24)}

Als Methode der Befreiung gilt der Dialog (vgl. Hoffmann 2018, 42). Pädagogik der Befreiung lässt sich nur gemeinsam mit den Unterdrückten und Ausgeschlossenen verwirklichen, und zwar durch die Sichtbarmachung ihrer subjektiven Perspektiven. So gilt es, den Subjekten durch das Zuhören eine Stimme zu geben: „Ihre Perspektiven müssen sichtbar gemacht und ihre Stimmen der Welt zu Gehör gebracht werden. Wo dies wirklich gelingt, verändert sich die gesellschaftliche Realität bereits" (ebd.). Bei Freire (1973) ist der Dialog als Praxis und handlungstheoretisch konzeptualisiert. Der Dialog umfasst bei Freire (ebd.) auch eine ethische Dimension. Auch Moser (2003) zeigt die ethische Dimension sonderpädagogischer Dialogik auf, was eine Verbindung zur Befreiungspädagogik erlaubt: „Das Dialogische bestimmt (...) einen ethischen Rahmen, der den Erziehungsprozess möglichst offenhält und dabei auf das Menschliche verweist, das erhalten und befördert werden soll“" (ebd., 96).

Pädagogik der Befreiung stellt Kindern und Bezugspersonen nicht Erzieher*innen im klassischen Sinn zur Verfügung, sondern geschulte Zuhörer*innen, die den Anspruch vertreten, auch selbst befreit von pädagogischen Machbarkeitsfantasien zu sein und die auch das Kind in sich zu erkennen versuchen. Die Fachpersonen sind Hörende, das Hören und Sprechen sind für Küchenhoff (2019) Erkenntniswege der Psychoanalyse. Diese Erkenntniswege im Sinne eines interpretativen Paradigmas möchte mit diesem Beitrag auch stärker der Inklusions- und Sonderpädagogik anempfohlen werden. Han $(2016,93)$ prognostiziert, dass es in der Zukunft womöglich einen neuen Beruf geben werde, „der Zuhörer heißt.“ Dieser schenke der*dem Anderen Gehör. Die Fähigkeit, zuzuhören, gehe zunehmend verloren. Die Psychoanalyse ist eine der wenigen Wissenschaften, die die Subjekte zum Zuhören befähigen kann: „Narziss erwidert die liebende Stimme der Nymphe Echo nicht, die eigentlich die Stimme des Anderen wäre" (ebd.). Zuhören im psychoanalytischen Verständnis ist eine aktive und nicht passive Handlung: „Ich muss zunächst den Anderen willkommen heißen, das heißt den Anderen in seiner Andersheit bejahen. Dann schenke ich ihm Gehör. Zuhören ist ein Schenken, ein Geben, eine Gabe. Es verhilft dem Anderen erst zum Sprechen“ (ebd.). Mit Han (ebd.) kann man die Bedeutung des Zuhörens für die menschliche Entwicklung noch etwas weiter entfalten: „Das Zuhören bringt den Anderen erst zum Sprechen. Ich höre schon zu, bevor der Andere spricht, oder ich höre zu, damit der Andere spricht.“ Zudem gilt, dass „,[d]er Zuhörer (...) ein Resonanzraum [ist], in dem der Andere sich freiredet. So kann das Zuhören heilend sein" (ebd., 94).

Inklusions- und Sonderpädagogik, die interdisziplinär um Perspektiven der Befreiungspädagogik, der Psychoanalyse und Philosophie erweitert ist, wird 
bemüht sein um „[d]ie gastfreundliche Aufnahme des Anderen“ (ebd., 95), die „den Anderen jedoch nicht einverleibt, sondern beherbergt und behütet“ (ebd.). Aus der Haltung der Geduld, der Passivität gegenüber der*dem Anderen sowie des Ausgesetzt-Seins gegenüber der*dem Anderen entwickelt Han (2016) Maximen einer Ethik des Zuhörens. Grund des Zuhörens ist die Sorge für und die Sorge um die*den Andere*n. Diese Form der Pädagogik ist auch normativ, sie hat eine politische Dimension: „Es ist eine Handlung, eine aktive Teilnahme am Dasein Anderer und auch an deren Leiden. Es verbindet, vermittelt Menschen erst zu einer Gemeinschaft" (ebd., 98). Dies ist doch seit über zehn Jahren immer noch aktuelles Ziel der Inklusions- und Sonderpädagogik: pädagogische Inklusionsbemühungen als Förderung der Teilhabe und Vergemeinschaftung des Menschen, insbesondere von besonders vulnerablen und leidenden Subjekten, zu verstehen. Voraussetzung für die Umsetzung dieses Bildungsideals ist das Eintreten der Prophezeiung von Han (ebd., 101): „Die kommende Gesellschaft könnte dagegen eine Gesellschaft der Zuhörenden und Lauschenden heißen.“

Gerade im Angesicht dessen, dass Erziehung eine vergessene pädagogische Verantwortung in der Gesellschaft darstellt, muss mit Ahrbeck $(2004,1)$ daran appelliert werden: „Kinder brauchen Erziehung“, sie bedürfen des Dialogs mit der*dem Anderen und sie haben ein Recht auf ihn. Wenn man von Erziehung spricht, kann durchaus von einer anthropologischen Notwendigkeit die Rede sein (vgl. Müller \& Stein 2018). Erziehung setzt voraus, dass jemand mir Gehör schenkt, sie ist es, vor allem im Sinne der Dialogischen Erziehung nach Freire (1977), die temporär von der Asymmetrie ihrer Beziehung lebt. Freires Bildungskonzept zielt auf eine Kultur des Dialogs hin: „Sie stellt die Pädagogik in den Dienst der Bewusstseinsbildung, der Reflexion der herrschenden Verhältnisse und ihrer ideologischen Verklärung, indem sie die konkrete Lebensform der unterdrückten Subjekte aufgreift und Bildung als Werkzeug der Befreiung und Werkzeug des politischen Kampfes vermittelt“ (Hoffmann 2018, 40).

Delgado (1998, 229f.) spricht mit Blick auf die Befreiungspädagogik und -theologie von Freire (1973 \& 1977) und Gutiérrez (1992) von einer, nachidealistischen ‘ und einer ,leidempfindlichen' Pädagogik. Dies kann und sollte Selbstanspruch der Inklusions- und Sonderpädagogik sein. Wenn sie keine leidempfindlichen Pädagogiken mehr sind, stellt sich die Frage nach ihrer Legitimation verschärft. Angesichts des zunehmenden Rechtspopulismus ist für Delgado „[e]ine Rückgewinnung des echt christlichen, befreienden Universalismus“ (Delgado 1998, 238) unumgänglich. Voraussetzung dafür ist, was zu Beginn mit Dederich (2013a) auf die sonderpädagogische Wissenschaft allgemein herausgearbeitet wurde: „eine 'Bewusstmachung, eine kritische Durchleuchtung der ambivalenten Rolle des Christentums in der Geschichte" (Delgado 1998, 238), die im Rahmen dieses Beitrags nicht mehr geleistet werden kann. Delgado hinterfragt mit Blick auf die Befreiungspädagogik ihre Rezeption im Westen kritisch: 
„Begnügen wir uns nicht allzuoft mit einem romantischen Blick auf die fernen Länder, wo der Glaube oder die pädagogische Phantasie ’lebtı, statt den großen Skandal der europäischen Geschichte aufzuarbeiten, daß nämlich Völker, die seit mehr als einem Jahrtausend evangelisiert wurden, bis in die Gegenwart hinein dem menschenverachtenden Rassismus, dem Kolonialismus (...), dem Nationalismus huldigen? Erst wenn wir auch vor der eigenen Haustür gründlich kehren, werden wir die befreienden Impulse eines Las Casas (Freire und Gutiérrez) wirklich verstanden und die Grundlage für eine halbwegs solidarische [inklusive; Anm. d. Autors] Weltgesellschaft unter den brüchigen Bedingungen der menschlichen Daseinsverfassung gelegt haben“" (ebd.).

Auch wenn die Dialektik von Herrschaft und Befreiung letztlich nicht aufhebbar und somit immer wirksam ist (vgl. Baquero et al. 1998, 16), können sich Inklusions- und Sonderpädagogik disziplinär selbst dazu verpflichten, gegenüber den adressierten Subjekten „die Offenhaltung des Blicks für den anderen Menschen“ (Dederich 2013b, 24) zu gewährleisten.

„Hör mir zu heißt: Berühre mich, wisse, dass ich existiere“ (Barthes 1989, 255).

\section{Literatur}

Ahrbeck, B. (2004): Kinder brauchen Erziehung. Stuttgart.

Ahrbeck, B. (2020): Was Erziehung heute leisten kann. Pädagogik jenseits von Illusionen. Stuttgart.

Baquero, P., Knauth, T. \& Schröder, J. (1998): Befreiung als Paradigma in Pädagogik, Theologie und Philosophie. In: Knauth, T. \& Schröder, J. (Hrsg.): Über Befreiung: Befreiungspädagogik, Befreiungsphilosophie und Befreiungstheologie im Dialog. Münster u.a., 11-92.

Barthes, R. (1989): Die Helle Kammer. Bemerkung zur Photographie. Frankfurt am Main.

Baumann, M. (2012/2019): Kinder, die Systeme sprengen. 2 Bde. Baltmannsweiler.

Dederich, M. (2013a): Inklusion und das Verschwinden der Menschen. Über Grenzen der Gerechtigkeit. In: Behinderte Menschen, 36, H.1, 32-43.

Dederich, M. (2013b): Philosophie in der Heil- und Sonderpädagogik. Stuttgart.

Delgado, M. (1998): Befreiung durch Erziehung? Überlegungen zum Gespräch zwischen Paulo Freires Pädagogik und der Theologie. In: Knauth, T. \& Schröder, J. (Hrsg.): Über Befreiung: Befreiungspädagogik, Befreiungsphilosophie und Befreiungstheologie im Dialog. Münster u.a., 227-240.

Dornes, M. (2010): Die Modernisierung der Seele. Frankfurt am Main.

Foucault, P.-M. (2009): Hermeneutik des Subjekts: Vorlesungen am Collège de France 1981/82. Frankfurt am Main.

Freire, P. (1973): Pädagogik der Unterdrückten. Bildung als Praxis der Freiheit. Reinbek bei Hamburg. Freire, P. (1977): Erziehung als Praxis der Freiheit. Reinbek bei Hamburg.

Gutiérrez, G. (1992): Theologie der Befreiung. Mainz.

Han, B.-C. (2016): Die Austreibung des Anderen: Gesellschaft, Wahrnehmung und Kommunikation heute. 2. Aufl. Frankfurt am Main.

Hoffmann, T. (2018): Inklusive Pädagogik als Pädagogik der Befreiung. Fünf Thesen. In: Hoffmann, T., Jantzen, W. \& Stinkes, U. (Hrsg.): Empowerment und Exklusion: Zur Kritik der Mechanismen gesellschaftlicher Ausgrenzung. Gießen, 19-48.

Küchenhoff, J. (2019): Verständigung und Selbstfindung. Basel.

Moser, V. (2003): Konstruktion und Kritik. Sonderpädagogik als Disziplin. Opladen. 
Müller, T. \& Stein, R. (Hrsg.) (2018): Erziehung als Herausforderung. Grundlagen für die Pädagogik bei Verhaltensstörungen. Bad Heilbrunn.

Ofner, F. (2009): Selbstbefreiung oder Inklusion. In: schulheft, 34, 9-20.

Stenger, U. (2002): Schöpferische Prozesse. Phänomenologisch-anthropologische Analysen zur Konstitution von Ich und Welt. Weinheim/München.

Trescher, H. (2019): Sonderpädagogik als Erfahrungswissenschaft aus poststrukturalistischer Perspektive. In: Dederich, M., Ellinger, S. \& Laubenstein, D. (Hrsg.): Sonderpädagogik als Erfahrungsund Praxiswissenschaft. Opladen/Berlin \& Toronto, 35-50. 


\section{Zwischen Individualisierungsversprechen und Vermessungsgefahr - Die Rolle der Schlüsseltechnologie Künstliche Intelligenz in der inklusiven Schule}

"AI $I^{1}$ is one of the most important things humanity is working on.
It is more profound than (...) electricity or fire."

Sundar Pichai, CEO von Google, spricht eines der wesentlichen Merkmale der neuen Schlüsseltechnologie Künstliche Intelligenz (KI) an: Sie ist solch eine „machtvolle Technologie“, dass sie „unsere Möglichkeiten des Wissenserwerbs, der Kommunikation, des Zusammenlebens und unsere Arbeitswelt" (Lenzen 2018, 147) massiv verändert. Sie wird einen radikalen gesellschaftlichen Wandel provozieren, der ausnahmslos alle (!) gesellschaftlichen Teilbereiche tangieren wird; selbstverständlich auch den des Bildungssystems.

$\mathrm{Da}$ „diese neuen Technologien grundlegende Veränderungen hervorbringen werden und potenziell mit positiven als auch negativen Effekten eng verknüpft sein dürften“" (Hamisch \& Kruschel 2019, 397), gilt es, sich tiefergehend mit ihnen im Kontext von Schule auseinanderzusetzen. Der vorliegende Beitrag geht der Frage nach, welche Herausforderungen, aber auch Chancen aus einer inklusiven Perspektive heraus mit dem Einsatz von KI verbunden sind. Insbesondere die im Inklusionsdiskurs häufig diskutierte Anforderung der Individualisierung steht dabei im Vordergrund, also die Frage danach, wie Lernprozesse an die individuellen Erfahrungen, das Vorwissen der Lernenden und das gemeinsame Lernen anknüpfen und so individuelle Lernwege ermöglicht werden. Zusätzlich soll das Thema anhand der möglichen Gefahr der Vermessung der Lernenden diskutiert werden.

Nach einem Einblick in den Begriff, Künstliche Intelligenz' werden zwei Beispiele zum Einsatz von KI im Lehr-Lern-Setting vorgestellt. Diese dienen als Analysevorlage zur überblicksartigen Betrachtung der Individualisierungsmöglichkeiten und Vermessungstendenzen von KI-Anwendungen für die (Hoch-)Schule.

$1 \mathrm{AI}=$ engl. Abk. für Künstliche Intelligenz

2 Zitat von Sundar Pichai (2016), siehe online unter: cnb.cx/2zTCSiC (letzter Zugriff: 22.06.2020) 


\section{Künstliche Intelligenz - Was ist das eigentlich?}

Bei ,Künstlicher Intelligenz' handelt es sich generell um einen sehr weiten Begriff, der kontrovers diskutiert wird. Unter ihm werden aktuell eine Reihe von Ansätzen subsumiert, die ein zentraler Aspekt eint: „Es ist der Versuch, ein System zu entwickeln, das eigenständig komplexe Probleme bearbeiten kann" (Kirste \& Schürholz 2019, 21). Dabei nutzen die Entwickler*innen unterschiedliche Techniken, wie ,Maschinelles Lernen' oder ,Tiefes Lernen' (,Deep Learning'). Intelligent ist ein System nicht - wie es der Begriff impliziert -, wenn es das Handeln von Menschen imitiert, sondern ,wenn es selbstständig und effizient Probleme lösen kann. Der Grad der Intelligenz hängt vom Grad der Selbstständigkeit, dem Grad der Komplexität des Problems und dem Grad der Effizienz des Problemlösungsverfahrens ab" (Mainzer 2016, 3).

Bei den meisten KI-Anwendungen handelt es sich um selbstoptimierende Systeme, die in großen Datenmengen mithilfe umfangreicher statistischer Auswertung Korrelationen erkennen - jedoch keine Kausalitäten. Auch wenn daran gearbeitet wird, verschiedene Bereiche zu vernetzen, so sind die meisten KIAnwendungen Experten in einem Gebiet. Sie können entweder Fotos auswerten oder Sprache erkennen oder ein bestimmtes Brettspiel spielen.

\section{Künstliche Intelligenz im Unterrichtssetting - Das Schulbuch ,Hypermind' und der Roboter ,Yuki'}

KI ist im schulischen Kontext Deutschlands bisher selten anzutreffen. In ,technologiebegeisterten' Industrienationen hingegen ist diese Technik in der Praxis bereits angekommen: So existieren KI-Systeme, die Schüler*innen in den USA im Mathematikunterricht täglich leistungsabhängige Stundenpläne vorschlagen, oder KI-Roboter in Japan mit dem Ziel, die Aufmerksamkeit der Schüler*innen im Unterricht zu steigern (vgl. Gonsch 2019). Im Folgenden werden zwei der seltenen Beispiele aus Deutschland skizziert, um sie anschließend vor dem Hintergrund der Frage nach dem Potenzial der Individualisierung und der Gefahr von Vermessung zu diskutieren.

Im Projekt ,Hypermind ${ }^{\text {‘3 }}$ wird ein dynamisch adaptives und somit intelligentes Schulbuch für das Schulfach Physik entwickelt. Dieses läuft als Software auf einem Tablet. Das Programm erkennt den Wissensstand sowie die Interessen der Schüler*innen und adaptiert die Schulbuchinhalte (vgl. Kuhn 2019, o.S.). Auf Grundlage von Daten, die mit Eyetracking-Technologie, Temperaturmessung im Gesicht über Infrarot sowie Pulsmessung per Smartwatch erhoben werden, wählt

3 Siehe online unter: bit.ly/31ao3n5 (letzter Zugriff: 22.06.2020) 


\section{Katharina Hamisch und Robert Kruschel}

Hypermind aus, welche Materialien und welches Medium der*dem Lernenden zur Verfügung gestellt werden. Auch wenn aktuell noch Menschen dem System beistehen, soll es in Zukunft eigenständig Entscheidungen treffen.

Im Projekt H.E.A.R.T. wird der Einsatz von Robotern als Lehrassistenten erforscht (vgl. Zeaiter 2017, 112). Der humanoide KI-basierte Roboter Yuki wird in Veranstaltungen des Sprachwissenschaftlers Handke eingesetzt. Nach einer Einrichtung gestaltet und strukturiert Yuki Lehrveranstaltungen, wodurch die Lehrperson mehr Zeit zur Interaktion mit den Lernenden erhält. Dazu wird der Roboter mit den entsprechenden Informationen zu Aufgaben oder Zeitspannen für bestimmte Arbeitsphasen programmiert. Er kann dann Abläufe koordinieren, Informationen bereitstellen, mündliche Prüfungen abnehmen oder beratend tätig sein. Die Kommunikation zwischen Roboter und Lernenden findet über Sensorik wie zuvor festgelegte visuelle oder taktile Reize oder über sogenannte Trigger-Wörter statt. Von Vorteil für den Einsatz des Geräts ist eine veränderte Lehre im Sinne des Inverted oder Flipped Classrooms (vgl. Handke 2019).

\section{Künstliche Intelligenz zwischen Individualisierung und Vermessung}

Die Frage, die an dieser Stelle interessiert, ist die Bedeutung von KI-geleiteten Individualisierungstechniken im pädagogischen Setting und deren potenziellen Vermessungsgefahren der Nutzenden.

Eine der zentralen Forderungen im pädagogischen Inklusionsdiskurs ist die nach der Veränderung von schulischen Lehr-Lernsettings (vgl. exempl. Carle 2017), um den ,vielfältigen und unterschiedlichen Ausgangs- und Umfeldbedingungen der Schüler*innen gerecht zu werden und es allen zu ermöglichen, an Bildungsund sozialen Prozessen gleichberechtigt zu partizipieren" (Ziemen 2018, 9). Insbesondere Schulbücher in ihrer tradierten Form stehen bereits länger in der Kritik (vgl. Lutz 2017, 38).

An dieser Kritik setzt das intelligente Lehrbuch Hypermind ${ }^{4}$ an. Es ist ein Beispiel für hochindividualisierte Lernsysteme, die versprechen, Lernende durch den Einsatz von KI exakt anhand derer Fähigkeiten und Bedarfe zu unterstützen und so inklusionsorientierten Forderungen nachzukommen. Ein ineffizienter, weil normalisierender, frontaler und lehrpersonenzentrierter Unterricht wird de facto überflüssig, da alle Schüler*innen in ihrem eigenen Tempo und nach eigenem Interesse an ,ihrem' eigenen Schulbuch lernen können. Den Versprechen der Macher*innen glaubend ist Hypermind auf der höchsten - der proaktiven - der

4 Datenschutzbezogene Diskussionen sollen an dieser Stelle keine Rolle spielen. Die Macher*innen versprechen diesbezüglich die Einhaltung der entsprechenden gesetzlichen Vorgaben. 
vier Stufen nach Weinert $(1997,51 f$.$) anzusiedeln: Es findet eine adaptive Unter-$ richtsgestaltung und individuelle Förderung der Lernenden entsprechend ihrer Voraussetzungen statt.

Mit Blick auf die möglichen Unterrichtsformen im Umgang mit Heterogenität nach Bohl, Batzel und Richey (2011) bedient Hypermind die Form der Individualisierung - die Lernangebote werden an einzelne Lernende angepasst. Die im tradierten Lernsetting die Lehrkraft stark beanspruchende Mammutaufgabe der Anpassung der Angebote an - im Optimalfall - jedes Individuum übernimmt nun die KI. Das Team um Hypermind betont aber, dass die Lehrperson nicht überflüssig wird. Sie wird weiterhin benötigt, um die Schüler*innen zu begleiten, zu beraten und zudem soziale Interaktion zwischen ihnen zu fördern.

Gleiches gilt für die Rolle der Lehrperson im Beispiel des Assistenzroboters Yuki. Der Roboter übernimmt sämtliche organisatorische und direktive Aufgaben im Unterrichtsgeschehen, wodurch sich die Lehrkraft intensiv auf die Lernenden einlassen kann (vgl. DW Deutsch 2019). Inwiefern dabei das gemeinsame Lernen Beachtung findet, wird nicht ausgeführt und bildet somit einen ,blinden Fleck'.

Im Gegensatz zu Hypermind bietet der Roboter jedoch keine Unterstützung für die generelle Unterrichtsgestaltung. Die Individualisierung findet weiterhin durch die didaktische Planung der Lehrperson statt. Der Roboter kann Daten über Nutzungsverhalten und Informationseingaben der Lernenden erheben. Beispielsweise überprüft er die Qualität der Ergebnisse von Rechercheaufgaben. Über Aufgaben, Umfang und geplanten Zeitpunkt entscheidet jedoch die Lehrperson vorab. Im Gegensatz zu Hypermind ermöglicht die Einbettung Yukis in ein Flipped Classroom-Konzept die Förderung der Interaktion zwischen den Lernenden im Sinne des kooperativen Lernens (vgl. Altemueller \& Lindquist 2017, 349), jedoch scheint hier die individuelle Ausgestaltung der Lehrperson von hoher Relevanz.

Auch wenn diese Vorzüge auf den ersten, inklusiven Blick' für die KI-Systeme sprechen, gibt es kritische Aspekte. So ist es beispielsweise fraglich, inwiefern beim Lernen mit Systemen wie Hypermind Forderungen nach einer Intensivierung des konstruktivistischen Charakters (vgl. Reich 2016) von Lernen nachgekommen werden könnte. Viel eher besteht die Gefahr, dass Lernen ein mitunter noch stärker rezeptiver, fremdgesteuerter und einseitig kognitiver Prozess würde. Die Forderung nach Individualisierung im inklusionswissenschaftlichen Diskurs bedeutet nicht, dass jede* $\mathrm{r}$,für sich allein arbeitet. [Die] Umsetzung ist angewiesen auf den Austausch untereinander" (Brügelmann 2011, 357) und einem Lernen am gemeinsamen Gegenstand (vgl. Feuser 1989). Im Gegenteil: Nicht technikbasierten Formen individualisierten Unterrichts, die weniger lehrer*innenzentriert sind, werden eher Chancen zugesprochen, persönliche Kontakte zwischen den Lernenden zu fördern und so zu einem positiven Unterrichtsklima beizutragen (vgl. Hess \& Lipowsky 2017, 27). Systeme wie Hypermind könnten hingegen die Gefahr 


\section{Katharina Hamisch und Robert Kruschel}

der „Individualisierungsfalle“ (Burow 1999) bedienen, indem Schüler*innen nur noch neben- statt mit- und voneinander lernen. Offen bleibt, wie lernwirksam diese Form des individualisierten Lernens ist, denn wie auch bei tradierten Formen ist sie von den Tiefenstrukturen des Unterrichts abhängig.

Spätestens seit dem ,PISA-Schock' von 2003 hatte sich die Orientierung der Bildungspolitik und -wissenschaft am Paradigma der Standardisierung und Evidenzorientierung deutlich verstärkt. Aus dem Bestreben nach Inklusion ergeben sich eher gegenteilige Implikationen (vgl. Rödler 2012, 38), wie etwa die am Beispiel des RTI-Ansatzes kritisch herausgearbeiteten Aspekte (vgl. Hinz 2016, 254f.), die sich nun augenscheinlich auch im Hypermind-Lehrbuch wiederfinden lassen. Das Programm misst physiologische Daten, um Rückschlüsse im Hinblick auf Interesse und Motivation der Lernenden ziehen zu können. Die Basis scheint in der These eines Zusammenhangs von körperlichen Reaktionen oder beobachtbarem Verhalten auf kognitive, psychische und emotionale Vorgänge zu liegen - eine Annahme, der sicherlich in Ansätzen gefolgt werden kann, die jedoch für den Menschen als komplexes Wesen deutlich zu kurz greift. So werden die Effekte der sozialen Eingebundenheit der Lernenden oder gar das Technologie-Defizit im Feld der Pädagogik außer Acht gelassen.

Die Berechnungen der Algorithmen basieren auf statistischen Werten der erhobenen Lernenden-Daten, welche Prognosen zu Lernprozessen zulassen sollen, um selbige zu optimieren. Jene ,algorithmische Steuerung kann als Fortführung und letztlich Intensivierung und Ausweitung bürokratischer Organisation verstanden werden" (Mohabbat Kar \& Parycek 2018, 24), denn die Handlungsoptionen werden vorstrukturiert und lassen bestimmte Handlungen zu, andere wiederum nicht (vgl. ebd.). Die ,gefühlte Freiheit' ist durch die Programmierung der KI deutlich begrenzt.

\section{Künstliche Intelligenz als Instrument für pädagogische Settings im inklusiven Kontext?}

Wie die vorangegangenen Ausführungen zeigen, steht die Pädagogik vor der Herausforderung, die KI-Technik sowohl für individuelle als auch gemeinschaftliche Lehr-Lern-Prozesse zu nutzen, ohne sich ihr zu unterwerfen.

Da sich die Entwicklung in Deutschland gegenwärtig am Anfang befindet, ist jetzt der Zeitpunkt, diese aus inklusiver Perspektive mitzubegleiten und zu beeinflussen. Dafür bedarf es der engen Kooperation mit Informatiker*innen. Die Entwickler*innen sind aufgefordert, Aspekte inklusiver Bildung, wie „exkludierende und marginalisierende Praktiken und Strukturen in Schule und Unterricht zu erkennen und zugunsten egalitärer Praktiken und Strukturen zu überwinden“ 
(Sturm 2016, 171), in der Konzeption der Software für Schulen zu berücksichtigen und gleichzeitig KI-Entscheidungen transparent sowie für Laien nachvollziehbar darzustellen. Zudem bedarf es Pädagog*innen, die ein Bewusstsein für mögliche Gefahren entwickeln, denn die Entscheidungen, die durch KI getroffen werden, sind „nicht per se neutraler oder objektiver“ (Beck, Grunwald, Jacob \& Matzner 2019, 16) als von Menschen gefällte Entscheidungen. Die diagnostischen, Verlockungen' KI gestützter Systeme, die im Extremfall gläserne Lernende produzieren und ihnen somit jeglichen Subjektstatus absprechen bzw. sie verobjektivieren, sind vor der Folie ihrer tatsächlichen pädagogischen Nutzbarkeit sowie ethischer Überlegungen kritisch zu hinterfragen. Auch wenn diese Systeme neutral und objektiv wirken, sind systematische und strukturelle Diskriminierungen dieser Technologie nicht fremd.

Ob die Frage nach einer Modernisierung von Lehr-Lern-Settings, die zudem dem inklusiven Anspruch gerecht wird, mit der KI-Technik beantwortet werden kann, bleibt an dieser Stelle offen. Deutlich geworden ist, dass die Algorithmen steuernd auf Lehrende und Lernende einwirken. Die Themen „Autonomie und Kontroll“" (Stubbe, Wessels \& Zinke 2019, 248) sind zwar im schulischen Kontext keine neuen Themen, jedoch werden sie im Kontext von KI als ethische Fragen „potenziert“ (ebd.). Eine entsprechende Reflexionsfähigkeit von Möglichkeiten und Grenzen algorithmischer Steuerung scheint somit unerlässlich.

Die Einbettung der KI-Technik in den pädagogischen Kontext kann mit der Suggestion einer Technologie für das Bezwingen der Unbestimmbarkeit des pädagogischen Handelns einhergehen. Jedoch kann vielmehr davon ausgegangen werden, dass sich eine Vielzahl neuer Forschungsfragen ergibt. Wie kann mit den Spannungsfeldern Fremdsteuerung - Selbststeuerung, Systematik - Erlebnis oder Gemeinschaftsbezug - Individuumsbezug umgegangen werden und wie können inklusive Lehr-Lern-Settings konstruiert werden?

So sollten nicht die Möglichkeiten der Technologie die Entwicklungen definieren, sondern der Einsatz von KI durch Ziele der Pädagogik oder des Unterrichts bestimmt werden (vgl. DGI 2019, o.S.). Sundar Pichai gibt im eingangs angeführten Zitat nicht nur zu bedenken, dass KI in seiner Bedeutung für die Menschheit grundlegender ist als Elektrizität und Feuer, sondern diese ebenso potenzielle Gefahren beinhaltet und es daher um das Erlernen eines reflexiven Umgangs damit geht:

"Well, it kills people, too. (...) We have learned to harness fire for the benefits of humanity but we had to overcome its downsides too. So my point is, AI is really important, but we have to be concerned about it." 


\section{Katharina Hamisch und Robert Kruschel}

\section{Literatur}

Altemueller, L. \& Lindquist, C. (2017): Flipped classroom instruction for inclusive learning. In: British Journal of Special Education, 44, 341-358.

Beck, S., Grunwald, A., Jacob, K. \& Matzner, T. (2019): Künstliche Intelligenz und Diskriminierung. Herausforderungen und Lösungsansätze. Online unter: https://bit.ly/3mYofNN (letzter Zugriff: 18.06.2020).

Bohl, T., Batzel, A. \& Richey, P. (2011): Öffnung - Differenzierung - Individualisierung - Adaptivität. Charakteristika, didaktische Implikationen und Forschungsbefunde verwandter Unterrichtskonzepte zum Umgang mit Heterogenität. In: Schulpädagogik heute, 2, 40-69.

Brügelmann, H. (2011): Den Einzelnen gerecht werden - in der inklusiven Schule. Mit einer Öffnung des Unterrichts raus aus der Individualisierungsfalle! In: Zeitschrift für Heilpädagogik, 9, 355-362.

Burow, O.-A. (1999): Die Individualisierungsfalle: Kreativität gibt es nur im Plural. Stuttgart.

Carle, U. (2017): Individuelles Lernen. In: Ziemen, K. (Hrsg.): Lexikon Inklusion. Göttingen, 99-100. DGI (Deutsche Gesellschaft für Information und Wissen e.V.) (2019): Positionspapier der DGI-Fachgruppe Bildung und Informationskompetenz. Im Spannungsfeld von Künstlicher Intelligenz und Bildung. Online unter: https://bit.ly/3p1 rea7 (letzter Zugriff: 17.05.2020).

DW Deutsch (2019): Deutschland: Wenn Roboter unterrichten | Reporter (Laufzeit: 12:36). YouTube-Kanal: DW Deutsch (24.02.2019). Online unter: https://bit.ly/38dxxBG (letzter Zugriff: 13.03.2020).

Feuser, G. (1989): Allgemeine integrative Pädagogik und entwicklungslogische Didaktik. In: Behindertenpädagogik, 1, 4-48.

Gonsch, V. (2019): Chancen und Risiken von KI im Klassenzimmer. Online unter: https://bit. ly/38jk1fX (letzter Zugriff: 09.09.2019).

Hamisch, K. \& Kruschel, R. (2019): Schlüsseltechnologie ,Künstliche Intelligenz - Überlegungen zur Zukunft schulischer Bildung. In: Huber, S. G. (Hrsg.): Jahrbuch Schulleitung 2019. Köln, 381-402.

Handke, J. (2019): Humanoide Roboter als Partner in der Lehre. Vortragsvideo (Laufzeit: 04:21). YouTube-Kanal: Digitalisierung der Lehre (09.04.2019). Online unter: https://bit.ly/38jk8Ip (letzter Zugriff: 13.03.2020).

Hess, M. \& Lipowsky, F. (2017): Lernen individualisieren und Unterrichtsqualität verbessern. In: Heinzel, F. \& Koch, K. (Hrsg.): Individualisierung im Grundschulunterricht. Anspruch, Realisierung und Risiken. Wiesbaden, 23-31.

Hinz, A. (2016): Response-to-Intervention - eine (Schein-)Lösung für die Herausforderung inklusionsorientierter Diagnostik?! In: Amrhein, B. (Hrsg.): Diagnostik im Kontext inklusiver Bildung: Theorien, Ambivalenzen, Akteure, Konzepte. Bad Heilbrunn, 243-257.

Kirste, M. \& Schürholz, M. (2019): Einleitung: Entwicklungswege zur KI. In: Wittpahl, V. (Hrsg.): Künstliche Intelligenz. Technologie, Anwendung, Gesellschaft. Berlin/Heidelberg, 21-35.

Kuhn, J. (2019): Revolution in der Schule. Online unter: https://bit.ly/2TS9vUi (letzter Zugriff: 18.06.2020).

Lenzen, M. (2018): Künstliche Intelligenz. Was sie kann und was uns erwartet. München.

Lutz, S. (2017): Mathematikschulbücher im Förderschwerpunkt Lernen. Die Relevanz des Mathematikschulbuchs im Unterricht aus Sicht von Lehrkräften. Bad Heilbrunn.

Mainzer, K. (2016): Künstliche Intelligenz - Wann übernehmen die Maschinen? Berlin/Heidelberg.

Mohabbat Kar, R. \& Parycek, P. (2018): Berechnen, ermöglichen, verhindern: Algorithmen als Ordnungs- und Steuerungsinstrumente in der digitalen Gesellschaft. In: Mohabbat Kar, R., Thapa, B. E. P. \& Parycek, P. (Hrsg.): (Un)berechenbar? Algorithmen und Automatisierung in Staat und Gesellschaft. Berlin, 7-39.

Reich, K. (2016): Die konstruktivistische und inklusive Didaktik. In: Porsch, R. (Hrsg.): Einführung in die Allgemeine Didaktik. Münster/New York, 177-206. 
Rödler, P. (2012): Inklusion und Standards - eine verständlich, aber paradoxe Forderung. In: Gemeinsam leben, 1, 33-40.

Stubbe, J., Wessels, J. \& Zinke, G. (2019): Neue Intelligenz, neue Ethik? In: Wittpahl, V. (Hrsg.): Künstliche Intelligenz. Technologie - Anwendung - Gesellschaft. Berlin/Heidelberg, 239-254.

Sturm, T. (2016): Lehrbuch Heterogenität in der Schule. 2. überarb. Aufl. München/Basel.

Weinert, F. E. (1997): Notwendige Methodenvielfalt. Unterschiedliche Lernfähigkeiten erfordern variable Unterrichtsmethoden. In: Friedrich Jahresheft, 15, 50-52.

Zeaiter, S. (2017): Roboter trifft Menschen mit Behinderung: Robotereinsatz zur Lehr-Lernunterstützung für Lerner mit Behinderung. In: Zeaiter, S. \& Handke, J. (Hrsg.): Inverted Classroom - The Next Stage. Lehren und Lernen im 21. Jahrhundert. Baden-Baden, 105-114.

Ziemen, K. (2018): Didaktik und Inklusion. Göttingen. 


\section{Zur Kontextualisierung der Inklusionsforschung}

\section{Einleitung}

Der Anteil von Drittmitteln in der Finanzierung der Hochschulen ist deutlich gestiegen und damit auch der Druck auf die Wissenschaftler*innen, diese erfolgreich einzuwerben. Hinzu getreten ist in den letzten Jahren die verstärkte Forderung danach, dass die so gewonnenen Forschungsergebnisse auch ihren Niederschlag in einer - veränderten - Schulpraxis finden sollen. Man mag diese Entwicklungen begrüßen oder ihnen skeptisch gegenüberstehen, sie markieren aber die Rahmenbedingungen, unter denen auch die Inklusionsforschung arbeitet. Im vorliegenden Beitrag wird den damit einhergehenden Ambivalenzen nachgegangen.

\section{Erste Rahmung der Inklusionsforschung: veränderte Forschungsförderung}

Im Jahr 1998 betrug die Drittmittelquote an bundesdeutschen Hochschulen ca. 16\%, bis 2013 ist sie kontinuierlich auf einen Wert zwischen 27 und $28 \%$ angestiegen, und hat sich seither auf diesem Niveau stabilisiert (vgl. DFG 2018; siehe auch Dohmen \& Wrobel 2018; Dzwonnek 2014; Richter 2016). Damit einhergehend hat sich die „Drittmittelbilanz“ als mittlerweile „wichtigste symbolische Währung im Wissenschaftssystem“ (Dzwonnek 2014, 92) etabliert und scheint für die Reputation mindestens so bedeutsam zu sein wie die eigentliche Forschungsleistung. So dienen Drittmittel „nicht mehr primär der Forschung selbst - also als Mittel zum Zweck - sondern die Einwerbung von Mitteln wird zum Zweck an sich“ (ebd.), mit der Folge, dass sich die Forschung und das damit befasste wissenschaftliche Personal nicht mehr an einem Thema und dessen Durchdringung orientieren, sondern an der, Währungseinheit' des (Drittmittel-) Projekts (vgl. Torka 2006; Zillmann \& Kolb 2013). 
Im Jahr 2018 betrug der von Bund, Ländern, EU und DFG erbrachte Anteil der Drittmitteleinnahmen der Universitäten ${ }^{1}$ bundesweit insgesamt mehr als 75\% des gesamten Drittmittelvolumens (vgl. Destatis 2018, eigene Berechnung). Die damit von politischen Akteur*innen zumindest mitbestimmten Schwerpunktsetzungen, die sich insbesondere in der Ausschreibung und Weiterentwicklung von Förderprogrammen materialisieren, haben daher einen großen Anteil an Prozessen der Steuerung der universitären Forschungsagenda. Es ist deshalb durchaus fraglich, inwiefern die Möglichkeit des freien Forschens mit der selbstbestimmten Setzung eigener Themen tatsächlich noch besteht, wie dies z.B. Dzwonnek (2014) mit dem Hinweis auf die jederzeit bestehende Möglichkeit zur Einreichung offener Förderanträge bei der DFG unterstellt. Ihrer Ansicht nach ist das Problem nicht die Finanzierung von Forschung durch Drittmittel, sondern der durch die Unterfinanzierung der Universitäten bedingte Zwang zur Einwerbung von Drittmitteln. Unter den gegebenen ökonomischen Bedingungen lässt sich das Risiko der vorrangigen Ausrichtung von Forschungsthemen an der Ressourcenakquise und weniger an Forschungsinteressen allerdings nicht mehr von der Hand weisen (vgl. Richter 2016; Webler 2016).

Argumentationen, die darauf zielen, dass durch thematische Vorgaben von Drittmittel vergebenden Institutionen „innerwissenschaftliche Fehlentwicklungen“" (Dilger 2018, 3) ausgeglichen und Themen mit höherer politischer, wirtschaftlicher oder gesellschaftlicher Relevanz gefördert werden könnten, erscheinen unterkomplex und naiv. So postuliert etwa Dilger, dass „bestimmte Themen (...) für die Gesellschaft, Steuerzahler und Unternehmen wichtiger [sind] als andere“ (ebd.) und übersieht, dass gerade die gesellschaftlichen Innovations- und Reflexionspotenziale des Wissenschaftssystems verloren gehen, wenn man es zum Dienstleister außerwissenschaftlicher Systeminteressen und Intentionen trimmen wolle. Abgesehen davon, dass alle Steuerungsintentionen immer mit den differenten Prozesslogiken der beteiligten Systemkontexte konfrontiert sind, also politische Steuerungsintentionen eher politisch als wissenschaftlich sinnvoll sind und im Wissenschaftssystem nicht anders als rekontextualisiert werden können, werden doch durch solche Steuerungsimpulse Aufmerksamkeiten und Beobachtungspotenziale gebunden. Ein Problem kann dies aber insbesondere dann werden, wenn bestimmte Themen oder auch Methoden aufgrund gesellschaftlicher, politischer und ökonomischer Interessen stark forciert werden, während andere als blinde Flecken bzw. Desiderata für entsprechende Zeiträume nicht gesehen und in der Konsequenz auch kaum beforscht werden können (vgl. ebd.). Für die Integrations-/Inklusionsforschung traf dies bis zur Ratifizierung der UN-BRK weitgehend zu und steht ihr bei wieder abflauendem öffentlichem und politischem Interesse an der Thematik möglicherweise auch wieder bevor.

1 Ohne medizinische Einrichtungen/Gesundheitswissenschaften der Universitäten 


\section{Zweite Rahmung der Inklusionsforschung: Transferforderungen und Transferenttäuschungen}

In diesen Zusammenhang ist auch die im wissenschaftspolitischen Kontext zunehmend eingeforderte Anschlussfähigkeit von Forschung an Praxis einzuordnen. So war und ist der Anspruch der empirischen Bildungsforschung, „zu einer Analyse und der Verbesserung des Bildungswesens“ beizutragen, indem sie ,aus verschiedenen Disziplinen theoretische und methodische Anregungen [entnimmt] " (Gräsel 2015, 16f.). Als zentrales Ergebnis soll so empirisch gesichertes Wissen für politische Steuerungsprozesse des Bildungssystems zur Verfügung gestellt werden, das idealerweise über Top-down-Strategien in die Fläche des Bildungssystems transferiert werden soll (vgl. Gräsel 2010, 14). Empirische Bildungsforschung wird dabei eng mit dem Konzept der Evidenzorientierung verknüpft, wie sich beispielsweise in dem vom deutschen Bildungsministerium für Bildung und Forschung (BMBF) aufgelegten ersten Rahmenprogramm zeigt: „Für eine evidenzbasierte Steuerung wird eine exzellente empirische Bildungsforschung benötigt (...). Verlässliche wissenschaftliche Erkenntnisse über Bildungsprozesse und das Bildungssystem werden in dem Rahmenprogramm als Bedingungen für die Qualität eines Bildungswesens betrachtet" (Bromme, Prenzel \& Jäger 2016, 130).

Dieser große Optimismus und die nach wie vor hohen Erwartungen an die Ergebnisse der empirischen Bildungsforschung kontrastieren nun eigentümlich mit der Beobachtung, wie wenig Beachtung deren Erkenntnisse in der Bildungspraxis finden, und zwar auf allen Ebenen: von der Bildungspolitik über die Bildungsverwaltung bis hin zur einzelnen Lehrkraft vor Ort. Steffens, Heinrich und Dobbelstein $(2019,15)$ stellen in diesem Zusammenhang nüchtern fest:

„Obwohl in den letzten Jahren zahlreiche Forschungsbefunde vorliegen, die Anregun-
gen für eine Berücksichtigung in der Praxis auf der System-, Schul- und Unterrichts-
ebene liefern, so fällt gleichzeitig auf, dass sie kaum einen praktischen Niederschlag
erfahren haben. Die Gründe dafür dürften sicherlich vielschichtig sein. Beeindruckend
ist dabei allerdings, wie wenig die dargebotenen Forschungsbefunde bei den potenziel-
len Adressaten Beachtung finden und wie selten sie in der Bildungsplanung, Lehrerbil-
dung sowie Schul- und Unterrichtsentwicklung ausführlicher rezipiert werden."

Die Gründe hierfür mögen in der Programmatik der Evidenzbasierung selbst zu suchen sein, insbesondere in deren beschränkter ökologischer Validität (vgl. Heinrich \& Klewin 2019; Sandkühler 2011), sie reichen nach unserer Einschätzung allerdings tiefer. Was mit den Ergebnissen der Bildungsforschung in der pädagogischen Praxis passiert - soweit sie dort überhaupt ankommen -, ist nämlich alles andere als klar. Denn unabhängig von der wissenschaftlichen Relevanz und Evidenz, entscheidet sich die "tatsächliche Relevanz empirischer Bildungsforschungsaktivitäten für pädagogische Handlungsfelder (...) maßgeblich auf der 
Mikroebene über das Tun bzw. Unterlassen der relativ autonom urteilenden und handelnden pädagogischen PraktikerInnen", so Hetfleisch, Goeze und Schrader (2017, 183; Hervorhebung im Original). Der Anspruch der evidenzbasierten Steuerung des Schulsystems, durch die Zunahme an Erklärungswissen eine effektivere und effizientere Qualitätsentwicklung auf allen Ebenen (Schulsystem - Einzelschule - Unterricht) erreichen zu können, wird in der Realisierung der Steuerungsintentionen mit der Differenz der Systemlogiken von Politik, Wissenschaft und Schulsystem konfrontiert und läuft auf „Implementationsbrüche“ (van Ackeren, Zlatkin-Troitschanskaia, Binnewies, Clausen, Dormann, Preisendörfer, Rosenbusch \& Schmidt 2011, 174) auf, sodass der praktische Nutzen der umfangreichen Ergebnisse der empirischen Bildungsforschung grundlegend infrage gestellt werden kann. Mit Tenorth $(2015,276)$ ist von „überzogenen Versprechen" empirischer Bildungsforschung an die Politik zu sprechen, die nicht eingelöst werden konnten.

\section{Ansätze zur Erweiterung der Rahmung empirischer (Inklusions-)Forschung}

Es scheint eine Neubestimmung des Verhältnisses zwischen Forschung und Praxis notwendig, welche die Differenz von Wissenschaftssystem und Schulsystem berücksichtigt. Altrichter (2019) verweist in diesem Zusammenhang zunächst auf das DFG-Programm zur gesellschaftlichen Verwendung sozialwissenschaftlichen Wissens in den 80er-Jahren (vgl. Beck \& Bonß 1989) und fasst dessen abschließendes Konzept wie folgt zusammen: „Die Nutzung wissenschaftlichen Wissens im Praxissystem erfordert eine aktive Veränderung dieses Wissens im Sinne der Logik des Praxissystems durch die dort Handelnden" (Altrichter 2019, 28). Es kann demnach nicht um eine bloße Anwendung des evidenten Wissens gehen, sondern die Eigenlogik des Praxissystems erfordert eine „Transformation oder Reinterpretation" (ebd.) des wissenschaftlichen Wissens. Diese Eigenlogiken der Systeme bleiben in vielen - auch aktuellen - Ansätzen zum Wissenstransfer unbeachtet und auch der Versuch der evidenzbasierten Steuerung im Rahmen von Topdown-Modellen bleibt unterkomplex und ist zu sehr medizinischen oder Management-Modellen verhaftet, ohne die Besonderheiten der pädagogischen Systeme und Profession in Rechnung zu stellen (vgl. van Ackeren et al. 2011; Farley-Ripple, May, Karpyn, Tilley \& McDonough 2018; Steffens et al. 2019).

Diese Schwierigkeiten im Verhältnis von Wissenschaft und Praxis wurden von der Forschungsförderung mittlerweile aufgegriffen und haben beispielsweise ihren Niederschlag im zweiten Rahmenprogramm zur empirischen Bildungsforschung des BMBF gefunden. Dies stellt nicht mehr die output- und evidenzbasierte Steu- 
erung ins Zentrum, sondern die Förderung von und Forderung nach der Kooperation von Forschung und Praxis (vgl. BMBF 2018).

Im Hinblick auf den Anspruch, künftig eine effektive bzw. für beide Seiten gewinnbringende Kooperation zu gestalten, stellen sich jedoch eine Reihe offener Fragen, auch wenn hier bereits zahlreiche konzeptionelle Ansätze zur Verfügung stehen. Als wesentliches Hindernis in der Kooperation zwischen Forschung und Praxis kann die in der Wissenschaft weithin bestehende systemimmanente Selbstreferenzialität angesehen werden: Für den Transfer des Wissens fehlt den Wissenschaftler*innen häufig die Zeit (und zuweilen auch die Lust). Vor allem wird dieser Transfer allenfalls als (lästiges) Anhängsel der eigentlichen Forschung betrachtet. Und auch im Schulsystem muss mit gewohnter Praxis gebrochen werden, wenn systematisch Erkenntnisse aus einem anderen Systemkontext rezipiert und bezogen auf die eigenen Systemprozesse übersetzt werden sollen. Erkenntnisse der Innovations- und Transferforschung legen hier nahe, „dass Merkmale, die einen Transfer fördern, während der Innovation - also während des Forschungsprozesses - systematisch erzeugt werden müssen" (Hahn, Klewin, Koch, Kuhnen, Palowski \& Stiller 2019, 142).

Alternativen zu evidenzbasierten und Top-down-Strategien des Wissenstransfers stellen sowohl neuere Konzeptionen zu einer performativen Folgenforschung (vgl. Dollinger 2018), die ein erweitertes Verständnis von Evidenz mit dem Konzept der performativen Kausalität an die Prozessierung sozialer Interventionen in der Praxis bindet, als auch die Ansätze der designbasierten Forschung und der Praxisforschung dar, in deren Rahmen auch lokale (Forschungs-)Partnerschaften zwischen Forschung und Praxis eingegangen werden (vgl. Asbrand \& Bietz 2019; Asbrand, Demmer, Heinrich \& Martens 2019; Heinrich \& Klewin 2019; Hetfleisch et al. 2017; Mintrop 2019). Diese Konzepte greifen Ansätze der Handlungsforschung auf und haben damit eine hohe Affinität zur Integrationsforschung, die ja gerade in ihren Anfängen in ihrem Selbstverständnis als wissenschaftliche Begleitung stets auf Praxisveränderung gezielt hatte (vgl. Feuser \& Meyer 1987; Reiser, Gutberlet, Klein, Kreie \& Kron 1984).

Gleichwohl bleiben auch hier Fragen offen: Grundlegend wäre zu klären, inwiefern ein Forschungstyp, der seinen Ausgangspunkt bzw. seine Fragestellungen aus den konkreten Problemlagen der kooperierenden Einzelschule bezieht, sich eigentlich von klassischer Organisationsentwicklung bzw. Schulentwicklungsberatung unterscheidet und warum Wissenschaftler*innen die Schulen hierbei besser unterstützen können sollen als (professionelle) Organisationsentwickler*innen? Wenig bedacht ist zudem, was bei diesem Forschungstyp eigentlich von den Partnerinstitutionen, den Schulen und Lehrkräften, verlangt wird. Asbrand et al. (2019) machen hier unter anderem auf die hohe Gefahr der Kränkung der konkret beteiligten Personen aus der Praxis aufmerksam, denn sie sind es - entgegen 
aller Beschwörung des Dialogs auf Augenhöhe -, die ihre Praxis öffentlich und sich selbst damit verletzlich machen.

Zudem muss diskutiert werden, welcher Typus von Erkenntnis sich mit diesem Forschungsformat gewinnen lässt. Denn wenn die gemeinsam von Forschung und Praxis entwickelten Lösungen passgenau zur Einzelschule sein sollen, dann stellt sich unmittelbar die Frage nach deren Generalisier- und Transferierbarkeit. Mit anderen Worten: Das Transferproblem stellt sich jetzt nicht mehr von Wissenschaft zu Praxis, sondern von Schule A zu Schule B. Ein Lösungsansatz hierfür liegt unseres Erachtens in der Etablierung intermediärer Institutionen, deren Aufgabe es bleibt, in diesem Sinne eine Schnittstelle zwischen Forschung und Praxis zu bilden. Sie sind in den Ansätzen des Brokerage konzeptionalisiert (vgl. Gould \& Fernandez 1989; Watling Neal, Neal, Kornbluh, Mills \& Lawlor 2015) und beispielsweise in den Landesinstituten und Qualitätseinrichtungen der Länder in Deutschland bereits strukturell angelegt (vgl. Bieber, Egyptien, Klein, Oechslein \& Pikowsky 2018). Wie erfolgreich solche intermediären Institutionen Brücken zwischen den Welten der Praxis und der Wissenschaft zu bauen vermögen, ist allerdings eine noch offene Frage.

\section{Anmerkung}

Diese Publikation ist im Rahmen des vom Bundesministerium für Bildung und Forschung geförderten Projekts ,Metavorhaben Qualifizierung der pädagogischen Fachkräfte für inklusive Bildung' (Förderkennzeichen: 01NV1701) entstanden.

\section{Literatur}

van Ackeren, I., Zlatkin-Troitschanskaia, O., Binnewies, C., Clausen, M., Dormann, C., Preisendörfer, P., Rosenbusch, C. \& Schmidt, U. (2011): Evidenzbasierte Schulentwicklung. Ein Forschungsüberblick aus interdisziplinärer Perspektive. In: Die Deutsche Schule, 103, 170-184.

Altrichter, H. (2019): Transfer ist Arbeit und Lernen. In: Schreiner, C., Wiesner, C., Breit, S., Dobbelstein, P., Heinrich, M. \& Steffens, U. (Hrsg.): Praxistransfer Schul- und Unterrichtsentwicklung. Münster, 27-34.

Asbrand, B. \& Bietz, C. (2019): Wissenschaftliche Begleitung und Versuchsschule: Was man aus der Evaluation schulischer Projekte über Schulentwicklung lernen kann. In: Die Deutsche Schule, 111, 78-90.

Asbrand, B., Demmer, C., Heinrich, M. \& Martens, M. (2019): Praxisforschung revisited - zum Potenzial rekonstruktiver Zugänge. In: WE_OS Jahrbuch, Bd. 2: Praxisforschung und Transfer, 2, 42-54.

Beck, U. \& Bonß, W. (Hrsg.) (1989): Weder Sozialtechnologie noch Aufklärung? Analysen zur Verwendung sozialwissenschaftlichen Wissens. Frankfurt am Main.

Bieber, G., Egyptien, E., Klein, G., Oechslein, K. \& Pikowsky, B. (2018): Positionspapier der Landesinstitute und Qualitätseinrichtungen der Länder zum Transfer von Forschungswissen. Online unter: https://bildung-rp.de/fileadmin/user_upload/bildung-rp.de/pl/Newsletter/2018/8/12/Positionspapier_Transfer_31.10.18.pdf (letzter Zugriff: 19.02.2021).

BMBF (Bundesministerium für Bildung und Forschung) (2018): Rahmenprogramm empirische Bildungsforschung. Bonn. 


\section{Felix Buchhaupt, Dieter Katzenbach, Deborah Lutz und Michael Urban}

Bromme, R., Prenzel, M. \& Jäger, M. (2016): Empirische Bildungsforschung und evidenzbasierte Bildungspolitik. Zum Zusammenhang von Wissenschaftskommunikation und Evidenzbasierung in der Bildungsforschung. In: Baumert, J. \& Tillmann, K.-J. (Hrsg.): Empirische Bildungsforschung: Der kritische Blick und die Antwort auf die Kritiker. Wiesbaden, 129-146.

Destatis (2018): Bildung und Kultur. Finanzen der Hochschulen. Fachserie 11, Reihe 4.5. Online unter: https://www.destatis.de/DE/Themen/Gesellschaft-Umwelt/Bildung-Forschung-Kultur/ Bildungsfinanzen-Ausbildungsfoerderung/Publikationen/Downloads-Bildungsfinanzen/finanzenhochschulen-2110450187004.pdf?_blob=publicationFile (letzter Zugriff: 19.02.2021).

DFG (Deutsche Forschungsgemeinschaft) (2018): Förderatlas 2018. Kennzahlen zur öffentlich finanzierten Forschung in Deutschland. Bonn.

Dilger, A. (2018): Vor- und Nachteile verschiedener Arten von Drittmitteln. In: Hochschulmanagement, 13, 2-5.

Dohmen, D. \& Wrobel, L. (2018): Entwicklung der Finanzierung von Hochschulen und Außeruniversitären Forschungseinrichtungen seit 1995. Berlin.

Dollinger, B. (2018): Paradigmen sozial- und erziehungswissenschaftlicher Wirkungsforschung: Eine Analyse kausaltheoretischer Annahmen und ihrer Folgen für die Soziale Arbeit. In: Soziale Passagen, 10, 245-262.

Dzwonnek, D. (2014): Gefahr oder Garant? Drittmittelforschung und Forschungsfreiheit - Anmerkungen zu einem unvermuteten Zusammenhang. In: Forschung \& Lehre, 14, 92-94.

Farley-Ripple, E., May, H., Karpyn, A., Tilley, K. \& McDonough, K. (2018): Rethinking Connections between Research and Practice in Education: A Conceptual Framework. In: Educational Researcher, 47, 235-245.

Feuser, G. \& Meyer, H. (1987): Integrativer Unterricht in der Grundschule. Ein Zwischenbericht. Solms-Oberbiel.

Gould, R. V. \& Fernandez, R. M. (1989): Structures of Mediation: A Formal Approach to Brokerage in Transaction Networks. In: Sociological Methodology, 19, 89-126.

Gräsel, C. (2010): Stichwort: Transfer und Transferforschung im Bildungsbereich. In: Zeitschrift für Erziehungswissenschaft, 13, 7-20.

Gräsel, C. (2015): Was ist Empirische Bildungsforschung? In: Reinders, H., Ditton, H., Gräsel, C. \& Gniewosz, B. (Hrsg.): Empirische Bildungsforschung. Strukturen und Methoden. 2. Aufl. Wiesbaden, 15-30.

Hahn, S., Klewin, G., Koch, B., Kuhnen, S. U., Palowski, M. \& Stiller, C. (2019): Über Praxisforschung zum Transfer von Innovationen? In: Schreiner, C., Wiesner, C., Breit, S., Dobbelstein, P., Heinrich, M. \& Steffens, U. (Hrsg.): Praxistransfer Schul- und Unterrichtsentwicklung. Münster, 141-152.

Heinrich, M. \& Klewin, G. (2019): Evidenzbasierte Steuerung ohne „Evidenztransfer“? Zum Problem der mangelnden Professionssensibilität des Programms der Evidenzbasierung sowie den Chancen und Grenzen von Praxisforschung als Alternative oder Ergänzung. In: Schreiner, C., Wiesner, C., Breit, S., Dobbelstein, P., Heinrich, M. \& Steffens, U. (Hrsg.): Praxistransfer Schul- und Unterrichtsentwicklung. Münster, 61-78.

Hetfleisch, P., Goeze, A. \& Schrader, J. (2017): Wie PraktikerInnen wissenschaftliche Befunde verwenden. Selektions- und Rezeptionsprozesse bei der Implementation eines evidenzbasierten Trainingskonzepts. In: Zeitschrift für Pädagogik, 63, 182-205.

Mintrop, R. (2019): Designbasierte Schulentwicklung - ein kurzer Abriss. In: Schreiner, C., Wiesner, C., Breit, S., Dobbelstein, P., Heinrich, M. \& Steffens, U. (Hrsg.): Praxistransfer Schul- und Unterrichtsentwicklung. Münster, 35-48.

Reiser, H., Gutberlet, M., Klein, G., Kreie, G. \& Kron, M. (1984): Sonderschullehrer in Grundschulen. Ergebnisse eines Schulversuchs zur integrativen Betreuung bei Lern- und Verhaltensstörungen. Weinheim. 


\section{Zur Kontextualisierung der Inklusionsforschung}

Richter, C. (2016): Finanzierungsflexibilität für Hochschulen: Fluch oder Segen? In: Hochschulmanagement, 11, 28-31.

Sandkühler, H. J. (2011): Kritik der Evidenz. In: Bellmann, J. \& Müller, T. (Hrsg.): Wissen, was wirkt. Kritik evidenzbasierter Pädagogik. Wiesbaden, 33-55.

Steffens, U., Heinrich, M. \& Dobbelstein, P. (2019): Praxistransfer Schul- und Unterrichtsforschung - eine Problemskizze. In: Schreiner, C., Wiesner, C., Breit, S., Dobbelstein, P., Heinrich, M. \& Steffens, U. (Hrsg.): Praxistransfer Schul- und Unterrichtsentwicklung. Münster, 11-26.

Tenorth, H.-E. (2015): Bildungsforschung und Bildungspolitik im Dialog - Lernprozesse und Irritationen. In: Die Deutsche Schule, 107, 264-284.

Torka, M. (2006): Die Projektförmigkeit der Forschung. In: die hochschule, 15, 63-83.

Watling Neal, J., Neal, Z. P., Kornbluh, M., Mills, K. J. \& Lawlor, J. (2015): Brokering the ResearchPractice Gap: A typology. In: American Journal of Community Psychology, 56, 422-435.

Webler, W.-D. (2016): Ist das der Strukturwandel, den die deutschen Hochschulen brauchen? In: Hochschulmanagement, 11, 47-60.

Zillmann, N. \& Kolb, M. (2013): Drittmittel: Vom Mittel zum Zweck zum (zweifelhaften) Zweck der Mittel. In: Ze-phir, 20/21, 3-7. 
Lisa-Katharina Möhlen, Helena Deiß, Seyda Subasi Singh und Michelle Proyer

\section{Lebenswelt(en der) Schule. Internationale Perspektiven zur Inklusion von Schüler*innen mit Fluchterfahrungen. Eine Perspektive aus der Praxis}

\section{Einleitung}

Mit Mandelas Imperativ ,Education is the most powerful weapon to change the world' zeigt sich die Bedeutung, welche Bildungserfahrungen sowohl für subjektive Handlungswirksamkeit als auch für gesellschaftliche Veränderungen beigemessen wird. Der folgende Beitrag fokussiert die Bildungssituation von Schüler*innen mit Fluchterfahrungen.

Der aktuelle Forschungsstand zeigt zwar eine zunehmende Sensibilisierung in Bezug auf die Bildungsbedürfnisse von Schüler*innen mit Fluchterfahrungen (vgl. Wiseman, Damaschke-Deitrick, Galegher \& Park 2019), jedoch beziehen sich die bisher vorhandenen Forschungsergebnisse primär auf spezifische Altersgruppen und/oder institutionelle Rahmenbedingungen.

Eine Ausnahme bildet die wegweisende Forschungsarbeit zur schulischen Inklusion von Schüler*innen mit Fluchterfahrung von Guo-Brennan und Guo-Brennan (2019). Die Forscher*innen haben Leitlinien zur Inklusion auf verschiedenen Ebenen konzipiert: Der bildungspolitischen und administrativen Ebene, zu der sich z.B. Schulbehörden zuordnen lassen, wird dabei die Organisation und Bereitstellung von ausreichenden Ressourcen und inklusiv-pädagogischen Rahmenbedingungen zugeschrieben (vgl. ebd., 81). Auf der operativen Ebene gestalten Lehrer*innen vor dem Hintergrund globaler Perspektiven einen inklusiven, bedürfnisorientierten Unterricht durch modifizierbare Lehr- und Lernmöglichkeiten. Hierbei wird die aktive Partizipation der Schüler*innen selbst proklamiert (vgl. ebd.). In der Professionalisierung von Lehrpersonen wird interkulturelle Professionalisierung sowie eine Sensibilisierung hinsichtlich individueller Bedürfnisse von Schüler*innen mit Fluchterfahrungen gefordert (vgl. Nordgren 2017, 83).

Exklusionserfahrungen von Schüler*innen mit Fluchterfahrung aus dem Regelschulsystem sind bisher wenig erforscht. Ausnahmen bilden die Forschungsarbeiten von Atanasoska und Proyer (2018) zur Exklusion von Schüler*innen, die bereits das Pflichtschulalter überschritten haben, und von Alpagu, Dausien, Draxl und Thoma (2019) zu geflüchteten Schüler*innen in Übergangsklassen. 
Auch mangelt es an internationalen Forschungsperspektiven zu diesem Thema (vgl. Dovigo 2018).

Vor diesem Hintergrund widmet sich das Erasmus+ Projekt ITIRE ,Improving Teacher Education to Improve Refugee Education' (ITIRE) ${ }^{1}$ einer länderübergreifenden Unterrichtskonzeption für den Unterricht von Schüler*innen mit Fluchterfahrungen. Der vorliegende Beitrag vertieft das Forschungsinteresse des Projektes und orientiert sich an der Frage: Wie lässt sich schulische Praxis unter besonderer Berücksichtigung von Schüler*innen mit Fluchterfahrungen gestalten?

\section{Method(olog)ische Zugänge}

Der Beitrag ist der qualitativen Migrationsforschung (vgl. King 2018) zuzuordnen. Um soziale Phänomene in der Lebenswelt Schule zu identifizieren (vgl. Meuser \& Nagel 1991), wird das Forschungsfeld durch Expert*inneninterviews erschlossen. Expert*innen werden als Personen bezeichnet, „die selbst Teil des Handlungsfeldes sind, das den Forschungsgegenstand ausmacht" (ebd., 445), sie stehen stellvertretend für die Zusammenhänge des zu untersuchenden institutionellen Kontextes (vgl. ebd., 444). Expert*innen verfügen dabei über Wissen zu Entscheidungsprozessen, inhärente Verantwortungsübernahme und Problemlösungsstrategien (vgl. ebd., 445). Die Erschließung dieses Kontextwissens ermöglicht den Zugang zu Aufgaben, Eigenschaften und Strukturen des Feldes (vgl. ebd., 449).

Der Datensatz besteht aus Audiomaterial eines Expert*inneninterviews in Gruppenformat, welches im April 2020 im Rahmen eines Projektevents abgehalten wurde. Hierzu diskutierten insgesamt zehn Expert*innen aus drei (Dänemark, Großbritannien und Österreich) der fünf Projektländer über ihre Erfahrungen im Umgang von Schüler*innen mit Fluchterfahrungen in unterschiedlichen Schultypen, -stufen und -fächern.

Das Datenmaterial wurde mittels einer konstruktivistischen Variante der Grounded Theory Methodologie in Anschluss an Charmaz (2014) ausgewertet. Nach Charmaz (ebd.) können diskursive Strukturen in der zu erforschenden Lebenswelt nur durch die Berücksichtigung von Entstehungskontexten und -bedingungen identifiziert werden. Dabei werden Annahmen von subjektiv entstehenden Wissenshorizonten betrachtet, in denen Wahrheit(en) plural, zeitlich und örtlich begrenzt ist/sind.

1 Erasmus+ ITIRE Projektpartner: Aarhus Universitet (Dänemark), Háskólinn á Akureyri (Island), UiT Norges Artic Universitet (Norwegen), Universität Wien (Österreich) und University of Winchester (Großbritannien). 


\section{Empirische Erkenntnisse: Erfahrungen in/aus der Lebenswelt Schule}

\subsection{Gelingensbedingungen für pädagogische Praxis}

Aus dem Datenmaterial geht hervor, dass Partizipation eine grundlegende Bedingung für inklusive pädagogische Praxis darstellt. Hierbei deckt sich das Datenmaterial mit den Ergebnissen von Guo-Brennan und Guo-Brennan (2019). Partizipation wird „als Mittel zum Einbringen eigener Interessen und als Beteiligung an der Fortentwicklung der Gesellschaft" (Krämer 2013, 11) verstanden. Dabei gilt es zum einen zu thematisieren, inwieweit sich das betroffene Kind in der Klasse als partizipierend erfährt und zum anderen, wie es von Mitschüler*innen und pädagogischem Personal erlebt wird. Hierzu merken die Expert*innen der Diskussion kritisch an, dass Partizipation kaum messbar sei, was die Grenzen der quantitativer Bildungsforschung verdeutlicht. Daran anschließend stellt sich die Frage nach der Förderung von Partizipation, bspw. durch die Lehrkräfte. Im Rahmen der Ermöglichung von Partizipation werden Lehrende mit unterschiedlichen Herausforderungen konfrontiert (aktive Einbindung der Schüler*innen, Umgang mit Heterogenität, Aufbereitung des Unterrichts und Reflexion der Auswirkungen pädagogischer Praxis auf das Empowerment des Kindes). Diesbezüglich wird z.B. die Berücksichtigung und Integration von (Erst-)Sprachkompetenzen von mehrsprachigen Schüler*innen als wegweisende Grundlage diskutiert, um benachteiligenden Faktoren des monolingualen Schulsystems entgegenzuwirken (vgl. Gogolin 2010; Nordgren 2017; Proyer, Atanasoska \& Sriwanyong 2017, 272ff.). Essenziell sei generell auch die Förderung einer interkulturell sensibilisierten Haltung von Lehrer*innen (vgl. Nordgren 2017, 83), um die Partizipation und das aktive Empowerment der Schüler*innen zu stärken (vgl. von Unger 2017).

Eine weitere zentrale Bedingung verorten die Expert*innen in interdisziplinäre Expertisen in der Lebenswelt Schule. Dazu konnte aus einem isländischen GoodPractice-Beispiel die Notwendigkeit der Kooperation von Lehrer*innen und Unterrichtsberater*innen entnommen werden. Unterrichtsberater*innen sind externe Personen, die in regelmäßigen Abständen Schulen in Island besuchen und beratend fungieren. Diesen Erkenntnissen folgend lässt sich Kompetenzaufteilung als zentraler Faktor für eine gelingende Praxis benennen. Ein solches Unterstützungsangebot ist auch in Kanada mit einer sogenannten ,teaching consultation'verfügbar (vgl. Köpfer \& Óskarsdóttir 2019). Darüber hinaus gibt es zudem „case-based consulting teams in schools (principal, methods \& resource team, teachers, parents, guidance team)" (ebd., 884). Die Begründung für solche Kooperationen ist, dass Lehrpersonen nicht alle Expertisen (z.B. transkulturelle Kompetenzen, traumapädagogisches Wissen etc.) aufweisen können, um den heterogenen Bedürfnissen von Schüler*innen adäquat zu begegnen (vgl. Bačáková 2009, 167; Otto, Migas, Austermann \& Bos 2016, 28; Proyer, Biewer, Kreuter \& Weiß 2019, 10). 
Dies ist besonders relevant, da Schüler*innen nicht nur sprachlichen und kulturellen Barrieren, sondern auch einer hohen Diskrepanz zwischen psychischer Verarbeitung und (schulischem) Leistungsdruck ausgesetzt sind (vgl. Alpagu et al. 2019, 5; Biewer, Proyer \& Kremsner 2019, 10). Letzteres drückt sich darin aus, „sozialen Erwartungen“ zu entsprechen, der „alltäglichen Lebensrealität“ gerecht zu werden sowie "Handlungsmöglichkeiten“ innerhalb der Schule zu nutzen (Alpagu et al. 2019, 5; Hervorhebungen im Original).

Ein weiteres Good-Practice-Beispiel aus Großbritannien stellt das Konzept sogenannter ,schools of sanctuary' dar (vgl. City of Sanctuary 2020). Dabei geht die Aufgabe einer Lehrperson „über das Lehren hinaus“ zu einer „Vermittlung eines Ethos, welcher Sicherheit und Inklusion bedeutet" (Interview 1, S.7, Z.15-17). Das Ziel ist es, außerschulische Erfahrungen bewusst in die Schulwelt miteinzubeziehen.

Dem folgend wird der Einbezug der Eltern bzw. Obsorgepersonen genannt. Ein Good-Practice-Beispiel aus Island zeigt Kooperationsmöglichkeiten mit Eltern respektive Obsorgepersonen auf, indem diese bei der Entwicklung von Lernmaterial miteinbezogen werden, um einen niederschwelligen Zugang zur isländischen Sprache zu schaffen sowie eine Übertragung von schulischem Lernen ins Private zu ermöglichen. Diese Kooperation unterstützt Lern- und Bildungsprozesse für Kinder sowie eine Förderung der Beziehungen zwischen Eltern/Obsorgepersonen und dem Lehrpersonal (vgl. Gunnpórsdóttir, Barillé \& Meckl 2018; Guo-Brennan \& Guo-Brennan 2019, 84).

Eine dritte Bedingung bezieht sich auf politische Rahmenbedingungen. Die Lehrer*innen bestätigen in der Gruppendiskussion, dass individuelles Engagement allein nicht ausreicht, sondern eine strukturelle Unterstützung nötig ist. Zudem konnte ein Wunsch nach (mehr) finanzieller Förderung identifiziert werden. Lehrkräfte fühlen sich oftmals in ihrem Engagement für geflüchtete Kinder ohne institutionelle, finanzielle und politische Unterstützung alleingelassen (vgl. Alpagu et al. 2019; Bačáková \& Closs 2013; Gunnpórsdóttir et al. 2018; Guo-Brennan \& Guo-Brennan 2019, 77). Eine strukturelle Unterstützung wäre die systematische Forderung und Förderung interkultureller Kompetenzen für pädagogisches Personal. Fakt ist, dass in Österreich Interkulturelle Pädagogik zwar seit den 1990erJahren curricular angeordnet, dennoch nach wie vor nicht gesetzlich verankert ist (vgl. Luciak \& Khan-Svik 2008, 493).

Eine weitere Erkenntnis in Bezug auf die politischen Rahmenbedingungen ist die mangelnde internationale Vergleichbarkeit von Bildungsplänen, die bereits seit Anfang der 2000er-Jahre gefordert wird (vgl. Artiles \& Dyson 2005; Dovigo 2018; Luciak \& Khan-Svik 2008).

Gegenwärtig sind keine internationalen Strategien zur Inklusion von Schüler*innen mit Fluchterfahrungen bekannt (vgl. Grin \& Schwob 2002; Luciak \& KhanSvik 2008), die aber erforderlich wären (vgl. Artiles \& Dyson 2005; Guo-Brennan \& Guo-Brennan 2019). 
Zusammenfassend sollte den Expert*innen zufolge die Idee einer ,Bildung für alle - unabhängig von Herkunft, Fähigkeiten etc. - vertreten werden. Diese Idee kann mit der Deklaration des Sustainable Development Goals 4 ,Education for All' verglichen werden, mit welchem die UNESCO bis 2030 die Grundbildung von jedem Kind kostenfrei gewährleisten will (vgl. UNESCO 2015). Biewer et al. $(2019,23)$ zufolge prägen Thematiken rund um Flucht und Migration die Lebenswelt vieler Schüler*innen in Österreich, weshalb diese als inhärente Herausforderungen von Inklusiver Pädagogik zu betrachten sind.

\subsection{Kriterien für inklusiven Unterricht mit dem Fokus auf Schüler*innen mit Fluchterfahrungen}

Die Expert*innen halten fest, dass es an wissenschaftlichen Konzeptionen zur didaktischen Implementierung von Refugee Education im Unterricht mangelt. In den Expert*innenaustausch flossen neun Kriterien der WHO (2008) für GoodPractice-Beispiele ein, die jedoch ursprünglich für den fachlichen Hintergrund im Pflegebereich entwickelt wurden (siehe Tab. 1).

Tab. 1: Darstellung der WHO Kriterien - umgesetzt für ein inklusives Unterrichtssetting

\begin{tabular}{|l|l|l|}
\hline $\begin{array}{l}\text { Kriterium der } \\
\text { WHO }\end{array}$ & $\begin{array}{l}\text { Beschreibung des } \\
\text { Kriteriums }\end{array}$ & $\begin{array}{l}\text { Diskussionspunkte/Anmerkungen der } \\
\text { interviewten Expert*innen }\end{array}$ \\
\hline Effektivität & $\begin{array}{l}\text { Messbarkeit des definierten } \\
\text { Ziels }\end{array}$ & $\begin{array}{l}\text { Fehlende Vereinheitlichung von Ziel- } \\
\text { dimensionen } \rightarrow \text { Schwierigkeit, um inter- } \\
\text { nationale Gültigkeit zu erwirken }\end{array}$ \\
\hline Effizienz & $\begin{array}{l}\text { Ausgewogenes Verhältnis zwi- } \\
\text { schen Input, Output und den } \\
\text { investierten Ressourcen }\end{array}$ & $\begin{array}{l}\text { Fokus auf die Qualität der Lehre } \rightarrow \\
\text { allerdings nur schwer messbar }\end{array}$ \\
\hline Relevanz & $\begin{array}{l}\text { Inhaltliche und methodische } \\
\text { Bedeutung für Praxis und } \\
\text { Zielgruppe }\end{array}$ & $\begin{array}{l}\text { Wirkung von Inklusion über schulische } \\
\text { Praktiken hinaus } \rightarrow \text { gesamtgesellschaft- } \\
\text { liche Verantwortung relevant }\end{array}$ \\
\hline $\begin{array}{l}\text { Ethische } \\
\text { Aspekte }\end{array}$ & $\begin{array}{l}\text { Berücksichtigung der und } \\
\text { Reflexion im Umgang mit } \\
\text { Zielgruppe }\end{array}$ & $\begin{array}{l}\text { kritisch, da ethische Bildungsimperative } \\
\text { im Verhältnis zu vorherrschenden segre- } \\
\text { gierenden Strukturen und neoliberalen } \\
\text { bildungspolitischen Agenden stehen } \\
\text { können }\end{array}$ \\
\hline $\begin{array}{l}\text { Nachhaltigkeit } \\
\text { Möglichkeit } \\
\text { Rer }\end{array}$ & $\begin{array}{l}\text { Einsatz muss vor dem Hinter- } \\
\text { grund verfügbarer Ressourcen } \\
\text { nachhaltig sein }\end{array}$ & $\begin{array}{l}\text { abhängig von administrativer und poli- } \\
\text { tischer Ebene, je nachdem, in welchem } \\
\text { international gestaltbar sein }\end{array}$ \\
\hline $\begin{array}{l}\text { Maße Ressourcen vorhanden sind } \\
\text { Differenz zwischen urbanen und länd- } \\
\text { lichen Gebieten; sehr heterogene Schul- } \\
\text { landschaft auf nationaler Ebene }\end{array}$ \\
\hline
\end{tabular}




\begin{tabular}{|l|l|l|}
\hline $\begin{array}{l}\text { Kriterium der } \\
\text { WHO }\end{array}$ & $\begin{array}{l}\text { Beschreibung des } \\
\text { Kriteriums }\end{array}$ & $\begin{array}{l}\text { Diskussionspunkte/Anmerkungen der } \\
\text { interviewten Expert*innen }\end{array}$ \\
\hline $\begin{array}{l}\text { Involvierung } \\
\text { von Part- } \\
\text { ner*innen- } \\
\text { schaften }\end{array}$ & $\begin{array}{l}\text { Einbezug von Stakeholdern } \\
\text { aus unterschiedlichen Ge- } \\
\text { bieten und Ebenen }\end{array}$ & $\begin{array}{l}\text { Bildungspartner*innenschaften auf } \\
\text { administrativer Ebene, außerschulische } \\
\text { Bildungsmöglichkeiten }\end{array}$ \\
\hline $\begin{array}{l}\text { Einbindung } \\
\text { der Zielgruppe }\end{array}$ & $\begin{array}{l}\text { Einbezug betroffener Perso- } \\
\text { nen }\end{array}$ & Lehrkräfte mit Migrationsgeschichte \\
\hline $\begin{array}{l}\text { Politisches } \\
\text { Commitment }\end{array}$ & $\begin{array}{l}\text { Bekenntnis politischer Ak- } \\
\text { teur*innen zu Good-Practice }\end{array}$ & $\begin{array}{l}\text { Grundvoraussetzung zur Etablierung von } \\
\text { Refugee Education }\end{array}$ \\
\hline
\end{tabular}

Die Tabelle bietet einen Überblick zu den WHO-Kriterien für Good-PracticeBeispiele in Bezug auf die Anwendung im Rahmen schulischer Bildung für Schüler*innen mit Fluchterfahrungen. Es stellte sich heraus, dass nicht allen Teilnehmer*innen die Kriterien der WHO bekannt waren. Sie bemängeln das Fehlen international bekannter Fachdidaktiken, welche den Expert*innen als Hintergrundfolie dienen, um Praktiken zu erarbeiten. Zudem wurde die fehlende Einbettung verschiedener Perspektiven von den beteiligten Akteur*innen als mangelhaft bewertet. Abschließend thematisierten die Expert*innen der Diskussion mit der internationalen Etablierung von Konzepten die Möglichkeit der internationalen Vergleichbarkeit. Sie berücksichtigen reflexiv, dass nationale Schullandschaften sehr heterogen strukturiert sind, sodass die Vergleichbarkeit internationaler Systeme erschwert wird.

\section{Fazit}

Mittels einer Expert*innendiskussion wurden aktuelle internationale Herausforderungen und Problemstellungen zur Entwicklung von Unterricht unter besonderer Berücksichtigung von Schüler*innen mit Fluchterfahrungen besprochen. Die Datenauswertung und -analyse erfolgte durch eine konstruktivistisch ausgelegte Variante der Grounded Theory Methodologie nach Charmaz (2014).

Festgehalten wurde, dass schulische und außerschulische Lern- und Bildungsprozesse interdependent betrachtet werden müssen, um durch interdisziplinäre und ebenenübergreifende Kooperationen pädagogische Praktiken wirksam durchzuführen. Als zweite Erkenntnis sind die Grundbedingungen zu nennen, die für eine gelingende pädagogische Praxis nötig sind. Schließlich konnte eine Forschungslücke zu fehlenden wissenschaftlichen Erkenntnissen in Bezug auf international gültige methodische und didaktische Konzepte zur Inklusion identifiziert werden. 
Abschließend kann Bildung - erneut bezugnehmend auf Mandelas einleitend angeführten Imperativ - als empowerndes und gesellschaftlich veränderndes Mittel betrachtet werden, um sozialer Benachteiligung infolge von Migration oder Flucht entgegenzuwirken. Dies schließt an die Forderung und Förderung eines Rechts auf ,Bildung für alle' an. Um dieses Recht umsetzen zu können, benötigt es internationale Konzepte, um die Inklusion von Schüler*innen mit Fluchterfahrungen zu fördern.

\section{Literatur}

Alpagu, F., Dausien, B., Draxl, A.-K. \& Thoma, N. (2019): Exkludierende Inklusion - eine kritische Reflexion zur Bildungspraxis im Umgang mit geflüchteten Jugendlichen einer Übergangsstufe. In: schulheft, 176, 51-63.

Artiles, A. J. \& Dyson, A. (2005): Inclusive education in the globalization age. The promise of comparative cultural-historical analysis. In: Mitchell, D. R. (Hrsg.): Contextualizing inclusive education: evaluating old and new international perspectives. London/New York, 37-68.

Atanasoska, T. \& Proyer, M. (2018): On the brink of education: Experiences of refugees beyond the age of compulsory education in Austria. In: European Educational Research Journaluropean Educational Research Journal, 17, H.2, 271-289.

Bačákoá, M. (2009): Access to education of refugee children in the Czech Republic. Report to the UNHCR Prague Office. Prague.

Bačáková, M. \& Closs, A. (2013): Continuing professional development (CPD) as a means to reducing barriers to inclusive education: research study of the education of refugee children in the Czech Republic. In: European Journal of Special Needs Education, 28, H.2, 203-216. Online unter: https://www.tandfonline.com/doi/abs/10.1080/08856257.2013.778108 (letzter Zugriff: 01.02.2021).

Biewer, G., Proyer, M. \& Kremsner, G. (2019): Inklusive Schule und Vielfalt. Stuttgart.

Charmaz, C. (2014): Constructing Grounded Theory. Thousand Oaks, California.

City of Sanctuary (2020): Schools of Sanctuary. Online unter: https://schools.cityofsanctuary.org/ (letzter Zugriff: 26.03.2020).

Dovigo, F. (Hrsg.) (2018): Challenges and Opportunities in Education for Refugees in Europe: From Research to Good Practices. Leiden, The Netherlands.

Gogolin, I. (2010): Stichwort: Mehrsprachigkeit. In: Zeitschrift für Erziehungswissenschaft, 13, H.4, 529-547.

Grin, F. \& Schwob, I. (2002): Bilingual Education and Linguistic Governance: The Swiss experience. In: Intercultural Education, 13, H.4, 409-426.

Gunnpórsdóttir, H., Barillé, S. \& Meckl, M. (2018): The education of students with immigrant background in Iceland: a parents' and teachers' voices. In: Scandinavian Journal of Educational Research, 63, H.4, 605-616.

Guo-Brennan, L. \& Guo-Brennan, M. (2019): Building Welcoming and Inclusive Schools for Immigrant and Refugee Students: Policy, Framework and Promising Praxis. In: Arar, K., Brooks, J. S. $\&$ Bogotch, I. (Hrsg.): Education, Immigration and Migration. Policy, Leadership and Praxis for a Changing World. Bingley, UK, 73-93.

King, R. (2018): Context-Based Qualitative Research and Multi-sited Migration Studies in Europe. In: Zapata-Barrero, R. \& Yalaz, E. (Hrsg.): Qualitative Research in European Migration Studies. Cham, 35-56.

Köpfer, A. \& Óskarsdóttir, E. (2019): Analysing support in inclusive education systems - a comparison of inclusive school development in Iceland and Canada since the 1980s focusing on policy and 
in-school support. In: International Journal of Inclusive Education, 23, H.7-8, 876-890. Online unter: https://www.tandfonline.com/doi/full/10.1080/13603116.2019.1624844 (letzter Zugriff: 08.02.2021).

Krämer, M. (2013): Inklusion - Integration - Partizipation. Drei Seiten einer Medaille. In: Berufsverband Deutscher Psychologinnen und Psychologen e.V. (Hrsg.): Inklusion, Integration, Partizipation. Psychologische Beiträge für eine humane Gesellschaft. Berlin, 11-16.

Luciak, M. \& Khan-Svik, G. (2008): Intercultural education and intercultural learning in Austria critical reflections on theory and practice. In: Intercultural Education, 19, H.6, 493-504.

Meuser, M. \& Nagel, U. (1991): ExpertInneninterviews - vielfach erprobt, wenig bedacht: ein Beitrag zur qualitativen Methodendiskussion. In: Garz, D. \& Kraimer, K. (Hrsg.): Qualitativ-empirische Sozialforschung: Konzepte, Methoden, Analysen. Opladen, 441-471.

Nordgren, R. D. (2017): Cultural Competence and Relational Closeness: Examining Refugee Education. In: Journal of Research in Innovative Teaching \& Learning, 10, H.1, 79-92.

Otto, J., Migas, K., Austermann, N. \& Bos, W. (2016): Integration neu zugewanderter Kinder und Jugendlicher ohne Deutschkenntnisse. Möglichkeiten, Herausforderungen und Perspektiven. Münster/New York.

Proyer, M., Atanasoska, T. \& Sriwanyong, S. (2017): Forces in Non-Linear Transitions: On the Impact of Escape on Educational Pathways in Young Refugees' Lives. In: Fasching, H., Geppert, C. \& Makarova, E. (Hrsg.): Inklusive Übergänge: (inter)nationale Perspektiven auf Inklusion im Übergang von der Schule in weitere Bildung, Ausbildung oder Beschäftigung. Bad Heilbrunn, 211-228.

Proyer, M., Biewer, G., Kreuter, L. \& Weiß, J. (2019): Instating settings of emergency education in Vienna: temporary schooling of pupils with forced migration backgrounds. In: International Journal of Inclusive Education, 25, H.2, 1-16. Online unter: https://www.tandfonline.com/doi/full/10 .1080/13603116.2019.1707299 (letzter Zugriff: 08.02.2021).

UNESCO (2015): Incheon Declaration and Framework for Action for the Implementation of Sustainable Development Goal 4. Paris.

von Unger, H. (2017): Junge Geflüchtete, Bildung und Arbeitsmarkt. Ein Lehrforschungsprojekt in München. München.

WHO (2008): Guide for Documenting and Sharing "Best Practices in Health Programmes". India, WHO Regional Office for Africa. Online unter: https://www.afro.who.int/sites/default/files/2017-06/ Guide_for_documenting_and_Sharing_Best_Practice_-_english_0.pdf (letzter Zugriff: 08.02.2021).

Wiseman, A., Damaschke-Deitrick, L., Galegher, E. \& Park, M. (2019): Comparative Perspectives on Refugee Youth Education. New York. 


\section{Helfen als individuelle Erfahrung und soziale Praxis zwischen Deautonomisierung und Befähigung}

\section{Hilfssubjekte}

In unserem Beitrag stellen wir konzeptionelle Überlegungen und erste Zwischenergebnisse eines neuen Forschungsschwerpunkts an der Universität Innsbruck zum Thema „Inklusion und Exklusion als bildende Erfahrung und soziale Praxis“"vor. ${ }^{1}$ Da ,Inklusion“ und ,Exklusion` als soziologische Kategorien zunächst abstrakte wissenschaftliche Begriffe sind, die sich nicht unmittelbar fassen lassen, werden die damit verbundenen Erfahrungen und Praktiken anhand konkreter Situationen des Helfens untersucht. Der Titel unseres Beitrags spielt dabei mit der Doppeldeutigkeit des Subjektbegriffs, der abgeleitet von dem lateinischen Wort subjectum sowohl mit ,das Zugrundeliegende' als auch ,das Unterworfene 'übersetzt werden kann (vgl. Althusser 2016, 98). In diesem Sinne beinhaltet der Begriff „Hilfssubjekte', wie wir ihn verstehen, sowohl die individuellen Erfahrungen und Praktiken seitens der Hilfegebenden und Hilfeempfangenden als auch das dem Helfen unterworfene Subjekt, dem ein bestimmter Hilfebedarf zugeschrieben und dem eine bestimmte gesellschaftliche Praxis aufgezwungen wird.

In diesem Zusammenhang stellen sich verschiedene Forschungsfragen, die zugleich als Desiderate im aktuellen Inklusionsdiskurs angesehen werden können: (1) Welche Phänomene werden überhaupt als Hilfe wahrgenommen? (2) Wie werden Situationen des Helfens von Helfenden und Hilfsbedürftigen subjektiv erfahren? (3) Wie werden Schüler*innen durch Praktiken des Helfens als Helfende und Hilfsbedürftige subjektiviert? (4) Welche ,Distinktionsgewinne` (vgl. Bourdieu 2018) und Verluste gehen damit einher? (5) Wie viel Unterstützung und Sorge um das Wohl und die Bedürfnisse der*des Anderen sind notwendig und wo werden Schüler*innen durch Praktiken des Helfens beim Erreichen von Unabhängigkeit und Autonomie auf ihren Bildungswegen behindert?

1 Ein besonderer Dank gilt an dieser Stelle unseren Kolleg*innen vom Institut für LehrerInnenbildung und Schulforschung: Herrn Univ.-Prof. Mag. Dr. Michael Schratz und Frau PD Dr. Johanna F. Schwarz, MA, die von Anfang an unverzichtbare Impulsgeber*innen und wichtige Diskussionsund Gesprächspartner*innen für die Konzeption und Weiterentwicklung dieses Forschungsschwerpunkts waren und sind. 
In den Praxiswelten der Inklusiven Pädagogik ist das Thema ,Helfen' allseits präsent und wurde dennoch bisher wenig systematisch untersucht. Einander zu helfen gilt als zutiefst menschliche Eigenschaft und als Grundlage für Lernen und Entwicklung (vgl. Tomasello 2014). Bestimmte gesellschaftliche Gruppen wie Kinder, Menschen mit Behinderungen, chronisch Kranke oder ältere Menschen sind zeitweise oder dauerhaft in besonderer Weise auf Hilfe und Unterstützung angewiesen (vgl. Rödler 2000). Hilfe und unterschiedliche Formen des Helfens sind dabei sowohl für die Geschichte der Sonderschule und der Behindertenpädagogik als auch für die Inklusive Pädagogik von zentraler Bedeutung, was an Begriffen wie „Hilfsschule‘, ,Behindertenhilfe', „Hilfe zur Selbsthilfe‘ oder der Forderung nach einer ,neuen Kultur des Helfens' (vgl. Zimpel 2012) deutlich wird.

Die autonome Behindertenbewegung kritisiert seit den 1980er Jahren die in der Behindertenhilfe organisierte „Wohltätermafia“ (Sierck \& Radtke 1984) und die durch die Helfenden ausgeübte "Gewalt der Menschlichkeit" (Christoph 1983). Institutionalisierte Formen von Hilfe sind oft eng verwoben mit Macht- und Herrschaftsverhältnissen, in denen nicht nur Hilfe organisiert, sondern zugleich Hilflosigkeit allererst produziert wird. Praktiken des Helfens ziehen eine Grenze zwischen Hilfe-Gebenden und Hilfe-Nehmenden. Es besteht eine Asymmetrie, die ein widersprüchliches Verhältnis von Einflussnahme einerseits und Vermeidung von Abhängigkeiten sowie stigmatisierenden Effekten andererseits hervorbringt (vgl. Krappmann \& Oswald 1995).

Helfen im Kontext von Schule und Unterricht bewegt sich somit in einem Spannungsfeld von Unterstützung als Befähigung (Empowerment) und Deautonomisierung als Zementierung bestehender Macht- und Herrschaftsverhältnisse, wobei die Übergänge oft fließend sind. Diese Spannungsfelder sollen in den beiden folgenden Abschnitten anhand erster empirischer Zwischenergebnisse aus dem Kontext von Unterricht und Schule etwas genauer betrachtet werden.

\section{Helfen unter Schüler*innen}

Untersuchungen zu asymmetrischen Hilfesituationen zwischen Schüler*innen, die sich nach Breidenstein $(2006,196)$ aufgrund von ungleich verteilten Wissensbeständen und Kompetenzen konstituieren, zeigen, dass diese eine hohe Komplexität aufweisen und nicht immer unproblematisch verlaufen. Bereits 1995 haben Krappmann und Oswald in ihrer ethnografischen Studie zum „Alltag der Schulkinder" darauf hingewiesen, dass in dem von ihnen ausgewerteten Datenmaterial ungefähr 40\% der Hilfesituationen konfliktträchtig verliefen (vgl. Krappmann \& Oswald 1995, 163). Nach Oevermann $(2009,138)$ erhöht sich zudem in unsicheren Sozialbeziehungen - bspw. dann, wenn Schüler*innen untereinander nicht befreundet sind - das Risiko erheblich, dass Hilfe scheitert bzw. problematisch 
verläuft. Darüber hinaus nutzen Schüler*innen, die Hilfe geben, die Asymmetrie zwischen ihnen und den Hilfe-Empfangenden nicht selten zum eigenen Vorteil. Solche Hilfen können dann soziale Dominanzen zum Ausdruck bringen, wodurch sich die Differenz von Stärke und Bedürftigkeit verfestigt. Schüler*innen mit sonderpädagogischem Förderbedarf (SPF) sind von solchen Hilfesituationen

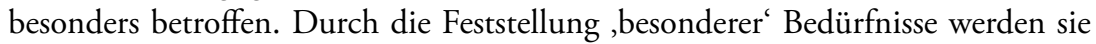
nach außen hin für andere erkennbar als hilfebedürftige Subjekte etikettiert.

Im Folgenden soll eine Hilfesituation im inklusiven Unterricht zwischen zwei Schülern einer 7. Jahrgangsstufe einer österreichischen Mittelschule analysiert und dabei das Spannungsverhältnis herausgearbeitet werden, in dem sich schulische Hilfe befinden kann. Die Situation findet in einer mathematischen Unterrichtseinheit für die Hälfte der Klasse statt. Für den Unterricht müssen die Schüler*innen jeweils an eigenen Arbeitsplätzen sitzen. Neben Frau Bauer, der Klassen- und Mathematiklehrerin, ist auch Frau Wagner, die Integrationslehrerin, anwesend:

„Es ist die letzte Stunde. Die eine Hälfte der Klasse hat bereits frei, die andere sitzt hier im Klassenzimmer. Jede*r Schüler*in an einem eigenen Tisch. Die Schüler*innen sind in ihre Zeichnungen geometrischer Formen vertieft. Mal wird das Geodreieck benutzt, mal der Zirkel. Orkan, der im Gegensatz zu seinen Mitschüler*innen bereits alle Formen auf Papier gebracht hat, wird von Frau Wagner, der Integrationslehrerin der Klasse, aufgefordert, sich neben Dejan, ein Schüler mit sonderpädagogischem Förderbedarf, zu setzen und ihm bei der Aufgabe zu helfen. Orkan kommt der Aufforderung kommentarlos nach. Auch Dejan nimmt die unerbetene Hilfesituation ohne Widerrede an. Dann zieht Frau Wagner ihre Jacke an. Sie wird den Rest der Stunde nicht mehr anwesend sein. ,Orkan, bleib dann sitzen und hilf dem Dejan.' Orkan beginnt, Dejan die einzelnen Schritte der Zeichnungen zu erklären. Doch nach kurzer Zeit wird dieser unterbrochen: ,Nicht so viel. Der Dejan soll das allein machen. Und nicht so schnell!', mahnt Frau Bauer, die sich neben den Tisch von Dejan gestellt hat" (FN 204412). ${ }^{2}$

Orkan ist mit der gestellten Aufgabe, verschiedene geometrische Formen zu zeichnen, bereits fertig. Der Abschluss der Aufgabe signalisiert den Lehrerinnen, dass Orkan vorübergehend beschäftigungslos ist und daher für eine andere Aufgabe zur Verfügung steht. Frau Wagner fordert Orkan deswegen auf, sich neben Dejan zu setzen, ein Schüler mit sonderpädagogischem Förderbedarf, mit dem Orkan ansonsten nichts - auch nicht in den Pausen oder seiner Freizeit - zu tun hat, um ihm bei den grafischen Zeichnungen zu helfen.

Durch die Anweisung der Lehrerin, die hier als Autoritätsperson die Hilfesituation zwischen den beiden Schülern festlegt, wird Dejan für die Gruppe (erneut) als hilfsbedürftiges Subjekt markiert. Dejan wird hier erst gar nicht nach seiner eigenen Einschätzung einer möglichen Hilfsbedürftigkeit gefragt, sondern diese

2 Feldnotiz 204412 (anonymisiert) aus dem Dissertationsprojekt zu Praktiken der Integration im schulischen Alltag von Hendrik Richter. 
wird über ihn hinweg bestimmt. Er ist damit nicht mehr in der Lage, den unterrichtlichen Anforderungen selbstständig nachzukommen. Seine (vermeintliche) Hilfsbedürftigkeit wird zusätzlich verstärkt, indem die Sitzordnung (jede*r Schüler*in an einem eigenen Tisch) nur für ihn zeitlich begrenzt aufgehoben wird. Dass bei Hilfestellungen die Sitzordnung während einer Einzelarbeitsphase gelockert wird, stellt für die Schüler*innen eine gewöhnliche Praktik dar und zeigt ihnen an, dass ein*e Schüler*in besondere Hilfe benötigt. Auch der Umstand, dass Orkan und Dejan die Situation unkommentiert lassen und auch ihre Mitschüler*innen keine eigenen Ansprüche stellen, neben einer bestimmten Person sitzen zu wollen, deutet auf eine eingeübte Interaktion hin, die allerdings nur möglich wird, weil die Lehrperson diese einrichtet.

Im Hinblick auf das baldige Verlassen des Klassenraums versucht Frau Wagner nun, im weiteren Verlauf die Hilfesituation zwischen Orkan und Dejan von ihrer Person loszulösen und zugleich abzusichern. Der Fortgang der Lehrerin soll eben nicht die Aufhebung der Hilfe bedeuten, sondern diese soll von den Schülern weitergeführt werden. Als Orkan mit der Erklärung der einzelnen Arbeitsschritte beginnt, wird er bereits nach kurzer Zeit von Frau Bauer unterbrochen. Die Lehrerin mahnt den Schüler: Er habe $z u$ viel und zu schnell erklärt. Außerdem soll Dejan das allein machen. Dass Orkan zwar als Hilfegeber eingesetzt wird, Dejan die Aufgabe allerdings selbstständig lösen soll, erzeugt ein Spannungsverhältnis zwischen einer Hilfe, die auf die selbstständige Lösung einer Aufgabe zielt, und einer Hilfe, die deautonomisierend wirkt, d.h. den Hilfenehmenden vom Hilfegebenden abhängig macht. Orkan vermag diesen Widerspruch nicht aufzulösen, weshalb die Hilfesituation auch für Orkan zur Überforderung wird und er den an ihn gerichteten Erwartungen nicht entspricht. Doch nicht nur für Schüler*innen, sondern auch für Lehrer*innen sind die Grenzen dieses Spannungsverhältnisses fließend. Darüber hinaus wird Dejan, indem er hier nicht nach seiner eigenen Einschätzung der Hilfe gefragt wird, sondern die Lehrerin die Hilfe als $z u$ viel und zu schnell bewertet, erneut als Hilfssubjekt der durch die Lehrerin festgelegten Hilfeform unterworfen. Die Hilfe wird damit ein weiteres Mal, ohne Dejan anzuhören, über ihn hinweg bestimmt. Durch die Zuschreibung als grundsätzlich hilfsbedürftige Subjekte laufen Hilfesituationen bei Schüler*innen mit SPF daher besonders Gefahr, dass das Spannungsverhältnis zur Seite der Deautonomisierung hin kippt und Hilfen damit keinen Zustand der Selbstbestimmung, sondern vielmehr Abhängigkeiten und Heteronomien erzeugen. 


\section{Helfen als Aufgabe der Schulassistenz}

Lenkt man den Blick von asymmetrischen Hilfesituationen zwischen Schüler*innen auf institutionalisierte Hilfekontexte im Zusammenhang mit Inklusiver Bildung, kommt Schulassistent*innen eine bedeutende Rolle zu. Durch das Zur-Verfügung-Stellen von Assistenz vonseiten des Staates betreffend sowohl lebenspraktische Dinge (Körperpflege oder Einhaltung der Tages- und Zeitstruktur) als auch schulische Belange (von der Aufrechterhaltung von Motivation und Lernfreude bis hin zur Unterstützung der sozialen Teilhabe oder Konfliktvermeidung) soll mittels Schulassistenz „die Bildung von Schülerinnen mit Behinderungen verbessert werden und insbesondere gemeinsames Lernen mit Schülerinnen ohne Behinderungen ermöglicht werden" (Tiroler Landesregierung 2018, $\$ 2$ ). Loos $(2014,7)$ fasst das Ziel der Schulassistenz als „Hinführung der Schüler zur größtmöglichen Selbstständigkeit und deren sozialer Integration in die Gemeinschaft der Klasse und in das Schulleben" zusammen. Die Verwendung des Begriffs ,Assistenz' verweist dabei auf die Abgrenzung selbstbestimmter von fremdbestimmter Hilfe, da die Begriffe ,Behindertenhilfe', ,Pflege' oder ,Betreuung' oftmals mit Fremdbestimmung und Bevormundung assoziiert werden (vgl. auch Feyerer, Prammer \& Prammer-Semmler 2017).

Ähnlich wie bei der oben analysierten Hilfesituation zwischen den beiden Schülern sind auch Assistenzleistungen in der Schule durch Ambivalenzen und Spannungsfelder geprägt. In alltäglichen Hilfesituationen werden inkludierende und exkludierende Praktiken sichtbar, die trotz eines grundsätzlichen Bemühens vonseiten der Schulassistenz verdeutlichen, dass Assistenzleistungen eine hohe Komplexität aufweisen, ihre Wirkungsweisen nicht immer mit der intendierten Wirkung übereinstimmen müssen und dabei auch problematisch verlaufen können. Dieses zentrale Spannungsfeld der beschriebenen Hilfesituationen ist dadurch gekennzeichnet, dass durch die Hilfestellung einerseits ein Beitrag zur Herstellung der Autonomie von Hilfsbedürftigen geleistet und andererseits ihre Autonomie aufgrund der erzeugten Abhängigkeit von dieser Hilfe beeinträchtigt wird. Das bedeutet, dass sich die Hilfestellung zwischen den Polen einer Unterstützung im Sinne von Empowerment und einer Deautonomisierung als erlernte Hilflosigkeit bewegt. Durch die Gefahr einer „Überhilfe“ (Breidenstein 2006, 195) kann es zu einer Verunmöglichung der Eigen- und Selbstständigkeit des Kindes kommen, wobei die Hilfe ursprünglich die Voraussetzung für die Realisierung von Autonomiepotenzialen ist. Die Assistenzleistung kann dabei sowohl förderlich als auch hinderlich für Entwicklung, Lernen oder auch soziale Kontakte mit Gleichaltrigen sein (vgl. u.a. auch Böing 2017; Hemmingsson, Borell \& Gustavsson 2003; Skär \& Tam 2001).

Folgende, von einer Schulassistentin verfasste, sehr positiv konnotierte, Narration zeigt beispielhaft auf, wie bei der Hilfeleistung trotzdem sowohl zwischen den 
Polen Befähigung und Unselbstständig-Machen als auch zwischen notwendigem ,Andersbehandeln“ und der Produktion von Besonders-Sein abgewogen werden muss:

„Das Schöne an dieser Woche war einfach, dass wir zwei uns nicht zu den Sportkursen eingetragen haben, [sondern das machten,] auf das wir Lust hatten (Stand-up-Paddling, Zumba, Tretbootfahren, Radeln, Trampolin, Minigolf) und wir waren immer im Geschehen der anderen dabei. Wir beide haben einfach abgesprochen, auf was er Lust hat und wir haben diese Sportarten und Tätigkeiten einfach versucht. Wie sich gezeigt hat, war sehr viel möglich und hat unheimlich Spaß gemacht (...)“ (EG 9).

Auffallend an dieser Narration ist einerseits, mit wie viel persönlicher Freude die Schulassistentin von dieser Sportwoche berichtet, andererseits aber auch, dass es ganz klar ist, dass für das Kind - anders als bei den Mitschüler*innen - Sonderregelungen für das Eintragen zu Sportkursen getroffen und diese auch nicht hinterfragt werden. Das Kind hat die Freiheit, spontan diese Entscheidung zu fällen, für die anderen gibt es eine Liste, um sich bereits im Vorfeld einzutragen.

Diese dem pädagogischen Handeln allgemein und besonders den Hilfesituationen in der Schule inhärenten Antinomien lassen sich in der Regel nicht vollständig auflösen. Wie mit ihnen umgegangen werden kann, zeigt beispielhaft folgende Narration einer Schulassistentin:

„Ich hole ein kleines Mädchen immer zu Hause ab und begleite es auf ihrem Schulweg. Sie fährt meistens mit dem Rad. Wir brauchen oft ziemlich lange, weil ihre Beine nicht so kräftig sind. Ich überlege mir immer wieder, ob ich nicht einfach anschieben soll, damit wir schneller sind. Meistens entscheide ich mich dagegen, weil es ein gutes Training ist und sie es doch alleine schaffen soll. Als es so kalt war, habe ich aber schon gleich zu ihrer Mutter gesagt, dass ich heute mithelfen werde, damit wir schneller sind“ (EG 31).

Sichtbar wird in dieser Narration, dass die eigene Rolle und die Hilfestellung laufend - nämlich „immer wieder“ - selbst reflektiert und angepasst wird, wobei darüber hinaus die Schulassistentin auch den Dialog mit der Mutter andeutet. Dabei zeigt sich eine Bewusstheit über das Spannungsfeld der Selbsttätigkeit versus schulischer oder eigener Zeiterfordernisse.

Die Reflexion der eigenen Rolle und der Hilfeleistung, der Dialog zwischen Kind, Eltern, Assistenz und Lehrperson sowie Kooperation und Austausch in multiprofessionellen Teams ermöglichen einen professionellen Umgang mit den aufgezeigten Antinomien zum Wohle des Kindes (vgl. auch Böing 2017, 25). Als Richtlinie kann dabei gelten, dass die Assistenzleistung ,frei von Zwang sowie Bevormundung, zeit- sowie bedarfsgerecht und veränderbar, also flexibel in der Inanspruchnahme“ (More, Kocnik \& Sigot 2020, 161) sein muss. In der Reflexion sollte dabei besonderes Augenmerk darauf gelegt werden, das pädagogische Handeln 


\section{Thomas Hoffmann, Cathrin Reisenauer und Hendrik Richter}

als Assistenzperson laufend zu reflektieren und anzupassen, da eine bestimmte Hilfestellung nicht nur für unterschiedliche Kinder und in unterschiedlichen Situationen befähigend oder deautonomisierend wirken kann, sondern sich die Wirkweisen im Laufe der Zeit verändern können.

\section{Literatur}

Althusser, L. (2016): Ideologie und Ideologische Staatsapparate. 1. Halbband. 2. Aufl. Hamburg.

Böing, U. (2017): „... und dann hab’ ich das ruckzuck fertig gemacht“. Zur Qualifizierung von Schulassistentinnen und -assistenten in an Inklusion orientierten Schulen - Impulse aus der Perspektive von Schülerinnen und Schülern. In: Feyerer, E., Prammer, W. \& Prammer-Semmler, E. (Hrsg.): Inklusion konkret. Assistenz und Bildung. Linz, 19-27.

Bourdieu, P. (2018): Die feinen Unterschiede: Kritik der gesellschaftlichen Urteilskraft. 26. Aufl. Frankfurt am Main.

Breidenstein, G. (2006): Teilnahme am Unterricht. Ethnographische Studien zum Schülerjob. Wiesbaden.

Christoph, F. (1983): Krüppelschläge: Gegen die Gewalt der Menschlichkeit. Reinbek bei Hamburg.

Feyerer, E., Prammer, W. \& Prammer-Semmler, E. (Hrsg.) (2017): Inklusion konkret. Assistenz und Bildung. Linz.

Hemmingsson, H., Borell, L. \& Gustavsson, A. (2003): Participation in School: School Assistants Creating Opportunities and Obstacles for Pupils with Disabilities. In: OTJR Occupation Participation Health, 23, 88-98.

Krappmann, L. \& Oswald, H. (1995): Alltag der Schulkinder. Beobachtungen und Analysen von Interaktionen und Sozialbeziehungen. Weinheim.

Loos, S. (2014): Assistenz in der Schule ist Teil des Menschrechtes auf Bildung. Rechtliche Grundlagen und (menschen-)rechtlicher Anspruch. In: Barth, U. \& Maschke, T. (Hrsg.): Inklusion - Vielfalt gestalten. Ein Praxisbuch. Stuttgart, 496-509.

More, R., Kocnik, E. \& Sigot, M. (2020): Persönliche Assistenz und Partizipation im schulischen Kontext. In: Gerhartz-Reiter, S. \& Reisenauer, C. (Hrsg.): Partizipation und Schule: Perspektiven auf Teilhabe und Mitbestimmung von Kindern und Jugendlichen. Wiesbaden, 153-166.

Oevermann, U. (2009): Die Problematik der Strukturlogik des Arbeitsbündnisses und der Dynamik von Übertragung und Gegenübertragung in einer professionalisierten Praxis von Sozialarbeit. In: Becker-Lenz, R., Busse, S., Ehlert, G. \& Müller-Hermann, S. (Hrsg.): Professionalität in der Sozialen Arbeit: Standpunkte, Kontroversen, Perspektiven. Wiesbaden, 113-142.

Rödler, P. (2000): geistig behindert: Menschen, lebenslang auf Hilfe anderer angewiesen. Grundlagen einer allgemeinen basalen Pädagogik. 2. Aufl. Neuwied/Berlin.

Sierck, U. \& Radtke, N. (1984): Die WohlTÄTERmafia: Vom Erbgesundheitsgericht zur humangenetischen Beratung. Hamburg.

Skär, L. \& Tam, M. (2001): My Assistant and I: Disabled children's and adolescents' roles and relationships to their assistants. In: Disability \& Society, 16, 917-931.

Tiroler Landesregierung (2018): Richtlinie des Landes Tirol nach $₫ 18$ Tiroler Teilhabegesetz über die Zuschüsse für Lohnkosten der Schulassistenz (Schulassistenz-Richtlinie). Beschluss der Tiroler Landesregierung vom 10.07.2018.

Tomasello, M. (2014): Die Ursprünge menschlicher Kommunikation. 3. Aufl. Frankfurt am Main. Zimpel, A. F. (2012): Einander Helfen: Auf dem Weg zur inklusiven Lernkultur. Göttingen. 
Entwicklungen 

Kathrin te Poel

\section{Zur Bedeutsamkeit eigener schüler*innenbiografischer Erfahrungen von angehenden Lehrpersonen für den sich anbahnenden Lehrer*innenhabitus und seine Anerkennungsbezüge}

\section{Zur Bildungsrelevanz von Anerkennung und zur Anerkennungsrelevanz von Lehrer*innenorientierungen}

Das Erfahren von Anerkennung gilt als essenzielle Voraussetzung für Identitätsentwicklungs- (vgl. Honneth 1992) und Bildungsprozesse (vgl. Stojanov 2006). Auch empirische Studien zeigen die hohe bildungsbiografische Bedeutsamkeit, die Anerkennungserfahrungen und -problematiken u.a. im Kontext Schule für Schüler*innen haben (vgl. bspw. Wiezorek 2005). Daneben zeichnen sich Zusammenhänge zwischen Anerkennung und Schulkultur ab (vgl. Helsper 2001), die darauf hindeuten, dass Anerkennung einzelschulspezifische Ausformungen erhält (vgl. Moldenhauer 2017). Und auch die impliziten Vorstellungen und Bilder, die angehende Lehrpersonen von Schüler*innen haben, weisen anerkennungsrelevante Facetten auf bzw. sind eng mit latenten Anerkennungsorientierungen verknüpft (vgl. te Poel 2020). Anhand der Rekonstruktion von zwei Interviewausschnitten, in denen angehende Lehrpersonen das Fehlen von Motivation bei Schüler*innen thematisieren, zeigt te Poel (ebd.), dass sich in einem Fall ausgeprägte Erklärungsmuster für einen Motivationsmangel finden lassen, mit denen empathische Handlungsmuster verknüpft sind. Solche Erklärungsmuster fehlen im zweiten Fall mit der Konsequenz, dass auch diesbezügliche Handlungsideen fehlen und sich die anerkennungsrelevanten Handlungsmuster auf Schüler*innen richten, bei denen Motivation vorhanden ist. Dieser Beitrag knüpft an letztgenannte Ergebnisse empirisch und theoretisch an, indem zunächst ausgehend von einer habitustheoretischen Perspektivierung die Bildungsgerechtigkeitsrelevanz solcher Anerkennungsfacetten impliziter Schüler*innenbilder von (angehenden) Lehrpersonen aufgezeigt wird. Davon ausgehend werden Professionalisierungsbedarfe und -chancen in der Lehrer*innenbildung markiert (Kap. 2). Anschließend wird anhand empirischer Fallrekonstruktionen der Frage nachgegangen, inwiefern sich Verknüpfungen zwischen impliziten Anerkennungsorientierungen 
angehender Lehrpersonen und ihren jeweils vorausgehenden schulbiografischen Erfahrungen als Schüler*in finden lassen (Kap. 3). Ausgehend von den Ergebnissen werden Ansatzpunkte für eine anerkennungssensible Professionalisierung angehender Lehrpersonen formuliert (Kap. 4).

\section{Habitustheoretische Perspektivierung impliziter Anerkennungsorientierungen}

Der Habitus als erfahrungs- und strukturell geprägte sowie feldspezifische „Systeme von Wahrnehmungs-, Bewertungs- und Handlungsschemata" (Bourdieu 2017, 177) wird in seiner Einverleibung nicht kognitiv eingeholt (vgl. ebd.), findet aber Ausdruck in den Praktiken einer Person (vgl. Kramer 2019). Demnach können sich auch habituell geprägte Anerkennungsorientierungen angehender Lehrpersonen in Handlungspraktiken niederschlagen.

Helsper (2019) differenziert mit einem auf das Feld Schule und den Lehrerberuf fokussierten Blick Bourdieus Habitusbegriff aus und unterscheidet analytisch den Herkunfts-, individuellen, Schüler*innen- und Lehrer*innenhabitus als Teile des Gesamthabitus einer Lehrperson. Diese sind prozessual verbunden (vgl. Helsper 2018), wobei der Herkunftshabitus in seinen familiären und milieuspezifischen Bezügen den prägenden Ausgangspunkt des Prozesses darstellt. Der individuelle Habitus entwickelt sich im Wechselspiel zwischen biografischem Verlauf und Partizipation an außerfamiliären Milieuräumen (vgl. Helsper 2019). Schüler*innenund Lehrer*innenhabitus sind als feldspezifische Ausformung des Gesamthabitus zu verstehen.

Helsper (ebd.) zufolge entwerfen Lehrpersonen dem eigenen Lehrer*innenhabitus äquivalente Schüler*innenbilder, die eher latent bleiben und eine „Repräsentanz des Schülerhabitus im Lehrerhabitus“ (ebd., 57) darstellen. Die (impliziten) Schüler*innenbilder, über die auch angehende Lehrpersonen verfügen, verweisen damit auf den sich anbahnenden Lehrer*innenhabitus, sie sind Teil dessen (vgl. ebd.). Wenn nun diese jeweils habitusspezifischen Schüler*innenbilder unmittelbar mit handlungsrelevanten Anerkennungsorientierungen symbiotisiert sind (siehe te Poel 2020), lässt sich daraus schließen, dass zugleich auch für die daraus resultierenden Anerkennungshandlungen eine implizite Passungsspezifik zum eigenen sich anbahnenden Lehrer*innenhabitus angenommen werden kann. Das ist insofern bildungsgerechtigkeitsrelevant, als diese Passungsspezifik den Erhalt von Anerkennung für Schüler*innen je nach habitueller Nähe oder Ferne erschweren oder erleichtern kann. Im ersten in Kap. 1 genannten Fall passt das an Erklärungsmuster geknüpfte empathische Handlungsmuster dann zu dem Habitus des gegenüberstehenden Schülers bzw. der gegenüberstehenden Schülerin oder aber 
nicht. Hier könnte eine Reflexion der eigenen Erklärungs- und Handlungsmuster mögliche Fokussierungen aufdecken und ggf. die Handlungsoptionen erweitern. Im zweitgenannten Fall fehlen Erklärungs- wie Handlungsmuster, was zu Begrenzungen wertschätzenden und damit anerkennenden Handelns führt. Eine Auseinandersetzung mit wissenschaftlichen Erklärungsmustern oder den Erklärungen anderer Studierender könnte in diesem Fall die eigenen Erklärungs- und damit die anerkennungsbezogenen Handlungsmuster erweitern. In beiden Fällen kann also eine Selbstreflexion professionalisierungsförderlich sein, wobei auch eine reflektierte Wissensaneignung Weiterentwicklungen anstoßen kann. Um konkretere Ansatzpunkte für solche anerkennungsbedeutsamen Reflexionen ausmachen zu können, geht das folgende Kapitel der Frage nach, inwiefern sich Verknüpfungen zwischen anerkennungsrelevanten Erklärungs- und Handlungsmustern angehender Lehrpersonen und deren (Schüler*innen-)Biografie ausmachen lassen. Den anerkennungstheoretischen Bezugspunkt bildet die anerkennungstheoretische Linie ausgehend von Honneth (1992), in deren Weiterführung Stojanov (2006) u.a. die Anerkennungsformen Empathie, Respekt und soziale Wertschätzung ausbuchstabiert. Als bildungs- und entwicklungsbedeutsame Beziehungsqualität gefasst kommt Anerkennung in dieser Linie ein normatives Moment zu (vgl. ebd., 9; 12).

\section{Anerkennungsrelevante Deutungen von angehenden Lehrpersonen und die Fremde oder Nähe zur eigenen Schüler*innenbiografie}

Die folgenden Rekonstruktionen erfolgten sequenzanalytisch mit der Methode der objektiven Hermeneutik (vgl. Wernet 2009). Nach deren Methodologie verweisen Sprechakte einer Person auf Handlungsakte und sind als Teil ihres Habitus aufzufassen (vgl. ebd.). Die Sequenzen stammen aus Interviews, die mit angehenden Lehrpersonen vor Beginn ihres Praxissemesters geführt wurden. Es haben acht Studierende teilgenommen.

Studentin 1 resümiert zunächst ihre bisherigen Erfahrungen in der praktischen Arbeit mit Schüler*innen ${ }^{1}$ :

S1: äh also mein jahrespraktikum war definitiv eine herausforderung (.) weil es ja eben eine schule war direkt in der innenstadt (.) sozialer brennpunkt [...] ähm es war auch eine recht große grundschule (.) die ich halt von mir damals selbst so nicht kannte.

1 Auf eine Glättung der Interviewpassagen im Sinne einer Einfügung von Groß- und Kleinschreibung wurde verzichtet. 
Das Erleben schulischer Praxis als „herausforderung“ begründet (,weil") die Studentin mit einer Beschreibung der Schule, an der sie tätig war. Damit verortet sie das Herausforderungserleben in den Merkmalen der Schule. Das ,ja eben " deutet eine Selbstverständlichkeit an, als läge das Dargelegte auf der Hand. Deutlich wird eine Differenz zwischen der beschriebenen Schule und der eigenen Schulzeit („von mir damals selbst so nicht kannte"). Dass diese Differenz von selbst thematisiert wird, verweist auf ihre subjektive Bedeutsamkeit. Das Differenzerleben, das im Zuge der Begründung des Herausforderungserlebens angeführt wird, scheint mit dem Herausforderungserleben verbunden und Letzteres zu begründen. Nach dem o.g. Jahrespraktikum war die Studentin noch einmal an der gleichen Schule tätig, worüber sie berichtet:

S1: ich hab' selbst schon gemerkt, dass ich ANDERS mit den schülern jetzt umgehen kann ähm es war nicht nur positive erfahrungen die ich gemacht habe ähm aber durch die bank weg bin ich schon froh (.) dass ich das gemacht hab und auch ne schule kennengelernt habe (.) die eben doch sehr viele ich sag jetzt ma schwierige kinder ähm hat.

Zusammenfassend zeigt sich eine an die Schüler*innen gerichtete Zuschreibung des Schwierig-Seins, die dieses Attribut in den Status einer Persönlichkeitseigenschaft hebt (,schwierige kinder"). Die Zuschreibung stellt eine Wertung dar, da der Begriff, „schwierig“" nicht näher durch wahrnehmbare Merkmale operationalisiert und konkretisiert wird. Die Wertung ist negativ konnotiert, da der Begriff Vorstellungen von Anstrengung impliziert. Die Zuschreibung nimmt die Studentin hier anstelle eines reflexiven Blickes auf sich selbst und auf mögliche eigene Schwierigkeiten im Umgang mit den Schüler*innen vor. Dass die Studierende die Schüler*innen als ,schwierige“ wahrnimmt, lässt eine Unpassung zwischen dem eigenen Schüler*innenhabitus im sich anbahnenden Lehrer*innenhabitus und dem erlebten Schüler*innenverhalten vermuten. Weil das Unpassende den eigenen impliziten Orientierungen fremd ist, wird der Umgang damit als schwierig erlebt, er kostet Anstrengung. Diese Lesart entspricht dem obigen Zusammenhang zwischen Differenz- und Herausforderungserleben. Es deutet sich an, dass das Sich-Hineinversetzen in die als "schwierige" erlebten Schüler*innen und eine entsprechende Anerkennung ihrer Bedürfnisse für die Studentin eine Herausforderung darstellen könnten, da über die zugeschriebene Wertung „schwierig“ hinausgehende Versuche eines verstehenden Nachvollziehens nicht artikuliert bzw. durch die direkte Wertung ggf. sogar blockiert werden. Wenn die Studentin auf die anschließende Frage, was dazu geführt habe, dass sie inzwischen ANDERS mit den Schüler*innen „umgehen kann“, eher selbstfokussiert antwortet: „ich meine ich bin seitdem auch jetzt fünfjahre älter geworden [...] ich bin selbstbewusster geworden 
im umgang mit den schülern“, bestätigt sich ein (noch) fehlendes Sich-Hineinversetzen in die Schüler*innen.

Dass die eigene Bildungs- und Schulbiografie in der Deutung von Praxiserfahrungen eine Rolle spielt, wird auch anhand eines zweiten Interviews deutlich. Studentin 2 resümiert bezugnehmend darauf, dass ihr das „BENEHMEN“ (Hervorhebung im Original) einiger Schüler*innen "negativ aufgefallen“ sei:

S2: wo ich dann auch an grenzen gekommen bin wo ich nicht wusste [I: klar] weil ich das NICHT KANNTE von mir selber.

Auch in diesem Fall wird eine Differenz zum eigenen Schüler*innenhabitus markiert, die hier als Begründung (,weil $\left.{ }^{*}\right)$ von Grenzerleben fungiert. Das Differenzerleben scheint mit der Negativwertung des erlebten Schüler*innenverhaltens ebenso verbunden wie es fehlende Erklärungs- oder Handlungsmuster (,wo ich nicht wusste") begründet. Da Schüler*innenbilder und Anerkennungsorientierungen unmittelbar verknüpft sind, lassen die fehlenden Erklärungs- und Handlungsmuster sowie die Negativdeutung auch hier darauf schließen, dass ein empathisches Nachvollziehen des „BENEHMEN[s]“ eine Herausforderung darstellen könnte. Empathie scheint somit gerade dann eine Herausforderung für angehende Lehrpersonen darzustellen, wenn sie mit einer Zielgruppe konfrontiert sind, die keine unmittelbaren Anknüpfungspunkte für Identifikationen mit der eigenen Bildungs- und Schüler*innenbiografie bietet und Erlebtes eine Art Fremde darstellt.

Gegenüber dieser Verknüpfung zwischen biografischer Fremdheit und fehlenden Erklärungs- und Handlungs- sowie damit auch Anerkennungsmustern verweist der folgende Fall auf Verknüpfungen zwischen konkreten Erfahrungen während der eigenen Schulzeit und den Denk- und Handlungsmustern der angehenden Lehrperson. So berichtet Studentin 3, die als Kind aufgrund eines Migrationshintergrundes selbst mit zwei Sprachen und auch mit „Sprachprobleme[n]“ konfrontiert war, von ihren ersten Praxiserfahrungen mit Schüler*innen:

S3: also die kinder die ZWEISPRACHIG aufgewachsen sind bei denen habe ich gearbeitet [...] das war interessant (.) da hatte ich ähm ganz unterschiedliche schüler aus afrika ähm syrien äh italien [...] mit den zu arbeiten war sehr interessant.

Es wird deutlich, dass ein Interesse an der Arbeit mit solchen Schüler*innen besteht, die Parallelen zur eigenen biografischen Erfahrung aufweisen. Die Frage, ob dieses Interesse in der biografischen Nähe begründet liegt, kann damit nicht beantwortet werden, da die Studentin an dieser Stelle selbst keine Verknüpfung zur eigenen Biografie herstellt. Da sie sich aber für diesen Tätigkeitsschwerpunkt selbst entschieden hat, fällt eine der eigenen Biografie nahe Fokussetzung auf. Die 
Betonung „ZWEISPRACHIG hebt genau diese Fokussetzung noch einmal hervor und unterstreicht deren Bedeutsamkeit. Von ihrer eigenen Schulzeit berichtet die Studentin später im Interview:

S3: ICH bin immer aufgefallen weil i ja [I: ((lacht))] ((lacht)) ganz schlechte Leistungen hatte UND sehr zurückhaltend.

„Aufl]fallen“ kann zunächst neutral als ,besonders wahrgenommen werden“ verstanden werden. Wenn die Studentin aber ihre Selbstschilderung als „zurückhaltend[e]“ Schülerin mit ,ganz schlechte[n] Leistungen“ als Grund anführt, „aufgefallen" zu sein, lässt dies - bedingt durch die Negativkonnotation letzterer - darauf schließen, dass das Auffallen eine negative Erfahrung für sie darstellte. Da die Studentin diese Erfahrung zudem von sich aus anspricht, scheint diese subjektiv bedeutsam für sie zu sein. Interessant ist in diesem Zusammenhang die etwas später formulierte Perspektive der Studentin auf das, was Lehrpersonen können sollten:

S3: empathievermögen ähm wenn man mit dem kind über äh die leistung spricht (.) sowohl stärken als auch schwächen ansprechen (.) nicht nur auf die schwächen eingehen [...] das kind nicht das gefühl geben, du bist jetzt EIN SONDERFALL:

Hier zeigen sich Parallelen zwischen den eigenen schüler*innenbiografischen Erfahrungen und der Fokussetzung als Lehrperson, wobei konkrete Handlungsoptionen deutlich werden. Diese sind eng, sogar manifest, mit Anerkennungsorientierungen, hier insbesondere Empathie, verknüpft. Es kann vermutet werden, dass die eigene Negativerfahrung des Auffallens als Schülerin ein Sich-Hineinversetzen in ähnliche Situationen von Schüler*innen und das Entwerfen von Handlungsoptionen, die dem eigenen Erleben entsprechen, begünstigen kann. Ebenso wie sich also ein Zusammenhang zwischen biografischer Fremdheit und fehlenden Handlungs- und Anerkennungsmustern in den Interviews mit angehenden Lehrpersonen andeutet, kann auch zwischen schüler*innenbiografisch bedeutsamen Erfahrungen und jeweils gesetzten Anerkennungsfoki der angehenden Lehrpersonen eine Verknüpfung angenommen werden. Die Fremde oder Nähe von (erlebten) Praxissituationen zu eigenen Erfahrungen als Schüler*in und zum eigenen Schüler*innenhabitus zeigt sich dabei insbesondere mit Blick auf Anerkennung in Form von Empathie für den späteren Lehrberuf bedeutsam. 


\section{Ansatzmöglichkeiten für die Reflexion von anerkennungsrelevanten habituellen Orientierungen im Lehramtsstudium}

Dass sich die Reflexion von sich anbahnenden anerkennungsrelevanten Lehrer*innenorientierungen als professionalisierungsförderlich erweist, zeigt te Poel (2020). Dass diese Reflexion zudem eine Bildungsgerechtigkeitsdimension hat, da unbewusste Anerkennungsfoki Passungsspezifika aufweisen können, die den Erhalt bildungsrelevanter Anerkennung für einzelne Schüler*innen erschweren oder erleichtern, zeigt Kap. 2. Die Ergebnisse in Kap. 3 geben nun konkrete Hinweise auf Ansatzpunkte für eine solche Reflexion, denn sie verweisen auf die Möglichkeit, zunächst den eigenen Schüler*innenhabitus wie auch bedeutsame (schüler*innen-)biografische Erfahrungen der angehenden Lehrpersonen in den Reflexionsfokus zu rücken. Im Seminar Habitus - Habituspassung - Habitusreflexion, das an der Universität Bielefeld angeboten wird, geschieht dies zunächst in Form einer schriftlichen Einzelarbeit: Die Studierenden reflektieren, was sie als Schüler*in ausgemacht hat und welche Erfahrungen prägend waren. In Kleingruppen tauschen sie sich anschließend aus, sodass eine Konfrontation mit anderen Erfahrungen stattfinden kann. Die Studierenden regulieren dabei selbst, was sie der Gruppe preisgeben. Da alle angehenden Lehrpersonen lange Zeit Schüler*in waren, ist davon auszugehen, dass ein reflexives Ansetzen an diesen Erfahrungen einer Überforderung vorbeugt. Um ein ganzheitliches Lernen zu ermöglichen, werden die Selbstreflexionen im Seminar mit Wissensinhalten verknüpft, sodass ein Wechselspiel zwischen Wissensaneignung und Reflexion entsteht. Die Studierenden können die Relevanz des Wissens anhand der Auseinandersetzung mit sich selbst und anderen erschließen. Nach der ersten biografischen Reflexion findet bspw. eine inhaltliche Auseinandersetzung mit dem Thema Schüler*innenhabitus statt. An diese schließt eine Reflexionseinheit an, in der die Studierenden - mit einer Verhaltensbeschreibung und einem Bild eines Schülers bzw. einer Schülerin konfrontiert - die Aufgabe erhalten, spontane Assoziationen zu notieren sowie - wenn möglich - erste Deutungen zu versuchen. Auch nach dieser Aufgabe tauschen sich die Studierenden aus, um in der Konfrontation mit anderen Assoziationen und Deutungen je eigene Deutungen zu hinterfragen und ggf. biografische Verknüpfungen zu erkennen. Es folgt eine inhaltliche Auseinandersetzung mit den Phänomenen Lehrer*innenhabitus und Anerkennung, um das Seminar mit Reflexionen sich anbahnender Lehrer*innenorientierungen in ihrer Anerkennungsrelevanz abzuschließen. Diese Reflexionen erfolgen anknüpfend an die rekonstruktiv kasuistische Bearbeitung konkreter Praxisfälle, die Lehrer*innenhandlungen fokussieren. Im Spiegel der rekonstruktiven Fallarbeit werden eigene, sich anbahnende Lehrer*innenorientierungen und ihre Anerkennungsre- 
levanz in den Blick genommen und ihr Verhältnis zum eigenen - zu Beginn des Seminars reflektierten - Schüler*innenhabitus betrachtet.

\section{Literatur}

Bourdieu, P. (2017): Meditationen. Zur Kritik der scholastischen Vernunft. 4. Aufl. Frankfurt am Main.

Helsper, W. (2001): Schülerpartizipation und Schulkultur - Bestimmungen im Horizont schulischer Anerkennungsverhältnisse. In: Böhme, J. \& Kramer, R. T. (Hrsg.): Partizipation in der Schule. Theoretische Perspektiven und empirische Analysen. Opladen, 37-48.

Helsper, W. (2018): Vom Schülerhabitus zum Lehrerhabitus - Konsequenzen für die Lehrer- professionalität. In: Leonhard, T., Kosinar, J. \& Reintjes, C. (Hrsg.): Praktiken und Orientierungen in der Lehrerbildung. Potenziale und Grenzen der Professionalisierung. Bad Heilbrunn, 17-40.

Helsper, W. (2019): Vom Schüler- zum Lehrerhabitus. Reproduktions- und Transformationspfade. In: Kramer, R. T. \& Pallesen, H. (Hrsg.): Lehrerhabitus. Theoretische und empirische Beiträge zu einer Praxeologie des Lehrerberufs. Bad Heilbrunn, 49-72.

Honneth, A. (1992): Kampf um Anerkennung. Zur moralischen Grammatik sozialer Konflikte. Frankfurt am Main.

Kramer, R. T. (2019): Sequenzanalytische Habitusrekonstruktion. In: Kramer, R. T. \& Pallesen, H. (Hrsg.): Lehrerhabitus. Theoretische und empirische Beiträge zu einer Praxeologie des Lehrerberufs. Bad Heilbrunn, 307-330.

Moldenhauer, A. (2017): Zum Verhältnis von Schulkultur, Partizipation und Milieu. In: Baader, M. S. \& Freytag, T. (Hrsg.): Bildung und Ungleichheit in Deutschland. Wiesbaden, 255-270.

te Poel, K. (2020): Anerkennung und Beziehungen. Didaktische Umsetzungen? Anfragen ausgehend von theoretischen und empirischen Analysen zum Zusammenhang von Menschen- bzw. Schülerbild, Anerkennungshandeln und Lehrerhabitus. In: Zeitschrift für Inklusion online, 2, o.S. Online unter: https://www.inklusion-online.net/index.php/inklusion-online/article/view/571 (letzter Zugriff: 28.01.2021).

Stojanov, K. (2006): Bildung und Anerkennung. Soziale Voraussetzungen von Selbst-Entwicklung und Welt-Erschließung. Wiesbaden.

Wernet, A. (2009): Einführung in die Interpretationstechnik der Objektiven Hermeneutik. Wiesbaden.

Wiezorek, C. (2005): Schule, Biographie und Anerkennung. Eine fallbezogene Diskussion der Schule als Sozialisationsinstanz. Wiesbaden. 


\section{Alina Quante und Oliver Danner}

\section{Grenzsetzungen bei Aufgabenbereichen von sonderpädagogischen und allgemeinen Lehrkräften in inklusiven Settings}

\section{Lehramtsspezifische Rollen und Aufgaben im Wandel}

Im Zusammenhang mit inklusiven Schulentwicklungsprozessen wandeln sich auch die schulstrukturellen Bedingungen. Da Schüler*innen mit sonderpädagogischem Förderbedarf vermehrt an inklusiven Schulen unterrichtet werden, kommt es zur Verlagerung sonderpädagogischer Ressourcen in das System der allgemeinen Schulen, was in einer Kooperation von allgemeinen und sonderpädagogischen Lehrkräften resultiert. Aufgrund dieser Wandlungsprozesse ist zu hinterfragen, inwiefern sich die Rollen und Aufgaben von Lehrkräften der allgemeinen Schulen und sonderpädagogischen Lehrkräften in inklusiven Schulen abgrenzen und welche Überschneidungen bestehen.

Insbesondere von den sonderpädagogischen Lehrkräften wird gefordert, sich in inklusiven Settings auf neue Rollen einzulassen, damit Inklusion gelingt (vgl. z.B. Weiss \& Lloyd 2002). Sie brauchen dafür ein hohes Maß an Flexibilität, da sie in unterschiedlichen organisatorischen Formen mit verschiedenen Lerngruppen und Lehrkräften zusammenarbeiten (vgl. Dorniak 2019). Daraus ist zu schließen, dass es aktuell vielfältige Rollenausformungen der sonderpädagogischen Lehrkräfte in inklusiven Settings gibt. Allerdings zeigen Ergebnisse von Kreis, Wick und Kosorok Labhart (2014), dass der sonderpädagogische Förderbedarf in der Schulpraxis weiterhin als Basis zur Regelung der Zuständigkeiten dient. So werden Aktivitäten mit Bezug zu Schüler*innen mit sonderpädagogischem Förderbedarf deutlich im Zuständigkeitsbereich der sonderpädagogischen Lehrkraft gesehen, während Aktivitäten, welche die gesamte Klasse betreffen, eher der allgemeinen Lehrkraft zugeschrieben werden (vgl. ebd.). Gerade mit Blick auf die Lehrkräfte allgemeiner Schulen scheint es, als seien diese nicht besonders flexibel in ihrer Rollen- und Aufgabengestaltung, da sie ihre Praxis lediglich bedingt den veränderten Anforderungen anpassen (vgl. Kreis, Wick \& Kosorok Labhart 2016). Es besteht daher eine Diskrepanz zwischen den normativen Forderungen und der Umsetzung von Inklusion in der Schulpraxis. 
Einen möglichen Faktor, der zur Erklärung dieser Diskrepanz dienen könnte, stellt die gemeinsam zur Verfügung stehende Zeit bei der Kooperation in inklusiven Settings dar. Diese wird als eine der wesentlichen Voraussetzungen für eine intensive Zusammenarbeit gesehen (vgl. Richter \& Pant 2016). Den theoretischen Annahmen folgend ist davon auszugehen, dass viel gemeinsam zur Verfügung stehende Zeit eher zu Kokonstruktionsprozessen bei der Kooperation und gemeinsamer Verantwortungsübernahme für die Lerngruppe führt (vgl. Grosche, Gräsel \& Fussangel 2020). Dies würde implizieren, dass ein Großteil der anstehenden Aufgaben auch als gemeinsam zu bewältigen angesehen wird und sich dadurch lehramtsspezifische Zuständigkeitsgrenzziehungen auflösen. In diesem Beitrag wird daher der Frage nachgegangen, welche Tätigkeiten oder Aufgabenbereiche in inklusiven Settings kooperierende Lehrkräfte in wessen Zuständigkeit sehen. Darüber hinaus wird analysiert, welchen Einfluss der Faktor der gemeinsamen zeitlichen Ressourcen auf die Zuständigkeitszuweisungen hat.

\section{Empirische Untersuchung}

\subsection{Forschungskontext}

Die hier dargestellten Daten wurden im Rahmen des Forschungsprojekts P-ink ${ }^{1}$ erhoben, welches die Professionalisierung von Lehrkräften für die Kooperation in inklusiven Settings fokussiert. Dafür wurde eine Professionalisierungsmaßnahme in Form einer Fortbildung konzipiert, durchgeführt und evaluiert, welche sich an sonderpädagogische und allgemeine Lehrkräfte richtete, die gemeinsam in inklusiven Settings unterrichten. Die Wirkungen dieser Maßnahme werden in Form einer Interventionsstudie untersucht (vgl. Quante, Danner, Rank \& Munser-Kiefer 2020). Die hier berichteten Daten stammen vom ersten Messzeitpunkt (MZP 1) und bilden die Aufgabenzuständigkeit in Zusammenhang mit den verfügbaren zeitlichen Ressourcen der Lehrkräfte ab.

\subsection{Methodik}

Um zu erfassen, welche Aufgaben und Tätigkeiten der kooperierenden Lehrkräfte in wessen Zuständigkeit liegen, wird ein Fragebogen von Kreis et al. (2014) adaptiert. Die Lehrkräfte werden zu insgesamt 22 Items (Beispielitem: ,Konkrete

1 P-ink (Professionalisierung für ein inklusives Schulsystem in der dritten Phase der Lehrer*innenbildung) wird durch das Bildungsministerium für Bildung und Forschung im Rahmen der Förderrichtlinie, Qualifizierung der pädagogischen Fachkräfte für inklusive Bildung' (FKZ: 01NV1703) gefördert (01.10.2017-30.09.2020). Am Projekt beteiligt sind Prof. Dr. Astrid Rank, Prof. Dr. Meike Munser-Kiefer, Alina Quante, Oliver Danner, Sabine Sroka, Dr. Claudia Urbanek und Prof. Dr. Anne Frey. 
Vorbereitung des gemeinsamen Unterrichts') befragt, welche fünf verschiedene Aufgabenbereiche abdecken. Anhand einer fünfstufigen Skala können die Lehrkräfte angeben, ob die Verantwortung vollständig oder tendenziell bei einer der Lehrkräfte liegt oder ob es sich um eine gemeinsame Aufgabe handelt.

\subsection{Stichprobe}

An der Fragebogenstudie beteiligten sich insgesamt 73 Lehrkräfte (MZP 1). Die Stichprobe setzte sich aus sonderpädagogischen Lehrkräften $(n=27)$ und Lehrkräften allgemeiner Schulen $(n=46)$ zusammen. Im Durchschnitt waren die Lehrkräfte 46,17 Jahre alt $(S D=10,63)$, wobei das Geschlechterverhältnis bei 6\% Männern zu $94 \%$ Frauen lag.

\subsection{Auswertung}

Es wurde erhoben, welche Aufgaben von den sonderpädagogischen Lehrkräften $(S)$ und den Lehrkräften allgemeiner Schulen (A) jeweils in welcher Verantwortung gesehen werden, wobei über den Ist-Stand der Kooperation berichtet wird. Anhand der Skalenwerte ergibt sich die Zuordnung der wahrgenommenen Zuständigkeit (Abb. 1). Aus den errechneten Skalenwerten zeichnet sich tendenziell ab, wie stark die jeweilige Einschätzung der Lehrkräfte ist. Je mehr der Wert Richtung -2 tendiert, desto stärker wird die Aufgabe der Lehrkraft allgemeiner Schulen zugeordnet, je näher der Wert Richtung +2 tendiert, desto eher wird die Zuständigkeit bei der sonderpädagogischen Lehrkraft gesehen. Liegt der Wert um $+/-0$, so handelt es sich um eine gemeinsame Aufgabe.

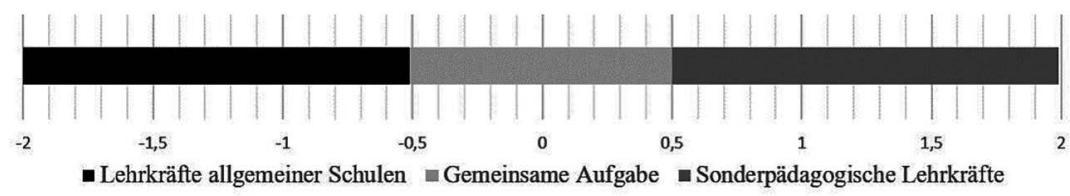

Abb. 1: Interpretation der Skalenwerte

Der zweite Faktor, der zur Beantwortung der Fragestellung herangezogen wird, ist der Einfluss der wöchentlich gemeinsam zur Verfügung stehenden Zeit in Form von Schulstunden pro Woche, welche sich bei den befragten Lehrkräften stark unterscheiden $(M W=6,72 ; S D=7,29 ;$ Spanne $=0-23)$. Aus dem vorliegenden Datensatz ergibt sich eine Unterteilung in $\leq 6$ und $>6$ Stunden als sinnvoll, um etwa gleich starke Untergruppen bilden zu können. Die Unterteilung der Daten anhand der Faktoren Lehramt und Zeit lässt aufgrund der Stichprobengröße nur eine deskriptive Analyse zu. 


\section{Alina Quante und Oliver Danner}

\section{Ergebnisse}

Beim Kompetenzbereich Diagnostik wird nur die Tätigkeit ,Lernentwicklungsstand für die gesamte Klasse (unabhängig von der Anzahl der gemeinsamen Stunden) von beiden Lehrämtern in der Zuständigkeit der allgemeinen Lehrkräfte gesehen. Bei mehr als sechs gemeinsamen Stunden pro Woche werden die Aufgaben, sonderpädagogischen Förderbedarf festzustellen, Förderziele zu überprüfen und den Lernentwicklungsstand der Kinder mit individuellem Förderbedarf zu erheben von den sonderpädagogischen Lehrkräften in der eigenen Zuständigkeit gesehen. Bei weniger als sechs gemeinsamen Stunden fällt die Aufgabe, Förderziele zu überprüfen, jedoch in die gemeinsame Zuständigkeit. Auch den Lernentwicklungsstand zu erheben, fällt bei weniger gemeinsamen Stunden zumindest aus Sicht der sonderpädagogischen Lehrkräfte in den gemeinsam verantworteten Bereich. Somit zeigt sich tendenziell eine gemeinsame Zuständigkeit für diagnostische Tätigkeiten bei wenig zeitlichen Ressourcen, während bei mehr gemeinsam verbrachter Zeit diagnostische Tätigkeiten der sonderpädagogischen Lehrkraft zugeschrieben werden.

Bei weniger als sechs gemeinsamen Stunden werden diese Tätigkeiten im Kompetenzbereich Gestaltung von Lerngelegenheiten für Schüler*innen mit sonderpädagogischem Förderbedarf von beiden Lehrkräften als gemeinsam erachtet. Ein ,Mehr an Stunden geht überwiegend mit einer stärkeren Zuständigkeit der sonderpädagogischen Lehrkräfte einher. Während die Aktivitäten ,Förderziele umsetzen“ sowie ,Unterrichtsinhalte festlegen ' bei weniger gemeinsamen Stunden von den Lehrkräften allgemeiner Schulen als eigene Zuständigkeit verortet werden, fallen diese bei mehr als sechs gemeinsamen Stunden in die Verantwortung der sonderpädagogischen Lehrkräfte. Somit ergibt sich bei mehr gemeinsamen Stunden eine Ausdifferenzierung der Zuständigkeiten zugunsten der sonderpädagogischen Lehrkraft.

Alle Lehrkräfte mit weniger als sechs gemeinsamen Stunden pro Woche verorten die Gestaltung von Lerngelegenheiten für die ganze Klasse in der Zuständigkeit der Lehrkräfte allgemeiner Schulen. Insbesondere Aufgaben, welche im Zusammenhang mit Differenzierung stehen, werden bei mehr als sechs gemeinsamen Stunden von beiden Lehrämtern als gemeinsame Tätigkeit erachtet. Die sonderpädagogischen Lehrkräfte sehen bei mehr gemeinsamen Stunden zudem die Beschaffung von Unterrichtsmaterialien sowie die Quartals- und Jahresplanung der Unterrichtsinhalte als gemeinsam verantwortet an.

Ein ähnlicher Befund zeigt sich auch beim Kompetenzbereich Handeln im Klassenraum. Lehrkräfte mit mehr als sechs gemeinsamen Stunden pro Woche nehmen die Aufgaben dieses Bereiches überwiegend als gemeinsam wahr. Das Intervenieren bei unangepasstem Verhalten sehen alle Lehrkräfte unabhängig von der Anzahl der gemeinsamen Stunden als gemeinsame Aufgabe an. Die ,konkrete 
Vorbereitung des gemeinsamen Unterrichts' wird bei weniger als sechs Stunden den allgemeinen Lehrkräften zugeschrieben. Bei mehr als sechs Stunden sehen die allgemeinen Lehrkräfte eine gemeinsame Zuständigkeit, während die sonderpädagogischen Lehrkräfte weiterhin die allgemeinen Lehrkräfte für zuständig erachten. Im Kompetenzbereich der Eltern und Kooperationsarbeit werden die Aktivitäten ,Beratungsgespräche' und ,kollegiale Beratung' unabhängig von den zur Verfügung stehenden zeitlichen Ressourcen von allen Lehrkräften als gemeinsame Tätigkeit beschrieben. ,Elternabende` werden bei wenig gemeinsamer Zeit in der Zuständigkeit der Lehrkraft allgemeiner Schulen gesehen, wobei sich bei mehr gemeinsamen Stunden eine Tendenz vonseiten der sonderpädagogischen Lehrkräfte hin zu einer gemeinsamen Verantwortung zeigt.

Tab. 1: Auszug der wahrgenommenen Zuständigkeiten aus Sicht der sonderpädagogischen Lehrkräfte $(S)$ und der Lehrkräfte an allgemeinen Schulen (A) (in Anlehnung an Kreis et al. 2014). Angegeben sind jeweils die Mittelwerte $(M W)$ und die Standardabweichung $(S D)$. Die Skalierung reicht von Min. $=-2,00$ bis $\operatorname{Max} .=2,00$.

\begin{tabular}{|c|c|c|c|c|c|c|c|}
\hline \multirow[b]{2}{*}{ Anzahl gemeinsame Stunden pro Woche } & & \multicolumn{2}{|c|}{$\begin{array}{l}\text { Aufgabe allg. LK } \\
\text { MW (SD) }\end{array}$} & \multicolumn{2}{|c|}{$\begin{array}{c}\text { Gemeinsame Aufgabe } \\
\text { MW (SD) }\end{array}$} & \multicolumn{2}{|c|}{$\begin{array}{c}\text { Aufgabe sp. LK } \\
\text { MW (SD) }\end{array}$} \\
\hline & & $\leq 6$ & $>6$ & $\leq 6$ & $>6$ & $\leq 6$ & $>6$ \\
\hline $\begin{array}{l}\text { Lernentwicklungsstand für die gesamte } \\
\text { Klasse erheben }\end{array}$ & $\begin{array}{l}\mathrm{A} \\
\mathrm{S}\end{array}$ & $\begin{array}{l}-1,64(0,64) \\
-1,40(0,66)\end{array}$ & $\begin{array}{l}-0,89(0,87) \\
-0,83(0,69)\end{array}$ & & & & \\
\hline $\begin{array}{l}\text { Sonderpädagogischen Förderbedarf } \\
\text { feststellen }\end{array}$ & $\begin{array}{l}\mathrm{A} \\
\mathrm{S}\end{array}$ & & & & & $\begin{array}{l}1,91(0,29) \\
1,60(0,66)\end{array}$ & $\begin{array}{l}1,38(0,70) \\
1,43(0,73)\end{array}$ \\
\hline Förderziele überprüfen & $\begin{array}{l}\mathrm{A} \\
\mathrm{S}\end{array}$ & & & $\begin{array}{l}0,00(1,04) \\
0,20(0,60)\end{array}$ & & & $\begin{array}{l}0,88(0,60) \\
0,86(0,64)\end{array}$ \\
\hline $\begin{array}{l}\text { Lernentwicklungsstand der Kinder mit } \\
\text { individuellem Förderbedarf erheben }\end{array}$ & $\begin{array}{l}\mathrm{A} \\
\mathrm{S}\end{array}$ & & & $0,40(0,80)$ & & $0,54(1,15)$ & $\begin{array}{l}0,88(0,93) \\
0,86(0,64)\end{array}$ \\
\hline Förderziele umsetzen & $\begin{array}{l}\mathrm{A} \\
\mathrm{S}\end{array}$ & $-0,75(0,72)$ & & $-0,10(0,54)$ & $0,29(0,88)$ & & $0,78(0,79)$ \\
\hline Unterrichtsinhalte festlegen & $\begin{array}{l}\mathrm{A} \\
\mathrm{S}\end{array}$ & $-0,55(1,23)$ & & $-0,11(0,57)$ & & & $\begin{array}{l}0,89(0,74) \\
1,29(0,45)\end{array}$ \\
\hline $\begin{array}{l}\text { Differenzierungsmaterialien beschaffen } \\
\text { Niveaudifferenzierung }\end{array}$ & $\begin{array}{l}\text { A } \\
\text { S } \\
\text { A } \\
\text { S }\end{array}$ & $\begin{array}{l}-1,00(0,96) \\
-1,00(0,63) \\
-0,86(0,99) \\
-1,10(0,70)\end{array}$ & & & $\begin{array}{r}0,30(0,64) \\
-0,14(0,64) \\
0,00(0,63) \\
-0,14(0,64)\end{array}$ & & \\
\hline $\begin{array}{l}\text { Gemeinsamen Unterricht konkret } \\
\text { vorbereiten } \\
\text { Elternabende }\end{array}$ & $\begin{array}{l}\mathrm{A} \\
\mathrm{S} \\
\mathrm{A} \\
\mathrm{S}\end{array}$ & $\begin{array}{l}-1,00(0,85) \\
-0,90(0,70) \\
-1,46(0,63) \\
-1,10(0,54)\end{array}$ & $\begin{array}{l}-0,71(0,70) \\
-0,60(0,92)\end{array}$ & & $\begin{array}{l}-0,40(0,66) \\
-0,29(0,45)\end{array}$ & & \\
\hline
\end{tabular}




\section{Alina Quante und Oliver Danner}

\section{Diskussion}

Die Annahme, dass ein Mehr an gemeinsamen Stunden mit mehr gemeinsamer Verantwortung bei den Aufgabenbereichen einhergeht, scheint sich nicht ohne Einschränkungen bestätigen zu lassen. Stattdessen ergeben sich, wie die Daten zeigen, Distinktionen. Grob skizziert lassen sich Aufgaben, welche die gesamte Klasse betreffen, sowohl bei wenig als auch bei mehr gemeinsam zur Verfügung stehenden Stunden pro Woche eher der Zuständigkeit der Lehrkraft allgemeiner Schulen zuordnen, was den Ergebnissen von Kreis et al. (2014) entspricht. Bei mehr gemeinsamen Stunden pro Woche zeigt sich aber die Tendenz, dass Aufgaben, die sich auf die Differenzierung und die Beschaffung von differenzierten Unterrichtsmaterialien beziehen, gemeinsam erledigt werden. Somit scheint sich bei mehr gemeinsamen Stunden zumindest in Bezug auf diese speziellen Tätigkeiten eine gemeinsame Verantwortung für die gesamte Klasse abzuzeichnen, wodurch lehramtsspezifische Grenzziehungen aufgelöst werden.

Insbesondere Aufgaben, die die Gestaltung von Lerngelegenheiten für Schüler*innen mit sonderpädagogischem Förderbedarf betreffen, werden in Form einer verstärkten Zuständigkeit der sonderpädagogischen Lehrkräfte ausdifferenziert, was den Ergebnissen von Kreis et al. (ebd.) entspricht. Überraschend ist, dass diagnostische Aufgaben bei einem geringeren gemeinsamen Stundendeputat eher als gemeinsame Zuständigkeit angesehen werden und sich ausgerechnet bei mehr gemeinsamen Stunden in die alleinige Zuständigkeit der sonderpädagogischen Lehrkraft verlagern. Eine mögliche Erklärung bietet die „Zuständigkeitsdiffusitätsproblematik“ (Kunze 2016, 273), mithilfe derer beschrieben werden kann, dass es bei wenigen gemeinsamen Stunden zu einer „Entdifferenzierung“ (ebd., 285) und bei mehr gemeinsam verfügbarer Zeit zu einer "Ausdifferenzierung“" (ebd.) kommt. Gerade der Befund zur Ausdifferenzierung der Aufgabenzuständigkeiten ist vor dem Hintergrund der Hoffnung auf kokonstruktive Prozesse in der interdisziplinären Kooperation, die mit einer gemeinsamen Verantwortungsübernahme einhergehen (vgl. Grosche et al. 2020), kritisch einzuordnen.

Da sich die Ausdifferenzierung der Zuständigkeiten zudem eher bei den Aufgabenbereichen ereignet, die Schüler*innen mit sonderpädagogischem Förderbedarf oder diagnostische Aufgaben mit Bezug auf sonderpädagogische Förderung betreffen, zeigt sich noch immer eine klientelspezifische Verantwortung, wie sie seit Langem in der Kooperationsforschung beschrieben wird (vgl. z.B. Reiser 1998). Dieses Vorgehen veranlasst zur Frage, ob es sich um eine „Problemdelegation “ (Urban \& Lütje-Klose 2014, 284) der Lehrkräfte allgemeiner Schulen an die sonderpädagogischen Lehrkräfte handelt, welche ein asymmetrisches Rollenverhältnis implizieren würde. Gleichzeitig kann die Zuschreibung dieser diagnostischen Tätigkeiten auch in der speziellen sonderpädagogischen Expertise liegen (vgl. Köpfer \& Lemmer 2020). Es ist daher von Bedeutung, ob die Zustän- 
digkeitszuschreibungen gemeinsam ausgehandelt werden und anhand welcher Begründungslinien dies jeweils geschieht. Qualitative Zugänge, die insbesondere die Aushandlung der Aufgaben und Zuständigkeiten in den Blick nehmen, könnten daher eine Möglichkeit bieten, diese Frage zu klären. Ergänzend ist anzumerken, dass die Beschaffenheit der hier berichteten Daten nur eine deskriptive Analyse zuließ, sodass eine quantitativ ausgerichtete empirische Untersuchung zu den hier aufgeworfenen Annahmen wünschenswert wäre.

\section{Literatur}

Dorniak, M. (2019): Akteur*innen in der Inklusion - Veränderte Rollenbilder am Beispiel der Sonderpädagogik. In: Baumert, B. \& Willen, M. (Hrsg.): Zwischen Persönlichkeitsbildung und Leistungsentwicklung. Fachspezifische Zugänge zu inklusivem Unterricht. Bad Heilbrunn, 239-250.

Grosche, M., Gräsel, C. \& Fussangel, K. (2020): Kokonstruktive Kooperation zwischen Lehrkräften. In: Zeitschrift für Pädagogik, 66, H.4, 461-479.

Köpfer, A. \& Lemmer, K. (2020): Die „perfekte“ Kooperationssituation. In: Herausforderung Lehrer*innenbildung - Zeitschrift zur Konzeption, Gestaltung und Diskussion, 3, H.1, 80-93.

Kreis, A., Wick, J. \& Kosorok Labhart, C. (2014): Wahrgenommene Zuständigkeiten von pädagogischem Personal in integrativen Schulen des Kantons Thurgau. In: Empirische Sonderpädagogik, 6, H.4, 333-349.

Kreis, A., Wick, J. \& Kosorok Labhart, C. (Hrsg.) (2016): Kooperation im Kontext schulischer Heterogenität. Münster/New York.

Kunze, K. (2016): Multiprofessionelle Kooperation - Verzahnung oder Differenzierung? Einige Einwände gegen die Polarisierungstendenz einer Diskussion. In: Idel, T.-S., Dietrich, F., Kunze, K., Rabenstein, K. \& Schütz, A. (Hrsg.): Professionsentwicklung und Schulstrukturreform: Zwischen Gymnasium und neuen Schulformen in der Sekundarstufe. Bad Heilbrunn, 261-277.

Quante, A., Danner, O., Rank, A. \& Munser-Kiefer, M. (2020): Kooperation zwischen Regelschulund Sonderschullehrkräften im inklusiven Setting - eine Interventionsstudie zur Professionalisierung. In: Grosche, M., Decristan, J., Urton, K., Bruns, G., Ehl, B. \& Jansen, N. C. (Hrsg.): Sonderpädagogik und Bildungsforschung - Fremde Schwestern? Bad Heilbrunn, 216-222.

Reiser, H. (1998): Sonderpädagogik als Service-Leistung? Perspektiven der sonderpädagogischen Berufsrolle zur Professionalisierung der Hilfsschul- bzw. Sonderschullehrerinnen. In: Zeitschrift für Heilpädagogik, 49, H.2, 46-54.

Richter, D. \& Pant, H. A. (2016): Lehrerkooperation in Deutschland. Eine Studie zu kooperativen Arbeitsbeziehungen bei Lehrkräften der Sekundarstufe I. Gütersloh.

Urban, M. \& Lütje-Klose, B. (2014): Professionelle Kooperation als wesentliche Bedingung inklusiver Schul- und Unterrichtsentwicklung. Teil 2: Forschungsergebnisse zu intra- und interprofessioneller Kooperation. In: Vierteljahresschrift für Heilpädagogik und ihre Nachbargebiete, 83, H.4, 283294.

Weiss, M. P. \& Lloyd, J. W. (2002): Congruence between roles and actions of secondary special educators in co-taught and special education settings. In: The Journal of Special Education, 36, H.2, 58-68. 


\section{Das Boot: Eine Fluchtgeschichte - Design-Based-Research in der Primarstufe}

Flucht und Migration sind derzeit viel diskutierte Themen. Nicht nur im gesellschaftlich-politischen, sondern auch im schulischen Kontext, insbesondere in Schulbüchern, werden diese Themen meist stereotypisiert und problembehaftet dargestellt.

Um den vorherrschenden Problemdiskurs kritisch zu hinterfragen, ist es notwendig, Flucht und Migration explizit zum Bildungsgegenstand zu machen und Schüler*innen eine intensivere Beschäftigung mit diesen Themen zu ermöglichen. Der vorliegende Beitrag widmet sich einem Projekt, das sich zum Ziel setzte, das Thema Flucht in der Primarstufe explorativ zu erforschen und innovative Unterrichtsmaterialien zu diesem Thema zu entwickeln. Das Buch ,Das Boot: Eine Fluchtgeschichte bildete dafür die Ausgangsbasis. Das Projekt wurde im Rahmen eines Design-Based-Research-Ansatzes (DBR) verwirklicht.

\section{Einleitung}

Migration ist kein Phänomen des 21. Jahrhunderts, Wanderungsbewegungen über Grenzen hinweg waren und sind Teil des gesellschaftlichen sozialen Gefüges (vgl. Mecheril 2016). Im gesellschaftlich-politischen Kontext werden Migrationsphänomene aber trotzdem häufig stereotypisiert und über einen Problem- oder Kosten-Nutzen-Diskurs beschrieben. So wurden Flucht und Migration nach einer ,Welle der Solidarität' im Jahr 2015 schnell zu einer ,Sicherheitsfrage' (vgl. Scheibelhofer 2017).

Dieser Diskurs ist auch im schulischen Kontext, insbesondere in Schulbüchern, sichtbar. Im deutschsprachigen Raum wird das Thema Migration in Schulbüchern überwiegend mit einem diskriminierenden und exkludierenden Sprachgebrauch behandelt und durch mit Stereotypen aufgeladene Abbildungen illustriert (vgl. u.a. Hintermann, Markom, Weinhäupl \& Üllen 2014; Niehaus, Georgi, Hoppe \& Otto 2015). Zudem werden auch ein Differenzparadigma (,Wir' und ,NichtWir ${ }^{`}$ ) und die Legitimierung von Hierarchisierung sichtbar (vgl. ebd.).

Diese überwiegend problematisierende Darstellung spiegelt jedoch weder die Realität von Einwanderungsgesellschaften noch die der migrationsbedingten hetero- 
genen Schulklassen wider (vgl. Ahlrichs 2015). Um diese Nachteile von Schulbüchern auszugleichen, setzen laut Ahlrichs (ebd.) Lehrkräfte häufig selbst erstellte Materialien oder Materialien aus dem Internet ein (vgl. auch Neumann 2015). Bei Internetmaterialien wird besonders ihre Aktualität, einfache Verfügbarkeit und Angebotsbreite geschätzt (vgl. ebd.). Diese Materialien haben aber zumeist keinen Bezug zu Kerncurricula, kaum theoretische Fundierung, sind zeitlich begrenzt (partikularer Einsatz) und erlauben selten Rückschluss auf den Kontext und die Zielsetzung des Entstehens (vgl. Kater-Wettstädt 2018). Internetmaterialien zum Thema Flucht und Migration richten sich zudem überwiegend an die Sekundarstufe und vernachlässigen die Primarstufe (vgl. ebd.). Kinder mit Fluchterfahrung machen jedoch nicht erst in der Sekundarstufe Diskriminierungserfahrungen im schulischen Kontext (vgl. IDB 2020). Die Thematik durchzieht alters- und schulstufenunabhängig den pädagogischen Schulalltag in seiner Gesamtheit. Insbesondere ethnische Herkunft und Religionszugehörigkeit stellen Angriffspunkte dar (vgl. Sauer \& Ajanovic 2012), die durch negative und tendenziöse mediale Darstellung von Menschen auf der Flucht begünstigt werden (vgl. Bešić, GasteigerKlicpera, Buchart, Hafner \& Stefitz 2020). Das Recht auf Bildung ohne jede Diskriminierung aufgrund von unterschiedlichen Identitätsmerkmalen steht laut der Inklusionsdefinition der UNESCO (2005) allen Personen zu. Daher ist es wichtig, die Themen Flucht und Migration frühzeitig, also bereits in der Primarstufe, zum Bildungsgegenstand zu machen und den vorherrschenden Problemdiskurs kritisch zu hinterfragen. Die angesprochenen Problematiken wurden im Rahmen des Projektes ,Das Boot' explizit für das Grundschulalter adressiert, welches nach einer nachfolgenden einleitenden Beschreibung des zentralen Projektgegenstandes, dem Buch ,Das Boot: Eine Fluchtgeschichte', erläutert wird.

\section{Das Buch}

,Das Boot: Eine Fluchtgeschichte' (vgl. Bešić \& Bešić 2019) ist ein Bilderbuch im Umfang von 19 Seiten mit einem Textvolumen von 206 Wörtern, illustriert mit 13 abstrakt gehaltenen Bildern. Die Erzählung folgt der Reise eines Babys, das von seiner Mutter in ein Boot gelegt wird und darin davonsegelt, um ein besseres Leben zu finden (siehe Abb. 1).

Diese zeitlose Hoffnung auf ein besseres Leben wird am Ende des Buches mit dem Satz „Wir wollen doch nur ein besseres Leben“ (ebd., 20) manifestiert. Dieser Schlusssatz wird zum Ausgangspunkt eines neuerlichen Aufbruchs ins Ungewisse, an dessen Beginn die Frage steht: ,Was passiert als Nächstes?'. Hierauf möchte das Bilderbuch keine endgültige Antwort bieten, sondern explizit neue Fragen provozieren, die die Leser*innen zu einer tieferen Reflexion der eigenen Werte und 


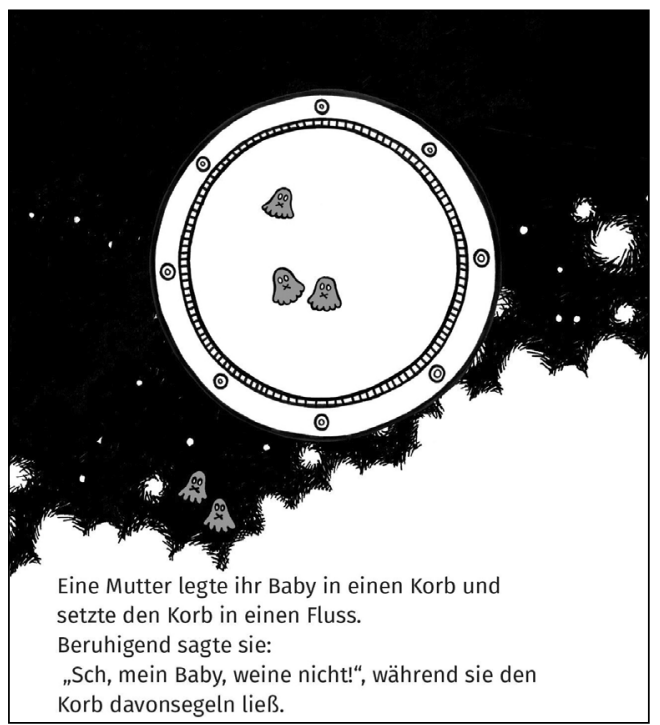

Abb. 1: ,Das Boot: Eine Fluchtgeschichte - Beginn der Erzählung (Bešić \& Bešić 2019, 1)

Normen sowie der gesellschaftlichen Wirklichkeit anregen sollen (vgl. Melrose 2019, o.S.): „Was denkst du, passiert als Nächstes?“ oder „Was sollte als Nächstes passieren?"“

Die Geschichte greift klassische Narrative auf (Moses-Zyklus) und bietet einen schulischen Thematisierungsrahmen für aktuelle Ereignisse und Berichte, mit denen Schüler*innen aller Altersstufen tagtäglich konfrontiert werden, wie z.B. Pressemeldungen über den dreijährigen Aylan Kurdi, der im Jahr 2015 an der türkischen Küste ertrank (vgl. Faller 2019).

\section{Das Forschungsprojekt}

Dieses Buch bildet den zentralen Gegenstand im begleitenden Forschungsprojekt (2019-2020) sowie die Ausgangsbasis für die Auseinandersetzung mit dem Thema Flucht. Das vorrangige Ziel bestand darin, die Thematik spezifisch in der Primarstufe explorativ zu erforschen und dazu innovative, praxisrelevante und wissenschaftlich fundierte Unterrichtsmaterialien zu entwickeln. Dabei wurden die Möglichkeiten und Grenzen, die sich aus der Arbeit mit dem Buch für Lehrpersonen (im Folgenden: LP) ergeben, sowie die Frage, ob bzw. wie das Thema Flucht in der Primastufe erfolgreich adressiert werden kann, fokussiert. 


\subsection{Methodischer Hintergrund und Setting}

Für die Realisierung dieser forschungsleitenden Zielsetzungen wurde der DBRAnsatz gewählt (vgl. McKenney \& Reeves 2018), weil bei diesem, neben der grundlegenden Beforschung des Themenfeldes und der Gewinnung von wissenschaftlichen Erkenntnissen, explizit auch das Erzielen von bildungspraktisch relevanten Ergebnissen und das Designen von einsatzfähigen bzw. praxisrelevanten Artefakten zentral sind (vgl. ebd.).

Folgend der DBR-Methodik wiederholten sich im Laufe des Projekts im Rahmen eines iterativen Vorgehens die systematische Entwicklung, Durchführung, Überprüfung und Neugestaltung (vgl. ebd.) der Unterrichtsmaterialien. Dieser Prozess wurde über das erste Halbjahr des Schuljahres 2019/20 hinweg durchgeführt. Dabei standen die beteiligten LP in der Rolle von Designerinnen im Vordergrund. Die Forschungsteammitglieder fungierten primär als Inputgeberinnen, indem sie wissenschaftliche Erkenntnisse zur Design-Methodik einbrachten und gezielte Impulse für den Fortgang des Entwicklungsprojektes und der Begleitforschung setzten. Die Schüler*innen (im Folgenden: SuS) waren durch ihr unmittelbares Feedback zu den Materialien ebenfalls in der Rolle von Inputgeber*innen aktiv. Die systematische wissenschaftliche Begleitung realisierte sich mittels teilnehmender Unterrichtsbeobachtungen und dokumentierter Reflexionsgespräche. Die Unterrichtsbeobachtungen $(n=5)$ fanden zu Beginn der Auseinandersetzung im Unterrichtsgeschehen mit dem Buch bzw. begleitend zum Einsatz der ersten entwickelten Materialien statt. Ziel der Beobachtungen war es, einerseits die Interaktion der SuS mit dem Buch und den Materialien zu erfassen und andererseits ihre offenen Fragen zu dokumentieren, die in weiteren zu entwickelnden Unterrichtsmaterialien aufgegriffen und behandelt werden sollten.

Die Reflexionsgespräche $(n=5)$ fanden zwischen den LP und den Forscherinnen während des Schulhalbjahres einmal im Monat statt. In diesen Gesprächen wurden die Rückmeldungen der SuS und die Erfahrungen der LP während der Arbeit mit dem Buch und der Materialerstellung diskutiert und seitens der Forscherinnen schriftlich dokumentiert.

\subsection{Stichprobe}

Am Projekt waren fünf LP aus drei steirischen Volksschulen mit jeweils einer Klasse (mit insgesamt $110 \mathrm{SuS}$ ) sowie zwei Forscherinnen beteiligt. Jeweils zwei LP unterrichteten eine zweite bzw. eine dritte Klasse und eine LP eine erste Klasse. Den Zugang zu den beteiligten LP erhielten die Forscherinnen durch eine LP, mit der sie bereits in anderen Projekten zusammenarbeiteten. In diesem Projekt fungierte diese LP gewissermaßen als, Gatekeeper'. Dadurch wurde nicht nur der Zugang zum Forschungsfeld erleichtert, sondern auch die Selbstaktivierung für das Projekt sowie eine konstruktive Zusammenarbeit zwischen den Beteiligten gewährleistet. 


\section{Edvina Bešić und Katharina Maitz}

Die involvierten LP berichteten, dass das Thema Flucht in ihren Klassen nicht nur aufgrund der Klassenzusammensetzung (in jeder Klasse gab es mindestens ein Kind mit Fluchterfahrung), sondern auch aufgrund der medialen und gesellschaftlichen Präsenz relevant sei. Daher war es ihnen wichtig, dieses Thema in ihren Klassen zu bearbeiten.

\subsection{Entwicklung und Implementierung der Unterrichtsmaterialien}

Der Designprozess der Unterrichtsmaterialien begann mit einem Treffen mit den beteiligten LP im September 2019, in dessen Rahmen das Buch vorgestellt, der Ablauf besprochen und Ideen für eine mögliche Integration des Buches in den Unterricht entwickelt wurden. Im Oktober 2019 begannen die LP - zunächst jede für sich - die ersten Materialien bzw. Methoden zu entwickeln und im Unterricht zu erproben. Die so entstandenen Materialien wurden in ständigem Austausch mit den anderen teilnehmenden LP im Anschluss zur weiteren Erprobung zur Verfügung gestellt. Während dieser Zeit trafen sich die LP einmal pro Woche für ca. eine Stunde, um die entwickelten Materialien und das weitere Vorgehen zu besprechen. Zentral war hierbei, dass alle entwickelten Materialien in direktem Bezug zu den Inhalten und Zielen des VS-Lehrplans (vgl. BMBWF 2020) standen, um eine sinnvolle Integration in den Unterricht zu ermöglichen.

Nach dem jeweiligen Einsatz der einzelnen Materialien im Unterricht wurde das Feedback der SuS im Rahmen eines Sesselkreises eingeholt und dokumentiert. Dabei wurde gefragt, was den SuS bei der Implementierung gefallen hat, was nicht und was sie anders machen würden. Von den SuS kamen zahlreiche Vorschläge, sowohl thematischer als auch methodischer Natur (z.B. Stunde zur Bildnerischen Erziehung, in der sie ,Willkommensplakate malen können; Partner*innen- vs. Gruppenarbeit). Die LP machten sich außerdem Notizen zur Eigenreflexion der Unterrichtseinheiten (was hatte ihrer Meinung nach gut funktioniert, was nicht?). Neben dem Feedback der SuS und der eigenen Unterrichtsreflexion flossen auch die Mitschriften aus den Unterrichtsbeobachtungen in die Materialentwicklung ein.

So wurde beispielsweise, als das Buch vorgelesen wurde, beobachtet, dass die SuS spezifisch die Frage ,Wieso ein besseres Leben, was war falsch mit dem alten?" stellten. Daraus entstanden Diskussionen darüber, was Menschen dazu bewegt, ihr Heimatland zu verlassen oder eine Mutter, ihr Kind allein auf den Weg in ein neues Land zu schicken. Als Reaktion darauf wurden Materialien entwickelt, die explizit diese Fragen aufgriffen (z.B. zum Thema ,Push- und Pull-Faktoren').

Im Dezember 2019/Januar 2020 wurden die entstandenen und erprobten Materialien von der LP in der Gatekeeper-Funktion zu einem Materialpaket zusammengestellt und mit dem Forschungsteam in dieser präfinalen Version kritisch diskutiert, evaluiert und schlussendlich konsensual vor dem Hintergrund der Zielsetzungen des Forschungsprojektes validiert. 


\section{4 ,Das Boot ${ }^{\star}-$ Materialien}

Das Materialpaket bietet in seiner aktuellen Fassung Produkte, die jeweils zu unterschiedlichen Phasen in der Arbeit mit dem Buch (vor, während und nach der Arbeit mit dem Buch) eingesetzt werden können. Sie bestehen aus einseitigen Beschreibungen von Unterrichtsstunden im A4-Format und beinhalten die Rubriken ,Übersicht', ,Ablauf der Stunde', ,Lehrplanbezug', ,Methodische Überlegungen' und ,Materialien' (Abb. 2).

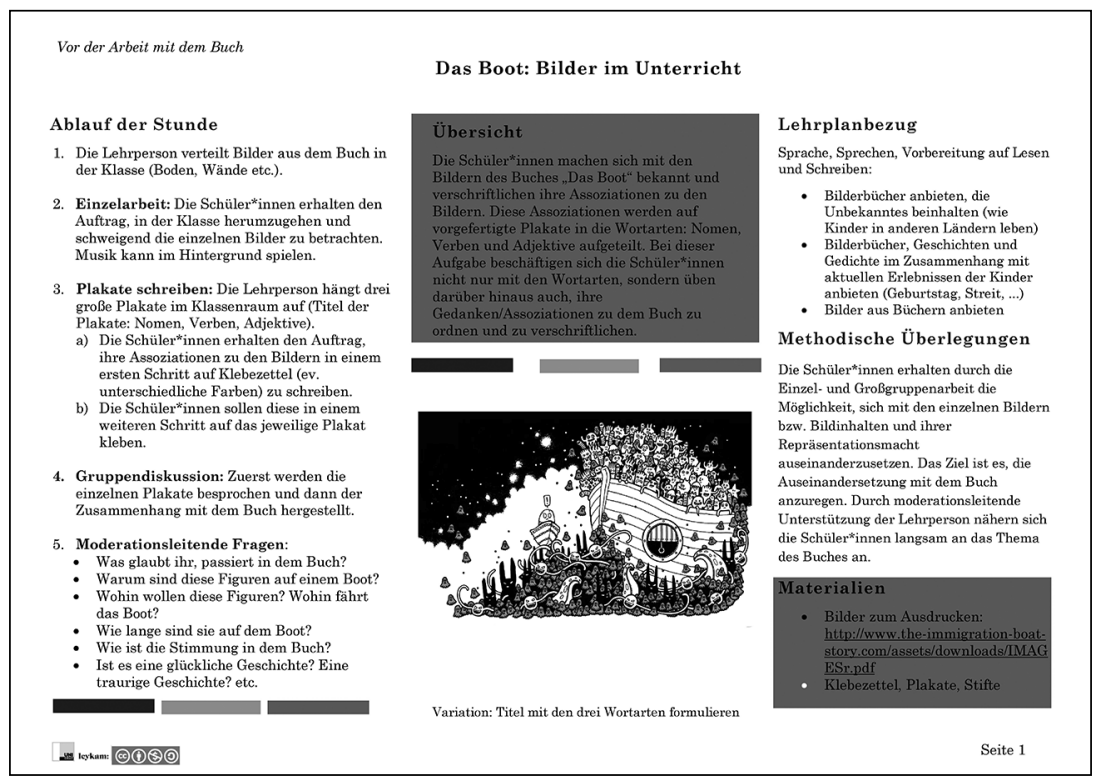

Abb. 2: Beispiel Materialien

Es wurden neun Produkte entwickelt, die sich auf den VS-Lehrplan (vgl. ebd.) beziehen und die Themenbereiche ,Erfahrungs- und Lernbereich Gemeinschaft ${ }^{\star}$ und ,Erfahrungs- und Lernbereich Zeit' im Kontext des Pflichtgegenstandes Sachunterricht fokussieren. Bei den Bildungs- und Lehraufgaben liegt der Fokus auf ,Sprache, Sprechen, Vorbereitung auf Lesen und Schreiben' und ,Deutsch, Lesen, Schreiben' im Kontext des Pflichtgegenstandes Deutsch. Daraus ergibt sich auch ein zeitgemäßes primarpädagogisches Setting für die Realisierung eines sprachsensiblen Fachunterrichts. Welche Materialien gut aufeinander aufbauen bzw. nacheinander eingesetzt werden sollten, wurde in den veröffentlichten Materialien als Empfehlung für eine zielführende methodisch-didaktische Umsetzung vermerkt. 


\section{Erfahrungen mit dem Buch und DBR}

\subsection{Auswertung}

Die während der Reflexionsgespräche entstandenen Protokolle und die von den LP verfassten schriftlichen Dokumentationen der Unterrichtseinheiten sowie der Feedbackrunden mit den SuS wurden als textliches Ausgangsmaterial definiert und computergestützt mittels strukturierender qualitativer Inhaltsanalyse induktiv ausgewertet (vgl. Kuckartz 2016). Die Codierung der Texte erfolgte im Forschungsteam in einem ersten Schritt unabhängig voneinander und in einem zweiten Schritt als ,Intercoder-Prozess' mit dem Ziel der Bearbeitung von Codierdifferenzen. Dabei wurden Kategorien gebildet, die sich entweder auf das Buch oder den DBR-Prozess beziehen. Die buchbezogenen Kategorien sind: 1) kindgerechte Darstellung von Flucht; 2) keine stereotype Darstellung von Menschen auf der Flucht; 3) Raum für (eigene) Interpretation; 4) Förderung von Empathie; und 5) Wahrnehmung von Vielfalt als Bereicherung. DBR-prozessbezogene Kategorien sind: 1) kooperatives Arbeiten mit den SuS; und 2) kooperatives Arbeiten zwischen den LP.

\subsection{Ergebnisse}

Besonders relevant für diesen Beitrag sind die Ergebnisse hinsichtlich der Frage, ob das Thema Flucht im Unterricht in der Primarstufe erfolgreich behandelt werden kann. Diese Frage wurde von den LP positiv beantwortet. Die Arbeit mit dem Buch repräsentiere eine Möglichkeit, das Thema explizit im Kontext des Grundschulalters erfolgreich behandeln zu können. Dabei wurden besonders die geringe Wortzahl und die Illustrationen positiv hervorgehoben. Außerdem zeigte sich, dass die LP durchaus überrascht waren, welches Diskussionsniveau in den Dialogen mit den SuS durch die Arbeit mit dem Buch realisiert werden konnte und dass diese auch Verbindungen zwischen dem Buch und neueren medialen Berichterstattungen über Flüchtlinge selbstständig herstellten. Die initiierten Unterrichts- und Bildungsprozesse stellten sowohl für die beteiligten $\mathrm{SuS}$ als auch LP wirkungsvolle Lernanlässe dar, die in dieser Form neuartig waren.

Des Weiteren wurden den LP zufolge die SuS dazu angeregt, einen Perspektivenwechsel vorzunehmen und so ein Verständnis für die Gründe von Flucht zu entwickeln. Somit wurden Geflüchtete entsprechend den Empfehlungen für die Unterrichtsgestaltung zum Thema Flucht und Migration von Padberg, Pichler, Hintermann und Baumann $(2016,202)$ „nicht nur als ,Objekte' im Unterricht besprochen". Zusammengefasst empfanden die LP das Buch als vielschichtige und praktische Unterrichtsgrundlage für die Bearbeitung der komplexen Thematik. Dieses repräsentiert aus ihrer Sicht eine gute Ausgangsbasis für die Entwicklung und den Einsatz von kooperativ designten Unterrichtsmaterialien und -methoden. 
Hinsichtlich der Entwicklung dieser Materialien zeigte sich, dass der DBR-Ansatz die Überwindung von Grenzen zwischen Praxis und Wissenschaft ermöglicht, indem im Rahmen des iterativen Prozesses das Feedback und somit die Interessen der $\mathrm{SuS}$ an einem Thema direkt in den Unterricht eingebracht wurden. Dies erlaubte auch, dass die Grenzen zwischen LP als Lehrende und SuS als Lernende aufgelöst wurden, da beide Gruppen in der Materialentwicklung beteiligt waren. Dadurch wurde die unmittelbare (Praxis-)Relevanz der Materialien erhöht. Ein weiterer positiver Aspekt des DBR-Ansatzes aus der Sicht der LP war die erhöhte Kooperation untereinander, welche als bereichernd empfunden wurde. Als Grenze des Ansatzes kann der große zeitliche Aufwand gesehen werden, der durch den iterativen Prozess notwendig ist.

\section{Fazit}

Im Rahmen des hier vorgestellten explorativen DBR-Projektes entstanden in enger Zusammenarbeit mit Lehrpersonen, Schüler*innen und Forscherinnen Materialien, die einen Einstieg in das Thema Flucht ermöglichen. Diese bearbeiten ein weithin präsentes, aber teilweise noch wenig bearbeitetes Thema im Schulalltag der Primarstufe. Sie zielen darauf ab, variantenreiche Möglichkeiten zu eröffnen, um mit Volksschüler*innen in einen Dialog über Flucht und Migration zu treten, die Thematik aus verschiedenen Perspektiven zu beleuchten und gerahmt vom VS-Lehrplan (vgl. BMBWF 2020) zu bearbeiten.

Die Ergebnisse des Forschungsprojektes lassen den Schluss zu, dass die Thematik Flucht in der Primarstufe zielführend, wirksam und nachhaltig im Unterricht behandelt werden kann, was langfristig die Entwicklung hin zu einer offenen, reflektierten und inklusiven Gesellschaft unterstützen kann.

\section{Literatur}

Ahlrichs, J. (2015): Migrationsbedingte Vielfalt im Unterricht: Lehrerhandeln zwischen theoretischen Ansprüchen und praktischen Herausforderungen. Braunschweig.

Bešić, E. \& Bešić, A. (2019): Das Boot: Eine Fluchtgeschichte. Graz.

Bešić, E., Gasteiger-Klicpera, B., Buchart, C., Hafner, J. \& Stefitz, E. (2020): Refugee students' perspectives on inclusive and exclusive school experiences in Austria. In: International Journal of Psychology, 55, H.5, 723-731. Online unter: https://doi.org/10.1002/ijop.12662 (letzter Zugriff: 01.02.2021).

BMBWF (Bundesministerium für Bildung, Wissenschaft und Forschung) (2020): Lehrplan der Volksschule. BGBl. 134/1963 idF II 303/2012. Online unter: https://www.bmbwf.gv.at/Themen/ schule/schulpraxis/lp/lp_vs.html (letzter Zugriff: 10.02.2021).

Faller, H. (2019): Im Namen des Sohnes. In: Zeitmagazin, Nr. 42, o.S. Online unter: https://www.zeit.de/zeit-magazin/2019/42/alan-kurdi-fluechtling-familie-erbil (letzter Zugriff: 15.11.2020). 


\section{Edvina Bešić und Katharina Maitz}

Hintermann, C., Markom, C., Weinhäupl, H. \& Üllen, S. (2014): Debating migration in textbooks and classrooms in Austria. In: Journal of Educational Media, Memory, and Society, 6, 79-106.

IDB (Initiative für ein diskriminierungsfreies Bildungswesen) (2020): Diskriminierung im österreichischen Bildungswesen: Bericht 2019. Wien.

Kater-Wettstädt, L. (2018): Unterricht zum Thema „Flucht und Flüchtlinge“? Eine Analyse von Lehrmaterialien aus dem Internet. In: Zeitschrift für Bildungsforschung, 8, 137-152.

Kuckartz, U. (2016): Qualitative Inhaltsanalyse. Methoden, Praxis, Computerunterstützung. Weinheim.

McKenney, S. \& Reeves, T. C. (2018): Conducting educational design research. London.

Mecheril, P. (2016): Migrationspädagogik - ein Projekt. In: Mecheril, P. (Hrsg.): Handbuch Migrationspädagogik. Weinheim/Basel, 8-31.

Melrose, A. (2019): The Boat. Word \& image \& refugees inhabiting the language of migration and hospitality is the ongoing story of being and becoming. In: Axon: Creative Explorations, 9, o.S.

Neumann, D. (2015): Bildungsmedien Online. Kostenloses Lehrmaterial aus dem Internet. Marktsichtung und empirische Nutzungsanalyse. Bad Heilbrunn.

Niehaus, I., Georgi, V., Hoppe, R. \& Otto, M. (2015): Schulbuchstudie Migration und Integration. Berlin.

Padberg, S., Pichler, H., Hintermann, C. \& Baumann, S. (2016): Flucht und Migration bewegt Schüler/innen, Studierende und Lehrpersonen! Geographiedidaktik und Geographieunterricht für Menschenrechte und gegen Rassismus. In: GW-Unterricht, 142, 197-205.

Sauer, B. \& Ajanovic, E. (2012): Schools as a "Protected Space"? Good Practices but Lack of Resources: the Case of Austria. In: Medarc, Z. \& Sedmak, M. (Hrsg.): Children's Voices: Interethnic Violence in the School Environment. Koper, 85-121.

Scheibelhofer, P. (2017): 'It won't work without ugly pictures': images of othered masculinities and the legitimisation of restrictive refugee-politics in Austria. In: NORMA, 12, 96-111.

UNESCO (United Nations Educational, Scientific and Cultural Organization) (2005): Guidelines for Inclusion: Ensuring Access to Education for All. Paris. 
Brigitte Kottmann

\section{Der Übergang von der Grundschule zur weiterführenden Schule als (Soll-)Bruchstelle des Gemeinsamen Lernens?}

Der Übergang von der Grundschule zur weiterführenden Schule kann als eine zentrale Gelenkstelle in der schulischen Bildungskarriere betrachtet werden. In der Bundesrepublik Deutschland (BRD) ist nach der Klasse 4 dafür ein sehr früher Zeitpunkt institutionell angelegt, in den meisten anderen europäischen Ländern erfolgt der erste Übergang nach den Klassen 6 oder 8. Im folgenden Beitrag wird das empirische Forschungsprojekt ,Kinderperspektiven auf den Übergang 4-5' beschrieben, in dem Viertklässler*innen ( $\mathrm{N}=83$ ) einer inklusiven Grundschule mittels eines Fragebogens zu ihren Perspektiven auf den bevorstehenden Übergang befragt wurden.

\section{Einführung}

An der Thematik des Übergangs von der Grundschule in die Sekundarstufe I besteht ein mehrfaches Interesse: Aus der Perspektive von Bildungsbenachteiligung unterliegt er primären und sekundären Herkunftseffekten (vgl. z.B. Dumont, Maaz, Neumann \& Becker 2014). Transitionen bergen für Kinder zudem ein erhöhtes Risiko, etikettiert und gegebenenfalls selektiert zu werden (vgl. Griebel \& Niesel 2015; Urban, Schulz, Meser \& Thoms 2015). Der Übergang wird im Kontext inklusiver Schule m.E. zu wenig berücksichtigt, damit korrespondiert eine Kritik am Konstrukt sonderpädagogischer Förder- bzw. Unterstützungsbedarf (vgl. Kottmann 2006; Kottmann, Miller \& Zimmer 2018), da die Entscheidung hierüber häufig eng mit der generellen Übergangsproblematik verbunden ist. Für Kinder mit einem sonderpädagogischen Förderbedarf (SPF) besteht somit zum Zeitpunkt des Übergangs eine erhöhte Gefahr, vom Gemeinsamen Lernen, mit dem in Nordrhein-Westfalen (NRW) inklusiver Unterricht bezeichnet wird, ausgeschlossen und einer Förderschule zugewiesen zu werden. Klemm $(2015,11)$ formuliert zuspitzend: „Nach der Grundschule ist Inklusion oft noch ein Fremdwort." Der Übergang kann auch als (Soll-)Bruchstelle des Gemeinsamen Lernens bezeichnet werden, da der Anteil der inklusiv beschulten Kinder und Jugendlichen an dieser Stelle deutlich nachlässt: Die Inklusionsanteile im Bildungssystem entsprechen bildlich einer Pyramide, so werden 67\% der Kinder mit einem SPF 
im Kita-Alter im allgemeinen System bzw. integrativ/inklusiv betreut, dann noch 44,9\% im Grundschulalter, in der Sekundarstufe I nur noch 35,3\% (vgl. Hollenbach-Biele 2016, 15, Daten für das Schuljahr 2014/15). Dementsprechend steht bei der Betrachtung der Anteile im Förderschul- bzw. sonderpädagogischen System die Pyramide auf dem Kopf.

Weiterhin ist die Thematik aus der Perspektive der Kindheitsforschung relevant. So merkt Gerarts (2017) im ,Handbuch Inklusive Kindheiten' an, dass Kinder verstärkt als Akteur*innen und Expert*innen ihre Sichtweisen und Wünsche einbringen sollten, um die Pluralität ihrer Lebenswelten erfassen zu können. Auch bei der Entscheidung über ihre zukünftige Schule werden kindliche Sichtweisen kaum, zumindest nicht systematisch, einbezogen. Mit Blick auf Inklusion fordert die Initiative ,Gemeinsam Leben, Gemeinsam Lernen NRW` (o.J., o.S.), „allen Kindern, die bereits am Gemeinsamen Unterricht teilnehmen, zukünftig einen Platz im Gemeinsamen Unterricht einer weiterführenden Schule anzubieten." Das Anbieten eines Platzes erfüllt jedoch nicht die Forderung, die Perspektive der Kinder einzubeziehen, da Kinder partizipativ an dieser Entscheidung beteiligt und ihre individuellen Situationen stärker berücksichtigt werden sollten.

\section{Der Übergang in rechtlicher und statistischer Hinsicht}

Für den Übergang werden die Weichen für Kinder mit einem SPF bereits kurz nach Klasse 3 gestellt: In NRW findet bei den Grundschulen zu Beginn des vierten Schuljahres seitens der Schulämter eine Abfrage darüber statt, bei welchen Kindern in der Klasse 5 mit einem Bedarf an sonderpädagogischer Unterstützung zu rechnen ist. Auf der Verwaltungsebene werden alsdann die Bedarfe, Ressourcen und Förderorte geklärt. Der Übergang der Kinder ohne SPF erfolgt auf der Grundlage einer (nicht verbindlichen) Empfehlung der Klassenlehrkräfte, die mit dem Halbjahreszeugnis der Klasse 4 ausgestellt wird. Im Jahr 2018 wechselten die meisten Kinder (41,7\%) zum Gymnasium, das - vermutlich aufgrund der Möglichkeit des Abiturs und des im Vergleich höheren Ansehens - die beliebteste Schulform darstellt, 27,9\% zur Gesamtschule, 20,3\% zur Realschule, 5,6\% zur Sekundar- und lediglich 3,6\% zur Hauptschule, die in NRW eine auslaufende Schulform darstellt (vgl. Ministerium für Schule und Bildung des Landes NRW 2019, 246).

Der Übergang fordert von allen Kindern erhebliche Bewältigungsressourcen, stellt aber gerade für Kinder mit einem SPF eine starke Zäsur dar. Das Ministerium formuliert:

Die „zuständige Schulaufsicht [bietet] den Eltern von Kindern mit sonderpädagogischem Unterstützungsbedarf mindestens eine geeignete allgemeine Schule an - 
wobei dies nicht immer die gewünschte Schule sein kann. Die Eltern können aber auch weiterhin die Förderschule für ihr Kind wählen. Kinder mit formal festgestelltem Bedarf an sonderpädagogischer Unterstützung (...) haben Anspruch auf Aufnahme in die von der Schulaufsicht vorgeschlagene wohnortnächste Schule der gewünschten Schulart, an der Gemeinsames Lernen eingerichtet ist" (Ministerium für Schule und Bildung des Landes NRW 2013, o.S.).

Der Vorschlag erfolgt häufig aus eher organisatorischer Perspektive. So zeigen sich u.U. gegensätzliche Interessen: Bei zieldifferenter Beschulung findet kaum Partizipation der Kinder und Eltern statt, sie bekommen in der Regel lediglich einen Vorschlag des Schulamtes. Das vermeintliche Recht des Kindes auf sonderpädagogische Förderung wiegt höher als das elterliche und kindliche Interesse an einer wohnortnahen, gut erreichbaren Schule, an einem selbstständig zu bewältigenden Schulweg, z.B. mit Nachbar*innenkindern, sowie dem persönlichen Wohlbefinden des Kindes durch den Erhalt von Peer-Beziehungen. Nach den aktuellen Regelungen ist es möglich, dass ein Kind mit SPF eine Schule vorgeschlagen bekommt, zu der kein anderes Kind aus der Klasse wechselt, und an der es niemanden kennt. Es muss einen Wechsel im Hinblick auf die Lehrer*innen- und Peer-Beziehungen, die vertrauten Gesichter, das Schulgebäude und -gelände, den Ganztag und die Mensa, den (häufig längeren) Schulweg und die Wohnortnähe bewältigen, was wiederum bedeutet: Die generellen Belastungen durch den Übergang in die Sekundarstufe I sind für ein Kind mit einem SPF deutlich größer.

\section{Der Übergang in empirischer Hinsicht}

Der Übergang ist aus der Perspektive von Lehrkräften und Eltern oder im Hinblick auf die Herstellung von Bildungsungleichheit vielfach erforscht (vgl. Dumont et al. 2014; Porsch 2018). Es zeigt sich aber, dass nur wenige Studien explizit die Perspektiven der Kinder einbeziehen. Übereinstimmend werden Freund*innen von den Kindern „als protektive Faktoren“ (Storck 2015, 99) betrachtet. Baar (2018, 44) fordert offensiv Strategien zum Erhalt der Freundschaften, diese wurden von befragten Kindern als „das Wichtigste“ (ebd., 45) bezeichnet. Es liegen meines Wissens in der BRD jedoch keine Studien vor, die explizit Kinder mit einem SPF einbeziehen. Für diese ist „der Übergang von der Grundschule auf eine weiterführende Schule mit all den beschriebenen Herausforderungen noch schwieriger zu bewältigen als für Schulkinder ohne Förderbedarf" (Mays, Zielemann, Franke, Wichmann \& Metzner 2018, 152). Der Übergang erfordert starke Anpassungsleistungen und Bewältigungsressourcen, darauf haben die individuelle, die interaktionale und die kontextuelle Ebene einen großen Einfluss (vgl. ebd.; Griebel \& Niesel 2015). Internationale Studien zeigen, dass Kinder mit SPF „spezifischere, 
auf die Transition bezogene Sorgen und Ängste entwickelten“ (Mays et al. 2018, 153), Sorgen um die Freundschaften aus der Grundschulzeit und sowohl Ängste (beispielsweise vor Isolation) als auch Vorfreude (beispielsweise auf neue Kontakte oder eine größere Bandbreite an Aktivitäten) äußerten (vgl. ebd.). Nach dem Übergang zeigen sie häufig schwächere Schulleistungen als vorher und haben ein z.T. signifikant niedrigeres schulisches Selbstkonzept (vgl. ebd., 154). Gerade das Finden neuer Freundschaften innerhalb des ersten Monats nach dem Übergang erweist sich jedoch als bedeutsam für positive Effekte u.a. auf das Selbstkonzept und die Motivation (vgl. Hattie 2014; Mays et al. 2018). Umso wichtiger ist es, diese Erkenntnisse frühzeitig einzubeziehen und positive Peer-Kontakte möglichst zu erhalten.

\section{Das Forschungsprojekt: ,Kinderperspektiven auf den Übergang 4-5}

Bei dem hier beschriebenen explorativen Projekt handelt es sich um einen ersten Zugang mit einer überschaubaren Stichprobe, insofern ist die Aussagekraft sicherlich eingeschränkt. Gleichwohl wird die aktuelle Situation von Schüler*innen im vierten Schuljahr thematisiert und ein Fokus auf Kinder mit einem ausgewiesenen SPF gerichtet. Für die Befragung wurde ein vierseitiger Paper-PencilFragebogen mit insgesamt 45 geschlossenen und offenen Fragen entwickelt (vgl. Heinzel 2012). Die Dauer der Bearbeitung variiert zwischen 10 und 40 Minuten, ist also so konzipiert, dass eine Schulstunde ausreicht; den Kindern mit Unterstützungsbedarf muss gegebenenfalls Assistenz ermöglicht werden. Konkrete Forschungsfragen sind u.a.: Wie ist die kindliche Perspektive auf den Übergang 4-5? Wie kann die aktuelle Situation der Kinder, z.B. im Hinblick auf Wohlbefinden und Selbstwirksamkeit, beschrieben werden? Welche Wünsche, Ängste und Ziele äußern sie? Der Fragebogen wurde an einer vierzügigen Grundschule in NRW in einer eher städtischen Lage pilotiert, die seit 2009/10 eine inklusive Schule ist. An der Befragung haben 83 Viertklässler*innen teilgenommen, 55 Jungen und 28 Mädchen. Sieben Kinder (fünf Jungen und zwei Mädchen) haben einen ausgewiesenen SPF mit den Förderschwerpunkten Lernen, Lernen und Emotionale und soziale Entwicklung, Geistige Entwicklung und Sprache, von ihnen sind fünf bereits im fünften Schulbesuchsjahr. Die Klassengröße variiert von 22-24 Kindern. 


\section{Ausgewählte Ergebnisse}

Emotionale Perspektive: Auf die Frage nach dem schulischen Wohlbefinden geben 64\% der Gesamtgruppe auf einer Skala von 1-10 die hohen Werte von 8-10 an, dabei äußern sich die Jungen positiver als die Mädchen. Von den Kindern mit einem SPF kreuzen vier Kinder den höchsten Wert 10 an (zwei wählen 7, eines 5). Bei der Aussage ,Ich freue mich auf die neue Schule' antworten 83\% der Gesamtgruppe in einer vierstufigen Likert-Skala mit stimmt' oder ,stimmt ziemlich', sechs der Kinder mit einem SPF äußern ,stimmt', eines ,stimmt nicht'. Die hohe Vorfreude korrespondiert vermutlich damit, dass mit dem Übergang auch ein Imagewechsel verknüpft wird und man dann,zu den Großen' gehört. Gleichwohl bedeuten die Ergebnisse aber auch, dass 32,5\% ein weniger ausgeprägtes Wohlbefinden und $15,7 \%$ weniger Vorfreude äußern. Die Aussage ,Ich habe Angst vor dem Wechsel' beantworten immerhin 19,3\% der Gesamtgruppe mit stimmt ziemlich' und 9,6\% mit ,stimmt', was fast einem Drittel der Kinder entspricht. Soziale Perspektive: Der Erhalt der Freundschaften und Beziehungen ist den Kindern sehr wichtig: Erwartungsgemäß bekräftigen $72,3 \%$ der Gesamtgruppe, 69,1\% der Jungen und 78,6\% der Mädchen sowie 100\% der Kinder mit einem SPF mit ,stimmt' die Aussage ,Ich möchte vor allem mit meinen Freunden und Freundinnen zusammen bleiben'. Antworten wie „weil da geht es mir gut und es gehen viele Freude hin“ oder „weil meine Geschwister da sind und meine Freunde“ zeigen, dass vertraute Gesichter einen hohen Stellenwert haben. Dieser Wunsch des Erhalts bestehender Kontakte findet sich auch hinsichtlich der Lehrkräfte: ,Ich würde gerne meine Lehrerinnen und Lehrer von der Grundschule behalten beantworten 67,5\% der Gesamtgruppe mit stimmt', 60\% der Jungen, 82,1\% der Mädchen und 85,7\% der Kinder mit einem SPF. Gleichzeitig formulieren sie auch Offenheit gegenüber den neuen Lehrkräften: ,Ich freue mich auf neue Lehrerinnen und Lehrer an der neuen Schule ' wird von 71,1\% mit stimmt' oder ,stimmt ziemlich' beantwortet, ohne Unterschiede hinsichtlich des Geschlechts oder eines SPFs.

Gewünschte Schulform: Von der Gesamtgruppe strebt jeweils ein Drittel (34,9\%) das Gymnasium oder die Gesamtschule an, 15,7\% die Realschule und 7,2\% die Sekundarschule, 7,2\% antworten ,weiß nicht'. Die Haupt- und die Förderschule werden von keinem Kind benannt. Weniger Jungen (29,1\%) als Mädchen $(46,4 \%)$ präferieren die Gesamtschule. Von den Kindern mit einem SPF wünschen sich drei eine Gesamtschule, zwei eine Realschule und ein Kind eine Sekundarschule, ein weiteres Kind antwortet mit ,weiß nicht'. Die Gründe variieren zwischen dem Image: „weil da schlaue Kinder sind“, dem Selbstkonzept: „weil sie zu mir passt“ sowie dem Wunsch nach Überschaubarkeit: „weil sie nur wenig Kinder hat". Im Zusammenhang mit der erhobenen Bildungsaspiration der Kinder zeigten die World-Vision-Studien mehrmals, dass Kindern die Wertigkeit bereits 
bewusst ist: „Offenbar ist den Kindern schon recht früh klar, dass die Wahl der weiterführenden Schule eine Weichenstellung für ihr weiteres Leben ist - vom Hauptschulabschluss haben sie kein positives Bild“ (Pupeter \& Wolfert 2018, 82). Dieser Aspekt wird hier bestätigt.

Insgesamt unterscheiden sich die Motive der Kinder bei der offenen Frage, Warum willst du zu dieser Schule gehen?' kaum, vor allem vertraute Gesichter spielen eine große Rolle. Die Kinder formulieren sehr konkrete Dinge, die sie mit den z.T. bereits besichtigten Schulen verknüpfen: „weil es da viel Sport gibt“ oder „weil in der Schule viel mit Musik gemacht wird“. Deutlich wird, dass die Kinder sich den Erhalt von Freundschaften und verlässliche Beziehungen wünschen, wie etwa die Äußerung: „meine Schwester ist auf der Schule“ exemplarisch zeigt. Mehrere wünschen sich aber auch eine Schule mit einem Image, ausgedrückt durch Formulierungen wie: „weil die Schule groß ist, weil die Schule schön ist, weil die Schule cool ist“. Aber auch pragmatische Gründe spielen hier eine Rolle: „ich habe schon Freunde da und die Fahrt ist besser. Die ist am nächsten“.

\section{Fazit und Limitierungen}

Auch wenn diese ersten Ergebnisse vorsichtig betrachtet werden müssen und erst wenige Rückschlüsse zulassen, zeigen sie doch, dass Kinder vor allem Kontinuität und Freundschaft zu Gleichaltrigen anstreben. Ihnen ist der bevorstehende Übergang als soziale Transition sehr bewusst. Um den Kindern mit einem SPF diesen zu erleichtern, sollte also nicht lediglich eine Schule mit Gemeinsamem Lernen vorgeschlagen werden, sondern die beteiligten professionellen Akteur*innen sollten explizit die Perspektiven der Kinder einbeziehen und deren Partizipation stärken: Die Wünsche der Kinder sollten bei der Empfehlung einen Einfluss haben, da sonst massive Diskontinuitäten und eventuell Segregation entstehen können. Ein Bruch im Gemeinsamen Lernen, wenn die Kinder nach der Klasse 4 zu einer Förderschule wechseln, kann sich zudem negativ auf ihr Selbstkonzept und ihre Peer-Beziehungen auswirken. Das Interesse einer inklusiven Schulentwicklung sollte darin liegen, Kinder und deren Partizipation zu stärken, die negativen Auswirkungen des Übergangs zu verringern bzw. ihn gänzlich abzuschaffen. Das derzeit durchgeführte Übergangsverfahren erzeugt jedoch vielmehr eine (Soll-) Bruchstelle für Kinder mit einem SPF.

Somit ist die Etablierung inklusions- und transitionssensibler Konzepte und Rahmenstrukturen notwendig, an denen Verwaltungen, Schulen, Lehrkräfte, Eltern und Kinder beteiligt sind. Welche konkreten Maßnahmen erleichtern den Kindern den Übergang, wie können Wohlbefinden, Resilienz und Selbstkonzepte gestärkt und unterschiedliche Handlungsoptionen entwickelt werden? Transi- 
tionen sollten reduziert bzw. Implikationen für gelungene Transitionen entwickelt (vgl. Mays et al. 2018) und Kooperationen der multiprofessionellen Teams gestärkt werden. Das gegliederte Schulsystem steht als exkludierendes System insgesamt infrage. So zeigt z.B. der Primus-Schulversuch in NRW, dass die Lösung des Problems weniger in einer ausdifferenzierten Gestaltung des Übergangs liegen sollte, sondern vielmehr in der Umsetzung längeren gemeinsamen Lernens, auch unter der Prämisse von Jahrgangsmischung (vgl. Stähling 2019).

Eine Fortführung bzw. Ausweitung des Forschungsprojekts wird angestrebt, um durch einen qualitativen Zugang die Perspektiven der Kinder tiefergehend zu erforschen, als dieses mit einem quantitativen Instrument möglich ist. Es wäre zudem gewinnbringend, die Sichtweisen der Kinder vor und nach dem Übergang bzw. längsschnittlich zu erheben und gegebenenfalls weitere Beteiligte (Eltern, Lehrkräfte, Assistenzen) einzubeziehen. Letzten Endes sollte das Ziel sein, Partizipation zu maximieren und Ausgrenzung zu minimieren (vgl. Booth \& Ainscow 2017).

\section{Literatur}

Baar, R. (2018): Der Übergang von der Primarstufe in den Sekundarbereich. Herausforderungen und Chancen für die Kinder. In: Lassek, M. \& Gutzmann, M. (Hrsg.): Kinder beim Übergang begleiten. Von der Anschlussfähigkeit zur gemeinsamen Verantwortung. Frankfurt, 37-46.

Booth, T. \& Ainscow, M. (2017): Index für Inklusion. Ein Leitfaden für Schulentwicklung. Weinheim/Basel.

Dumont, H., Maaz, K., Neumann, M. \& Becker, M. (2014): Soziale Ungleichheiten beim Übergang von der Grundschule in die Sekundarstufe I: Theorie, Forschungsstand, Interventions- und Fördermöglichkeiten. In: Zeitschrift für Erziehungswissenschaft, 17, 141-165.

Gemeinsam Leben, Gemeinsam Lernen NRW (o.J.): Eckpunkte für eine zukunftsfähige Schulpolitik. Online unter: http://www.gemeinsam-leben-nrw.de/sites/default/files/Schulpolitische-Eckpunkte. pdf (letzter Zugriff: 28.01.2021).

Gerarts, K. (2017): Inklusive Forschung über Kinder mit Kindern. In: Amirpur, D. \& Platte, A. (Hrsg.): Handbuch Inklusive Kindheiten. Opladen, 131-144.

Griebel, W. \& Niesel, R. (2015): Übergänge verstehen und gestalten. Transitionen in der Bildungslaufbahn von Kindern. 3. Aufl. Berlin.

Hattie, J. (2014): Lernen sichtbar machen. 2. Aufl. Baltmannsweiler.

Heinzel, F. (Hrsg.) (2012): Methoden der Kindheitsforschung. Ein Überblick über Forschungszugänge zur kindlichen Perspektive. 2. Aufl. Weinheim/Basel.

Hollenbach-Biele, N. (2016): Inklusion statt Förderschule? Zum Stand des Gemeinsamen Unterrichts in Deutschland. In: Bertelsmann-Stiftung (Hrsg.): Inklusion kann gelingen! Forschungsergebnisse und Beispiele guter schulischer Praxis. Gütersloh, 11-33.

Klemm, K. (2015): Inklusion in Deutschland, Daten und Fakten. Gütersloh.

Kottmann, B. (2006): Selektion in die Sonderschule. Das Verfahren zur Feststellung von sonderpädagogischem Förderbedarf als Gegenstand empirischer Forschung. Bad Heilbrunn.

Kottmann, B., Miller, S. \& Zimmer, M. (2018): Macht Diagnostik Selektion? In: Zeitschrift für Grundschulforschung, 11, H.1, 23-38.

Mays, D., Zielemann, H., Franke, S., Wichmann, M. \& Metzner, F. (2018): Der Übergang von der Grundschule auf die weiterführende Schule im Kontext inklusiver Bildung. Ein Blick in die For- 


\section{2 | Brigitte Kottmann}

schung und Praxis. In: Porsch, R. (Hrsg.): Der Übergang von der Grundschule auf weiterführende Schulen. Stuttgart, 139-164.

Ministerium für Schule und Bildung des Landes NRW (2013): Erstes Gesetz zur Umsetzung der VN-Behindertenrechtskonvention in den Schulen (9. Schulrechtsänderungsgesetz). Online unter: https://www.schulministerium.nrw.de/docs/Schulsystem/Schulformen/Grundschule/Gemeinsames-Lernen/index.html (letzter Zugriff: 28.01.2021).

Ministerium für Schule und Bildung des Landes NRW (2019): Das Schulwesen in Nordrhein-Westfalen aus quantitativer Sicht. 2018/2019. Statistische Übersicht Nr. 404. Düsseldorf.

Porsch, R. (Hrsg.) (2018): Der Übergang von der Grundschule auf weiterführende Schulen. Stuttgart. Pupeter, M. \& Wolfert, S. (2018): Schule: Frühe Weichenstellung. In: World-Vision Deutschland e.V. (Hrsg.): Kinder in Deutschland 2018. 4. World Vision Kinderstudie. Weinheim/Basel, 76-94.

Stähling, R. (2019): Wieso Schulen ohne Brüche von Jahrgang 1 bis 10? In: Grundschule aktuell, $147,30-33$.

Storck, J. (2015): Auswirkungen des Übergangs von der Grundschule in die Sekundarstufe I auf das Wohlbefinden und Selbstkonzept von Schülerinnen und Schülern. Kassel. Online unter: https:// kobra.uni-kassel.de/bitstream/handle/123456789/2015071348756/StorckGrundschule.pdf?sequence=1\&isAllowed=y (letzter Zugriff: 02.02.2021).

Urban, M., Schulz, M., Meser, K. \& Thoms, S. (Hrsg.) (2015): Inklusion und Übergang. Perspektiven der Vernetzung von Kindertageseinrichtungen und Grundschulen. Bad Heilbrunn. 
Anne Goldbach und Nico Leonhardt

\section{Elemente von Macht im Kontext einer inklusionssensiblen Hochschulentwicklung}

Erfahrungen aus dem Hochschulprojekt der Universität Leipzig ,Qualifizierung von Bildungs- und Inklusionsreferent*innen in Sachsen' (QuaBIS)

\section{Einleitung}

Hochschulen zeichnen sich durch viele Traditionen aus. Sie werden z.B. als Quelle von Wissen und als Innovationsmotor wahrgenommen. Zudem gilt die Hochschule als eine der beständigsten Institutionen überhaupt. Diese Beständigkeit zeigt sich jedoch auch in den unhinterfragten Traditionslinien standfester Barrieren, die durch selbstverständliche Leistungsmythen all denen einen Zugang verwehren, die nicht als ,fähig' kategorisiert werden (vgl. Powell 2016, 35ff.). Besonders deutsche Universitäten seien von einer „Aura der Exklusivität“ umgeben, so Alheit $(2014,196)$.

Ziel des QuaBIS-Projektes in Leipzig ist eine Veränderung dieser Machtstrukturen und damit eine veränderte Wissensproduktion, die zur Verankerung von inklusiver Bildung beiträgt.

Somit soll der Versuch gewagt werden, bisherige Grenzen zu überschreiten und Gänge aus einer abgeschotteten in eine bisher exklusive Welt zu ermöglichen. Im folgenden Beitrag sollen Aspekte eines alltäglichen Umgangs mit Machtfragen reflektiert und diskutiert werden, da diese unumgänglich zur gemeinsamen Arbeit gehören.

\section{Macht im Diskurs einer Pädagogik bei zugeschriebener Behinderung}

Macht ist „weder positiv noch negativ besetzt, sondern schlicht präsent. Eine Wertung kann erst im Einklang mit folgender Frage vorgenommen werden: Wie, durch wen und auf welche Weise kommt Macht zum Einsatz (und wirkt sich in weiterer Folge auf konkrete Personen aus) “ (Kremsner 2020, 10)? Dennoch wird schon mit Goffmans (1973) Beschreibungen zu ,Totalen Institutionen` für den 
Bereich der sogenannten Behindertenhilfe deutlich, dass Bewohner*innen solcher Institutionen über vergleichsweise sehr wenig Macht verfügen, gleichzeitig jedoch subtiler und direkter Machtausübung ausgesetzt sind (vgl. Kremsner 2020), worüber sich weder die Menschen mit zugeschriebener Behinderung noch die Professionellen bewusst sind (vgl. Kremsner 2017). Es gilt deshalb umso mehr, und insbesondere für inklusionssensible Entwicklungen auch innerhalb der exklusiven Institution Hochschule, eben diese Prozesse aufzudecken, um damit Reflexionsund Veränderungsmöglichkeiten zu schaffen.

Aufgrund der großen Vielfalt an theoretischen Zugängen zu Macht im Kontext einer Pädagogik bei zugeschriebener (geistiger) Behinderung ist es für die hier vorgenommene Auseinandersetzung zielführend, sich auf zwei machtspezifische Phänomene zu konzentrieren.

In einem ersten Fokus stehen demnach Othering-Prozesse, die zu einer kategorialen Zuschreibung und Konstruktion fundamentaler Andersheit (vgl. Boger \& Textor 2016) führen, wodurch ableistische Diskriminierung hervorgerufen wird. Solche Prozesse der Veranderung ${ }^{1}$ gründen in einer gesellschaftlich fest verwurzelten, hierarchischen und asymmetrischen Differenzordnung sowie damit verbundenen Herrschaftsverhältnissen (vgl. Riegel 2016). So kann bspw. mit Foucault davon ausgegangen werden, dass die Markierung einer Behinderung als Abweichung von der Norm erst durch den (wissenschaftlichen) Diskurs hervorgebracht wird und nicht per se besteht (vgl. Foucault, Seitter \& Konersmann 1996).

Der Begriff Othering beschreibt diesen Prozess des ,Different-Machens' (vgl. Castro Varela \& Dhawan 2005) und die damit verbundene Konstruktion der Anderen.

Für Menschen mit zugeschriebenen Behinderungen folgt aus dieser Zuschreibung häufig ein Ausschluss aus der allgemeinen normalisierten Gesellschaft und ein damit verbundener Einschluss in Hegemonialapparate der sogenannten Behindertenhilfe (vgl. Kremsner 2017), die, wie oben bereits beschrieben, selbst wiederum von asymmetrischen Beziehungen, Abhängigkeitsverhältnissen und Unterdrückung geprägt sind (vgl. u.a. Flieger 2015; Kremsner 2017; Kremsner 2020). Butler (2006) weist darauf hin, dass die Aufrechterhaltung und Reproduktion dieser Machtverhältnisse gesichert sind, weil Menschen, die immer wieder als ,Andere' markiert werden, sich selbst folglich auch als anders wahrnehmen. Im Sinne Spivaks kann dem hinzugefügt werden, dass ,im imperialen Macht- und Wissensdiskurs die Perspektiven und Stimmen von marginalisierten Anderen nicht gehört werden bzw. diese sprachlos gemacht werden" (Riegel 2016, 53) und sie dementsprechend auch wenig Möglichkeiten haben, auf ihre Situation aufmerksam zu machen, geschweige denn diese zu verändern.

1 Veranderung ist als deutscher Begriff zu Othering zu verstehen. 
Damit soll auf Subalternität als ein zweites machtspezifisches Phänomen (vgl. Spivak 2008) eingegangen werden. Subalterne (unterdrückte Gruppen) sind Menschen, die sich aufgrund ihrer Vorerfahrungen nicht trauen, zu sprechen, die nicht sprechen dürfen oder deren Stimme(n) einfach nicht gehört oder ernstgenommen werden (vgl. Kremsner 2017). Sprache wird dabei als ein Machtinstrument im Hinblick auf verschiedene Facetten theoretisch beleuchtet. Poststrukturalistisch lässt sich argumentieren, dass durch Sprache Realitäten erst hergestellt werden und Sprache somit ein großes Machtpotenzial innehat. Auch aus diskurstheoretischer Perspektive wird argumentiert, dass erst in (sprachlichen) Diskursen „implizit Dominanzordnungen, Normen und Ideologien zum Ausdruck gebracht [werden], die auf den hegemonialen Kontext und Herrschaftsverhältnisse verweisen“" (Riegel 2016, 28). Auch Butler (2006) beschreibt Sprache als ein Mittel der Macht zur Ausgrenzung und Herabsetzung von Menschen, gleichzeitig verweist sie jedoch auch darauf, dass eben jene performative Reproduktion von Differenzordnungen durch Sprache auch Potenzial hat, Widerstand und Veränderung hervorzurufen. Riegel (2016) verweist auf dieses Widerstandspotenzial beispielweise insofern, als dass „absichtliche Irritationen der Norm“ (ebd., 31) dazu beitragen können, eben solche Normalitätsgrenzen zu verschieben und zu verändern.

\section{Inklusionssensible Hochschulentwicklung}

So könnte beispielsweise inklusionssensible Hochschulentwicklung eine Möglichkeit darstellen, diese Performanz zu leisten, damit die Subalternität einzelner Menschen mit Behinderungserfahrung aufzuheben und ihre Stimme bedeutsamer werden zu lassen. Subjektperspektiven von Menschen mit Behinderungserfahrungen sollten ein ,Mehr' an gesellschaftlicher Sichtbarkeit und politischer Wirkmacht entfalten. Erst dann können gesellschaftlich gesetzte Standards, die Exklusion an sich erst reproduzieren (vgl. Kronauer 2002), stärker infrage gestellt und verändert werden.

Der Aufforderung zur Umsetzung inklusiver Hochschulentwicklung stellen sich die Hochschulen als Bildungs-, Ausbildungs- und Forschungseinrichtungen mit Bezug zur UN-Behindertenrechtskonvention zunehmend und bringen sich in diesen Diskurs allmählich ein: durch veränderte barrierefreie Studienbedingungen, inklusionsbezogene Hochschulforschungen, die Entwicklung verschiedener Hochschulstrategien/-aktionspläne und verschiedene andere Öffnungsprozesse (vgl. Dannenbeck, Dorrance, Moldenhauer, Oehme \& Platte 2016). Der Fokus der Maßnahmen in Deutschland beschränkt sich derzeit jedoch noch weitgehend auf das Feld Studium und Behinderung (vgl. Schuppener, Goldbach, Leonhardt, Langner \& Mannewitz 2020). Auch Hochschulen sind Räume pädagogischer Pra- 
xen. Diese „repräsentieren und konstituieren Machtverhältnisse, und sie werden von ihnen bestimmt. Darüber hinaus sind diese Praktiken in sozialen Räumen organisiert und gestalten soziale Räume“ (Bramberger \& Plaute 2018, 18).

Um der Hochschule inhärente Machtstrukturen und -verhältnisse offenzulegen, braucht es einen reflexiven Blick auf eben diese Differenzverhältnisse (vgl. ebd., 23).

Diversität und Partizipation werden im Kontext inklusiver (Hochschul)Bildung bislang primär auf der Ebene von Lernenden betrachtet (Heterogenität der Schüler*innenschaft, der Studierenden), weniger auf der Ebene der Lehrenden. Mit dem Anspruch einer Weiterentwicklung und diversitätsbewussten Öffnung von Hochschule sollte der Blick jedoch auch darauf gelenkt werden, welche Rolle beispielsweise Menschen mit Behinderungserfahrungen als Wissensvermittler*innen und damit Produzent*innen im akademischen Raum im Rahmen von Lehr- und Forschungstätigkeiten leisten können. Dieser Perspektiverweiterung widmet sich das Projekt QuaBIS, in welchem seit Mai 2019 u.a. an der Universität Leipzig sechs Menschen, die überwiegend vorher in einer Werkstatt für behinderte Menschen (WfbM) gearbeitet haben, zu sogenannten Bildungs- und Inklusionsreferent*innen in einer dreijährigen Qualifizierung ausgebildet werden. Sie sollen zukünftig Studierenden sowie Lehr-, Fach- und Führungskräften beispielhaft vermitteln, wie Inklusion und Diversität theoretisch zu verstehen sind, reflektiert werden können und praktisch funktionieren (vgl. Schuppener et al. 2020). Indem im Projekt QuaBIS sechs Menschen mit Behinderungserfahrungen eine Stimme im Kontext der Hochschullehre erhalten, tragen sie dazu bei, hochschultypische Machtstrukturen aufzubrechen und universitäre Machtansprüche durch in Gang gesetzte Empowermentprozesse besser zu verteilen (vgl. Noack 2003, 4f.). Ziel des Projektes ist es daher, eine Veränderung von Machtstrukturen und damit eine veränderte Wissensproduktion, die zur Verankerung inklusiver Bildung beiträgt und die von Butler (2006) beschriebenen ,Irritationen der Norm` befördern.

\section{Projekterfahrungen im Kontext machtvoller Strukturen}

Im Folgenden soll anhand von Aussagen der Bildungs- und Inklusionsreferent ${ }^{*}$ innen über eigene Machterfahrungen im Rahmen der Qualifizierung an der Hochschule reflektiert werden. Insbesondere auch darüber, inwiefern die derzeitigen Strukturen einer inklusionsorientierten Hochschulentwicklung eine Möglichkeit bieten, Machtverhältnisse zu hinterfragen und zu verändern. 


\subsection{Gewollt oder ungewollt stattfindende Othering-Prozesse}

Ein Teilnehmer der Qualifizierung schildert wahrgenommene Othering-Prozesse im Kontext der Hochschule wie folgt:

„Mein Empfinden ist, gerade in den Seminaren war es nur in einem Seminar, wo das Gefühl aufkam, dass man so klein ist, weil doch die meisten Studenten sowas Überlegenes hatten. Da hat man gemerkt, dass die einen Gymnasiumabschluss hatten und das haben die auch gezeigt, dass die so den Blick von oben herab haben. Es gibt aber auch andere Seminare, wo man das Gefühl hatte, dass man den gleichen Stand hatte."

Sehr deutlich kommt in diesem, aber auch in allen anderen noch folgenden Zitaten ein verinnerlichtes ,Anderssein“ zum Ausdruck (vgl. Kremsner 2020). Das Gefühl zu haben, „dass man so klein ist“, deutet auf vielfältige Erfahrungen von Unterdrückung hin. Die eigene Zuschreibung zur Gruppe der Anderen kann dabei als eine Folge der sozialen Wirkkraft hegemonialer Diskurse gedeutet werden (vgl. Butler 2006). Die als ,anders' Markierten bedienen sich dabei in gleicher Weise der Argumentationen derer, die sie als anders konstruieren (vgl. Riegel 2016). Ebenso wird ersichtlich, dass das eigene Anderssein erst in Bezug auf das Normalitäts-Dispositiv des Gymnasialabschlusses als relevant beschrieben und davon abgeleitet wird (vgl. ebd.). Trotz aller Kritik an der gesellschaftlich etablierten Praxis einer Unterscheidung in ,die Normalen' und ,die Anderen' ist zu hinterfragen, wie realistisch eine Auflösung von Normalitätsgrenzen im Sinne eines Transnormalismus tatsächlich ist (vgl. Boger 2019), oder ob es nicht vielmehr eine fortwährende Aufgabe ist, flexibelnormalistische Grenzen kritisch zu hinterfragen und anzupassen. So wird am oben genannten Zitat genauso deutlich, dass bestehende Differenzen nicht zwangsläufig zu einer wahrgenommenen Veranderung führen, sondern ein gemeinsames Studieren auf Augenhöhe ebenso möglich ist.

Eine Teilnehmerin beschreibt in ihren Gedanken über wahrgenommene Machterfahrungen im Bereich der Hochschule:

„Bei den jüngeren Studenten merkt man auch, dass die versuchen, ihre Macht auszuloten, unbewusst. Wenn die mich mit Dingen überbieten können, wenn die was sagen, wo ich dann nicht mitreden kann, weil ich kein Verständnis dafür hab.“

Neben dem auch hier deutlich werdenden verinnerlichten eigenen Anderssein verweist sie auf die von ihr wahrgenommene asymmetrische Beziehung und damit verbundene Machtverhältnisse zwischen „den jüngeren Studenten“ und ihr selbst als Bildungs- und Inklusionsreferentin. Sie erlebt, dass dieses Machtungleichgewicht zur Abgrenzung von ihr als ,Andere“, die scheinbar „kein Verständnis dafür“ hat, genutzt wird. Hierin verweist sie auch auf das Machtpotenzial von nicht angemessener Sprache, durch welche Ausgrenzung und damit Othering erfolgt. 


\section{Anne Goldbach und Nico Leonhardt}

\subsection{Erfahrungen mit Subalternität oder deren Auflösung}

In einigen Aussagen der Bildungs- und Inklusionsreferent*innen werden Erfahrungen mit Subalternität deutlich, die sowohl im derzeitigen Kontext der Qualifizierung an der Hochschule sichtbar werden als auch ihren Ursprung in früheren biografischen Erfahrungen haben. In Bezug auf Subalternität im Hochschulkontext sagt eine Teilnehmerin:

„Ich hab's halt schon gemerkt, dass einige Dozenten schon zeigen, dass sie hier Macht haben und mir zeigen: Da ist dein Platz, du hast in dem Sinne gar nichts zu sagen."

Auf der anderen Seite weist sie jedoch auch darauf hin, dass es ungewohnt ist und zum Teil eine Überforderung darstellt, plötzlich gehört und geachtet zu werden:

„Das Krasse war für mich, wo Saskia [Professorin im Fachbereich] mich als Kollegin hingestellt hat. Das hat noch nie jemand zu mir gesagt. Ich kannte das nicht, sonst hatte ich immer jemanden, der höher über mir stand und mich das hat auch spüren lassen und hier wird man so gleich behandelt. Ich hab auch Angst, den Anforderungen hier nicht gerecht zu werden, weil wenn mich Studentinnen fragen, wo ich mich machtlos fühle, wo ich nicht weiß, wie ich reagieren soll.“

In diesen Aussagen wird deutlich, dass sich durch die Erfahrungen von performativer Unterdrückung ein subalternes Selbstbild entwickeln kann (vgl. Butler 2006; Kremsner 2017; Spivak 2008), welches dazu führt, dass die Verschiebung bisheriger Grenzen bzw. Ordnungen gleichzeitig mit einem Moment der Ohnmacht einhergehen kann und Zugehörigkeit möglicherweise als brüchig wahrgenommen wird.

\section{Fazit/Ausblick}

Die untersuchten Zitate haben gezeigt, dass Differenzwahrnehmungen und eine damit verbundene Zuschreibung als ,Andere* $r^{*}$ eine Bedeutung für die erlebte Praxis im inklusionsorientierten Hochschulprojekt QuaBIS haben. Vor dem Hintergrund dessen, dass ohnehin zu hinterfragen ist, ob die Auflösung von Normalitätsgrenzen im Sinne des Transnormalismus möglich ist, sollte viel eher versucht werden, die Bedingungen herauszuarbeiten, die dazu führen können, dass Differenzen keine Otheringprozesse auslösen, sondern anerkennendes Miteinander ermöglichen. Die Zugehörigkeit zu einem bisher exklusiven System wie der Hochschule allein garantiert noch keine reale Verschiebung von Grenzen bzw. Ordnungen. Um diese Gänge über Grenzen hinweg zu durchschreiten, bedarf es viel mehr einer Reflexion der genannten Machtmechanismen. 
Es wurde deutlich, dass Sprache dabei als Mittel der Macht fungieren kann, um Ausgrenzung zu produzieren und Beteiligung zu verwehren. Butler sieht jedoch in der Performation auch eine Möglichkeit für Veränderung und Widerstand, durch performative „Irritation der Norm, der bewussten Verschiebung von Grenzen oder der Inszenierung von Zugehörigkeiten bzw. Nicht-Zugehörigkeiten bis hin zu Veränderungen vorherrschender Ordnungen“" (Riegel 2016, 31).

Durch die Beteiligung der Bildungs- und Inklusionsreferent*innen sollen eben solche Veränderungen angestoßen und reflektiert werden. Eine Aussage eines weiteren Teilnehmers zeigt, dass die Möglichkeit einer solchen (empowernden) Grenzverschiebung durchaus wahrgenommen wird:

„Das ist emotional die Uni. So wie ich das ganze [als] Komplex angucke, dann ist sie richtig machtvoll. Dann würd ich das so sehen, das ist ein Komplex. Eh ... die Fakultät ... und die Fakultät ... wo ich das Projekt, Gemeinsam Forschen ${ }^{2}$ gemacht hatte, ich habe dann irgendwie, irgendwann gemerkt, am ersten Tag: Uni: ,Wow, das ist ein riesen Teil!' Ich habe mich dann selbst - mich als Macht empfunden ... Wenn ich mir den ganzen Bereich der Universität angucke. Sie strahlt mir in dem Blickwinkel, strahlt genau dasselbe aus wie bei uns. Das wirkt bei uns mächtig und daraufhin strahlen wir diese selbe Macht aus, wie die Universität."

\section{Literatur}

Alheit, P. (2014): Die Exklusionsmacht des universitären Habitus. Exemplarische Studien zur „neuen deutschen Universität“. In: Ricken, N., Koller, H.-Ch. \& Keiner, E. (Hrsg.): Die Idee der Universität - revisited. Wiesbaden, 195-208.

Boger, M.-A. (2019): Theorien der Inklusion. Die Theorie der trilemmatischen Inklusion zum Mitdenken. Münster.

Boger, M.-A. \& Textor, A. (2016): Das Förderungs-Stigmatisierungs-Dilemma - Oder: Der Effekt diagnostischer Kategorien auf die Wahrnehmung durch Lehrkräfte. In: Amrhein, B. (Hrsg.): Diagnostik im Kontext inklusiver Bildung - Theorien, Ambivalenzen, Akteure, Konzepte. Bad Heilbrunn, 79-97.

Bramberger, A. \& Plaute, W. (2018): Bildungstheoretische Begründung einer inklusiven Hochschule. In: Harter-Reiter, S., Plaute, W. \& Schneider-Reisinger, R. (Hrsg.): Inklusive Hochschule. Diskursbausteine offener Hochschulbildung aus Theorie, Praxis und Forschung. Innsbruck, 17-24.

Butler, J. (2006): Hass spricht: Zur Politik des Performativen. Frankfurt am Main.

Castro Varela, M. d. M. \& Dhawan, N. (2005): Postkoloniale Theorie. Eine kritische Einführung. Bielefeld.

Dannenbeck, C., Dorrance, C., Moldenhauer, A., Oehme, A. \& Platte, A. (2016): Inklusionssensible Hochschule. Zur Einführung in diesen Band. In: Dannenbeck, C., Dorrance, C., Moldenhauer, A., Oehme, A. \& Platte, A. (Hrsg.): Inklusionssensible Hochschule. Grundlagen, Ansätze und Konzepte für Hochschuldidaktik und Organisationsentwicklung. Bad Heilbrunn, 9-21.

Flieger, P. (2015): Verteilt Leicht Lesen die Macht neu? In: Candussi, K. \& Fröhlich, W. (Hrsg.): Leicht Lesen. Der Schlüssel zur Welt. Wien/Köln \& Bonn, 143-154.

Foucault, M., Seitter, W. \& Konersmann, R. (1996): Die Ordnung des Diskurses. Frankfurt am Main.

2 Partizipatives Forschungsprojekt (2009-2011) an der Universität Leipzig (ESF) 


\section{Anne Goldbach und Nico Leonhardt}

Goffman, E. (1973): Asyle. Über die soziale Situation psychiatrischer Patienten und anderer Insassen. Frankfurt am Main.

Kremsner, G. (2017): Vom Einschluss der Ausgeschlossenen zum Ausschluss der Eingeschlossenen. Biographische Erfahrungen von so genannten Menschen mit Lernschwierigkeiten. Bad Heilbrunn.

Kremsner, G. (2020): Gewalt und Machtmissbrauch gegen Menschen mit Lernschwierigkeiten in Einrichtungen der Behindertenhilfe. In: Teilhabe, 59, 10-15.

Kronauer, M. (2002): Exklusion. Die Gefährdung des Sozialen im hoch entwickelten Kapitalismus. Frankfurt am Main/New York.

Noack, W. (2003): Wir müssen uns den Machtverhältnissen in der sozialen Arbeit stellen! In: Theorie und Praxis der Sozialen Arbeit, 54, H.6, 4-10.

Powell, J. J. W. (2016): Von Ableismus zur universal design Universität. In: Dannenbeck, C., Dorrance, C., Moldenhauer, A., Oehme, A. \& Platte, A. (Hrsg.): Inklusionssensible Hochschule. Grundlagen, Ansätze und Konzepte für Hochschuldidaktik und Organisationsentwicklung. Bad Heilbrunn, 34-51.

Riegel, Ch. (2016): Bildung - Intersektionalität - Othering. Pädagogisches Handeln in widersprüchlichen Verhältnissen. Bielefeld.

Schuppener, S., Goldbach, A., Leonhardt, N., Langner, A. \& Mannewitz, K. (2020): Inklusion inklusiv vermitteln: Menschen mit Behinderungserfahrungen als Lehrende an der Hochschule. In: Schneider-Reisinger, R. \& Oberlechner, M. (Hrsg.): Diversitätssensible PädagogInnenbildung in Forschung und Praxis: Utopien, Ansprüche und Herausforderungen. Leverkusen-Opladen, 108117.

Spivak, G. C. (2008): Can the Subaltern Speak? Postkolonialität und subalterne Artikulation. Wien. 


\section{Michaela Kaiser}

\section{Inklusionsbezogene Anforderungswahrnehmung - Regulativ für (kunstpädagogische) Professionalisierung}

\section{Umgang mit Offenheit, Ungewissheit und Widersprüchlichkeit in der Lehrkräftebildung}

Gleichwohl es sich bei dem Professionalisierungsbegriff in der Kunstpädagogik noch immer um ein streitbares und kontrovers diskutiertes Konzept handelt, konnte sich in der letzten Dekade der kunstpädagogischen Forschung eine schmale Debatte um die Professionalität von Kunstlehrkräften etablieren. Doch obwohl die Integration der erziehungswissenschaftlichen Professionalisierungsforschung eine zentrale Forderung darstellt (vgl. Bos, Wendt \& van Holt 2010), bezieht sich ein Gros der Kunstpädagog*innen auf die Annahme, dass „sich Kunst und Kunsterfahrung einer bildungswissenschaftlich begründeten Professionalisierungsdebatte entziehen" (Dreyer 2005, 21). Uneindeutigkeiten ergaben sich insofern im Hinblick auf die fachspezifisch begründete Professionalität von angehenden Kunstlehrkräften, insbesondere im Hinblick auf die Versiertheit für die Diversität von Kindern und Jugendlichen (vgl. Kaiser \& Brenne 2019). Der Erfahrung von Uneindeutigkeit, Offenheit und Ereignishaftigkeit, d.h. der Erfahrung von Kontingenz, wird im Lehrkräftehandeln allgemein eine relevante Rolle zugewiesen, was sich unter anderem in ihrer konzeptuellen Anerkennung in den struktur- (vgl. u.a. Helsper 2014) und kompetenztheoretischen (vgl. u.a. Baumert \& Kunter 2006) Ansätzen der Professionalisierungsforschung zeigt; und auch in das Gebiet der Inklusionsforschung haben struktur- und kompetenztheoretische Annäherung an ein diesbezüglich professionalisiertes Lehrkräftehandeln bereits Eingang gefunden (vgl. u.a. Häcker \& Walm 2015; Lütje-Klose, Miller \& Ziegler 2014; Moser, Schäfer \& Redlich 2011). Doch stellen die Trennlinien zwischen diesen Annäherungen eine Herausforderung für die akademische Professionalisierung von Kunstlehrkräften dar - vor allem im Hinblick auf ihre inklusionsbezogenen Professionalisierungsprozesse. Indem mit der Theorie der Anforderungsbearbeitung (vgl. Košinár 2014) danach gefragt wird, inwiefern die Bearbeitung inklusionsbezogener Rollenanforderungen sowobl durch individuell entwickelte Kompetenzen als auch strukturelle Rahmenbedingungen beeinflusst wird, wird die Frage der Bewältigung von Kontingenz in einem Mittelweg zusammenge- 
führt. Mit diesem Zugang zur Professionalisierung von Kunstlehrkräften wird an das Modell des Erfahrungslernens angeschlossen (vgl. ebd.), welches die Deutung und Bearbeitung von beruflichen Anforderungen über den Erwerb standardisierter Kompetenzen stellt - ein Befund, der sich auch auf dem Feld der Kunstpädagogik replizieren ließ (vgl. Kaiser 2019; Kaiser \& Brenne 2020). Den Wahrnehmungs- und Deutungsmustern kommt dabei eine Scharnierfunktion zu, um sich mit den Anforderungssituationen in Beziehung zu setzen (vgl. ebd.). Sowohl auf dem Feld der Kunstpädagogik als auch auf dem der Erziehungswissenschaft zeigt sich jedoch, dass die Lehrkräftebildung gegenüber den berufsbiografisch früh erworbenen Überzeugungen und Deutungsmustern nur wenig irritierendes und transformatorisches Potenzial entfaltet. Infolgedessen ist es von Interesse, auf einer Mikroebene der Frage nachzugehen, was genau sich professionalisierungsbezogen im Zuge des Erlebens von Kontingenz ereignet.

\section{Offenheit, Ungewissheit und Widersprüchlichkeit in hochschuldidaktischen Settings}

Im Projekt ,Expertise und Kooperation für eine Basisqualifikation Inklusion ' an der Universität Osnabrück (Land Niedersachsen 2015-2018) wurde im Rahmen einer kombinierten Professionalisierung in den bildungswissenschaftlichen Lehrveranstaltungen Basiswissen vermittelt, während in einem Zertifikatskurs Möglichkeiten zur Vertiefung und Spezialisierung eröffnet wurden (vgl. Kunze 2019, VI). Die hier angebotenen Lehrveranstaltungen unterliegen zwei Prämissen: erstens der interdisziplinären Kooperation von Fachdidaktik und Erziehungswissenschaft und zweitens der Verknüpfung von universitärer, wissenschaftlicher Expertise mit der beruflichen Expertise aus der schulischen und außerschulischen Bildungspraxis (vgl. ebd.). Das vorgestellte Projekt wurde in der Kooperation aus Kunstdidaktik, Erziehungswissenschaft und der Kunstwerkstatt der Heilpädagogischen Hilfe umgesetzt (vgl. dazu Brenne, Ossowski \& Seidel 2020) und basiert auf unterschiedlichen Begründungslinien des forschenden (vgl. u.a. Fichten 2017) und kasuistischen (vgl. u.a. Combe \& Gebhard 2012) Lernens. Es zielt auf den Aufbau fachdidaktischer und inklusionsdidaktischer Expertise sowie auf die Anbahnung von inklusiven Überzeugungen und Haltungen auf der Basis von Erfahrung und Reflexion. Die hier verortete empirische Begleitforschung basiert auf Daten aus 23 problemzentrierten Interviews mit angehenden (Kunst-)Lehrkräften, die mithilfe der Grounded Theory (vgl. Strauss \& Corbin 2010) analysiert wurden. Im Zentrum der Analysen steht dabei die Rekonstruktion der Wahrnehmung inklusionsbezogener Anforderungen. Forschungsleitend ist die Frage, inwiefern kontingente Wahrnehmungsmuster und deren Bearbeitung inklusionsbezogene Professionalisierungsprozesse anregen. Anhand einer exem- 
plarisch ausgewählten Interviewsequenz wird in der folgenden Darstellung die Wahrnehmung und Deutung von Kontingenz nachgezeichnet.

\title{
3 Professionalisierung innerhalb von Offenheit, Ungewissheit und Widersprüchlichkeit
}

Zur Beantwortung dieser Frage konnten die Prozessmomente Diskontinuität Irritation - Dekonstruktion - Rekonstruktion und Transformation herausgearbeitet werden, die Erfahrungen von Kontingenz prägen und damit Professionalisierungsprozesse be- oder verhindern.

Das Erleben von Diskontinuität impliziert dabei, dass die Kontinuität des professionsbezogenen Selbstverständnisses unterbrochen wird, indem es zu Differenzerfahrung von eingeschliffenen Wahrnehmungs- und Deutungsweisen und inklusionsbezogenen Anforderungen kommt, die an angehende Kunstlehrkräfte herangetragen und als subjektiv relevant wahrgenommen werden. Kurzum: eine Differenzerfahrung zwischen professionellem Habitus und situativem sozialen Feld (vgl. Koller 2018):

\begin{abstract}
„Also, ich muss schon sagen, dass mich die Arbeit mit Maria da schon völlig aus dem Takt gebracht hat (lacht). Also jetzt nur mal auf unser Werk [Kunstwerk; Anm. d. Autorin] bezogen war das echt schon was anderes. Maria hatte da 'nen ganz anderen Blick drauf und hatte sich wahrscheinlich auch so gedacht, sie will da in 'ne andere Richtung als ich (...) Das war ja so, dass wir da eigentlich abgesprochen hatten, dass wir 'ne Art Mobile bauen wollten. Wir hatten schon die unterschiedlichsten Sachen gesammelt, ja, alles mögliche eigentlich und ich dachte noch: ,Das wird gut!'. Wir hatten dann so ähm, ja im Prinzip alles schon gut zurechtgelegt und zusammengestellt und alles war eigentlich ähm, ja auch ganz gut besprochen. Und da war ich nur 'nen kurzen Moment nicht da und plötzlich fängt die dann an und laminiert die ganzen Teile ein. Da dachte ich mir nur: ,Das kann doch jetzt nicht wahr sein! So war das aber nich' abgesprochen ‘ (...)“ (Interview 16, Absatz 243-252).
\end{abstract}

In dem hier vorgestellten Praxisforschungsprojekt fiel das Moment der Diskontinuität mit ästhetischen Erfahrungen zusammen, denn es zeigte sich analog zu den künstlerisch-ästhetischen Erfahrungen eine Irritation der gewohnten Wahrnehmungs- und Deutungsweisen:

„Das hat mich schon ziemlich sauer gemacht, weil sie sich da einfach nich' an meine Absprachen gehalten hat. Das war schon ziemlich ärgerlich. Und ich hab' das einfach nicht verstanden. Dachte mir auch: ,Das kann ja wohl nicht so schwer sein, zu verstehen, was wir hier geplant hatten'. Da lief halt mal so gar nichts nach Plan. (...) Ich hab' dann nichts gesagt und dachte: ,Dann muss ich mich halt darauf einlassen “" (Interview 16, Absatz 253-258). 
Als entscheidend erweist sich, dass die Situationswahrnehmung nicht mit den bis dahin entwickelten Präkonzepten zu beantworten ist, sodass es neuere Wege der Deutung der Situationswahrnehmung bedarf. Das Moment der Irritation fordert demnach dazu auf, eingeschliffene Wahrnehmungs- und Deutungsweisen zu hinterfragen und Räume für Reflexionsprozesse aufzuschließen (vgl. u.a. Liebau \& Zirfas 2008). Das Moment der Irritation ist damit unmittelbar mit der Entnormalisierung der professionsbezogenen Selbstwahrnehmung verwoben, indem es darauf drängt, (berufs-)biografisch erworbene Präkonzepte infrage zu stellen.

„Ich muss schon sagen, einfach war das nich'. Klar, hätt' ich mir gewünscht, dass wir an dem Werk auch so arbeiten, wie wir das besprochen hatten, aber das war eben nich' so. (..) Ich bin da für mich dann erstmal 'n bisschen auf Distanz gegangen und hab' mir das alles so angeguckt. Is' halt n' anderes Rangehen, hab' ich mir gedacht. Is' halt so, wenn mehrere Leute zusammenkommen mit ihren Vorstellungen und die von Maria war'n dann ja auch noch mal speziell. (.) Ja, das so zu merken, war schon mal wichtig für mich. Am Ende fand' ich's dann aber auch ganz witzig, was da bei uns entstanden ist. Irgendwie war's halt Kraut und Rüben, aber auch irgendwie Kraut und Rüben mit Konzept, wenn man's dann mal genauer betrachtet" (Interview 16, Absatz 253-265).

Die Redewendung ,Kraut und Rüben` legt offen, dass gewohnte Wahrnehmungsweisen durchbrochen und in gewisser Weise dekonstruiert werden, indem Präkonzepte von inklusivem Unterricht hinterfragt werden. An dieser Stelle erschließt sich die als Irritation erlebte Diskontinuität des professionellen Selbstverständnisses, die Anlass für eine Neubestimmung des Verhältnisses von professionsbezogenem Selbstverständnis und wahrgenommenen Anforderungssituationen ist. Eine solche Neubestimmung entwickelt sich im Rahmen dieses aufgeworfenen Frage-Antwort-Verhältnisses durch die Interaktion mit der Studierendengruppe, demnach der sozialen Umwelt. Innerhalb dieser Interaktion wird durch die narrative Nachzeichnung gemeinsame Sinnbildung und in gewisser Weise die Rekonstruktion des aufgeworfenen Frage-Antwort-Verhältnisses angestoßen:

„Und später ham' wir noch unsere Erfahrungen im Seminar besprochen und da hab” ich mich dann auch zum ersten Mal gefragt, wie das eigentlich im richtigen Unterricht wär'. Hätt' ich da auch die Offenheit wie heute und könnte da zulassen, dass nich' alles nach Plan läuft? (.) Aber vorbereiten darauf, in 'nem inklusiven Setting zu arbeiten, könnt' ich mich schon. So schlimm war's ja jetzt auch nich', auch wenn's erstmal ziemlich drunter und drüber gegangen is' (lacht). Aber ich sag' mal so, da wächst man ja auch an seinen Aufgaben" (Interview 16, Absatz 278-283).

So wird die gemeinsame Sinnbildung auch zu einer Bewegung des Verstehens, oder mit Rosa (2016) gesprochen zu einem dialogisch-analytischen Prozess der Anverwandlung, der für die Reflexion konstitutiv ist. Die Irritation des professionsbezogenen Selbstverständnisses genügt also nicht, um Professionalisierungs- 
prozesse anzustoßen. Es bedarf ebenso, sich durch das Suchen und Finden von Antworten auf aufgeworfene Fragen zu den Anforderungen ins Verhältnis zu setzen (vgl. Kaiser 2020). Konstitutiv für die reflexiven Prozesse der De- und Rekonstruktion des professionsbezogenen Selbstverständnisses ist ihr transformatorisches Potenzial, das sich auch als Aneignungs- bzw. Anverwandlungspotenzial beschreiben lässt. Insofern lässt sich die De- und Rekonstruktion des professionsbezogenen Selbstverständnisses vor dem Hintergrund inklusionsbezogener Anforderungssituationen auch als reflexiver Anverwandlungsprozess verstehen, der Professionalisierungsprozesse in Gang setzt (vgl. Abb. 1).

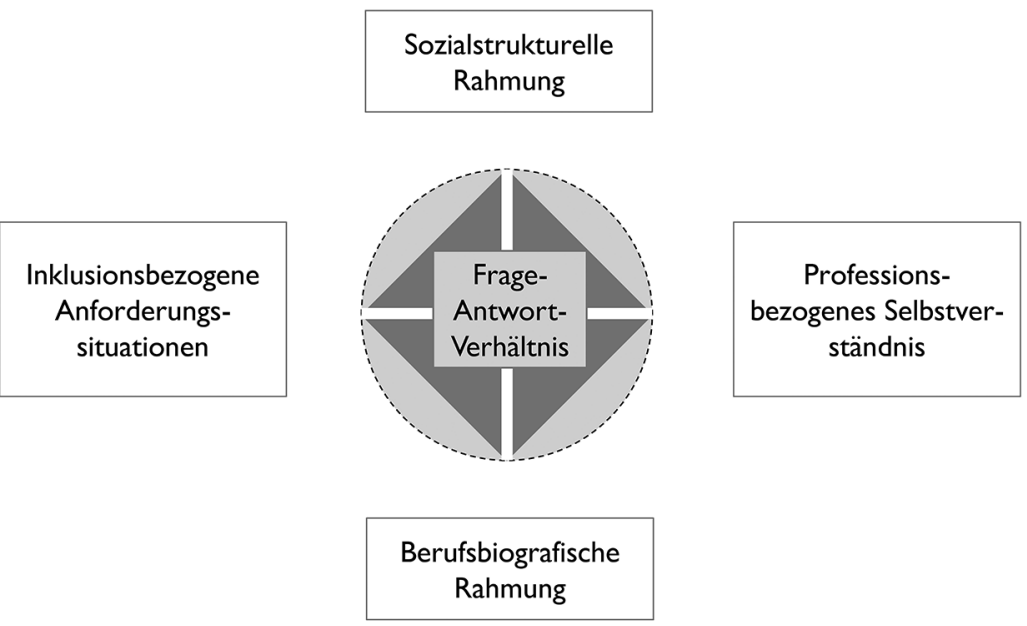

Abb. 1: Reflexive Professionalisierung im Frage-Antwort-Verhältnis

\section{Offenheit, Ungewissheit, Widersprüchlichkeit - Implikationen für die inklusionsbezogene Lehrkräftebildung}

Exemplarisch konnte anhand der vorausgegangenen Analyse gezeigt werden, dass das Konzept der inklusionsbezogenen Anforderungswahrnehmung und -bearbeitung aus professionalisierungstheoretischer Perspektive an den Entwurf transformatorischer Bildungs- und entsprechend transformatorischer Professionalisierungsprozesse (vgl. Koller 2018) anschließt. Professionalisierung wird somit durch transformatorische Erfahrungen angestoßen. Angehende Kunstlehrkräfte werden - so verstanden - als im (Professionalisierungs-)Prozess begriffen und damit biografisch (vgl. Terhart 2014) wie sozial imprägniert gefasst (vgl. Leonhard 
2018; Reh \& Ricken 2012), was den Wirkungsbereich standard- und kompetenzorientierter ebenso wie die strukturtheoretischen Konzeptionen der Professionalisierung relativiert. Es stellt sich dementsprechend die Frage, unter welchen hochschulischen Voraussetzungen ebensolche Erfahrungsfelder eröffnet werden, in denen Studierende sich auf Erfahrungen von Kontingenz, d.h. auf Ungewissheit, Ereignishaftigkeit und Unbestimmtheit, einlassen, anstatt sich am Modus Operandi zu orientieren. Für die akademische Kunstlehrkräftebildung ergibt sich hieraus die Aufgabe, die Wahrnehmung für Diskontinuitäten sowie die Dekodierung und Enkodierung von Kontingenzerfahrungen zu schulen, damit mittels Irritation die Dekonstruktion eingeschliffener Wahrnehmungs- und Deutungsweisen in Gang gesetzt wird. Jedoch ist für Professionalisierungsprozesse neben der Dekonstruktion auch die Rekonstruktion im Sinne der narrativen Nachzeichnung im Austausch mit der sozialen Umwelt notwendig, damit Sinnbildungsprozesse angestoßen werden. Damit sich diese als sinnhaft erweisen können, sollten die hochschuldidaktischen Zugänge sowohl kasuistisch-rekonstruktiv als auch reflexiv sein.

Dass eine kasuistisch angelegte Lehrer*innenbildung habitusbildende Reflexivität ermöglicht, ist eine Erkenntnis, die sich inzwischen in der Forschung zur Lehrkräftebildung theoretisch wie empirisch durchsetzen konnte. Doch zeigen die Analysen, dass die Irritation und in der Folge Reflexion anregende Momente nicht per se hochschuldidaktisch vorgesehen waren, sondern vielmehr zufällig im Rahmen ästhetischer Erfahrungen ausgelöst wurden. An dieser Stelle ist weiteres Innovationspotenzial im Hinblick auf die akademische Kunstlehrkräftebildung zu sehen, als sich der Modus Operandi des professionellen Handelns und die normative Zielperspektive inklusiven Kunstlehrkräftehandelns nicht als deckungsgleich erweisen (vgl. Kramer \& Pallesen 2018), sondern professionstheoretisch weiterzuführen sind.

An dieser Stelle zeichnet sich ab, dass weder das berufsnahe Handeln in den praktischen Elementen der Kunstlehrkräftebildung noch der Einsatz rekonstruktiver Kasuistik zur Entwicklung des kritisch-reflexiven Berufshabitus führen, diesen aber sehr wohl wahrscheinlicher machen, indem praktische Handlungserfahrungen in Verbindung mit rekonstruktiver Kasuistik dazu führen, sich in Momente der Kontingenz zu begeben. Auch zeichnet sich an dieser Stelle ab, wie sehr das kompetenzorientierte Streben nach Verfüg- und Kontrollierbarkeit mit reflexiven Professionalisierungsprozessen in Widerspruch geraten ist. Welche Konsequenzen hieraus für eine inklusionsbezogene Professionalisierung zu ziehen wären, ist bislang nicht systematisch diskutiert, doch ist anzunehmen, dass eine Stärkung dieser Elemente über die Kunstlehrkräfte hinweg und weiterführend in der zweiten und dritten Phase eine professionalisierende Wirkung zeitigen dürfte. 


\section{Literatur}

Baumert, J. \& Kunter, M. (2006): Stichwort. Professionelle Kompetenz von Lehrkräften. In: Zeitschrift für Erziehungswissenschaft, 9, H.4, 469-520.

Bos, W., Wendt, H. \& van Holt, N. (2010): Kompetenzmessung im Fach Kunst: Herausforderungen und Perspektiven. In: Bering, K., Höxter, C. \& Niehoff, R. (Hrsg.): Orientierung: Kunstpädagogik (Artificium, Bd. 35). Oberhausen, 407-430.

Brenne, A., Ossowski, E. \& Seidel, C. (2021): Come Together. Künstlerische Kollaborationen im Kontext der Implementation inklusiver Kompetenzen. München.

Combe, A. \& Gebhard, U. (2012): Verstehen im Unterricht. Die Rolle von Phantasie und Erfahrung. In: ZISU, 1, 174-178.

Dreyer, A. (2005): Kunstpädagogische Professionalität und Kunstdidaktik. Eine qualitativ-empirische Studie im kunstpädagogischen Kontext. München.

Fichten, W. (2017): Forschendes Lernen in der Lehrerbildung. In: Schüssler, R., Schöning, A., Schwier, V., Schicht, S., Gold, J. \& Weyland, U. (Hrsg.): Forschendes Lernen im Praxissemester Zugänge, Konzepte, Erfahrungen. Bad Heilbrunn, 30-38.

Häcker, T. \& Walm, M. (2015): Inklusion als Herausforderung an eine reflexive Erziehungswissenschaft. Anmerkungen zur Professionalisierung von Lehrpersonen in ,inklusiven“ Zeiten. In: Erziehungswissenschaft, 26, H.51, 81-89.

Helsper, W. (2014): Lehrerprofessionalität - der strukturtheoretische Professionsansatz zum Lehrberuf. In: Terhart, E., Bennewitz, H. \& Rothland, M. (Hrsg.): Handbuch der Forschung zum Lehrerberuf. 2. Aufl. Münster, 216-240.

Kaiser, M. (2019): Kunstpädagogik im Spannungsfeld von Inklusion und Exklusion. Oberhausen.

Kaiser, M. (2020): Wie Kunst und Kultur in die Schule kommen. In: Transfer Praxis - Schule, 4, 111-118.

Kaiser, M. \& Brenne, A. (2019): Typisch Kunstpädagogik? Inklusionsbezogene Überzeugungen angehender Kunstlehrkräfte. In: Skorsetz, N., Bonanati, M. \& Kucharz, D. (Hrsg.): Diversität und soziale Ungleichheit - Herausforderungen an die Integrationsleistung der Grundschule. Wiesbaden, 290-295.

Kaiser, M. \& Brenne, A. (2020): Inklusionsbezogene Anforderungsbearbeitung als Regulativ kunstpädagogischer Professionalisierung. In: Qf - Qualifizierung für Inklusion. Online-Zeitschrift zur Forschung über Aus-, Fort- und Weiterbildung pädagogischer Fachkräfte, Bd.2, H.1, o.S. Online unter: https://www.qfi-oz.de/index.php/inklusion/article/view/22 (letzter Zugriff: 09.10.2020).

Koller, H. C. (2018): Bildung anders denken: Einführung in die Theorie transformatorischer Bildungsprozesse. 2. Aufl. Stuttgart.

Košinár, J. (2014): Professionalisierungsverläufe in der Lehrerausbildung. Anforderungsbearbeitung und Kompetenzentwicklung im Referendariat. Opladen.

Kramer, R. T. \& Pallesen, H. (2018): Lehrerhandeln zwischen beruflichem und professionellem Habitus - Praxeologische Grundlegungen und heuristische Schärfungen. In: Leonhard, T., Košinár, J. \& Reintjes, C. (Hrsg.): Praktiken und Orientierungen in der Lehrerbildung. Bad Heilbrunn, 41-52.

Kunze, I. (2019): Vorwort. In: Falkenreck, D., Götzl, N., Hollen, M. \& Ossowski, E. (Hrsg.): Lehrerausbildung für Inklusion. Fragen und Konzepte zur Hochschulentwicklung. Baltmannsweiler, V-VII.

Leonhard, T. (2018): Potenziale einer praxistheoretischen Perspektive auf die Lehrpersonenbildung. In: Leonhard, T., Košinár, J. \& Reintjes, C. (Hrsg.): Praktiken und Orientierungen in der Lehrerbildung. Bad Heilbrunn, 81-92.

Liebau, E. \& Zirfas, J. (2008): Die Sinne und die Künste. Perspektiven ästhetischer Bildung. Bielefeld. Lütje-Klose, B., Miller, S. \& Ziegler, H. (2014): Professionalisierung für die inklusive Schule als Herausforderung für die LehrerInnenbildung. In: Soziale Passagen, 6, H.1, 69-84. 


\section{Michaela Kaiser}

Moser, V., Schäfer, L. \& Redlich, H. (2011): Kompetenzen und Beliefs von Förderschullehrkräften in inklusiven Settings. In: Lütje-Klose, B., Langer, M.-T., Serke, B. \& Urban, M. (Hrsg.): Inklusion in Bildungsinstitutionen. Eine Herausforderung an die Heil- und Sonderpädagogik. Bad Heilbrunn, 143-149.

Reh, S. \& Ricken, N. (2012): Das Konzept der Adressierung. Zur Methodologie einer qualitativempirischen Erforschung von Subjektivation. In: Miethe, I. \& Müller, H. R. (Hrsg.): Qualitative Bildungsforschung und Bildungstheorie. Opladen, 35-56.

Rosa, H. (2016): Resonanz: Eine Soziologie der Weltbeziehung. Frankfurt am Main.

Strauss, A. \& Corbin, J. (2010): Grounded Theory. Grundlagen qualitativer Sozialforschung. Weinheim.

Terhart, E. (2014): Forschung zu Berufsbiographien von Lehrerinnen und Lehrern: Stichworte. In: Terhart, E., Bennewitz, H. \& Rothland, M. (Hrsg.): Handbuch der Forschung zum Lehrerberuf. Münster, 433-437. 


\section{Patrick Gollub, Silvia Greiten, Teresa Schkade und Marcel Veber}

\section{Schulpraktische Professionalisierung für den Umgang mit Heterogenität - ein interdisziplinärer Blick aus hochschuldidaktischen Projekten}

\section{Einleitung}

Schulpraktische Professionalisierung wird häufig singulär aus fachdidaktischer oder bildungswissenschaftlicher Perspektive betrachtet und auf die Durchführung der Praxisphasen in Lehramtsstudiengängen reduziert. Dieser Beitrag nimmt einen transdisziplinären Blick ein, beleuchtet erziehungswissenschaftliche Ansprüche an schulpraktische Lehrer*innenbildung und legt den Schwerpunkt auf den Umgang mit Heterogenität als aktuelle Herausforderung (vgl. Hascher \& de Zordo 2015) - auch mit Blick auf Inklusion.

Studierende stehen (mindestens) vor einer Professionalisierungstrias: Sie sollen Praxisphasen nutzen, um Schulpraxis aus Lehrer*innenwarte verstehen zu lernen, sich die Verbindung von schulpraktischer Profession und wissenschaftlicher Disziplin vergegenwärtigen und v.a. Unterrichten erproben. Diese Ziele werden durch curriculare Vorgaben, diverse Reflexionsinstrumente und hochschuldidaktische Formate der Praxisbegleitung gestützt und unterschiedlich gewichtet (vgl. Gröschner \& Klaß 2020). Wie die Professionalisierungstrias in den jeweiligen bildungspolitischen Rahmenbedingungen hochschuldidaktisch bearbeitet werden kann, ist nach wie vor von (akutem) Forschungsinteresse (vgl. Reh 2020).

Im Folgenden werden hochschuldidaktische Projekte schulpraktischer Professionalisierung skizziert, die Perspektiven auf Heterogenität, Professionalisierung und Reflexivität thematisieren. Den Begriff Heterogenität verwenden wir u.a. in Bezug auf unterschiedliche Dimensionen von Lerner*innenmerkmalen (vgl. Trautmann \& Wischer 2011, 40), die für Studierende in Praxisphasen zur Planung und Durchführung von Unterricht relevant sind. In diesem Praxisfeld bieten sich Ansatzpunkte für Professionalisierungsprozesse, die die Professionalisierungstrias betreffen: a) Entwicklung von Kompetenzen zum Umgang mit Heterogenität, b) Initiierung von Reflexion, um auf Heterogenität bezogenes Wissen und Können und eine Haltung weiterzuentwickeln sowie c) letztlich Weiterlernen in beruf- 
licher Praxis als berufsbiografische Aufgabe grundzulegen (vgl. Terhart 2014, 11). Die vorgestellten Projekte stützen sich u.a. auf das ALACT-Modell nach Korthagen (vgl. Korthagen \& Wubbels 2002, 49), nach welchem Reflexion als erfahrungsbasiertes Lernen in einem zyklischen Prozess verstanden wird.

\section{Bildungswissenschaftliches Konzept der Begleitung im Praxissemester}

Der Umgang mit Heterogenität in der schulischen Praxis wird von einem Gros der Studierenden als Ziel und Herausforderung gleichermaßen wahrgenommen. Erfolgt die theoretische Auseinandersetzung in den ,klassischen ' fachdidaktischen und bildungswissenschaftlichen Lehrveranstaltungen, bieten die praxisbezogenen Studien als Begleitseminare zum Praxissemester die Chance, das erworbene Theoriewissen (im Sinne des Forschenden Lernens) zu erproben, zu hinterfragen und zu erforschen (vgl. Häcker 2019, 83).

An der Universität Münster sind die bildungswissenschaftlichen Begleitseminare als Kleingruppen mit 15 Teilnehmer*innen konzipiert, wodurch ein engerer Austausch und eine persönliche Betreuung während der Praxisphase möglich sind (vgl. Gollub \& Veber 2018). In Abständen von einigen Wochen finden drei Blocktermine statt. Während der Vorbereitungstermine werden allgemeindidaktische Fragestellungen unter der Berücksichtigung von Heterogenität thematisiert: Wie plane ich Unterricht? Wie werde ich und wie wird mein Unterricht einer heterogenen Lerngruppe gerecht? Welche Methoden sind in welcher Lerngruppe und in welchem Setting sinnvoll? Der Unterricht, in dem Studierende während des fünfmonatigen schulpraktischen Aufenthaltes hospitieren, kann zu einem Beobachtungs- und Erfahrungsraum werden, wenn in den Schulen Mentor*innen Partizipation am Unterrichtsgeschehen und dessen Planung anbieten und Studierende dieses Angebot annehmen. Die zu planenden Unterrichtsvorhaben - das können sowohl ganze Stunden als auch einzelne Phasen oder eine ganze Reihe sein - dienen der Entwicklung der individuellen Professionalisierung und stellen gleichzeitig eine Überprüfung subjektiver didaktischer Konzepte dar. Vor dem Hintergrund didaktischer Ansätze können in den universitären Begleitveranstaltungen Unterrichtsplanung und -durchführung vielschichtig reflektiert werden (vgl. Häcker 2019, 84ff.). Die Praxisphasenbegleitung in den Bildungswissenschaften kann so einen großen Anteil schulpraktischer Professionalisierung von Studierenden im Hinblick auf Heterogenität leisten. 


\section{Co-Peer-Learning als bildungswissenschaftliches Konzept schulpraktischer Professionalisierung}

Praxisphasen in der Lehrer*innenbildung bieten Lerngelegenheiten, sich mit Unterrichtssettings und Unterrichtsplanung für heterogene und explizit inklusive Lerngruppen auseinanderzusetzen. In Kombination mit reflexiv angelegter Begleitung können sie so für Studierende einen Beitrag zur Professionalisierung für Unterrichtsplanung und -durchführung leisten. Die darauf bezogene reflexive Begleitung zielt darauf ab, implizites Wissen zu beschreiben, Theoriewissen heranzuziehen, sich auf dieser Basis mit Unterrichtsplanung und -gestaltung auseinanderzusetzen (vgl. Greiten 2019) und so professionell zu handeln (vgl. Korthagen \& Wubbels 2002).

An der Bergischen Universität Wuppertal wurde im Rahmen des vom BMBF geförderten QLB-Projektes KOLBI (Kohärenz in der Lehrerbildung) ein Reflexions- und Feedbackformat entwickelt, das sich auf die soziale Ressource von Tandems oder Tridems als Co-Peer-Learner stützt (vgl. Greiten 2019). Unter ,CoPeers' (vgl. Falchikov 2001) werden hier Studierende verstanden, die mit einem vergleichbaren Ausbildungs-, Kenntnis- und Kompetenzstand gleichrangig auf einer sozialen Ebene stehen (vgl. Treidler, Westphal \& Stroot 2014). In einem bildungswissenschaftlich ausgerichteten Blockseminar zur Vorbereitung auf das Praxissemester setzen sich Studierende aus unterschiedlichen Unterrichtsfächern u.a. mit Inklusion, Umgang mit heterogenen Lerngruppen und Konzepten der Unterrichtsplanung im Sinne von Unterrichtsreihen auseinander.

Während des Vorbereitungsseminars finden sich Studierende als Tandems oder Tridems zusammen und begleiten sich gegenseitig während des Praxissemesters im Rahmen eines Co-Peer-Learning-Konzepts (vgl. Greiten 2019 \& 2020). Dieses umfasst u.a. ein Gesprächssetting mit Impulsfragen, die auf Reflexion des eigenen Konzepts zur Unterrichtsplanung und Grundprinzipien der individuellen Förderung, inklusiven Unterrichts und Umgang mit heterogenen Lerngruppen abzielen. Darüber hinaus nutzen sie im Vorbereitungsseminar verwendete Materialien zu entsprechenden Planungskategorien (vgl. Greiten 2020). Nach dem Beginn des Praxissemesters vereinbaren die Co-Peers vor, während und nach einem Unterrichtsvorhaben Gesprächstermine, um sich auf der Basis des gemeinsamen didaktischen und weiteren bildungswissenschaftlichen Wissens aus dem Vorbereitungsseminar im Rahmen des Co-Peer-Learning-Konzepts in der Unterrichtsplanung zu begleiten, Reflexion und Feedback zu ermöglichen und sich gegenseitig in der Professionalisierung im Sinne der Kompetenzentwicklung (vgl. Terhart 2014) zu unterstützen. Das Konzept des Co-Peer-Learning intendiert eine Kopplung individueller Lernsituationen der Studierenden und Lerninhalten des Praxissemesters v.a. im Hinblick auf Inklusion und Heterogenität. 


\section{4 , Teilhabe und Schulsport ${ }^{\text {' }}$ - ein fachdidaktisches Konzept in der Sportlehrer*innenbildung}

Inklusion im Sportunterricht umzusetzen, wird im Sinne der Planung und Durchführung eines Unterrichts verstanden, der allen Schüler*innen in ihrem Sein individuell(e) Wertschätzung zuteilwerden lässt (vgl. Tiemann 2015). Sport nimmt als körper- und bewegungsbezogenes Fach in Bezug auf Heterogenität eine Sonderrolle ein (vgl. Thomas \& Leineweber 2018). Im Rahmen des Forschungsprojekts ,Schulsport2030“ wird an der Deutschen Sporthochschule Köln u.a. untersucht, welche Einstellung Sportstudierende im Lehramt zu inklusivem Schulsport haben, da diese als relevante Einflussgröße für gelingenden inklusiven Unterricht betrachtet werden (vgl. Avramidis \& Norwich 2002). Auch deren Selbstwirksamkeit hinsichtlich eines inklusiven Sportunterrichts wird dabei empirisch erfasst. Von Interesse ist, ob die Einstellung der Studierenden für inklusiven Schulsport eher positiv oder negativ ausfällt und inwiefern sie das eigene Unterrichten eines inklusiven Schulsports für gut umsetzbar halten.

In der genannten Untersuchung steht v.a. der Lehramtskurs, Teilhabe und Schulsport' im Fokus. Dieser wird über 15 SWS als Pflichtveranstaltung angeboten und nähert sich dem inklusiven Schulsport methodisch-didaktisch mit konkreten Unterrichtsbeispielen an. Im Kurs erproben die Lehramtsstudierenden unterschiedliche Ansätze eines inklusiven Schulsports, reflektieren Unterrichtssequenzen und setzen sich kritisch mit Möglichkeiten sowie Barrieren einer gleichberechtigten Teilhabe aller Schüler*innen auseinander. Im Kontext der Professionalisierung leiten die Studierenden dabei selbst Unterrichtssequenzen, die sie anschließend im Plenum reflektieren.

Basierend auf empirischen Ergebnissen zur Einstellung und Selbstwirksamkeit von Studierenden zu Inklusion im Schulsport (vgl. Odipo, Thomas \& Abel 2018) zeigt sich, dass diese unterschiedlich ausfallen. Während Studierende die Selbstwirksamkeit im Bereich der Koedukation sehr hoch einschätzen, lässt sich in Bezug auf die Förderschwerpunkte Sehen und geistige Entwicklung ein wesentlich geringeres Selbstwirksamkeitsempfinden feststellen. Generell zeigt sich eine eher positive Einstellung zu inklusivem Schulsport, auch wenn sich hier hinsichtlich verschiedener Heterogenitätsdimensionen ebenfalls Unterschiede erkennen lassen. Koedukativer Schulsport geht mit einer positiveren Einstellung einher, als es bei deren Einstellung zum gemeinsamen Unterrichten von Schüler*innen mit und ohne Förderschwerpunkt emotionale und soziale Entwicklung der Fall ist. Im Sinne einer schulpraktischen Professionalisierung kommt dieser hochschuldidaktische Kurs demnach dem Ziel nahe, angehende Sportlehrer*innen für eine gleichberechtigte Teilhabe im Schulsport zu sensibilisieren und deren Professionalisierung und Reflexivität im inklusiven Schulsport anzubahnen. 


\section{Reflexion und Rekonstruktion von Praxisphasen als interdisziplinärer hochschuldidaktischer Zugang}

Im letzten Kapitel wird kein Lehrprojekt, sondern eine theoretische Perspektive auf Reflexion und kasuistische Rekonstruktion von Praxisphasen entfaltet. Aus Erkenntnissen aus einem Forschungswerkstattseminar zur schulpraktischen Professionalisierung, in dem Praxisphasenreflexionen mit Studierenden rekonstruiert wurden (vgl. Grummt \& Veber 2019), kristallisierte sich die Frage heraus, wie Theorie und schulische Praxis sowie die Disziplin Erziehungswissenschaft und die Professionalisierung in der Lehrer*innenbildung im Kontext der Gestaltung von schulpraktischen Studien mit inklusivem Fokus relationiert werden können. Die Bearbeitung dieser hochschuldidaktischen Frage dieser doppelten Relationierung führte zu einer theoretischen Argumentation, in die Hochschullehrerfahrungen eingeflochten wurden.

Zwei Vorbemerkungen:

a) Inklusion hat im hier zugrunde liegenden Verständnis eine normative Komponente (vgl. Kullmann, Lütje-Klose \& Textor 2014), mit Auswirkungen auf die genannte doppelte Relationierung von Theorie und Praxis sowie Disziplin und Profession; tradierte Muster und Strukturen des Bildungssystems werden kritisch hinterfragt, was angehende Lehrkräfte zusätzlich herausfordern kann (vgl. Hascher $\&$ de Zordo 2015, 178). b) In der aktuellen Diskussion zur Professionalisierung für Inklusion wird deutlich, dass der Aspekt der Rekonstruktion viel Beachtung erfährt (vgl. u.a. Fabel-Lamla, Kunze, Moldenhauer \& Rabenstein 2020). In diesem Rahmen wird oft der Weg beschritten, Reflexion über Rekonstruktion (meist im Rahmen von Kasuistik) anzuregen, wie es bereits Kraimer (1995) einbrachte. Da Reflexion Teil der Lehrer*innenbildung ist, stellt sich die Frage, wenn ein Aspekt der Rekonstruktion - die Kasuistik - herausgenommen wird, inwieweit mittels Kasuistik ein Sprung von Rekonstruktion zu Reflexion (vgl. Modell von Korthagen \& Wubbels 2002) geleistet werden kann. Die normative Komponente von Inklusion wird in der Diskussion zur kasuistischen Lehrer*innenbildung teilweise reflektiert, u.a. damit, wie genau Normativität zurückgehalten werden könne (zur Vertiefung: vgl. Grummt \& Veber 2019). So lässt sich diskutieren, ob eine direkte Verbindung von Rekonstruktion und Reflexion in der Lehrer*innenbildung mit dem Fokus auf Heterogenität und Inklusion vollzogen werden kann. Und zudem, ob nicht Reflexion (unter Berücksichtigung professionalisierungsbezogener Begründungsebenen: vgl. Häcker 2019, 85ff.) und Rekonstruktion notwendig sind, um die beschriebene doppelte Relationierung anzubahnen.

Reflexion mittels Rekonstruktion von Praxisphasen als hochschuldidaktischer Zugang bietet zusätzlich zu grundlegenden Professionalisierungsprozessen auch Chancen für Professionalisierung mit dem Fokus auf Heterogenität und Inklu- 
sion: Angelehnt an die objektive Hermeneutik (vgl. Oevermann 2002) wurde im Rahmen des o.g. Forschungswerkstattseminars aus den von Studierenden angefertigten Praxisphasenberichten die Gesamtreflexion rekonstruiert. Eine Vorgabe für das Rekonstruieren der Gesamtreflexion war - im Sinne der kasuistischen Lehrer*innenbildung - das „mimetische Nachvollziehen einzelner Fälle [und] das erkennende Deuten von Strukturzusammenhängen“" (Kraimer 1995, 52). In der Rekonstruktion stellte sich für die teilnehmenden Studierenden als Kernfrage heraus, wie sie den Umgang mit den inklusionsbezogenen Widersprüchen (vgl. Hascher \& de Zordo 2015, 175) zu tradierten Mustern und Strukturen im Schulsystem erfassen und in ihren individuellen Professionalisierungsprozessen gerade in Bezug zu Praxisphasen durch das Abstand-Nehmen von normativen Ebenen von Inklusion in Rekonstruktionsprozessen integrieren können.

\section{Ausblick}

Aus den skizzierten Projekten und den theoretisch-konzeptionellen Überlegungen zur doppelten Relationierung ergeben sich für uns u.a. folgende Fragen zur schulpraktischen Professionalisierung für Heterogenität: Welche persönlichen Erfahrungen haben Studierende mit selektiven Mechanismen des Schulsystems, mit Heterogenität und deren Bearbeitung in ihrem Professionalisierungsprozess? Wie ist ihre Einstellung gegenüber partizipativen Bildungsprozessen und wie lässt sich ihr theoretisches Wissen dahingehend erweitern? Ist der Einsatz von Kasuistik als hochschuldidaktische Methode eine Möglichkeit, den individuellen Professionalisierungsprozess der Studierenden nachhaltig zu unterstützen? Welche hochschuldidaktischen Konzepte eignen sich in besonderem Maße, um Heterogenität und Inklusion thematisch und schulpraktisch zu bearbeiten? Und letztlich die übergeordnete Frage danach, wie Studierende die Theorie-Praxis-Relation in ihre schulischen Praxisphasen integrieren.

\section{Literatur}

Avramidis, E. \& Norwich, B. (2002): Teachers' attitudes towards integration/inclusion. A Review of literature. In: European Journal of Special Needs Education, 17, H.2, 129-147.

Fabel-Lamla, M., Kunze, K., Moldenhauer, A. \& Rabenstein, K. (Hrsg.) (2020): Kasuistik - Lehrer*innenbildung - Inklusion. Empirische und theoretische Verhältnisbestimmungen. Bad Heilbrunn.

Falchikov, N. (2001): Learning together. Peer tutoring in higher education. London.

Gollub, P. \& Veber, M. (2018): Das Praxissemester am Hochschulstandort Münster - organisatorische Rahmungen und Chancen vor dem Hintergrund inklusiver Bildung. In: Gollub, P., Paulus, D., Rott, D. \& Veber, M. (Hrsg.): Studentische Forschung im Praxissemester. Berlin u.a., 29-40.

Greiten, S. (2019): Das „Co-Peer-Learning-Gespräch“ als Reflexions- und Feedbackformat zur Unterrichtsplanung im Praxissemester. In: Degeling, M., Franken, N., Freund, S., Greiten, S., Neuhaus, 
D. \& Schellenbach-Zell, J. (Hrsg.): Herausforderung Kohärenz: Praxisphasen in der universitären Lehrerbildung. Bildungswissenschaftliche und fachdidaktische Perspektiven. Bad Heilbrunn, 209221.

Greiten, S. (2020): Szenarien zum Co-Peer-Learning als Reflexionsanlässe zur Unterrichtsplanung in Praxisphasen. In: Die Materialwerkstatt. Zeitschrift für Konzepte und Arbeitsmaterialien für Lehrer*innenbildung und Unterricht, 2, H.2, 40-48.

Gröschner, A. \& Klaß, S. (2020): Praxissemester und Langzeitpraktikum. In: Cramer, C., König, J., Rothland, M. \& Blömeke, S. (Hrsg.): Handbuch Lehrerinnen- und Lehrerbildung. Bad Heilbrunn, 629-635.

Grummt, M. \& Veber, M. (2019): Kasuistische Analyse von Unterrichtsreflexionen. Ein neues Konzept der reflexionsorientierten, kasuistischen Lehrer_innenbildung in der Diskussion. In: Pädagogische Horizonte, 3, H.1, 63-77.

Häcker, T. (2019): Reflexive Professionalisierung. Anmerkungen zu dem ambitionierten Anspruch, die Reflexionskompetenz angehender Lehrkräfte umfassend zu fördern. In: Degeling, M., Franken, N., Freund, S., Greiten, S., Neuhaus, D. \& Schellenbach-Zell, J. (Hrsg.): Herausforderung Kohärenz: Praxisphasen in der universitären Lehrerbildung. Bildungswissenschaftliche und fachdidaktische Perspektiven. Bad Heilbrunn, 81-96.

Hascher, T. \& de Zordo, L. (2015): Praktika und Inklusion. In: Häcker, T. H. \& Walm, M. (Hrsg.): Inklusion als Entwicklung: Konsequenzen für Schule und Lehrerbildung. Bad Heilbrunn, 165184.

Korthagen, F. \& Wubbels, T. (2002): Aus der Praxis lernen. In: Korthagen, F., Kessels, J., Koster, B., Lagerwerf, B. \& Wubbels, T. (Hrsg.): Schulwirklichkeit und Lehrerbildung. Reflexion der Lehrertätigkeit. Berlin, 41-54.

Kraimer, K. (1995): Die soziale Diagnose in der qualitativen Sozialforschung. In: Eberwein, H. \& Mand, J. (Hrsg.): Forschen für die Schulpraxis: Was Lehrer über Erkenntnisse qualitativer Sozialforschung wissen sollten. Weinheim, 38-56.

Kullmann, H., Lütje-Klose, B. \& Textor, A. (2014): Eine Allgemeine Didaktik für inklusive Lerngruppen - fünf Leitprinzipien als Grundlage eines Bielefelder Ansatzes der inklusiven Didaktik. In: Amrhein, B. \& Dziak-Mahler, M. (Hrsg.): Fachdidaktik inklusiv. Auf der Suche nach didaktischen Leitlinien für den Umgang mit Vielfalt in der Schule. Münster u.a., 89-103.

Odipo, T., Thomas, M. \& Abel, T. (2018): Heterogenität und Inklusion als Teil des Lehramtsstudiums - „Teilhabe und Schulsport“ ein Lehramtskurs im Fokus. In: Kleinert, J. \& Wolf, J. (Hrsg.): Schulsport 2020: Aktuelle Forschung und Perspektiven in der Sportlehrerbildung. St. Augustin, 49-70.

Oevermann, U. (2002): Klinische Soziologie auf der Basis der Methodologie der objektiven Hermeneutik - Manifest der objektiv hermeneutischen Sozialforschung. Frankfurt am Main.

Reh, S. (2020): Brauchen wir besondere qualitative Ansätze und Methoden für eine Inklusionsforschung? Kritische Bemerkungen zur Entwicklung der erziehungswissenschaftlichen Inklusionsforschung. In: Budde, J., Dlugosch, A., Herzmann, P., Rosen, L., Panagiotopoulou, A., Sturm, T. \& Wagner-Willi, M. (Hrsg.): Inklusionsforschung im Spannungsfeld von Erziehungswissenschaft und Bildungspolitik. Opladen, 189-198.

Terhart, E. (2014): Umgang mit Heterogenität: Anforderungen an Professionalisierungsprozesse. In: Lehren und Lernen, 40, H.8/9, 7-12.

Thomas, M. \& Leineweber, H. (2018): Erfassung von Einstellung, Selbstwirksamkeit und Belastungsempfinden von Sportlehrkräften bezogen auf heterogene Lerngruppenzusammensetzungen: Reliabilität und Validität der drei Messinstrumente. In: Empirische Sonderpädagogik, 10, H.3, 273-293.

Tiemann, H. (2015): Inklusiven Sportunterricht gestalten - didaktisch-methodische Überlegungen. In: Giese, M. \& Weigelt, L. (Hrsg.): Inklusiver Sportunterricht in Theorie und Praxis. Aachen, 53-66. 
196 Patrick Gollub, Silvia Greiten, Teresa Schkade und Marcel Veber

Trautmann, M. \& Wischer, B. (2011): Heterogenität in der Schule. Eine kritische Einführung. Wiesbaden.

Treidler, M., Westphal, P. \& Stroot, T. (2014): Peer-Learning. In: Westphal, P., Stroot, T., Lerche, E.-M. \& Wiethoff, C. (Hrsg.): Peer Learning durch Mentoring, Coaching \& Co. Köln, 15-20. 


\section{Pierre-Carl Link, Cedric Steinert und Susanne Jurkowski}

\section{Implementierung von Inklusion als Querschnittsthema an der Universität Erfurt durch das Kompetenz- und Entwicklungszentrum für Inklusion. Inklusionsspezifische Professionalisierung der Lehrer*innenbildung durch Team-Teaching, Fortbildung und Online-Lernumgebung}

Inklusive Schulentwicklung ist sowohl eine theoretische als auch eine handlungspraktische Herausforderung für die Lehrer*innenbildung (vgl. Stellbrink 2012). Die Sonderpädagogik mit ihren spezialisierten Fachrichtungen stellt einen Baustein dar, um diese Entwicklung disziplinär und professionell auszugestalten (vgl. Müller 2020). Deshalb ist an der Erfurt School of Education, die für die Lehrer*innenbildung an der Universität Erfurt verantwortliche Organisationseinheit, und am Lehrstuhl für Inklusive Bildungsprozesse mit Schwerpunkt emotionale und soziale Entwicklung das Kompetenz- und Entwicklungszentrum für Inklusion bereits in der zweiten Förderphase als Teilprojekt der Qualitätsoffensive Lehrer*innenbildung (QUALITEACH II) des Bundesministeriums für Bildung und Forschung angesiedelt. Aus sonderpädagogischer Perspektive liegt der Fokus des Kompetenz- und Entwicklungszentrums für Inklusion auf den Lernvoraussetzungen von Kindern und Jugendlichen mit einem sonderpädagogischen Förderbedarf in Thüringen. Ziel ist es, sonderpädagogische Inhalte in allen lehramtsbezogenen Studiengängen als Querschnittsthema (vgl. Heinrich, Urban \& Werning 2013) zu verankern. Gegenstand sind dabei einzelne sonderpädagogische Fachrichtungen und die Wahrnehmung und Analyse von individuellen Lernvoraussetzungen sowie Unterstützungsmöglichkeiten durch die Lehrpersonen. Beispiele sind die Themen Förderdiagnostik, differenzierende Unterrichtsgestaltung, ClassroomManagement, Lehrer*innen-Schüler*innen-Beziehung und multiprofessionelle Kooperation. Die Umsetzung dieser Inhalte in der Hochschullehre erfolgt über drei Zugangswege:

1) Team-Planning/Team-Teaching,

2) videobasierte Lerneinheiten und

3) Fort- und Weiterbildungen. 
Beim Team-Planning/Team-Teaching erhalten Lehrende der Bildungswissenschaften sowie der Fachdidaktiken bzw. Fachwissenschaften Unterstützung durch Lehrende aus der Sonderpädagogik, um inklusionsbezogene förderpädagogische Fragestellungen für die Hochschullehre aufzuarbeiten und sie zukünftig dauerhaft in ihren Lehrveranstaltungen zu verankern. Durch die Entwicklung videobasierter Lerneinheiten mit authentischen Unterrichtsvideos und deren Einbettung in eine Online-Lernumgebung sollen Förderbedarfe und Fördermöglichkeiten für die Studierenden sichtbar werden. Darüber hinaus werden zur Vernetzung innerhalb der regionalen Bildungslandschaft Symposien, Workshops und Diskussionsrunden zum Thema Inklusion in der Lehrer*innenbildung organisiert. Diese Angebote richten sich an Studierende ebenso wie an Akteur*innen der zweiten und dritten Phase der Lehrer*innenbildung. Der vorliegende Beitrag fokussiert diese drei Umsetzungswege einer querschnittlichen Implementierung von Inklusion in a) der Hochschullandschaft für Studierende und b) für Lehrende gleichermaßen sowie c) in der Vernetzung mit der regionalen Bildungspraxis, jeweils unter einer professionalisierungstheoretischen Perspektive.

\section{Integrierte Vermittlung sonderpädagogischer und bildungswissenschaftlicher Inhalte durch Team-Teaching}

Durch interdisziplinäres Team-Teaching wird eine grundsätzliche Qualitätsverbesserung des Lehrens und Lernens erwartet (vgl. Kricke \& Reich 2016). Zudem eignet sich Team-Teaching, um die in der Lehrer*innenbildung erforderlichen multiprofessionellen Inhalte zu integrieren. Die Lehrpersonen ergänzen ihre spezifischen Kompetenzen, können das Lernen der Studierenden durch Differenzierung und Individualisierung unterstützen und fungieren als Vorbilder für die multiprofessionelle Kooperation in der inklusiven Schule (vgl. Jurkowski \& Müller 2018; Lütje-Klose \& Urban 2014).

An der Universität Erfurt kooperieren Mitarbeitende des Kompetenz- und Entwicklungszentrums für Inklusion mit Lehrenden aus Bildungswissenschaften, Fachdidaktiken, Fachwissenschaften und Psychologie, analog dem Modell des Team-Teaching von Lehrperson und Sonderpädagog*in in integrativen und inklusiven Schulkonzepten. Mit dem Ziel des Transfers sonderpädagogischen und inklusionsspezifischen Wissens in die lehrer*innenbildenden Veranstaltungen und Module entwickeln und planen die Lehrenden gemeinsam das Seminarkonzept, die Seminarsitzungen und führen diese gemeinsam durch.

Im Folgenden soll am Beispiel des Seminars, Offene Lernumgebungen, reciprocal teaching und cooperative learning' die Kooperation von Mitarbeiter*innen des Kompetenzzentrums mit Lehrenden der Bildungswissenschaften umrissen werden. Der bildungswissenschaftliche Schwerpunkt des Seminars lag in Lernmetho- 
den (wie beispielsweise differenzierendes Unterrichten, traditioneller Gruppenunterricht, kooperatives Lernen, reziprokes Lernen und offene Unterrichtsformen). Diese Inhalte wurden im Team-Teaching um folgende sonderpädagogische und inklusionsspezifische Inhalte erweitert: historische und rechtliche Hintergründe, Heterogenitätsdimensionen, Behinderung als Konstrukt, Entwickeln eines Inklusionsbegriffs, Bedeutung inklusiven Unterrichts, Differenzierungs- und Handlungsmöglichkeiten, inklusive Bildungsprozesse, (sonder-)pädagogische Förderbedarfe. In den Seminarsitzungen wird je eine Lernmethode vorgestellt, in einer Gruppenaktivierung erprobt und mithilfe einer Fallvignette zu einer prototypischen Situation im gemeinsamen Unterricht erweitert. Im Team-Teaching werden sodann gemeinsam mit den Studierenden die Fallvignette analysiert und beurteilt, situationsentsprechende Handlungsweisen erarbeitet und weitere pädagogische Möglichkeiten diskutiert. Auf diese Weise lernen die Studierenden die Notwendigkeit der Anpassung von Lernmethoden an heterogenitätsbedingte Voraussetzungen der Schüler*innen kennen, erarbeiten Ansätze der Förderdiagnostik und Förderplanung sowie grundlegendes Wissen über Unterstützungsnetzwerke. Sie erfahren außerdem, dass die Expertise von Sonderpädagog*innen sowie eine interdisziplinäre Kooperation essenziell sind für die erfolgreiche Planung und Umsetzung inklusiven Unterrichts.

Um durch Team-Teaching Studierende effektiv auf die inklusive Unterrichtspraxis vorzubereiten, ist es ein Anliegen der aktuellen Forschung, geeignete Team-Teaching-Formate zu entwickeln. Beispielsweise wurde im Kompetenz- und Entwicklungszentrum für Inklusion ein Konzept mit einem kontinuierlichen TeamTeaching über mehrere Seminarstunden hinweg erarbeitet, während bei einem weiteren Konzept die sonderpädagogischen und inklusionsspezifischen Inhalte nur punktuell einfließen, indem Team-Teaching in wenigen einzelnen Sitzungen stattfindet. Diese Konzepte wurden in einem Prä-Post-Kontrollgruppen-Design im Hinblick auf die folgenden Variablen bei den Studierenden verglichen: Verständnis von Heterogenität, Wissen über Möglichkeiten des differenzierenden Unterrichtens und Überzeugungen sowie Einstellungen gegenüber Förderbedarfen und inklusivem Unterricht.

Für den vorliegenden Tagungsband liegen die ersten Ergebnisse zu den Einstellungen der Studierenden vor (vgl. Kunz, Luder \& Moretti 2010). Für die Skala Schulische Integration' ergab sich weder ein signifikanter Haupteffekt der Zeit $(\mathrm{F}(2,112)<1, \mathrm{p}>.05)$ noch eine signifikante Wechselwirkung zwischen Zeit und Bedingung $(\mathrm{F}(2,112)=2.36, \mathrm{p}>.05)$. Die deskriptiven Werte verweisen jedoch auf einen leichten Anstieg der Werte im kontinuierlichen Team-Teaching. Dagegen ergaben sich für die Skala ,Schulische Förderung und Unterstützung' sowohl ein signifikanter Haupteffekt der Zeit $(\mathrm{F}(2,112)=10.67, \mathrm{p}<.01)$ als auch eine signifikante Wechselwirkung zwischen Zeit und Bedingung $(\mathrm{F}(2,112)=3.24$, $\mathrm{p}<.05)$. Post-hoc T-Tests zeigten in der Kontrollgruppe ohne Team-Teaching kei- 
nen Anstieg der Werte $(\mathrm{t}(24)=-1.10, \mathrm{p}>.05)$, ebenso wenig in der Bedingung mit punktuellem Team-Teaching ( $\mathrm{t}(39)=-.56, \mathrm{p}>.05)$. Der Anstieg in den Einstellungen zur schulischen Förderung und Unterstützung wird jedoch in der Bedingung mit kontinuierlichem Team-Teaching signifikant $(\mathrm{t}(49)=-4.73, \mathrm{p}<.01)$.

Die bereits vorliegenden Ergebnisse legen somit nahe, sonderpädagogische und inklusionsspezifische Fragen querschnittlich in einem kontinuierlichen TeamTeaching-Format zu verankern und damit Lernprozesse der Studierenden interdisziplinär, multiperspektivisch und kontinuierlich anzuregen. Die umfassende Auswertung der qualitativen Daten, beispielsweise der Mindmaps zum Verständnis von Heterogenität, wird weitere Erkenntnisse über die Wirksamkeit der Seminarformate erbringen.

\section{Eine Online-Lernumgebung zur Schulung der professionellen Wahrnehmung}

Auf einer Online-Lernumgebung haben Studierende und Dozierende Zugang $\mathrm{zu}$ authentischen Unterrichtsvideos und digitalen Workbooks zu verschiedenen inklusionsspezifischen Themen. Das Ziel der digitalen Lernumgebung ist, die Studierenden für die Heterogenität von Lerngruppen zu sensibilisieren und damit die Entwicklung ihrer professionellen Wahrnehmung des Unterrichtsgeschehens, insbesondere mit Blick auf inklusionsspezifische Fragestellungen, zu fördern. Den wissenschaftlichen Hintergrund bilden das Verständnis über ,Professionelle Wahrnehmung' und Studien zur Wirksamkeit von Unterrichtsvideos in der Lehrer*innenbildung (vgl. Ratzka, Lipowsky, Krammer \& Pauli 2005).

Unterricht ist ein komplexes Geschehen, da die Schüler*innen untereinander und mit der Lehrperson in vielfältigen Interaktionen stehen und mehrere Arbeits- und Lernaktivitäten der Schüler*innen zeitgleich ablaufen können (vgl. Doyle 1986). Für die Lehrperson ist der weitere Verlauf des Unterrichts relativ unvorhersehbar (vgl. ebd.). Gleichzeitig ist die Lehrperson gefordert, unmittelbar zu handeln (vgl. ebd.). Eine wesentliche Fähigkeit erfahrener Lehrer*innen ist es daher, die Wahrnehmung zahlreicher Eindrücke zu kanalisieren und den Blick auf das Wesentliche im Unterricht zu lenken, um das Geschehen im Klassenraum im Sinne einer guten Klassenführung zu leiten. Diese Fähigkeit wird als ,Professionelle Wahrnehmung' bezeichnet (vgl. Sherin, Russ, Sherin \& Colestock 2008). Unter dem Aspekt der professionellen Wahrnehmung erfordert inklusiver Unterricht die Sensibilität der Lehrer*innen für die Heterogenität der Schüler*innen und das Erkennen ihrer individuellen Lernstände und Lernprozesse sowie die Notwendigkeit einer individualisierten Unterstützung und Förderung. 
Authentische Unterrichtsvideos werden von Studierenden wie auch Lehrer*innen in der Weiterbildung als kognitiv und motivational anregend erlebt und können zum Verständnis des Unterrichtsgeschehens beitragen (vgl. Blomberg 2010). Speziell $360^{\circ}$-Videos bieten die Möglichkeit, dass sich Studierende im Klassenraum bewegen, in das Geschehen ,eintauchen', auf bestimmte Personen fokussieren oder sie eine geöffnete Perspektive auf den gesamten Klassenraum einnehmen können. Durch diese besondere Technik sollen Studierende leichter die Komplexität im Klassenraum erfassen, wichtige Ereignisse identifizieren und verstehen sowie das pädagogische Handeln der Lehrperson nachvollziehen können (vgl. Gold \& Holodynski 2017).

Gemeinsam mit schulischen Kooperationspartner*innen hat das Kompetenzund Entwicklungszentrum für Inklusion in den vergangenen vier Jahren über 40 Videosequenzen aus inklusiven Unterrichtssettings mit der $360^{\circ}$-Technik gedreht und didaktisch aufbereitet. Diese didaktische Aufbereitung ist von zentraler Bedeutung, damit Studierende, gestützt durch die Videos, unterrichtsrelevante Themen erarbeiten und Wahrnehmungsfähigkeiten erwerben können (vgl. Blomberg 2010). Daher wurde von einer Softwareentwicklungsfirma eine Online-Lernumgebung entwickelt, in der die Studierenden in ihrer Arbeit mit den Unterrichtsvideos angeleitet werden. Studierende können so ihnen als wichtig erscheinende Sequenzen markieren und direkt mit Notizen versehen oder für ausgewählte Abschnitte bestimmte Beobachtungscodes vergeben. Darüber hinaus enthält die Online-Lernumgebung Arbeits- und Lernmaterial zu inklusionsspezifischen Fragestellungen des Lehrens und Lernens. Herzstück sind dabei digitale Workbooks, mit denen die Studierenden wie in einem Arbeitsheft die Videos begleitende Texte und Aufgaben bearbeiten können und durch Reflexionsfragen zu einer vertieften selbstständigen Auseinandersetzung angeregt werden.

Die Umsetzung der Online-Lernumgebung wird wissenschaftlich begleitet. Forschungsfragen beziehen sich hierbei etwa darauf, wie aktivierend und motivierend die Studierenden die Arbeit am Material erleben, wie sie die Möglichkeiten der $360^{\circ}$-Technik nutzen und inwiefern sie ein vertieftes Verständnis inklusiver Unterrichtsgestaltung entwickeln.

\section{Sonderpädagogische Professionalisierung durch Fortbildungsangebote und interdisziplinäre Netzwerkarbeit}

Eine stärkere Vernetzung von Forschung, Theorie und Praxis sonderpädagogischer und inklusionspädagogischer Provenienz erfolgt über Fortbildungsangebote für regionale schulische und außerschulische Bildungsakteur*innen, Studierende und Lehrende gleichermaßen. Dadurch können Studierende frühzeitig über ein 
fachlich relevantes Thema in Kontakt mit Bildungspraktiker*innen treten. Für Bildungsforscher*innen und Lehrende besteht die Möglichkeit eines intensiveren Austauschs und der Anbahnung von Kooperationsaktivitäten mit der Praxis. Das Kompetenz- und Entwicklungszentrum für Inklusion organisiert diese Professionalisierungsmaßnahmen zur Öffnung der Universität für Praxispartner*innen und begleitet die Umsetzung eines stärker inklusiven Bildungssystems aus einer wissenschaftlichen Expertise heraus. In Symposien, Workshops und weiteren Fortbildungsveranstaltungen wird der aktuelle Forschungsstand zu Inklusion aufgearbeitet und einer breiten Öffentlichkeit zur Verfügung gestellt.

Gemeinsam mit Kooperationspartner*innen aus der regionalen Bildungslandschaft werden praxisrelevante Schwerpunkte und Bedarfe für Fortbildungsangebote und Symposien identifiziert. Ziel dieser Vorhaben ist der sukzessive Aufbau eines interdisziplinären und multiprofessionellen Netzwerks pädagogischer Akteur*innen, die ihre Interessen auch bildungspolitisch effektiver bündeln und voranbringen können. Ein Netzwerk, vergleichbar mit den inklusiven Modellregionen in Österreich (vgl. Feyerer 2016), kann als regionale Lerngemeinschaft für schulische und außerschulische Zielsetzungen eine nachhaltige Entwicklungsplattform sein, mit der Hilfe- und Unterstützungsstrukturen - etwa für besonders komplexe Fallkonstellationen, aber auch für rechtliche oder pädagogischfachwissenschaftliche Fragen - etabliert werden.

Da die Weiterentwicklung von Inklusion im regionalen Bildungswesen auch ein gesetztes Ziel des Kompetenzzentrums ist, wird jährlich das Erfurter Symposium für frühkindliche Entwicklung themenspezifisch ausgerichtet. Die Erfurter Symposien zeigen Möglichkeiten der Inklusion, der Prozessdiagnostik und des pädagogischen Handelns sowie der ganzheitlichen Förderung zur Vorbereitung auf den Übergang in die Schule als auch für das dortige erfolgreiche Lehren und Lernen auf. In den Vorträgen und Workshops sind durchgängig interdisziplinäre Perspektiven vertreten. Seit 2021 finden die Erfurter Symposien für frühkindliche Entwicklungsförderung unter dem Akronym ESEL - Erfurter Symposium für Entwicklung und Lernen statt und erweitern hiermit das inhaltliche Spektrum im Hinblick auf die Gesamtheit der Entwicklungs- und Lernprozesse im Kindesalter. Deutlich werden soll damit die enge Verknüpfung zwischen Entwicklung und Lernen und die Notwendigkeit, Übergänge zu gestalten und anzuleiten.

Außerdem werden durch Kongressbeiträge, Fortbildungsangebote, eine wöchentlich stattfindende Inklusionssprechzeit und wissenschaftliche Publikationen die Ergebnisse internen und externen Lehrer*innenbildungsakteur*innen zur Verfügung gestellt. Fortbildungsformate im hochschuldidaktischen Programm Akademische Qualifizierung und Weiterbildung der Universität Erfurt, die vom Kompetenzzentrum für Inklusion verantwortet werden, bilden hierbei einen weiteren Ansatz im thematischen Kontext. 


\section{Fazit und Ausblick}

Die Vernetzung unterschiedlicher Disziplinen sowie die Kooperationen multiprofessioneller Expertisen sind grundlegend für das Gelingen von frühen Entwicklungsprozessen und Inklusion. Mit dem Kompetenz- und Entwicklungszentrum für Inklusion in der Lehrer*innenbildung wird an der Universität Erfurt die Vorbereitung auf die inklusive Schule querschnittlich in den lehrer*innenbildenden Studiengängen verankert. Eine dauerhafte curriculare Implementation der durch die Forschung positiv evaluierten Bausteine inklusiver Pädagogik ist das Ziel der weiteren Arbeit im Kompetenz- und Entwicklungszentrum für Inklusion. Die Konstellation dieses Projekts der Lehrer*innenbildung, die die Vermittlung von inklusionsspezifischem Professionalisierungswissen interdisziplinär und querschnittlich vornimmt, wäre im deutschsprachigen akademischen Raum einerseits auf Lehrstuhl- und Studiengangsebene, andererseits aber auch auf Ebene der universitären Lehrer*innenbildungseinrichtungen übertragbar.

\section{Literatur}

Blomberg, G. A. (2010): Der Einsatz von Unterrichtsvideos in der universitären Lehrerbildung. München. (Dissertation). Online unter: https://mediatum.ub.tum.de/1078234 (letzter Zugriff: 12.04.2020).

Doyle, W. (1986): Classroom organization and management. In: Wittrock, M. (Hrsg.): Handbook of research on teaching. New York, 392-431.

Feyerer, E. (2016): Mit Inklusiven Modellregionen auf dem Weg zur inklusiven Schule? Österreichische Bildungspolitik zwischen Vision und Pragmatismus. In: Zeitschrift für Inklusion-online. net, H.2, o.S. Online unter: https://www.inklusion-online.net/index.php/inklusion-online/article/ view/361 (letzter Zugriff: 04.02.2021).

Gold, B. \& Holodynski, M. (2017): Using digital video to measure the professional vision of elementary classroom management: Test validation and methodological challenges. In: Computers \& Education, 107, 13-30.

Heinrich, M., Urban, M. \& Werning, R. (2013): Grundlagen, Handlungsstrategien und Forschungsperspektiven für die Ausbildung und Professionalisierung von Fachkräften für inklusive Schulen. In: Döbert, H. \& Weishaupt, H. (Hrsg.): Inklusive Bildung professionell gestalten. Münster, 69-133.

Jurkowski, S. \& Müller, B. (2018): Teaching in inclusive settings: The development of multi-professional cooperation in teaching dyads. In: Teaching and Teacher Education, 75, 224-231.

Kricke, M. \& Reich, K. (2016): Teamteaching. Eine neue Kultur des Lehrens und Lernens. Basel.

Kunz, A., Luder, R. \& Moretti, M. (2010): Die Messung von Einstellungen zur Integration (EZI). In: Empirische Sonderpädagogik, 2, H.3, 83-94.

Lütje-Klose, B. \& Urban, M. (2014): Kooperation als wesentliche Bedingung inklusiver Schul- und Unterrichtsentwicklung. Teil 1: Grundlagen und Modelle inklusiver Kooperation. In: Vierteljahrsschrift für Heilpädagogik und ihre Nachbargebiete, 83, 112-123.

Müller, T. (2020): Schule und verhaltensauffällige Kinder im Spagat von Individualisierung und Standardisierung. In: Herz, B., Hoyer, J. \& Liesebach, J. (Hrsg.): Brennpunkt Erziehungshilfe: Dialogpartner Technik? Bad Heilbrunn, 23-34.

Ratzka, N., Lipowsky, F., Krammer, K. \& Pauli, C. (2005): Lernen aus Unterrichtsvideos. Ein Fortbildungskonzept zur Entwicklung von Unterrichtsqualität. In: Pädagogik, 57, H.5, 30-33. 


\section{Pierre-Carl Link, Cedric Steinert und Susanne Jurkowski}

Sherin, M. G., Russ, R. S., Sherin, B. L. \& Colestock, A. (2008): Professional vision in action: An exploratory study. In: Issues in Teacher Education, 17, H.2, 27-46.

Stellbrink, M. (2012): Inklusion als Herausforderung für die Entwicklung von Unterricht, Schule und Lehrerbildung. In: Fürstenau, S. (Hrsg.): Interkulturelle Pädagogik und sprachliche Bildung. Wiesbaden, 83-100. 
Perspektiven 



\author{
Timm Albers, Agnes Filipiak, Katja Franzen und \\ Frank Hellmich
}

\title{
Kompetenzentwicklung im inklusiven Unterricht (KinU) - eine internationale Perspektive
}

\section{Einleitung}

Die Anforderungen inklusiver Bildungssysteme und die Frage nach Aus-, Fortund Weiterbildung (angehender) Lehrer*innen für die inklusive Schule zeigen sich global herausfordernd und lösen eine Renaissance des Internationalisierungsgedankens in einer eher lokal orientierten Lehrer*innenbildung aus: Erfahrungen und Forschungsergebnisse - z.B. aus Skandinavien - werden herangezogen und diskutiert, um Konzepte auf- und auszubauen. Im Forschungsprojekt ,Kompetenzentwicklung im inklusiven Unterricht (KinU) ${ }^{`}$ an der Universität Paderborn wird, in Kooperation mit der Freien Universität Bozen und der PH Linz, die internationale Perspektive und deren Einfluss auf eine inklusionsorientierte Kompetenzentwicklung bei Lehramtsstudierenden fokussiert. In diesem Artikel wird Kin $U$ mit seinen Zielen und Fragestellungen, seiner theoretischen Grundlegung und Methodik vorgestellt sowie erste Ergebnisse diskutiert.

\section{Theorien}

KinU fußt auf zwei ineinandergreifende Diskurslinien: Internationalisierung in der Lehrer*innenbildung im Spannungsfeld von Bildungsföderalismus und Globalisierungstrend sowie der Kompetenzorientierung in der Lehrer*innenbildung.

\subsection{Internationalisierung in der Lehrer*innenbildung}

Eine internationale Ausrichtung spielt in der Lehrer*innenbildung traditionell keine große Rolle. Das Bildungssystem ist auf nationaler Ebene bundeslandspezifisch organisiert. Entsprechend ist die Aus- und Weiterbildung von Lehrkräften durch lokale Organisationsstrukturen gekennzeichnet. Im Vergleich mit anderen Professionen ist der Wettbewerbsvorteil durch Auslandsaufenthalte für Lehrkräfte gering (vgl. Kämper van den Boogaart 2019; Leutwyler, Mantel \& Tremp 2011). 
In der Forschung lassen sich verschiedene Argumente ausmachen, die eine Auseinandersetzung mit der internationalen Perspektive in der Lehrer*innenbildung legitimieren:

a. Die Professionalisierungsdiskussion: Besonders in den USA gibt es richtungsweisende Diskurse um Kompetenzstandards und Outcome-Orientierung in der Lehrer*innenbildung (vgl. Gonon 2011, 23f.).

b. Reform der Lehrer*innenbildung: internationale Vergleichsstudien, wie z.B. PISA (vgl. ebd.).

c. Das interkulturelle Argument (vgl. HRK 2014; Leutwyler et al. 2011): Heterogene Klassen erfordern Kompetenzen im Umgang mit diversen Vielfaltsdimensionen (vgl. DAAD 2013).

Insbesondere c) macht deutlich, dass diese Aspekte in besonderem Maß auf eine inklusionsorientierte Lehrer*innenbildung zutreffen. Die Forderung nach Inklusion hat durch die UN-Behindertenrechtskonvention (2006) sowie die Salamanca-Erklärung $(1994)^{1}$ internationale Tragweite. Deshalb stellt die Umsetzung von Inklusion eine internationale Aufgabe dar, die im Rahmen der nationalen Bildungssysteme und teilweise auch Inklusionsverständnisse (vgl. Bürli 2010; Europäische Kommission 2002) unterschiedlich bearbeitet wird. Hier lohnt, trotz der nur bedingten Übertragbarkeit auf andere Systeme, der Blick über den eigenen Tellerrand hinaus. Im Hinblick auf die konkrete Berücksichtigung einer internationalen Perspektive in der Lehrer*innenbildung rät die Hochschulrektorenkonferenz (HRK 2014, 97) zu einer „weiten Arbeitsdefinition“ von Internationalisierung, die ,klassische' Internationalisierungsstrategien, z.B. Studierendenmobilität, mit Strategien ,at home' - also ohne einen Auslandsaufenthalt - koppelt. Zentral für die Effektivität solcher Strategien ist laut Leutwyler (2013) die konzeptionelle Einbettung und Begleitung der Maßnahmen.

\subsection{Kompetenzen in der inklusionsorientierten Lehrer*innenbildung}

Der Bologna-Prozess führte zu einer nachhaltigen Kompetenzorientierung in der Lehrer*innenbildung. In Anlehnung an Weinert (2001, 27f.) sind Kompetenzen „die bei Individuen verfügbaren oder durch sie erlernbaren kognitiven Fähigkeiten und Fertigkeiten, um bestimmte Probleme zu lösen, sowie die damit verbundenen motivationalen, volitionalen und sozialen Bereitschaften und Fähigkeiten, um die Problemlösungen (...) erfolgreich und verantwortungsvoll nutzen zu können.“ Auch im Bereich der inklusionsorientierten Lehrer*innenbildung stellt sich die

1 Originaldokumente online unter https://www.behindertenrechtskonvention.info/uebereinkommen-ueber-die-rechte-von-menschen-mit-behinderungen-3101/ (letzter Zugriff: 02.02.2021) sowie https://unesdoc. unesco.org/ark:/48223/pf0000110753?.posInSet=4\&queryId=N-EXPLORE7df84d79-d0bd-42b0-9c5d-286f3d53cc85 (letzter Zugriff: 02.02.2021). 
Frage nach spezifischen Kompetenzen. Gemäß der Hypothese, Inklusion generiere kein grundständig neues Berufsbild und erfordere daher kein eigenes Kompetenzprofil (vgl. Gebhardt, Kuhl, Wember \& Wittich 2018, 282f.), knüpft die Forschung hier an bestehende Erkenntnisse aus der allgemeinen Lehrer*innenbildung an, z.B. an das ,COACTIV-Modell' nach Baumert und Kunter (2011). Für Inklusion müssen lediglich bestimmte Kompetenzbereiche (z.B. Diagnostik) ausdifferenziert werden (vgl. Hillenbrand, Melzer \& Hagen 2013; Melzer, Hillenbrand, Sprenger \& Hennemann 2015; Moser 2014). Solche Spezifizierungen finden sich z.B. im Rahmen von international vergleichenden Projekten wie, Teacher Education for Inclusion (TE41)‘ (vgl. Merz-Atalik \& Franzkowiak 2011). Die ,European Agency for Development in Special Needs Education' (2012) formuliert vier zentrale Kompetenzen von Lehrkräften in inklusiven Settings:

- Wertschätzung von Verschiedenheit,

- Kooperationsfähigkeit,

- Bereitschaft zur persönlichen Weiterentwicklung und

- Unterstützung für alle Lernenden.

Aufbauend darauf sollen Rückschlüsse auf die Aus- und Weiterbildung von Lehrkräften möglich werden, die z.B. Curricula an die Kompetenzanforderungen aus der Praxis anpassen und effektive Lehr-/Lernformate entwickeln. Doch obwohl es in diesem Bereich Ansätze gibt, fehlt es an evidenzbasierten Formaten. Hier setzt das $K i n U$ an.

\section{Fragestellungen und Ziele}

KinU leistet einen Beitrag zur inklusionsorientierten Lehrer*innenbildung, indem es die internationale Perspektive integriert, um Studierende für die Arbeit an inklusiven Schulen zu qualifizieren: Auf der Makro-Ebene wird der Einfluss der nationalen Bildungs- und Ausbildungssysteme auf die Entwicklung einer ,Inklusionskompetenz' (Kap. 2.2) untersucht. Wie prägen nationale Bildungssysteme und Studienordnungen in der Lehrer*innenbildung Einstellungen, Konzepte und Kompetenzen hinsichtlich Inklusion und an welchen Stellen lassen sich im internationalen Austausch konstruktive Synergieeffekte erzeugen? Auf der Meso-Ebene wird unter Berücksichtigung unterschiedlicher Internationalisierungsstrategien (vgl. Leutwyler 2013) ein Lehr-/Lernkonzept entwickelt, das den inklusionsorientierten Kompetenzentwicklungsprozess der Studierenden fördern soll. Hier sollen verschiedene Maßnahmen, wie z.B. Hospitation an Schulen im Ausland oder internationales Arbeiten an inklusionsbezogenen Fragestellungen, erprobt und ihre Wirksamkeit auf die Kompetenzentwicklung untersucht werden: Führt die reale Konfrontation im Rahmen der Exkursionen zu stärkeren Lerneffekten als die inter- 
nationale Perspektive, at home? Bringen Hospitationen im Ausland einen Mehrwert in der inklusionsorientierten Lehrer*innenbildung? Welche didaktischen Strategien fördern die Kompetenzentwicklung? Auf der Chrono-Ebene wird die Kompetenzentwicklung der Studierenden, z.B. im Hinblick auf Selbstwirksamkeitsüberzeugungen und Einstellung gegenüber Inklusion, über den Verlauf der einzelnen Interventionen in den Blick genommen: Wie nehmen Studierende ibre eigene Kompetenzentwicklung wahr? Wie entwickeln sich Einstellungen gegenüber Inklusion und ihrer Umsetzung innerhalb des Seminarverlaufs?

\section{Forschungsdesign und -methodik}

Kin $U$ ist in zwei parallele Blockveranstaltungen organisiert, an denen jeweils 40 Studierende des Lehramts für sonderpädagogische Förderung (SPF) und an Grundschulen (GS) teilnehmen. Forschungsmethodisch werden die Lerngruppen in ,Experimental-‘ (EG) und ,Kontrollgruppe (KG) verteilt: Während erstere an Exkursionen nach Linz und Brixen teilnimmt, hospitieren die Studierenden der KG an deutschen, inklusiv arbeitenden Schulen. Die theoretischen Seminarinhalte bleiben dabei gleich. Kin $U$ ist auf zwei Kohorten ausgelegt. Die folgende Tabelle verdeutlicht Forschungsdesign sowie Ablauf der Lehrveranstaltungen:

Tab. 1: KinU Forschungsdesign

\begin{tabular}{|c|c|c|c|c|c|c|}
\hline $\begin{array}{l}\text { Lehramts- } \\
\text { studierende } \\
\text { SPF/GS }\end{array}$ & \multirow{3}{*}{ 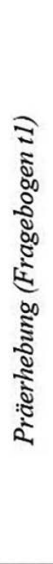 } & Lehrveranstaltung & \multirow{3}{*}{ 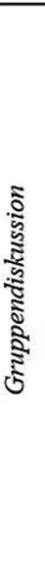 } & Intervention & \multirow{3}{*}{ 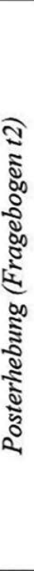 } & \multirow{3}{*}{ 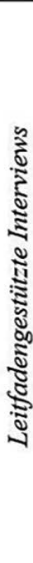 } \\
\hline $\mathrm{EG}(\mathrm{N}=40)$ & & $\begin{array}{l}\text { Infoveranstaltung } \\
\text { 1. Sitzung: Inklusion aus } \\
\text { erziehungs-wissenschaftlicher } \\
\text { Perspektive/soziale } \\
\text { Partizipation in der inklusiven } \\
\text { Schule }\end{array}$ & & $\begin{array}{l}\text { Exkursion und } \\
\text { Hospitation mit EG } \\
\text { nach/in Italien und } \\
\text { Österreich }\end{array}$ & & \\
\hline $\mathrm{KG}(\mathrm{N}=40)$ & & $\begin{array}{l}\text { 2. Sitzung: Beobachtung von } \\
\text { Erziehungs- und } \\
\text { Bildungsprozessen in der } \\
\text { inklusiven Schule/Inklusion in } \\
\text { Österreich und Südtirol } \\
\text { - Exkursion bzw. Hospitation } \\
\text { inkl. Reflexion } \\
\text { - 3. Sitzung: Abschlussreflexion } \\
\text { und Prüfung } \\
\text { (Posterpräsentation) }\end{array}$ & & $\begin{array}{l}\text { KG hospitiert in } \\
\text { Schulen vor Ort } \\
\text { und besucht die } \\
\text { Laborschule } \\
\text { Bielefeld. }\end{array}$ & & \\
\hline
\end{tabular}

In Anlehnung an den Mixed-Methods-Ansatz werden quantitative (Fragebogenerhebung) und qualitative Methoden (Interviews, Gruppendiskussionsverfahren) kombiniert. Der Präfragebogen (t1) kommt in der ersten Seminarsitzung zum 
Einsatz, der Postfragebogen (t2) nach der Intervention. Der Fragebogen beinhaltet Skalen zum Inklusionsverständnis (z.B. in Anlehnung an Wild, Krapp, Schiefele, Lewalter \& Schreyer 1995), zu Einstellungen und Überzeugungen gegenüber inklusiven Unterricht und Inklusion (z.B. nach Bergren 1997; Hang \& Rabren 2009; Langner 2015; Mahat 2008), Vorerfahrungen (vgl. z.B. Bosse \& Spörer 2014) sowie Selbstwirksamkeitsüberzeugungen im inklusiven Unterricht (vgl. z.B. Kopp 2009).

Es werden Gruppendiskussionen zum Inklusionsverständnis und den unterschiedlichen Schul- und Bildungssystemen in Deutschland, Südtirol und Oberösterreich geführt. Diese werden in Anlehnung an Bohnsack (2008) ausgewertet, um kollektive Orientierungsmuster im Umgang mit inklusionsbezogenen Fragestellungen sichtbar zu machen. Diese qualitativen Daten ergänzen die Ergebnisse der Fragebogenerhebung auf Makro- und Chrono-Ebene. Zum Ende werden mit einer kleinen Stichprobe Studierender $(\mathrm{N}=10)$ leitfadengestützte Interviews geführt, um neben der individuellen Kompetenzentwicklung (Chrono-Ebene) die Fragestellungen auf der Meso-Ebene (Kap. 3) zu erheben und so Erkenntnisse zur Wirksamkeit der vorgenommenen Interventionen zu gewinnen. Die Interviews werden mithilfe qualitativer Inhaltsanalysen nach Mayring (2010) ausgewertet.

\section{Erste Ergebnisse}

Zum aktuellen Zeitpunkt ist die Auswertung nicht abgeschlossen, sodass hier nur erste Ergebnisse vorgestellt werden können:

- Makro-Ebene: Das nationale Bildungssystem ist prägend für Einstellungen und Perspektiven in Bezug auf Inklusion, inklusiven Unterricht und inklusionsbezogene Professionalisierung. Es zeigt sich, dass wenig Bewusstsein für diesen Einflussfaktor gegeben ist. Die internationale Perspektive scheint im Lehramtsstudium eine untergeordnete Rolle zu spielen, sodass hier wenig Vorwissen verfügbar ist, diese Perspektive gleichzeitig als sehr bereichernd empfunden wird:

"Ich fand das [die Hospitationen an Schulen in Österreich und Italien] sehr spannend, weil das mir noch in keinem anderen Seminar verdeutlicht wurde. Und einfach den Unterschied zu Deutschland zu sehen, oder den Vergleich ziehen zu können. Denn da gibt es ja dann doch Unterschiede, zum Beispiel, dass Südtirol ja schon einige Schritte voraus ist." 


\section{Timm Albers, Agnes Filipiak, Katja Franzen und Frank Hellmich}

- Meso- und Chrono-Ebene $e^{2}$ :

Die Kompetenzentwicklung der Studierenden zeigt, dass Hospitationserfahrungen positive Auswirkungen auf das Selbstwirksamkeitserleben haben:

„Gerade die Hospitation hat gezeigt, dass Inklusion wirklich machbar ist (...) und das hat mir einfach nochmal mehr Sicherheit gegeben (...) gerade auch im Hinblick auf meinen späteren Beruf."

Der systematische und informelle Austausch über Inklusion wird als zentral für die Kompetenzentwicklung wahrgenommen:

„Ähm, ich würd' noch sagen, dass es auf jeden Fall mit der Exkursion richtig gut war (...) Dass man mit den anderen Studierenden in den Austausch gekommen ist, auch außerhalb, ähm, von den Seminaren."

Die Effektstärke ist bei der Experimentalgruppe tendenziell größer, hier ist ein positiver Einfluss der Auslandsexkursion zu vermuten. Der direkte Kontakt mit anderen Schulsystemen und Studierenden erleichtert die Auseinandersetzung mit einer internationalen Perspektive. Studierende geben z.B. an, dass sie nach der Exkursion Erleichterung verspüren, da „woanders auch nicht alles besser" ist. Offenbar vermittelt die theoretische Auseinandersetzung mit der internationalen Perspektive im Abgleich mit den eigenen Erfahrungen einen idealisierten Eindruck.

Die folgende Abbildung fasst weitere Teilergebnisse aus den Einzelinterviews zusammen:

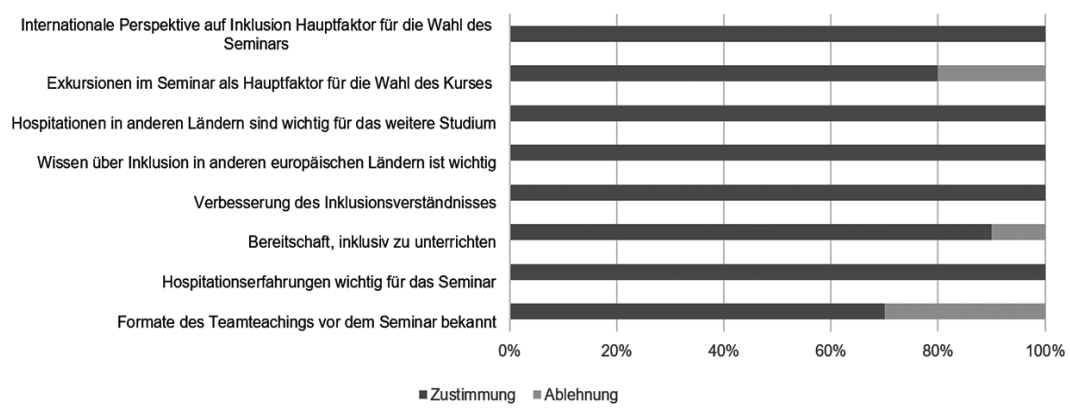

Abb. 1: Ergebnisse Einzelinterviews

2 Da bei der Befragung von Studierenden in die Beantwortung der Fragestellungen auf der MesoEbene stets Informationen zur individuellen Kompetenzentwicklung (Chrono-Ebene) einfließen, werden diese Ebenen in dieser ersten Ergebnisdarstellung zusammengefasst. 


\section{Ausblick}

Die Ergebnisse werden durch weitere Daten einer zweiten Kohorte ergänzt und spezifiziert. Bereits jetzt zeichnet sich ab, dass das Potenzial einer internationalen Perspektive in der Kompetenzentwicklung zukünftiger Lehrkräfte für inklusive Settings bisher kaum ausgeschöpft wird und dass der Austausch über Einstellungen und Problemstellungen sowie Reflexion eine wesentliche Rolle spielen. Daraus ergeben sich neue Forschungsfragen:

- Wie kann die internationale Perspektive stärker im Studium verankert werden? Da die Möglichkeiten für Exkursionen aufgrund begrenzter Ressourcen über den Projektzeitraum hinaus eingeschränkt sind: Wie lassen sich Internationalisierungs-strategien ,at home ' und Kooperationen stärker etablieren und welche Möglichkeiten sind effektiv?

- Wie können kollaborative Austauschprozesse zwischen den Studierenden stärker in den Lehr-/Lernprozess eingebunden werden, wie lässt sich dabei das Potenzial informellen Austausches nutzen?

Kin $U$ kann diese Fragen sicherlich nicht erschöpfend beantworten. Dennoch erscheint ein besonderer Fokus auf kooperative Austauschformate unter Einbindung von E-Learning-Formaten sowohl auf Ebene des Seminars als auch in der Kooperation mit den Hochschulen in Linz und Südtirol zur Überprüfung der bisherigen Ergebnisse sinnvoll.

\section{Literatur}

Baumert, J. \& Kunter, M. (2011): Das Kompetenzmodell von COACTIV. In: Kunter, M., Baumert, J., Blum, W., Klusmann, U., Krauss, S. \& Neubrand, M. (Hrsg.): Professionelle Kompetenz von Lehrkräften. Münster, 29-53.

Bergren, B. A. (1997): Teacher attitudes toward included special education students and co-teaching. Online unter: https://files.eric.ed.gov/fulltext/ED408754.pdf (letzter Zugriff: 09.11.2020).

Bohnsack, R. (2008): Rekonstruktive Sozialforschung. Einführung in qualitative Methoden. Opladen/Farmington Hills.

Bosse, S. \& Spörer, N. (2014): Erfassung der Einstellung und der Selbstwirksamkeit von Lehramtsstudierenden zum inklusiven Unterricht. In: Empirische Sonderpädagogik, 4, 279-299.

Bürli, A. (2010): Wie hast du’s, Europa, mit der Integration Behinderter? In: Zeitschrift für Inklusion, 4, H.2, o.S. Online unter: http://www.inklusion-online.net/index.php/inklusion-online/article/ view/137 (letzter Zugriff: 08.10.2019).

DAAD-Fachkonferenz ,'Internationalisierung der Lehrerbildung“ (Hrsg.) (2013): Lehrerbildung muss internationaler werden. Resolution zur Internationalisierung der Lehramtsausbildung. Online unter: https://www.daad.de/medien/veranstaltungen/lehrerbildung/2013_pressemeldung_resolution_25141.de.pdf (letzter Zugriff: 08.10.2019).

Europäische Kommission (2002): Definitionen des Begriffs ,Behinderung' in Europa: Eine vergleichende Analyse. Online unter: www.arbeitundbehinderung.at/downloads/complete_report_de-1. pdf (letzter Zugriff: 02.02.2021).

European Agency for Development in Special Needs Education (2012): Inklusionsorientierte Lehrerbildung. Ein Profil für inklusive Lehrerinnen und Lehrer. Online unter: https://www.europeanagency.org/sites/default/files/profile_of_inclusive_teachers_de.pdf (letzter Zugriff: 14.08.2019). 


\section{Timm Albers, Agnes Filipiak, Katja Franzen und Frank Hellmich}

Gebhardt, M., Kuhl, J., Wember, F. \& Wittich, C. (2018): Inklusives Modell in der Lehramts-ausbildung nach den Anforderungen der UN-BRK. In: Hußmann, S. \& Welzel, B. (Hrsg.): DoProfiL. Das Dortmunder Profil für inklusionsorientierte Lehrerinnen und Lehrerbildung. Münster, 279292.

Gonon, P. (2011): Die Bedeutung des internationalen Arguments in der Lehrerbildung. In: Beiträge zur Lehrerbildung, 29, H.1, 20-26.

Hang, Q. \& Rabren, K. (2009): An examination of co-teaching: Perspectives and efficacy indicators. In: Remedial and Special Education, 30, H.5, 259-268.

Hillenbrand, C., Melzer, C. \& Hagen, T. (2013): Bildung schulischer Fachkräfte für inklusive Bildungssysteme. In: Döbert, H. \& Weishaupt, H. (Hrsg.): Inklusive Bildung professionell gestalten. Situationsanalyse und Handlungsempfehlungen. Münster, 33-68.

HRK-Audit „Internationalisierung der Hochschulen“ und Internationale Abteilung (2014): Auf internationale Erfolge aufbauen. Beispiele guter Internationalisierungspraxis an deutschen Hochschulen. Online unter: https://www.hrk.de/fileadmin/redaktion/hrk-audit/Infothek/HRK-Audit_Auf_ internationale_Erfolge_aufbauen.pdf (letzter Zugriff: 08.10.2019).

Kämper van den Boogaart, M. (2019): Lehramtsausbildung - das Elend einer pädagogischen Provinz in einer globalisierten Welt? In: Falkenhagen, C., Grimm, N. \& Volkmann, L. (Hrsg.): Internationalisierung des Lehramtsstudiums. Modelle, Konzepte, Erfahrungen. Paderborn, 17-34.

Kopp, B. (2009): Inklusive Überzeugung und Selbstwirksamkeit im Umgang mit Heterogenität. Wie denken Studierende des Lehramts für Grundschulen? In: Empirische Sonderpädagogik, 1, 5-25.

Langner, A. (2015): Kompetent für einen inklusiven Unterricht. Eine empirische Studie zu Beliefs, Unterrichtsbereitschaft und Unterricht von LehrerInnen. Wiesbaden.

Leutwyler, B. (2013): Internationalisierung in der Lehrerinnen- und Lehrerbildung. Ein Überblick über Aktionsfelder und Herausforderungen. In: Kentron - Journal zur Lehrerbildung, 26, 7-15. Online unter: https:/www.uni-potsdam.de/fileadmin01/projects/zelb/Dokumente/Publikationen/kentron/kentron_26-2013.pdf (letzter Zugriff: 08.10.2019).

Leutwyler, B., Mantel, C. \& Tremp, P. (2011): Lokale Ausrichtung - internationaler Anspruch: Lehrerinnen- und Lehrerbildung an pädagogischen Hochschulen. In: Beiträge zur Lehrerbildung, 29, H.1, 5-19.

Mahat, M. (2008): The development of a psychometrically-sound instrument to measure teachers' multidimensional attitudes toward inclusive education. In: International Journal of Special Education, 23, H.1, 82-92.

Mayring, P. (2010): Qualitative Inhaltsanalyse. Grundlagen und Techniken. Weinheim/Basel.

Melzer, C., Hillenbrand, C., Sprenger, D. \& Hennemann, T. (2015): Aufgaben von Lehrkräften in inklusiven Bildungssystemen - Review internationaler Studien. In: Erziehungswissenschaft, 26, H.51, 61-80.

Merz-Atalik, K. \& Franzkowiak, T. (2011): Das Projekt „Teacher Education for Inclusion (TE41)“ Lehrerbildung für Inklusion der European Agency for Development in Special Needs Education. In: Zeitschrift für Inklusion, 6, H.3, o.S. Online unter: http://www.inklusion-online.net/index. php/inklusion-online/article/view/85/85 (letzter Zugriff: 08.10.2019).

Moser, V. (2014): Forschungserkenntnisse zur sonderpädagogischen Professionalität in inklusiven Settings. In: Trumpa, S., Seifried, S., Franz, E. \& Klauß, T. (Hrsg.): Inklusive Bildung. Erkenntnisse und Konzepte aus Fachdidaktik und Sonderpädagogik. Weinheim, 92- 106.

Weinert, F. E. (2001): Leistungsmessung in Schulen. Eine umstrittene Selbstverständlichkeit. In: Weinert, F. E. (Hrsg.): Leistungsmessung in Schulen. Weinheim/Basel, 17-31.

Wild, K.-P., Krapp, A., Schiefele, U., Lewalter, D. \& Schreyer, I. (1995): Dokumentation und Analyse der Fragebogenverfahren und Tests (Berichte aus dem DFG-Projekt Bedingungen und Auswirkungen berufsspezifischer Lernmotivation, Nr. 2). Neubiberg. 
Katharina Maria Pongratz

\section{Sehnsucht nach Bildung?}

\section{Über den Einsatz systemischer Fragetechniken in der qualitativen Erwachsenenbildungs-/Weiterbildungsforschung bei Biografieträger*innen mit einer zugeschriebenen geistigen Behinderung}

Sehnen sich erwachsene Menschen mit einer zugeschriebenen geistigen Behinderung (mzgB) nach Bildung? Gibt es ein Verständnis von Bildung, welches die bisher befragten und noch zu befragenden Biografieträger*innen verbindet und wenn ja, ist dieses mit den bisher verwendeten Bildungsbegriffen innerhalb der Erwachsenenbildung/Weiterbildung übereinstimmend? Diese und weitere Fragen werden in Form des Forschungsvorhabens Sehnsucht nach Bildung? - Behinderung, Teilhabe und Partizipation in der Erwachsenen- und Weiterbildung aktuell untersucht und diskutiert. Für den folgenden Beitrag wird sich auf den Prozess der Datenerhebung biografisch auswertbaren Materials fokussiert, welcher bisherige Interviewformen und Interviewtechniken für Menschen mit einer zugeschriebenen geistigen Behinderung durch die Integration systemischer Fragetechniken im Feld erprobt(e) und ergänzt. Es wird sich auf Daten und Erfahrungswerte von 11 Interviews bezogen, welche im Zeitraum von 2017-2020 durchgeführt wurden. Dabei wird der folgenden Frage nachgegangen: (Wie) kann eine qualitative Erwachsenenbildungs-/Weiterbildungsforschung (noch mehr) biografisch auswertbares Material von und mit erwachsenen Menschen mit einer zugeschriebenen geistigen Behinderung erheben, um - u.a. bezugnehmend auf das Gesamtvorhaben - zukünftig Bildungsorientierungen dieser zu rekonstruieren?

\section{Eckdaten des Forschungsprojekts}

Untersucht wurde im Rahmen einer dreijährigen Pretestphase des laufenden Promotionsvorhabens ein an der Universität durchgeführter Kurs Schriftspracherwerb für erwachsene Menschen mit einer sogenannten geistigen Behinderung. Die Teilnehmenden sind in einer Werkstatt für behinderte Menschen (WfbM) in regionaler Nähe der den Kurs durchführenden Universität beschäftigt. Ein materialistischdialektisches Behinderungsverständnis nach Jantzen (2002, 312), welches die 
Vorstellung einer bio-psycho-sozialen Einheit Mensch beinhaltet (vgl. Moser \& Sasse 2008, 76), bildet die Grundlage des begleiteten Kursangebotes. Konzeptionell liegt das Verständnis des Lernens am Gemeinsamen Gegenstand diesem Weiterbildungsangebot zugrunde (vgl. Feuser 1989). Mitarbeitende der WfbM und Studierende des Masterstudiengangs Bildungswissenschaft belegen diesen Kurs gemeinsam; die Mitarbeitenden der WfbM als berufliche Weiterbildung, die Studierenden als Seminar mit Prüfungsleitung und Erwerb von Credit Points (4 SWS, $10 \mathrm{CP})$.

Die dreijährige wissenschaftliche Begleitung (2017-2020), die Pretestphase des diesem Beitrag zugrunde liegenden Forschungsprojektes, erfolgte mittels teilnehmender Beobachtung, Bild- und Videodokumentation, Motivationsabfragen, biografischer- und leitfadengestützter Interviews als Formate empirischer Datenerhebung. Das Promotionsvorhaben untersucht dabei Bildungsorientierungen von Biografieträger*innen und nimmt Angebote der Erwachsenenbildung/Weiterbildung und deren (Nicht)Teilnehmende in den Blick. Begrifflichkeiten wie Behinderung, Teilhabe und Partizipation werden aus der Perspektive der Erwachsenenbildung/Weiterbildung betrachtet und bezugnehmend auf aktuelle Debatten diskutiert. Grundlagentheoretisch wird sich der praxeologischen Wissenssoziologie (vgl. Bohnsack 2017) unter Heranziehung der Theorien Bourdieus (vgl. Bourdieu 1982 \& 1985; Bourdieu, Pialoux \& Schwibs 2015) bedient. Inklusion wird dabei als soziale Praxis gedacht. Der Feldzugang erfolgt in Form der Erhebung empirischen Materials von Biografieträger*innen msgB, welche (nicht) an Angeboten der Erwachsenenbildung/Weiterbildung teilnehmen. Methodologisch ist das Projekt in der Dokumentarischen Methode verortet (vgl. Bohnsack 2013 \& 2017; Schäffer \& Dörner 2012).

\section{Geistige Behinderung und qualitative Erwachsenenbildungs-/Weiterbildungsforschung}

Im Jahr 1970 wurde Erwachsenenbildung als „Fortsetzung oder Wiederaufnahme organisierten Lernens nach Abschluss einer unterschiedlich ausgedehnten Ausbildungsphase" (Deutscher Bildungsrat 1970, 197) definiert. Die Erwachsenen-/ Weiterbildung blickt dazu auf einen langjährigen Erfahrungsschatz differenzierter Angebote für heterogene Gruppen zurück (vgl. Lauber-Pohle 2019, 16). Die Volkshochschulen bspw. haben mit Setzung der Definition für Erwachsenenbildung durch den deutschen Bildungsrat im Kontext der Zielgruppenorientierung bereits vor 50 Jahren begonnen, Menschen mit zugeschriebener geistiger Behinderung als Zielgruppe zu identifizieren (vgl. Heimlich \& Behr 2018, 1211; Schäffer, Schemmann \& Dörner 2012; Tenorth \& Tippelt 2007; Wittpoth 2013). Das Thema Inklusion wirkt dennoch in der Erwachsenenbildung/Weiterbildung, ins- 
besondere mit Blick auf Erwachsene mit einer geistigen Behinderung, empirisch nach wie vor als eher randständig erforscht, da keine Studien vorzuliegen scheinen, welche den Fokus auf das Phänomen, geistige Behinderung' in der Erwachsenen-/Weiterbildung richten und dabei die untersuchte Personengruppe zu Wort kommen lassen.

Ausgehend von - unter anderem - den Arbeiten von Buchner (2008) bzw. Buchner, Koenig und Schuppener (2011) sowie Demmer (2013 \& 2014) wurde im Jahr 2017 begonnen, eigenes empirisches Material von Biografieträger*innen zu erheben und mit einer qualitativen Erwachsenen- und Weiterbildungsforschung (vgl. Schäffer \& Dörner 2012) in Bezug zu setzen. Dies erfolgte zunächst im Rahmen der wissenschaftlichen Begleitung des oben aufgeführten Schriftspracherwerbkurses, welcher während der Vorlesungszeit einmal wöchentlich als Lehrveranstaltung an einer Universität angeboten wird, und ging im Anschluss daran in ein eigenständiges Forschungsprojekt über. Das Ziel der qualitativen Bildungsforschung wird nach hiesigem Verständnis nicht als ein empathisches Nachvollziehen von Situationen oder Verhalten verstanden, sondern als eine Rekonstruktion typischer Muster in bestimmten Situationen, bspw. genauer zu untersuchender Milieus, welche es zu analysieren gilt (vgl. ebd.). Inklusive Bildung wird dazu im erziehungswissenschaftlichen Diskurs nach Köpfer, Powell und Zahnd (2020) als ein konzeptueller Bezugspunkt verstanden. Inklusive Bildung erfolgt, wenn Fragen von Teilhabe und Partizipation mit Blick auf Bildung, Erziehung und Sozialisation verhandelt werden (vgl. ebd., 89). Ein Inklusionsbegriff im Kontext der Erwachsenen-/Weiterbildung wird im laufenden Promotionsvorhaben unter Einbeziehen der Theorien Bourdieus und einer daran anschließenden praxeologischen Perspektive als soziale Praxis entwickelt. Unter einer inklusiv qualitativen Erwachsenenbildungs-/Weiterbildungsforschung wird an dieser Stelle zunächst verstanden, dass gängige Erhebungs- und Auswertungsverfahren bei unterschiedlichen Biografieträger*innen bei Bedarf modifiziert Anwendung finden, um eine Generierung empirischen Materials auch dann zu ermöglichen, wenn sich Herausforderungen im Feld zeigen. Hierbei ist ein weiterer Fokus auf u.a. traumatisch vorbelastete Biografieträger*innen zu richten, um diese vor einer potenziellen (Re-)Traumatisierung zu schützen; die Schadensvermeidung nach von Unger (2014) bildet die Grundlage im gesamten Forschungsprozess.

\section{Feldzugang, Datenerhebung, Herausforderungen}

Der Feldzugang gestaltet(e) sich problemlos; die zu Befragenden stimmten einer wissenschaftlichen Begleitung/Befragung zu und der Austausch mit allen Beteiligten verlief reibungslos. Allen Biografieträger*innen wurde, orientiert an den Konzepten der leichten Sprache, vermittelt, worum es sich bei den unterschied- 
lichen Erhebungsformaten handelt, zudem wurden die datenschutzrechtlichen Hinweise angemessen erörtert, um ein informiertes Einverständnis sicherzustellen (vgl. ebd., 28f. ). Die Erhebung gestaltete sich jedoch in ersten Interviews herausfordernd, da kaum bis gar keine Erzählteile zustande kamen. Das methodische Vorgehen wurde deshalb im Verlauf der Erhebung modifiziert. Erschwerend kam für die Erhebungsphase hinzu, dass alle befragten Biografieträger*innen auf verschiedene Weise von traumatischen Erfahrungen in ihrem (Schul-)Leben berichteten. Bedingt durch eine u.a. systemisch therapeutische Ausbildung der Forschenden wurden deshalb präventiv einzelne Interviews abgebrochen, da die Gefahr einer (Re-) Traumatisierung der Befragten vonseiten der durchführenden Forscherin wahrgenommen wurde und die Stabilisierung der befragten Person Vorrang hatte. Die Notwendigkeit einer noch besser an die Biografieträger*innen angepassten Gesprächsführung in Form von modifizierten Fragetechniken wurde deutlich; dies zeigte sich u.a. anhand folgender Entwicklung im Feld: Die ersten durchgeführten Interviews endeten bereits nach wenigen Minuten, wiesen praktisch eher ein Frage-Antwort-Spiel mit langen Pausen auf und beinhalteten keine bis sehr wenige Narrative. Durch Einsetzen der u.a. von Demmer (2013 \& 2014) und Buchner (et al.) (2008 \& 2011) erarbeiteten Modifikationen in der Interviewführung, wie bspw. dem Legen einer Linie mit biografischen Eckdaten und dem individuellen Einlassen auf die zu interviewenden Gesprächspartner*innen, wurde bei weiteren Interviews eine erste Veränderung hinsichtlich der Zunahme der Erzählteile und Dauer der Interviews beobachtet. Dennoch ist die erste Phase der Datenerhebung als fortlaufend schwierig und herausfordernd zu bewerten. Dies führte zwischenzeitlich bei der Forschenden zu Überlegungen, den Feldversuch (frühzeitig) zu beenden. Ein Schlüsselerlebnis weckte dann erneut den Ehrgeiz der Forschenden, weiter nach einem Zugang zu dem biografisch auswertbaren Material zu suchen und die Datenerhebung nicht als gescheitert aufzugeben: Auf die Frage, warum eine Kursteilnehmende jede Woche zum Kurs Schriftspracherwerb an die Universität komme, erfolgte folgende Antwort: „Ich möchte in die Schule gehen und die Sachen lernen, die ich verlernt habe. Ich gebe nicht auf. Man muss es probieren. Es macht Spaß und ich gehe weiter zur Schule." Dieser Satz prägte die hier auszugsweise vorgestellte Erhebungsphase nachhaltig. Wenn die zu befragende Personengruppe den Ehrgeiz zeigte, nicht aufzugeben, musste es einen Weg geben, biografisch auswertbares Material der befragten Biografieträger*innen msgB zu generieren. Die Forschende entschied sich deshalb zunächst dafür, die bereits befragten Biografieträger*innen in einem persönlichen Gespräch zu fragen, warum diese in den Interviews deutlich weniger erzählten als bspw. im Rahmen des Kurses selbst. So wurde angemerkt, dass ein*e Biografieträger*in Angst vor dem Mikrofon und der Kamera hatte; andere wiesen darauf hin, dass vonseiten der Forschenden nicht ausreichend Zeit/Möglichkeiten zum Antworten eingeräumt wurde/n, wieder andere meinten, die Fragen seien schlicht ,blöd und 
unverständlich' gewesen. Alle Hinweise vereinten jedoch, dass es scheinbar um äußere Einflüsse der Interviewsituation ging, welche einen Erzählfluss verhinderten, und nicht um eine scheinbar mögliche Unfähigkeit des Erzählens bei den zu Befragenden. Ohne die Befragung und das Zu-Wort-Kommen der Biografieträger*innen selbst wäre eine Fortführung des Vorhabens nicht möglich gewesen. Diese Beispiele und Erkenntnisse führten letztendlich zu einer Überarbeitung der Gesprächs- und Fragetechniken, auf welche im Folgenden eingegangen wird.

\section{Systemische Fragetechniken in biografischen und leitfadengestützten Interviews}

Systemische Fragen scheinen aufgrund ihres Interviewstils auf den ersten Blick vielen Regeln der psychotherapeutischen Orthodoxie zu widersprechen (vgl. Simon \& Rech-Simon 2018, 7); die*der Gesprächsführende ist dabei aktiv und verfügt über einen vorbereiteten Fragenkatalog, welcher je nach Verlauf der Sitzung zum Einsatz kommt. Der Begriff der zirkulären Fragen als wichtiges Werkzeug systemischer Therapeut*innen (vgl. Patrzek 2017; Penn 1983; Selvini Palazzoli, Boscolo, Cecchin \& Prata 1981; Simon \& Rech-Simon 2018; Tomm 2018) wird in aktuellen Debatten und Therapieverfahren unterschiedlich verwendet. Oftmals wird dieser gar als Überbegriff für alle systemischen Gesprächs- und Fragetechniken verwendet, wenngleich innerhalb der systemischen Therapie zahlreiche Fragetechniken existieren und gezielt - je nach Kontext - zum Einsatz kommen (vgl. Simon \& Rech-Simon 2018, 8). Für das hiesige Forschungsvorhaben fanden bisher folgende systemische Interventionen Anwendung:

- triadische Fragen inkl. einhergehendem Einführen einer Außenperspektive,

- angewendete Verflüssigungen von Eigenschaften in der Fragestellung,

- gezieltes Erfragen von Unterschieden,

- Fragen nach qualitativen und quantitativen Differenzierungen,

- hypothetische Fragen in Bezug auf die Biografie der Befragten.

Es wurde dabei untersucht, welche Impulse die Biografieträger*innen benötigten, um innerhalb einer vertrauensvollen Interviewsituation in das Erzählen zu gelangen. Hierbei zeigte sich: Die triadischen Fragetechniken und die qualitative und quantitative Differenzierung wirkten besonders erzählstimulierend. Inwieweit dabei für die Auswertung kritische Eingriffe in die Eingangserzählung (vgl. Nohl 2017, 20f. ) oder bspw. die Zugzwänge des Erzählens vonseiten der befragten Interviewträger*innen zu verzeichnen sind, muss im Rahmen einer detaillierten Auswertung analysiert und reflektiert werden. Dennoch: Durch das Einführen systemischer Fragen in unterschiedliche Interviewphasen und Formate konnte bisher eine maßgebliche Verbesserung hinsichtlich der Anzahl der generierten 
Erzählteile erzielt werden. Die eingesetzte Eingangserzählung, welche durch eine autobiografisch orientierte Erzählaufforderung nach Schütze $(1983,285)$ aufrechterhalten werden konnte, setzte eine Erzählung in Gang; wenn es stockte, fanden systemische Fragetechniken impulsgebend ihren Einsatz. Die Interviewten erzählten mit Anwendung der modifizierten Fragetechniken zunehmend autobiografisch. Diese Modifizierung der empirischen Erhebungsinstrumente mittels eigens für Biografieträger*innen mit besonderen Bedürfnissen entwickelter Gesprächs- und Fragetechniken wird zukünftig im Verlauf einer weiteren Erhebungsphase erprobt, aufbereitet und in Bezug auf die Gütekriterien qualitativer Bildungs- und Sozialforschung (vgl. Przyborski \& Wohlrab-Sahr 2014) weiter ausgewertet. Die Frage danach, inwieweit zukünftig das biografische Interview oder ein leitfadengestütztes Interview dazu, unter Berücksichtigung der modifizierten Gesprächs- und Fragetechniken als Erhebungsinstrument, Anwendung findet, gilt es ebenfalls weiter zu untersuchen. Sicher scheint jedoch, dass eine Datenerhebung mittels modifizierter Gesprächstechniken in Interviewform mit der untersuchten Personengruppe fortgeführt wird und werden kann.

\section{Zusammenfassung}

Das Durchführen von Interviews als Erhebungsinstrument qualitativer Bildungsforschung mit Biografieträger*innen mit einer zugeschriebenen geistigen Behinderung wird in Bezug auf eine qualitative Erwachsenenbildungs-/Weiterbildungsforschung zum jetzigen Stand des Forschungsvorhabens als grundsätzlich durchführbar bewertet, so wie es bereits andere Studien (siehe Kap. 2 und 3) anderer Disziplinen im Feld bestätigten. Es wurde für dieses Vorhaben herausgefunden, dass sich insbesondere eine Modifizierung der Erhebungsinstrumente durch das Anwenden systemischer Fragetechniken als hilfreich erweisen kann, um biografisch auswertbares empirisches Material zu generieren, wenn die Biografieträger*innen besondere Bedürfnisse aufweisen. Dies kann bei Menschen mit einer zugeschriebenen geistigen Behinderung zutreffen, aber auch bei anderen befragten Personen (bspw. Interviews mit Menschen mit einer anderen Muttersprache, Interviews mit hochaltrigen Personen usw.). Die Bereitschaft der Forschenden, sich auf einen zielgruppenorientierten Einsatz eben dieser Erhebungsinstrumente einzulassen und sich in der Anwendung dieser auszuprobieren und die Befragten aktiv mit einzubeziehen, wird dabei als Grundhaltung für einen gelingenden Forschungsprozess vorausgesetzt. Die Anwendung der Erhebungsinstrumente könnte je nach Interviewpartner*in und Qualifikation der Forschenden sicher auch um andere Frage- und Gesprächstechniken erweitert werden, wenn Erhebungsinstrumente unter Anleitung der klassischen Erzählaufforderungen oder zu entwickelnder Leitfragen keine Narrative zur Folge haben. Hierbei muss sich der Fokus nicht 
nur auf systemische Fragetechniken richten; die Psychoanalyse bietet sicher ebenfalls interessante Alternativen, um nur eine weitere zu nennen. Es scheint, dass das gesamte Feld der qualitativen Forschung - bezugnehmend auf das Integrieren von eigens dafür entwickelten und gezielt eingesetzten Fragetechniken in etablierte Erhebungsinstrumente der qualitativen Bildungs- und Sozialforschung - eine einladende Anzahl an Desideraten aufweist.

Vielleicht könnte zukünftig grundsätzlich überlegt werden, welche Methoden/ Instrumente es (weiter) zu entwickeln gilt, um empirische Forschung mit bis zu diesem Zeitpunkt vielleicht gering empirisch erforschten Personengruppen zu ermöglichen - herausfordernde Interviewpartner*innen finden sich gegebenenfalls auch losgelöst von der Zuschreibung einer geistigen Behinderung.

\section{Literatur}

Bohnsack, R. (Hrsg.) (2013): Die dokumentarische Methode und ihre Forschungspraxis. Grundlagen qualitativer Sozialforschung. 3. aktual. Aufl. Wiesbaden.

Bohnsack, R. (2017): Praxeologische Wissenssoziologie. Opladen.

Bourdieu, P. (1982): Die feinen Unterschiede. Kritik der gesellschaftlichen Urteilskraft. 1. Aufl. Frankfurt am Main.

Bourdieu, P. (1985): Sozialer Raum und „Klassen“. Leçon sur la leçon/Pierre Bourdieu. 1. Aufl. Frankfurt am Main.

Bourdieu, P., Pialoux, C. \& Schwibs, B. (2015): Entwurf einer Theorie der Praxis. Auf der ethnologischen Grundlage der kabylischen Gesellschaft. 4. Aufl. Frankfurt am Main.

Buchner, T. (2008): Das qualitative Interview mit Menschen mit so genannter geistiger Behinderung. Ethische, methodologische und praktische Aspekte. In: Biewer, G., Luciak, M. \& Schwinge, M. (Hrsg.): Begegnung und Differenz: Menschen, Länder, Kulturen. Beiträge zur Heil- und Sonderpädagogik. Bad Heilbrunn, 516-528.

Buchner, T., Koenig, O. \& Schuppener, S. (2011): Gemeinsames Forschen mit Menschen mit intellektueller Behinderung. Geschichte, Status quo und Möglichkeiten im Kontext der UN-Behindertenrechtskonvention. In: Teilhabe - die Fachzeitschrift der Lebenshilfe, 50, H.1, 4-10.

Demmer, C. (2013): Biografien bilden. Lern- und Bildungsprozesse von Frauen mit Behinderung im Spannungsfeld von Teilhabe und Ausschluss. Bochum.

Demmer, C. (2014): Autobiografisch narrative Interviews - (k)ein Erhebungsformat für Menschen mit kognitiver Beeinträchtigung? Siegen. Online unter: https://www.bildung.uni-siegen.de/forschung/workingpapers/wopa/narrative_intervies.pdf (letzter Zugriff: 20.05.2019).

Deutscher Bildungsrat (Hrsg.) (1970): Empfehlungen der Bildungskommission - Strukturplan für das Bildungswesen. Stuttgart.

Feuser, G. (1989): Allgemeine integrative Pädagogik und entwicklungslogische Didaktik. In: Behindertenpädagogik, 28, 4-48.

Heimlich, U. \& Behr, I. (2018): Inklusion von Menschen mit Behinderung in der Erwachsenenbildung/Weiterbildung. In: Tippelt, R. \& von Hippel, A. (Hrsg.): Handbuch Erwachsenenbildung/ Weiterbildung. 6. überarb. und aktual. Aufl. Wiesbaden, 1207-1223.

Jantzen, W. (2002): Identitätsentwicklung und pädagogische Situation behinderter Kinder und Jugendlicher. In: Sachverständigenkommission 11. Kinder- und Jugendbericht (Hrsg.): Gesundheit und Behinderung im Leben von Kindern und Jugendlichen, Bd. 4. 5 Bände. München, 319380. 
Köpfer, A., Powell, J. W. \& Zahnd, R. (Hrsg.) (2020): Handbuch Inklusion international. Globale, nationale und lokale Perspektiven auf Inklusive Bildung. 1. Aufl. Leverkusen.

Lauber-Pohle, S. (2019): Dimensionen einer inklusiven, allgemeinen Erwachsenenbildung. In: Hessische Blätter für Volksbildung, 1, 7-17.

Moser, V. \& Sasse, A. (2008): Theorien der Behindertenpädagogik. München.

Nohl, A.-M. (2017): Interview und Dokumentarische Methode. Wiesbaden.

Patrzek, A. (2017): Systemisches Fragen. Professionelle Fragetechnik für Führungskräfte, Berater und Coaches. 2. Aufl. Wiesbaden.

Penn, P. (1983): Zirkuläres Fragen. In: Familiendynamik - Interdisziplinäre Zeitschrift für Praxis und Forschung, 8, 198-220.

Przyborski, A. \& Wohlrab-Sahr, M. (2014): Qualitative Sozialforschung. Ein Arbeitsbuch. 4. erw. Aufl. München.

Schäffer, B. \& Dörner, O. (Hrsg.) (2012): Handbuch Qualitative Erwachsenen- und Weiterbildungsforschung. Leverkusen.

Schäffer, B., Schemmann, M. \& Dörner, O. (Hrsg.) (2012): Erwachsenenbildung im Kontext. Theoretische Rahmungen, empirische Spielräume und praktische Regulative. Bielefeld.

Schütze, F. (1983): Biographieforschung und narratives Interview. In: Neue Praxis, 13, 283-293.

Selvini Palazzoli, M., Boscolo, L., Cecchin, G. \& Prata, G. (1981): Hypothetisieren - Zirkularität Neutralität: Drei Richtinien für den Leiter der Sitzung. In: Familiendynamik - Interdisziplinäre Zeitschrift für Praxis und Forschung, 6, 123-139.

Simon, F. B. \& Rech-Simon, C. (2018): Zirkuläres Fragen. Systemische Therapie in Fallbeispielen: ein Lernbuch. 13. Aufl. Heidelberg.

Tenorth, H.-E. \& Tippelt, R. (Hrsg.) (2007): Beltz Lexikon Pädagogik. 1. Aufl. Weinheim.

Tomm, K. (2018): Die Fragen des Beobachters. Schritte zu einer Kybernetik zweiter Ordnung in der systemischen Therapie. 6. Aufl. Heidelberg.

von Unger, H. (2014): Partizipative Forschung. Einführung in die Forschungspraxis. Wiesbaden.

Wittpoth, J. (2013): Einführung in die Erwachsenenbildung. 4. überarb. und aktual. Aufl. Opladen. 


\section{Laura Schwörer, Hannah van Ledden, Pia Algermissen und Mandy Hauser}

\section{Zusammenarbeit und Mediennutzung in einer Partizipativen Forschungsgruppe}

\section{Das ParLink-Forschungsprojekt}

Partizipative Forschungsarbeit ist eine echte Herausforderung - insbesondere dann, wenn die Forschenden weit voneinander entfernt leben und sich nur selten treffen können. Im ParLink-Projekt versuchen wir, partizipativ zu forschen. Darum arbeiten wir mit einer sogenannten Partizipativen Forschungsgruppe zusammen. Sowohl in unserer direkten Zusammenarbeit als auch bei der Zusammenarbeit über unterschiedliche digitale Medien ist es uns wichtig, dass die Forschungsgruppe wirklich partizipieren kann. Durch die Corona-Pandemie hat die digitale Mediennutzung für das Projekt nochmals an Bedeutung gewonnen. Das Risiko der Pseudopartizipation (vgl. Hauser 2020) zwingt uns dazu, viel über unsere Interaktion und unsere Mediennutzung zu reflektieren.

Das Forschungsprojekt ParLink (,Partizipative Lehre im Kontext inklusionssensibler Hochschulentwicklung') ist ein interdisziplinäres. Wir untersuchen gemeinsam Möglichkeiten inklusionssensibler Hochschulentwicklung auf der Ebene der Dozierenden. Dabei liegt unser Fokus auf der Gestaltung von Hochschullehre durch sogenannte Bildungsfachkräfte ${ }^{1}$ mit Lernschwierigkeiten. Wir gehen sowohl der Frage nach, inwiefern durch partizipative Lehre mit qualifizierten Bildungsfachkräften die inklusive Qualität der Hochschulbildung erhöht werden kann, als auch der, welche (didaktischen) Gestaltungsmöglichkeiten partizipativer Lehre es gibt. Unsere Arbeitsweise wollen wir im Folgenden näher vorstellen. Ein partizipatives Forschungsprojekt in einem Text angemessen zu präsentieren, ist jedoch gar nicht so einfach und es gibt unterschiedliche Möglichkeiten, darüber zu schreiben. In den folgenden Absätzen sind zwei unterschiedliche Heran-

1 Gemeint sind hier Dozierende mit Lernschwierigkeiten. Diese werden an mehreren Standorten in Deutschland und in unterschiedlichen Projekten für die Hochschullehre qualifiziert. Die Bezeichnung für diese Lehrpersonen ist jeweils unterschiedlich. In den Projekten des Instituts für Inklusive Bildung (IIB) Kiel werden sie ,Bildungsfachkräfte‘ genannt, im sächsischen Qualifizierungsprojekt (QuaBiS) in Leipzig und Dresden hingegen ,Bildungs- und Inklusionsreferent*innen‘. Die am ParLink-Projekt beteiligten Dozierenden bezeichnen sich als Bildungsfachkräfte. 
gehensweisen mit unterschiedlich einfacher Sprache zu finden, die durch die Verwendung von gerader und kursiver Schrift unterschieden werden können.

\section{Einblick in die Partizipative Forschungsgruppenarbeit}

\subsection{Zusammensetzung der Partizipativen Forschungsgruppe}

Die Partizipative Forschungsgruppe setzt sich aus Menschen unterschiedlicher Gruppen zusammen: Wir sind sechs hauptamtlich Forschende, die im Forschungsprojekt angestellt sind und acht sogenannte Ko-Forschende (vgl. von Unger 2014). Die Ko-Forschenden sind sechs forschungsbegeisterte Studierende unterschiedlicher Fachrichtungen und zwei Bildungsfachkräfte. Das bedeutet, unsere Forschungsgruppe besteht aus Menschen mit und ohne Behinderungserfahrung. Die Studierenden verteilen sich auf die von uns untersuchten Fachrichtungen Kindheitspädagogik, Grundschulpädagogik und Sozialpädagogik. So kommen in der Partizipativen Forschungsgruppe ganz unterschiedliche Perspektiven zusammen.

Die Teilnehmenden der Forschungsgruppe sind Menschen der Vielfalt, die sich in ihrem individuellen Wesen gegenseitig prägen und inspirieren.

\subsection{Warum arbeiten wir mit einer Partizipativen Forschungsgruppe?}

Momentan sind Hochschulen sehr exklusive Orte, an denen vor allem Menschen ohne offizielle Beeinträchtigung studieren, lehren und forschen. Soll sich die Hochschule inklusionssensibel entwickeln, dann bedeutet das, dass auch Forschungsprojekte inklusionsorientiert arbeiten und die von ihrer Forschung betroffenen Menschen als Expert*innen einbeziehen sollten (vgl. Reisel, Egloff \& Hedderich 2016). Die von unserer Forschung zu partizipativer Lehre Betroffenen sind vor allem Studierende und Dozierende mit Lernschwierigkeiten.

Der größte Sinn der Partizipativen Forschungsgruppe besteht darin, dass wir uns gegenseitig neues Wissen schenken und voneinander lernen. Besonders lernen wir dabei, im Team zu arbeiten und dadurch Ergebnisse für die Themenfelder zu erzielen. Das sind wertvolle Erkenntnisse, die wir herausfinden möchten. Es ist eine sehr bereichernde und wissenschaftliche Arbeit, deren Ergebnisse offiziell anerkannt werden sollen.

Die Teilnehmenden mit ihren unterschiedlichen Erfahrungshintergründen stellen unterschiedliche Fragen, nehmen unterschiedliche Positionen ein und haben vielfältige Ideen bei der Auswertung und Interpretation unserer Forschungsergebnisse. 
Die Aufgabe der Partizipativen Forschungsgruppe ist es, den gesamten Forschungsprozess von der Fragestellung bis zur Ergebnispräsentation zu begleiten und zu lenken. Wichtige Entscheidungen werden gemeinsam in der Forschungsgruppe getroffen.

Aktuell arbeitet die Partizipative Forschungsgruppe an der Auswertung des umfangreichen Datenmaterials. Hierbei nimmt die Nutzung digitaler Medien einen zentralen Stellenwert für die Forschungsarbeit ein.

\section{Mediennutzung}

\subsection{Warum nutzen wir digitale Medien?}

Seit Beginn des ParLink-Projektes nutzen wir digitale Medien, um Wiederholungen der Forschungsgruppenarbeit anzubieten, um die Gruppe über die aktuellen Geschehnisse auf dem Laufenden zu halten, um vorbereitend Methoden und Inhalte vorzustellen oder um organisatorische Abstimmungen zu treffen. So ist es möglich, die Zeit bei den örtlichen Forschungsgruppentreffen gut zu nutzen und den Kontakt innerhalb der Gruppe zu halten. Außerdem werden die Ko-Forschenden für ihre Forschungsarbeit nicht bezahlt, sondern bloß geringfügig finanziell entschädigt ${ }^{2}$. Daher ist es wichtig, dass über digitale Medien die Möglichkeit besteht, im Alltag spontan an kleineren Aufgaben mitzuwirken.

Die Auswahl an möglichen Online-Tools ist groß und dennoch gibt es nur wenige, die den Ansprüchen an die finanziellen und strukturellen Bedarfe unserer Projektarbeit gerecht werden können. Im Folgenden wird daher erläutert, welche Medien sich für das ParLink-Projekt bewährt haben.

\subsection{Welche Medien nutzen wir im ParLink-Forschungsprozess?}

\subsubsection{Der ParLink-Mailverteiler}

Von Beginn an haben wir das klassische Medium Mailverteiler zur Kommunikation genutzt. Der Mailverteiler ist für uns die Basis aller Kommunikation mit der Forschungsgruppe. Hierüber wurden Protokolle sowie Links zu Umfragen (vgl. 3.2.2) und Videos versendet.

Ich finde es wunderbar, dass es immer wieder wichtige Informationen im Verteiler gibt, besonders dann, wenn es um die Vorbereitungen eines zukünftigen Termins oder die Zusammenfassung des letzten Fokustreffens geht. Dadurch ist es mir dann

2 Eine Anstellung oder Honorartätigkeit ist aufgrund von Beschäftigungsvorgaben (bspw. bezüglich Qualifikationen) nicht möglich und in den Projektgeldern nicht vorgesehen. Für die Beteiligung von Ko-Forschenden in unserem drittmittelgeförderten Projekt bleibt daher bislang nur die Möglichkeit einer Aufwandsentschädigung. 
möglich, bestimmte Aufgaben für das nächste Treffen gründlich zu erledigen und einen vergangenen Termin noch einmal für mich persönlich zu verinnerlichen, um mir meine wichtigsten Erkenntnisse aus der Forschungsarbeit und die für mich schönsten Momente noch einmal durch den Kopf gehen zu lassen.

Von Anfang an war der Mailverteiler auch zur Kommunikation innerhalb der Forschungsgruppe gedacht, sodass alle Forschungsgruppenmitglieder Mails an den Verteiler senden können. In jeder Mail ist ein Hinweis enthalten, wie eine Mail an einzelne oder alle Mitglieder des Verteilers gesendet werden kann. Davon wird jedoch kaum Gebrauch gemacht. Die meisten Mails im Mailverteiler werden von den hauptamtlich Forschenden versendet.

\subsubsection{Typeform}

Auf der Suche nach einer Möglichkeit, die Forschungsgruppe auch trotz räumlicher Distanz in Entscheidungen einbeziehen zu können, Informationen zu vermitteln, unseren Arbeitsstand mitzuteilen und Ideen zu sammeln, entdeckten wir Typeform. Es handelt sich dabei um ein Online-Umfragetool, das sehr flexibel an die Nutzer*innen angepasst werden kann und bezüglich der Integrationsfähigkeit von Bildern und Videos ästhetisch und einfach ist. Mit Typeform gelingt es uns relativ gut, kommunikative Barrieren abzubauen: Hier können Antwortmöglichkeiten mit Bildern versehen und große Bilder und Videos einfach eingebaut werden; alle Schriften und Buttons sind frei anpassbar.

Es fühlte sich zauberhaft für mich an, meine Stimme abgeben zu können, weil ich dadurch etwas zur Entwicklung der Gruppe beitrug. Den Aufbau der Abstimmung fand ich sehr leicht zu verstehen und die dazugegebenen Bilder bereicherten mich sehr!

Aus technischen Gründen funktionierte die Abstimmung nicht immer einwandfrei. Für mich persönlich ist es wichtig, viel Ruhe und Zeit für eine Abstimmung zu haben, weil es mir schwerfällt, eine Entscheidung unter Zeitdruck treffen zu müssen.

Wir nutzen Typeform sehr häufig und für ganz unterschiedliche Zwecke: Wir haben damit Wiederholungsmöglichkeiten über ein Quiz angeboten, über methodische Fragen abgestimmt und die Beteiligung für ein bevorstehendes Forschungsgruppentreffen abgefragt.

\subsubsection{Videobotschaften}

Da wir uns leider nur sehr selten im Jahr zum Forschungsgruppentreffen sehen, müssen einige Informationen als Voraussetzung für eine Abstimmung auch zwischen den Treffen vermittelt werden. Wir haben also über Videos Themen erklärt, damit die Forschungsgruppe dann mitentscheiden kann. Dazu haben wir sowohl Zeichentrickfilme verwendet als auch allein oder mit Kolleg*innen vor der Kamera gestanden. 
Videos erfüllen für uns jedoch noch weitere Funktionen: Sie stellen kollegiale Nähe zwischen den hauptamtlich Forschenden und den Ko-Forschenden her und sind sehr motivierend. Es wurden deshalb auch häufig Videos aus dem Büro verschickt, um für die Forschungsgruppe transparent zu machen, wie die Arbeitsprozesse der hauptamtlich Forschenden ablaufen und wie die durch die Partizipative Forschungsgruppe getroffenen Entscheidungen umgesetzt werden.

\section{Arbeitsmodus während Corona}

\subsection{Veränderungen in der Zusammenarbeit}

Seit dem Beginn der Pandemie können wir uns nicht mehr persönlich begegnen. Dadurch sind schon zwei Termine ausgefallen, die vor Ort hätten stattfinden sollen. Dies bedauere ich zutiefst und ich sehne mich sehr danach, dass diese nachgeholt werden können.

Die Corona-Pandemie bedeutet für ein Forschungsprojekt mit partizipativem Anspruch eine große Einschränkung. Die Beteiligungsmöglichkeiten für die KoForschenden sind über viele Monate hinweg auf die Nutzung digitaler Medien beschränkt. Es wird noch schwieriger, echten Mitgestaltungsraum für die Ko-Forschenden zu schaffen - und nicht bloß Pseudopartizipation (vgl. Hauser 2020).

Bei unseren Forschungsgruppentreffen konnten wir Forschungsmethoden vermitteln und gemeinsam anwenden, Forschungsfragen erarbeiten, die Datenerhebungen vor- und nachbereiten und mit der Auswertung der Daten beginnen. Dabei konnten wir konzentriert zusammenarbeiten, uns gegenseitig motivieren und uns als Forschungs-Team erleben. Alle Ko-Forschenden konnten sich aktiv beteiligen und individuell aufeinander eingehen. Ohne die Möglichkeit solcher Präsenztreffen mussten wir unsere Arbeitsmethoden umstellen.

Wir investieren nun mehr Zeit, um barrierearme Medien wie z.B. Filme zu erstellen. Außerdem haben wir bereits ein Forschungsgruppentreffen, das allerdings statt zwei Tagen nur zwei Stunden dauern konnte, komplett online durchgeführt.

\subsection{Auswertungs- und Interpretationsarbeit online}

Die größte Aufgabe für die Forschungsgruppe ist momentan die Auswertung und Interpretation des Datenmaterials aus unseren Erhebungen. Dabei handelt es sich um Transkripte von drei Gruppendiskussionen zum Thema ,Erfahrungen und Vorstellungen von Hochschulakteur*innen zu inklusiver Hochschulentwicklung und partizipativer Lehre', die nun Stück für Stück gelesen und inhaltsanalytisch interpretiert werden. 
Bei unserer Online-Zusammenarbeit beschäftigen wir uns schon länger mit Transkripten, wofür es immer verschiedene Kleingruppen gibt. Je nachdem, wer wann Zeit hat, entstehen diese Gruppen, in denen wir uns dann Abschnitt für Abschnitt voranarbeiten. Dabei bearbeiten wir die aufgenommenen Gruppendiskussionen, indem wir die einzelnen Sätze verschiedenen Themenschildern zuordnen.

Diese Interpretationsarbeit, die wir ohne die Corona-Pandemie sowohl während der Forschungsgruppentreffen als auch bei einer extra dafür ins Leben gerufenen einwöchigen Forschungswerkstatt gemeinsam vorgenommen hätten, findet nun online statt. Wir arbeiten dabei per Videokonferenz in Kleingruppen von zwei bis vier Personen. Die Kleingruppen arbeiten das Material Satz für Satz mit der Methode der qualitativen Inhaltsanalyse (vgl. Kuckartz 2018) durch. Dazu arbeiten wir gemeinsam über einen in der Videokonferenz geteilten Bildschirm, auf dem die Kodierungsarbeit in MAXQDA durchgeführt wird. Nach etwa anderthalb bis zwei Stunden beendet die Kleingruppe ihre Arbeit und gibt den neuen Arbeitsstand an die nachfolgende Kleingruppe weiter. So arbeiten wir uns kontinuierlich durch das Datenmaterial.

Diese Art der Online-Kleingruppenarbeit kostet sehr viel Zeit und ist technischen Schwierigkeiten unterworfen. Allerdings hat sie auch Vorteile: Die Ko-Forschenden können sich je nach Zeit und Interesse unterschiedlich in das Forschungsprojekt einbringen. Außerdem haben wir so eine Möglichkeit gefunden, das gesamte Datenmaterial partizipativ durchzuarbeiten. Ohne die Online-Kleingruppenarbeit müssten die hauptamtlich Forschenden vermutlich einige Abschnitte des Datenmaterials alleine und ohne die Forschungsgruppe analysieren.

\section{Reflexion unserer Zusammenarbeit und Mediennutzung}

Für das ParLink-Projekt war und ist es eine Herausforderung, trotz der Kontaktbeschränkungen durch die Corona-Pandemie den Kontakt innerhalb der Gruppe zu halten und die Forschungsarbeit weiter voranzutreiben. Dabei bewähren sich im Alltag die Kommunikationswege, die wir in den vorhergehenden eineinhalb Jahren gemeinsamer Forschungsarbeit etabliert haben. Dennoch fehlen der persönliche Kontakt und der intensive Austausch, sowohl im Hinblick auf inhaltliche als auch motivationale und soziale Aspekte.

Dadurch, dass wir uns zurzeit nicht vor Ort treffen können, arbeiten und forschen wir natürlich auch anders als vorher, wodurch unsere Zusammenarbeit eine völlig neue Dimension bekommt. Online-Termine sind nicht das Gleiche wie örtliche Termine. Bei Online-Terminen kann man natürlich nicht ganz so intensiv zusammenarbeiten wie bei Treffen vor Ort, aber dennoch arbeiten und forschen wir natürlich im Rahmen unserer Möglichkeiten weiter mit Videokonferenzen, indem 
wir uns dabei in den Ozean der Transkripte hineinvertiefen, um in dessen Meeresspiegel auf Entdeckungsreise zu gehen.

Ich wünsche mir, dass wir mehr als nur zwei Tage nacheinander ein Fokusgruppentreffen vollziehen, wenn örtliche Begegnungen wieder möglich sind, weil ich glaube, dass wir dann viel intensiver und wirkungsvoller zusammenarbeiten und reflektieren können. Denn das gemeinsame Interpretieren der Transkripte zeigt mir immer wieder, welche wertvollen Ergebnisse zustande kommen können, wenn wir genug Zeit und (Raum) dafür haben.

Neben diesen alltäglichen Herausforderungen in der Zusammenarbeit beschäftigen uns auch die dahinterliegenden forschungsmethodischen Fragestellungen, die direkt unseren partizipativen Anspruch betreffen und daher permanent mitgedacht und reflektiert werden müssen:

Im Forschungsalltag müssen verschiedene Entscheidungen getroffen und Fragen geklärt werden, die mal größere, mal kleinere Auswirkungen auf den Verlauf des Projektes haben. Da der partizipative Anspruch für unser Forschungsanliegen von zentraler Bedeutung ist, müssen die Perspektiven aller Projektbeteiligten einbezogen werden. Der konstante Kontakt zu den Ko-Forschenden führt dazu, dass Aspekte bedacht werden, die innerhalb der Gruppe der hauptamtlich Forschenden nicht präsent gewesen wären.

Dass wir manchmal unterschiedliche Ansichten zu verschiedenen Aussagen haben, beflügelt mich auch sehr, denn wenn ich in einer bestimmten Aussage eine Behinderung oder Voraussetzung sehe, kann ein*e Andere ${ }^{*}$ wieder etwas ganz anderes darin sehen.

Für eine gute Zusammenarbeit ist aber wichtig, dass der Kontakt nicht nur punktuell ist, sondern dass die Ko-Forschenden auch zwischen den Treffen an dem Forschungsprojekt beteiligt sind. Nur so ist es möglich, aktuelle Entscheidungen und Prozesse mit-steuern zu können und inhaltlich informiert zu sein. Letzteres bildet die Grundlage, auf der die Ko-Forschenden ihre Meinung und Perspektive einbringen können, anstatt nur scheinbar zu partizipieren.

Manchmal habe ich das Gefühl, nicht aktiv genug im Projekt sein zu können. Das ist der Fall, wenn es zu wenig Zeit für bestimmte Aufgaben gibt, sodass es mir dadurch dann manchmal schwerfällt, meine Ideen zu äußern.

Es ist in vielerlei Hinsicht eine Herausforderung für alle Projektbeteiligten, einen Nachvollzug der aktuellen Prozesse im Forschungsprozess in einem Maß zu ermöglichen, das eine wirkliche Partizipation erlaubt: Zum einen ist eine ständige Reflexion der verschiedenen Rollen in der Forschungsgruppe notwendig, zum anderen brauchen wir Kommunikationswege, die regelmäßigen Austausch schaffen, ohne die ehrenamtlich Ko-Forschenden zu sehr zu belasten. 
Durch die Typeform-Umfragen bekommen wir oft wichtige Hinweise und können Entscheidungen, die bereits vorbesprochen wurden, gut gemeinsam treffen. Schwierig ist dabei, dass in diesem Rahmen und Format nur wenig Austausch und Diskussion entstehen können. Wenn viele Rückmeldungen kommen, ist das sehr hilfreich, um im Forschungsprozess sicher sein und im Alltag agieren zu können.

Hilfreich wäre es, zusätzlich ein Medium zu finden, über das auch auf die Distanz Diskussionen geführt werden können. Wir sind weiterhin auf der Suche nach Wegen, die Beteiligung an und Übernahme von Verantwortung für Projektentscheidungen zu fördern.

Das ParLink-Projekt arbeitet mit einem partizipativen Anspruch. Darunter verstehen wir, dass wir uns jederzeit um die Beteiligung der Partizipativen Forschungsgruppe bemühen und an neuen Möglichkeiten der Mit-Bestimmung arbeiten. Gleichzeitig gehen wir jedoch davon aus, dass die Beteiligungsmöglichkeiten, die wir bereitstellen, noch nicht ausreichend sind und dass wir unsere Zusammenarbeit permanent sensibel reflektieren müssen (vgl. Kremsner, Buchner \& Koenig 2016). Auf Fragen rund um Macht und Privilegien, darüber, wie man sie abbauen kann, wie wirkliche Partizipation möglich ist und wann Scheinpartizipation droht, gibt es oft keine einfachen Antworten. Ebenso gibt es für die Dilemmata, die sich auftun, keine perfekten Lösungen, sondern Annäherungen an die Umsetzung eines partizipativen Anspruchs.

Die Zusammenarbeit in der Forschungsgruppe und das Arbeiten an möglichst weitreichender Partizipation sind ein sich stetig weiterentwickelnder Prozess, der nicht nur die Forschungsergebnisse beeinflusst, sondern auch das Potenzial hat, die umgebende Hochschul- und Forschungslandschaft zu verändern.

\section{Literatur}

Hauser, M. (2020): Qualität und Güte im gemeinsamen Forschen mit Menschen mit Lernschwierigkeiten. Qualitätskriterien Partizipativer und Inklusiver Forschung. Bad Heilbrunn.

Kremsner, G., Buchner, T. \& Koenig, O. (2016): Inklusive Forschung. In: Hedderich, I., Hollenweger, J., Biewer, G. \& Markowetz, R. (Hrsg.): Handbuch Inklusion und Sonderpädagogik. Bad Heilbrunn, 645-649.

Kuckartz, U. (2018): Qualitative Datenanalyse. Methoden, Praxis, Computerunterstützung. Weinheim.

Reisel, M., Egloff, B. \& Hedderich, I. (2016): Partizipative Forschung. In: Hedderich, I., Hollenweger, J., Biewer, G. \& Markowetz, R. (Hrsg.): Handbuch Inklusion und Sonderpädagogik. Bad Heilbrunn, 636-644.

von Unger, H. (2014): Partizipative Forschung. Einführung in die Forschungspraxis. Wiesbaden. 


\section{David Paulus, Patrick Gollub und Marcel Veber}

\section{Forschendes Lernen und Kasuistik. Grenzwelten und Zwischengänge bezogen auf Reflexivität in der inklusionssensiblen Lehrer*innenbildung ${ }^{1}$}

Wissen und Können, Theorie und Praxis - mit diesen Begriffspaaren ist eine, wenn nicht sogar die zentrale Thematik der Lehrer*innenbildung angesprochen. Bei aller Vielfalt der Konzepte und normativen Setzungen kreist die bildungswissenschaftliche Debatte im Kern um die Frage, wie die Professionalisierung der (angehenden) Lehrpersonen unterstützt und entwickelt werden kann. Der Versuch der Beantwortung dieser Frage hat sich in den letzten Jahren weiter verkompliziert. Durch die Ratifizierung der UN-Behindertenrechtskonvention ändern sich nicht nur die Professionsansprüche an Lehrpersonen und es findet eine Erweiterung der schulischen Aufgabenfelder statt. Das Recht auf Inklusive Bildung trifft auch auf ein segregierendes, eher exkludierendes Schulsystem mit in der Breite wenig Inklusionserfahrungen - v.a. abgesehen von den langjährigen Leuchtturmschulen (u.a. die Gesamtschule Köln-Holweide: vgl. Schwager 2016) oder den (auch frühen) regionalen Schulversuchen (u.a. in Hamburg: vgl. Wocken, Antor \& Hinz 1988). Geht man davon aus, dass die Umsetzung gemeinsamen Unterrichts für alle sich nicht in der Ausweitung des didaktisch-methodischen Repertoires der Lehrkräfte erschöpft, ist die Bedeutung der Reflexion in Bezug auf das professionelle Selbstverständnis zu betonen (vgl. Reiser 1998, 46). Es ist ein „Wandel in den professionellen Orientierungen" (Budde \& Hummrich 2014, o.S.), unter Berücksichtigung des Twin-Track-Approaches (vgl. Lindmeier 2018), angezeigt. Infolgedessen sind Überlegungen anzustellen, wie dieser Wandlungsprozess angestoßen und systematisiert werden kann.

Hier möchte der Beitrag inhaltlich ansetzen und eine wenig thematisierte Verbindung in der inklusionsorientierten Lehrer*innenbildung aufgreifen (vgl. Heinzel \& Krasemann 2019): In Bezug auf inklusive Professionalisierung soll diskutiert werden, wie Forschendes Lernen und Kasuistik, die für sich genommen als Ansätze etabliert sind ${ }^{2}$, als Brücken zwischen hochschulischer Lehrer*innenbildung und

1 Für die wertvollen Rückmeldungen zu diesem Text sei Andreas Hinz herzlich gedankt.

2 An dieser Stelle sei gerade auf Erfahrungen aus der (frühen) Integrationsforschung zur Relationierung von erziehungswissenschaftlicher Disziplin und professionsbezogener Schulpraxis verwiesen (vgl. Eberwein \& Mand 1995): Dort weist bspw. Kraimer (1995) auf die Bedeutung von kasu- 
inklusionsorientierter Praxis fungieren können. In der Verknüpfung der beiden Ansätze liegen Potenziale zur Ausbildung eines kritisch-reflexiven Habitus, der (nicht nur) für die inklusive Professionalisierung von Bedeutung ist. Dabei gehen wir, die Autoren dieses Beitrags, von der These aus, dass die Notwendigkeit eines systematischen fallorientierten Denkens vor dem Hintergrund vielfältiger, systemverändernder Inklusionsansprüche - bspw. im Vergleich zu den eher systemimmanenten Bemühungen, individuelle Förderung in die Lehrer*innenbildung $\mathrm{zu}$ implementieren - virulenter geworden ist. In diesem Zusammenhang wird beleuchtet, wie Ambivalenzen hinsichtlich zunehmender Reflexionsanforderungen in der Lehrer*innenbildung produktiv gewendet werden können. Das ist auch als Antwort auf die (u.E. berechtigte) Kritik am Forschenden Lernen zu lesen.

\section{Eine Sprache für Inklusion und Exklusion finden}

Gelegentlich wird der Eindruck erweckt, Diskriminierungen seien im Schulsystem erst zum Problem geworden, als mit der Ratifizierung der UN-Behindertenrechtskonvention die Partizipation aller Menschen am gesellschaftlichen Leben als Menschenrecht konnotiert wurde. Dem ist natürlich nicht so. Vielmehr ist die Ratifizierung als politische Maßnahme zu verstehen, die das Recht auf Partizipation vulnerabler Gruppen stärkt. Die Konvention verweist auf eine gesellschaftliche Lücke: Auch wenn Behinderungen und Diskriminierungen zuvor existierten, müssen diese auch als solche sprachlich gefasst (u.a. anknüpfend an den inklusionsbezogenen Dekategorierungsdiskurs: vgl. Katzenbach 2015) und, unter Berücksichtigung der Perspektive der Exkludierten, verstanden werden. So können die Exklusionen an theoretischer und praktischer Geltung gewinnen. Mit der UN-Behindertenrechtskonvention verbreitete sich ein spezifisches Verständnis von Inklusion, sodass Ungerechtigkeit(en) und Exklusion gesellschaftlich als Missstände problematisiert werden konnten (frei nach Gümüsay 2020, 46). Das ist eine Voraussetzung, um gegen sie in der Folge aufbegehren und effektive Maßnahmen zu ihrer Beendigung einleiten zu können. An diesem Punkt kann eine Verknüpfung aus Kasuistik und Forschendem Lernen ansetzen, um eine inklusionssensible Lehrer*innenbildung zu ermöglichen, die die (vor)herrschenden Perspektiven systematisch infrage stellt. Denn in der Rekonstruktion schulischunterrichtlicher Praktiken und Strukturen liegt eine Möglichkeit, um Exklusion und Diskriminierung sichtbar zu machen, sodass Reifizierungen vermieden werden und auf ein pädagogisches Verständnis (bspw. von Behinderung) aufmerksam gemacht wird (vgl. Dederich 2016). Hiermit ist auch das Wechselverhältnis von

istischen Zugängen in hochschulischen Professionalisierungsprozessen - gerade mit Bezug zu als auffällig deklariertem Verhalten - hin. 
Inklusion und Exklusion angesprochen (vgl. Kronauer 2018), um den prozessualen, ambivalenten Momenten von Inklusion auf die Spur zu kommen.

Kasuistik in der Lehrer*innenbildung ist von der Überlegung geleitet, dass durch die Arbeit an und mit Fällen ,praxisrelevante' Kompetenzen erlernt werden. Durch das Lernen am Fall (vgl. Combe \& Kolbe 2008, 872) sollen sich die Lehramtsstudierenden der Komplexität des Feldes bewusst sowie „verinnerlichte Deutungs- und Handlungsmuster über Schule und Unterricht" (Hummrich 2016, 21) irritiert werden. Es geht um ein theoriebezogenes Hinterfragen von Kulturen, Strukturen und Praktiken. Auch wenn die Definitionen, was Forschendes Lernen ist, divers sind und in ihren konkreten Ausprägungen auseinandergehen (vgl. Bellmann 2020, 11), sind kollektive Anknüpfungspunkte zur Fallarbeit auszumachen: Indem die Studierenden die Schule bzw. den Unterricht empirisch beforschen und die erlebte Berufspraxis kritisch befragen, sollen sie einen wissenschaftlich-reflexiven Habitus entwickeln (vgl. Klewin, Schüssler \& Schicht 2017, 133). Dabei ist dem Grundgedanken des Forschenden Lernens auch inhärent, dass das fallspezifische Denken und Handeln gefördert werden soll.

Gerade in verlängerten Praxisphasen, wie z.B. dem sog. ,Praxissemester', ist die Reflexion der Erfahrungen von Virulenz, da der längere Aufenthalt im schulischen Feld zu Problematiken führen kann, wie unreflektierte Übernahme berufskultureller Praktiken (vgl. Rothland \& Boecker 2014, 391) oder berufliche ,Einsozialisation', wenn sich in der Schule am vermeintlich Möglichen orientiert wird. Dem „reflexionslosen Aufgehen in Praxiszwängen“ (Häcker 2017, 38f.) ist vorzubeugen, sodass sich in Praxisphasen die Rückkopplung der Schulpraxis an das akademische Studium als Herausforderung darstellt (vgl. Hascher 2014, 557f.).

Aber auch in der Konzipierung des Forschenden Lernens treten, in Verknüpfung mit dem Praxissemester, ambige Momente zutage. Denn Forschendes Lernen ist (zumindest hier) eher als hochschuldidaktisches Prinzip einzuschätzen denn als wissenschaftsorientierte Forschung (vgl. Bellmann 2020, 17). So ist das Praxissemester bedingt als Forschungssemester konzipiert. Darauf verweist schon der Name. Forschendes Lernen erscheint dementsprechend als Mittel zum Zweck. Es geht nicht primär um Forschungsprozesse und somit um einen wissenschaftlichen Erkenntnisgewinn. Zuvorderst soll das Forschende Lernen professionelles Handeln in der Praxis anbahnen. Dahinter steckt auch die Annahme, dass die Unterrichtsqualität, in Zeiten gesellschaftlicher Singularisierungsprozesse (vgl. Reckwitz 2017), weiterhin wesentlich vom Wissen und Können der Lehrpersonen getragen wird. Strukturelle Problematiken können da, unter dem Deckmantel expansiver Reflexivitätsanforderungen an die Individuen, in Vergessenheit geraten. Das gilt auch für die praktische Bearbeitung von Inklusion, wenn Inklusionsansprüche an die Akteur*innen vor Ort delegiert werden (vgl. Budde, Blasse, Rißler \& Wesemann 2019). 


\section{Chancen und Grenzen einer reflexiv-inklusionssensiblen Lehrer*innenbildung}

Vor allem der strukturtheoretische Professionsansatz sieht in der Fallarbeit einen Beitrag zu einer reflexiven Lehrer*innenbildung (vgl. Combe \& Kolbe 2008, 871f.). Grundlegend ist die Idee, dass die Studierenden, ausgehend von Einzelfällen, sich der Praxis distanzierend-verstehend annähern. Hierbei wird implizitem Wissen und immanenten Normen der Praxis nachgegangen, die einen wesentlichen Stellenwert für das pädagogische Handeln besitzen. Dieser Aspekt erscheint insbesondere bei der Gestaltung schulischer Inklusion zentral, um Inklusionsund Exklusionsdynamiken sichtbar zu machen. Durch die methodisch kontrollierte Reflexion von Praxis wird idealerweise eine „Kultur der Distanz“ (Neuweg 2010, 38) etabliert, die die „Kultur der Einlassung auf Praxis“ (ebd.) ergänzt. Kasuistisches Arbeiten kann ein kontextsensibles Handeln bewusstmachen, das durch die (wahrgenommene) Zunahme an Heterogenität in den Lerngruppen an Bedeutung gewinnt.

Gleichwohl ist das Verhältnis zwischen Wissen und Handeln in der Lehrer*innenbildung reichlich diffizil und empirisch weitgehend ungeklärt. Reflexivität wird, wie Forschendes Lernen und Kasuistik auch, als ein Scharnier zwischen wissenschaftlichem Wissen und Handeln konzipiert, da unumstritten ist, dass kein direkter Transfer existiert. Reflexionen und Reflexivität sind unentbehrlich für pädagogische Professionalität und werden von Häcker (2017, 23; Hervorhebungen im Original) wie folgt definiert: „Reflexion wird dabei im weitesten Sinne als ein besonderer Modus des Denkens betrachtet, und zwar als eine rekursive, referenzielle bzw. selbstreferenzielle, d.h. rückbezügliche bzw. selbstbezügliche Form. Reflexivität bezeichnet entsprechend die habitualisierte bzw. institutionalisierte Form eines solchen Denkens." Reflexionen brauchen also Referenzpunkte, wie z.B. Theorien und Konzepte der Inklusiven Bildung: denn es ist fraglich, inwiefern Reflexivität domänenunspezifisch erworben werden kann. Plausibler erscheint, dass Reflexivität an Inhalte gebunden ist. Inklusion könnte als analytische Denkfigur, als konkrete Utopie fungieren, mit der domänenspezifische Bezüge hergestellt werden und Praxis wissenschaftsorientiert reflektiert wird. Reflexion erscheint so als „begreifende Durchdringung“ (Holzkamp 1995, 394), mit der „exklusionsrelevante Differenzkategorien“ (Budde \& Hummrich 2014, o.S.) identifiziert werden.

Die Idee der Reflexivität geht aber noch weiter. Da beim pädagogischen Handeln grundsätzlich Unsicherheit bezüglich des Handlungserfolgs besteht, ist die Kompetenz zum Lernen aus Erfahrungen für die Professionalität von Lehrkräften wesentlich. Indem das Handeln kontinuierlich reflektiert wird, sollen professionelle Kompetenzen angebahnt werden. Das implizite Wissen ist zu transformieren in ein explizites, sodass wiederum ein wissenschaftlich fundiertes Handeln 
ermöglicht wird. Hierin liegt der pädagogische Impuls für die Praxis: Aus den Reflexionen sollen begründete Folgerungen für das Handeln erwachsen. Damit geht keineswegs eine Abwertung des impliziten Wissens oder eine Hierarchisierung der Wissensformen einher. Wissenschaftliches Wissen ist dem praktischen Wissen nicht überlegen. Vielmehr haben die unterschiedlichen Wissensformen ihre jeweilige Berechtigung und sind produktiv miteinander zu verbinden (vgl. Hechler 2016).

Trotz dieser Argumentationen ist die Steigerung der Reflexivität nicht als ,Allheilmittel' anzusehen. Häcker, Berndt und Walm $(2016,271)$ warnen vor einer „Überbürdung durch individualistische Reflexivitätsanforderungen“. Denn eine Erlahmung der Reflexionskräfte und „Formen defensiven Reflektierens" (Häcker 2017, 27) auf Seiten der Studierenden sind angesichts der Expansion der Reflexionsanforderungen durchaus zu diagnostizieren (vgl. ebd., 23). Zudem ist fraglich, inwieweit Reflexivität hochschuldidaktisch förderbar ist. Zusätzlich gilt es, Folgendes zu bedenken: die Gefahr des Ignorierens von sozialen Ungleichheitsbedingungen und Exklusionsrisiken (z.B. Armut). Auch wenn Forderungen nach einer Förderung eines reflexiven Habitus nachvollziehbar sind, ist Vorsicht gegenüber „Machbarkeitsmythen' bei eindimensionaler Steigerung der individuellen Reflexivität angebracht. Vielmehr sind die schulisch-unterrichtlichen Praktiken und Strukturen zum Gegenstand gesellschaftlicher Aushandlungs- und Veränderungsprozesse zu machen. Auch die Pädagogisierung sozioökonomischer Probleme ist in diesem Zusammenhang kritisch zu betrachten: Bildungspolitik ersetzt keine Sozialpolitik. Erstrebenswert ist eine Verbindung aus individueller und bildungspolitischer Verantwortung, sodass aus Strukturen (re-)produzierenden Akteur*innen systemverändernde Lehrpersonen werden (vgl. Dannenbeck \& Hinz 2017).

\section{Ein Ausblick in die Zwischenwelt der Bedeutsamkeit}

Fallorientiertes Arbeiten ist eine Option, die beansprucht, zur Ausbildung eines kritisch-reflexiven Habitus beizutragen. Dieser ist grundlegend für den Erwerb und die Weiterentwicklung von (inklusions)pädagogischer Professionalität. Fallarbeit als hochschuldidaktische Methode ermöglicht einen Praxisbezug zweiter Ordnung und eine Relationierung von Wissenschaft und Praxis. Dabei liegen u.E. die Potenziale der Fallarbeit auch in der Anvisierung ,realitätsnahen', authentischen Lernens: Fälle aus der eigenen Unterrichtspraxis bieten vermutlich mehr Potenzial, dass die Studierenden für sich Bedeutsamkeit konstituieren. Das ist auch aus bildungstheoretischer Warte zu begrüßen: Wenn die Studierenden am wissenschaftlichen Erkenntnisprozess partizipieren, wird ihre Autonomie im Kontext eines zunehmend verschulten Lehramtsstudiums gestärkt. Seminare können 
als handlungsentlastende Räume fungieren, in denen sich „kommunikative Reflexivität" (Reh 2004, 368) realisiert.

Gleichzeitig ist zu betonen: Eine Steigerung der individuellen Reflexivität kann nicht allein die Verantwortung für Innovationen im deutschen Bildungssystem, auch mit Blick auf Inklusive Bildung, übernehmen. Erstens garantiert Reflexivität keinen inklusionspädagogischen Handlungserfolg in der Praxis, sondern kann sich sogar als hinderlich erweisen. Zweitens sind strukturelle Widersprüche und Problemlagen gleichsam aufzuklären und mit ihnen ist wiederum reflexiv umzugehen. Hierfür kann die Verbindung aus Forschendem Lernen und Kasuistik eine gewinnbringende Zwischenwelt repräsentieren.

\section{Literatur}

Bellmann, J. (2020): „Teacher as Researcher“? Forschendes Lernen und die Normalisierung des pädagogischen Blicks. In: Brinkmann, M. (Hrsg.): Forschendes Lernen. Pädagogische Studien zur Konjunktur eines hochschuldidaktischen Konzepts. Wiesbaden, 11-37.

Budde, J., Blasse, N., Rißler, G. \& Wesemann, V. (2019): Inklusion als Professionalisierungsdilemma? In: Zeitschrift für Inklusion, 14, H.3, o.S. Online unter: https://www.inklusion-online.net/index. php/inklusion-online/article/view/512/391 (letzter Zugriff: 13.01.2021).

Budde, J. \& Hummrich, M. (2014): Reflexive Inklusion. In: Zeitschrift für Inklusion, 8, H.4, o.S. Online unter: https://www.inklusion-online.net/index.php/inklusion-online/article/view/193/199 (letzter Zugriff: 13.01.2021).

Combe, A. \& Kolbe, F.-U. (2008): Lehrerprofessionalität: Wissen, Können, Handeln. In: Helsper, W. \& Böhme, J. (Hrsg.): Handbuch der Schulforschung. Wiesbaden, 857-875.

Dannenbeck, C. \& Hinz, A. (2017): Das Politische (in) der Gestaltung inklusionsorientierter Bildungsprozesse. In: Boban, I. \& Hinz, A. (Hrsg.): Inklusive Bildungsprozesse gestalten. Nachdenken über Horizonte, Spannungsfelder und Schritte. Seelze, 52-69.

Dederich, M. (2016): Behinderung. In: Dederich, M., Beck, I., Antor, G. \& Bleidick, U. (Hrsg.): Handlexikon der Behindertenpädagogik: Schlüsselbegriffe aus Theorie und Praxis. Stuttgart, 107110.

Eberwein, H. \& Mand, J. (Hrsg.) (1995): Forschen für die Schulpraxis: Was Lehrer über Erkenntnisse qualitativer Sozialforschung wissen sollten. Weinheim.

Gümüsay, K. (2020): Sprache und Sein. Berlin.

Häcker, T. (2017): Grundlagen und Implikationen der Forderung nach Förderung von Reflexivität in der Lehrerinnen- und Lehrerbildung. In: Berndt, C., Häcker, T. \& Leonhard, T. (Hrsg.): Reflexive Lehrerbildung revisited. Traditionen - Zugänge - Perspektiven. Bad Heilbrunn, 21-45.

Häcker, T., Berndt, C. \& Walm, M. (2016): Reflexive Lehrerinnen- und Lehrerbildung in ,inklusiven Zeiten'. In: Amrhein, B. (Hrsg.): Diagnostik im Kontext inklusiver Bildung. Theorien, Ambivalenzen, Akteure, Konzepte. Bad Heilbrunn, 261-278.

Hascher, T. (2014): Forschung zur Wirksamkeit der Lehrerbildung. In: Terhart, E., Bennewitz, H. \& Rothland, M. (Hrsg.): Handbuch der Forschung zum Lehrerberuf. 2. Aufl. Münster, 542-571.

Hechler, O. (2016): Evidenzbasierte Pädagogik. Von der verlorenen Kunst des Erziehens. In: Ahrbeck, B., Ellinger, S., Hechler, O., Koch, K. \& Schad, G. (Hrsg.): Evidenzbasierte Pädagogik. Sonderpädagogische Einwände. Stuttgart, 42-83.

Heinzel, F. \& Krasemann, B. (2019): Fallarbeit im Praxissemester - Bewährung und Reibungslosigkeit als Richtschnur der Reflexion. In: Zeitschrift für interpretative Schul- und Unterrichtsforschung, 8, 67-80. 
Holzkamp, K. (1995): Lernen. Subjektwissenschaftliche Grundlegung (Studienausgabe). Frankfurt am Main.

Hummrich, M. (2016): Was ist der Fall? Zur Kasuistik in der Erziehungswissenschaft. In: Hummrich, M., Hebenstreit, A., Hinrichsen, M. \& Meier, M. (Hrsg.): Was ist der Fall? Kasuistik und das Verstehen pädagogischen Handelns. Wiesbaden, 13-38.

Katzenbach, D. (2015): De-Kategorisierung inklusive? Über Risiken und Nebenwirkungen des Verzichts auf Etikettierungen. In: Huf, C. \& Schnell, I. (Hrsg.): Inklusive Bildung in Kita und Grundschule. Stuttgart, 33-55.

Klewin, G., Schüssler, R. \& Schicht, S. (2017): Forschend lernen - Studentische Forschungsvorhaben im Praxissemester. In: Schüssler, R., Klewin, G., Schicht, S. \& Schwier, V. (Hrsg.): Das Praxissemester im Lehramtsstudium: Forschen, Unterrichten, Reflektieren. Bad Heilbrunn, 131-171.

Kraimer, K. (1995): Die soziale Diagnose in der qualitativen Sozialforschung. In: Eberwein, H. \& Mand, J. (Hrsg.): Forschen für die Schulpraxis: Was Lehrer über Erkenntnisse qualitativer Sozialforschung wissen sollten. Weinheim, 38-56.

Kronauer, M. (2018): Was kann die Inklusionsdebatte von der Exklusionsdebatte lernen? In: Feyerer, E., Prammer, W., Prammer-Semmler, E., Kladnik, C., Leibetseder, M. \& Wimberger, R. (Hrsg.): System. Wandel. Entwicklung: Akteurinnen und Akteure inklusiver Prozesse im Spannungsfeld von Institution, Profession und Person. Bad Heilbrunn, 40-54.

Lindmeier, C. (2018): Implikationen der internationalen Fachdiskussion über einen „Twin-Track Approach“ der inklusiven Erziehung und Bildung. In: Zeitschrift für Heilpädagogik, 69, H.4, 156-166.

Neuweg, G. (2010): Fortbildung im Kontext eines phasenübergreifenden Gesamtkonzepts der Lehrerbildung. In: Müller, F., Eichenberger, A., Lüders, M. \& Mayr, J. (Hrsg.): Lehrerinnen und Lehrer lernen: Konzepte und Befunde zur Lehrerfortbildung. Münster, 35-50.

Reckwitz, A. (2017): Die Gesellschaft der Singularitäten. Frankfurt am Main.

Reh, S. (2004): Abschied von der Profession, von Professionalität oder vom Professionellen? Theorien und Forschungen zur Lehrerprofessionalität. In: Zeitschrift für Pädagogik, 50, H.3, 358-372.

Reiser, H. (1998): Sonderpädagogik als Service-Leistung? Perspektiven der sonderpädagogischen Berufsrolle. Zur Professionalisierung der Hilfsschul- bzw. Sonderschullehrerinnen. In: Zeitschrift für Heilpädagogik, 2, 46-54.

Rothland, M. \& Boecker, S. K. (2014): Wider das Imitationslernen in verlängerten Praxisphasen. Potenzial und Bedingungen des Forschenden Lernens im Praxissemester. In: Die Deutsche Schule, 106, H.4, 386-397.

Schwager, M. (2016): Die Gesamtschule Köln-Holweide. Zum Umgang mit Migration und Armut unter dem Gesichtspunkt einer Orientierung an Inklusion. In: Ottersbach, M., Platte, A. \& Rosen, L. (Hrsg.): Soziale Ungleichheiten als Herausforderung für inklusive Bildung. Wiesbaden, 173188.

Wocken, H., Antor, G. \& Hinz, A. (Hrsg.) (1988): Integrationsklassen in Hamburger Grundschulen. Hamburg. 


\section{Dietlind Gloystein und Ulrike Barth}

\section{Divers denken und handeln! - Theoretische Orientierungen und Handlungsperspektiven für die Lehrkräftebildung}

Dieser Beitrag stellt theoretische sowie methodologische Grundlagen und Einsatzmöglichkeiten einer phänomenologischen wahrnehmungsorientierten Vignettenarbeit in der Lehrkräfteausbildung im Bereich der entwicklungsbezogenen Diagnostik dar. Zunächst werden Grundzüge einer an Inklusion orientierten pädagogischen Diagnostik und phänomenologischen Erfahrungstheorie hervorgehoben. Neue Ansprüche, die sich daraus ergeben, und didaktisch-methodische Erfordernisse fließen konsequent - und in Form von Wahrnehmungsvignetten - in die Ausbildung zukünftiger Lehrkräfte im Bereich der adaptiven diagnostischen Kompetenz ein.

\section{Lehrkräftebildung für Inklusion - Diagnostik im Umbruch}

Die Realisierung eines inklusiven Schulsystems stellt Anforderungen an die institutionelle Weiterentwicklung, aber auch an die Aus- und Weiterbildung von Lehrkräften. Um der skizzierten Vielfalt oder Diversität, einhergehend mit dem Anspruch auf Chancengerechtigkeit, gerecht zu werden, bedarf es einer Neujustierung grundlegender pädagogischer Aufgaben - auch im Bereich der Diagnostik. Neben fachbezogenen, individuellen und lerngruppenbezogenen Anlässen, die wiederkehrend auf die sorgsame Erhebung vielfältiger Ausgangslagen (vgl. Gloystein \& Moser 2018, 65ff.) angewiesen sind, erfordert eine an Inklusion orientierte Diagnostik eine konstruktive Auseinandersetzung mit Fragestellungen, die u.a. auf die Überwindung von Barrieren beim Lernen, die Ermöglichung von Partizipation und das komplexe Zusammendenken und -führen von individuellen, fachlichen und curricularen Perspektiven zielt (vgl. Gloystein \& Frohn 2020, 66).

\subsection{Grundzüge einer an Inklusion orientierten pädagogischen Diagnostik}

Der Paradigmenwechsel in der Sonderpädagogik und die aktuellen Entwicklungen in der Allgemeinen Pädagogik haben eine breite Diskussion um die Funktion von Diagnostik ausgelöst (vgl. u.a. Meyer \& Jansen 2016; Selter, Hußmann, 
Hößle, Knipping, Lengnink \& Michaelis 2017), die sich zunehmend in Richtung individualisierter Diagnose und Lernprozessbegleitung entwickelt, eher qualitative als quantitative Verfahren einsetzt und sich an Entwicklungsbedingungen von Kindern und Jugendlichen orientiert. Diesem Trend folgend wird im Rahmen der universitären Ausbildung zunehmend auf die „Schulung diagnostischer Kompetenzen“ (Meyer \& Jansen 2016, 18f.) gesetzt. Die Veränderung grundsätzlicher Leitlinien in der Pädagogik, verbunden mit einer Schwerpunktsetzung hinsichtlich personalisierter Lernprozesse, führt in der Lehrkräfteausbildung vornehmlich zu einer inhaltlichen und methodisch-didaktischen Neuausrichtung innerhalb der fachbezogenen (vgl. Selter et al. 2017) als auch der klassenführungsbezogenen (vgl. González, Hövel, Hennemann \& Schlüter 2019) Diagnostik.

Weiterhin stellen die verschiedenen Voraussetzungen von Kindern und Jugendlichen die größten Herausforderungen für die Ausbildungssituation und für die Planung pädagogischer Angebote dar. Dabei besteht in der hochschuldidaktischen Umsetzung diagnostischer Themenstellungen und auch in der einschlägigen Fachliteratur kein Mangel an wissenschaftlichen Belegen und Hinweisen auf die divergenten Ausgangsbedingungen von Schüler*innen. Vielmehr bedarf es auf allen Seiten der Bereitschaft und der Fähigkeit, „das Potenzial, das in den Unterschiedlichkeiten liegt, zu erkennen und daraus resultierende Möglichkeiten zum Wohle der Einzelnen und der Gesamtheit zu nutzen. Ziel ist dabei immer, die Teilhabe aller, unabhängig von ihren unterschiedlichen Ausgangsbedingungen, in optimalem Maß zu ermöglichen" (Gloystein \& Barth 2020, 116f.). Derart diversitätskompetente Menschen verfolgen das Ziel, soziale Vielfalt konstruktiv zu nutzen, Diskriminierungen zu vermeiden und Chancengleichheit zu erhöhen. Dazu gehört auch, einen Umgang mit der Wirkung bestimmter und fundamentaler Diversitätsdimensionen, wie etwa Entwicklung (vgl. Gloystein \& Barth 2020), Sprache, Migration zu finden. Diversitätskompetenz, verstanden als Schlüssel- und gleichzeitig Teilkompetenz einer adaptiven diagnostischen Kompetenz, welche grundlegend und mehrperspektivisch den Fokus auf die Erstellung von Ausgangslagen als Voraussetzung für die Planung pädagogischer Angebote legt, kommt situationsspezifisch zum Einsatz, muss systematisch erworben, erweitert und gestärkt werden.

\subsection{Grundzüge einer phänomenologischen Erfahrungstheorie}

Eine in diesem Zusammenhang methodologische und qualitative Herangehensweise der Erkenntnisgewinnung ist die phänomenologisch orientierte. Phänomenologie hat in der Pädagogik eine Tradition von mehr als 100 Jahren. Kernthemen - Zeit, Leib, Welt, Andere*r - werden bereits zu Beginn von Husserls Phänomenologie beeinflusst und mit Theorie und Praxis von Bildung und Erziehung in Bezug gesetzt. Anhand phänomenologischer Zugänge wird versucht, (pädagogische) Erfahrung im Vollzug in ihren zeitlichen, sinnlichen und weltlichen Dimen- 
sionen zu erfassen und zu beschreiben. Dafür hat die Phänomenologie eine eigene Methodologie entwickelt, die aus Deskription, Reduktion und Variation besteht (vgl. Fischer 2019). Es geht um die „Sache selbst“; als reflexiv-skeptischen Zugang zum Phänomen und dem, was sich zeigt, und wie es sich zeigt (Brinkmann 2017, 17). Aus phänomenologisch orientierter Perspektive erhalten die Theorien der Bildung und Erziehung einen neuen Zugriff - den der Erfahrung (vgl. ebd.). „Das methodische Mittel, die Wirklichkeitserfahrung zu erfassen, ist die Deskription“ (ebd., 19). Es gibt verschiedene Zugänge zu Phänomenologie und Pädagogik. In diesem Beitrag werden explizit zwei spezifische gewählt: die Praxen - orientiert am Wert phänomenologischer Betrachtungsweisen für die Erforschung institutionalisierten Lehrens und Lernens (vgl. ebd., 4) - und die Methodologie. Im Sinne inklusiven Lernens und Lehrens (vgl. Frohn, Brodesser, Moser \& Pech 2019) halten wir den Zugang über die Phänomenologie für eine wichtige qualitative, auf subjektive Erfahrungen gerichtete Forschungsperspektive, die die ,first person perspective` in Bildung und Erziehung stärkt (vgl. ebd., 8).

\subsection{Konsequenzen für die Ausbildung angehender Lehrkräfte}

Mit dem Aufbau eines inklusiven Schulsystems wird die Entwicklung und Umsetzung eines pädagogischen Ansatzes intendiert, dessen wesentliches Prinzip die Wertschätzung und Anerkennung von Diversität in Bildung und Erziehung ist. Eine Umorientierung in der Pädagogik hat auch einen Paradigmenwechsel in der Diagnostik bewirkt. Für den diagnostischen Prozess des Erkennens, Verstehens und Erklärens (vgl. Lanwer 2006), als bestimmenden Moment individualisierter und gruppenbezogener Entwicklungs- und Lernprozesse, bedarf es unter Beachtung strukturierender, orientierender und zum Handeln auffordernder Werte (vgl. Booth 2012, 186) einer theoretischen Anbindung. Sowohl bildungstheoretische als auch entwicklungs- und lerntheoretische Konzepte bilden hierfür den Bezugsrahmen (vgl. Lanwer 2006, 39ff.) und stellen damit das Fundament für diagnostisches Denken und Handeln mit entsprechendem Einfluss auf die Wahl von Methoden und Werkzeug. Demgemäß orientiert sich die noch zu entfaltende fundamentale Diversitätsdimension Entwicklung an entwicklungstheoretischen Konzepten und nutzt entsprechend einer inklusionspädagogischen Ausrichtung qualitative und partizipative Methoden und Verfahren. Die Einführung und Übung einer phänomenologischen Herangehensweise kann einen Baustein in dieser relevanten Neuausrichtung der Lehrkräftebildung darstellen. 


\section{Einsatz von Vignetten}

Pädagogik ist ohne Erkenntnisse über die zu erziehenden, zu bildenden oder zu beratenden Personen undenkbar (vgl. Prengel 2003). Es geht also darum, in der Ausbildung von zukünftigen Lehrkräften eine Sensibilität für diese Erkenntnisse und eine Verknüpfung mit ihrem jeweils erlangten Wissen zu ermöglichen. Phänomenologische Zugänge können hierfür Entscheidendes leisten, denn dieser Forschungsstil setzt beim Subjekt an (vgl. Brinkmann 2015), wobei das Ziel dieses Verfahrens ist, sich ,etwas zeigen zu lassen' (vgl. ebd., 38). Diese Forschungsrichtung entsteht, indem sich Forschende auf einen Prozess der Erfahrung einlassen und diese Momente sprachlich in sogenannten Vignetten formulieren (vgl. Agostini, Eckart, Peterlini \& Schratz 2017, 334). Eine Vignette zeigt „Abschattungen dessen, was sich (...) ereignet; es ist nicht zwingend mehr, aber auch nicht weniger als sich durch Tests, Messmethoden (...) erfahren lässt" (Peterlini 2018, 26). Durch das Lesen der Vignetten und den Austausch darüber entstehen unterschiedliche Sichtweisen auf das Lernen. Im Austausch über Sprachvignetten oder Videodokumente wird versucht, die durch Affizierung festgehaltenen Momente aufzugreifen und zu beschreiben. Diese phänomenologische Herangehensweise zeichnet sich „durch Offenheit für andere Zugänge“ (Agostini et al. 2017, 343) aus, denn Vignetten dienen nicht „der Wahrheitsfindung über den beschriebenen ,Fall' (...), der von seiner Anamnese her zur Diagnose und Prognose führen würde. Vielmehr ist Vignettenforschung in immer neuen Suchbewegungen auf Phänomene von Lernen und Leben gerichtet, die sich im beschriebenen Geschehen zeigen und auf erweiterte Verstehensmöglichkeiten hin reflektiert werden“" (ebd., 347). Es geht in der Vignettenforschung darum, das Mögliche sichtbar zu machen und ein weites Spektrum an Verstehenszugängen zu eröffnen (vgl. ebd., 348), denn der italienische Philosoph D'Arcais (1995) beschreibt die drei zentralen Aufgaben der Erziehungswissenschaft als „die Wahrnehmung konkreter Situationen, gefolgt von kritischer Reflexion derselben“" (Agostini et al. 2017, 350f.). Daraus erst könnten „Orientierungen für das zukünftige erzieherische Handeln abgeleitet werden“ (ebd., 351). Die Vignette dient der ersten Aufgabe. Das Lesen/ Anschauen und der Dialog über die Vignette dienen der zweiten Aufgabe und daraus wiederum können Impulse für die dritte Aufgabe entstehen (vgl. ebd., 350f.). 


\section{Beispiele zur Arbeit mit phänomenologischen Wahrnehmungsvignetten in der 1. Phase der Lehrkräftebildung}

Im Folgenden beschreiben wir zwei erprobte Zugänge zur Arbeit mit Wahrnehmungsvignetten. In Verbindung mit einem Professionalisierungsansatz, wobei das Konstrukt der adaptiven Lehrkompetenz (vgl. Beck, Baer, Guldimann, Bischoff, Brühwiler, Müller, Niedermann, Rogalla \& Vogt 2008; Brühwiler 2014) in Ergänzung um Diversitätskompetenz (vgl. Gloystein \& Barth 2020) vielversprechend erscheint, bedienen wir uns der Textvignetten, um Wahrnehmung und Reflexion zu schärfen und um Studierende für einen Perspektivwechsel und neue Impulse in der Diagnostik zu inspirieren.

\subsection{Erfahrungen mit der Arbeit mit Wahrnehmungsvignetten mit Studierenden im Bachelor Waldorfpädagogik}

Die ,Soziale Arbeit` ist im Bachelor-Studium der Waldorfpädagogik Teil des Kernstudiums im Bereich der pädagogischen Praxis und findet meist in Anbindung an eine Bildungseinrichtung statt. Die Studierenden begleiten drei Jahre lang wöchentlich während des Semesters für ca. drei Stunden ein Kind im Alter zwischen 1-16 Jahren, das einen sogenannten ,Förder- oder Unterstützungsbedarf ${ }^{\circledR}$ hat. In 14-tägigen seminaristischen Übungen werden die Erfahrungen reflektiert; dabei ist ein Mittel die Wahrnehmungsvignette. Diese Textvignetten im Rahmen der ,Sozialen Arbeit' zu schreiben, ist wie ein Bild malen, eine künstlerische Schreibübung. Die Studierenden nehmen einen für sie persönlich bedeutsamen Moment in den Blick, der sie affiziert, verdichten und versprachlichen ihn. In Anlehnung an die Vignettenforschung der Innsbrucker Forschungsgruppe wird an unserem Institut die Arbeit mit Wahrnehmungsvignetten mit Studierenden zum Zweck der Reflexion kompetenzorientiert und im Sinne des Konzepts ,Forschendes Lernen' (vgl. Fichten \& Meyer 2014) pädagogisches Beobachten und Handeln entwickelt und erforscht.

Die Studierenden schreiben kleine Textvignetten aus dem Alltag oder zu kurzen Videoaufzeichnungen. Die Beobachtung und das Schreiben erfordern einen Blick, ein Miterfahren und schlussendlich einen sprachlichen Ausdruck dieses Moments. Durch diese regelmäßige Übung stellen wir eine Intensivierung individueller wertfreier Beobachtungsschulung der Studierenden fest. In den Reflexionsgesprächen, in denen Wahrnehmungsmomente dem Wissen kindlicher Entwicklungsgesten fachlich zugeordnet werden, ergibt sich eine Haltungs- und Einstellungsänderung bei Studierenden bezüglich Entwicklungswissen und Bedarfen. 


\subsection{Videovignetten im diagnostischen Grundlagenseminar ,Vom Zusammenhang Motorik, Wahrnehmung, Sprache, Lernen und Verhalten'}

An der Humboldt Universität konzentriert sich die diagnostische Grundausbildung zukünftiger Pädagog*innen zunehmend auf ein Sichtbarmachen grundlegender Orientierungen hinsichtlich inklusiv-diagnostischer Vorstellungen und Haltungen, auf die Aneignung von spezifischem Fachwissen und die Einführung in adäquate Instrumente, Methoden und Handlungswerkzeuge.

Kommen Vignetten zum Einsatz, haben die Studierenden Grundzüge und Leitideen einer werte- und prozessorientierten sowie partizipativen Diagnostik entlang der Diversitätsdimension Entwicklung erarbeitet. Studierende beobachten und beschreiben videografierte Situationen zum Themenschwerpunkt. Im Rahmen einer professionellen Lerngemeinschaft (Studierende aller Fächer und Schulstufen) setzen sie sich anschließend mit dem Beobachteten auseinander, analysieren in entwicklungslogisch-strukturierten Prozessen die erfassten Erfahrungen, nehmen eine Ausgangslagenbestimmung und den Bezug zum (fachlichen) Lernen vor, planen ein pädagogisches Angebot. Im Fazit lernen die Studierenden (Video-) Vignetten als diagnostische Methode für die Arbeit im schulischen Feld kennen. Ihr Einsatz unterstützt bereits in der Ausbildung die Orientierung an einem erfahrungsorientierten und lernseitigen Blick. Bezugstheoretisches Wissen und selbstreflexive Erfahrungen erleichtern eine Deutung und das Verstehen diagnostischer Situationen von Kindern und Jugendlichen im Bereich der allgemeinen und besonderen Entwicklung und damit in ihrer entwicklungsbezogenen Diversität.

\section{Ausblick}

Dieser Beitrag führt in den relevanten Themenkomplex ,Dimensionen der Vielfalt und diagnostisches Handeln' ein. Diversitätsbewusste Diagnostik will für das Vorhandensein verschiedener Perspektiven sensibilisieren. Sie zielt darauf ab, bestehende Diversität bewusst zu machen und sie im Rahmen von inklusiver Bildung und insbesondere einer an Diversität und Partizipation orientierten Diagnostik zu nutzen, um Ausschluss zu vermeiden, Barrieren abzubauen und optimale Lernund Entwicklungsmöglichkeiten innerhalb der sozialen Gemeinschaft zu realisieren.

Im Kontext eines inklusionsorientierten Bezugsrahmens eröffnen wir eine diagnostische Herangehensweise, welche vom Kind ausgeht. Eine Pädagogik für alle kann ihr volles Potenzial entfalten, wenn es ihr gelingt, Individualität und damit Diversität zu erkennen und zu verstehen - nicht nur fach-, sondern auch entwicklungs- und kontextbezogen. Dafür braucht es für alle wiederkehrend - und im 
Zusammenspiel aller Beteiligten - die Bestimmung von (diagnostisch) vielfältigen Ausgangslagen.

Das Zusammendenken von Entwicklungs- und Fachperspektive wird zunehmend erörtert und erprobt (vgl. Gloystein \& Barth 2020). In der universitären Ausbildung werden verstärkt überfachliche Seminare in Kooperation von Fachdidaktik und Sonderpädagogik angeboten und Konzepte entwickelt, in denen Fachunterricht für heterogene Gruppen ohne Grenzen geplant, durchgeführt und reflektiert wird (vgl. Barth \& Gloystein 2018). Zwangsläufig geht diese Entwicklung zugunsten einer Allgemeinen Pädagogik mit der Aufhebung von Sonder- und Regelpädagogik einher (vgl. Feuser 2013, 49) - auch in der Diagnostik.

\section{Literatur}

Agostini, E., Eckart, E., Peterlini, H. P. \& Schratz, M. (2017): Responsives Forschungsgeschehen zwischen Phänomenologie und Pädagogik: „Lernseits“ von Unterricht am Beispiel phänomenologischer Vignettenforschung. In: Brinkmann, M., Buck, M. F. \& Rödel, S. S. (Hrsg.): Pädagogik - Phänomenologie. Verhältnisbestimmungen und Herausforderungen. Wiesbaden, 323-356.

Barth, U. \& Gloystein, D. (2018): Adaptiv kompetent - Potentiale gemeinsamer Lehre. In: Langner, A. (Hrsg.): Inklusion im Dialog: Fachdidaktik - Erziehungswissenschaft - Sonderpädagogik. Bad Heilbrunn, 247-253.

Beck, E., Baer, M., Guldimann, T., Bischoff, S., Brühwiler, C., Müller, P., Niedermann, R., Rogalla, M. \& Vogt, F. (2008): Adaptive Lehrkompetenz. Münster.

Booth, T. (2012): Der aktuelle „Index for Inklusion“ in dritter Auflage. In: Reich, K. (Hrsg.): Inklusion und Bildungsgerechtigkeit. Weinheim, 180-204.

Brinkmann, M. (2015): Phänomenologische Methodologie und Empirie in der Pädagogik. In: Brinkmann, M., Kubac, R. \& Rödel, S. S. (Hrsg.): Pädagogische Erfahrung. Theoretische und empirische Perspektiven. Wiesbaden, 33-87.

Brinkmann, M. (2017): Phänomenologische Erziehungswissenschaft. Ein systematischer Überblick von ihren Anfängen bis heute. In: Brinkmann, M., Buck, M. F. \& Rödel, S. S. (Hrsg.): Pädagogik - Phänomenologie. Verhältnisbestimmungen und Herausforderungen. Wiesbaden, 17-46.

Brühwiler, C. (2014): Adaptive Lehrkompetenz und schulisches Lernen. Münster.

D’Arcais, P. F. (1995): Pädagogik - warum und für wen? In: Böhm, W. (Hrsg.): Pädagogik - wozu und für wen? Stuttgart, 24-42.

Feuser, G. (2013): Grundlegende Dimension einer Lehrerinnen-Bildung für die Realisierung einer inklusionskompetenten Allgemeinen Pädagogik. In: Feuser, G. \& Maschke, T. (Hrsg.): Lehrerbildung auf dem Prüfstand. Gießen, 11-66.

Fichten, W. \& Meyer, H. (2014): Skizze einer Theorie forschenden Lernens in der Lehrer_innenbildung. In: Feyerer, E., Hirschenhauser, K. \& Soukup-Altrichter, K. (Hrsg.): Last oder Lust? Forschung und Lehrer_innenbildung. Münster, 11-42.

Fischer, A. (2019): Deskriptive Pädagogik (1914). In: Brinkmann, M. (Hrsg.): Phänomenologische Erziehungswissenschaft von ihren Anfängen bis heute. Eine Anthologie. Wiesbaden, 43-60.

Frohn, J., Brodesser, E., Moser, V. \& Pech, D. (Hrsg.) (2019): Inklusives Lehren und Lernen. Bad Heilbrunn.

Gloystein, D. \& Barth, U. (2020): Diversitätskategorien denken - Ein neues Grundprinzip in der Diagnostik. In: Dietze, T., Gloystein, D., Moser, V., Piezunka, A., Röbenack, L., Schäfer, L., Wachtel, G. \& Walm, M. (Hrsg.): Inklusion - Partizipation - Menschenrechte: Transformationen in die Teilhabegesellschaft? Bad Heilbrunn, 112-120. 
Gloystein, D. \& Frohn, J. (2020): Der Baustein adaptive diagnostische Kompetenz: ein Selbstversuch und inklusionssensible pädagogische Diagnostik als Impuls für Perspektivwechsel und professionelle Reflexion. In: Brodesser, E., Frohn, J., Welskop, N., Liebsch, A.-C., Moser, V. \& Pech, D. (Hrsg.): Inklusionsorientierte Lehr-Lern-Bausteine für die Hochschullehre. Bad Heilbrunn, 62-75.

Gloystein, D. \& Moser, V. (2018): Ausgangslage. In: Frohn, J., Brodesser, E., Moser, V. \& Pech, D. (Hrsg.): Inklusives Lehren und Lernen. Bad Heilbrunn, 65-67.

González, L., Hövel, D., Hennemann, T. \& Schlüter, K. (2019): Auswirkungen des gezielten Einsatzes von Classroom-Management-Strategien im inklusiven Fachunterricht Biologie auf das Unterrichtsverhalten von Schülern unter erhöhten Risiken aus Perspektive der Lehrperson. In: Empirische Sonderpädagogik, 11, H.1, 53-70.

Lanwer, W. (2006): Methoden in der Heilpädagogik und Heilerziehungspflege. Troisdorf.

Meyer, M. \& Jansen, C. (Hrsg.) (2016): Schulische Diagnostik. Bad Heilbrunn.

Peterlini, H.-P. (2018): Fenster zum Lernen. In: Baur, S. \& Peterlini, H.-P. (Hrsg.): An der Seite des Lernens. Erfahrungsprotokolle aus dem Unterricht an Südtiroler Schulen - Ein Forschungsbericht. Innsbruck, 21-29.

Prengel, A. (2003): Kinder akzeptieren, diagnostizieren, etikettieren? - Kulturen- und Leistungsvielfalt im Bildungswesen. In: Warzecha, B. (Hrsg.): Heterogenität macht Schule. Beiträge aus sonderpädagogischer und interkultureller Perspektive. Münster, 27-39.

Selter, C., Hußmann, S., Hößle, C., Knipping, C., Lengnink, K. \& Michaelis, J. (2017): Diagnose und Förderung heterogener Lerngruppen. Theorien, Konzepte und Beispiele aus der MINT-Lehrerbildung. Münster. 


\section{Wie verändern sich die subjektiven Theorien von Lehramtsstudierenden über guten inklusiven Unterricht?}

\section{Einleitung}

Die Realisierung einer inklusiven Bildung erfordert, dass Lehrkräfte „anschlussfähige allgemeinpädagogische und sonderpädagogische Basiskompetenzen für den professionellen Umgang mit Vielfalt in der Schule (...) entwickeln können“ (Hochschulrektorenkonferenz \& Kultusministerkonferenz 2015, 3). Um die Kompetenzentwicklung von Studierenden zu unterstützen, sind neben dem Professionswissen auch handlungsleitende subjektive Theorien ${ }^{1} \mathrm{zu}$ adressieren, da sie die Planung, Wahrnehmung, Durchführung und Reflexion von Unterricht beeinflussen (vgl. Baumert \& Kunter 2006; Wahl 2013). Im Zusammenhang mit inklusivem Unterricht stellen subjektive Theorien eine besondere Herausforderung dar: Die subjektiven Theorien der Studierenden basieren oftmals auf Erfahrungen aus der eigenen Schulzeit (vgl. Pajares 1992). Viele Studierende haben keine Erfahrungen mit inklusivem Unterricht gesammelt, sodass sich ihre subjektiven Theorien möglicherweise als undifferenziert erweisen. Zudem können Prinzipien inklusiven Unterrichts dem eigenen Weltbild und Wertesystem widersprechen, was eine Modifizierung subjektiver Theorien erschweren kann (vgl. Wilde $\&$ Kunter 2016).

\section{Die Bedeutung subjektiver Theorien für professionelles Handeln}

Subjektive Theorien werden definiert als „Kognitionen der Selbst- und Weltsicht, als komplexes Aggregat mit zumindest impliziter Argumentationsstruktur, das auch die zu objektiven (wissenschaftlichen) Theorien parallelen Funktionen der Erklärung, Prognose und Technologie erfüllt" (Groeben, Wahl, Schlee \& Scheele 1988, 19). Im Gegensatz zu Wissen, welches im Fachdiskurs validiert werden und dem Anspruch der Widerspruchsfreiheit genügen muss, ist es für subjektive Theorien hinreichend, dass ihre Träger*innen an ihren Wahrheitsgehalt glauben (vgl.

1 Der Begriff subjektive Theorien wird aufgrund der großen Ähnlichkeit der Konstrukte synonym zu Überzeugungen und Beliefs verwendet (vgl. Reusser \& Pauli 2014). 
Wilde \& Kunter 2016). Da Theoriewissen nur dann handlungsleitend wird, wenn es in subjektive Theorien integriert wird (vgl. Blömeke, Eichler \& Müller 2003), erscheint ein weiter Wissensbegriff sinnvoll, der ,objektives' Wissen als ,wahren“ Teil subjektiver Theorien definiert. Wenn subjektive Theorien handlungsleitend sind, stellt sich die Frage, was ,professionelle' subjektive Theorien kennzeichnet. Professionelle subjektive Theorien weisen eine differenzierte Struktur auf, sind reflektiert und begründet und stehen im Einklang mit wissenschaftlichen Theorien und Erkenntnissen (vgl. Wilde \& Kunter 2016). Dies erfordert, dass subjektive Theorien, die im Widerspruch zu wissenschaftlichen Theorien stehen, bearbeitet werden müssen. Eine weitere Herausforderung besteht darin, dass sich das Handeln von Lehrkräften in widersprüchlichen Handlungsanforderungen - Antinomien - vollzieht, die gleichermaßen Gültigkeit beanspruchen und sich daher nicht auflösen lassen (vgl. Helsper 2002). Professionalität besteht in dieser Perspektive darin, die Widersprüche aushalten und reflektieren zu können (vgl. ebd.). Diese widersprüchlichen Handlungsanforderungen können als kognitiv repräsentierte Konzepte in subjektive Theorien einfließen.

\section{Forschungsinteresse}

Diese Studie hat zum Ziel, die inhaltlichen Veränderungen subjektiver Theorien von Lehramtsstudierenden im Verlauf eines Seminars längsschnittlich zu untersuchen. Dabei ist von Interesse, welche inhaltlichen Aspekte inklusiven Unterrichts durch die Intervention neu hinzutreten oder sich ausdifferenzieren. Auf diese Weise soll überprüft werden, ob die Studierenden konkretere und differenziertere subjektive Theorien über guten inklusiven Unterricht entwickeln. Um die Veränderungen der subjektiven Theorien möglichst offen zu erfassen, wurden Strukturlegepläne ausgewählt, die zugleich als Reflexionsanlass dienten und damit zusätzlich eine hochschuldidaktische Funktion erfüllten. Auf dieser Grundlage sollen Konsequenzen für die Weiterentwicklung des Seminarkonzepts gezogen werden, um die Professionalisierung der Studierenden noch gezielter zu unterstützen.

\section{Methodik}

\subsection{Seminarbeschreibung}

Die Daten wurden im Rahmen des Seminars Ist das guter inklusiver Unterricht? erhoben. Das Seminar wurde in zwei Wintersemestern von jeweils einer Hochschullehrerin der Psychologie und der Erziehungswissenschaft im Teamteaching durchgeführt. Ziel war es, Studierende u.a. durch die Auseinandersetzung mit 
ihren subjektiven Theorien für inklusiven Unterricht zu professionalisieren. ${ }^{2}$ Dazu arbeiteten die Studierenden zunächst ihre subjektiven Theorien über guten inklusiven Unterricht mithilfe einer Strukturlegetechnik heraus, die dann mit wissenschaftlichen Theorien aus den Perspektiven der Pädagogischen Psychologie, der Schulpädagogik und der Inklusiven Pädagogik konfrontiert wurden. Auf dieser Basis haben die Studierenden ein Raster für die Beobachtung inklusiven Unterrichts erarbeitet und an Unterrichtsvideos erprobt. Das Raster kam schließlich in Hospitationen in inklusiven, Good Practice'-Schulen zum Einsatz. Die Hospitationserfahrungen wurden in einer abschließenden Sitzung reflektiert.

\subsection{Sample}

Für die vorliegende Studie wurden die Strukturlegepläne von insgesamt 55 Studierenden analysiert. Davon haben 42 Studierende am ersten und 13 Studierende am zweiten Seminardurchlauf teilgenommen. 35 Studierende waren weiblich, 19 männlich, eine weitere Person ordnete sich keinem Geschlecht zu. 32 Studierende strebten das Lehramt für Gymnasien und Gesamtschulen, 20 das Lehramt für Haupt-, Real-, Sekundar- und Gesamtschulen an, eine Person studierte ein anderes Fach als Lehramt und zwei weitere Personen machten keine Angaben zu ihrem Studiengang. Die Studierenden waren im ersten bis neunten Fachsemester $(M=4,87, S D=1,41)$.

\subsection{Datenerhebung}

Zur Erfassung ihrer subjektiven Theorien haben die Studierenden vor und nach dem Seminar jeweils einen Strukturlegeplan zu gutem inklusivem Unterricht erstellt. Strukturlegepläne sollen subjektive Theorien möglichst adäquat abbilden, indem Inhalte durch Begriffe auf Klebezetteln und Beziehungen durch beschriftete Pfeile repräsentiert werden. Dazu wurden in einem ersten Schritt Begriffe gesammelt und auf den Klebezetteln notiert. In einem zweiten Schritt haben die Studierenden die Zettel auf einer Unterlage sortiert und in eine für sie sinnvolle Struktur gebracht. Die Beziehungen zwischen den Begriffen wurden durch Pfeile kenntlich gemacht.

Um die Art des Zusammenhangs näher zu beschreiben, haben sie die Pfeile mit Relationen beschriftet, die dem Inventar der alltagssprachlichen Flexibilisierungsvariante für Strukturlegetechniken (vgl. Scheele, Groeben \& Christmann 1992) entnommen wurden. Dabei wurden Relationen verschiedener Strukturlegetechniken in Alltagssprache ,übersetzt', sodass sie leichter zugänglich sein sollen (z.B. ,das

2 Für eine ausführliche Beschreibung des Seminarkonzepts: vgl. Faix, Lütje-Klose, Textor \& Wild 2019. Bi ${ }^{\text {professional }}$ wird im Rahmen der gemeinsamen Qualitätsoffensive Lehrerbildung von Bund und Ländern aus Mitteln des Bundesministeriums für Bildung und Forschung gefördert (Förderkennzeichen 01JA1908). 
heißt', ,und', ,erkennbar an', ,notwendige Voraussetzung', ,führt zu'). Bei Bedarf konnten die Studierenden auch eigene Relationen hinzufügen.

Die Arbeitsschritte des Sammelns und Sortierens wechselten sich stetig ab, bis es zu einer Sättigung kam. Am Ende des Seminars haben die Studierenden ihren ursprünglichen Strukturlegeplan überarbeitet, indem sie neue Begriffe auf andersfarbigen Klebezetteln ergänzt haben. Den Studierenden war es freigestellt, die ursprüngliche Struktur beizubehalten und neue Begriffe zu ergänzen oder eine neue Struktur zu wählen (vgl. Faix, Lütje-Klose, Textor \& Wild 2020). Zudem konnten alte Klebezettel vom Strukturlegeplan entfernt und nicht weiter verwendet werden. Aus forschungspraktischen Gründen war es nicht möglich, alle Strukturlegepläne im Dialog-Konsens-Verfahren zu validieren.

\subsection{Datenauswertung}

Die inhaltliche Auswertung der Strukturlegepläne erfolgte in Anlehnung an die zusammenfassende qualitative Inhaltsanalyse nach Mayring (2010). Ziel der Analyse war es, das Material zusammenzufassen und zu reduzieren, um zu zentralen Kategorien zu gelangen. Dazu wurden am Material induktiv Kategorien gebildet. Als Analyseeinheit wurde ein Klebezettel festgelegt, auf welchem meistens nur ein Begriff notiert wurde. Angrenzende Relationen und Klebezettel wurden als Kontexteinheiten in die Analyse einbezogen. Eine Reduktion des Materials erfolgte überwiegend durch die Streichung bedeutungsgleicher Inhalte. Die induktiv am Material gebildeten Kategorien wurden schließlich zu Oberkategorien gruppiert. Das so generierte Kategoriensystem wurde an einem Teil des Materials entwickelt und schließlich am gesamten Material überprüft. Durch die Ermittlung der Kategorienhäufigkeiten wurde das Kategoriensystem quantitativ weiterverarbeitet. Die Anzahl der Kodierungen zu den beiden Erhebungszeitpunkten erlaubt Rückschlüsse im Hinblick auf die Lernentwicklung der Studierenden, da ersichtlich wird, welche Kategorien sich ausdifferenzieren oder neu hinzutreten.

\section{Ergebnisse}

\subsection{Hauptkategorien}

Auf Basis der qualitativen Inhaltsanalyse wurden die fünf Hauptkategorien Inklusionsverständnis, Professionalität der Lehrkraft, Unterricht, Rahmenbedingungen und Ressourcen und (Multiprofessionelle) Kooperation gebildet. Die Kategorie Inklusionsverständnis umfasst Grundorientierungen und Werthaltungen sowie Dimensionen von Heterogenität. Unter der Kategorie Professionalität der Lehrkraft werden mentale Dispositionen (z.B. Wissen, Einstellungen), Persönlichkeitsmerkmale (z.B. Geduld, Konfliktfähigkeit) und Aus-, Fort- und Weiterbildung als Gelegenheiten 
der Professionalisierung für inklusiven Unterricht zusammengefasst. Die Kategorie Rahmenbedingungen und Ressourcen umfasst Rahmenbedingungen (z.B. Lehrpläne, Schulsystem) und Ressourcen (z.B. Geld, Material, Ausstattung). Da beide nicht immer voneinander abgegrenzt werden können, werden sie zu einer Kategorie zusammengefasst. Die Kategorie (Multiprofessionelle) Kooperation umfasst die Zusammenarbeit verschiedener Akteur*innen wie z.B. Sonderpädagog*innen, Schulbegleiter*innen und Eltern. Unter der Kategorie Unterricht werden Dimensionen der konkreten Gestaltung inklusiven Unterrichts zusammengefasst.

Insgesamt ist ein erheblicher Zuwachs der Kodierungen über alle Kategorien hinweg erkennbar. Die quantitativ größten Zuwächse finden sich in der Hauptkategorie Unterricht, deren Kodierungen sich gegenüber dem ersten Erhebungszeitpunkt mehr als verdoppeln. Auch die Anzahl der Kodierungen in den Kategorien Professionalität der Lehrkraft und (Multiprofessionelle) Kooperation verdoppelt sich gegenüber dem ersten Erhebungszeitpunkt nahezu, wobei beide Kategorien deutlich weniger Kodierungen als die Kategorie Unterricht aufweisen. Die Kategorie Inklusionsverständnis weist zu beiden Erhebungszeitpunkten die meisten Kodierungen nach der Kategorie Unterricht auf, wobei die Anzahl der Kodierungen im Vergleich zum ersten Erhebungszeitpunkt nur wenig ansteigt. Die wenigsten Kodierungen weist zu beiden Zeitpunkten die Kategorie Rahmenbedingungen und Ressourcen auf.

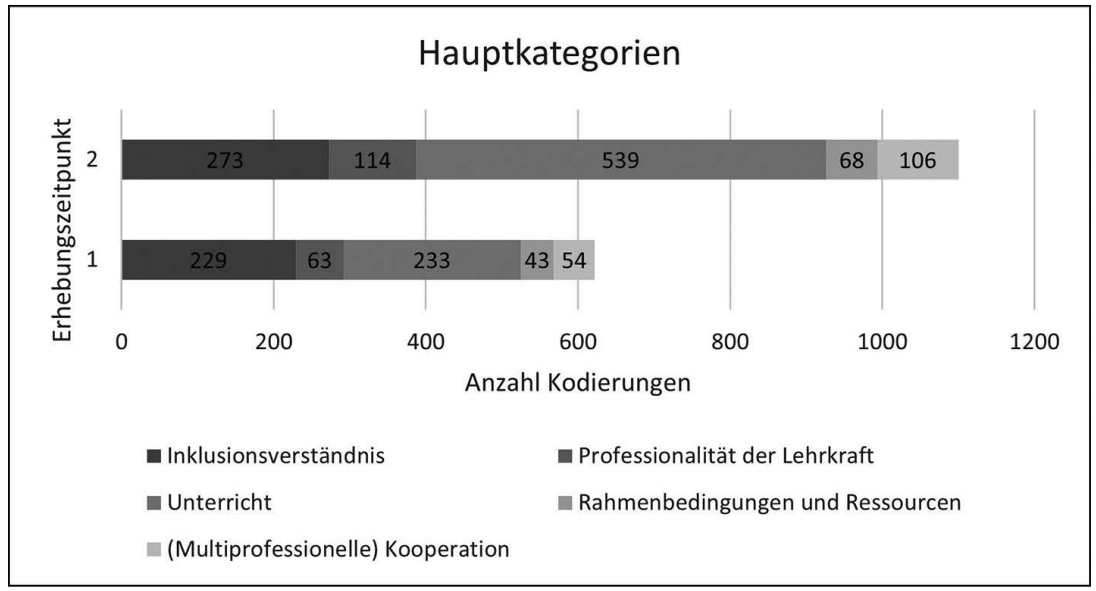

Abb. 1: Kodierungshäufigkeiten der Hauptkategorien zum ersten und zweiten Erhebungszeitpunkt

\subsection{Unterricht}

Da die Hauptkategorie Unterricht für die Fragestellung dieser Studie zentral ist, wird sie im Folgenden gesondert betrachtet. Hier konnten die Unterkategorien 
pädagogisch-psychologische Dimensionen, Differenzierung und Förderung, Herstellung von Gemeinsamkeit, alternative Unterrichtsformen und Klassenklima herausgearbeitet werden. Unter der Kategorie pädagogisch-psychologische Dimensionen wurden Dimensionen zusammengefasst, die von der pädagogisch-psychologischen Forschung als lernwirksam identifiziert wurden (z.B. Klassenführung, kognitive Aktivierung). Die Kategorie Differenzierung und Förderung umfasst differenzierende, individualisierende Maßnahmen, die auch auf den Strukturlegeplänen häufig mit Förderung und Unterstützung verbunden werden. Die Kategorie Herstellung von Gemeinsamkeit umfasst sozialintegrative Maßnahmen, die neben akademischer auch soziale Partizipation ermöglichen sollen (z.B. kooperatives Lernen, gemeinsamer Unterrichtsgegenstand). Unter der Kategorie alternative Unterrichtsformen werden Unterrichtsformen zusammengefasst, die von einem ,traditionellen' Frontalunterricht abweichen (z.B. Wochenpläne, Projektunterricht, offener Unterricht). Da die Kategorie Klassenklima teilweise schwer von Grundorientierungen und Werthaltungen im Unterricht abgegrenzt werden kann, werden hier Aspekte der Lernatmosphäre und des sozialen Miteinanders erfasst (z.B. kein Zeitdruck, Beziehungsarbeit). Zur besseren Übersicht wurden alle Kategorien, die zum zweiten Erhebungszeitpunkt weniger als 40 Kodierungen aufwiesen, unter Sonstige zusammengefasst (z.B. Methoden, Medien und Sozialformen, (Förder-)Diagnostik). Die größten Zuwächse finden sich in der Kategorie pädagogisch-psychologische Dimensionen, die sich gegenüber dem ersten Erhebungszeitpunkt mehr als versechsfacht. Zugleich wird diese Kategorie zum zweiten Erhebungszeitpunkt am häufigsten kodiert. Ein deutlicher Zuwachs findet sich auch für die Kategorie Differenzierung und Förderung, welche zum zweiten Erhebungszeitpunkt am zweithäufigsten kodiert wird. Des Weiteren finden sich Zuwächse für die Kategorien Herstellung von Gemeinsamkeit, alternative Unterrichtsformen und Klassenklima, wobei diese zu beiden Erhebungszeitpunkten deutlich weniger häufig kodiert werden als pädagogisch-psychologische Dimensionen und Differenzierung und Förderung. 


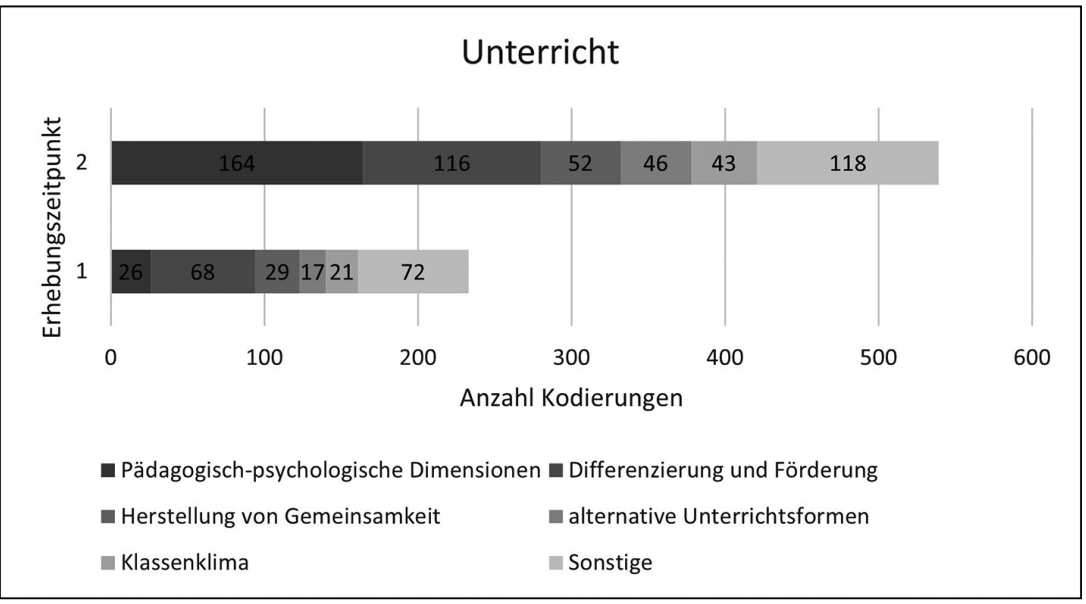

Abb. 2: Kodierungshäufigkeiten innerhalb der Kategorie Unterricht zum ersten und zweiten Erhebungszeitpunkt

\section{Diskussion}

Die Ergebnisse deuten darauf hin, dass die Studierenden zu Beginn der Lehrveranstaltung noch über unspezifische subjektive Theorien verfügten, die sich im Verlauf der Seminare deutlich ausdifferenzierten. Dies zeigt sich an einem deutlichen Zuwachs der Kategorie Unterricht. Die Studierenden verfügen nach den Seminaren über konkretere Vorstellungen, welche Dimensionen für die Gestaltung guten inklusiven Unterrichts relevant sind. Dieses Ergebnis steht im Einklang mit den Zielen des Seminars. Innerhalb der Kategorie Unterricht sind die stärksten Zuwächse in der Kategorie pädagogisch-psychologische Dimensionen zu verzeichnen. Diese enorme Steigerung lässt sich darauf zurückführen, dass psychologische Inhalte im Lehramtsstudium an der Universität Bielefeld vergleichsweise wenig verankert sind, sodass der Lernzuwachs für die Studierenden in diesem Bereich besonders hoch ist. Auffällig ist zu beiden Zeitpunkten das quantitativ ungleiche Verhältnis der Kategorien Differenzierung und Förderung und Herstellung von Gemeinsamkeit. Diesem Befund sollte mit Blick auf die Professionalisierung der Studierenden Aufmerksamkeit geschenkt werden: Differenzierung und die Herstellung von Gemeinsamkeit stehen in einem Spannungsverhältnis (vgl. Werning \& Lütje-Klose 2016). Für inklusiven Unterricht ist die individuelle Förderung von Schüler*innen allein nicht hinreichend, da diese zu Isolation und Stigmatisierung führen kann (vgl. Lütje-Klose 2018). Aus diesem Grund bedarf es einer aktiven und bewussten Herstellung von Gemeinsamkeit, um nicht nur akademische, 
sondern auch soziale Teilhabe zu ermöglichen. Um die Professionalisierung der Studierenden gezielt zu unterstützen, muss ein Bewusstsein für das Spannungsfeld geschaffen werden, um es reflektierend bearbeiten zu können (vgl. Helsper 2002). Gleiches gilt für weitere Spannungsfelder wie z.B. Anerkennung von Differenz und Dekonstruktion von Differenz, die sich auf Ebene untergeordneter Kategorien finden und ebenfalls unterschiedlich häufig benannt werden. Dabei ist zu berücksichtigen, dass das quantitative Verhältnis der Kategorien allein noch nicht aussagt, ob sich die Studierenden dieser Spannungsfelder bewusst sind und ob sie diese reflektieren. Allerdings zeigt sich, dass nur wenige Studierende auf ihren Strukturlegeplänen Relationen verwenden, die ein Spannungsverhältnis anzeigen oder die Spannungsfelder anderweitig kennzeichnen (vgl. Faix 2020). Grundsätzlich gilt, dass die Ergebnisse nicht unabhängig von den Seminarinhalten betrachtet werden können und sich inhaltliche Schwerpunktsetzungen in den Strukturlegeplänen widerspiegeln. Auf dieser Grundlage sollten die Spannungsfelder in zukünftigen Seminaren gezielt thematisiert werden, um die Studierenden dafür zu sensibilisieren und ihre Professionalisierung für inklusiven Unterricht weiter zu unterstützen.

\section{Literatur}

Baumert, J. \& Kunter, M. (2006): Stichwort: Professionelle Kompetenz von Lehrkräften. In: Zeitschrift für Erziehungswissenschaft, 9, 469-520.

Blömeke, S., Eichler, D. \& Müller, C. (2003): Rekonstruktion kognitiver Strukturen von Lehrpersonen als Herausforderung für die empirische Unterrichtsforschung: Theoretische und methodologische Überlegungen zu Chancen und Grenzen von Videostudien. In: Unterrichtswissenschaft, 31, 103-121.

Faix, A.-C. (2020): „Lehrersein ist ja irgendwie von Widersprüchen irgendwo gekennzeichnet«. Eine längsschnittliche Untersuchung Subjektiver Theorien zu gutem inklusivem Unterricht. In: Pädagogische Horizonte, 4, 57-79.

Faix, A.-C., Lütje-Klose, B., Textor, A. \& Wild, E. (2019): Ist das guter inklusiver Unterricht? Mit Videoanalysen und Hospitationen von der Theorie zur Praxisreflexion. In: Herausforderung Lehrer_innenbildung, 2, 1-19.

Faix, A.-C., Lütje-Klose, B., Textor, A. \& Wild, E. (2020): Strukturlegepläne als hochschuldidaktisches Instrument zur Lehrevaluation und Reflexion Subjektiver Theorien. In: Herausforderung Lehrer_innenbildung, 3, 523-537.

Groeben, N., Wahl, D., Schlee, J. \& Scheele, B. (1988): Das Forschungsprogramm Subjektive Theorien: eine Einführung in die Psychologie des reflexiven Subjekts. Tübingen.

Helsper, W. (2002): Lehrerprofessionalität als antinomische Handlungsstruktur. In: Kraul, M., Marotzki, W. \& Schweppe, C. (Hrsg.): Biographie und Profession. Bad Heilbrunn, 64-102.

Hochschulrektorenkonferenz \& Kultusministerkonferenz (2015): Lehrerbildung für eine Schule der Vielfalt. Gemeinsame Empfehlung von Hochschulrektorenkonferenz und Kultusministerkonferenz. Online unter: https://www.kmk.org/fileadmin/Dateien/veroeffentlichungen_beschluesse/2015/2015_03_12-Schule-der-Vielfalt.pdf (letzter Zugriff: 29.05.2019).

Lütje-Klose, B. (2018): Konzeptualisierung von Inklusion und Sonderpädagogik. In: Lütje-Klose, B., Riecke-Baulecke, T. \& Werning, R. (Hrsg.): Basiswissen Lehrerbildung: Inklusion in Schule und Unterricht. Grundlagen in der Sonderpädagogik. Seelze, 9-28. 


\section{Ann-Christin Faix}

Mayring, P. (2010): Qualitative Inhaltsanalyse. In: Mey, G. \& Mruck, K. (Hrsg.): Handbuch Qualitative Forschung in der Psychologie. Wiesbaden, 601-613.

Pajares, M. F. (1992): Teacher's Beliefs and Educational Research: Cleaning Up a Messy Construct. In: Review of Educational Research, 62, 307-332.

Reusser, K. \& Pauli, C. (2014): Berufsbezogene Überzeugungen von Lehrerinnen und Lehrern. In: Terhart, E., Bennewitz, H. \& Rothland, M. (Hrsg.): Handbuch der Forschung zum Lehrerberuf. Münster, 642-661.

Scheele, B., Groeben, N. \& Christmann, U. (1992): Ein alltagssprachliches Struktur-Lege-Spiel als Flexibilisierungsversion der Dialog-Konsens-Methodik. In: Scheele, B. (Hrsg.): Struktur-LegeVerfahren als Dialog-Konsens-Methodik. Ein Zwischenfazit zur Forschungsentwicklung bei der rekonstruktiven Erhebung Subjektiver Theorien. Münster, 152-195.

Wahl, D. (2013): Lernumgebungen erfolgreich gestalten. Vom trägen Wissen zum kompetenten Handeln. Bad Heilbrunn.

Werning, R. \& Lütje-Klose, B. (2016): Einführung in die Pädagogik bei Lernbeeinträchtigungen. München/Basel.

Wilde, A. \& Kunter, M. (2016): Überzeugungen von Lehrerinnen und Lehrern. In: Rothland, M. (Hrsg.): Beruf Lehrer/Lehrerin. Ein Studienbuch. Münster, 299-315. 
Katja Baucke

\section{Internationaler Vergleich als Reflexionsangebot. Eine explorative Studie zur Sicht von Hochschullehrenden auf schulische Inklusion in Deutschland und Kanada}

\section{Einleitung}

„In Canada in the early twenty-first century, being a classroom teacher means that you are certain to have exceptional children or adolescents in your classes. This is because, as a country, we have made a commitment to the inclusion and participation of persons with disability in Canadian society" (Hutchinson \& Martin 2012, XIV).

Das Zitat aus der Einleitung eines kanadischen Lehrbuchs zu inklusivem Unterricht stellt unmissverständlich dar, dass Inklusion keine Option, sondern eine gesetzte Norm im Klassenzimmer darstellt. Lehramt zu studieren bedeutet für kanadische Studierende die grundsätzliche Bereitschaft, in inklusiven Settings zu arbeiten. Verschiedene Studien belegen, dass die Bereitschaft deutscher Lehrkräfte, in inklusiven Settings zu unterrichten, weniger selbstverständlich ist und von verschiedenen Faktoren abhängt (vgl. Textor 2015, 74f.). Ein wesentlicher Aspekt stellt die Selbsteinschätzung der Lehrpersonen dar. Sehen sie sich selbst als kompetent in diesem Bereich an und haben gegebenenfalls auch bereits praktische Erfahrungen mit Heterogenität, so steigt die Bereitschaft, in inklusiven Klassen zu unterrichten. Die Selbsteinschätzung von Regelschulkräften ist jedoch überwiegend negativ. Als Begründung werden häufig fehlende Kompetenzen und eine diesbezüglich unzureichende Vorbereitung im Zuge der akademischen Ausbildung gesehen (vgl. ebd., 75).

Angesichts der unterschiedlichen Selbstverständlichkeit von inklusivem Unterricht für Lehrkräfte in Deutschland und Kanada fokussiert diese Studie die subjektive Einschätzung zu Inklusion von Hochschullehrenden, die in der universitären Ausbildung von Lehrkräften tätig sind, und nimmt eine international-vergleichende Perspektive ein. In Deutschland geführte Interviews mit Lehrenden unterschiedlicher Universitäten werden mit Interviews mit Lehrenden an Universitäten der kanadischen Provinz Saskatchewan kontrastiert. Durch den Fokus auf Saskatchewan betrachtet die Studie eine Provinz, die bislang in deutschen Publikationen

1 Der Vortrag 2020 in Wien wurde noch unter dem Namen Katja Klose gehalten. 
kaum Beachtung findet (vgl. Rousseau, Borri-Anadon \& St-Vincent 2019). Im Gegensatz zu sehr dicht bevölkerten Provinzen wie Ontario oder Québec und sehr dünn besiedelten Provinzen wie New Brunswick oder Newfundland and Labrador wird den Provinzen in der Mitte des demografischen Spannungsfeldes, wie Saskatchewan oder Manitoba, insgesamt wenig Aufmerksamkeit geschenkt. Die Berücksichtigung unterschiedlicher kanadischer Provinzen und Territorien ${ }^{2}$ ist jedoch wesentlich, da sich die Schulsysteme unterscheiden: Die vier dünner besiedelten Provinzen New Brunswick, Nunavut, North West Territories und Yukon stehen für ,full inclusion', wohingegen in den anderen Provinzen mit einem Kaskadenmodell gearbeitet wird, bei welchem auch separierende Aspekte in Betracht kommen, wenn dies der Förderung dienlich scheint. AuCoin und Vienneau (2019, 164) verdeutlichen das Wesensmerkmal des Prinzips ,full inclusion‘:

„Die schulische Integration beruhte auf dem Prinzip der optimalen Normalisierung der Schulerfahrung möglichst vieler Schüler*innen mit Behinderungen oder mit Adaptions- oder Lernschwierigkeiten. Das Modell der schulischen Inklusion (full inclusion) hingegen schlug eine Ausweitung dieses Prinzips auf alle diese Schüler*innen vor, und zwar unabhängig von ihrer Behinderung, aber vor allem unter Anerkennung bzw. Wertschätzung des einmaligen Charakters aller Lernenden und unter Berücksichtigung aller Aspekte der Person bei den verfolgten Lernzielen."

Ähnlich wie in Kanada sind auch in den jeweiligen Bundesländern Deutschlands unterschiedlich weitreichende Umsetzungsstrategien im Hinblick auf inklusiven Unterricht zu konstatieren. Eine vollumfängliche Inklusion stellt hier allerdings nach wie vor eher eine Ausnahme dar (vgl. Autorengruppe Bildungsberichterstattung 2020). Vor dem Hintergrund der unterschiedlichen Ausgangslagen lohnt ein vergleichender Blick auf das subjektive Selbstverständnis von Hochschullehrenden zu inklusivem Unterricht in Deutschland und der kanadischen Provinz Saskatchewan. Nach einer Skizzierung des Forschungsdesigns werden ausgewählte Ergebnisse der Studie dargestellt, wobei der Fokus auf Gemeinsamkeiten in den Deutungen der Hochschullehrenden in beiden Ländern gerichtet wird.

\section{Methodisches Vorgehen}

Um die subjektiven Deutungen der Hochschullehrenden zu Inklusion und zu inklusivem Unterricht zu erheben, wurden Expert*inneninterviews durchgeführt. Die Stichprobe umfasst 44 Interviews, davon 19 in Deutschland und 25 in Saskatchewan, und setzt sich nach dem Convenience Sample zusammen: „A conve-

2 Kanada besteht aus Provinzen und Territorien, aus Gründen der Leserlichkeit wird im Weiteren nur von Provinzen gesprochen. 
nience sample selects research participants based on their ease of availability, and lacks any clear sampling strategy. The selection process relies on including those who are the most eager and able to participate in the study" (Aurini, Heath \& Howells 2016, 55). Dies bedeutete in Bezug auf die in Deutschland geführten Interviews ein nicht stringentes Akquirieren von Teilnehmenden und die Nutzung diverser Feldzugänge. ${ }^{3}$ Die Stichprobe in Saskatchewan wurde ebenfalls mit der Methode des Convenience Sample gewonnen, allerdings gezielt im Zuge einer Forschungsreise zur Datenerhebung.

Die Durchführung der Expert*inneninterviews orientierte sich an den Gütekriterien der qualitativen Forschung nach Steinke (2017, 324ff.). Um den subjektiven Deutungen der Hochschullehrenden einen möglichst breiten Raum zu eröffnen, standen weit und offen formulierte Fragen im Kern des Erhebungsinstruments. Die Leitfragen fokussierten (1) das Inklusionsverständnis der Hochschullehrenden, (2) die Vermittlung von Inklusion in der Hochschullehre sowie (3) die Reflexion der aktuell aus Sicht der Interviewten größten Umsetzungsschwierigkeiten von inklusivem Unterricht in Schulen. Da auf die systeminterne Handlungsexpertise der Interviewten vertraut wurde, wurde bewusst auf umfangreiche Hinführungen und Erläuterungen zum Interviewthema verzichtet.

Ausgewertet wurden die Interviews inhaltsanalytisch nach Mayring (2002). Kombiniert wurde die Analyse mit der Strategie der Thematic Analysis, um die Kategorien stärker induktiv aus den Narrativen der Interviewten zu generieren (vgl. Braun \& Clarke 2006, 89). Die in diesem Beitrag nur exemplarisch mögliche Vorstellung von Forschungsergebnissen bezieht sich auf ausgewählte Gemeinsamkeiten der in Deutschland und Kanada geführten Interviews.

\section{Gemeinsamkeiten der Interviews in Deutschland und Kanada}

„Also auch, dass Differenz möglich ist, aber gemeinsam auch zu gucken, wie bekommen wir's denn hin (...), dass jeder gesellschaftliche Teilhabe hat, ohne eben die Differenzen in den Fokus zu stellen" (Interview 1, Z.14-17).

Grundsätzlich können in den Interviews Heterogenität und Differenz als Kernund Ausgangspunkte ausgemacht werden, wenn die interviewten Lehrenden an Universitäten nach ihrem Inklusionsverständnis gefragt werden. Darüber, dass der Umgang mit Heterogenität einen immanenten Teil von Inklusion darstellt, herrscht Einigkeit zwischen den unterschiedlichen Lehrenden, ungeachtet ihres jeweiligen Lehrortes. Dies knüpft an die Studie von Piezunka, Schaffus und Gro-

3 So wurde beispielsweise die Teilnahme an einer einschlägigen wissenschaftlichen Tagung genutzt, um Interviewpartner*innen zu gewinnen. 
sche an, in der als zugrunde liegendes Fundament der untersuchten Inklusionsdefinitionen „die Überwindung von Diskriminierung aufgrund sozial konstruierter Gruppenzugehörigkeit“ $(2017,216)$ sichtbar wurde. Der Umgang, mit welchem Heterogenität aus Sicht der meisten interviewten Hochschullehrenden begegnet werden soll, wird in folgendem Ausschnitt deutlich:

„I guess, I'd define inclusion as the ability to make sure that every student (...) has a place in the classroom and ability to feel safe and recognized (...) And that is, the students would have the capability to participate in learning fully. So that (...), ideally activities are remodified, or content may sometimes be modified and preferably will be modified for all students" (Interview 29, Z.3-9).

Der Deutung nach sollen prinzipiell alle Schüler*innen in ihrer Heterogenität am Unterricht teilnehmen können. Dies realisiert sich durch Modifizierungen in der Unterrichtsgestaltung. Diese Prämisse spiegelt sich in Deutschland in der inzwischen häufigen Adressierung von Differenzierung im Unterrichtsgeschehen in Forschung und Lehre wider (vgl. u.a. Jürgens 2019; Textor 2015, 119ff.).

In diesem Zusammenhang ist bemerkenswert, dass - entgegen der in Deutschland verbreiteten Vorstellungen zum kanadischen Schulsystem - auch in den meisten kanadischen Provinzen separierende Praktiken die Heterogenität der Zusammensetzung der Schüler*innenschaft beeinträchtigen können. Boban und Hinz (2019, 445f.) erläutern in diesem Zusammenhang: „Wer sich in Provinzen mit dem Kaskaden-Modell bewegt, kann eigentlich nicht überrascht sein, wenn er das ganze Spektrum integrativer, teilintegrativer und segregativer Organisationsformen im Schulsystem vorfindet."

Einer der signifikantesten Unterschiede zwischen den in Deutschland und Kanada Interviewten zeigt, dass die Lehrenden an deutschen Universitäten das vertikal gegliederte Schulsystem, und den politischen Unwillen, dieses zu verändern, als eines der größten Probleme wahrnehmen, das die praktische Umsetzung von Inklusion erschwert. Kritik am Schulsystem findet sich in den kanadischen Interviews demgegenüber nicht, dennoch werden aus Sicht der Interviewten auch dort Inklusionsbestrebungen durch die mangelnde Bereitschaft zur Veränderung gebremst:

„I think it's because schools are sights of conservatism. So, schools are basically spaces where the state or the local communities are trying to reproduce a certain social space or social world and if you try to include people in the name of ... by expanding the boundaries of equality, you can nearly always have a pushback. You always get to get people who don't want the boundaries expanded“ (Interview 20, Z.35-39). 
Auch an den Universitäten selbst ist der Paradigmenwandel zu Inklusion aus Sicht von Interviewten in Kanada noch nicht abgeschlossen:

„I would define it [Inklusion; Anm. d. Autorin] first of all broadly. And not (...) in narrow terms. So, I think, before I joined this institution, inclusion historically for a number of people, has been focused on issues that relate physically to individuals who may have physical or mental exceptionalities" (Interview 28, Z.3-6).

Auch in Kanada wurde der Begriff ,Inklusion' lange Zeit nur in einem engen Inklusionsverständnis verstanden. Gegenwärtig wird der Begriff für ein breites Spektrum von Aspekten der Heterogenität verwendet (vgl. Di Giorgio 2019, 56). Zugleich führt das Kaskaden-Modell dazu, dass „Schüler*innen ganz selbstverständlich etikettiert [werden], um an Hautfarbe, Ethnizität, Migrationserfahrung oder Behinderung besondere Bedürfnisse festzumachen" (Schroeder 2019, 89). Entsprechend wird Inklusion zwar nicht mehr eng am Konzept der Behinderung festgemacht, jedoch gründen Unterstützung und Empowerment im schulischen Kontext in Kategorisierungen (vor allem im Kaskadenmodell). Ressourcenzuweisungen sind auch in deutschen Schulen häufig (bundeslandabhängig) an Diagnosen und festgestellte sonderpädagogische Förderbedarfe gekoppelt.

In der Einschätzung der interviewten Hochschullehrenden in Bezug auf die zur Umsetzung von inklusivem Unterricht notwendigen Ressourcen überwiegen ebenfalls Gemeinsamkeiten in den in Deutschland und Kanada geführten Interviews. Kurz und prägnant bringt es folgendes Interviewzitat auf den Punkt: „School settings are under resourced" (Interview 24, Z.31). Die Aussage fasst exemplarisch die übereinstimmende Einschätzung der Interviewten zu den institutionellen Voraussetzungen in beiden Schulsystemen zusammen. Der in den Interviews als signifikant evaluierte Mangel an Ressourcen bezieht sich auf verschiedene Ebenen. Es reicht von eher allgemeinen Einschätzungen zu unzureichenden finanziellen Ressourcen (vgl. u.a. Interview 26, Z.41-70), zeitlichen Kapazitäten von Lehrkräften (vgl. u.a. Interview 29, Z.27-30) über konkreter formulierte Forderungen nach mehr Personal (vgl. u.a. Interview 14, Z.50-59) bis zur Benennung konkreter Unterstützungsbedarfe im Klassensetting, wie bspw. ein „loudspeaker system maybe for somebody who is hard of hearing" (Interview 34, Z.18). Die bildungspolitische Strategie, Inklusion möglichst kostengünstig zu realisieren, scheint den Interviews in Kanada folgend somit nicht nur im deutschen Bildungssystem ein akutes wie langwieriges Problem darzustellen (vgl. Becker 2015, 30ff.). Auch aus Sicht der Interviewten in der kanadischen Provinz Saskatchewan ist dies eine wesentliche Hürde, an der die praktische Umsetzung inklusiven Unterrichts zu scheitern droht. In Kanada variiert das Ressourcenproblem je nach räumlicher Lage der Schulen: Di Giorgio (2019) nennt neben politischen und schulkonzeptionellen Unterschieden explizit auch durch Bevölkerungsdichte oder geografische Lage bedingte Unterschiede in der Ressourcenzuteilung. So profitieren 
Schulen in kleineren Orten häufig mehr von vorhandenen Ressourcen als jene in bevölkerungsreichen Gebieten (vgl. ebd., 50f.). Als flächenmäßig große Provinz mit vergleichsweise dünner Besiedlung scheint das Schulsystem in Saskatchewan somit strukturell schlechter gestellt zu sein als die Systeme in anderen Provinzen.

\section{Ausblick}

Die für diesen Beitrag exemplarisch ausgewählten Befunde aus den Analysen von Interviews mit Hochschullehrenden in Deutschland und der kanadischen Provinz Saskatchewan zu inklusiver Bildung in Schulen zeigen, dass die im wissenschaftlichen Diskurs bereits angeklungenen kritischen Perspektiven an einer einseitigen ,Heroisierung des kanadischen Schulsystems' empirisch Bestätigung finden (vgl. u.a. Jahr \& Kruschel 2019b; Schroeder 2017). Zudem zeigt sich, dass der Fokus von Inklusion in beiden Ländern inzwischen mehr auf ein weit gefasstes Verständnis von Heterogenität als auf Behinderung im engeren Sinne gelegt wird. Die interviewten Lehrenden an den Hochschulen sind sich einig darin, dass die Zuständigkeit für den Umgang mit und die Wertschätzung von Heterogenität bei den Lehrkräften in den Schulen liegt, gleichzeitig herrschen in beiden Ländern weiterhin separierende Praktiken im Schulalltag.

Eine der wesentlichen strukturellen Ursachen dürfte die Konservativität der Bildungssysteme und somit auch der institutionellen Routinen in den Schulen darstellen. Die Tendenz, bestehende Strukturen und etablierte Gegebenheiten aufrechtzuerhalten, um die Stabilität von Schule zu wahren, lässt sich sowohl in den Deutungen der interviewten Hochschullehrenden in Deutschland als auch in Saskatchewan feststellen.

All dies wird in den Interviews mit den Hochschullehrenden durch Forderungen nach besserer und umfassenderer Ressourcenausstattung gerahmt. Der übereinstimmend hervorgehobene Geld-, Zeit- und Personalmangel macht deutlich, dass innerhalb der Bildungspolitik aus Sicht der Interviewten in beiden Ländern in Bezug auf die Umsetzung der durch die UN-BRK gesetzlich verankerten inklusiven Beschulung Reflexionsbedarf besteht (vgl. Beauftragte der Bundesregierung für die Belange von Menschen mit Behinderungen 2017, Art. 24). Der Vergleich der beiden Länder dient nicht dazu, empirische Erkenntnisse zum kanadischen Schulsystem unkritisch auf das deutsche Schulsystem zu übertragen, sondern durch den „Charakter des länderübergreifenden Austauschs“ (Jahr \& Kruschel 2019a, 12) den Stand inklusiver Beschulung in beiden Kontexten kritisch zu reflektieren. Der räumlich präzisere Fokus auf die Provinz Saskatchewan eröffnet zudem neue Möglichkeiten des Vergleichs, da dadurch der in Deutschland häufig übersehene Facettenreichtum der kanadischen Forschungslandschaft in Bezug 
auf Inklusion sichtbar wird. Die institutionellen Voraussetzungen zur schulpraktischen Umsetzung von Inklusion unterscheiden sich in Saskatchewan sowohl von denen in sehr dicht besiedelten als auch von denen in sehr dünn besiedelten Provinzen. Künftige vergleichende Studien könnten die Reflexionsmöglichkeiten erweitern, indem weitere Provinzen sowie die Differenziertheit der deutschen Bildungslandschaft in den Vergleich einbezogen werden.

\section{Literatur}

AuCoin, A. \& Vienneau, R. (2019): Schulische Inklusion und Denormalisierung: Vorschlag für ein neues Paradigma. In: Jahr, D. \& Kruschel, R. (Hrsg.): Inklusion in Kanada. Internationale Perspektiven auf heterogenitätssensible Bildung. Weinheim/Basel, 161-174.

Aurini, J. D., Heath, M. \& Howells, S. (2016): The how to of qualitative research. Los Angeles/London/New Delhi/Singapore/Washington DC \& Melbourne.

Autorengruppe Bildungsberichterstattung (Hrsg.) (2020): Bildung in Deutschland 2020. Ein indikatorengestützter Bericht mit einer Analyse zu Bildung in einer digitalisierten Welt. Bielefeld.

Beauftragte der Bundesregierung für die Belange von Menschen mit Behinderungen (2017): Die UNBehindertenrechtskonvention. Übereinkommen über die Rechte von Menschen mit Behinderungen. Online unter: https://www.behindertenbeauftragte.de/SharedDocs/Publikationen/UN_Konvention_deutsch.pdf?_blob=publicationFile\&v=2 (letzter Zugriff: 18.04.2020).

Becker, U. (2015): Die Inklusionslüge. Behinderung im flexiblen Kapitalismus. Bielefeld.

Boban, I. \& Hinz, A. (2019): Menschenrechtsbasierte Pädagogik - inklusiv und ,appropriate? Fragen nach Besuchen in Toronto und New Brunswick. In: Jahr, D. \& Kruschel, R. (Hrsg.): Inklusion in Kanada. Internationale Perspektiven auf heterogenitätssensible Bildung. Weinheim/Basel, 445458.

Braun, V. \& Clarke, V. (2006): Using thematic analysis in psychology. In: Qualitative Research in Psychology, 3, H.2, 77-101.

Di Giorgio, C. (2019): Inklusive Erziehung und Bildung in Kanada: Überblick über aktuelle Forschungslinien, Strategien und Praxis. In: Jahr, D. \& Kruschel, R. (Hrsg.): Inklusion in Kanada. Internationale Perspektiven auf heterogenitätssensible Bildung. Weinheim/Basel, 47-58.

Hutchinson, N. L. \& Martin, A. K. (2012): Inclusive Classrooms in Ontario Schools. Toronto.

Jahr, D. \& Kruschel, R. (2019a): Einleitung: Inklusion in Kanada als Lern- und Reflexionsangebot für Bildungsreformen. In: Jahr, D. \& Kruschel, R. (Hrsg.): Inklusion in Kanada. Internationale Perspektiven auf heterogenitätssensible Bildung. Weinheim/Basel, 12-28.

Jahr, D. \& Kruschel, R. (Hrsg.) (2019b): Inklusion in Kanada. Internationale Perspektiven auf heterogenitätssensible Bildung. Weinheim/Basel.

Jürgens, E. (2019): Jedem Schüler gerecht werden - so gelingt es! Umgang mit Heterogenität und Differenzierung in der Unterrichtspraxis. Köln.

Mayring, P. (2002): Einführung in die Qualitative Sozialforschung. Eine Anleitung zu qualitativem Denken. 5. Aufl. Weinheim/Basel.

Piezunka, A., Schaffus, T. \& Grosche, M. (2017): Vier Definitionen von schulischer Inklusion und ihr konsensueller Kern. Ergebnisse von Experteninterviews mit Inklusionsforschenden. In: Unterrichtswissenschaft, 45, H.4, 207-222.

Rousseau, N., Borri-Anadon, C. \& St-Vincent, L.-A. (2019): Inklusive Bildung in Kanada: gemeinsame Schwerpunkte und provinzspezifische Besonderheiten. In: Jahr, D. \& Kruschel, R. (Hrsg.): Inklusion in Kanada. Internationale Perspektiven auf heterogenitätssensible Bildung. Weinheim/ Basel, 59-78. 


\section{Katja Baucke}

Schroeder, J. (2017): Kanada kann auch anders. Vielfältige Schulangebote im „gelobten Land der Inklusion“. Online unter: https://www.inklusion-online.net/index.php/inklusion-online/article/ view/397 (letzter Zugriff: 18.04.2020).

Schroeder, J. (2019): Ein ,Gewimmel von Diskursen' zu Inklusion in Kanada. In: Jahr, D. \& Kruschel, R. (Hrsg.): Inklusion in Kanada. Internationale Perspektiven auf heterogenitätssensible Bildung. Weinheim/Basel, 79-91.

Steinke, I. (2017): 4.7 Gütekriterien qualitativer Forschung. In: Flick, U., von Kardoff, E. \& Steinke, I. (Hrsg.): Qualitative Forschung. Ein Handbuch. 12. Aufl. Reinbek bei Hamburg, 319-331.

Textor, A. (2015): Einführung in die Inklusionspädagogik. Bad Heilbrunn. 
Bettina Amrhein, Benjamin Badstieber und René Schroeder

\section{Zum Umgang mit als störend wahrgenommenen Handlungsweisen von Schüler*innen in einem inklusionsorientierten Unterricht - Perspektiven für die Lehrer*innenbildung (im Förderschwerpunkt emotionale und soziale Entwicklung)}

Innerhalb institutionalisierter Lehr-Lernprozesse fallen Schüler*innen ,immer wieder durch psychosoziale Handlungsweisen auf, die in ihrer personalen Umwelt als nicht mehr tolerierbar (...) angesehen werden" (Störmer 2013, 314). Dies gilt offenbar insbesondere für Schüler*innen, denen ein sonderpädagogischer Unterstützungsbedarf im Förderschwerpunkt emotionale und soziale Entwicklung (SPU-ESE) zugesprochen wird.

Dabei werden die in dem Verhalten sichtbar werdenden ,Problemlagen' - medizinisch-psychologischer Denktradition folgend - wiederholt als individuelle Dispositionen der Schüler*innen markiert und Möglichkeiten ihrer Überwindung durch pädagogisch-psychologische ,Verhaltensmodifikationen' propagiert. In der wissenschaftlichen und ,pädagogischen' (Ratgeber*innen-)Literatur ist die Rede vom Umgang mit ,schwierigen', , auffälligen', ,verhaltensgestörten' Schüler*innen (etc.) allgegenwärtig.

Der vorliegende Beitrag skizziert vor diesem Hintergrund mögliche Alternativen in der pädagogischen Adressierung der als störend wahrgenommenen Handlungsweisen von Schüler*innen und fokussiert auf die Ausgestaltung pädagogischer Beziehungen innerhalb einer Schule für alle Schüler*innen. Dabei richtet sich der Blick insbesondere auf die Professionalisierung der Lehrkräfte und es wird thematisiert, wie sie unterstützt werden können, die hinter den störenden Handlungsweisen liegenden, (un-)sichtbaren sozialen Verhältnisse und darin hervortretenden emotionalen und sozialen Bedarfe der Schüler*innen zu erkennen und pädagogisch zu adressieren.

Dazu werden in einem ersten Schritt die Bedeutung pädagogischer Beziehung in Schule und in einem zweiten Schritt empirische Befunde zur Notwendigkeit der Unterstützung von Lehrkräften hinsichtlich eines beziehungskompetenten Umgangs mit als störend wahrgenommenen Handlungsweisen von Schüler*innen (mit SPU-ESE) skizziert. 
Schließlich werden Perspektiven zur Professionalisierung von (angehenden) Lehrkräften hinsichtlich ihrer Beziehungskompetenz anhand eines im BMBF-Projekt RAISE entwickelten Qualifizierungsprogramms sowie einer Seminarkonzeption umrissen.

\section{Zur Bedeutung pädagogischer Beziehungen}

Als Kontrapunkt zu einer stark individualisierenden und potenziell pathologisierenden Sichtweise auf als störend wahrgenommene Handlungsweisen von Schüler*innen (vgl. Störmer 2013) kann die Frage nach gestörten bzw. konflikthaften Interaktionen in der Lehrer*innen-Schüler*innen-Beziehung (L-S-Beziehung) neue Perspektiven eröffnen. Aus internationalen Befunden lässt sich ein interdependentes Bedingungsgefüge erkennen, indem eine positive L-S-Beziehung einerseits als wichtiger protektiver Faktor im Kontext sozial-emotionaler Problemlagen hervortritt (vgl. z.B. Nemer, Sutherland, Chow \& Kunemund 2019; Sabol \& Pianta 2013), andererseits jedoch eben solche Problemlagen mit einer Zunahme negativer Interaktionsverläufe verbunden sind, die zu einer Belastung der L-S-Beziehung führen können (vgl. etwa Baker, Grant \& Morlock 2008; Birch \& Ladd 1998; Sabol \& Pianta 2013). Ein Teufelskreis negativer Interaktionsverläufe mit eskalierenden L-S-Konflikten sowie ein steigendes Exklusionsrisiko (vgl. Doumen, Verschueren, Buyse, Germeijs, Luyckx \& Soenens 2008) können die Folge sein. Die positive Beziehungsgestaltung muss daher als Notwendigkeit wie auch als zentrale pädagogische Herausforderung im Umgang mit als störend wahrgenommenen Handlungsweisen angesehen werden (vgl. Herz \& Zimmermann 2015). So findet sich die Forderung eines Primats der Beziehung auch in der Literatur des Förderschwerpunktes ESE (vgl. Hillenbrand 2008), womit Akzeptanz, Vertrauen oder Geborgenheit (vgl. z.B. Stein \& Stein 2020) zum Rahmen pädagogischen Handelns werden sollen, ohne jedoch eine individualisierende und potenziell pathologisierende Perspektivierung der Problemlagen dabei grundlegend infrage zu stellen (vgl. Störmer 2013). Unter dem Anspruch schulischer Inklusion ergeben sich damit Notwendigkeiten, diesen Primat im Sinne einer beziehungssensiblen Bearbeitung gestörter bzw. als gestört wahrgenommener Interaktionen in der Schule weitergehend zu fokussieren, allgemeinpädagogisch zu wenden und in einer struktur- und systemorientierten Perspektive den Abbau potenzieller Barrieren bzw. den (Wieder-)Aufbau sicherer Räume des emotionalen und sozialen Lehrens und Lernens zu ermöglichen. 


\section{Empirische Befunde}

Im Kontext aktueller bildungspolitischer Reformimpulse zur Umsetzung der UN-Behindertenrechtskonvention zeigt sich, dass die Anzahl der Schüler*innen, denen ein SPU-ESE im Regelschulbereich zugesprochen wird, stetig steigt (vgl. KMK 2020, 6). Dabei handelt es sich bei der Bezeichnung dieses Personenkreises nachweislich um eine sehr heterogene Schüler*innengruppe (vgl. Stein \& Müller 2015, 12). In Studien zur sozialen Integration von Schüler*innen mit SPU-ESE wurde wiederholt berichtet, dass diese im Alltag stärker ausgegrenzt werden als ihre Mitschüler*innen ohne einen solchen SPU (vgl. Huber 2008; Huber \& Wilbert 2012), wobei dieser Zusammenhang von Klasse zu Klasse sehr stark variierte (vgl. Huber \& Wilbert 2012; Shapiro, Miller, Sawka, Gardill \& Handler 1999).

Die empirischen Befunde deuten auf entsprechende Herausforderungen für Lehrkräfte hin (vgl. Stein \& Müller 2015, 39; Willmann 2015, 63). Diese stehen Kindern mit sogenannten ,Verhaltensauffälligkeiten' im Bereich Aufmerksamkeit, Kommunikation und sozial-emotionale Kompetenzen jedoch offensichtlich weniger positiv gegenüber als Kindern mit anderen SPU, wie auch internationale Studien belegen (vgl. z.B. Avramidis \& Norwich 2002; Male 2011, 183; Scanlon \& Barnes-Holmes 2013). Sie reagieren häufig ablehnend (vgl. Dyson 2010) bzw. halten den Umgang mit diesen für besonders schwierig (vgl. Clough \& Lindsay 1991).

Damit ergibt sich sowohl mit Blick auf die Lern- und Entwicklungsbedürfnisse der Schüler*innen (mit einem SPU-ESE) als auch bzgl. des Kompetenzerlebens und der Gesundheit der Lehrkräfte (vgl. Amrhein \& Badstieber 2015) eine Qualifikationsnotwendigkeit für eine (bedingungslose) L-S-Beziehung in der Aus- und Fortbildung von Lehrkräften im Kontext sozial-emotionaler Problemlagen (vgl. Becker \& Prengel 2016; Garwood \& Van Loan 2019; Leggio \& Terras 2019). Dies knüpft an zentrale Befunde der aktuellen Unterrichtsforschung an, wonach die L-S-Beziehung bzw. die sozial-emotionale Unterstützung als bedeutsame Merkmale der Unterrichtsqualität gelten müssen (vgl. Praetorius, Klieme, Herbert \& Pinger 2018; Sabol \& Pianta 2013). Wie diese Perspektive in Professionalisierungsprozessen der Aus- und Fortbildung integriert werden kann, soll nachfolgend exemplarisch skizziert werden.

\section{Perspektive für die Lehrer*innenfortbildung}

Ein hier vorzustellendes Programm der Lehrer*innenfortbildung wird zurzeit im Rahmen eines vom BMBF geförderten Forschungsprojektes (RAISE - Restorative Approaches in Inclusive School Environments) entwickelt und erprobt. Die Konzeption orientiert sich an einem im anglo-amerikanischen Raum vorliegenden 
und evaluierten Professionalisierungsansatz. Der sogenannte Restorative Practice Approach (RPA) soll Lehrkräften bzw. Schulen helfen, eine wertschätzende Beziehungsarbeit auch und gerade im Falle von Konflikten zwischen allen Beteiligten zu entwickeln und zu erhalten. Drewery $(2013,48)$ fasst die Leitidee des Ansatzes zusammen, indem sie festhält: „restorative approaches are about managing relationships rather than behavior." Die an Schule Beteiligten werden dabei angeleitet, eine gemeinsame Unterrichtskultur und -praxis zu etablieren,

- die von der Sinnhaftigkeit jedweden Handelns ausgeht,

- die Notwendigkeiten der (bedingungslosen) Akzeptanz und des Verstehens der hinter dem Verhalten aufscheinenden emotionalen und sozialen Bedürfnisse der Schüler*innen reflektiert und

- die darauf abzielt, durch eine dialogische Kommunikation mit den Schüler*innen in konflikthaften Situationen Prozesse der,Wiedergutmachung', des in Beziehung-Bleibens und des gemeinsamen emotionalen und sozialen Lernens pädagogisch zu adressieren (vgl. Wong, Cheng, Ngan \& Ma 2011).

So konzentriert sich das Programm konzeptionell in einer inklusionspädagogischen Perspektive nicht (ausschließlich) auf die Unterstützung einzelner Schüler*innen, sondern sensibilisiert Lehrkräfte für die allgemeinpädagogische Bedeutung von Konflikten und eröffnet Möglichkeiten einer (Wieder-) Herstellung positiv erlebter Beziehungen innerhalb konflikthafter Lehr- und Lernsituationen.

Im hier vorgestellten Fortbildungsansatz liegt der Fokus darauf, die Rolle der Teilnehmenden in diese Richtung zu stärken (Empowerment) (vgl. Hopkins 2003) und sie zur Anleitung entsprechender Schul- und Unterrichtsentwicklungsprozesse zu befähigen, und zwar ohne Verhaltensweisen einzelner Schüler*innen einseitig als störend zu interpretieren und zu sanktionieren (vgl. Thorsborne \& Blood 2013).

Qualitative und quantitative empirische Studien zur Anwendung dieses Ansatzes verweisen unter anderem auf eine erfolgreiche Reduzierung von Mobbing und Konflikten (vgl. McCluskey, Lloyd, Kane, Riddell, Stead \& Weedon 2008; Wong et al. 2011) sowie auf eine erfolgreiche Verbesserung der Beziehungen zwischen Lehrkraft und Schüler*innen (vgl. Gregory, Clawson, Davis \& Gerewitz 2014). Das noch weiter zu entwickelnde Qualifikationsprogramm im Rahmen des Projektes RAISE wird derzeit umfassend evaluiert.

\section{Perspektiven für die Lehrer*innenausbildung}

Auch im Kontext der Lehrer*innenausbildung ergeben sich Möglichkeiten zur Professionalisierung von Lehrkräften für die Gestaltung pädagogischer Beziehungen. Exemplarisch lässt sich hier eine universitäre Seminarkonzeption zu Didaktik und Unterricht zwischen Beziehungsgestaltung, Strukturgebung und Konfliktbearbei- 
tung für Studierende im Lehramt der integrierten Sonderpädagogik an der Universität Bielefeld skizzieren, die sich aktuell in der Erprobung befindet und ebenfalls evaluiert wird. Ziel ist die Stärkung der Fähigkeit, auf Basis von Empathie, Wärme und Echtheit eine vertrauensvolle Beziehung zu Schüler*innen aufzubauen, das Spannungsverhältnis von Nähe und Distanz alters- und entwicklungsbezogen zu regulieren, Engagement und Wertschätzung in die Arbeit mit Schüler*innen einzubringen sowie Konflikte reflexiv zu lösen.

Sowohl in der Zielsetzung wie auch der inhaltlich-methodischen Ausgestaltung knüpft das Seminar an bestehende Konzepte, wie sie etwa mit der Beziehungsdidaktik von Miller (2011) oder dem international verbreiteten STEP-Ansatz (vgl. Dinkmeyer, McKay \& Dinkmeyer 2011) bereits vorliegen, an und ergänzt diese um Spezifika gestörter Interaktions- und Beziehungsmuster (vgl. z.B. Baier, Reiter \& Winkler 2008; Herz \& Zimmermann 2015). Der Schwerpunkt liegt zunächst auf Aspekten der Beziehungspädagogik (vgl. Liegle 2017) bzw. -didaktik (vgl. Miller 2011), um Dynamiken und Kommunikationsprozesse mit Blick auf eine beziehungsförderliche Unterrichtsgestaltung, d.h. Aktivitäten zur Verbesserung der Beziehungsqualität innerhalb der Lerngruppe, zu erarbeiten und im Hinblick auf eigene schulische Beziehungserfahrungen zu reflektieren. Aufbauend findet eine kritische Auseinandersetzung mit pädagogisch-therapeutischen Prinzipien des sonderpädagogischen Fachdiskurses (vgl. etwa Baier et al. 2008; Stein \& Stein 2020) statt und wird durch eine mehrperspektivische Sicht auf unterrichtliche Konflikte (vgl. z.B. Winkel 2011) und eine Einordnung der L-S-Beziehung in Zusammenhänge von Unterrichtsqualität (vgl. Sabol \& Pianta 2013) erweitert. Dies schließt mit einer Reflexion zu beziehungskompetentem Handeln im Kontext des individuellen Professionalisierungsprozesses der Studierenden ab.

\section{5 (Zwischen-)Fazit}

Die Professionalisierung der Lehrkräfte im Umgang mit als störend wahrgenommenem Verhalten erscheint für einen inklusionsorientierten Unterricht von hoher Bedeutung.

Zur Unterstützung eines solchen Professionalisierungsprozesses wurde im vorliegenden Beitrag die Berücksichtigung der Beziehungen und ihrer potenziellen Verletzungen in der Bearbeitung als störend wahrgenommener Handlungsweisen hervorgehoben, in dessen Zentrum nicht die einseitige Anpassung der Verhaltensweisen bestimmter (etikettierter) Schüler*innen an bestehende organisationale und institutionelle Ordnungen, sondern vielmehr die gemeinsame (Wieder-)Herstellung unterstützender Interaktionsmöglichkeiten stehen.

Erste Erfahrungen im weiter oben beschriebenen Projekt RAISE machen deutlich, wie paradox die Anbahnung eines solchen Perspektivwechsels innerhalb der beste- 
henden Routinen und Strukturen des Erziehungs- und Bildungssystems erlebt werden kann. So zeigten sich deutliche Herausforderungen für die Aus- und Fortbildner*innen, die Kolleg*innen dabei zu unterstützen, eine inklusionsorientierte Ausgestaltung des eigenen unterrichtlichen Handelns anzubahnen, anstatt über sanktionierende und hochgradig fremdbestimmte, Verhaltensmodifikationen' bei den Schüler*innen vermeintlich schnelle Erfolge im Umgang mit Konflikten bzw. sogenannten ,Verhaltensauffälligkeiten' herbeiführen zu wollen (vgl. Amrhein \& Badstieber 2021/i.V.).

\section{Literatur}

Amrhein, B. \& Badstieber, B. (2015): Inklusion und Gesundheit: Motor für eine gesunde Schule. In: nds - Zeitschrift der Bildungsgewerkschaft, 9, 18-19.

Amrhein, B. \& Badstieber, B. (2021/i.V.): (Un-)mögliche Perspektiven auf herausforderndes Verhalten in der Schule - Theoretische, empirische und praktische Beiträge zur De- und Rekonstruktion des Förderschwerpunkts Emotionale und Soziale Entwicklung. Weinheim/Basel.

Avramidis, E. \& Norwich, B. (2002): Teachers' attitudes towards integration/inclusion: a review of the literature. In: European Journal of Special Needs Education, 17, 129-147.

Baier, S., Reiter, S. \& Winkler, C. (2008): Welches Verhalten stört die Didaktik. In: Grohnfeldt, M. (Hrsg.): Didaktik in der Sonderpädagogik. Würzburg, 153-181.

Baker, J. A., Grant, S. \& Morlock, L. (2008): The Teacher-Student Relationship as a Developmental Context for Children with Internalizing or Externalizing Behavior Problems. In: School Psychology Quarterly, 23, 3-15.

Becker, U. \& Prengel, A. (2016): Pädagogische Beziehung mit emotional-sozial beeinträchtigten Kindern und Jugendlichen - Ein Beitrag zur Inklusion bei Angst und Aggression. In: Zimmermann, D., Meyer, M. \& Hoyer, J. (Hrsg.): Ausgrenzung und Teilhabe. Perspektiven einer kritischen Sonderpädagogik auf emotionale und soziale Entwicklung. Bad Heilbrunn, 94-104.

Birch, S. H. \& Ladd, G. W. (1998): Children's Interpersonal Behaviors and the Teacher-Child Relationship. In: Developmental Psychology, 34, 934-946.

Clough, P. \& Lindsay, G. (1991): Integration and the Support Services: Changing Roles in Special Education. Windsor.

Dinkmeyer, D., McKay, G. D. \& Dinkmeyer, D. (2011): STEP - Das Buch für Lehrer/innen. Weinheim/Basel.

Doumen, S., Verschueren, K., Buyse, E., Germeijs, V., Luyckx, K. \& Soenens, B. (2008): Reciprocal Relations Between Teacher-Child Conflict and Aggressive Behavior in Kindergarten: A ThreeWave Longitudinal Study. In: Journal for Clinical Child \& Adolescent Psychology, 37, 588-599.

Drewery, W. (2013): Restorative approaches in New Zealand schools. In: Sellman, E., Cremin, H. \& McCluskey, G. (Hrsg.): Restorative Approaches to Conflict in Schools: Interdisciplinary perspectives on whole school approaches to managing relationships. London/New York, 40-50.

Dyson, A. (2010): Die Entwicklung inklusiver Schulen: drei Perspektiven aus England. In: Die Deutsche Schule, 102, 115-126.

Garwood, J. D. \& Van Loan, C. L. (2019): Pre-service educators' dispositions toward inclusive practices for students with emotional and behavioural difficulties. In: International Journal of Inclusive Education, 23, 1332-1347.

Gregory, A., Clawson, K., Davis, A. \& Gerewitz, J. (2014): The promise of restorative practices to transform teacher-student relationships and achieve equity in school discipline. In: Journal of Educational \& Psychological Consultation, 26, 325-353. 
Herz, B. \& Zimmermann, D. (2015): Beziehung statt Erziehung? Psychoanalytische Perspektiven auf pädagogische Herausforderungen in der Praxis mit emotional-sozial belasteten Heranwachsenden. In: Stein, R. \& Müller, T. (Hrsg.): Inklusion im Förderschwerpunkt emotionale und soziale Entwicklung. Stuttgart, 144-169.

Hillenbrand, C. (2008): Einführung in die Pädagogik bei Verhaltensstörungen. 4. Aufl. München/ Basel.

Hopkins, B. (2003): Just Schools: A Whole School Approach to Restorative Justice. London.

Huber, C. (2008): Jenseits des Modellversuchs. Soziale Integration von Schülern mit sonderpädagogischem Förderbedarf im Gemeinsamen Unterricht. Eine Evaluationsstudie. In: Heilpädagogische Forschung, 34, 2-14.

Huber, C. \& Wilbert, J. (2012): Soziale Ausgrenzung von Schülern mit sonderpädagogischem Förderbedarf und niedrigen Schulleistungen im gemeinsamen Unterricht. In: Empirische Sonderpädagogik, 4, 147-165.

KMK (Kultusministerkonferenz) (2020): Sonderpädagogische Förderung in allgemeinen Schulen (ohne Förderschulen) 2017/2018. Online unter: https://www.kmk.org/fileadmin/Dateien/pdf/ Statistik/Dokumentationen/Aus_SoPae_Int_2017.pdf (letzter Zugriff: 22.04.2020).

Leggio, J. C. \& Terras, K. L. (2019): An Investigation of the Qualities, Knowledge, and Skills of Effective Teachers for Students with Emotional/Behavioral Disorders: The Teacher Perspective. In: The Journal of Special Education Apprenticeship, 8, 1-15.

Liegle, L. (2017): Beziehungspädagogik: Erziehung, Lehren und Lernen als Beziehungspraxis. Stuttgart.

Male, D. B. (2011): The impact of a professional development program on teachers' attitudes towards inclusion. In: Support for Learning, 26, 183-186.

McCluskey, G., Lloyd, G., Kane, J., Riddell, S., Stead, J. \& Weedon, E. (2008): Can restorative practices in schools make a difference? In: Educational Review, 60, 405-417.

Miller, R. (2011): Beziehungsdidaktik. 5. Aufl. Weinheim/Basel.

Nemer, S. L., Sutherland, K., Chow, J. C. \& Kunemund, R. (2019): A Systematic Literature Review Identifying Dimensions of Teacher Attributions for Challenging Student Behavior. In: Education and Treatment of Children, 42, 557-578.

Praetorius, A.-K., Klieme, E., Herbert, B. \& Pinger, P. (2018): Generic dimensions of teaching quality: the German framework of Three Basic Dimensions. In: ZDM - Mathematics Education, 50, 407-426.

Sabol, T. J. \& Pianta, R. C. (2013): Relationship between teachers and children. In: Reynolds, W. M. \& Miller, G. E. (Hrsg.): Handbook of psychology: Educational psychology, 2. New York, 199-212.

Scanlon, G. \& Barnes-Holmes, Y. (2013): Changing attitudes: supporting teachers in effectively including students with emotional and behavioural difficulties in mainstream education. In: Emotional and Behavioural Difficulties, 18, 374-395.

Shapiro, E., Miller, D. N., Sawka, K., Gardill, M. C. \& Handler, M. (1999): Facilitating the Inclusion of Students with EBD into General Education Classrooms. In: Journal of Emotional and Behavioral Disorders, 7, 83-93.

Stein, R. \& Müller, T. (2015): Verhaltensstörungen und emotional-soziale Entwicklung: zum Gegenstand. In: Stein, R. \& Müller, T. (Hrsg.): Inklusion im Förderschwerpunkt emotionale und soziale Entwicklung. Stuttgart, 19-43.

Stein, R. \& Stein, A. (2020): Unterricht bei Verhaltensstörungen. Ein integratives didaktisches Modell. 3. Aufl. Bad Heilbrunn.

Störmer, N. (2013): Du störst! Herausfordernde Handlungsweisen und ihre Interpretation als „Verhaltensstörungen“. Berlin.

Thorsborne, M. \& Blood, P. (2013): Implementing Restorative Practices in schools: A practical guide to transforming school communities. London/Philadelphia. 


\section{0 | Bettina Amrhein, Benjamin Badstieber und René Schroeder}

Willmann, M. (2015): Emotional-soziale Schwierigkeiten und Verhaltensstörungen: Diagnostik und Assessment in der inklusiven Schule. In: Schäfer, H. \& Rittmeyer, C. (Hrsg.): Handbuch inklusive Diagnostik. Weinheim/Basel, 419-432.

Winkel, R. (2011): Der gestörte Unterricht. Diagnostische und therapeutische Möglichkeiten. Baltmannsweiler.

Wong, D. S., Cheng, C. H., Ngan, R. M. \& Ma, S. K. (2011): Program effectiveness of a restorative whole-school approach for tackling school bullying in Hong Kong. In: International Journal of Offender Therapy and Comparative Criminology, 55, 846-862. 


\section{Andrea Holzinger, Gerda Kernbichler, Silvia Kopp-Sixt, Mathias Krammer und Gonda Pickl}

\section{Profilierung für Inklusive Pädagogik (IP) im Lehramt der Primar- und Sekundarstufe Allgemeinbildung}

Gegenstand des Beitrags sind Ergebnisse des Forschungsprojektes ,Profilierung für Inklusive Pädagogik im Lehramtsstudium', das unterstützt vom Forschungsfonds der Pädagogischen Hochschule Steiermark von 2017-2020 durchgeführt wurde. Nach einer Darstellung der Rahmenbedingungen werden die Eckpunkte der Begleitforschung erläutert. Ziel der Ergebnisdarstellung ist es, die Gelingensfaktoren für die Profilierung für Inklusive Pädagogik im Lehramtsstudium zu identifizieren und strukturelle, inhaltliche, organisatorische und hochschuldidaktische Spannungsfelder aufzuzeigen, die sich in der Umsetzung der Curricula ergeben.

\section{Gesetzlicher Rahmen und Realisierung der Pädagog*innenbildung Neu - Fokus Inklusive Pädagogik}

Das Bundesrahmengesetz $2013^{1}$ zur Einführung einer neuen Ausbildung für Pädagog*innen sieht gemäß $\$ 42$ Abs. 10 vor, dass die Curricula die Zielsetzungen von Art. 24 der UN-Behindertenrechtskonvention zu beachten und Inklusive Pädagogik (IP) in einem angemessenen Ausmaß zu berücksichtigen haben. Weiters schreibt die für die Entwicklung der Curricula verbindliche Zielperspektive des Entwicklungsrates $(2013,3)$ den Erwerb von Basiskompetenzen für Diversität und Inklusion für alle Lehrpersonen vor: Pädagog*innen „haben eine inklusive Grundhaltung und fundierte wissenschaftliche Kenntnisse, [um] mit Diversität im Rahmen eines institutionellen Gesamtkonzepts umzugehen."

Darüber hinaus ist gemäß $\$ 38$ Abs. 2 des Bundesrahmengesetzes 2013 IP verpflichtend anzubieten, im Bereich der Primarstufe in Form eines Schwerpunktes im Umfang von 60-80 ECTS-Anrechnungspunkten (ECTS-AP), im Bereich der Sekundarstufe in Form der Spezialisierung anstelle eines zweiten Unterrichtsfaches im Umfang von 95 ECTS-AP. Erfolgt im Masterstudium in der Primar-

1 Details siehe online unter: https://www.ris.bka.gv.at/Dokumente/BgblAuth/BGBLA_2013_I_124/ BGBLA_2013_I_124.pdfsig (letzter Zugriff: 02.03.2021). 
stufe eine fachliche Vertiefung in einem Förderbereich, hat das Masterstudium 90 anstelle von 60 ECTS-AP zu betragen.

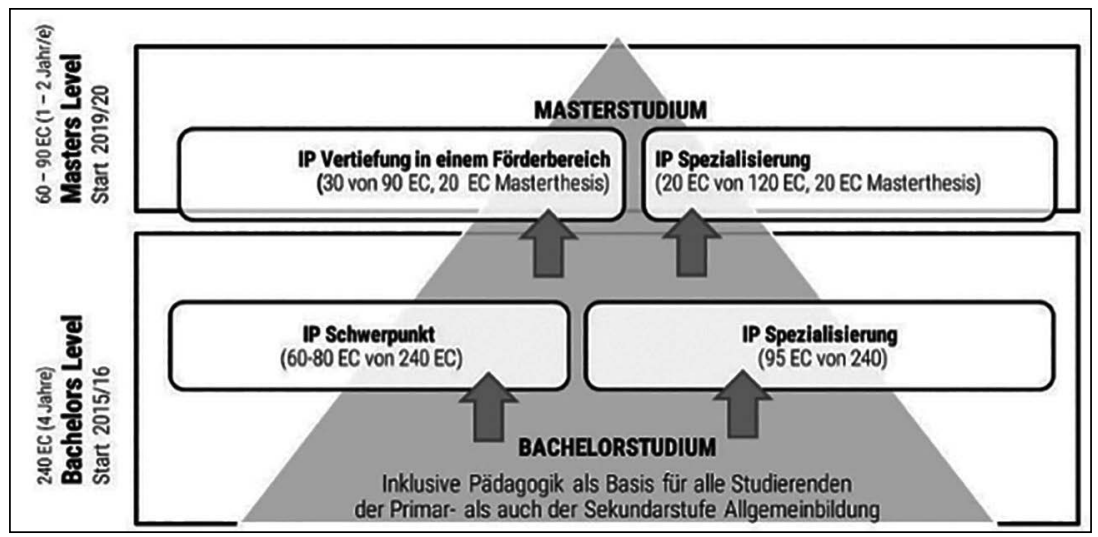

Abb. 1: Umsetzung der gesetzlichen Vorgaben zur Inklusiven Pädagogik im Entwicklungsverbund Südost

Mit dieser Struktur strebt Österreich vor dem Hintergrund von Modellen von Lehrer*innenbildungsprogrammen für Inklusion (vgl. Pugach \& Blanton 2009; Stayton \& McCollum 2002) einen hohen Grad der Zusammenführung der bisher getrennt geführten allgemeinen und sonderpädagogischen Studiengänge an. Diese Modelle orientieren sich an der Quantität der sonderpädagogischen Inhalte in der Lehrer*innenausbildung bzw. dem Grad der Zusammenführung von Sonder- und Regelschullehramt. Stayton und McCollum (2002) unterscheiden zwischen dem Infusion Model, dem Collaborative Training Model und dem Unification Model. Während im Infusion Model Studierende ausgewählte Veranstaltungen zu inklusiven Inhalten im Rahmen ihres gewählten Studiums besuchen, sind im Collaborative Training Model gemeinsam zu besuchende Veranstaltungen curricular verankert und es können auch gemeinsam zu absolvierende Praxisphasen vorgesehen sein. Im Unification Model basiert das Lehramt auf einem gemeinsamen Curriculum mit Fokus auf individuelle Lernentwicklung und Förderung ohne Differenzierung zwischen einem allgemeinen und einem sonderpädagogischen Lehramt.

Das österreichische Modell liegt an der Schnittstelle von Collaborative Training Model und Unification Model. Es besuchen alle Studierenden - vor allem in den Bildungswissenschaften - curricular verankerte, inklusionsbezogene Veranstaltungen. Die Möglichkeit der Wahl des Schwerpunktes bzw. der Spezialisierung IP innerhalb des jeweiligen Altersstufenlehramtes entspricht einem Dual-DegreeProgramm. In der Primarstufe erfolgt die Qualifizierung sowohl zur Generalistin*zum Generalisten für alle Fachbereiche gemäß dem Lehrplan der österrei- 
chischen Volksschule als auch zur Spezialistin*zum Spezialisten für IP. In der Sekundarstufe qualifizieren sich die Studierenden sowohl für ein Unterrichtsfach gemäß den Lehrplänen der Sekundarstufe als auch für die Spezialisierung IP.

\section{Eckpunkte der Begleitforschung vor dem Hintergrund der Curricula im Entwicklungsverbund Südost - Fokus Inklusive Pädagogik}

Das Design verfolgt einen multimethodalen Ansatz und umfasst quantitative und qualitative Erhebungseinheiten über zwei Studienjahre hinweg.

Im Zentrum des Forschungsinteresses stehen die Fragen,

- welche Faktoren für die Wahl des Schwerpunktes bzw. der Spezialisierung IP mit Fokus Behinderung als bedeutsam identifiziert werden,

- welche Elemente sich förderlich auf die Kompetenzentwicklung im Bereich des Wissens, des Handelns und der Haltung auswirken.

Dazu werden mittels einer Fragebogenstudie Beweggründe für die Wahl der Profilierung, berufsbezogene Persönlichkeitsmerkmale und allgemeine und berufsfeldbezogene Interessenslagen bei Studierenden erhoben. Weiters werden durch Interviews mit Lehrenden und Studierenden Erkenntnisse und Einsichten in die Wirkungszusammenhänge der curricular vorgegebenen Inhalte, der hochschuldidaktischen Umsetzung und der in den pädagogisch-praktischen Studien gemachten Erfahrungen gewonnen.

\section{Studierendencharakteristika, Wertesysteme und Einstellungen Lehramtsstudierender im Vergleich}

Die Fragebogenstudie zur Untersuchung von Studierendenmerkmalen hatte zum Ziel, deren Charakteristika wie auch mögliche Unterschiede in Einstellungen und Wertesystemen (Prioritäten im Leben bzw. Diversitätsorientierung) zu erfassen.

\subsection{Stichprobe}

Von den 2018/19 befragten 700 Studierenden der anfallenden Stichprobe studierten insgesamt über die Semester hinweg 87 (12,4\%) das Lehramt Primarstufe mit der Schwerpunktwahl Inklusive Pädagogik, 429 (64,4\%) eine andere Schwerpunktwahl. Bei den Studierenden im Lehramt Sekundarstufe Allgemeinbildung wurden 67 (9,6\%) Personen mit der Spezialisierung Inklusive Pädagogik und $117(16,7 \%)$ Personen mit einer anderen Fächerwahl befragt. Hinsichtlich 
der Geschlechterverteilung waren 573 (82,3\%) der Befragten weiblich und 121 $(17,7 \%)$ männlich.

\subsection{Instrumente}

Zur Erfassung der Lebenszufriedenheit der Studierenden wurde der FLZM-Fragebogen von Henrich und Herschbach (2000) verwendet. Die daraus verwendeten Skalen ,Zufriedenheit' und ,Priorität' fragen jeweils die Wichtigkeit bzw. Zufriedenheit von/mit acht verschiedenen Teilbereichen ab. Sie weisen mit einem Cronbach alpha von 0,83 bzw. 0,82 eine gute interne Konsistenz auf. Zur Erfassung der Diversitätsorientierung der befragten Studierenden wurde die Milville-Guzman Universality Diverse Scale - Short Form (MGUDS-S) von Fuertes, Milville, Mohr, Sedlacek und Gretchen (2000) verwendet. Diese besteht aus drei Subskalen: Diversity of Contact - DoC (Cronbach alpha: 0,68), Relativistic Appreciation RA (Cronbach alpha: 0,66) und Comfort with Difference - CwD (Cronbach alpha: 0,66). Daneben wurden auch noch eine Reihe von Variablen hinsichtlich des sozio-demografischen Hintergrunds, der persönlichen schulischen Laufbahn und der Herkunftsfamilie erhoben.

\subsection{Ergebnisse}

Die Auswertung der Herkunftsverhältnisse ergab eine erwartete Schichtkontinuität hinsichtlich der Berufswahl. 18\% der befragten Studierenden gaben an, dass die Mutter, 9\%, dass der Vater Lehrperson sei. Dieses Ergebnis ist vergleichbar mit Befunden nach Kühne (2006), der eine allgemeine Berufsvererbungsquote mit 24\% zur Diskussion stellt bzw. eine lehramtsspezifische von $11 \%$ für die Primarstufe und $18 \%$ für die Sekundarstufe.

Bemerkenswert ist, dass ca. $11 \%$ angeben, selbst eine Inklusionsklasse während ihrer schulischen Karriere besucht zu haben, und 22\% eine Tätigkeit mit inhaltlichem Bezug zum Studium ausüben. Weitere 52,2\% gehen ebenfalls einer beruflichen Tätigkeit neben dem Studium nach, die jedoch keinen unmittelbaren inhaltlichen Bezug zum Studium aufweist.

In Tabelle 1 sind die Mittelwerte der Ergebnisse des FLZM-Fragebogens jeweils getrennt nach den einzelnen Subpopulationen (Primarstufe und Sekundarstufe jeweils mit und ohne Schwerpunkt-/Spezialisierungswahl Inklusion) dargestellt.

Tab. 1: Prioritäten im Leben

\begin{tabular}{|l|c|c|c|c|}
\hline Wie wichtig ist (sind) dir...? & Primar + IP & Primar - IP & Sek AB + IP & Sek AB - IP \\
\hline Freund*innen/Bekannte & 3,76 & 3,68 & 3,66 & 3,67 \\
\hline Freizeitgestaltung und Hobbys & 3,25 & 3,36 & 3,42 & 3,28 \\
\hline Gesundheit & 3,67 & 3,71 & 3,75 & 3,69 \\
\hline
\end{tabular}




\begin{tabular}{|l|c|c|c|c|}
\hline Wie wichtig ist (sind) dir...? & Primar + IP & Primar - IP & Sek AB + IP & Sek AB - IP \\
\hline $\begin{array}{l}\text { Einkommen/finanzielle Sicher- } \\
\text { heit }\end{array}$ & 2,93 & 3,11 & 2,97 & 3,17 \\
\hline Beruf/Arbeit & 3,11 & 3,18 & 3,10 & 3,07 \\
\hline Wohnsituation & 2,97 & 3,19 & 3,13 & 3,16 \\
\hline Familienleben/Kinder & 3,43 & 3,55 & 3,48 & 3,23 \\
\hline Partner*innenschaft/Sexualität & 3,40 & 3,50 & 3,28 & 3,28 \\
\hline
\end{tabular}

Der deskriptive Vergleich der Mittelwerte zeigt, dass sich die Werte zwischen den Gruppen nur sehr geringfügig ändern. Ausnahmen bilden die Prioritäten für Einkommen und finanzielle Sicherheit sowie jene der Wohnsituation, welche von Studierenden der Primarstufe + IP als etwas weniger bedeutsam eingeschätzt werden. Da zudem auch die Streuung der Variablen zwischen den Gruppen relativ ähnlich ist, kann von einem ähnlichen Antwortverhalten der einzelnen Subpopulationen ausgegangen werden. Ein identes Ergebnis zeigt sich auch bei der Einschätzung der Zufriedenheit mit den angeführten acht Teilbereichen. Die Mittelwerte bzw. die Streuung sind sehr homogen, auf eine Berechnung der statistischen Signifikanz der Gruppenunterschiede wurde daher verzichtet, da auch in den einzelnen Teilbereichen das Antwortverhalten ein ähnliches Muster aufweist.

Hinsichtlich der Diversitätsorientierung der im Studienjahr 2018/19 befragten Personen ergab die Auswertung der MGUDS-S Subskalen mittels t-test keine signifikanten Gruppenunterschiede zwischen Personen, die eine Inklusionsklasse besucht hatten, und jenen Personen, die keine Inklusionsklasse besuchten. Signifikante Unterschiede ergaben sich nur bei Personen, die ein Praktikum bzw. einen Beruf mit inhaltlichem Bezug zum Studium ausüben.

Zusammenfassend lässt sich daher festhalten, dass es sich bei den befragten Studierenden der vier Subgruppen Primar vs. Sekundar in Kombination mit der Wahl IP vs. andere Schwerpunkte/Fächer an der Pädagogischen Hochschule Steiermark um eine relativ homogene Gruppe handelt.

\section{Auf dem Weg zu wirkungsvollen Expert*innen für Inklusion - die Perspektiven von Lehrenden und Studierenden}

Die Datenerhebung erfolgte mittels qualitativer Forschungsinterviews nach der ,Critical Incidence Technique' (vgl. Flanagan 1954), durchgeführt in den Studienjahren 2017/18 und 2018/19. Interviewpartner*innen waren acht unterschiedliche Lehrende, drei gleichbleibende Studierende der Primarstufe mit Schwerpunkt IP (um Perspektivenänderungen im Studienverlauf dokumentieren zu können) und drei Studierende der Sekundarstufe AB mit unterschiedlichen Fächern 
zusätzlich zur Spezialisierung IP. Die jeweiligen Leitfäden fragten im Sinne der Kompetenzbausteine nach Gonzáles und Wagenaar (2003) nach förderlichen sowie einschränkenden Faktoren in den Bereichen Wissen (theoretisches Wissen und Verständnis betreffend Fachbereiche), Handeln (praktische und operante Anwendung des Wissens) und Haltung (Werte im Hinblick auf Wahrnehmung und Zusammenleben im sozialen Umfeld) in für die Ausbildung relevanten Bereichen. Drei weitere Interviews mit dem Fokus auf die pädagogisch-praktischen Studien wurden mit Studierenden der Sekundarstufe AB durchgeführt: Die Kategorienbildung der durch die Interviews erhobenen Daten erfolgte gemäß der qualitativen Inhaltsanalyse nach Mayring (2010) unter Verwendung des Programms MAXQDA 11 (2014).

\subsection{Curriculare Strukturen}

Lehrende sehen im Curriculum einen profunderen Wissens- und Kompetenzerwerb in Bezug auf Inklusion und Förderbereiche gegeben als in der früheren Ausbildung zur*zum Sonderschullehrer*in. Der Einblick in alle Förderbereiche zu Beginn des Studiums führt zu breitem Basiswissen und erleichtert Studierenden die Wahl von spartenspezifischen Vertiefungen im Rahmen von Wahlpflichtmodulen. Studierende schätzen den crosskategorialen Ansatz des Curriculums, die inhaltlichen Schwerpunktsetzungen, das ausgewogene Verhältnis von Theorie und Praxis und die Möglichkeit, medizinisches und neurowissenschaftliches Wissen in Verbindung mit Handlungswissen zu erwerben. Kritisch angemerkt wird von ihnen, dass gewisse Inhalte nur dem inklusiven Schwerpunkt vorbehalten blieben, obwohl sie für alle Lehrer*innen relevant wären.

\subsection{Hochschuldidaktik}

Im Hinblick auf kontinuierlichen Wissenserwerb wünschen sich Lehrende, Lehrveranstaltungen mit aufbauenden Inhalten fortlaufend unterrichten zu können. Studierende profitieren von einem breiten Fachwissen Lehrender, das unter Einbeziehung von Erfahrungsberichten und mit Praxisbezug weitergegeben wird. Kooperative Lernmethoden fördern Wissen wie auch Handeln Studierender. Fallbeispiele und Rollenspiele bereiten sie auf die Realität des Unterrichtens vor und erlauben das Verknüpfen von erworbenem Fachwissen mit situationsrelevantem Handeln. Konkrete Erfahrungen mit assistierenden Technologien führen zur Sicherheit im Einsatz dieser Medien mit Schüler*innen, die diese benötigen. Als haltungsbildend sehen Lehrende dieses Studienschwerpunktes das authentische Vorleben der eigenen Einstellung im Hinblick auf inklusive Settings. Studierende profitieren vom direkten Kontakt mit Lehrenden, Gastreferent*innen oder Studierenden, die selbst von einer Behinderung betroffen sind. Sensibilisierungsund Selbsterfahrungsübungen in Bezug auf verschiedene Formen von Behinderungen ermöglichen ergänzende Sichtweisen. 


\subsection{Pädagogisch-praktische Studien}

Lehrende konstatieren eine erhöhte Motivation für Wissensvertiefung, wenn Studierende in ihren Praxisklassen Schüler*innen mit Bedarfen in Förderbereichen erleben, die in Lehrveranstaltungen thematisch behandelt wurden. Die durch Mentor*innen gewährte Einsicht in Aufgabenbereiche der Inklusionslehrer*innen wie Evaluierung, Förderplanerstellung oder Elternkooperation vertieft dieses Wissen weiter.

Die inklusive Haltung Studierender wächst mit dem Wahrnehmen kleiner Schritte in Lernprozessen. Studierende der Primarstufe profitieren durch die Inklusive Pädagogik in Hinblick auf Individualisierung und Methodik auch für ihren allgemeinen Unterricht in Volksschulklassen, Studierende der Sekundarstufe für den Unterricht in ihrem Zweitfach. Die Interaktion mit Schüler*innen mit Beeinträchtigungen bzw. mit unterschiedlichen sozialen Hintergründen trägt zum Abbau von Vorurteilen bei. Studierende erfahren in den pädagogisch-praktischen Studien eine Bestätigung ihrer Studienwahl.

Die Auswahl der Praxisklassen und die Qualifikation der Mentor*innen sowie ko-konstruktive Ansätze in der Zusammenarbeit von Studierenden und Mentor*innen sind bedeutsam für einen gelingenden Theorie-Praxis-Transfer. In der Sekundarstufe sichern vor allem die fachdidaktischen Begleitlehrveranstaltungen den Theorie-Praxis-Transfer.

\section{Limitation der Studie}

Die Resultate der Interviewstudie reflektieren die Perspektiven von jeweils acht Lehrenden im Bachelorstudium der Primarstufe und Sekundarstufe AB sowie von jeweils drei Studierenden der Primarstufe und der Sekundarstufe AB. Obwohl durch Triangulation (vgl. Eisner 2017) die Validität der Ergebnisse gewährleistet ist, könnte sie durch eine Langzeitstudie bzw. Replik der Studie in einem weiteren Durchgang noch erhöht werden.

Beim quantitativen Teil der Stichprobe handelt es sich um eine reine Fragebogenerhebung von Einschätzungen der Studierenden, Kausalaussagen sind daher nicht zulässig. Darüber hinaus bleibt anzumerken, dass die Studierenden insgesamt ein sehr homogenes Antwortverhalten in allen abgefragten Aspekten und Dimensionen aufweisen. Dies schränkt die interferenzstatistische Auswertung ein. 


\section{Zusammenfassung der Ergebnisse}

Die Gruppe der an dieser Studie partizipierenden Studierenden ist von einer auffallenden Homogenität hinsichtlich der Orientierungen zur Inklusion und Diversität geprägt.

Als förderliche Elemente für die Kompetenzentwicklung im Bereich des Wissens, des Handelns und der Haltung werden der Kontakt mit Menschen mit Behinderungen (z.B. Studienkolleg*innen oder Lehrende) sowie mit Personen mit Expert*innenstatus identifiziert. Lehrende werden als Expert*innen ihres Fachgebietes und als, role models' gesehen. Die förderliche Wirkung von Lehrenden mit Behinderung auf den eigenen Kompetenzerwerb wird von den Studierenden hervorgehoben. Für die Hochschulentwicklung bedeutet dies, dass Lehrende, die die gesellschaftliche Vielfalt spiegeln, eine wertvolle Ressource darstellen und wesentlich dazu beitragen können, persönliche Einstellungen und Haltungen zu reflektieren sowie Barrieren in den Köpfen abzubauen (vgl. Holzinger, Feyerer, Grabner, Hecht \& Peterlini 2019). Weiters bestätigen Studierende entwicklungsförderliche Effekte, wenn das in Lehrveranstaltungen erworbene Wissen in anschlussfähig konzipierten pädagogisch-praktischen Studien zu konkreten Handlungserfahrungen transformiert werden kann.

Hervorzuheben ist die Wirkung der spezifisch im Schwerpunkt bzw. in der Spezialisierung Inklusive Pädagogik erworbenen Kompetenzen auf die allgemeine - generalistische - Unterrichtstätigkeit der Primarstufe bzw. auf das zweite bzw. jedes weitere gewählte Fach der Sekundarstufe Allgemeinbildung. Dies betrifft vor allem Kompetenzen im Bereich der Individualisierung und Differenzierung sowie der Inklusiven Didaktik und dem Universal Design for Learning (vgl. Hall, Meyer \& Rose 2012).

Während formal das Ziel der Pädagog*innenbildung NEU in Curricula festgeschrieben ist, spiegeln sich Wirkung und Erfolg in den Ergebnissen der Befragungen der Studierenden der ersten Kohorten der neuen Lehramtsstudien wider und zeigen wirkungsvolle Strategien und hochschuldidaktische Elemente auf, die es nun stetig weiterzuentwickeln gilt.

\section{Literatur}

Eisner, E. W. (2017): The enlightened eye: Qualitative inquiry and the enhancement of educational practice. New York.

Entwicklungsrat (2013): Professionelle Kompetenzen von PädagogInnen. Zielperspektive. Online unter: https://www.qsr.or.at/dokumente/1869-20140529-092429-Professionelle_Kompetenzen_ von_PaedagogInnen_Zielperspektive.pdf (letzter Zugriff: 12.04.2020).

Flanagan, J. C. (1954): The critical incident technique. In: Psychological Bulletin, 51, H.4, 327-358.

Fuertes, J., Milville, M., Mohr, J., Sedlacek, W. \& Gretchen, D. (2000): Factor Structure and Short Form of the Milville-Guzman Universality-Diversity Scale. In: Measurement and Evaluation in Counselling and Development, 33, 157-169. 
Gonzáles, J. \& Wagenaar, R. (Hrsg.) (2003): Tuning Educational Structures in Europe, Final Report. Phase I. Bilbao.

Hall, T. E., Meyer, A. \& Rose, D. H. (2012): Universal Design for Learning in the Classroom. Practical Applications. New York.

Henrich, G. \& Herschbach, P. (2000): Questions on Life Satisfaction (FLZM). A Short Questionnaire for Assessing Subjective Quality of Life. In: European Journal of Psychological Assessment, 16, 150-159.

Holzinger, A., Feyerer, E., Grabner, R., Hecht, P. \& Peterlini, H. K. (2019): Kompetenzen für Inklusive Bildung -Konsequenzen für die Lehrerbildung. In: Breit, S., Eder, F., Krainer, K., Schreiner, Ch., Seel, A. \& Spiel, Ch. (Hrsg.): Nationaler Bildungsbericht 2018. Band 2. Fokussierte Analysen und Zukunftsperspektiven für das Bildungswesen. Graz, 63-98.

Kühne, S. (2006): Das soziale Rekrutierungsfeld der Lehrer. Empirische Befunde zur schichtspezifischen Selektivität in akademischen Berufspositionen. In: Zeitschrift für Erziehungswissenschaft, 9, 618-619.

MAXQDA 11 (2014): The Art of Data Analysis. VERBI Software. Consult. Berlin.

Mayring, P. (2010): Qualitative Inhaltsanalyse: Grundlagen und Techniken. 12. Aufl. Weinheim.

Pugach, M. \& Blanton, L. (2009): A Framework for Conducting Research on Collaborative Teacher Education. In: Teaching and Teacher Education: An International Journal of Research and Studies, 25, H.4, 575-582.

Stayton, V. D. \& McCollum, J. (2002): Unifying general and special education: What does the research tell us? In: Teacher Education and Special Education, 25, H.3, 211-218. 


\section{Kooperative Prozesse im technikbezogenen Unterricht}

Schüler*innen der Primarstufe begegnen technischen Themen im Allgemeinen mit Interesse und Neugier. Technische Sachverhalte erschließen sie sich meist unmittelbar in Tätigkeiten wie Bauen und Konstruieren, aber auch im Bedienen und Gebrauchen (vgl. Fast \& Finkbeiner 2019, 27). Im wissenschaftlichen Diskurs um frühe technische Bildung gewinnen Denk- und Handlungsweisen sowie Aspekte technischen Problemlösens von Kindern aktuell vermehrt an Bedeutung. Auffallend ist allerdings, dass insbesondere Schüler*innen mit der Zuschreibung eines sogenannten ,sonderpädagogischen Förderbedarfs' im Kontext technischer Bildung bislang weder in der Forschungs- noch in der Unterrichtspraxis als relevante Akteur*innen adressiert zu werden scheinen und somit weitgehend unsichtbar bleiben.

Im Sinne eines anzustrebenden inklusionskompetenten und entwickelnden Unterrichts (vgl. Siebert 2010, 124) zielt die aktuelle wissenschaftliche Auseinandersetzung der Autor*innen dieses Beitrags mit der Thematik primär auf eine unterrichtsentwicklungsrelevante Integration allgemein- und sonderpädagogischer sowie fachdidaktischer Aspekte. Besonderer Fokus liegt hierbei auf der Berücksichtigung kooperativer Prozesse eines gemeinsamen Handelns und Problemlösens im technikbezogenen Unterricht.

\section{Frühe technische Bildung}

Frühe Erfahrungen und handelnde Auseinandersetzungen mit Technik erscheinen als bedeutsamer Teil der kindlichen Lebenswelt. Bildungsprozesse im Zusammenhang mit Technik berücksichtigen, fördern und erweitern alltags- und lebensweltliche Handlungskompetenzen der Lernenden und zielen darauf, Aspekte des technischen Kulturbereichs kennenzulernen (vgl. DGTB 2018, 3; Wensierski \& Sigeneger 2015, 83).

Grundlegende Hinweise dazu finden sich insbesondere in wissenschaftlichen Betrachtungen technischer Bildung und problemlösender Strategien von regelschulkonformen Schüler*innen in der Primarstufe, die vorzugsweise im Bereich des Sachunterrichts (vgl. Mammes \& Zolg 2015; Möller \& Wiesenfarth 1992), Gestaltungsunterrichts (vgl. Wyss 2016) oder im Zusammenhang mit technik- 
bezogenem Unterricht im fächerverbindenden Kontext (vgl. Greinstetter, Fast \& Bramberger 2018) zu finden sind.

Als ein zentrales Merkmal von Technik gilt die Finalität. So wird allgemein immer dann von Technik gesprochen, „wenn Gegenstände von Menschen künstlich gemacht und für bestimmte Zwecke verwendet werden (...)“ (Ropohl 2009, 30). Die Ausrichtung auf den Zweck unterscheidet die Technik zudem von den Naturwissenschaften, welche sich eher kausalen Fragen widmen.

Mit Blick auf einen technikbezogenen und problemlösungsorientierten Unterricht rücken deshalb die Interessen von Schüler*innen und ihre auf den Zweck ausgerichteten Erklärungen und Ergebnisse auf dem Weg zu einer technischen Problemlösung vermehrt in den Mittelpunkt. Dies verlangt Bewertungen und Entscheidungen, die sowohl eigene als auch die Ideen anderer berücksichtigen. Neben sprachlichen und kognitiven Möglichkeiten sind das Erkennen und Lösen eines technischen Problems, bedingt durch das sich in der Entwicklung befindende Abstraktionsvermögen der Kinder, primär an konkrete Handlungen gebunden. Somit kommt der Wechselseitigkeit von Handeln und Denken in der Primarstufe eine große Bedeutsamkeit zu, da Aufgaben, die auf ein rein kognitionsdominiertes Problemlösen zielen, den Voraussetzungen der Lernenden widersprechen. Dies zeigt sich auch vor dem Hintergrund, dass Kinder im Grundschulalter Lösungswege unter Einbeziehung von Materialien und Werkzeugen häufig ,probierend bestreiten (vgl. Wiesenfarth 1992, 37). Für Lerngelegenheiten im technikbezogenen Unterricht ergibt sich daraus die Forderung, dass Lernhandlungen nicht allein vom Denken zum Handeln hin zu gestalten sind, sondern aufbauend auf der Wahrnehmung sowie den Vorerfahrungen der Kinder, um damit eine möglichst direkte Auseinandersetzung mit dem Lerngegenstand Technik zu ermöglichen (vgl. Binder 2014, 397).

Das mögliche Potenzial dahingehender vielfältiger kindlicher Lern- und Entwicklungswege, wie sie für heterogene Lerngruppen in der Primarstufe anzunehmen sind, findet im Zusammenhang mit einem frühen technikbezogenen Unterricht bis dato jedoch wenig Beachtung.

\section{Zur Praxis technikbezogenen Unterrichts}

An österreichischen Grundschulen ist der technikbezogene Unterricht im Lehrplan des Sachunterrichts (technischer Erfahrungs- und Lernbereich) und im Technischen Werken verortet. Beide Unterrichtsgegenstände bilden aus ihren jeweiligen Perspektiven heraus sowie in einem fächerverbindenden Kontext, ausgehend von lebensweltlichen Situationen und Anlässen, die Basis für eine technische Bildung in der Grundschule. 
Ein Kernproblem des Unterrichtsgegenstandes Technisches Werken, welches u.a. auf eine bis dato nicht konsensfähige Fachdidaktik zurückzuführen ist, zeigt sich im durch Lehrpersonen bevorzugten Einsatz vorgefertigter Materialsätze in Form sogenannter Werkpackungen, deren Zweck vor allem im rezepthaften Nachbau eines Produktes liegt (vgl. Hennerbichler, Sturm \& Finkbeiner 2017, 19).

Die damit verbundenen kleinschrittigen Bau- und Bastelanleitungen verunmöglichen oftmals das Erkennen und Lösen individueller technischer Probleme, anstatt sie zu fördern. Zudem werden Lerngelegenheiten in der Elementar- und Primarstufe häufig kreativen Aspekten zugeordnet, die überwiegend eher dekorative Zwecke in Form von Oberflächengestaltung durch Farben und Materialien verfolgen, anstatt technisch-kreative und lösungsoffene Möglichkeiten für die Lernenden zu bieten. Der Anerkennung kindlichen Problembewusstseins sowie der Bedeutung kooperativer und kommunikativer Prozesse wird somit wenig Beachtung geschenkt.

Dieser Reduktionismus erscheint umso tragender, je höher die den Kindern zugeschriebenen sonderpädagogischen Förderbedarfe sind. Infolgedessen gilt es, den Anspruch technischer Bildung als Allgemeinbildung (vgl. DGTB 2018, 3) in den Blick zu nehmen.

\section{Lernen und Kooperation in technischen Bildungsprozessen}

In der kulturgeschichtlichen Entwicklung kann das Verhältnis von Mensch und Technik - und damit die Herstellung und der Gebrauch technischer Artefakte als ein soziales Phänomen verstanden werden (vgl. Ropohl 2009, 39). Dies zeigt sich beispielsweise in der Sprache, der Weitergabe von Wissen sowie der Koordination gemeinsamer Handlungen (vgl. Poser \& Wiegerling 2016, 111). Kooperation als gemeinsame Handlungsregulation unterliegt somit einer intersubjektiven Konstruktion und Koordination von Vorhaben und Zielrichtungen, die über Prozesse der Kommunikation und des Dialogs vermittelt werden. Kooperation, als handelnder Austausch von Bedeutungen mit anderen (vgl. Rödler 2018, 80), kann als Einigungsprozess zwischen verschiedenen Bedürfnissen, Fähigkeiten und Sichtweisen betrachtet werden. Dieser beinhaltet Momente der Annäherung und Abgrenzung auf Basis einer Wertschätzung und Anerkennung der Individualität der jeweils anderen und ermöglicht die Übernahme der jeweiligen Leitungsfunktion durch alle Beteiligten (vgl. Prammer \& Prammer-Semmler 2011). Allen Beteiligten wird es dadurch ermöglicht, ihre Motive einzubringen und bisher aufgebautes Wissen und Fähigkeiten arbeitsteilig zu realisieren sowie neu zu erwerben (vgl. ebd., 39). Das gemeinsame Durchdringen eines Gegenstandes hat dabei die bewusste Lerntätigkeit der Schüler*innen im Blick, welche über eine formale Wissensaneignung hinausgeht (vgl. Langner 2016, 174; Siebert 2010). Unterricht 
versteht sich dadurch als Entwicklung induzierender Prozess durch und mit andere/n. Aneignung bzw. Lernen erfolgt dann in Kooperation bzw. in gemeinsam geteilter Tätigkeit, sowohl mit Lehrpersonen als auch mit anderen Kindern (vgl. Siebert 2010; Ziemen 2016).

Kooperative Prozesse werden somit, mit Blick auf Herausforderungen und Chancen inklusiven Unterrichts, zu einem zentralen Fundament gemeinsamen Lernens (vgl. Feuser 1995; Wittich 2017, 61). Dies ist für die Primarstufe insofern von Bedeutung, als Schüler*innen zwischen 6 und 10 Jahren als eine noch weitestgehend heterogene Gruppe betrachtet werden (vgl. Trautmann \& Wischer 2011).

Wissenschaftliche Arbeiten zum kooperativen Lernen im Kontext inklusiven Unterrichts fokussieren gegenwärtig überwiegend eine evaluative Überprüfung der Wirksamkeit kooperativer Lernsettings als Methode (vgl. Braunsteiner, Fischer, Kernbichler, Prengel \& Wohlhart 2018, 35). Jedoch geben Autor*innen wie Anja Hackbarth (2017) mit der Untersuchung zu Schüler*innenkooperationen im Kontext jahrgangsübergreifenden Lernens Hinweise auf die Bedeutung kooperativer Prozesse und ihrer intersubjektiven Realisierung, die es in zukünftigen Studien aufzugreifen gilt.

\section{Die Perspektive der Schüler*innen anerkennen}

Ausdrucksformen von Kindern unterliegen ihrer jeweiligen Entwicklungssituation und unterscheiden sich grundlegend von den Erklärungsmustern und Sinnstrukturen Erwachsener. Neben sprachlichen rücken u.a. auch dingliche Ausdrucksformen in den Blickpunkt (vgl. Nentwig-Gesemann \& Wagner-Willi 2007) und unterstreichen die zweckdienliche Beachtung kognitiver, sprachlicher und handelnder Aspekte. Hierin gründet auch das grundlegende Interesse des Autor*innenteams dieses Beitrags: den kooperativen Handlungen von Schüler*innen in der Primarstufe innerhalb technisch orientierter Lerngelegenheiten entscheidend Beachtung zu schenken.

Als generell forschungsleitend erweisen sich dabei Aspekte, die vor dem Hintergrund kooperativer Prozesse

- aktuelle Wahrnehmungs-, Denk- und Handlungskompetenzen der Schüler*innen berücksichtigen,

- mögliche Einschluss- und Ausschlussmomente beobachtbar werden lassen,

- die zum Einsatz kommenden Mittel (wie Sprache, Objekte usw.) und deren jeweilige Bedeutung aufzeigen.

Videografie als komplexes Verfahren könnte, wenngleich die Analyse von Sprache in der Ausrichtung dominiert (vgl. Fritzsche \& Wagner-Willi 2015, 131), in einem ersten Schritt den Beobachtungsprozess unterstützen (vgl. Schnettler \& Knob- 
lauch 2009). Umfassende Hinweise dafür finden sich bei Sujbert, Sunnen, Arend und Fixmer (2014) in ihren Darstellungen zu den Beobachtungen gemeinsam konstruierter Lernprozesse von Kindern. Ergebnisse der Mikroanalysen zeigen, dass Kinder ihre Interaktionen u.a. mit Gegenständen organisieren und neben der gesprochenen Sprache auch auf weitere kommunikative Ressourcen zurückgreifen. Die in diesem Zusammenhang von den Autor*innen des vorliegenden Beitrags in Planung befindliche Studie zielt darauf ab, technikbezogene, kooperative Lernarrangements auf Basis sequenzieller, videogestützter Beobachtungen $\mathrm{zu}$ analysieren und $\mathrm{zu}$ interpretieren. Grundlegend dabei ist die Annahme, „(...) dass den Forschenden noch nicht bekannt ist, welchen Aspekten und Zusammenhängen im beobachteten Unterrichtsgeschehen welche Bedeutung zukommt“ (Dinkelaker 2016, 55).

\section{Ausblick}

Unterricht als Vermittlungsform menschlicher Entwicklung (vgl. Siebert 2010, 118 ) berücksichtigt, wie von Feuser $(2019,154)$ beschrieben, Aspekte der Kommunikation und Kooperation, welche sich insbesondere in der Gegenseitigkeit von Handlungen zeigen. Anknüpfend daran bieten Lernarrangements, die die Bedeutung von Kooperation für Lernen und Entwicklung anerkennen, die Möglichkeit, individuelle und kollektive Voraussetzungen der Lernenden zu beachten, um dabei sowohl kognitive, soziale als auch emotionale Aspekte im Zusammenhang mit Lernen in die Zielsetzung miteinzubeziehen (vgl. Möller 1999, 133). Als anschlussfähig erweist sich hierbei die Mehrperspektivität technischer Bildung (vgl. DGTB 2018) als grundlegende Orientierung fachbezogener Lerngelegenheiten vor dem Hintergrund inklusiver Didaktik. Auf Basis der jeweiligen Aneignungsniveaus können die Kinder in der gemeinsam geteilten Tätigkeit ihre bisher aufgebauten Sinn- und Bedeutungsstrukturen erweitern (vgl. Rödler 2018). Dies könnte u.a. einen Beitrag leisten, wenn es darum geht, fachliches Lernen im Kontext sogenannter inklusiver und heterogener Lerngruppen zu realisieren. Seitz (2006, o.S.) hat dies in der Frage nach dem „Kern der Sache“ bereits früh im Rahmen der Diskussion um eine inklusive Didaktik aufgeworfen und in den vergangenen Jahren, insbesondere im Zusammenhang mit dem Sachunterricht (vgl. Seitz 2004 \& 2018), weiter erörtert. In der gemeinsamen Lerntätigkeit (vgl. Langner 2016) und der Kooperation am Gemeinsamen Gegenstand (vgl. Feuser 1995) werden Wissensbestände ausgetauscht, verhandelt und erweitert. Diesen, unter anderem als Mikroprozesse (vgl. Schulte, Kurnitzki, Lütje-Klose \& Miller 2019) beschriebenen, Aspekten gilt die Aufmerksamkeit im weiteren Vorgehen, um im Hinblick auf die Erfahrungen und Bedürfnisse der Lernenden mögliche Perspektiven aufzuzeigen und diese gemeinsam zu befragen. 


\section{Literatur}

Binder, M. (2014): Technisches Handeln - eine Studie zu einem grundlegenden Begriff Technischer Bildung. Inaugural-Dissertation zur Erlangung eines Doktorgrades der Philosophie der Pädagogischen Hochschule Weingarten. Online unter: https://hsbwgt.bszbw.de/files/147/Binder+-+Studie+zum+technischen+Handeln.pdf (letzter Zugriff: 01.01.2017).

Braunsteiner, M.-L., Fischer, C., Kernbichler, G., Prengel, A. \& Wohlhart, D. (2018): Erfolgreich lernen und unterrichten in Klassen mit hoher Heterogenität. In: Breit, S., Eder, F., Krainer, K., Schreiner, C., Seel, A. \& Spiel, C. (Hrsg.): Nationaler Bildungsbericht 2018. Band 2. Fokussierte Analysen und Zukunftsperspektiven für das Bildungswesen. Graz, 19-62.

DGTB (Deutsche Gesellschaft für Technische Bildung e.V.) (2018): Anliegen und Grundzüge Allgemeiner Technischer Bildung. Grundsatzpapier Nr. 1. Online unter: https://dgtb.de/wp-content/ uploads/2018/09/Grundsatzpapier-Nr_1_04-08-2018-final.pdf (letzter Zugriff: 01.04.2020).

Dinkelaker, J. (2016): Datengewinnung und -formate in der videobasierten Unterrichtsforschung. In: Rauin, U., Herrle, M. \& Engartner, T. (Hrsg.): Videoanalysen in der Unterrichtsforschung: Methodische Vorgehensweisen und Anwendungsbeispiele (Grundlagentexte Methoden). Weinheim/Basel, 50-75.

Fast, M. \& Finkbeiner, T. (2019): Technische Bildung im fächerverbindenden Unterricht der Primarstufe - Eine qualitative Untersuchung zu Interessenförderung. In: tu Zeitschrift für Technik im Unterricht, 44, H.1, 26-44.

Feuser, G. (1995): Behinderte Kinder und Jugendliche zwischen Integration und Aussonderung. Darmstadt.

Feuser, G. (2019): Lernen durch Kooperation am Gemeinsamen Gegenstand. In: Behrendt, A., Heyden, F. \& Hacker, T. (Hrsg.): „Das Mögliche, das im Wirklichen (noch) nicht sichtbar ist...“. Planung von Unterricht für heterogene Lerngruppen - im Gespräch mit Georg Feuser. Düren, 5-30.

Fritzsche, B. \& Wagner-Willi, M. (2015): Dokumentarische Interpretation von Unterrichtsvideografien. In: Bohnsack, R., Fritzsche, B. \& Wagner-Willi, M. (Hrsg.): Dokumentarische Video- und Filminterpretation. Methodologie und Forschungspraxis. Sozialwissenschaftliche Ikonologie: Qualitative Bild- und Videointerpretation. Band 3. 2. Aufl. Opladen/Berlin \& Toronto, 131-152.

Greinstetter, R., Fast, M. \& Bramberger, A. (2018): Technische Bildung im fächerverbindenden Unterricht der Primarstufe. Forschung - Technik - Geschlecht. Baltmannsweiler.

Hackbarth, A. (2017): Schülerkooperation in inklusiven und exklusiven Schulformen. In: Budde, J., Dlugosch, A. \& Sturm, T. (Hrsg.): (Re-)Konstruktive Inklusionsforschung. Differenzlinien. Handlungsfelder. Empirische Zugänge. Opladen/Berlin \& Toronto, 196-209.

Hennerbichler, S., Sturm, R. \& Finkbeiner, T. (2017): Praxishandbuch Grundschule für Technisches und Textiles Werken. Graz.

Langner, A. (2016): Lernschwierigkeit. In: Ziemen, K. (Hrsg.): Lexikon Inklusion. Göttingen, 173-175.

Mammes, I. \& Zolg, M. (2015): Technische Aspekte. In: Kahlert, J., Fölling-Albers, M., Götz, M., Hartinger, A., Miller, S. \& Wittkowske, S. (Hrsg.): Handbuch Didaktik des Sachunterrichts. 2. aktual. u. erw. Aufl. Stuttgart/Bad Heilbrunn, 143-149.

Möller, K. (1999): Konstruktivistisch orientierte Lehr- Lernprozessforschung im naturwissenschaftlichtechnischen Bereich des Sachunterrichts. In: Köhnlein, W. (Hrsg.): Vielperspektivisches Denken im Sachunterricht. Forschung zur Didaktik des Sachunterrichts. Band 3. Bad Heilbrunn, 125-191.

Möller, K. \& Wiesenfarth, G. (1992): Werkstatt Technik. In: Hameyer, U., Lauterbach, R. \& Wiechmann, J. (Hrsg.): Innovationsprozesse in der Grundschule: Fallstudien, Analysen und Vorschläge zum Sachunterricht. Bad Heilbrunn, 168-178.

Nentwig-Gesemann, I. \& Wagner-Willi, M. (2007): Rekonstruktive Kindheitsforschung. Zur Analyse von Diskurs- und Handlungspraxis bei Gleichaltrigen. In: Wulf, C. \& Zirfas, J. (Hrsg.): Pädagogik des Performativen: Theorien, Methoden, Perspektiven. Weinheim/Basel, 213-223. 
Poser, H. \& Wiegerling, K. (2016): Homo Creator: Technik als philosophische Herausforderung (Anthropologie - Technikphilosophie - Gesellschaft). Wiesbaden.

Prammer, W. \& Prammer-Semmler, E. (2011): Die Bedeutung von Kooperation für inklusive Pädagogik - eine Analyse differenzierter Handlungsmöglichkeiten im schulischen Feld. Unveröffentlichte Masterarbeit. Darmstadt.

Rödler, P. (2018): Die Forderung nach Inklusion in ihrer Bedeutung für Didaktik und Fachdidaktik. In: Langner, A. (Hrsg.): Inklusion im Dialog: Fachdidaktik - Erziehungswissenschaft - Sonderpädagogik. Bad Heilbrunn, 77-85.

Ropohl, G. (2009): Allgemeine Technologie. Eine Systemtheorie der Technik. 3. überarb. Aufl. Karlsruhe.

Schnettler, B. \& Knoblauch, H. (2009): Videoanalyse. In: Kühl, S., Strodtholz, P. \& Taffertshofer, A. (Hrsg.): Handbuch Methoden der Organisationsforschung. Wiesbaden, 272-297.

Schulte, F., Kurnitzki, S., Lütje-Klose, B. \& Miller, S. (2019): Mikroprozesse im inklusionsorientierten Sachunterricht: Gemeinsamkeit herstellen und den Lerngegenstand fokussieren. In: Pech, D., Schomaker, C. \& Simon, T. (Hrsg.): Inklusion im Sachunterricht. Perspektiven der Forschung. Forschungen zur Didaktik des Sachunterrichts. Band 10. Bad Heilbrunn, 21-35.

Seitz, S. (2004): Forschungslücke Inklusive Fachdidaktik - ein Problemaufriss. In: Schnell, I. \& Sander, A. (Hrsg.): Inklusive Pädagogik. Bad Heilbrunn/Obb, 215-232.

Seitz, S. (2006): Inklusive Didaktik: Die Frage nach dem ,Kern der Sache‘. In: Zeitschrift für Inklusion, 1, H.1, o.S. Online unter: https://www.inklusion-online.net/index.php/inklusion-online/ article/view/184 (letzter Zugriff: 01.04.2020).

Seitz, S. (2018): Forschung zu inklusivem Sachunterricht - Bestandsaufnahme und Perspektiven. In: Pech, D., Schomaker, C. \& Simon, T. (Hrsg.): Sachunterrichtsdidaktik \& Inklusion. Ein Beitrag zur Entwicklung. Baltmannsweiler, 96-111.

Siebert, B. (2010): Entwickelnder Unterricht und integrative Pädagogik. In: Siebert, B. (Hrsg.): Integrative Pädagogik und Kulturhistorische Theorie. Frankfurt am Main, 103-133.

Sujbert, M., Sunnen, P., Arend, B. \& Fixmer, P. (2014): Mit Video einen mikroanalytischen Blick auf gemeinsam konstruierte Lernprozesse von Kindern richten. In: Kopp, B., Martschinke, S., Munser-Kiefer, M., Haider, M., Kirschhock, E.-M. \& Ranger, G. (Hrsg.): Individuelle Förderung und Lernen in der Gemeinschaft. Jahrbuch Grundschulforschung. Band 17. Wiesbaden, 182-185.

Trautmann, M. \& Wischer, B. (2011): Heterogenität in der Schule. Eine kritische Einführung. Wiesbaden.

Wensierski, H.-J. \& Sigeneger, J.-S. (2015): Technische Bildung: Ein pädagogisches Konzept für die schulische und außerschulische Kinder- und Jugendbildung. Studien zur technischen Bildung. Band 1. Opladen.

Wiesenfarth, G. (1992): Zum technischen Handeln als Grundbegriff einer Technikdidaktik. In: Zeitschrift für Technik im Unterricht, 66, H.4, 31-44.

Wittich, C. (2017): Mathematische Förderung durch kooperativ-strukturiertes Lernen. Eine Interventionsstudie zur Ablösung vom zählenden Rechnen an Grund- und Förderschulen. Wiesbaden.

Wyss, B. (2016): Gestalterisch-konstruktives Problemlösen von Sechs- und Achtjährigen. Theoretische Grundlagen und empirische Studie zur Technischen Gestaltung in Kindergarten und Unterstufe. München. Online unter: https://opus.bibliothek.uniaugsburg.de/opus4/frontdoor/deliver/index/ docId/37902/file/Wyss_Dissertation.pdf (letzter Zugriff: 01.04.2020).

Ziemen, K. (2016): Unterricht, Lernen, Entwicklung - Herausforderungen mit Blick auf Inklusion. In: Böing, U. \& Köpfer, A. (Hrsg.): Be-Hinderung der Teilhabe. Soziale, politische, und institutionelle Herausforderungen inklusiver Bildungsräume. Bad Heilbrunn, 158-166. 


\section{Autor*innenverzeichnis}

Albers, Timm, Prof. Dr., Institut für Erziehungswissenschaften, Universität Paderborn, Deutschland. Arbeits- und Forschungsschwerpunkte: Mehrsprachigkeit, (früh)kindliche Bildung. timm.albers@upb.de

Algermissen, Pia, Universität Leipzig, Deutschland. Arbeits- und Forschungsschwerpunkte: Partizipative Lehre und Forschung, Inklusive Hochschule. piaalgermissen@gmail.com

Amrhein, Bettina, Prof. Dr., Universität Bielefeld, Deutschland. Arbeits- und Forschungsschwerpunkte: inklusive Schul- und Unterrichtsentwicklung mit Blick auf den Förderschwerpunkt emotionale und soziale Entwicklung, Internationalisierung der Lehrer*innenbildung und Forschung im Deutschen Auslandsschulwesen.bettina.amrhein@uni-bielefeld.de

Badstieber, Benjamin, Universität Bielefeld, Deutschland. Arbeits- und Forschungsschwerpunkte: Transformationsforschung in Schule unter Berücksichtigung von Heterogenität und Differenz, professionelle Rollen in inklusionsorientierter Schul- und Unterrichtsentwicklung, De- und Rekonstruktion im Förderschwerpunkt emotionale und soziale Entwicklung. benjamin.badstieber@ uni-bielefeld.de

Bärmig, Sven, Dr., Institut für Rehabilitationspädagogik, Arbeitsbereich Schulische Erziehungshilfe, Martin-Luther-Universität Halle/Wittenberg, Deutschland. Arbeits- und Forschungsschwerpunkte: Theorie der Sonderpädagogik, kritische Theorie und Pädagogik. sven.baermig@paedagogik.uni-halle.de

Barth, Ulrike, Prof. Dr., Institut für Waldorfpädagogik, Inklusion und Interkulturalität, Alanus Hochschule, Mannheim, Deutschland. Arbeits- und Forschungsschwerpunkte: Inklusive Didaktik und Methodik für den Unterricht, Diversitätskompetenzen, Diagnostische Fragestellungen.ulrike.barth@alanus.edu

Baucke, Katja, MA, Institut für Sonderpädagogik, Abteilung Berufsorientierung in inklusiven Kontexten, Leibniz Universität Hannover, Deutschland. Arbeitsund Forschungsschwerpunkte: International vergleichende Pädagogik, Inklusion im außerschulischen Bereich und Berufsorientierung. katja.baucke@ifs.uni-hannover.de 
Bešić, Edvina, HS-Prof., PhD, Institut für Sekundarstufe Allgemeinbildung, Forschungszentrum für Inklusive Bildung (FZIB), Pädagogische Hochschule Steiermark, Österreich. Arbeits- und Forschungsschwerpunkte: Inklusions- und Diversitätsforschung, Intersektionalitätsforschung an der Schnittstelle Migration und Behinderung.edvina.besic@phst.at

Boger, Mai-Anh, Dr. phil., Fakultät für Erziehungswissenschaft, Universität Bielefeld, Deutschland. Arbeits- und Forschungsschwerpunkte: Psychoanalytische Pädagogik, Philosophien der Differenz und Alterität, Inklusionspädagogik. mai-anh.boger@uni-bielefeld.de

Bömelburg, Lena, Dr., Fachgebiet Partizipation bei Beeinträchtigungen des Lernens, Fakultät Rehabilitationswissenschaften, Technische Universität Dortmund, Deutschland. Arbeits- und Forschungsschwerpunkte: Inklusive Berufsorientierung, Transitionsforschung, Jugendliche und junge Erwachsene mit Lern- und Entwicklungsstörungen in Schule und Arbeitswelt.

lena.boemelburg@tu-dortmund.de

Buchhaupt, Felix, Institut für Sonderpädagogik, Fachbereich Erziehungswissenschaften, Goethe-Universität Frankfurt, Deutschland. Arbeits- und Forschungsschwerpunkte: Professionalisierungsforschung, rekonstruktive Unterrichtsforschung, Schulentwicklungsforschung. f.buchhaupt@em.uni-frankfurt.de

Danner, Oliver, $M A$, ehemals Lehrstuhl für Grundschulpädagogik, Projekt P-ink, Universität Regensburg, aktuell Bayerisches Landesjugendamt, Deutschland. Arbeits- und Forschungsschwerpunkte: Multiprofessionelle Kooperation im Bildungssektor, Verhältnis Schul- und Sozialpädagogik. oliver.danner@zbfs.bayern.de

Deiß, Helena, BA MA, EDUCULT - Institute of Cultural Policy and Cultural Management, Institut für Bildungswissenschaft, Universität Wien, Österreich. Arbeits- und Forschungsschwerpunkte: soziale Ungleichheit und kritische Migrationsforschung, rekonstruktiv-qualitative Sozialforschung, Inklusive Pädagogik. helena.deiss@univie.ac.at

Eibl, Susanne, Mag. BEd, Volksschule Erdbergstraße; Pädagogische Hochschule Wien; Pädagogische Hochschule Oberösterreich, Österreich. Arbeits- und Forschungsschwerpunkte: Bereiche der Unterrichts- und Schulentwicklung mit Fokus auf kooperatives Handeln und Problemlösen, fachdidaktische und rekonstruktive Bildungsforschung. susanne.eibl@ph-ooe.at 
Faix, Ann-Christin, MEd, Fakultät für Psychologie und Sportwissenschaft, Arbeitseinheit 9 Pädagogische Psychologie, Universität Bielefeld, Deutschland. Arbeits- und Forschungsschwerpunkte: Professionalisierung für inklusiven Unterricht, Subjektive Theorien, Hochschuldidaktik. a.faix@uni-bielefeld.de

Feuser, Georg, Prof. em. Dr., Institut für Erziehungswissenschaft, Universität Zürich, Schweiz. Arbeits- und Forschungsschwerpunkte: Inklusion, Allgemeine Pädagogik und entwicklungslogische Didaktik, Behindertenpädagogik, Didaktik, Basis-Therapie SDKHT. gfeuser@swissonline.ch

Filipiak, Agnes, $M E d$, Fakultät für Erziehungswissenschaften, Pädagogik bei Behinderung und Benachteiligung (PBB), Universität Hamburg. agnes.filipiak@ uni-hamburg.de

Finkbeiner, Timo, Dipl. Päd. Mag. BEd, Kirchliche Pädagogische Hochschule Wien/ Krems, Österreich. Arbeits- und Forschungsschwerpunkte: Bereiche der fachdidaktischen und rekonstruktiven Bildungsforschung.timo.finkbeiner@kphvie.ac.at

Franzen, Katja, MEd, Institut für Erziehungswissenschaften, Universität Paderborn, Deutschland. Arbeits- und Forschungsschwerpunkte: Projekt ,Kompetenzentwicklung im inklusiven Unterricht' (KinU), Selbstwirksamkeitsüberzeugung von Grundschullehrkräften im Kontext inklusiver Erziehung und Bildung. katja. franzen@upb.de

Gerland, Juliane, Prof. Dr., Fachbereich Sozialwesen, Lehrgebiet Musik in kindheitspädagogischen und sozialen Handlungsfeldern, Fachhochschule Bielefeld, Deutschland. Arbeits- und Forschungsschwerpunkte: Musikpädagogik und Inklusion, Musikalische Bildung in der Kindheit, Qualitative Methoden im Kontext Musikalischer Bildung. juliane.gerland@fh-bielefeld.de

Gloystein, Dietlind, Institut für Rehabilitationswissenschaften, Professional School of Education, Humboldt-Universität zu Berlin, Deutschland. Arbeits- und Forschungsschwerpunkte: an Inklusion orientierte Veränderungsprozesse in den Bereichen Diagnostik, Schul- und Unterrichtsentwicklung, Lehrkräftebildung. dietlind. gloystein@hu-berlin.de

Goldbach, Anne, Dr., Institut für Förderpädagogik, Universität Leipzig, Deutschland. Arbeits- und Forschungsschwerpunkte: Leichte und Einfache Sprache in der beruflichen Teilhabe von Menschen mit Behinderungserfahrungen, Inklusionsorientierte Hochschulentwicklung, Partizipative Lehre und Forschung. goldbach@ uni-leipzig.de 
Gollub, Patrick, Dr., Institut für Erziehungswissenschaft, Westfälische WilhelmsUniversität Münster, Deutschland. Arbeits- und Forschungsschwerpunkte: Lehrer*innenprofessionsforschung, Schul- und Unterrichtsforschung, Praxisphasen in der Lehrer*innenbildung. p.gollub@uni-muenster.de

Greiten, Silvia, Prof. Dr., Institut für Erziehungswissenschaft, Pädagogische Hochschule Heidelberg, Deutschland. Arbeits- und Forschungsschwerpunkte: Schulund Unterrichtsentwicklung im Kontext von Heterogenität, Lehrer*innenprofessionalisierung, qualitative Forschungsmethoden. greiten@ph-heidelberg.de

Grubich, Rainer, HS-Prof. Mag. Dr., Büro für Inklusive Bildung, Institut für übergreifende Bildungsschwerpunkte. Pädagogische Hochschule Wien, Österreich. Arbeits- und Forschungsschwerpunkte: Inklusive Pädagogik, inklusive Schulentwicklung, Umgang mit Heterogenität, integrative Beschulung von Schüler*innen mit Autismus-Spektrum-Störung.

Grubich-Müller, Regina, Bildungsdirektion für Wien, Österreich. Arbeits- und Forschungsschwerpunkte: inklusive Schulentwicklung, altersheterogene Lerngruppen.

Hamisch, Katharina, MA, freie Bildungswissenschaftlerin, Deutschland. Arbeitsund Forschungsschwerpunkte: Innovationen und Transformationen im Bildungssystem, inklusionsorientierte und zukunftsorientierte Schulentwicklung, Bedeutung von Digitalisierung und Globalisierung für Schüler*innen, Lehrer*innen und Schule.kontakt@katharinahamisch.de

Hauser, Mandy, Dr., Universität Leipzig, Deutschland. Arbeits- und Forschungsschwerpunkte: Pädagogik im Kontext zugeschriebener geistiger Behinderung, Partizipative und Inklusive Forschung, Inklusive Hochschule. mandy.hauser@ uni-leipzig.de

Hellmich, Frank, Prof. Dr., Institut für Erziehungswissenschaften, Universität Paderborn, Deutschland. Arbeits- und Forschungsschwerpunkte: inklusiver (Anfangs-)Unterricht, Grundschulpädagogik und empirische Bildungsforschung. frank.hellmich@uni-paderborn.de

Hoffmann, Thomas, Univ.-Prof. Dr., Institut für LehrerInnenbildung und Schulforschung, Universität Innsbruck, Österreich. Arbeits- und Forschungsschwerpunkte: Inklusive Pädagogik, Bildungstheorie und entwicklungsorientierte Didaktik, Disability History und Geschichte der Behindertenpädagogik. thomas. hoffmann@uibk.ac.at 
Holzinger, Andrea, Dr., Institut für Elementar- und Primarpädagogik, Forschungszentrum für Inklusive Bildung (FZIB), Pädagogische Hochschule Steiermark, Österreich. Arbeits- und Forschungsschwerpunkte: Inklusive Bildung, Professionsforschung. Andrea.holzinger@phst.at

Hülsken, Julia, Fachbereich Sozialwesen, Lehrgebiet Musik in kindheitspädagogischen und sozialen Handlungsfeldern, Fachhochschule Bielefeld, Deutschland. Arbeits- und Forschungsschwerpunkte: Inklusive kulturelle Bildung, inklusive Kulturproduktionen. julia.hülsken@fh-bielefeld.de

Jochmaring, Jan, MA, Fachgebiet Rehabilitationssoziologie, Fakultät Rehabilitationswissenschaften, Technische Universität Dortmund, Deutschland. Arbeits- und Forschungsschwerpunkte: Übergangs- und Ungleichheitsforschung, Soziologie der Behinderung. jan.jochmaring@tu-dortmund.de

Jurkowski, Susanne, Prof. Dr., Erziehungswissenschaftliche Fakultät, Universität Erfurt, Deutschland. Arbeits- und Forschungsschwerpunkte: Diagnostik und Förderung sozial-emotionaler Fähigkeiten, Kooperatives Arbeiten und Lernen, Multiprofessionelle Unterrichtsteams. susanne.jurkowski@uni-erfurt.de

Kaiser, Michaela, Dr., Humanwissenschaftliche Fakultät, Department Ästhetische Bildung, Universität Potsdam, Deutschland. Arbeits- und Forschungsschwerpunkte: Kunstpädagogik und -didaktik, Inklusion, Begabung und Leistung. michaela.kaiser@uni-potsdam.de

Kappeller, Felix, $M A M E d$, Institut für Sonderpädagogik, Abteilung Allgemeine Behindertenpädagogik und -soziologie, Leibniz Universität Hannover, Deutschland. Arbeits- und Forschungsschwerpunkte: Visual Culture Studies, kulturwissenschaftliche Disability Studies und Geschlechterforschung, Verkörperung und (Assistenz-) Technik aus subjektivierungs- und praxistheoretischer Perspektive. felix.kappeller@ifs.uni-hannover.de

Katzenbach, Dieter, Prof. Dr., Institut für Sonderpädagogik, Fachbereich Erziehungswissenschaften, Arbeitsbereich „Inklusive Pädagogik und Didaktik bei kognitiven Beeinträchtigungen“, Goethe-Universität Frankfurt, Deutschland. Arbeits- und Forschungsschwerpunkte: Inklusion in Schule und Gemeinwesen, Psychoanalytische Pädagogik im Kontext geistiger Behinderungen. d.katzenbach@em.uni-frankfurt.de

Kernbichler, Gerda, Dr., Institut für Sekundarstufe Allgemeinbildung, Pädagogische Hochschule Steiermark, Österreich. Arbeits- und Forschungsschwerpunkte: Fachdidaktik, Professionsforschung. gerda.kernbichler@aon.at 
Kopp-Sixt, Silvia, BEd MA, Institut für Elementar- und Primarpädagogik, Forschungszentrum für Inklusive Bildung (FZIB), Pädagogische Hochschule Steiermark, Österreich. Arbeits- und Forschungsschwerpunkte: Professionsforschung, Inklusive Pädagogik und Mentoring. silvia.kopp-sixt@phst.at

Kottmann, Brigitte, Dr., Fakultät für Erziehungswissenschaft, Universität Bielefeld, Deutschland. Arbeits- und Forschungsschwerpunkte: Lernwerkstätten und Schüler*innenhilfeprojekte in der Lehrer*innenbildung, der Studiengang der Integrierten Sonderpädagogik, Sonderpädagogischer Förderbedarf und soziale Benachteiligung. brigitte.kottmann@uni-bielefeld.de

Krammer, Mathias, $P h D$, Institut für Bildungswissenschaften, Forschungszentrum für Inklusive Bildung (FZIB), Pädagogische Hochschule Steiermark, Österreich. Arbeits- und Forschungsschwerpunkte: Inklusive Bildung, quantitative Methodik. Mathias.krammer@phst.at

Kremsner, Gertraud, Dr., Institut für Förderpädagogik, Universität Leipzig, Deutschland und Zentrum für Lehrer*innenbildung, Universität Wien, Österreich. Arbeits- und Forschungsschwerpunkte: Dis/Ability Studies (in Education), Inklusive Forschung und Lehre, gesellschaftstheoretische Grundlagen. gertraud. kremsner@univie.ac.at

Kruschel, Robert, Institut für Förderpädagogik, Universität Leipzig, Deutschland. Arbeits- und Forschungsschwerpunkte: Governance und Steuerung von Inklusion im Bildungssystem, Inklusionsorientierte Schulentwicklung und Inklusive Bildungsprozesse im Sozialraum. robert.kruschel@uni-leipzig.de

van Ledden, Hannah, Universität Leipzig, Deutschland. Arbeits- und Forschungsschwerpunkte: Partizipative Lehre und Forschung, Inklusive Hochschule. Hannah.vledden@googlemail.com

Leonhardt, Nico, Institut für Förderpädagogik, Universität Leipzig, Deutschland. Arbeits- und Forschungsschwerpunkte: Inklusive Schulentwicklung und Sozialraumorientierung, Inklusionssensible Hochschulentwicklung, Partizipative Forschung und Lehre.nico.leonhardt@uni-leipzig.de

Link, Pierre-Carl, Prof., Professor für Erziehung und Bildung bei Beeinträchtigungen der sozio-emotionalen Entwicklung, Institut für Verhalten, sozio-emotionale und psychomotorische Entwicklung, Interkantonale Hochschule für Heilpädagogik, Zürich, Schweiz. Arbeits- und Forschungsschwerpunkte: Allgemeine Heilpädagogik, Pädagogik bei Verhaltensstörungen, Gestaltpädagogik und psychoanalytische Pädagogik. pierre-carl.link@hfh.ch 
Lutz, Deborah, PhD, Institut für Sonderpädagogik, Fachbereich Erziehungswissenschaften, Goethe-Universität Frankfurt, Deutschland. Arbeits- und Forschungsschwerpunkte: Inklusive Bildungsprozesse, „geistige“ Behinderung, persönliche Assistenz und Teilhabe.d.lutz@em.uni-frankfurt.de

Maitz, Katharina, $P h D$, Forschungszentrum für Inklusive Bildung (FZIB), Universität Graz, Österreich. Arbeits- und Forschungsschwerpunkte: Inklusive Bildung, Technology Enhanced Learning. Katharina.maitz@uni-graz.at

Möhlen, Lisa-Katharina, BA MA, Zentrum für Lehrer*innenbildung, Universität Wien, Österreich; Institut für Erziehungswissenschaften, Abteilung Schulpädagogik und allgemeine Didaktik, Technische Universität Braunschweig, Deutschland. Arbeits- und Forschungsschwerpunkte: inklusive Schulentwicklung, Digitalisierung im Schulsystem, partizipative Forschungsmethoden. lisa-katharina. moehlen@univie.ac.at

Niediek, Imke, Prof. Dr., Institut für Sonderpädagogik, Abteilung Pädagogik bei Beeinträchtigungen der geistigen Entwicklung, Leibniz Universität Hannover, Deutschland. Arbeits- und Forschungsschwerpunkte: Qualitative Forschungsmethoden im Kontext komplexer Behinderung, Unterstützte Kommunikation über die Lebensspanne, Interaktion und Partizipation in mixed abled Gruppen. imke. niediek@ifs.uni-hannover.de

Paudel, Florentine, Dr., Institut für Hochschulmanagement, Büro für Inklusive Bildung, Pädagogische Hochschule Wien, Österreich. Arbeits- und Forschungsschwerpunkte: Inklusion und Professionalisierung, digitale Inklusion, Schriftsprache. florentine.paudel@phwien.ac.at

Paulus, David, Dr., Arbeitsgruppe ,Historische Schul- und Curriculumforschung', Westfälische Wilhelms-Universität Münster, Deutschland. Arbeits- und Forschungsschwerpunkte: Wissenschaft-Praxis-Verhältnis und Praxisphasen, Fachlichkeit von Unterricht, Inklusionsorientierte Lehrer*innenbildung. d.paulus@ uni-muenster.de

Pickl, Gonda, Dr., Institut für Elementar- und Primarpädagogik, Pädagogische Hochschule Steiermark, Österreich. Arbeits- und Forschungsschwerpunkte: Inklusive Pädagogik, Professionsforschung. gonda.pickl@phst.at

te Poel, Kathrin, Dr., Universität Bielefeld, Deutschland. Arbeits- und Forschungsschwerpunkte: Anerkennung, Habitusforschung und -reflexion, Lehrer*innenprofessionalität. kathrin.te_poel@uni-bielefeld.de 
Pongratz, Katharina Maria, MA, Institut I: Bildung, Beruf und Medien, Ottovon-Guericke Universität Magdeburg, Deutschland. Arbeits- und Forschungsschwerpunkte: Erwachsenenbildung-/wissenschaftliche Weiterbildung, funktionelle (Hirn)systeme, qualitative Bildungsforschung. katharina.pongratz@ovgu.de

Proyer, Michelle, Ass. Prof. Dr., Zentrum für Lehrer*innenbildung/Institut für Bildungswissenschaft, Universität Wien, Österreich. Arbeits- und Forschungsschwerpunkte: Inklusive Pädagogik, Nexus Behinderung und Kultur, international (vergleichende) Perspektiven, forced migration studies. michelle.proyer@ univie.ac.at

Quante, Alina, MEd, Lehrstuhl für Pädagogik (Grundschulpädagogik), Universität Regensburg, Deutschland. Arbeits- und Forschungsschwerpunkte: Kooperation, Inklusion und Professionalisierung. alina.quante@ur.de

Reisenauer, Cathrin, MMag. Dr., Institut für LehrerInnenbildung und Schulforschung, Universität Innsbruck, Österreich. Arbeits- und Forschungsschwerpunkte: Inklusion in der Schule, pädagogisches Handeln, Lehrer*innenprofessionalisierung. cathrin.reisenauer@uibk.ac.at

Richter, Hendrik, Institut für LehrerInnenbildung und Schulforschung, Universität Innsbruck, Österreich. Arbeits- und Forschungsschwerpunkte: Inklusion und Exklusion, soziale Ungleichheit, Armut und Klassenzugehörigkeit, Unterdrückung und widerständige Praktiken. hendrik.richter@uibk.ac.at

Schimek, Bernhard, HS-Prof. Dr., Institut für allgemeine bildungswissenschaftliche Grundlagen und reflektierte Praxis. Pädagogische Hochschule Wien. Arbeitsund Forschungsschwerpunkte: Diversität und Bildungsgerechtigkeit.bernhard. schimek@phwien.ac.at

Schkade, Teresa, Institut für Bewegungs- und Neurowissenschaft, Deutsche Sporthochschule Köln, Deutschland. Arbeits- und Forschungsschwerpunkte: Inklusion und Heterogenität im Schulsport, Einstellung und Selbstwirksamkeit von angehenden Sportlehrkräften zu inklusivem Schulsport. t.schkade@dshs-koeln.de

Schroeder, René, Dr., Department für Heilpädagogik und Rehabilitation, Humanwissenschaftliche Fakultät der Universität zu Köln, Deutschland. Arbeits- und Forschungsschwerpunkte: inklusive (Sach-)Unterrichtsdidaktik, inklusionsorientierte Lehrer*innenprofessionalisierung, didaktische Entwicklungsforschung zu Konzepten formativen Assessments im inklusiven Unterricht. rene.schroeder@ uni-koeln.de 
Schwörer, Laura, Institut für Inklusive Bildung Kiel, Deutschland. Arbeits- und Forschungsschwerpunkte: Seminare und Vorlesungen zum Thema Inklusion für Studierende, Fach- und Führungskräfte, kreatives Engagement bei künstlerischen Vorhaben (privat).schwoerer@inklusive-bildung.org

Sieger, Marvin, Institut für Sonderpädagogik, Abteilung Pädagogik bei Beeinträchtigungen der geistigen Entwicklung, Leibniz Universität Hannover, Deutschland. Arbeits- und Forschungsschwerpunkte: Mensch-Ding-Relationen und Artefakte, Digitale Musiziergeräte, Qualitative Sozialforschung und Praxisforschung, Dis/ ability Studies. marvin.sieger@ifs.uni-hannover.de

Sponholz, Dirk, FöL, Institut für Sonderpädagogik, Fachbereich 5: Erziehungswissenschaften, Universität Koblenz-Landau, Campus Landau, Deutschland. Arbeits- und Forschungsschwerpunkte: Übergangs- und Ungleichheitsforschung, Inklusive Berufsorientierung.sponholz@uni-landau.de

Steinert, Cedric, MA, Erfurt School of Education, Universität Erfurt, Deutschland. Arbeits- und Forschungsschwerpunkte: Interdisziplinäre Kooperation in der Hochschullehre, Teamteaching in der Hochschullehre: Entwicklung und Implementation eines hochschuldidaktischen Konzepts zur gemeinsamen Vermittlung von inklusionsbezogenen Inhalten in (Fach-)Didaktik und Bildungswissenschaften. cedric.steinert@uni-erfurt.de

Subasi Singh, Seyda, Dr., Institut für Bildungswissenschaft, Universität Wien, Österreich. Arbeits- und Forschungsschwerpunkte: Inklusion, Intersektionalität, Bildungsungleichheit.seyda.subasi@univie.ac.at

Urban, Michael, Prof. Dr., Institut für Sonderpädagogik, Fachbereich Erziehungswissenschaften, Arbeitsbereich „Bildung und Erziehung im Kontext sozialer Marginalisierung", Goethe-Universität Frankfurt, Deutschland. Arbeits- und Forschungsschwerpunkte: schulische Inklusion, soziale Herstellung und Bearbeitung von „Verhaltensstörungen“, „Lernschwierigkeiten“ und verwandten Konstrukten im Schulsystem. m.urban@em.uni-frankfurt.de

Veber, Marcel, Jun.-Prof. Dr., Institut für Erziehungswissenschaft, Universität Osnabrück, Deutschland. Arbeits- und Forschungsschwerpunkte: inklusionsorientierte Lehrer*innenbildung, schulpraktische Studien und Professionalisierung. marcel.veber@uos.de

York, Jana, Dr., Fachgebiet Rehabilitationssoziologie, Fakultät Rehabilitationswissenschaften, Technische Universität Dortmund, Deutschland. Arbeits- und Forschungsschwerpunkte: Arbeitsweltbezogene Gesundheitspolitik, Gesundheitliche Ungleichheit. jana.york@tu-dortmund.de 

Der thematische Sammelband beruht auf der Konzeption der ,IF02020' in Wien - der 34. Jahrestagung der Inklusionsforscher*innen - und stellt den Versuch dar, die rund um diese Tagung begonnenen, weiterentwickelten oder vielleicht sogar in diesem Kontext überhaupt erst entstandenen Fragestellungen und Diskussionen gebündelt darzustellen. Ganz im Sinne der Themenstellung des Bandes gerät notwendigerweise die Inklusionsforschung selbst ins Zentrum der Auseinandersetzungen, wenn die ihr inhärenten Kontroversen, Perspektiven und Entwicklungen diskutiert werden. Daraus ergeben sich u.a. Hinweise auf empirische, theoretische und paradigmatische Ausrichtungen von Inklusion, interdisziplinäre Zusammenschlüsse, Kritik an der pädagogischen wie auch der (eigenen) akademischen Praxis, intersektionale Verwobenheiten oder auch Leerstellen, die zu diskutieren offen bleiben.

Die Herausgeber*innen

Geeint durch das Interesse an der Auseinandersetzung mit der Verortung der Inklusionsforschung hat sich für die IFO-Tagungsorganisation 2020 in Wien ein überinstitutionelles Team gebildet: Michelle Proyer und Gertraud Kremsner repräsentieren die Universität Wien; Bernhard Schimek, Florentine Paudel und Rainer Grubich stehen für die Pädagogische Hochschule Wien und Regina Grubich-Müller vertritt die Bildungsdirektion für Wien.

978-3-7815-2485-9

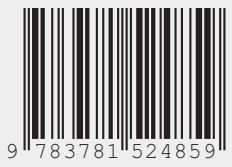

